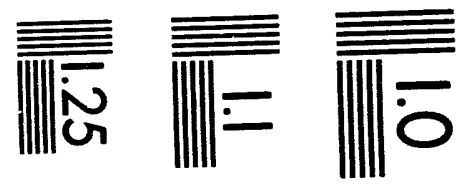

$$
\begin{aligned}
& \text { 竞兠 }
\end{aligned}
$$

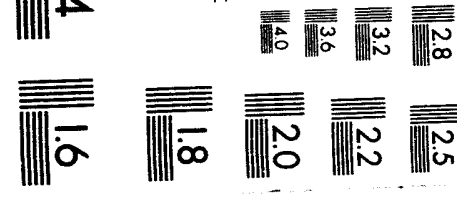



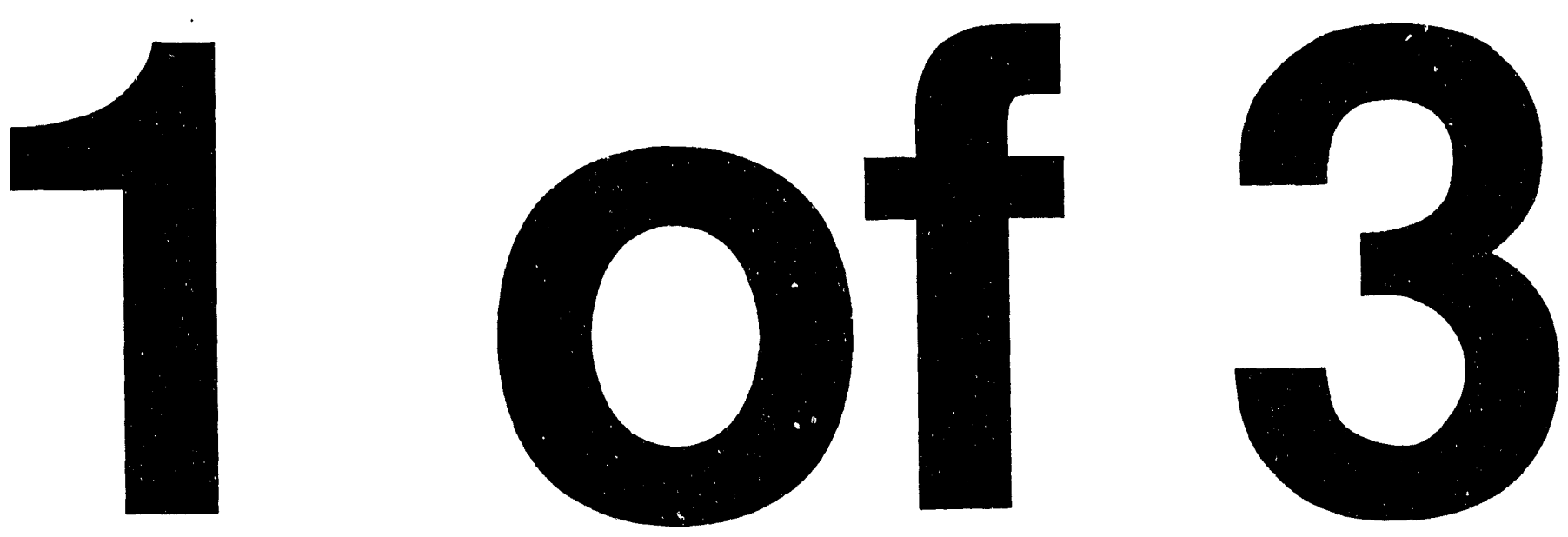
Models to Evaluate Magnicon

Architectures and Designs Suitable for High-Perveance Beams

Daniel E. Rees 


\section{Contents}

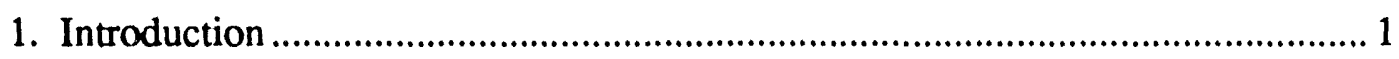

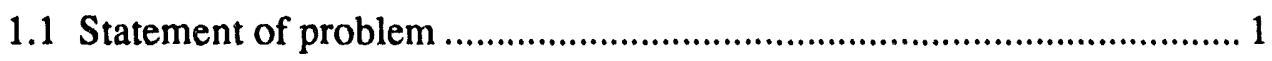

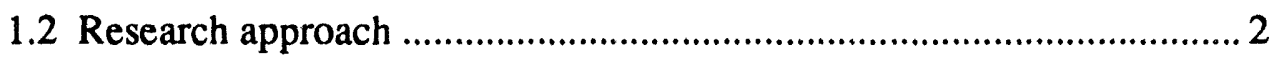

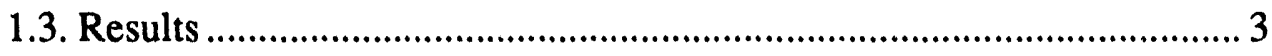

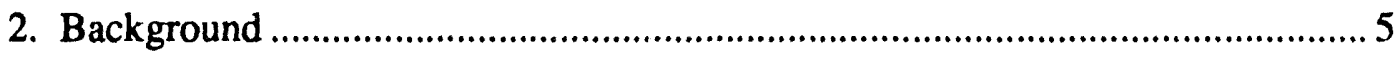

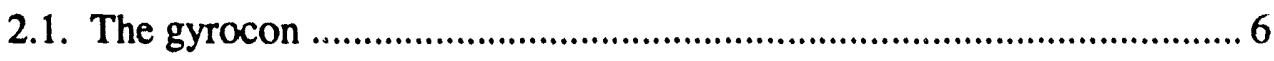

2.2. The magnicon ........................................................................... 9

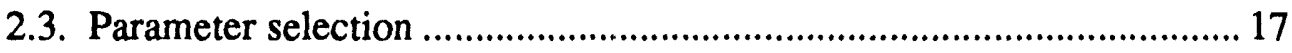

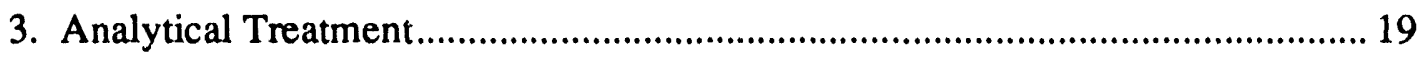

3.1. Magnicon rotating cavity fields ......................................................... 19

3.1.1. Generalized field expressions ............................................. 19

3.1.2. Small-signal field equations ................................................. 25

3.1.3. Cavity loss ......................................................................... 26

3.1.4. Stored energy and quality factor ............................................ 28

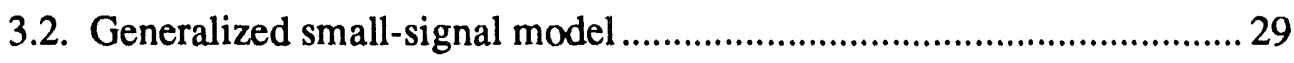

3.2.1. Drive cavity small-signal model and trajectories.................... 33

3.2.1.1. Optimum drive cavity length ................................... 33

3.2.1.2. Beam loading …….................................................... 34

3.2.2. Drift-space small-signal model and trajectories..................... 39

3.2.3. Passive-cavity small-signal model and trajectories ................ 41

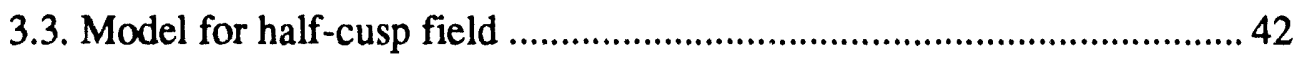

4. Large-Signal Numerical Model ....................................................................... 47

4.1. Generation of self-consistent solutions for beam-driven cavities ......... 47

4.1.1. Energy-balance approach ...................................................... 48

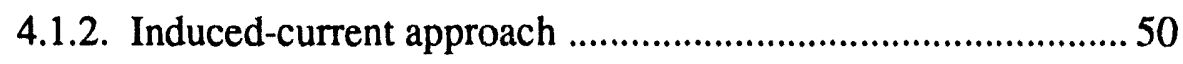

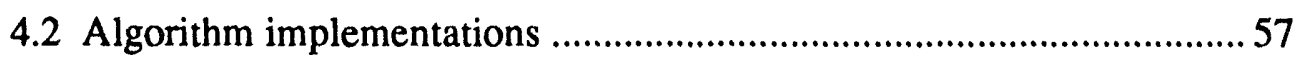

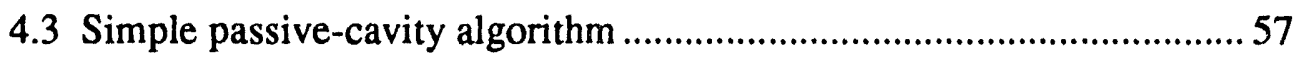

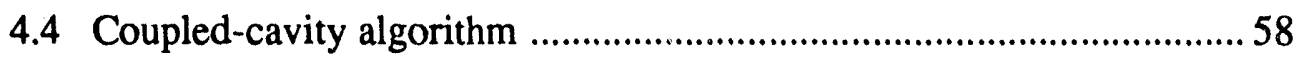

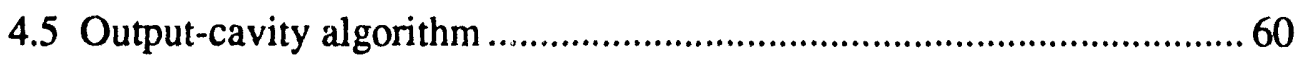

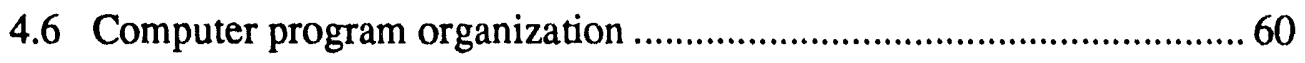

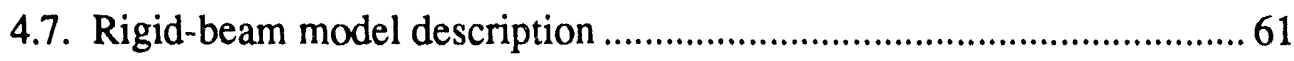


5. Applications of Rigid-Beam Model for Magnicon Structures. 62

5.1. Rigid-beam model validation 62

5.2. Optimum drive-cavity length 77

5.3. Investigation of parameter variation on simple passive cavity performance 85

5.3.1. Effect of variation of cavity length and focusing-field strength 85

5.3.2. Beam entrance position 103

5.4. Investigation of parameter variation on coupled-cavity performance 154

5.5. Comparison of coupled cavity and simple passive cavity 161

5.6. Investigation of parameter variation on output-cavity performance 163

6. Application of Rigid-Beam Model for Magnicon Design 188

6.1 Design rules 188

6.2 Comparison to Russian high-perveance magnicon design 189

6.3 Comparison with experimental results 200

6.4. New design for a standard magnicon 203

6.4.1 Geometry with POISSON half-cusp fields. 214

6.5. The uniform-field magnicon: an inıproved geometry 223

7. Finite-Beam Effects on Magnicon Efficiency 235

7.1 Modeling finite-beam effects 236

7.2. Efficiency degradation in standard magnicon 241

7.3. Efficiency degradation in uniform-field magnicon ................................ 248

7.4. Results of modeling finite-beam effects .............................................. 256

8. Conclusions 259

Appendix A 262

A.1 Particle-pushing algorithm 262

A.2 Bessel function algorithms 263

A.3 Lorentz force algorithms 265

A.4 Coupled-cavity equivalent-circuit model ........................................... 266 References 269 


\title{
MODELS TO EVALUATE MAGNICON ARCHITECTURES AND DESIGNS SUITABLE FOR HIGH-PERVEANCE BEAMS
}

by

\author{
Daniel E. Rees
}

\begin{abstract}
The magnicon, a new high-power, radio frequency ( $r f)$ deflection-modulated amplifier, was recently developed at the Institute for Nuclear Physics in Novosibirsk, Russia. The first magnicon achieved a peak output power of $2.6 \mathrm{MW}$ for $50-\mu$ s pulses at a frequency of $915 \mathrm{MHz}$ with a dc-to-rf conversion efficiency of $73 \%$. The conversion efficiency achieved by the original magnicon represents a significant improvement over state-of-the-art conventional velocity- and density-modulated devices. Therefore, if properly exploited, the magnicon could substantially reduce the operating expenses of industrial, scientific, and military facilities that require large amounts of $r f$ power. This dissertation describes the operational principles of the magnicon, provides small-signal analytical theory (where practical), presents a large-signal numerical model to characterize magnicon performance, and then utilizes this model to investigate the characteristics of the component magnicon structures. Using these modeling tools, the firstgeneration magnicon architecture is analyzed for its performance sensitivity to electron-beam size and is found to support beams of only limited diameter. Finally, an alternate magnicon geometry, called a "uniform-field" magnicon, is presented and shown to support beams of larger diameter.
\end{abstract}




\section{Introduction}

\subsection{Statement of problem}

Recently, the Institute for Nuclear Physics in Novosibirsk, Russia, developed a relatively new class of amplifiers called deflection-modulated amplifiers. These electron devices have to date shown a remarkable improvement in performance characteristics over velocity- and density-modulated amplifiers. Researchers in Russia have demonstrated efficiencies for these devices as high as $90 \%$ at frequencies up to $7 \mathrm{GHz}$ and at power levels exceeding $50 \mathrm{MW}$. The initial deflection-modulated amplifier developed at Novosibirsk was known as the "gyrocon." Due to its limiting geometry, the gyrocon could produce large amounts of power very efficiently, but only at a single power level. Essentially the gyrocon had no linear range. In an effort to overcome the limitations of the gyrocon, engineers at Novosibirsk developed a new class of linear, deflection-modulated amplifier known as the "magnicon." The original magnicon developed in Russia achieved 2.6 MW of output power at $915 \mathrm{MHz}$ with an electronic efficiency of $85 \%$ and an overall efficiency of $73 \%$ for short pulses $(50 \mu \mathrm{s})$. It is described in Ref. [1].

The performance characteristics of the magnicon show a distinct improvement over state-of-the-art velocity- or density-modulated high-power rf sources. Commercially available klystrons, for instance, can produce $1.1 \mathrm{MW}$ at $350 \mathrm{MHz}$ with a dc-to-rf conversio efficiency of only $67 \%$. (Their efficiency at $700 \mathrm{MHz}$ would likely be less.) Also, recent developments in klystrode amplifiers have improved their power output to 250-kW continuous-wave (CW) at $267 \mathrm{MHz}$ with a dc-to-rf conversion efficiency of $70 \%$. (It is not clear at this time whether grid technology would allow the klystrode's output to be extended to the higher frequency regime or allow for increased levels of $\mathrm{CW}$ power.) Despite the promise of deflection-modulated amplifiers, their development to date has concentrated on short-pulse amplifiers with low-perveance beams. The beam voltage and current for the first magnicon, for instance, was $300 \mathrm{kV}$ and $12 \mathrm{~A}$ for a perveance of $7.3 \times 10^{-8}$ pervs.

The question raised and answered by this research is: can the magnicon still provide the high efficiency demonstrated in its initial development when it is operated at perveances three to four times higher? For if it can successfully operate at higher perveances, the magnicon can become a very attractive alternative to conventional tube 
technology for proposed particle accelerators that require hundreds of megawatts of $\mathrm{CW}$ rf power at frequencies around $700 \mathrm{MHz}$. For these systems, even a $5 \%$ increase in efficiency could save over $\$ 50$ million annually in operating costs, according to current projections (Ref. [2]).

To answer the basic question, I have developed and applied large-signal numerical models to the magnicon in order to analyze the effects of parameter and geometry variations on performance. Then, once the attributes of a high-efficiency design are identified, this design will be evaluated for performance sensitivity to beam size. The magnicon geometry will then be modified to decrease performance sensitivity to beam diameter, and a new magnicon geometry and design will be proposed that has an improved tolerance to beam diameter.

\subsection{Research approach}

The research is organize $\mathrm{i}$ into four parts.

The first part (Chapter 2) consists of a summary of the history of the development of the magnicon. Information for this summary was gathered from the appropriate scientific literature, which is listed in the references. Then, using this information, I analyze the potential capabilities and shortcomings of the first magnicon, which was developed and demonstrated at the Institute for Nuclear Physics. Because the magnicon was initially developed to solve some problems encountered in the development of the first deflectionmodulated amplifier, the gyrocon, I also examine the gyrocon's shortcomings as part of the historical review. Current work underway on the next generation of magnicons in the United States and Russia is also described.

The second part of the investigation (Chapter 3) follows the development of a smallsignal theory for the magnicon and evaluates the usefulness of the theory, which proves to be suitable only for an examination of the magnicon's drive cavity. This is because small-signal theory cannot completely describe particle trajectories in the drift spaces and beam-driven cavities that follow the drive cavity. This shortcoming is due to the complexities involved in generating a small-signal theory for the arbitrary beam-entrance time and arbitrary beam-entrance position in passive cavities and drift spaces and in generating self-consistent solutions within beam-driven passive cavities. Large-signal numerical methods must be employed to solve these problems. Nevertheless, I do provide small-signal theory for drift spaces and passive cavities, but it is based on restrictive boundary conditions with respect to beam entrance time and position. 
Although I draw some conclusions from this theory, I present it here mainly as a reference to benchmark the large-signal numerical model.

Part three (Chapters 4, 5, 6, and 7) focuses on the generation of a large-signal, rigidbeam model and on the large-signal, finite-beam-size model. The rigid-beam model assumes that a beam has an infinitesimally small diameter. Due to the unique nature of the rotating $\mathrm{rf}$ fields within the magnicon, the rigid-beam model allows the behavior of the entire beam to be simulated by tracking a single particle through the cavities. I also use it to explore what effects varying the magnicon's geometry and operating parameters have on performance. Furthermore, I use it to generate high-efficiency magnicon designs and self-consistent solutions for cavity field amplitude and phase. Once this has been accomplished, the high-efficiency design can then be evaluated by a numerical model that uses the rigid-beam model's self-consistent solutions in order to calculate the performance of a beam with a finite diameter. I use this model to analyze the impact of beam size on magnicon performance, first for the Russian magnicon then for a new geometry that is much more tolerant of beams with larger diameters. Space-charge forces of the beam are discussed and evaluated, as well as the force applied to the beam by the cavity walls when the beam is deflected off-axis.

The fourth part of the dissertation (Chapter 8) consists of a summary of the work and the conclusions.

\subsection{Results}

The primary result of this research is to lay the groundwork for a new magnicon, one that preserves the high efficiency of the original magnicon but decreases the sensitivity of its performance to beam diameter. However, the analysis supporting this research also provided several intermediate results.

In modeling the rotating-fields in the magnicon cavities, the research demonstrates the effectiveness of certain modified modeling techniques in calculating self-consistent solutions for standing-wave, beam-driven cavities. Using a rigid-beam modeling technique, I show it is possible to calculate self-consistent solutions for the rotating magnicon fields. Specifically, I use the rigid-beam modeling technique to evaluate the effect of entrance position, entrance angle, and cavity length on the beam-driven cavity gain in the large-signal regime. Also, I compare the performance of simple cylindrical beam-driven cavities to coupled beam-driven cavities in order to determine which passive-cavity geometry can increase the conversion of longitudinal beam energy into the 
transverse direction with the lowest rf losses in the cavity walls. Optimum coupledcavity lengths and simple-cavity lengths are then determined in order to lessen rf cavity loss while maintaining the highest gain possible. The rigid-beam model also allowed me to examine output cavity geometry, focusing field, and decelerating voltage in order to determine the optimum geometry and operating parameters for converting the beam energy to the rf fields in the output cavity. These data represent a body of information that can be used to determine the optimum operating parameters and geometry for magnicon design.

Another tool that proved useful was the induced-current method for determining beam loading and cavity detuning. This tool for cavity analysis, which was originally developed for klystron modeling, was here modified and adapted for modeling the magnicon. I show that the induced-current technique may be used to calculate selfconsistent solutions for the rotating modes by decomposing the modes into their component standing-wave modes. Then, using an iterative approach, I arrive at the selfconsistent solution for the standing-wave fields. I also use the induced-current method to calculate, for different values in the focusing field, the beam-loaded quality factor $(Q)$ of the cavity and the frequency detuning caused by the beam in the beam-driven cavities. (The relationship between the strength of the focusing field, the cavity $Q$, and detuning frequency is discussed in Ref. [3].) 


\section{Background}

Most high-power microwave amplifiers can be classified as electron devices that are either velocity-modulated (klystron, traveling-wave tube [TWT], gyrotron) or densitymodulated (gridded-tube). In any of these devices, a continuous beam of electrons is transformed into a series of electron bunches that are separated in time by the length of the rf period. If these bunches were monoenergetic and infinitely dense, then each microwave amplifier would be able to achieve $100 \%$ efficiency. Unfortunately, neither velocity nor density modulation produces monoenergetic, infinitely dense bunches, and this factor limits the efficiency achievable by the generator. For example, in the case of the klystron, bunching is accomplished by modulating a continuous electron beam in a number of consecutive cavities using a time-varying electric field. The velocity distribution of the bunch limits the strength of the electric field that can be developed across the output cavity. If the electric field were high enough to decelerate the most energetic electrons of the bunch, then the less energetic electrons would be turned around and would manifest themselves as body current, which could potentially damage the klystron or cause instability. (This phenomenon has been verified at CERN through experiments on high-efficiency klystrons and documented in Ref. [1].)

Another limiting factor for velocity- and density-modulated high-power microwave generators is that the interaction region, where energy is extracted from the electrons, is limited to half an rf period (a transit angle of $180^{\circ}$ ), which limits the peakpower capability of high-frequency klystrons because of voltage breakdown in the output cavity. The limited size of the output cavity in higher-frequency klystrons can also limit the average-power capability because of thermal effects. The impetus for the development of deflection-modulated microwave amplifiers was to overcome these limitations.

The primary advantage of deflection-modulated devices is that they require no electron bunching. The interaction between the rf fields in the output cavity and the electron beam is nearly monoenergetic. Thus, much higher electronic efficiencies are theoretically possible.

The gyrocon, the first major deflection-modulated amplifier, was invented by G. I. Budker in Russia (Ref. [2]). Electronic efficiencies up to $90 \%$ have been demonstrated for it at $430 \mathrm{MHz}$. The gyrocon, how $\cdot$ ver, suffers from some of the limitations of 
conventional electron devices-mainly, the limited length of the interaction region. This limitation, along with others discussed in the next section, led to the development of an improved deflection-modulated amplifier, the magnicon.

The magnicon utilizes the input structure of the gyrocon, but its output structure has been modified to address some of the limitations of the gyrocon. The first magnicon, which was developed and tested by Dr. Oleg Nezhevenko in 1985 at the Institute for Nuclear Physics, demonstrated an efficiency of $73 \%$ at a frequency of $915 \mathrm{MHz}$ and at a power level of $2.6 \mathrm{MW}$, as shown in Ref. [3]. A second magnicon, designed to operate at $7 \mathrm{GHz}$ for linear-collider applications, is in the process of being tested at Novosibirsk. (As of this writing, no test results have been made available.) Another magnicon designed for high peak power is under development at the U.S. Naval Research Laboratory (NRL) for linear-collider applications (Refs. [4, 5]).

\subsection{The gyrocon}

The gyrocon, which is shown in Fig. 2.1, represented the first generation of deflection-modulated amplifiers. It consists of an electron source, a deflection cavity, a drift space, and an output cavity. Electrons leaving the source enter the deflection (or input) cavity, which is a circular cavity resonant in the $\mathrm{TM}_{110}$ mode. The rf energy is coupled into the cavity through two apertures separated in angle by $90^{\circ}$ and in excitation phase by $\pi / 2$. This configuration sets up a rotating $\mathrm{TM}_{110}$ mode at the if frequency (Fig. 2.2). (Throughout this report, the magnicon cavity mode is referred to as a rotating $\mathrm{TM}_{110}$ mode. This is not strictly true. Figure 2.2 illustrates that the rotating mode does not have the typical $\mathrm{TM}_{110}$ mode pattern. The correct, but cumbersome, way to refer to the mode present in the magnicon cavities would be to call it a rotating mode made up of two $\mathrm{TM}_{110}$ modes.)

Because the mode is rotating, interaction with the radially directed rf magnetic field converts a portion of the electrons' longitudinal velocity into transverse velocity. Therefore, when the electron beam enters the drift space, it contains a transverse velocity component that rotates in time with a frequency equal to the rf frequency. The result, as the beam enters the drift space, is an outward-spiraling beam traveling along a conical surface. 


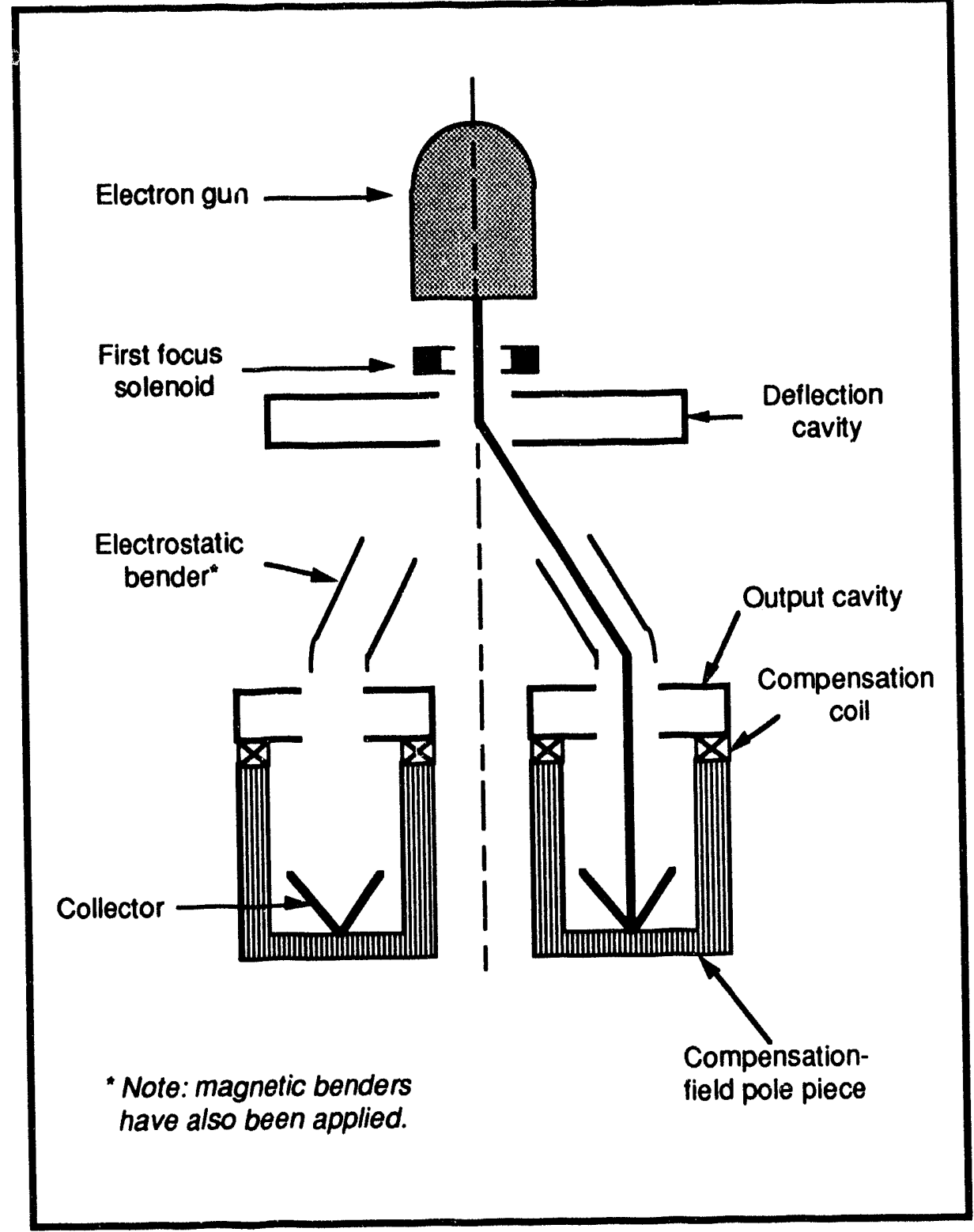

Figure 2.1. Schematic diagram of the gyrocon. 


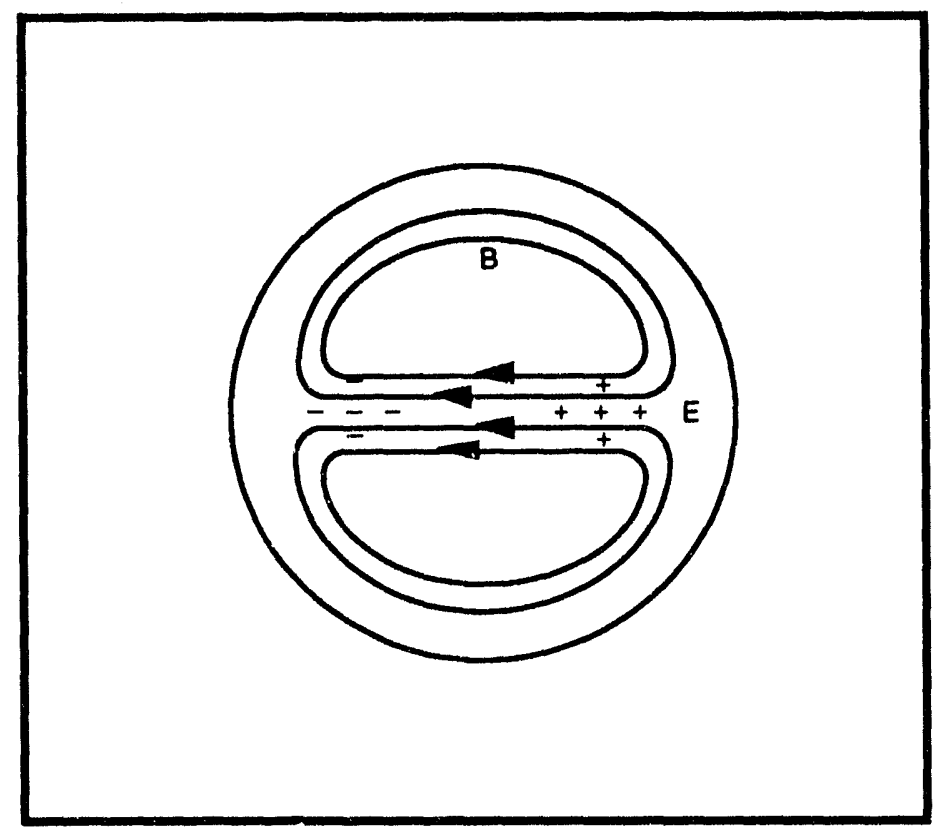

Figure 2.2. Input-cavity field of a gyrocon.

The system described above is the most rudimentary of possible deflection systems and was used only in early gyrocon designs. Later gyrocons (and the magnicon) employ multiple cavities that are classified as input cavities, passive cavities, and deflection cavities. All utilize rotating $\mathrm{TM}_{110}$ modes immersed in a static, axially directed magnetic field. All but the input cavity are excited by the electron beam. With this type of deflection system, which will be explained in detail in later sections, the gyrocon can achieve a gain of $60 \mathrm{~dB}$.

Once in the drift space, the deflected electrons travel along straight lines, tracing out a conical surface and passing through an azimuthal slot into the circular output cavity, which is resonant in the $\mathrm{TM}_{110}$ mode. The angle at which the electrons enter the output cavity rotates in time with a frequency equal to the if frequency. Energy is removed from the electron beam by interaction with the electric field of a rotating $\mathrm{TM}_{110}$ mode. The rf energy is then extracted through two windows with an angular separation of $90^{\circ}$. The phase of the rf output from the first window lags behind that from the second by $\pi / 2$. Table 2-1 summarizes gyrocon results obtained in Russia (Ref. [6]).

In the gyrocon, as in most conventional velocity-modulated or density-modulated electron devices, all the energy must be extracted from the electron beam within half a wavelength in the longitudinal direction. Therefore, problems connected with overheating and voltage breakdown become severe as power and frequency increase. 
Furthermore, the gyrocon is a nonlinear device; it has no range of linear amplification (Ref. [7]). If the rf drive is decreased, then the deflection angle is changed and the electron beam misses the entrance slot. (This factor limits its aplicability as an rf amplifier for accelerators.) Finally, because the entrance slot and the collector slot are coaxial, rf leakage can compromise beam quality in the drift space and complicate the design of an isolated collector. Efforts to overcome these difficulties led to the development of the magnicon.

Table 2-1. Gyrocon results from Russia.

\begin{tabular}{lcccc}
\hline & $\begin{array}{c}\text { Initial } \\
\text { gyrocon }\end{array}$ & $\begin{array}{c}\text { CW } \\
\text { (VEPP-4) }\end{array}$ & $\begin{array}{c}\text { Pulsed } \\
\text { (VEPP-4) }\end{array}$ & $\begin{array}{c}\text { Pulsed } \\
\text { (Balakin) }\end{array}$ \\
\hline Frequency (MHz) & 430 & 182 & 430 & 7000 \\
Power (MW) & 0.6 & 0.4 & 65 & 60 \\
Pulse width ( $\mu$ s) & 20 & CW & 0.7 & 50 \\
Rep. rate (Hz) & 0.1 & - & 1 & $\vdots$ \\
Beam voltage (kV) & 320 & 240 & 1600 & 1 \\
Efficiency (\%) & 65 & 60 & 75 & 1300 \\
Gain (dB) & 7 & 17 & 26 & 25 \\
Reference & {$[6]$} & {$[7]$} & {$[7]$} & {$[8]$} \\
\hline
\end{tabular}

\subsection{The magnicon}

The magnicon and the gyrocon have identical input structures; however, the output cavity of the magnicon is quite different and has none of the other's limitations. The output cavity of the magnicon couples the beam energy to the rf fields through an extended interaction with the same rotating $\mathrm{TM}_{110}$ field as in the gyrocon output cavity. Therefore, the magnicon is not constrairied to extract all beam energy within a half cycle. This feature addresses the gyrocon limitations of output-cavity overheating and voltage breakdown as the operating power and frequency increase. The magnicon output cavity is cylindrical rather than coaxial, and the beam entrance port in the output cavity is circular. The circilar nature of the entrance prevents propagation in the coaxial mode into the drift space, a feature that troubled the gyrocon. This improvement results in a linear range of amplification because it is no longer critical that the beam precisely passes through the output cavity entrance slot, as was the case with the gyrocon. 
Table 2.2 summarizes past and ongoing magnicon development efforts. Only one magnicon has been successfully developed and tested, the original one at the Institute for Nuclear Physics (Novosibirsk). A second magnicon is currently being developed there, and although it has not yet been tested, its design parameters are known. In addition, work on two other magnicons is progressing elsewhere (Ref. [9]). Both of these operate at high-frequencies where the magnicon is operated in the frequency-doubling mode (i.e., the extraction frequency is twice the drive-cavity excitation frequency.)

Table 2.2. Russian magnicon results and NRL design summary.

\begin{tabular}{lccc}
\hline & $\begin{array}{c}\text { Novosibirsk } \\
\text { First magnicon } \\
\text { measured results } \\
\text { (Ref. [10]) }\end{array}$ & $\begin{array}{c}\text { Novosibirsk } \\
\text { Second magnicon } \\
\text { design parameters design parameter } \\
\text { (Ref. [11])* }\end{array}$ & $\begin{array}{c}\text { NRL } \\
\text { magnicon }\end{array}$ \\
\hline Drive frequency (MHz) & 915 & 3500 & 5700 \\
Operating frequency (MHz) & 915 & $7000^{* *}$ & $11400^{* *}$ \\
Pulse width ( $\mu$ s) & 50 & 2 & 0.5 \\
Repetition rate (Hz) & 1 & 5 & single-shot \\
Gain (dB) & 30 & 50 & 50 \\
Efficiency (\%) & 73 & $60-70$ & 50 \\
Beam voltage (kV) & 300 & 420 & 500 \\
Beam current (A) & 12 & 240 & 200 \\
Power (MW) & 2.6 & $60-70$ & 50 \\
\hline \hline
\end{tabular}

* Magnicon has not yet entered test phase.

** Magnicon used in frequency-doubling mode of operation.

A mechanical drawing of a magnicon is shown in Fig. 2.3. This geometry, although not identical to the initial magnicon, does have similar features. It represents a design for a higher-gain magnicon. The greater gain comes from the fact that more and different types of beam-driven cavities in the input structure have been added. These additional cavities will be examined now because they reflect an enhanced magnicon structure currently being considered by research efforts both in this country and Russia. 


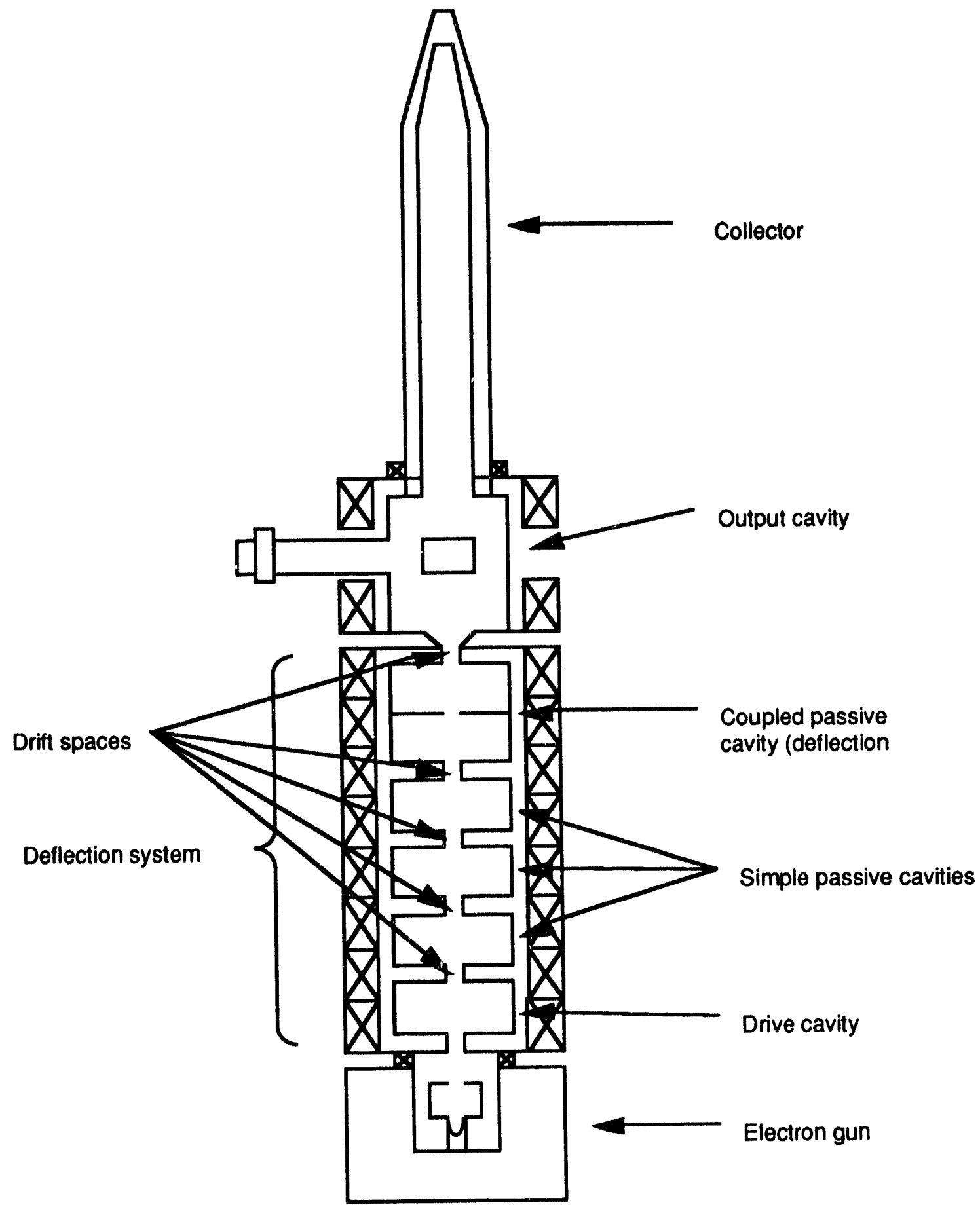

Figure 2.3. Drawing of possible magnicon geometry.

The magnicon in Fig. 2.3 consists of an electron gun; a deflection system comprising simple passive cavities, a coupled passive cavity, and drift spaces separating the individual cavities; a larger drift space separating the deflection system from output cavity; an output cavity; and a collector. The deflection system and output cavity each 
has its own separate solenoid magnet. Therefore, a sharp magnetic transition can exist in the drift-space region when different strength magnetic fields are being used. The deflection system consists of a drive cavity and a number of passive cavities that are driven by the beam. In the figure, two types of passive cavities can be seen: simple passive cavities and a coupled passive cavity. The coupled passive cavity, which operates in the pi mode, provides the majority of beam deflection before the beam departs the deflection system. It is sometimes referred to as the deflection cavity. The pi-mode coupled cavity is often used in such situations because it keeps the if wall losses associated with providing the beam deflection lower than if the equivalent deflection were accomplished using a simple cylindrical cavity.

The deflection system of the magnicon transfers the electron beam's initial longitudinal energy into the transverse direction. The output cavity extracts the transverse energy of the beam. Additional conversion of longitudinal-to-transverse anergy takes place in the drift space between the deflection system and output cavity. Upon exiting the deflection system, the beam is allowed to drift approximately one Larmor diameter off-axis. It then enters the output cavity through a sharp magnetic transition, ideally a half-cusp. The sharp magnetic transition further transfers the beam's longitudinal energy into the transverse direction. To a first approximation, the efficiency of the magnicon is determined by the ability of its input structure and half-cusp magnetic field to convert the electron beam's longitudinal energy into transverse energy.

The magnicon cavities are all cylindrical and resonant in the $\mathrm{TM}_{110}$ mode. These cavities support a rotating mode that is composed of two $\mathrm{TM}_{110}$ mode fields. The standing-wave mode and rotating-wave modes are illustrated in Fig. 2.4 and 2.5. 


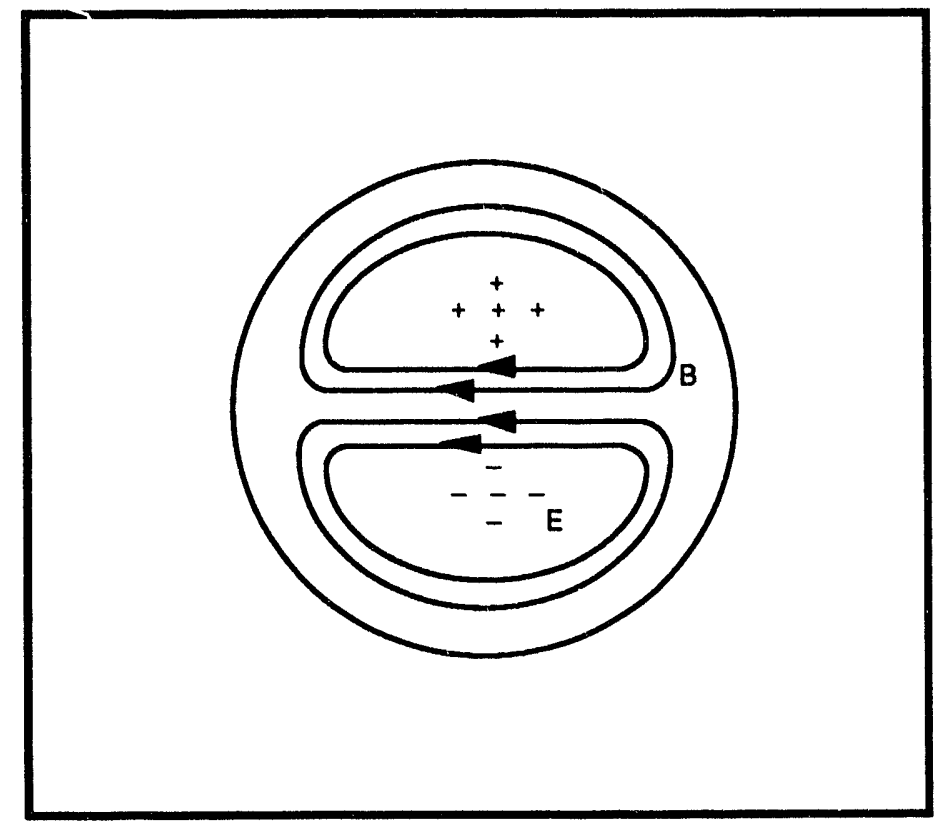

Figure 2.4. $\mathrm{TM}_{110}$ mode cavity fields.

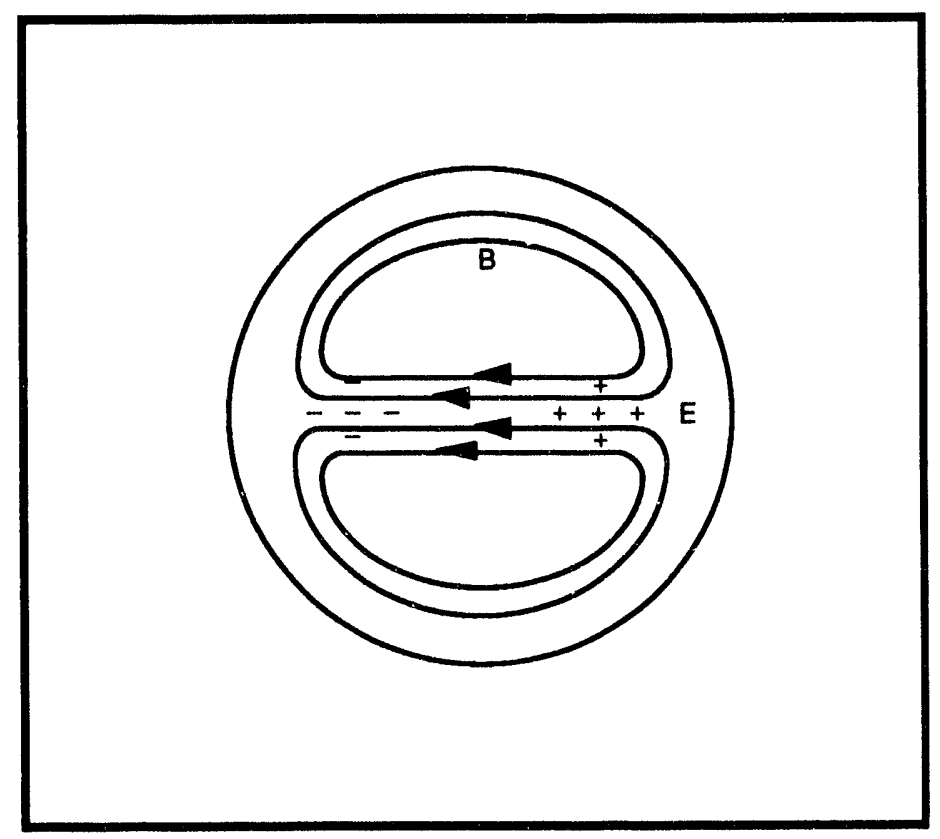

Figure 2.5. Rotating mode field pattern.

The rotating mode is excited in the drive cavity through two apertures separated in angle by $90^{\circ}$ and in excitation phase by $\pi / 2$. The rotating mode in the output cavity is coupled through two waveguide openings with similar spatial and phase characteristics. It is the interaction of the longitudinal velocity of the beam with the radial rf magnetic field near the axis that transforms the beam's longitudinal energy into transverse energy. 
The deflection-system cavities are immersed in a magnetic focusing field directed along the axis of the magnicon. The strength of the deflection-system focusing field is selected such that the ratio of the resulting cyclotron frequency, $\Omega$, to the if frequency, $\omega$, satisfies the expression

$$
0 \leq \frac{\Omega}{\omega} \leq 2
$$

Selection of this ratio determines the position of the rotating beam with respect to the rotating rf fields. This is illustrated in Figs. 2.6 and 2.7, provided in Ref. [12]. Figure 2.6 shows the trajectory of a single particle in a driven deflection cavity for different values of the magnetic field. It is shown from the viewpoint of an observer looking down the beam pipe. The particle starts at the center and moves outward. The paths followed by the electrons are indicated for various ratios of the cyclotron frequency to the $\mathrm{if}$ frequency. Figure 2.7 shows the azimuthal position of the beam relative to the zero electric-field line of the mode. In Fig. 2.7 the abscissa is the particle transit angle and the ordinate is the angle between the beam position and line of zero electric field. This figure illustrates the fact that the amount of energy the drive-cavity fields impart to the beam is a function of focusing-field strength. For example, for $\Omega / \omega=2$, the beam is always in the region of zero electric field, and the beam does not gain energy. This is equivalent to saying the cold-cavity quality factor, $Q$, is equal to the beam-loaded $Q$. For $\Omega / \omega<2$ the beam is exposed to accelerating fields and gains energy resulting in a beam-loaded $Q$ that is less than the cold-cavity $Q$. Figure 2.6 also shows that when $\Omega / \omega>2$, the beam is exposed to decelerating fields and gives up energy to the drive cavity. This could cause instabilities because the energy given up by the beam could excite an additional selfconsistent rotating mode in the drive cavity that would, in turn, modify the beam trajectory. 


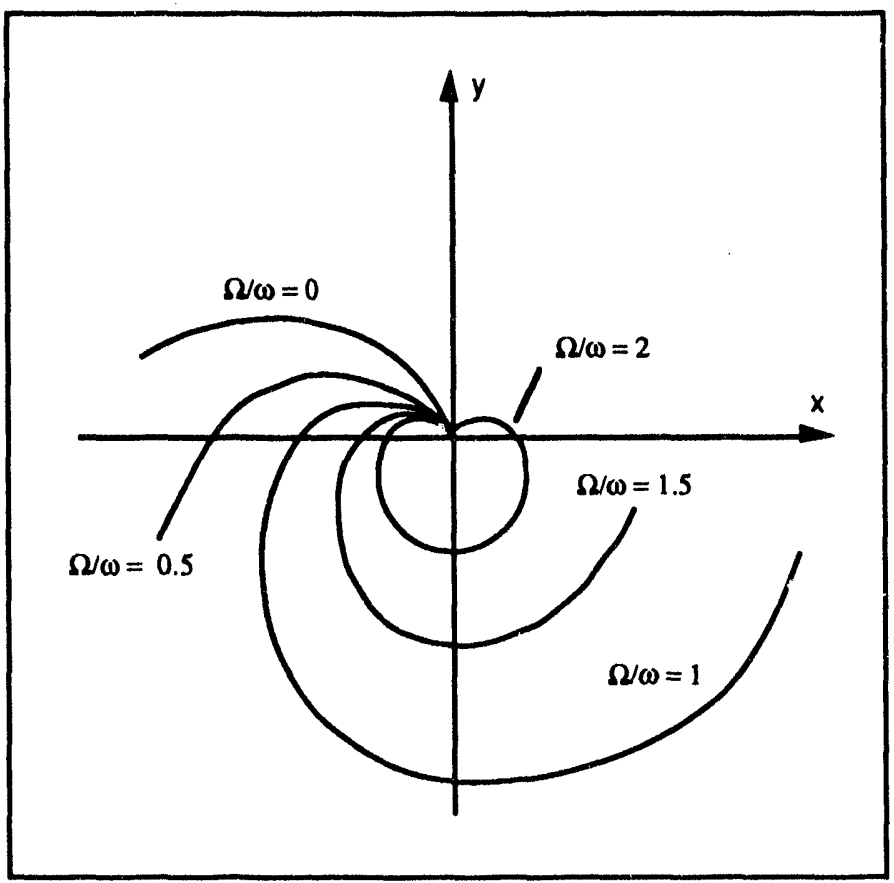

Figure 2.6. Particle trajectories in a deflection cavity for various values of magnetic field.

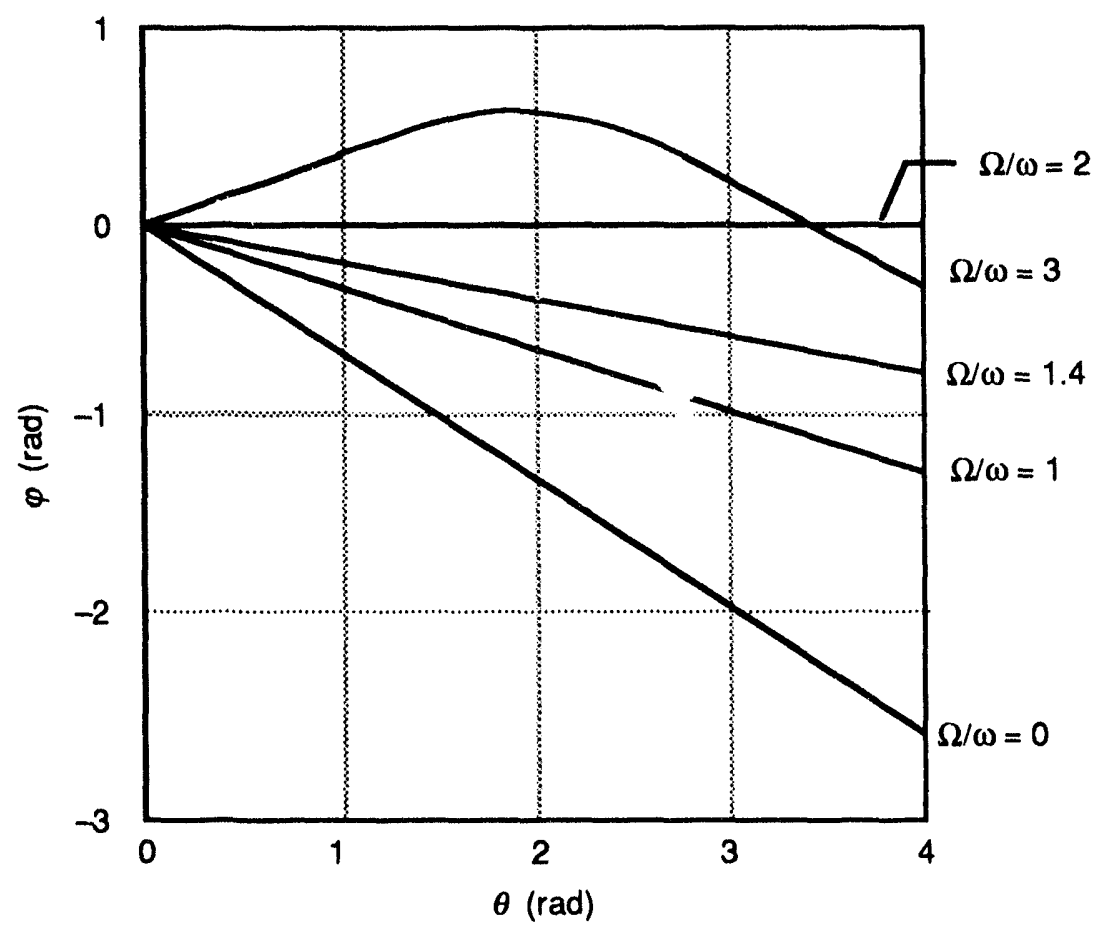

Figure 2.7. Azimuth position of electrons relative to zero-electric-field region.

The output-cavity focusing field is selected such that the rf frequency and cyclotron frequency are equal. Figure 2.8 illustrates the output-cavity field and one 
revolution of the cyclotron orbit of an individual electron. (The figure is used more for general understanding of the physics of the conversion of the beam energy to the rf fields than as an accurate depiction of particle trajectories in the output cavity.) The conversion of the rotating-beam energy to the rf fields is accomplished by an extended interaction with the steady-state if fields. With each cyclotron revolution, a fraction of the transverse energy of the beam is transferred to longitudinal energy, and interaction with the longitudinal rf electric field converts this energy to the rf field. When the electron orbit is near the axis of the output cavity in the region of a strong rf magnetic field and weak electric field, the $\left(V_{\text {radial }} \times B_{R F}\right)$ component of the Lorentz force converts a portion of the perpendicular velocity component into longitudinal velocity. Then, at the other extreme of the electron orbit, where the electron is in the region of the strong rf electric field, the interaction of the electrons with the longitudinal electric field removes this added longitudinal velocity. Through this interaction, the electrons give up their transverse energy to the rf fields over a number of wavelengths, and the spent beam contains only the longitudinal energy the electron beam possessed when it entered the output cavity. A representative trajectory of a single electron as it travels through the output cavity is shown in Fig. 2.9.

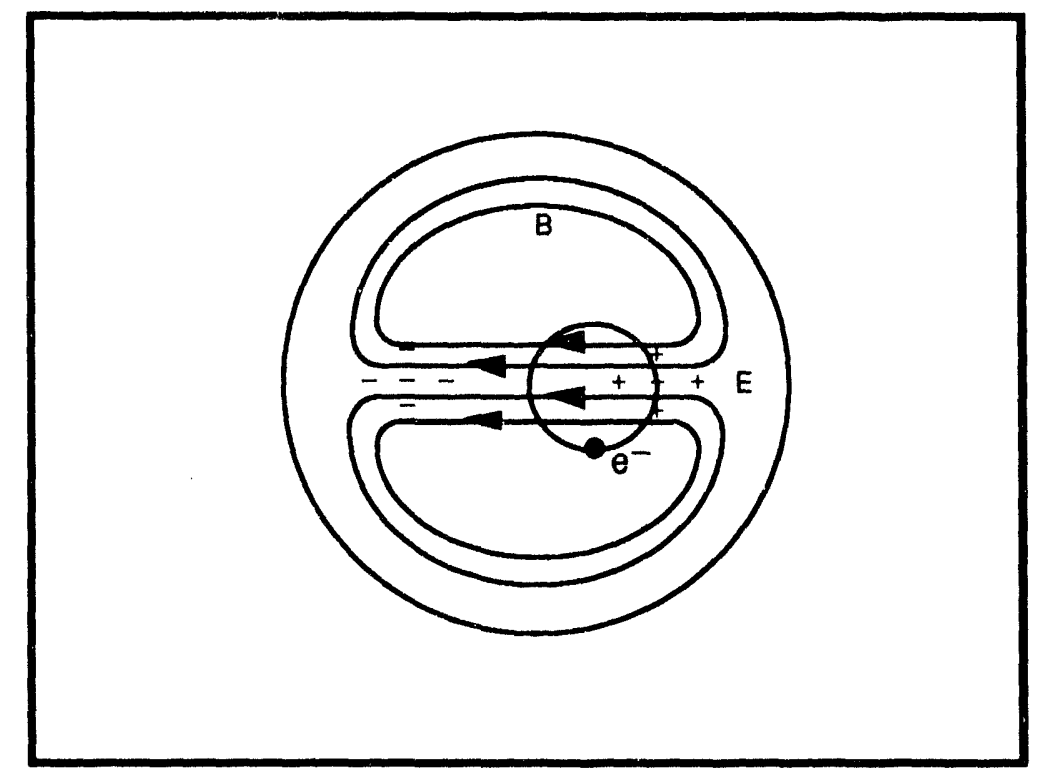

Figure 2.8. Magnicon output cavity field and cyclotron orbit of a single electron. 


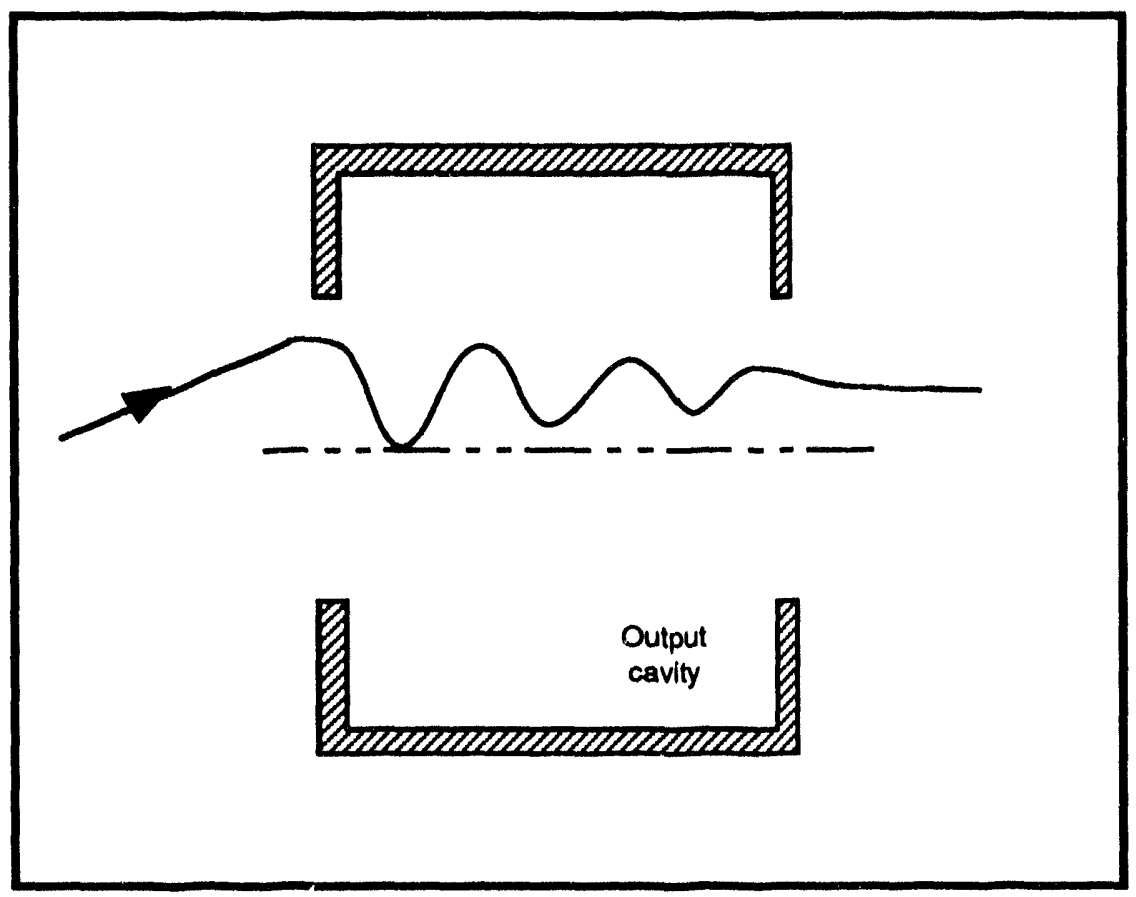

Figur 3.9. Trajectory of an electron moving through the magnicon output cavity.

It is also possible to use the magnicon as a frequency doubler by designing the output cavity to be resonant at twice the drive frequency and selecting the output-cavity focusing-field strength so that the cyclotron frequency in the output cavity is twice the drive frequency. This is the approach that NRL (Ref. [4]) and the Institute for Nuclear Physics (Ref. [11]) are using to design magnicons intended to provide rf power to the Next Linear Collider.

Because of the extended interaction between the if field and the beam, the magnicon, unlike the gyrocon, does not suffer the same power and frequency restrictions due to voltage breakdown and overheating. In addition, because of the nature of the interaction, the magnicon will function as a linear amplifier. The beam enters the output cavity through a circular aperture rather than a slot. Hence, as drive power is decreased, there is a corresponding decrease in the resulting deflection angle. The magnicon continues to serve as an $\mathrm{rf}$ amplifier, although at reduced efficiency (as a result of the reduced transverse velocity). Thus, the magnicon does have a linear range of operation.

\subsection{Parameter selection}

Whenever possible in this research, results will be presented in a normalized format so they can be translated to magnicon designs involving a variety of operating 
parameters. However, in some cases specific operating parameters must be selected. For instance, the beam current must be selected before self-consistent cavity field solutinns can be calculated. Therefore, a baseline set of parameters needs to be selected and used for the modeling. The results of the modeling, when possible, will be presented as normalized results.

The parameter selection for the magnicon is determined by its application. The application of interest to Los Alamos National Laboratory (LANL), the funding agent for this effort, is to use the magnicon as a high-efficiency, CW rf source for particle accelerator applications. LANL has ongoing development efforts for several projects that could make use of the magnicon: the Accelerator Transmutation of Waste (ATW) program and the Accelerator Production of Tritium (APT) program, among others. The baseline design for these efforts calls for $700-\mathrm{MHz}$ generators with beam power of 3 MW. The high beam power was chosen because of limitations in commercially available rf windows. Currently, commercial windows are available for power levels up to $1 \mathrm{MW}$. It is likely that the window technology could be extended to $1.2 \mathrm{MW}$. Because power is coupled out of the magnicon output cavity through two windows, this would mean the maximum possible magnicon output would be $2.4 \mathrm{MW}$. Assuming the amplifier has an $80 \%$ efficiency, the device's total beam power should be $3 \mathrm{MW}$ to achieve the $2.4 \mathrm{MW}$ output.

To achieve $3 \mathrm{MW}$ of beam power, the current and voltage beam parameters have been set at $170 \mathrm{kV}$ and $18 \mathrm{~A}$. The beam voltage was selected because it represented an incremental improvement over the $300 \mathrm{kV}$ beam voltage of the initial magnicon based on its perveance. The perveance of the LANL baseline design is $2.57 \times 10^{-7}$ pervs, compared to $0.73 \times 10^{-7}$ pervs used in the initial development. This difference represents a perveance 3.5 times as large as that in the initial development. Ideally, the magnicon voltage would be no higher than the voltage of a conventional klystron. However, for such a high value of perveance, 3-D space-charge modeling would be required. The 170 $\mathrm{kV}$ was selected as a intermediate step to determine if a magnicon geometry could be identified that would provide high efficiency at a greatly increased perveance. In addition, a $\mathrm{CW}$ gun at $170 \mathrm{kV}$ is feasible, and the baseline design could be experimentally verified if funding was available. 


\section{Analytical Treatment}

\subsection{Magnicon rotating cavity fields}

The analytical expressions for the rotating cavity fields are developed below.

\subsubsection{Generalized field expressions}

The geometry used for derivation of general field expressions is shown in Fig. 3.1 .

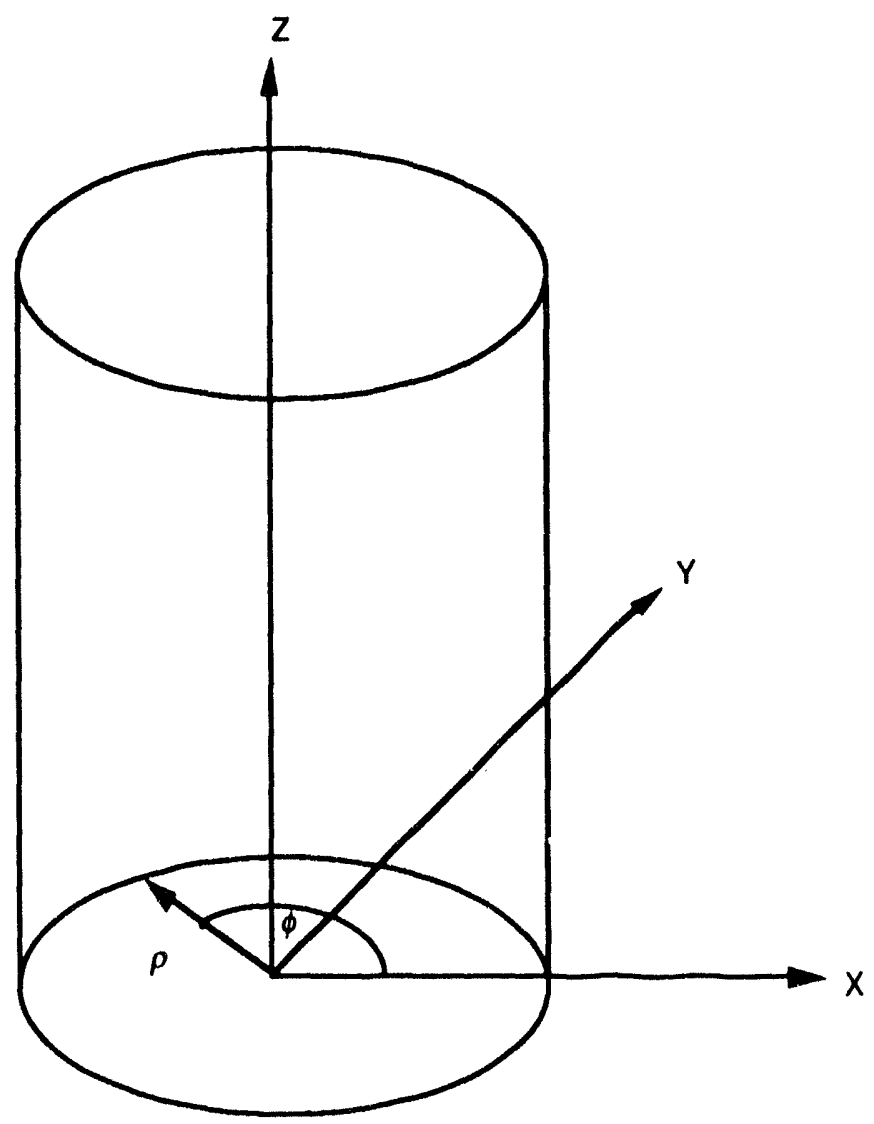

Figure 3.1. Geometry used for generalized field expressions.

The vector potential, $A$, expressed in cylindrical coordinates for the magnetic modes transverse to the $z$ axis, is gaven by Ref. [1] as

$$
\begin{aligned}
A_{z}(\rho, \phi, z)= & {\left[A_{1} J_{m}\left(\beta_{\rho} \rho\right)+B_{1} Y_{m}\left(\beta_{\rho} \rho\right)\right]\left[C_{2} \cos (m \phi)+D_{2} \sin (m \phi)\right] } \\
& {\left[C_{3} \cos \left(\beta_{z} Z\right)+D_{3} \sin \left(\beta_{z} Z\right)\right] . }
\end{aligned}
$$


The constant $B_{1}$ has a value of zero because $Y_{m}$ is unbounded at the origin, and $D_{2}$ can be set to zero by an arbitrary selection of the origin. The fields are given by

$$
\begin{aligned}
& E_{\rho}=-j\left(\frac{1}{\omega \mu \varepsilon}\right)\left(\frac{\partial^{2} A_{z}}{\partial \rho \partial z}\right), \\
& E_{\phi}=-j\left(\frac{1}{\omega \mu \varepsilon \rho}\right)\left(\frac{\partial^{2} A_{z}}{\partial \phi \partial z}\right), \\
& E_{z}=-j\left(\frac{1}{\omega \mu \varepsilon}\right)\left(\frac{\partial^{2}}{\partial^{2} z^{2}}+\beta_{\rho}^{2}+\beta_{z}^{2}\right) A_{z}, \\
& H_{\rho}=\left(\frac{1}{\mu \rho}\right)\left(\frac{\partial A_{z}}{\partial \phi}\right), \\
& H_{\phi}=-\left(\frac{1}{\mu}\right)\left(\frac{\partial A_{z}}{\partial \rho}\right),
\end{aligned}
$$

and

$$
H_{z}=0
$$

$A_{z}$ is then given by

$$
A_{z}=A_{m n p} J_{m}\left(\beta_{\rho} \rho\right) \cos (m \phi)\left[C_{3} \cos \left(\beta_{z} z\right)+D_{3} \sin \left(\beta_{z} z\right)\right] \text {. }
$$

Substituting Eq. (3.8) into Eqs. (3.2), (3.3), and (3.4) results in component values for $E$ given by

$$
\begin{aligned}
& E_{\rho}=-j \frac{\beta_{z} \beta_{\rho}}{\omega \mu \varepsilon} A_{m n p} J_{m}^{\prime}\left(\beta_{\rho} \rho\right) \cos (m \phi)\left[-C_{3} \sin \left(\beta_{z} z\right)+D_{3} \cos \left(\beta_{z} z\right)\right], \\
& E_{\phi}=j \frac{\beta_{z} m}{\omega \mu \varepsilon \rho} A_{m u p} J_{m}\left(\beta_{\rho} \rho\right) \sin (m \phi)\left[-C_{3} \sin \left(\beta_{z} z\right)+D_{3} \cos \left(\beta_{z} z\right)\right],
\end{aligned}
$$

and

$$
E_{z}=-j \frac{\beta_{\rho}^{2}}{\omega \mu \varepsilon} A_{m n p} J_{m}\left(\beta_{\rho} \rho\right) \sin (m \phi)\left[C_{3} \cos \left(\beta_{z} z\right)+D_{3} \sin \left(\beta_{z} z\right)\right]
$$

At the cavity radius, $a, E_{z}$ equals zero. Therefore, 


$$
\beta_{\rho}=\frac{\chi_{m n}}{a}
$$

where $X_{m n}$ is the $n$th zero of the $m$ th-order Bessel function. Also, $E_{\rho}$ must also equal zero at $z=0$ and $z=h$. Therefore, $D_{3}=0$ and

$$
\beta_{z}=\frac{p \pi}{h}
$$

where $p=1,2,3 \ldots$ Specializing these results to the magnicon mode of interest, the $\mathrm{TM}_{110}$ mode, results in field quantities given by

$$
\begin{aligned}
& E_{\rho}=0, \\
& E_{\phi}=0, \\
& E_{z}=-j \frac{\left(\frac{\chi_{11}}{a}\right)^{2}}{\omega \mu \varepsilon} A_{110} J_{1}\left(\frac{\chi_{11}}{a} \rho\right) \cos (\phi), \\
& H_{\rho}=-\frac{1}{\mu \rho} A_{110} J_{1}\left(\frac{\chi_{11}}{a} \rho\right) \sin (\phi), \\
& H_{\phi}=-\frac{1}{\mu} A_{110}\left(\frac{\chi_{11}}{a}\right) J_{1}\left(\frac{\chi_{11}}{a} \rho\right) \cos (\phi),
\end{aligned}
$$

and

$$
H_{3}=0 \text {. }
$$

Rewriting Eqs. (3.13) through (3.16) in terms of peak field magnitude, $E_{O}$, results in

$$
\begin{aligned}
& E_{\rho}=0, \\
& E_{\phi}=0, \\
& E_{\imath}=E_{o} J_{1}\left(\frac{\chi_{11}}{a} \rho\right) \cos (\phi), \\
& H_{\rho}=-j\left(\frac{\omega \varepsilon E_{o} a^{2}}{\chi_{11}^{2} \rho}\right) J_{1}\left(\frac{\chi_{11}}{a} \rho\right) \sin (\phi),
\end{aligned}
$$




$$
H_{\phi}=-j\left(\frac{\omega \varepsilon E_{o} a}{\chi_{11}}\right) J_{1}\left(\frac{\chi_{11}}{a} \rho\right) \cos (\phi),
$$

and

$$
H_{2}=0 \text {. }
$$

These are converted to instantaneous expressions for use in the simulations as

$$
\mathcal{E}=\operatorname{Re}\left(E e^{j a x}\right)
$$

and

$$
\mathcal{H}=\operatorname{Re}\left(H e^{j a x}\right),
$$

resulting in

$$
\begin{aligned}
& \mathcal{E}_{\mathrm{z}}=E_{o} J_{1}\left(\frac{\chi_{11}}{a} \rho\right) \cos (\phi) \cos (\omega t), \\
& \mathcal{H}_{\rho}=\frac{E_{o}}{\omega \rho \mu} J_{1}\left(\frac{\chi_{11}}{a} \rho\right) \sin (\phi) \sin (\omega t),
\end{aligned}
$$

and

$$
\mathcal{H}_{\phi}=E_{o} \sqrt{\frac{\varepsilon}{\mu}} J_{1}^{\prime}\left(\frac{\chi_{11}}{a} \rho\right) \cos (\phi) \sin (\omega t) .
$$

The rotating $\mathrm{TM}_{110}$ mode is excited in the magnicon drive cavity by coupling to the cavity through two drive loops equidistant from the axis, spatially displaced by $\pi / 2$. In addition, the field coupled into the first port differs by $\pi / 2$ from the excitation phase at port 2. The result on Eqs. (3.28) through (3.30) is that

$$
\omega t \rightarrow \omega t-\frac{\pi}{2}
$$

and

$$
\phi \rightarrow \phi-\frac{\pi}{2}
$$

Making these substitutions, the fields at port 2 are written as 


$$
\begin{aligned}
& \varepsilon_{z 2}=E_{0} J_{1}\left(\frac{\chi_{11}}{a} \rho\right) \sin (\phi) \sin (\omega t), \\
& \mathcal{H}_{\rho 2}=\frac{E_{o}}{\omega \rho \mu} J_{1}\left(\frac{\chi_{11}}{a} \rho\right) \cos (\phi) \cos (\omega t),
\end{aligned}
$$

and

$$
\mathcal{H}_{\phi 2}=E_{\circ} \sqrt{\frac{\varepsilon}{\mu}} J_{1}\left(\frac{\chi_{11}}{a} \rho\right) \sin (\phi) \cos (\omega t)
$$

The equations for the rotating $\mathrm{TM}_{110}$ mode are given by adding $\mathrm{Eq}(3.28)$ through (3.33), Eq. (3.29) through (3.34), and Eq. (3.30) through (3.35), as

$$
\begin{aligned}
& \mathcal{E}_{\mathrm{z}}=E_{o} J_{1}\left(\frac{\chi_{11}}{a} \rho\right) \cos (\omega t-\phi), \\
& \mathcal{H}_{\rho}=\frac{E_{o}}{\omega \rho \mu} J_{1}\left(\frac{\chi_{11}}{a} \rho\right) \cos (\omega t-\phi),
\end{aligned}
$$

and

$$
\mathcal{H}_{\phi}=E_{o} \sqrt{\frac{\varepsilon}{\mu}} J_{1}\left(\frac{\chi_{11}}{a} \rho\right) \sin (\omega t-\phi) .
$$

For the $\mathrm{TM}_{110}$ mode, the resonant frequency is found from

$$
\beta=\omega \sqrt{\mu \varepsilon}=\frac{\chi_{11}}{a}
$$

Substituting Eq. (3.39) into Eqs. (3.36), (3.37), and (3.38), and using the relations $c=1 / \sqrt{\mu \varepsilon}$ and $\eta=\sqrt{\mu / \varepsilon}$, gives

$$
\begin{aligned}
& \varepsilon_{z}=E_{o} J_{1}\left(\frac{\chi_{11}}{a} \rho\right) \cos (\omega t-\phi), \\
& \mathcal{H}_{\rho}=\frac{E_{o}}{\eta} \frac{J_{1}\left(\frac{\chi_{11}}{a} \rho\right)}{\frac{\chi_{11}}{a} \rho} \cos (\omega t-\phi),
\end{aligned}
$$

and 


$$
\mathcal{H}_{\phi}=\frac{E_{o}}{\eta} J_{1}^{\prime}\left(\frac{\chi_{11}}{a} \rho\right) \sin (\omega t-\phi) .
$$

This can be converted to Cartesian coordinates using

$$
\begin{aligned}
\mathcal{E}_{z} & =\mathcal{E}_{z}, \\
\mathcal{H}_{x} & =\mathcal{H}_{\rho} \cos (\phi)-\mathcal{H}_{\phi} \sin (\phi),
\end{aligned}
$$

and

$$
\mathcal{H}_{y}=\mathcal{H}_{\rho} \sin (\phi)+\mathcal{H}_{\phi} \cos (\phi) .
$$

Substituting Eq. (3.40) into (3.43), Eq. (3.41) into (3.44), and Eq. (3.42) into (3.45), and using the relation

$$
J_{1}\left(\frac{\chi_{11}}{a} \rho\right)=J_{0}\left(\frac{\chi_{11}}{a} \rho\right)-\frac{1}{\frac{\chi_{11}}{a} \rho} J_{1}\left(\frac{\chi_{11}}{a} \rho\right),
$$

results in

$$
\begin{aligned}
& \mathcal{E}_{z}=E_{o} J_{1}\left(\frac{\chi_{11}}{a} \rho\right) \cos (\omega t-\phi) \\
& \mathcal{H}_{x}=\frac{E_{o}}{\eta}\left[\frac{J_{1}\left(\frac{\chi_{11}}{a} \rho\right)}{\frac{\chi_{11}}{a} \rho} \cos (\omega t-2 \phi)-J_{0}\left(\frac{\chi_{11}}{a} \rho\right) \sin (\omega t-\phi) \sin (\phi)\right]
\end{aligned}
$$

and

$$
\mathfrak{H}_{y}=\frac{E_{0}}{\eta}\left[\frac{J_{1}\left(\frac{\chi_{11}}{a} \rho\right)}{\frac{\chi_{11}}{a} \rho} \sin (2 \phi-\omega t)+J_{0}\left(\frac{\chi_{11}}{a} \rho\right) \sin (\omega t-\phi) \cos (\phi)\right]
$$

Therefore, by converting $\mathcal{H}$ to $\mathrm{B}$, the full set of field equations for the rotating $\mathrm{TM}_{110}$ mode is given by

$$
\begin{aligned}
& \varepsilon_{x}=0, \\
& \varepsilon_{y}=0,
\end{aligned}
$$




$$
\begin{aligned}
& \varepsilon_{\mathrm{z}}=E_{0} J_{1}\left(\frac{\chi_{11}}{a} \rho\right) \cos (\omega t-\phi) \\
& \mathbf{B}_{x}=\frac{E_{0}}{c}\left[\frac{J_{1}\left(\frac{\chi_{11}}{a} \rho\right)}{\frac{\chi_{11}}{a} \rho} \cos (\omega t-2 \phi)-J_{0}\left(\frac{\chi_{11}}{a} \rho\right) \sin (\omega t-\phi) \sin (\phi)\right] \\
& \mathbf{B}_{y}=\frac{E_{0}}{c}\left[\frac{J_{1}\left(\frac{\chi_{11}}{a} \rho\right)}{\frac{\chi_{11}}{a} \rho} \sin (2 \phi-\omega t)+J_{0}\left(\frac{\chi_{11}}{a} \rho\right) \sin (\omega t-\phi) \cos (\phi)\right]
\end{aligned}
$$

and

$$
\mathbf{B}_{\mathbf{z}}=0
$$

\subsubsection{Small-signal field equations}

The small-signal forms of these equations are sometimes useful. The small-signal regime for the magnicon can be defined as occurring when the expression

$$
\frac{\chi_{11}}{a} \rho
$$

approaches zero. The resulting small-signal field equations are found by using

$$
J_{1}\left(\frac{\chi_{11}}{a} \rho\right) \cong \frac{\chi_{11}}{2 a} \rho \text { as } \quad \frac{\chi_{11}}{a} \rho \rightarrow 0
$$

and

$$
J_{0}(x) \rightarrow 1 \text { as } x \rightarrow 0
$$

to yield

$$
\begin{aligned}
& \varepsilon_{x}=0, \\
& \varepsilon_{y}=0, \\
& \varepsilon_{z}=\frac{E_{o}}{2}\left(\frac{\chi_{11}}{a}\right)[x \cos (\omega t)+y \sin (\omega t)],
\end{aligned}
$$




$$
\begin{aligned}
& \mathbf{B}_{x}=\frac{E_{o}}{2 c} \cos (\omega t), \\
& \mathbf{B}_{y}=\frac{E_{o}}{2 c} \sin (\omega t),
\end{aligned}
$$

and

$$
\mathbf{B}_{2}=0
$$

The derivation of Eqs. (3.58) through (3.63) used trigonometric identities for transforming products into sums or differences and trigonometric identities for transforming angle sums or differences into products. It also took advantage of the fact that $x$ and $y$ could be represented as $x=\rho \cos (\phi)$ and $y=\rho \sin (\phi)$.

\subsubsection{Cavity loss}

Both the $Q$ and the wall loss of the magnicon cavities are calculated by using the cylindrical cavity model. The perturbations caused by the beam pipe as it enters and exits the cavity are not considered. Assuming the cavity wall to be a good conductor, the current density on the side wall, top, and bottom is calculated to be

$$
J_{s}=\hat{n} \times\left. H\right|_{\text {sufface }},
$$

where $J_{S}$ is the current density and $\hat{n}$ is the outward unit normal. The cavity loss and quality factor are computed for one of the $\mathrm{TM}_{110}$ modes, and the result for the cavity loss is then doubled to account for the presence of the two modes that make up the rotating $\mathrm{TM}_{110}$ mode. The calculations will use the time-averaged form of the field expressions from Eqs. (3.20) through (3.25). The current densities on the side wall and bottom are given by

$$
J_{s(\text { side })}=\hat{a}_{\rho} \times\left[-j \frac{E_{o}}{\eta} J_{1}^{\prime}\left(\chi_{11}\right) \cos (\phi) \hat{a}_{\phi}\right]
$$

and

$$
J_{s(\text { bottom })}=-\hat{a}_{z} \times\left[-j\left(\frac{E_{o}}{\eta}\right) \frac{J_{1}\left(\frac{\chi_{11}}{a} \rho\right)}{\frac{\chi_{11}}{a} \rho} \sin (\phi) \hat{a}_{\rho}-j\left(\frac{E_{o}}{\eta}\right) J_{1}^{\prime}\left(\frac{\chi_{11}}{a} \rho\right) \cos (\phi) \hat{a}_{\phi}\right]
$$


or

$$
J_{s(\text { side })}=-j \frac{E_{0}}{\eta} J_{1}^{\prime}\left(\chi_{11}\right) \cos (\phi) \hat{a}_{z}
$$

and

$$
J_{s(\text { bothom })}=\left[j\left(\frac{E_{o}}{\eta}\right) \frac{J_{1}\left(\frac{\chi_{11}}{a} \rho\right)}{\frac{\chi_{11}}{a} \rho} \sin (\phi) \hat{a}_{\phi}-j\left(\frac{E_{o}}{\eta}\right) J_{1}\left(\frac{\chi_{11}}{a} \rho\right) \cos (\phi) \hat{a}_{\rho}\right]
$$

The power dissipated in the walls is found from

$$
P_{d}=\frac{R_{s}}{2} \iint_{A} J_{s} \bullet J_{s}^{*} d S,
$$

where $R_{S}$ is the surface resistivity. Substituting Eqs. (3.65) and (3.66) into Eq. (3.69) results in

$$
P_{d(\text { side })}=\frac{R_{s}}{2} \int_{z=0}^{h} \int_{\phi=0}^{2 \pi}\left[\left(\frac{E_{0}}{\eta}\right)^{2} J_{1}^{\prime 2}\left(\chi_{11}\right) \cos ^{2}(\phi)\right] a d \phi d z
$$

and

$$
P_{d(\text { bottom })}=\frac{R_{s}}{2} \int_{\rho=0}^{a} \int_{\phi=0}^{2 \pi}\left[\left(\frac{E_{o}}{\eta}\right)^{2} \frac{J_{1}^{\prime 2}\left(\frac{\chi_{11}}{a} \rho\right)}{\left(\frac{\chi_{11}}{a} \rho\right)^{2}} \sin ^{2}(\phi)+\left(\frac{E_{o}}{\eta}\right)^{2} J_{1}^{\prime 2}\left(\frac{\chi_{11}}{a} \rho\right) \cos ^{2}(\phi)\right] \rho d \rho d \phi .
$$

Solving first for the dissipated power in the side wall gives

$$
P_{d(\text { side })}=\frac{R_{s}}{2}\left(\frac{E_{o}}{\eta}\right)^{2} J_{1}^{\prime 2}\left(\chi_{11}\right) a h \pi .
$$

Using Eq. (3.46) to further simplify this expression yields

$$
P_{d(\text { side })}=\frac{R_{s}}{2}\left(\frac{E_{o}}{\eta}\right)^{2} a h \pi J_{o}^{2}\left(\chi_{11}\right)
$$

Solving for the dissipated power in the bottom wall gives 


$$
P_{d \text { (bottom })}=\frac{R_{s}}{2}\left(\frac{E_{o}}{\eta}\right)^{2} \pi \int_{\rho=0}^{a}\left[J_{1}^{2} \frac{\frac{\chi_{11}}{a} \rho}{\left(\frac{\chi_{11}}{a} \rho\right)^{2}}+J_{1}^{\prime 2}\left(\frac{\chi_{11}}{a} \rho\right)\right] \rho d \rho \text {. }
$$

Using Eq. (3.46) to further simply this expression results in

$$
P_{d(\text { bottom })}=\frac{R_{s}}{2}\left(\frac{E_{o}}{\eta}\right)^{2} \pi \frac{a^{2}}{2} J_{o}^{2}\left(\chi_{11}\right) .
$$

To account for the power dissipation of both the top and bottom of the cavity, the total power dissipation is equal to the sum of Eq. (3.73) and twice Eq. (3.75). The total dissipation in the walls is given by

$$
P_{d}=\frac{R_{s}}{2}\left(\frac{E_{o}}{\eta}\right)^{2} \pi J_{o}^{2}\left(\chi_{11}\right)\left[a^{2}+a h\right]
$$

Equation (3.76) represents the power dissipated in the wall for one of the two $\mathrm{TM}_{110}$ modes that make up the rotating $\mathrm{TM}_{110}$ mode. Therefore, the power dissipated due to the rotating $\mathrm{TM}_{110}$ mode would be twice that expressed in Eq. (3.76).

\subsubsection{Stored energy and quality factor}

The stored energy in the cavity is found, as above, for one of the two $\mathrm{TM}_{110}$ modes that make up the rotating $\mathrm{TM}_{110}$ mode. The electric field is used to calculate the time-averaged stored electric energy according to

$$
W_{e}=\frac{1}{2} \iiint_{V} \varepsilon|E|^{2} d V o l
$$

Using Eqs. (3.20) through (3.25), this stored energy is found to be

$$
W_{e}=E_{o}^{2} \frac{\varepsilon \pi h}{2} \int_{0}^{a} \rho J_{1}^{2}\left(\frac{\chi_{11}}{a} \rho\right) d \rho
$$

or

$$
W_{e}=E_{o}^{2} \frac{\varepsilon \pi h a^{2}}{4} J_{o}^{2}\left(\chi_{11}\right)
$$


Using Eq. (3.76) and Eq. (3.79), we can determine the $Q$ of the cavity as

$$
Q=\omega \frac{W_{d}}{P_{d}}=\frac{\omega \mu h a}{2 R_{s}(h+a)}
$$

Note that this derivation was completed using only one of the rotating modes. However, the result would be the same if both modes were included, considering that both modes double the stored energy and also double the power lost in the cavity walls.

\subsection{Generalized small-signal model}

Particle trajectories are determined from a relativistic form of the Lorentz force equations, Ref. [2], which consist of two equations: the Lorentz force equation and the Lorentz energy equation. The former equation is

$$
\frac{d p}{d t}=-e(E+v \times B),
$$

where $p$ is the momentum given by

$$
p=m v
$$

and the energy equation is

$$
m c^{2} \frac{d \gamma}{d t}=-e v \cdot E
$$

The time derivative of $p$ is

$$
\frac{d p}{d t}=\frac{d v}{d t} \gamma+v \frac{d \gamma}{d t}
$$

If we assume ideal fields, for a rotating $\mathrm{TM}_{110}$ magnicon mode in the presence of a solenoidal forusing field in the $z$ direction (along the axis of the magnicon), the only field components present are $B_{x}, B_{y}, B_{z}$, and $E_{z}$. Using Eqs. (3.81), (3.83), and (3.84), the component forms of the relativistic force equations are

$$
\begin{aligned}
& \frac{d v_{x}}{d t}=-\frac{e}{y m}\left(v_{y} B_{z}-v_{z} B_{y}-\frac{v_{x} v_{z} E_{z}}{c^{2}}\right), \\
& \frac{d v_{y}}{d t}=-\frac{e}{y m}\left(v_{z} B_{x}-v_{x} B_{z}-\frac{v_{y} v_{z} E_{z}}{c^{2}}\right),
\end{aligned}
$$




$$
\frac{d v_{z}}{d t}=-\frac{e}{m m}\left(E_{z}+v_{x} B_{y}-v_{y} B_{x}-\frac{v_{z}^{2} E_{z}}{c^{2}}\right),
$$

and

$$
\frac{d \gamma}{d t}=-\frac{e}{m c^{2}} \nu_{z} E_{z}
$$

These equations can be specialized to the small-signal regime by assuming that $\nu_{z}$ and $\gamma$ are constant. This results in

$$
\begin{aligned}
& \frac{d v_{x}}{d t}=-\frac{e}{m m}\left(v_{y} B_{z}-v_{z} B_{y}\right), \\
& \frac{d v_{y}}{d t}=-\frac{e}{m}\left(v_{z} B_{x}-v_{x} B_{z}\right),
\end{aligned}
$$

and

$$
\frac{d v_{z}}{d t}=0
$$

Equations (3.89), (3.90), and (3.91) are used to analytically derive small-signal trajectories in the deflection system.

Particle trajectories derived from small-signal analysis serve several functions. They describe particle trajectories in the drive cavity, they are used to determine the loaded $\mathrm{Q}$ of the drive cavity, they are used to predict optimum drive-cavity geometry, and they are used as an independent evaluation on the accuracy of the large-signal numerical simulation. However, small-signal analysis is limited in applicability to those cavities having a small radial displacement. In addition, the results of the analysis are not selfconsistent, and they are extremely complex for an arbitrary cavity entrance time. The analysis that follows assumes zero entrance time for passive cavities and drift spaces and is, therefore, useful only as a tool to benchmark the self-consistent numerical simulation and illustrate restrictive cases.

Substituting Eqs. (3.58) through (3.63) into Eqs. (3.89) through (3.91) results in

$$
\frac{d v_{x}}{d t}=-\Omega v_{y}+E_{2} \sin (\omega t)
$$


and

$$
\frac{d v_{y}}{d t}=\Omega v_{x}-E_{2} \cos (\omega t)
$$

where $E_{2}$ and $\Omega$, the cyclotron frequency, are given by

$$
\Omega=\frac{e B_{o}}{y m}
$$

and

$$
E_{2}=\frac{E_{0} e v_{8}}{2 m c}
$$

and where $B_{o}$ represents the solenoid focusing field. Equations (3.92) and (3.93) can be decoupled by taking their second derivatives. This results in

$$
\frac{d^{2} v_{x}}{d t^{2}}+\Omega^{2} v_{x}=E_{2}(\Omega+\omega) \cos (\omega t)
$$

and

$$
\frac{d^{2} v_{y}}{d t^{2}}+\Omega^{2} v_{y}=E_{2}(\Omega+\omega) \sin (\omega t)
$$

The solutions for Eqs (3.96) and (3.97) are given by

$$
v_{x}=A_{3} \cos (\Omega t)+A_{4} \sin (\Omega t)+A_{5} \cos (\omega t)
$$

and

$$
v_{y}=A_{0} \cos (\Omega t)+A_{1} \sin (\Omega t)+A_{2} \sin (\omega t)
$$

The constant for the particular solution is given by

$$
A_{2}=A_{5}=\frac{E_{2}}{(\Omega-\omega)} .
$$

The particle trajectories are given by

$$
x=\frac{A_{3}}{\Omega} \sin (\Omega t)-\frac{A_{4}}{\Omega} \cos (\Omega t)+\frac{A_{5}}{\omega} \sin (\omega t)+\text { constant }
$$


and

$$
y=\frac{A_{0}}{\Omega} \sin (\Omega t)-\frac{A_{1}}{\Omega} \cos (\Omega t)-\frac{A_{2}}{\omega} \cos (\omega t)+\text { constant }
$$

Equations (3.101) and (3.102) can be specialized for either a drive cavity, passive deflection cavity, or drift space by evaluating their constants at the proper boundary conditions.

The boundary conditions for the drive cavity, when $t=0$, are

$$
\begin{aligned}
& x=y=z=0, \\
& v_{x}=v_{y}=0, \\
& v_{z}=v_{z 0},
\end{aligned}
$$

and

$$
\frac{d v_{y}}{d t}=-E_{2}, \quad \frac{d v_{x}}{d t}=0
$$

The boundary conditions for a passive deflection cavity, when $t=0$, are

$$
\begin{aligned}
& x=x_{0}, \quad y=y_{0}, \quad z=0, \\
& v_{x}=v_{x o}, \quad v_{y}=v_{y 0}, \quad v_{z}=v_{z o}, \\
& \frac{d v_{y}}{d t}=\Omega v_{x o}-E_{2} \sin (\varphi),
\end{aligned}
$$

and

$$
\frac{d v_{x}}{d t}=-\Omega v_{y o}+E_{2} \cos (\varphi)
$$

(For simplicity, the conditions defined in Eq. [3.104] assume zero entrance time and that particles enter the cavity on-axis. The variable $\varphi$ is added to represent the if phase. Neither assumption is strictly valid, and the small-signal trajectories are not useful without the self-consistent phase, but they can be useful as a verification of the largesignal numerical simulation.) 
The boundary conditions for a drift space, when $t=0$, are

$$
\begin{aligned}
& x=x_{0}, \quad y=y_{0}, \\
& v_{x}=v_{x 0}, \quad v_{y}=v_{y 0}, \\
& \frac{d v_{y}}{d t}=\Omega v_{x 0}, \quad \frac{d v_{x}}{d t}=-\Omega v_{y o},
\end{aligned}
$$

and

$$
E_{2}=0
$$

To simplify the trajectories, these boundary conditions also assume zero entrance time and are useful as a benchmark on the large-signal numerical simulation routines. They also predict trajectories for particles entering a drift space at a time increment such that $\Omega t$ is an integer multiple of $2 \pi$.

\subsubsection{Drive-cavity small-signal model and trajectories}

Using the boundary conditions in Eq. (3.103), the velocities and trajectories in the drive cavity can be calculated as

$$
\begin{aligned}
& v_{x}=\frac{E_{2}}{(\Omega-\omega)}[\cos (\omega t)-\cos (\Omega t)], \\
& v_{y}=-\frac{E_{2}}{(\Omega-\omega)}[\sin (\Omega t)-\sin (\omega t)], \\
& x=-\frac{E_{2}}{(\Omega-\omega)}\left[\frac{1}{\Omega} \sin (\Omega t)-\frac{1}{\omega} \sin (\omega t)\right],
\end{aligned}
$$

and

$$
y=-\frac{E_{2}}{(\Omega-\omega)}\left[\frac{1}{\omega} \cos (\omega t)-\frac{1}{\Omega} \cos (\Omega t)\right]+\frac{E_{2}}{\Omega \omega} .
$$

\subsubsection{Optimum drive-cavity length}

The optimum length of the drive cavity is one that maximizes the conversion of longitudinal velocity to radial velocity at the output of the drive cavity. The magnitude of the radial velocity, $v_{p}$, is given by 


$$
v_{p}^{2}=v_{x}^{2}+v_{y}^{2}
$$

and is found, using Eqs. (3.106) and (3.107), to be

$$
v_{p}^{2}=-\left(\frac{E_{2}}{\Omega-\omega}\right)^{2}[-2+2 \cos (\Omega t) \cos (\omega t)+2 \sin (\Omega t) \sin (\omega t)] \text {. }
$$

This simplifies to

$$
v_{p}=\sqrt{2}\left(\frac{E_{2}}{\Omega-\omega}\right) \sqrt{1-\cos \{(\Omega-\omega) t\}} .
$$

To find the transit time of maximum radial velocity (or equivalent length), the first derivative of the velocity is calculated and set equal to zero. The derivative is

$$
\frac{d \nu_{p}}{d t}=\frac{E_{2}}{\sqrt{2}} \frac{\sin (\Omega-\omega) t}{\sqrt{1-\cos [(\Omega-\omega) t]}}
$$

After setting the derivative equal to zero, we find the cavity transit time that maximizes the conversion of longitudinal to radial velocity is

$$
t=\frac{\pi}{\Omega-\omega}
$$

which corresponds to a cavity length in the small-signal regime given by

$$
h=\frac{v_{20} \pi}{\Omega-\omega} .
$$

\subsubsection{Beam loading}

The cyclotron-to-rf-frequency ratio $(\Omega / \omega)$ determines the beam loading in the drive cavity. The difference between the rate of rotation of the cyclotron motion and the rate of rotation of the cavity fields affects the degree, to which the beam experiences any accelerating and decelerating forces from the if fields. It will be shown that for values of $\Omega / \omega$ less than 2.0 , power is absorbed by the beam. Furthermore, it will also be shown that the power absorbed increases for lower values of $\Omega / \omega$ and decreases for higher values. For values of $\Omega / \omega$ greater than 2.0 , the beam gives up energy and enters a potentially unstable operating regime. From the conservation of energy equation, Ref. [1], the instantancous supplied power is calculated from 


$$
P_{s}=-\iiint_{V \text { olume }} \mathcal{E} \cdot \mathcal{J} d V
$$

To determine the power provided to the beam, the small-signal regime assumes the beam to be infinitesimally thin. The small-signal form of the instantaneous, rotating electric field for the $\mathrm{TM}_{110}$ mode from Eq. (3.60) is

$$
\varepsilon_{2}=\frac{E_{o}}{2}\left(\frac{\chi_{11}}{a}\right)[x \cos (\omega t)+y \sin (\omega t)]
$$

Substituting $x$ from Eq. (3.108) and $y$ from Eq. (3.109) into Eq. (3.117) results in

$$
\varepsilon_{\mathrm{z}}=\frac{E_{o}}{2 \Omega}\left(\frac{\chi_{11}}{a}\right) \frac{E_{2}}{(\Omega-\omega)}\left\{\sin [(\Omega-\omega) t]+\frac{(\Omega-\omega)}{\omega} \sin (\omega t)\right\}
$$

Because the beam is assumed to be infinitesimally small, the current density is represented by

$$
\mathrm{J}=I_{0} \delta\left(x-x_{0}\right) \delta\left(y-y_{0}\right)
$$

where $I_{O}$ has a value equal to the beam current, and $x_{O}$ and $y_{O}$ represent the position of the beam. Using Eqs. (3.118) and (3.119), the power supplied by the beam can be calculated as

$$
\begin{aligned}
P_{s}= & -\frac{\chi_{11} I_{o} E_{o} E_{2}}{\Omega 2 a(\Omega-\omega)} \iiint_{\text {Volume }}\left\{\sin [(\Omega-\omega) t]+\frac{(\Omega-\omega)}{\omega} \sin (\omega t)\right\} \\
& \delta\left(x-x_{0}\right) \delta\left(y-y_{o}\right) d x d y d z
\end{aligned}
$$

or

$$
P_{s}=-\frac{\chi_{11} I_{o} E_{o} E_{2}}{\Omega 2 a(\Omega-\omega)} \int_{0}^{T}\left\{\sin [(\Omega-\omega) t]+\frac{(\Omega-\omega)}{\omega} \sin (\omega t)\right\} v_{z o} d t
$$

where $T$ is the cavity transit time. Equation (3.121) evaluates to

$$
\begin{aligned}
P_{s}= & -\frac{\chi_{11} I_{o} E_{o}^{2} v_{z o}^{2} e}{4 a \Omega(\Omega-\omega)^{2} m c} \\
& \left\{\frac{\Omega^{2}-2 \omega \Omega}{\omega^{2}}+\cos [(\Omega-\omega) T]-\frac{(\Omega-\omega)^{2}}{\omega^{2}} \cos (\omega T)\right\} .
\end{aligned}
$$


To demonstrate the effect of changing the strength of the focusing field, here is a numerical example. In it, the power absorbed by the beam is calculated as well as the stored energy, the power dissipated in the walls, the cold-cavity $Q$, and the beam-loaded Q. The beam-loaded, or hot, $Q$ is calculated according to

$$
Q_{\text {not }}=\frac{\omega W_{e}}{P_{d}+P_{s}}
$$

The numerical example is based on the following conditions:

$$
\begin{aligned}
& \chi_{11}=3.83128, \\
& I_{o}=18 \mathrm{~A}, \\
& E_{o}=2.0 \times 10^{5} \mathrm{~V} / \mathrm{m}, \\
& v_{2 o}=1.9808 \times 10^{8} \mathrm{~m} / \mathrm{s}, \\
& a=0.26 \mathrm{~m},
\end{aligned}
$$

and

$$
\omega=2 \pi \times 700.0 \times 10^{6} \mathrm{rad} / \mathrm{s} .
$$

Table 3.1 summarizes the calculations. 
Table 3.1. Summary of calculations

\begin{tabular}{|c|c|c|c|c|c|c|}
\hline$\Omega / \omega$ & $\Omega$ & $\begin{array}{c}\text { Pd } \\
\text { (watts) }\end{array}$ & $\begin{array}{c}\text { Ps } \\
\text { (watts) }\end{array}$ & $\begin{array}{c}\text { We } \\
\text { (joules) }\end{array}$ & Qcold & Qhot \\
\hline 0.8 & $3.52 \mathrm{E}+09$ & 118.6 & 5357.8 & $1.20 \mathrm{E}-03$ & 44631 & 966 \\
\hline 0.9 & $3.96 \mathrm{E}+09$ & 118.6 & 5044.0 & $1.20 \mathrm{E}-03$ & 44631 & 1025 \\
\hline 1.0 & $4.40 \mathrm{E}+09$ & 118.6 & 4623.3 & $1.20 \mathrm{E}-03$ & 44631 & 1115 \\
\hline 1.1 & $4.84 \mathrm{E}+09$ & 118.6 & 4123.9 & $1.20 \mathrm{E}-03$ & 44631 & 1247 \\
\hline 1.2 & $5.28 \mathrm{E}+09$ & 118.6 & 3571.9 & $1.20 \mathrm{E}-03$ & 44631 & 1434 \\
\hline 1.3 & $5.72 \mathrm{E}+09$ & 118.6 & 2989.1 & $1.20 \mathrm{E}-03$ & 44631 & 1703 \\
\hline 1.4 & $3.16 \mathrm{E}+09$ & 118.6 & 2403.2 & $1.20 \mathrm{E}-03$ & 44631 & 2096 \\
\hline 1.5 & $3.60 \mathrm{E}+09$ & 118.6 & 1848.7 & $1.20 \mathrm{E}-03$ & 44631 & 2690 \\
\hline 1.6 & $7.04 \mathrm{E}+09$ & 118.6 & 1338.0 & $1.20 \mathrm{E}-03$ & 44631 & 3633 \\
\hline 1.7 & $7.48 \mathrm{E}+09$ & 118.6 & 890.4 & $1.20 \mathrm{E}-03$ & 44631 & 5245 \\
\hline 1.8 & $7.92 \mathrm{E}+09$ & 118.6 & 513.2 & $1.20 \mathrm{E}-03$ & 44631 & 8337 \\
\hline 1.9 & $8.36 \mathrm{E}+09$ & 118.6 & 219.9 & $1.20 \mathrm{E}-03$ & 44631 & 15637 \\
\hline 2.0 & $8.80 \mathrm{E}+09$ & 118.6 & 0.0 & $1.20 \mathrm{E}-03$ & 44631 & 44631 \\
\hline 2.1 & $9.24 \mathrm{E}+09$ & 118.6 & -149.5 & $1.20 \mathrm{E}-03$ & 44631 & -171165 \\
\hline 2.2 & $9.68 \mathrm{E}+09$ & 118.5 & -238.4 & $1.20 \mathrm{E}-03$ & 44631 & -44163 \\
\hline 2.3 & $1.01 \mathrm{E}+10$ & 118.6 & -278.8 & $1.20 \mathrm{E}-03$ & 44631 & -33024 \\
\hline
\end{tabular}

Note the negative $Q h o t$ value for $\Omega / \omega$ ratios greater than 2 . Although the negative value has no meaning, it is caused by the beam providing power to the cavity, which can lead to a potentially unstable and oscillatory condition. Based on my personal experience, I cannot accept this. A minimum bandwidth of $0.05 \%$ is desirable for particle accelerator rf sources in order to minimize operational difficulties and the impact on amplifier performance of macnining tolerances and amplifier-cavity heating. Based on the hot $Q$ of Table 6-1, an $\Omega / \omega$ ratio of 1.4 would provide this approximate bandwidth in the drive cavity. It will be shown in subsequent sections that a similar relationship between the loaded $Q$ and magnetic-field strength exists in the passive cavities.

Figure 3.2 illustrates a top view of particle trajectories for various values of $\Omega / \omega$. The length for each trace is that which results in the maximum velocity conversion to the transverse direction. Figure 3.3 shows the corresponding radial velocity for the same values of $\Omega / \omega$ with the traces ending at the corresponding optimum lengths. 


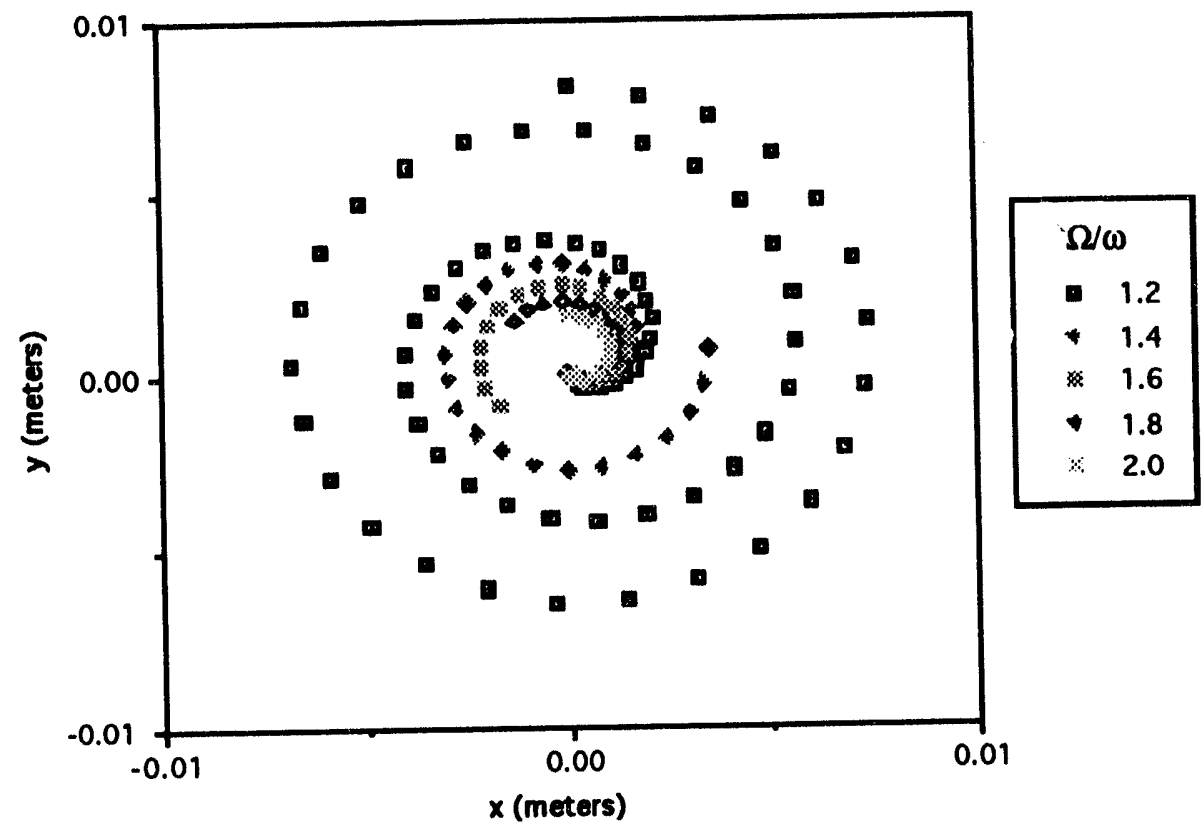

Figure 3.2. Drive-cavity particle trajectories (top view).

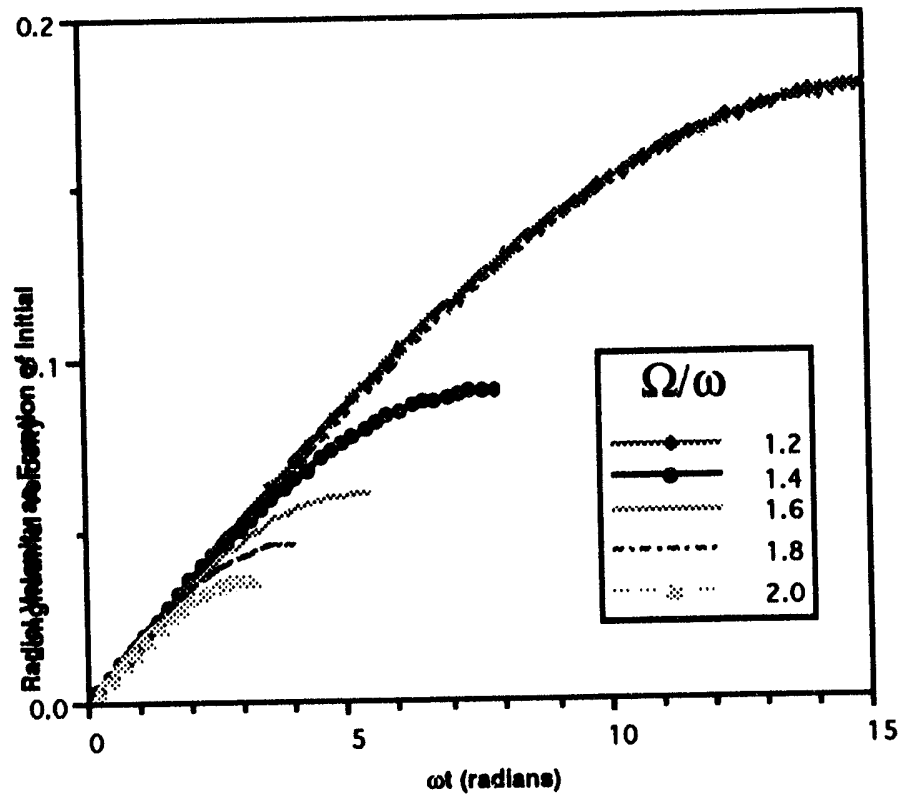

Figure 3.3. Radial velocity presented as a fraction of initial longitudinal velocity.

Figures 3.2 and 3.3 were generated at a frequency of $700 \mathrm{MHz}$ with the conditions described in Eq. (3.124), except that the peak cavity field, $E_{o}$, was $0.36 \mathrm{MV} / \mathrm{m}$, not 0.2 $\mathrm{MV} / \mathrm{m}$. The figures show that lower values for the focusing field $(\Omega / \omega)$ result in a greater transfer of initial longitudinal velocity into radial velocity. Passive-cavity large-signal analysis will demonstrate this to be true only in the drive cavity. In addition, the figures 
illustrate that the optimum electrical length may be greater than $2 \pi$ for lower focusingfield values.

\subsubsection{Drift-space small-signal model and trajectories}

Substituting the boundary conditions for a drift space in Eq. (3.105) into Eqs. (3.98) through (3.102) provides the trajectories in the drift space. These trajectories are given by

$$
\begin{aligned}
& v_{x}=v_{x o} \cos (\Omega t)-v_{y 0} \sin (\Omega t), \\
& v_{y}=v_{y o} \cos (\Omega t)+v_{x o} \sin (\Omega t), \\
& x=\frac{v_{x o}}{\Omega} \sin (\Omega t)+\frac{v_{y o}}{\Omega}[\cos (\Omega t)-1]+x_{o},
\end{aligned}
$$

and

$$
y=\frac{v_{y o}}{\Omega} \sin (\Omega t)-\frac{v_{x o}}{\Omega}[\cos (\Omega t)-1]+y_{0}
$$

Large-signal analysis will show that drift-space length should be selected to bring the beam into the passive cavities at a minimum radius. An attempt to generate a closedform solution for optimum length by taking the derivative of the expression for the radius leads to a transcendental equation and would only be valid for drift spaces with an entrance time that is an integer multiple of $2 \pi$. Thus, selecting the optimum length would rely more on numerical modeling than an analytical solution. A top and side view of the particle trajectories described by Eqs. (3.127) and (3.128) are presented in Figs. 3.4 and 3.5 for a range of magnet focusing-field values. The small-signal analysis for the drive cavity is used to calculate a set of initial conditions. The initial conditions are the output position and velocity of a drive cavity where $\Omega t$ is an integer multiple of $2 \pi$. The drivecavity conditions used to generate Fig. 3.3 were also used to determine the input conditions for Figs. 3.4 and 3.5. 


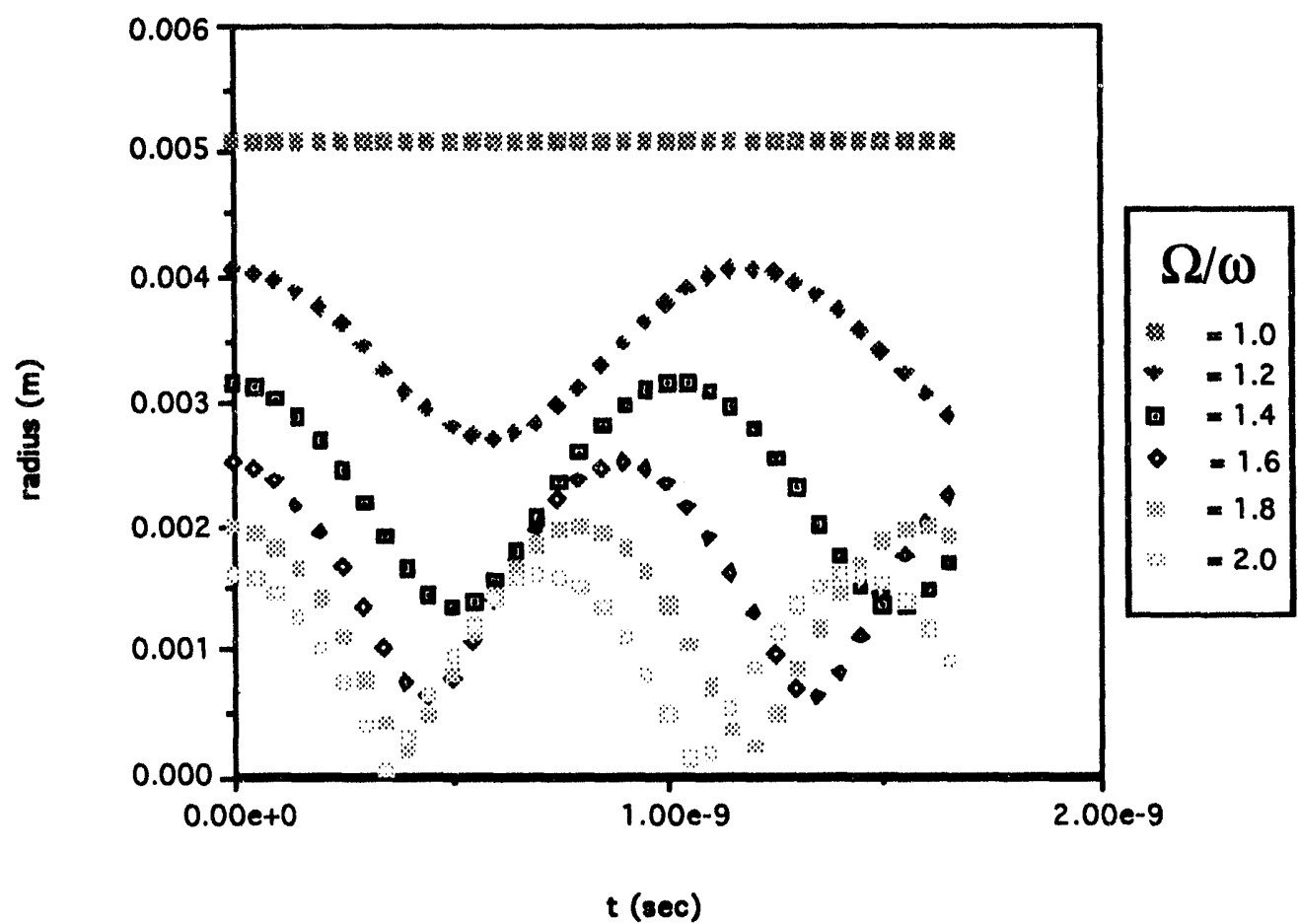

Figure 3.4. Drift-space particle trajectories.

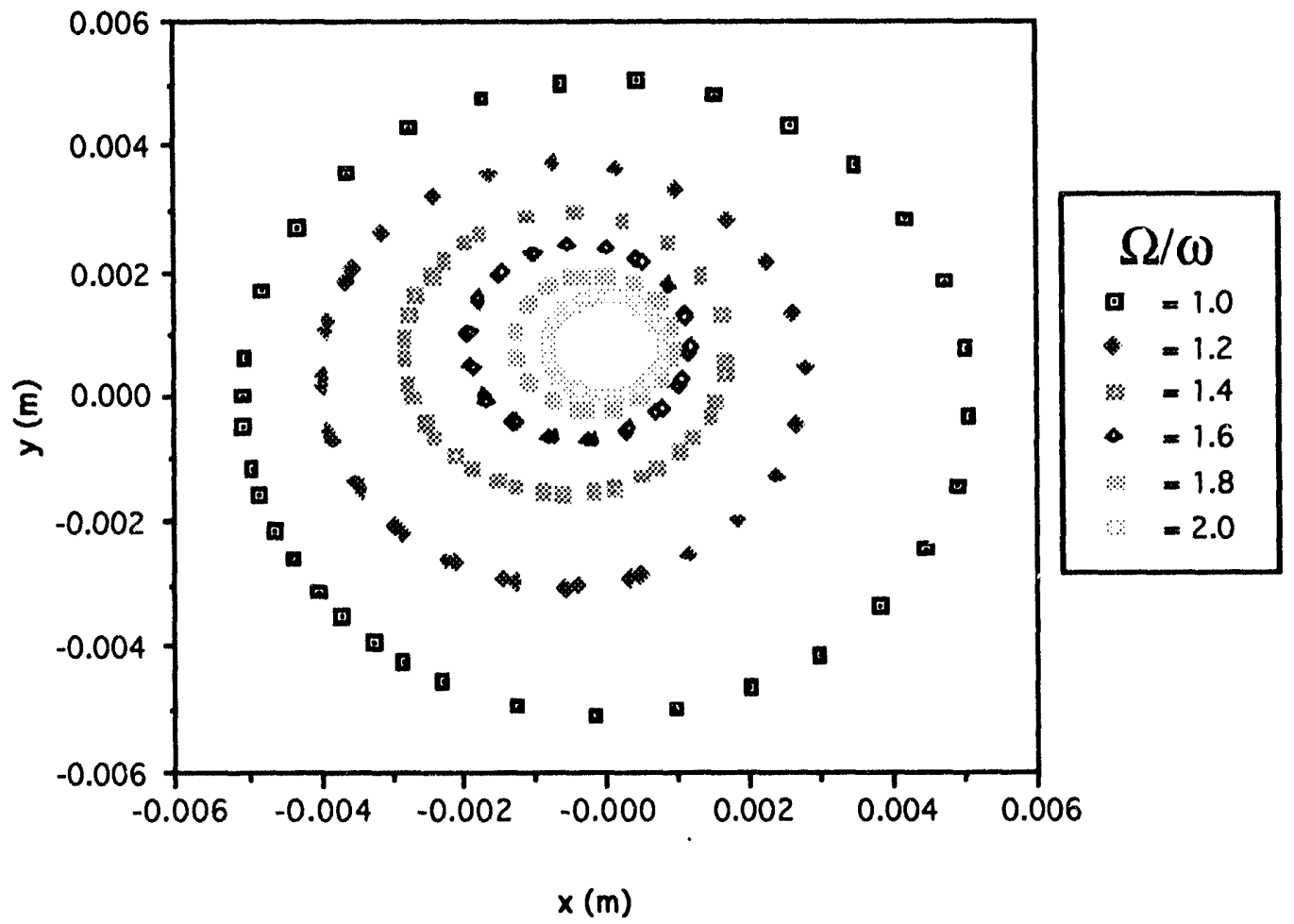

Figure 3.5. Displacement off-axis of a single electron as a function of time. 
Figures 3.4 and 3.5 provide a limited insight into magnicon behavior. The limitation arises from the fact that the initial conditions used to solve the equations governing particle trajectories in the figures were limited to cases where the entrance value of $\Omega t$ was equal to an integer multiple of $2 \pi$. For this restricted class of trajectories, the figures illustrate the cyclotron motion in the drift space. It has been noted that the cyclotron radius increases in the drift space as the strength of the magnetic focusing field decieases. The increase in the radius of the cyclotron motion results from the increase in transverse energy at the output of the drive cavity as the magnetic focusing field decreases in strength (see Fig. 3.2).

Figure 3.5 also indicates that it becomes increasingly difficult to focus the beam to the axis in the presence of a decreasing magnetic focusing field. For the case where $\Omega / \omega=1.0$, the beam maintains a constant radius in the drift space. In generating Fig. 3.5, which used the initial conditions that were determined from the small-signal drive-cavity trajectories where $\Omega t=2 \pi$, it was discovered that the center of the cyclotron motion turns out to be equal to the longitudinal axis of the magnicon. The result is a constant radius in the drift space. The large-signal theory will show that this phenomenon is a result of the exit time constraint on the drive cavity. In other words, it is incorrect to infer from Fig. 3.5 that the magnetic focusing field determines the quality of the focus achievable in the drift space. The quality of the focus is not only determined by the magnetic focusing field, it also depends on the initial conditions (or length of the cavity or cavities prior to the drift space). Large-signal analysis will further clarify this point and the importance of focusing the beam to the magnicon axis by demonstrating that passive-cavity gain decreases as the entrance position of the beam moves off-axis.

\subsubsection{Passive-cavity small-signal model and trajectories}

Substituting the boundary conditions for a passive cavity in Eq. (3.104) into Eqs. (3.98) through (3.102), the equations for the trajectories in the drift space are calculated to be

$$
\begin{aligned}
& \nu_{x}=B_{0} \cos \Omega t+B_{1} \sin \Omega t+B_{2} \cos (\omega t+\phi), \\
& v_{y}=B_{3} \cos (\Omega t)+B_{4} \sin (\Omega t)+B_{2} \sin (\omega t+\phi),
\end{aligned}
$$

and 


$$
x=\frac{B_{0}}{\Omega} \sin \Omega t-\frac{B_{1}}{\Omega}[\cos \Omega t-1]+\frac{B_{2}}{\omega}[\sin (\omega t+\phi)-\sin (\phi)]+x_{0},
$$

and also

$$
y=\frac{B_{3}}{\Omega} \sin \Omega t-\frac{B_{4}}{\Omega}[\cos \Omega t-1]+\frac{B_{2}}{\omega}[\cos (\phi)-\cos (\omega t+\phi)]+y_{0},
$$

with

$$
\begin{aligned}
& B_{0}=v_{x o}-B_{2} \cos (\phi), \\
& B_{1}=-v_{y o}+\frac{E_{2} \sin (\phi)}{\Omega}+\frac{B_{2} \omega \sin (\phi)}{\Omega}, \\
& B_{2}=\frac{E_{2}}{(\Omega-\omega)}, \\
& B_{3}=v_{y o}-B_{2} \cos (\phi),
\end{aligned}
$$

and

$$
B_{4}=v_{x o}-\frac{E_{2} \cos (\phi)}{\Omega}-\frac{B_{2} \omega \cos (\phi)}{\Omega}
$$

The quantity $\phi$ represents the rf phase of excitation of the passive cavity. Remember, as has been noted earlier, that the restrictive boundary condition necessary to solve these equations limits their usefulness to being an independent verification of the numerical model.

\subsection{Model for half-cusp field}

Two techniques are employed in this work to model the magnetic transitions in the drift space. One method uses a matrix of field values computed from a numerical code like POISSON. This method is best suited for final verification of a magnicon geometry. It is not ideally suited for the intermediate modeling because of the time associated with calculating POISSON magnetic fields for the necessary number of geometry variations. It would be most desirable if an analytical method for modeling magnetic transitions in this drift space could be tied in the code to the magnicon geometry. 
The method selected for modeling and simulating the magnetic field around the pole pieces at the exit of the deflection system and entrance of the output cavity is described in Ref. [3]. (Although this topic is slightly out of place, this method is described here to consolidate all analytical theory into a single chapter.) In Ref. [3] the mixed-boundary-condition problem is solved for an infinitely thin, grounded, perfectly conducting plane. In addition, the plane has a circular hole of radius $a$ cut in it. Furthermore, the electric field, which is normal to the plane, is far from the hole, has a constant magnitude, and has different values on either side of the plane. This derivation is analogous to the problem associated with the modeling of a pole piece. The geometry of the analogous problem is illustrated in Fig. 3.3.

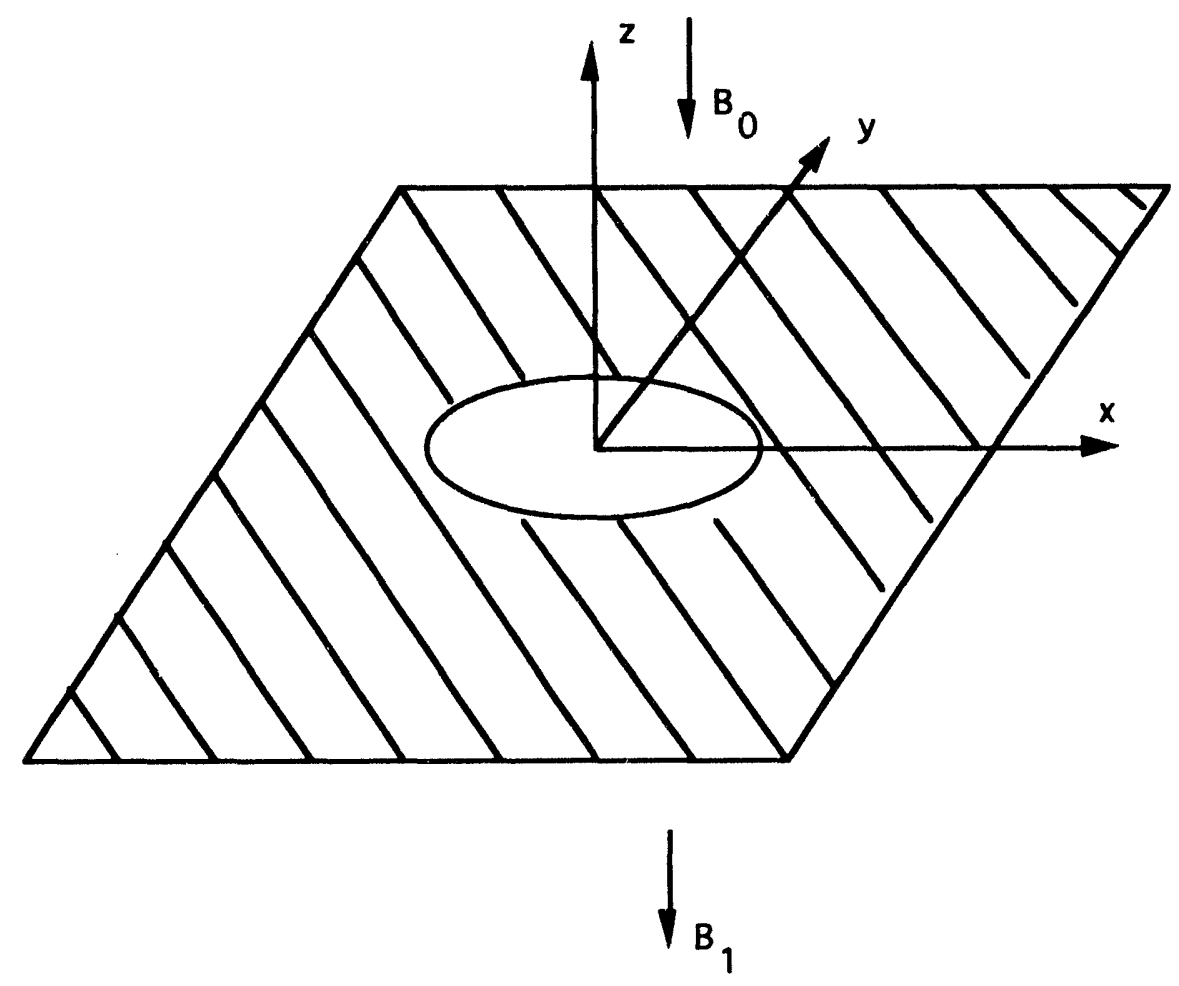

Figure 3.3. Magnetic-field-problem geometry.

Reference [3] determines the electric potential as

$$
\Phi=E_{o} z+\Phi^{(1)} \text { for } z>0
$$

and 


$$
\Phi=E_{1} z+\Phi^{(1)} \text { for } \quad z<0
$$

and gives a value for the added potential on the axis as

$$
\Phi_{m}^{(1)}(0, z)=\frac{\left(E_{o}-E_{1}\right) a}{\pi}\left[1-\frac{|z|}{a} \tan ^{-1}\left(\frac{a}{|z|}\right)\right] .
$$

In the magnetic analog for the deflection-cavity pole piece, the analog of $E_{0}, B_{0}$, is equal to zero, and the analog of $E_{1}, B_{1}$, equals the negative of the deflection-cavity focusing field, $B_{\text {def. }}$. With these substitutions, the magnetic potential, $\Phi_{m}$, for the deflection-cavity pole piece is

$$
\Phi_{m}=\Phi_{m}^{(1)} \text { for } z>0
$$

and

$$
\Phi_{m}=-B_{\text {def }} z+\Phi_{m}{ }^{(1)} \text { for } \quad z<0
$$

with the value for the added potential on-axis given by

$$
\Phi^{(1)}(0, z)=\frac{B_{d e f} a}{\pi}\left[1+\frac{z}{a} \tan ^{-1}\left(\frac{a}{-z}\right)\right] .
$$

With these equations, the magnetic field on-axis is found, using

$$
B(0, z)=-\nabla \Phi_{m},
$$

to be

$$
B(0, z)=\frac{B_{d e f} a}{\pi}\left[\frac{1}{a} \tan ^{-1}\left(\frac{a}{z}\right)-\frac{z}{z^{2}+a^{2}}\right] \text { for } z>0
$$

and

$$
B(0, z)=B_{d e f}+\frac{B_{d e f} a}{\pi}\left[\frac{1}{a} \tan ^{-1}\left(\frac{a}{z}\right)-\frac{z}{z^{2}+a^{2}}\right] \text { for } z<0 .
$$

Near the axis of the magnicon, the radial and longitudinal components of the focusing field can be found using a paraxial approximation for the magnetic field, which is given in Ref. [4] as 


$$
B_{\rho}(\rho, z)=-\left(\frac{\rho}{2}\right)\left[\frac{\partial B_{i}(0, z)}{\partial z}\right]
$$

and

$$
B_{z}(\rho, z)=B_{z}(0, z)-\left(\frac{\rho^{2}}{4}\right)\left[\frac{\partial^{2} B_{z}(0, z)}{\partial z^{2}}\right] .
$$

By using the paraxial approximation, $B$ is assumed to make only small angles with the axis so that $B_{\rho} \ll B_{z}$. This assumption is certainly valid for the exit from the deflection system because the beam is very near the magnicon axis. However, the assumption is not as valid at the entrance into the output cavity, where the beam has drifted off-axis and is approaching the pole piece. This fact partially motivates using the POISSON calculated fields to check the final geometry.

The radial component of the focusing field is given by Eq. (3.147) for all $z$ and $\rho$ near the axis as

$$
B_{\rho}(\rho, z)=\frac{\rho B_{d e f} a^{3}}{\pi\left(z^{2}+a^{2}\right)^{2}},
$$

and the longitudinal component is given as

$$
\begin{aligned}
B_{z}(\rho, z)= & B_{d e f}+\frac{B_{d e f} a}{\pi}\left[\frac{1}{a} \tan ^{-1}\left(\frac{a}{z}\right)-\frac{z}{z^{2}+a^{2}}\right] \\
& -\frac{2 \rho^{2} B_{d e f} z a^{3}}{\pi\left(z^{2}+a^{2}\right)^{3}} \text { for } z<0
\end{aligned}
$$

and

$$
\begin{aligned}
B_{z}(\rho, z)= & \frac{B_{d e f} a}{\pi}\left[\frac{1}{a} \tan ^{-1}\left(\frac{a}{z}\right)-\frac{z}{z^{2}+a^{2}}\right] \\
& -\frac{2 \rho^{2} B_{d e f} z a^{3}}{\pi\left(z^{2}+a^{2}\right)^{3}} \text { for } z>0 .
\end{aligned}
$$

The fields for the output-cavity pole piece are calculated using the same approach, except that $B_{l}$ is now set equal to zero, and $-B_{o}$ is equal to the output-cavity field, $B_{\text {out }}$. The magnetic potential is given by 


$$
\Phi_{m}=-B_{\text {out }} z+\Phi_{m}^{(1)} \text { for } z>0
$$

and

$$
\Phi_{m}=\Phi_{m}^{(1)} \text { for } z<0
$$

The on-axis magnetic field is given by

$$
B(0, z)=\frac{-B_{\text {out }} a}{\pi}\left[\frac{1}{a} \tan ^{-1}\left(\frac{a}{z}\right)-\frac{z}{z^{2}+a^{2}}\right] \text { for } z<0
$$

and

$$
B(0, z)=B_{\text {out }}-\frac{B_{\text {out }} a}{\pi}\left[\frac{1}{a} \tan ^{-1}\left(\frac{a}{z}\right)-\frac{z}{z^{2}+a^{2}}\right] \text { for } z>0,
$$

and the paraxial approximation finally results in the magnetic fields near the axis being given by

$$
\begin{aligned}
B_{\rho}(\rho, z)= & -\frac{2 \rho^{2} B_{o u t} z a^{3}}{\pi\left(z^{2}+a^{2}\right)^{3}}, \\
B_{z}(\rho, z)= & -\frac{B_{o u s} a}{\pi}\left[\frac{1}{a} \tan ^{-1}\left(\frac{a}{z}\right)-\frac{z}{z^{2}+a^{2}}\right] \\
& +\frac{2 \rho^{2} B_{o u t} z a^{3}}{\pi\left(z^{2}+a^{2}\right)^{3}} \text { for } z<0,
\end{aligned}
$$

and

$$
\begin{aligned}
B_{z}(\rho, z)= & B_{\text {out }}-\frac{B_{o u t} a}{\pi}\left[\frac{1}{a} \tan ^{-1}\left(\frac{a}{z}\right)-\frac{z}{z^{2}+a^{2}}\right] \\
& +\frac{2 \rho^{2} B_{o u t} z a^{3}}{\pi\left(z^{2}+a^{2}\right)^{3}} \text { for } z>0 .
\end{aligned}
$$




\section{Large-Signal Numerical Model}

This chapter describes the approach to large-signal modeling of the magnicon cavities. Large-signal modeling is used because small-signal modeling proved to be insufficient for several reasons:

- the boundary conditions are too restrictive,

- the solutions lack self-consistency, and

- the actual magnicon particle trajectories do not satisfy small-signal modeling criteria.

The generation of self-consistent solutions for the beam-driven cavities is discussed as well as the components of the large-signal model. This information will provide the details for implementing the large-signal model.

The application of the large-signal model to two regimes is described. One regime uses the rigid-beam approximation. This approximation is equivalent to assuming that a beam has an infinitesimally small radius. For this regime, none of the finite-beamsize effects are considered. The second regime uses the self-consistent solutions for cavity-field amplitude and phase taken from the rigid-beam model.

I will utilize the rigid-beam model to investigate the effects of parameter variation on the magnicon's simple and coupled passive cavities and on its output cavity. Comparisons will then be made between simple and coupled cavities in order to characterize their beam deflection. The rigid-beam model will finally be used to propose high-efficiency magnicon geometries and to evaluate a geometry for a high-efficiency CW magnicon proposed by its inventor, Dr. Oleg Nezhevenko.

I will utilize the finite-beam-size model to evaluate the impact of large beam radius. It will also be used to evaluate the sensitivity of the high-efficiency design created with the rigid-beam model to variations in beam size and to investigate methods for minimizing the performance degradation from increased beam size.

\subsection{Generation of self-consistent solutions for beam-driven cavities}

Two methods are presented here for calculating self-consistent solutions for the cavity field. One method, called the energy-balance approach, utilizes both the rf phase that extracts the most energy from the beam and the rf field amplitude that results in an 
energy balance between the power provided by the beam and the power dissipated in the cavity wall. The second method utilizes the beam's induced current to calculate the selfconsistent solutions. These methods will be briefly discussed below and then explored in depth in subsequent sections.

The main advantage of the energy-balance approach is the speed of its algorithm. The primary disadvantage is that the energy-balance approach is strictly an "onresonance" method. It does not consider the cavity detuning action of the beam. The effect of the cavity detuning on the self-consistent solution is such that the resulting solution is valid at the detuned resonance, which is very near the cold-cavity resonance and could be adjusted by tuning the cavity. Furthermore, the energy-balance method cannot be used to calculate the loaded $Q$ of the beam-driven cavity. In order to determine that, the frequency response of the cavity must be known. Unfortunately, the energybalance approach, because it is strictly a resonance calculation, cannot determine frequency response.

The induced-current method allows for the calculation of both beam loading and cavity detuning. These parameters are determined by calculating a range of selfconsistent solutions at frequencies around resonance. The loaded $\mathbf{Q}$ and cavity detuning are then determined from the frequency response. The disadvantage of the inducedcurrent method is the speed of its calculation. The convergence of the algorithm is quite slow, especially as conditions near resonance. However, when the self-consistent resonant solutions generated by the two approaches are compared, there is excellent agreement. Thus, the solution used for the bulk of the modeling was the energy-balance approach because of its speed. The induced-current method was used only to determine the attributes of the beam-driven cavity that required knowledge of the frequency response, such as the loaded $Q$ and the cavity detuning.

\subsubsection{Energy-balance approach}

The energy-balance approach to calculating the self-consistent solution for cavity amplitude consists of the following steps:

1. Select an initial value for cavity-field amplitude.

2. Determine the phase that extracts the most power from the beam. This phase is the self-consistent cavity phase. 
3. Calculate the power dissipated in the cavity walls for the current value of cavity-field amplitude.

4. If the power dissipated in the cavity walls is equal to the power provided by the beam, then the cavity-field amplitude is the self-consistent solution for the cavity amplitude. Using the self-consistent phase from Step 2, the self-consistent cavity field can be determined.

5. If the conditions of energy balance are not satisfied, then the cavity-field amplitude estimate is updated and the process repeats starting at Step 2.

Furthermore, a unique aspect of the nature of the beam/field interaction in the magnicon can be used to simplify the implementation of the energy-balance approach. In the beam-driven magnicon cavities, under the rigid-beam assumption of zero radius and in the steady state, each electron traverses the exact same if fields. This is due to the fact that both the entrance vector of the beam and the cavity fields are rotating at the rf frequency. Thus, although each subsequent particle that enters the cavity has an entrance vector that has shifted, the rf fields have shifted by the same amount. Therefore, the energy given up by each electron in the beam during the transit of the cavity is constant. Because each electron sees exactly the same if fields while in the cavity, a single electron trajectory can be used to calculate the self-consistent fields rather than an entire electron beam whose longitudinal length exceeds the cavity length. In addition, because the energy extracted from each electron as it passes through the cavity is a constant in time and is equal to the energy given up by the beam in the cavity at any moment in time, the time-averaged value of power provided by the beam is also a constant and equal to the energy provided by the integration of the electric field with the current of a single electron as it crosses the cavity. Thus, equating the energy lost by a single electron as it crosses the cavity to the time-averaged power dissipated in the walls provides the basis for the self-consistent solution of the energy-balance approach.

The initial guess at cavity-field amplitude for this approach should be as close as possible to the self-consistent amplitude in order to minimize the time required for the algorithm to converge on the self-consistent solution. The phase that extracts the most energy from the beam is determined from a component of the conservation of energy equation. From Ref. [1], the instantaneous real power supplied by the beam is given by

$$
P_{s}=-\iiint_{V o l u m e} \mathcal{E} \bullet \mathrm{J} d V
$$


where $\varepsilon$ is the instantaneous electric field and $\mathcal{J}$ is the instantaneous current density. For the rigid beam model, $\mathcal{J}$ is represented, according to Ref. [2], by

$$
\mathrm{J}=I_{0} \delta\left(x-x_{0}\right) \delta\left(y-y_{0}\right) \text {. }
$$

Substituting Eq. (4.2) into Eq. (4.1) results in

$$
P_{s}=-\int_{z} \mathcal{E}(x, y, z, t) I_{0} \delta\left(x-x_{0}\right) \delta\left(y-y_{0}\right) d x d y d z
$$

This equation is evaluated numerically as particles are advanced through the beam-driven cavity. For example, at a given cavity-field amplitude and phase, the electron is advanced through the cavity by a constant time step. The instantaneous electric-field value at the center of a time step is multiplied by the change in $z, \Delta z$, and the beam current. The variation in $\Delta z$ for the constant time step weights the numerical integral; as longitudinal velocity is transferred into the transverse direction, $\Delta z$ decreases, lessening its contribution to the numerical integral. This process is equivalent to a numerical integration with a constant step in $\Delta z$. Here, the beam current is weighted by the ratio of present longitudinal velocity to initial longitudinal velocity. The numerical integration can be represented as a summation by

$$
P_{S}=-\sum_{k} I_{0} \varepsilon_{k} \Delta z_{k}
$$

where $k$ represents the cavity transit time divided by the constant time step. The supplied power calculated in Eq. (4.4) is compared to the power dissipated in the cavity walls given in Eq. (3.76) as

$$
P_{d}=\frac{R_{d}}{2}\left(\frac{E_{o}}{\eta}\right)^{2} \pi J_{o}^{2}\left(\chi_{11}\right)\left[a^{2}+a h\right] .
$$

Note that Eq. (4.5) was developed for only one of the two standing-wave modes that make up the rotating mode, so the value of Eq. (4.5) is calculated from

$$
P_{d}=R_{d}\left(\frac{E_{o}}{\eta}\right)^{2} \pi J_{o}^{2}\left(\chi_{11}\right)\left[a^{2}+a h\right] .
$$




\subsubsection{Induced-current approach}

A numerical model based on the induced current leads to self-consistent solutions of the voltage excited by the presence of a beam in a passive cavity. This model is also useful for calculating beam-loading and cavity-detuning effects. Ramo's theorem, described in Refs. [3] and [4], gives the induced current for the dc case based on the motion of charge between two electrodes as

$$
i_{\text {ind }}=\frac{\int_{\text {volume }} J E d V}{V_{\text {norm }}} .
$$

In Eq. (4.7), $i_{i n d}$ is the induced current, $V_{\text {norm }}$ is a normalizing factor representing the voltage between the electrodes, $J$ is the current density, $d V$ is the cavity volume, and $E$ is the electric field between the electrodes. For the dc case, the induced current is a physically measurable quantity. It is the current at an instant in time flowing between the electrodes. For the rf case, the induced current no longer has a simple physical interpretation. It cannot be measured. Nonetheless, it provides a tool for the selfconsistent calculation of cavity fields driven by the beam. In the high-frequency case, the induced current can be described as a fictitious current source that can be combined with a lumped equivalent-circuit representation of a resonant cavity to simulate not only cavity wall loss but also the effects of loading and cavity detuning. These effects are due to the beam's influence on the cavity fields and the stored energy in the cavity. The definition of the induced current is not unique, but it is fixed by the cavity model and its cavity impedance.

The cavity voltage, $V_{c a v}$, is related to the induced current, $i_{i n d}$, by

$$
V_{c a v}=i_{i n d} Z_{c a v}
$$

where $Z_{C a v}$ is the impedance of the cavity with no beam present. The cavity voltage is the integral of the cavity field along the line of maximum cavity field. In Eq. (4.8), both $i_{i n d}$ and $V_{c a v}$ are in phasor notation with an $e^{j \omega_{n} t}$ time variation. An iterative solution of Eq. (4.8) gives the self-consistent beam-driven cavity voltage. This method requires an initial guess at the complex cavity voltage, $V_{0}$. Equation (4.7) then gives the induced current based on the beam distribution resulting from the field estimate. A new value for the cavity voltage, $V_{l}$, is calculated using Eq. (4.8). The initial guess is then replaced by 
a new cavity voltage, $V_{2}$, which is calculated from the initial guess and the value from Eq. (4.8) using

$$
V_{2}=\mathscr{F} V_{1}+(1-\mathcal{F}) V_{0},
$$

where

$$
-1 \leq \mathcal{F} \leq+1
$$

This process repeats until it converges upon the solution. The selection of $\mathcal{F}$ affects the stability and speed of convergence. While this approach has been used in the past to model klystron cavities (see Ref. [5]), this is the first application of this technique to modeling deflection-modulated amplifiers. The application of the induced-current method to the magnicon resulted from an effort to calculate the beam-loaded $Q$ and cavity detuning caused by the beam in the magnicon passive cavities.

The induced-current method is used to calculate the magnicon passive-cavity voltage at frequencies near the resonant frequency of the cold cavity. The bandwidth of the cavity in the presence of the beam gives the beam-loaded $Q$, and the peak of the voltage response indicates the frequency detuning. However, the nature of the magnicon cavity complicates this approach. The rf fields in the magnicon are a traveling wave, and it is necessary to decompose the traveling wave into two standing waves to use this approach. In addition, the unloaded and beam-loaded Q's of the magnicon cavities are much higher than those in a comparable velocity-modulated device like a klystron. This factor slows convergence.

The following proof demonstrates that Ramo's theorem, stated in Ref. [3], is valid in the steady state for cavities with large unloaded Q's. The method is similar to the development given in Ref. [5] but is only applicable for a current variation with a harmonic time variation. The development in Ref. [5] assumes only a periodic current density that can be decomposed into Fourier components as necessary for a velocitymodulated electron device with beam bunching. The results of the two models are identical.

Using normal-mode field expansions, Ref. [6] solves the steady-state excitation of fields in cavities by the volume distribution of currents. According to Ref. [6], the electric field of the $n$th mode resulting from the volume distribution of current follows from 


$$
e_{n}=-j \omega \mu \frac{\int_{v} J \cdot E_{n} d V}{k_{n}^{2}-k^{2}\left(1+\frac{1-j}{Q_{n}}\right)}
$$

and

$$
E=\sum_{n} e_{n} E_{n}
$$

$E$ represents the total field in the cavity from all modes, $k$ is the wave number, and the product of $e_{n}$ and $E_{n}$ represents that component of the total field associated with the $n$th mode. (For this derivation, all quantities are phasor representations with an implicit $e^{j \omega_{n} t}$ time variation. This accurately reflects the time variation of the current density for the magnicon.) We define the peak cavity voltage, $V_{n}$, for the $n$th mode as

$$
V_{n}=i_{\text {ind }} Z_{\text {cav }}
$$

or

$$
i_{\text {ind }}=\frac{V_{n}}{Z_{c a v}}
$$

where $Z_{c a v}$ is the empty cavity impedance. (Note: The definition for $V_{n}$ is not unique. It only needs to be consistent with the definition of $Z_{c a v}$. In this derivation, $V_{n}$ is defined as the peak cavity voltage.) Using a parallel RLC circuit representation of the resonant cavity, we can give the impedance by

$$
Z_{c a v}^{-1}=\frac{1}{R}+j\left(\frac{f}{f_{n}}-\frac{f_{n}}{f}\right) \frac{Q_{n}}{R},
$$

where $Q_{n}$ is the quality factor of the $n$th mode, $f$ is frequency, $R$ is the shunt resistance of the unloaded cavity for the $n$th mode, and $f_{n}$ is the resonant frequency of the $n$th mode. We can express the induced current as

$$
i_{\text {ind }}=\left(-\int_{l} e_{n} E_{n} \bullet d l\right)\left[\frac{1}{R}+j\left(\frac{f}{f_{n}}-\frac{f_{n}}{f}\right) \frac{Q_{n}}{R}\right] .
$$

Substitution of $e_{n}$ from Eq. (4.11) into Eq. (4.16) gives 


$$
i_{i n d}=j \omega \mu \frac{\left(\int_{l} E_{n} \bullet d l\right)\left[\frac{1}{R}+j\left(\frac{f}{f_{n}}-\frac{f_{n}}{f}\right) \frac{Q_{n}}{R}\right]\left(\int_{v} J \bullet E_{n} d V\right)}{k_{n}^{2}-k^{2}\left(1+\frac{1-j}{Q_{n}}\right)} .
$$

Expanding the denominator of Eq. (4.17) results in

$$
i_{i n d}=j \omega \mu \frac{\left[\int_{l} E_{n} \bullet d l\right]\left[\frac{1}{R}+j\left(\frac{f}{f_{n}}-\frac{f_{n}}{f}\right) \frac{Q_{n}}{R}\right]\left[\int_{v} J \bullet E_{n} d V\right]}{k_{n}^{2}-k^{2}\left(1-\frac{1}{Q_{n}}\right)+k^{2} \frac{j}{Q_{n}}} .
$$

For $Q_{n} \gg 1$, Eq. (4.18) becomes

$$
i_{i n d}=j \omega \mu \frac{\left[\int_{l} E_{n} \bullet d l\right]\left[\frac{1}{R}+j\left(\frac{f}{f_{n}}-\frac{f_{n}}{f}\right) \frac{Q_{n}}{R}\right]\left[\int_{v} J \bullet E_{n} d V\right]}{k_{n}^{2}-k^{2}+\frac{j k^{2}}{Q_{n}}} .
$$

Further simplification of the denominator results in

$$
i_{\text {ind }}=j \omega \mu \frac{\left[\int_{1} E_{n} \bullet d l\right]\left[\frac{1}{R}+j\left(\frac{f}{f_{n}}-\frac{f_{n}}{f}\right) \frac{Q_{n}}{R}\right]\left[\int_{V} J \bullet E_{n} d V\right]}{\frac{j \omega^{2}}{Q_{n} c^{2}}\left[1+j \frac{f_{n}}{f}\left(\frac{f}{f_{n}}-\frac{f_{n}}{f}\right) Q_{n}\right]} .
$$

For values of $f$ approximately equal to $f_{n}$, Eq. (4.20) becomes

$$
i_{\text {ind }}=\frac{\mu Q_{n} c^{2}}{\omega R}\left[\int_{l} E_{n} \bullet d l\right]\left[\int_{V} J \bullet E_{n} d V\right] .
$$

In Eq. (4.21), $Q_{n}$ is given by

$$
Q_{n}=\frac{\omega_{n} \frac{1}{2} \varepsilon \int_{v}\left|e_{n} E_{n}\right|^{2} d V}{\frac{1}{2 R}\left(\int_{l} e_{n} E_{n} \bullet d l\right)^{2}}
$$

This development is based on the normalized modes requiring 


$$
\int_{\nu}\left|E_{n}\right|^{2} d v=1
$$

Then, canceling the $e_{n}$ 's in Eq. (4.22), the equation simplifies to

$$
Q_{n}=\frac{\omega_{n} \varepsilon R}{\left(\int_{l} E_{n} \bullet d l\right)^{2}}
$$

Substitution into Eq. (4.21) gives

$$
i_{\text {ind }}=\frac{f_{n}\left[\int_{v} J \cdot E_{n} d V\right]}{f\left[\int_{l} E_{n} \bullet d l\right]} .
$$

When $f$ approaclies $f_{n}$, Eq. (4.25) reduces to

$$
i_{\text {ind }}=\frac{\left[\int_{v} J \bullet E_{n} d V\right]}{\left[\int_{l} E_{n} \bullet d l\right]} .
$$

Equation (4.26) is the same as Eq. (4.7), except it is in phasor form. There is an implicit $e^{j \omega_{n} t}$ time dependence in the equation, and Eq. (4.26) can be generalized to any of the $n$ modes as

$$
i_{\text {ind }}=\frac{\left[\int_{\nu} J \bullet E_{n} d V\right] e^{j \omega_{n} !}}{\left[\int_{l} E_{n} \bullet d l\right]}
$$

Equation (4.27) provides a guide to calculate the induced current. It is necessary to calculate the induced current from the instantaneous form of Eq. (4.27) at a number of time samples over a complete rf period. The current can then be decomposed into its phasor notation by evaluation of the Fourier coefficients. 
The induced-current method can be applied to the beam-driven magnicon cavities to determine the self-consistent solution for the cavity voltage (and consequently field). The nature of the beam/field interactions, already mentioned in the application of the energy-balance approach to calculating self-consistent solutions, also applies here. If a rigid-beam model is used (infinitesimally thin beam), then each particle in the beam sees the same passive cavity fields in the steady state because both the entrance vector of the beam and the rf fields rotate at the same rate. Therefore, it is possible to track only a single particle through the cavity and extract from the single-particle trajectory the position of the full beam at an instant in time. This characteristic also implies that the $J \cdot E$ integral in the rotating mode is a constant function of time. In order to apply the inducedcurrent method to the magnicon, the rotating mode is decomposed into the two standingwave modes. The self-consistent solutions for the two standing-wave modes are identical, with the expected phase shift.

The implementation of the induced-current method is quite similar to the energybalance approach. The primary steps are as follows:

1. Based on the initial guess or current computed value of the cavity field, a single electron is advanced across the cavity. The electron position at each time step is stored in an array.

2. The array is manipulated to determine the position of the beam at a time $t$. The manipulation involves rotating the angular polar coordinate of the array position by the product of the rf frequency (the speed of rotation of the entrance vector), the time interval separating the exit time of the cavity, and the time tag associated with the position being corrected. With this approach, the position that the rigid beam would occupy at time $t$ is extracted from the trajectory of a single electron. This extraction is repeated at a number of time steps evenly spaced between $t$ and $t+2 \pi / \omega$. A discrete Fourier analysis of these sample points is used to calculate the complex value of the induced current.

3. The induced current is multiplied by the cavity impedance to calculate a new estimate of the cavity voltage.

4. The new estimate of the cavity voltage is compared to the old estimate in order to determine if the solution has converged. If the solution has not converged, then the old estimate is updated by the new value and the process repeats. 


\subsection{Algorithm implementations}

The techniques used to advance the particles, calculate Bessel functions, and implement Bessel functions are described in Appendix A. The following sections address how these algorithms are used to model specific magnicon structures.

\subsection{Simple passive-cavity algorithm}

The passive-cavity algorithm serves two functions. It calculates the selfconsistent cavity-field amplitude and phase using the energy-balance approach, and it advances a single particle through the cavity. Section 4.1 , which described the energybalance approach, explained that due to the unique nature of the magnicon beam/field interactions, it was possible to characterize the behavior of the entire beam with a single particle.

The passive-cavity algorithm implements the energy-balance approach in this way. A user-defined search interval is selected for the determination of the selfconsistent phase. This interval divides the $2 \pi$ phase space into some number of equal intervals. For each of these intervals the particle is pushed through the cavity, and the energy extracted from the beam is calculated for each phase value. The energy extracted from the beam and corresponding phase value is stored in an array. The array entries are sorted, and the top two phases that extracted the most energy from the beam are identified. A phase between these values is calculated, and the energy extracted from the beam at this new phase is calculated. The new value is then inserted into the array at the third position, and the sort repeats. This process continues until the phase difference between the top two phases in the array is less than a user-defined threshold. When this happens, the average phase is then used to calculate the energy extracted from the beam again. This energy is then compared to that which is dissipated in the walls for the initial value of cavity amplitude. If agreement is within a user-defined threshold, the complete self-consistent solution for the cavity field has been determined. If the values are not within the threshold, then the cavity-field amplitude is adjusted and the process is repeated.

The energy extracted from the beam is calculated using Eq. (4.4). The energy supplied to the walls is twice the value calculated in Eq. (3.76). The particle position is advanced in accordance with the equations given in Appendixes A.1 and A.2, and the instantaneous form of the field expressions were presented in Eqs. (3.50) through (3.55). 
Another form of this algorithm was created to implement the induced-current method for a study of beam loading in passive cavities. However, this algorithm was never incorporated into the main program but was used as a stand-alone analysis tool for beam loading. (It was described in Section 4.1.2.)

\subsection{Coupled-cavity algorithm}

My approach to the coupled cavity can best be explained by considering an analogous system: two pendulums connected by a spring. These two pendulums, considered together, will either swing together with the same frequency (the zero mode) or opposite with the same frequency $\left(180^{\circ}\right.$ out of phase-the $\pi$ mode). The coupled cavity will operate in the same manner. The fields in the two cavities will either be in phase or $180^{\circ}$ out of phase, and the relative amplitude will depend on the coupling coefficient between the two cavities and how much energy is extracted in each cavity. (In Appendix A.4 an equivalent-circuit argument is provided to substantiate this assertion.) The description below considers only coupled cavities with the geometry shown in Figure 4.1 , which shows a cross section of a coupled cavity made up of two cylindrical cavities with an inductive coupling aperture at the outer radius of the cavity. In the figure, the two cavities have equal lengths. However, I have not limited the algorithm with this constraint. 


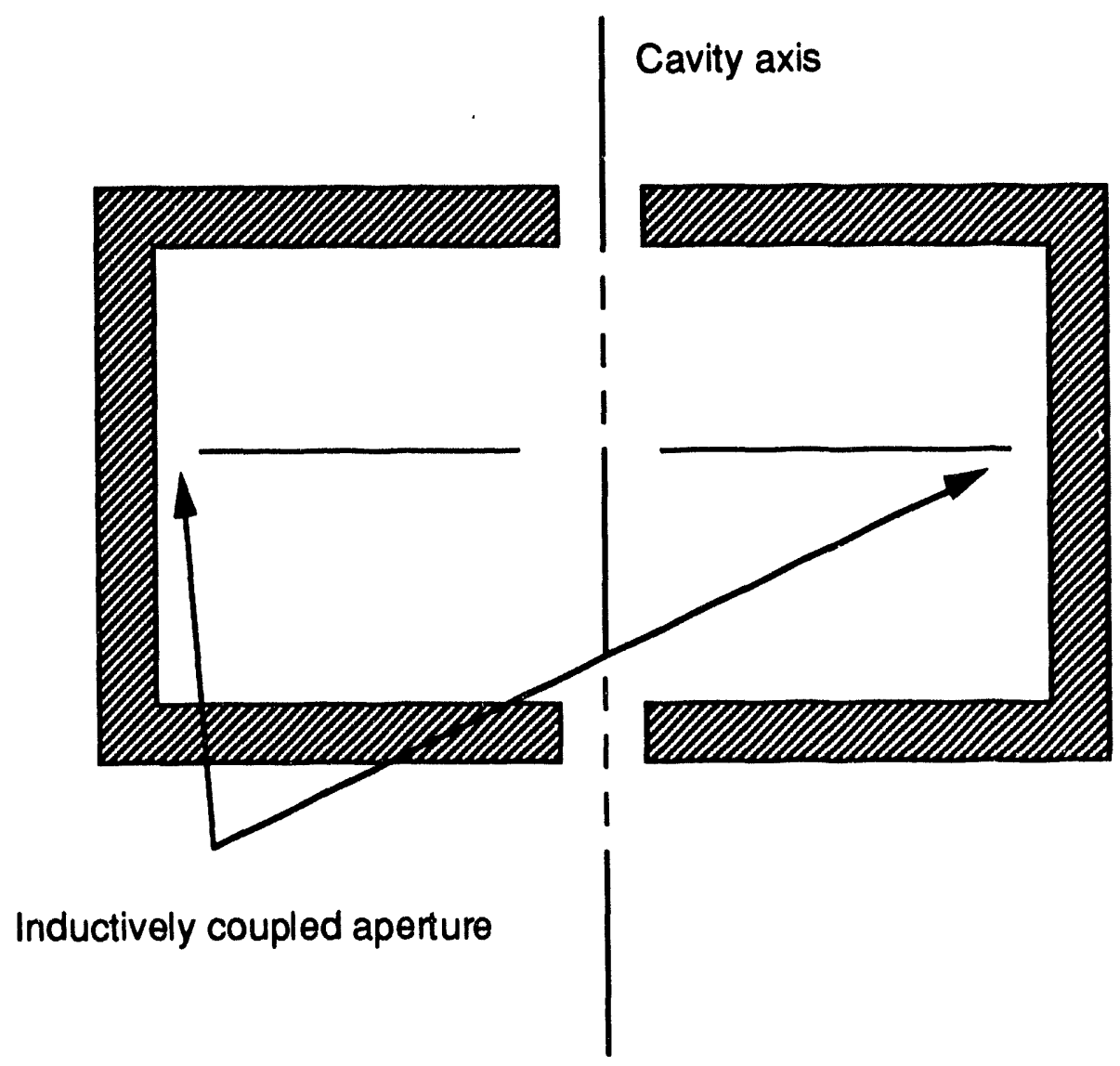

Figure 4.1. Coupled-cavity geometry.

The coupled-cavity algorithm assumes that the coupling coefficient is small. Therefore, from Eq. (A.49) it can be seen that the resonant frequency of the $\pi$ mode is approximately the resonant frequency of the simple cylindrical cavity; the coupled cavity would have the same radius and same field expressions as the simple cylindrical cavity. It is also assumed that the cavity-field amplitude is the same in each of the two cylindrical cavities that make up the coupled cavity. These two assumptions tie together and result in a third assumption: that the beam gives up approximately the same energy in each of the cavities. So the same field amplitude exists across the coupled cavity with only minimal coupling between the two individual cylindrical cavities.

The coupled-cavity algorithm is similar to the simple passive-cavity algorithm. The phase is selected that extracts the most energy from the beam, and then the energy extracted from the beam is balanced against the energy required by the wall for the field amplitude. The self-consistent solution is determined when the energies balance. The coupled-cavity algorithm uses the same approach as the simple cylindrical cavity algorithm to determine the phase of maximum energy extraction; however, when the 
particle passes the boundary between the two cavities, the rf phase is increased by $\pi$ in the second cavity.

\subsection{Output-cavity algorithm}

The output-cavity algorithm follows the passive-cavity algorithm, except that the routine does not iterate on the amplitude of the cavity field. Instead, the output cavity iterates on the phase as described in Section 4.3 in order to select the phase that extracts the most energy from the beam. The cavity-field amplitude is a user-defined input that is similar to those used in conventional design practices. In these conventional situations, the tube designer can select an output-cavity voltage by adjusting the coupling iris to the output cavity. The power extracted from the beam in the output cavity is the rf output power.

\subsection{Computer program organization}

The computer program I used to calculate beam dynamics is organized into subroutines called by the main program. The main program determines which subroutine to call based on the current longitudinal position of the particle. The main routine reads an input file, which, among other things, contains the start and stop coordinates of the drive cavity, simple passive cavities, coupled passive cavities, and the output cavity. The end coordinate of the deflection system is also read from the input file. The position of the particle at subsequent time steps is compared to these coordinates, and the appropriate subroutine call is executed based on the particle's longitudinal position. Program control resides in the main program until the particle enters a cavity (simple, coupled, or output), where computation of a self-consistent solution is required before the particle position can be advanced correctly. In this case, program control transfers to the subroutine, which calculates the self-consistent solution for the simple, coupled, or output cavity. Program control resides in these subroutines, which make calls to the other subroutines, until the self-consistent solution is determined. The subroutine remains in control until the particle is advanced to the end coordinate of the cavity. Control is then passed back to the main program.

The program treats all spaces between cavities as drift spaces where the beam experiences forces due only to the static $B_{z}$ component of the focusing field. However, the ending coordinate of the deflection system and the input coordinate of the output cavity are used in conjunction with the drift-space algorithm to model the fringe field associated with the pole piece at the input of the output cavity and the output of the 
deflection system. (The model was described in Section 3.3.) Provisions are also made within the output-cavity and coupled-cavity algorithms to include the effects of the fringe field at the output- and deflection-system solenoids.

\subsection{Rigid-beam model description}

The rigid-beam model is used to compute self-consistent solutions using the energy-balance approach described in Section 4.1.1 and the induced-current approach described in Section 4.1.2. The rigid-beam model was introduced in these sections and is described in more detail below.

The rigid-beam model is a modeling technique in which the beam is assumed to have an infinitesimally thin radius and is modeled by a spatial impulse of current. In other words, through any transverse cross section of the magnicon, the current would have a finite value at only one point. The rigid-beam model assumes a steady-state. The transient response is not considered. The model is three-dimensional and can utilize all field components. However, for the results presented here only the fundamental-mode field components are utilized, while the effects of the holes where the beam enters and exits the cavities are ignored. The model considers neither space-charge effects nor the effects of finite-beam diameter. Because of the unique nature of the magnicon's rotating fields and the rotating entrance vector of the beam into the cavities, the rigid-beam model for it is essentially a single-particle model, where the behavior of the magnicon can be fully characterized by the behavior of a single electron. 


\section{Applications of Rigid-Beam Model for Magnicon Structures}

In this chapter, the rigid-beam model is used to evaluate, characterize, and develop insight into the beam-field interaction in the component structures that make up the magnicon: the drive cavity, single passive cavity, coupled passive cavity, and output cavity. The beam-field interactions are evaluated several ways: by varying initial beam velocity vectors and positions as the beam enters these structures, by varying the geometry of the cavities, by varying the focusing-field strength, and, in the case of the output cavity, by varying the output-cavity voltage. The results of this chapter will provide the basis to generate new or improved magnicon designs.

Large amounts of data and performance plots have been provided in these sections. They are included to provide a tool for generating magnicon designs when an approximate geometry will suffice, or when establishing an initial geometry, which can then be further refined through large-scale modeling. Insights gained from the plots are presented immediately following each set of graphs.

\subsection{Rigid-beam model validation}

The material in this section serves to benchmark the code. Specific numerical examples are calculated using the large-signal rigid-beam model. These results are then compared to results calculated from the small-signal model. They show excellent agreement.

Figures 5.1 through 5.4 demonstrate the agreement between the small-signal theory and the large-signal simulation for a drive cavity. Figures 5.1 and 5.2 illustrate transverse displacement of the particle off-axis ( $x$ and $y$ ), while Figs. 5.3 and 5.4 illustrate transverse velocity ( $V x$ and $V y$ ). All of the data plotted in these figures was generated for the conditions listed in Table 5.1. The drive-cavity length is approximately the optimum length for $\omega / \Omega=1.4$ 
Table 5.1 Simulation parameters for Figs. 5.1 through 5.4.

\begin{tabular}{|c|c|}
\hline Beam Voltage & $170 \mathrm{kV}$ \\
\hline Beam Current & $18 \mathrm{~A}$ \\
\hline Frequency & $700 \mathrm{MHz}$ \\
\hline Time Step & $0.5 \times 10^{-12} \mathrm{~s}$ \\
\hline Drive Cavity Field & $0.12 \times 10^{6} \mathrm{~V} / \mathrm{m}$ \\
\hline$\Omega / \omega$ Ratio & 1.4 \\
\hline $\mathbf{v}_{\mathbf{z o}}$ & $1.981 \times 10^{8} \mathrm{~m} / \mathrm{s}$ \\
\hline
\end{tabular}




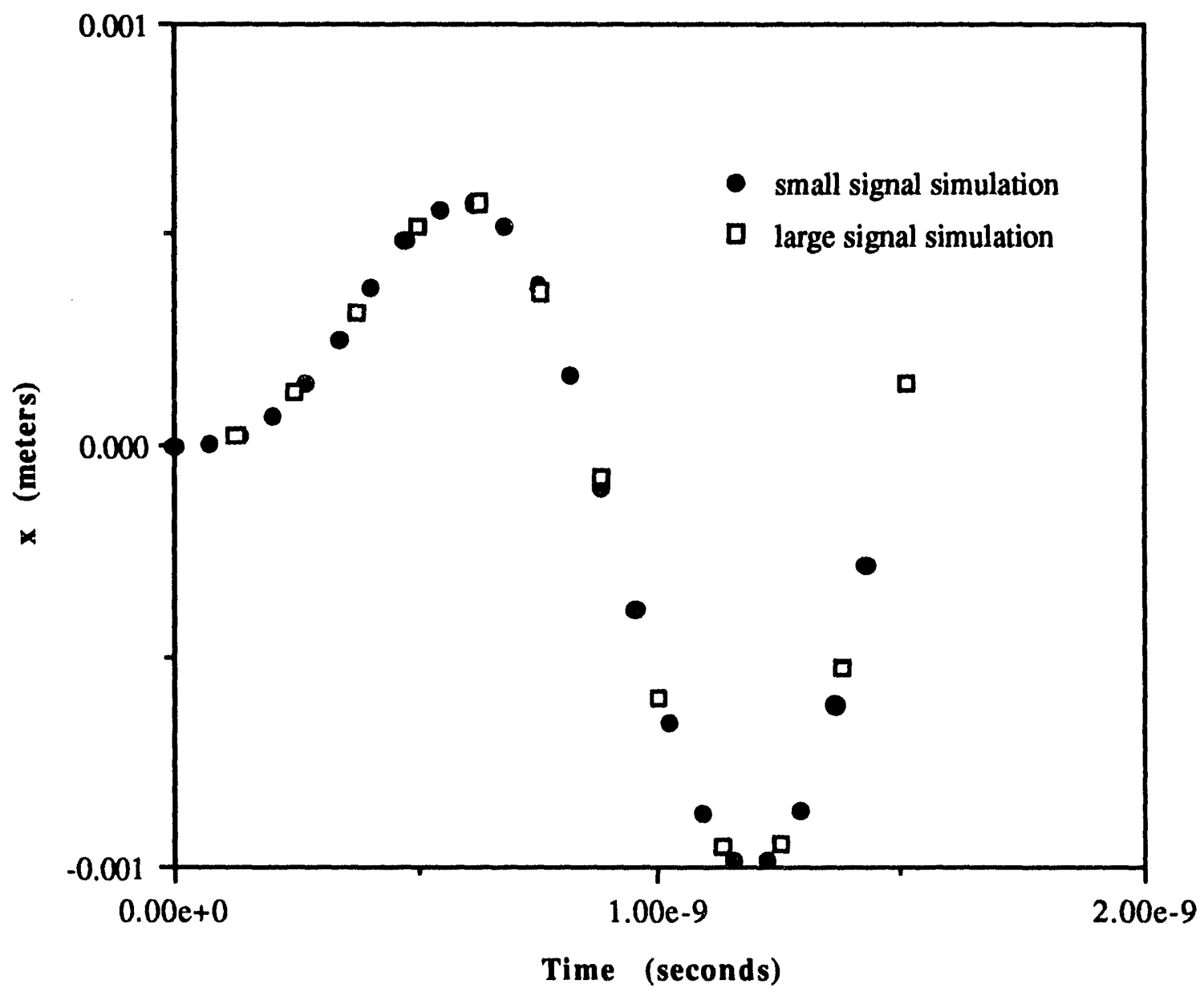

Figure 5.1. Drive-cavity comparison for displacement in $x$. 


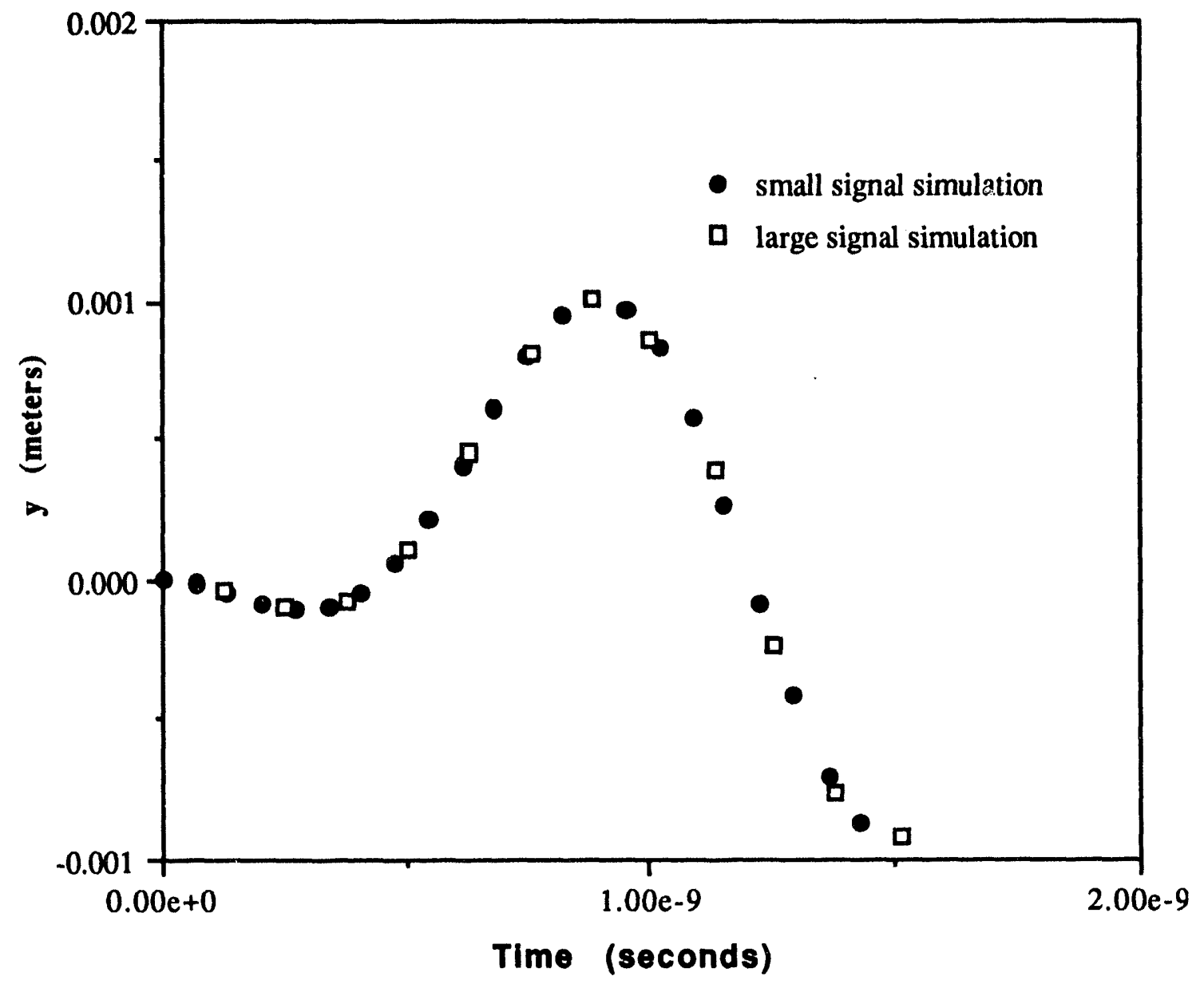

Figure 5.2. Drive-cavity comparison for displacement in $y$. 


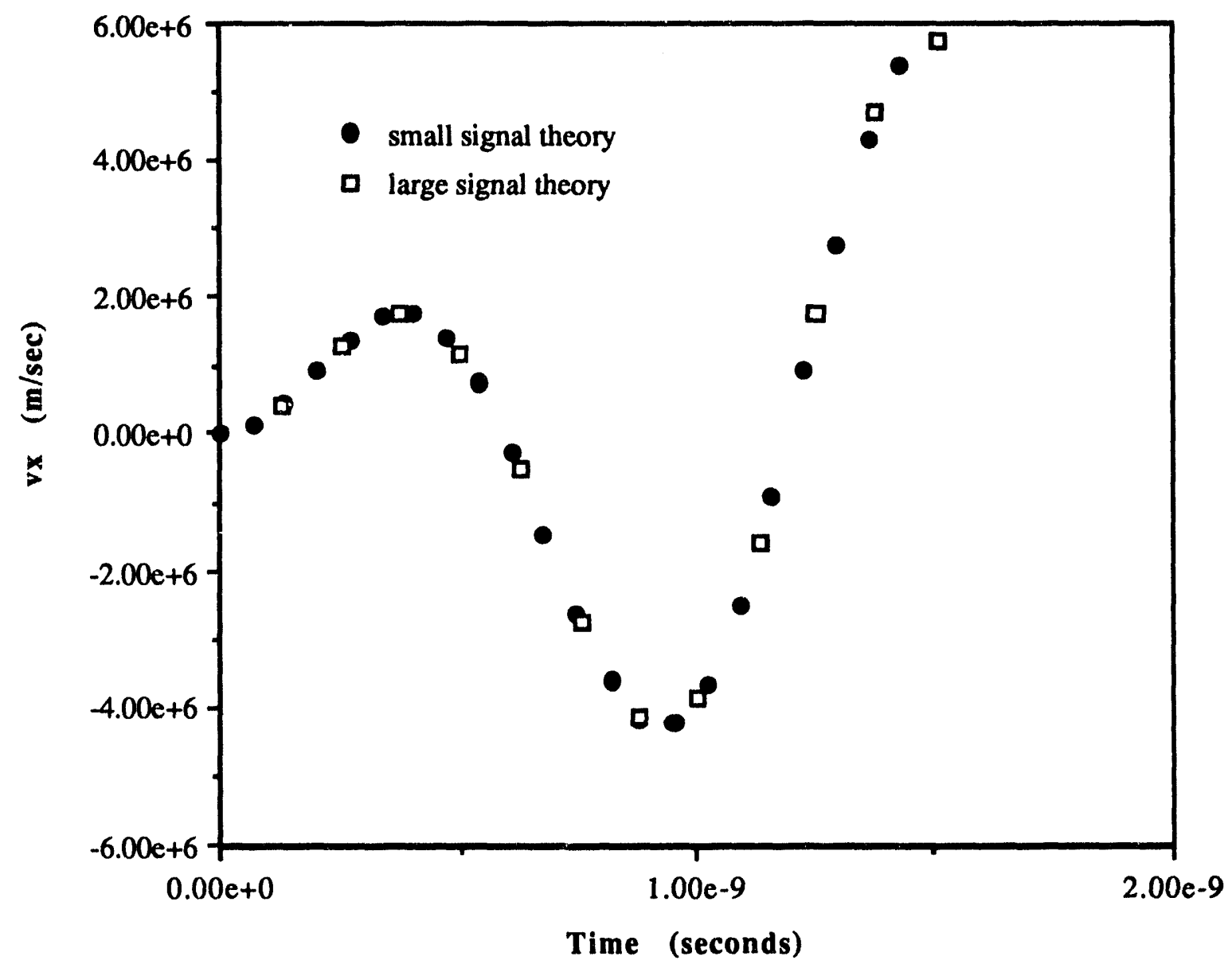

Figure 5.3. Drive-cavity comparison for velocity in $x$. 


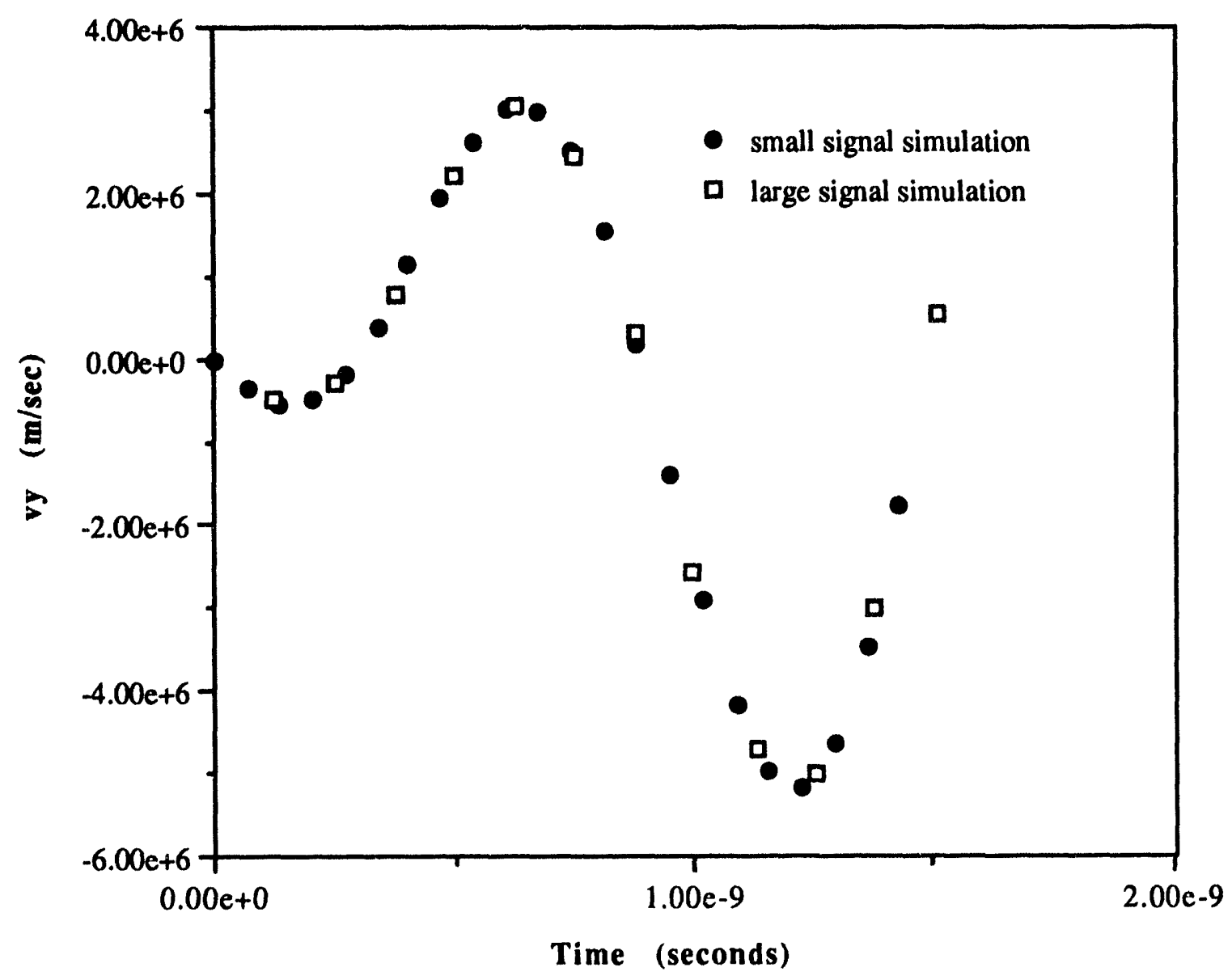

Figure 5.4. Drive-cavity comparison for velocity in $y$.

Figures 5.5 through 5.8 demonstrate the agreement between the small-signal theory and the large-signal simulation for the drift spaces. Figures 5.5 and 5.6 illustrate transverse displacement, while Figs. 5.7 and 5.8 illustrate transverse velocity. Figures 5.5 through 5.8 were generated under the conditions listed in Table 5.2. The drift-space length was approximately $0.2 \mathrm{~m}$. 
Table 5.2 Conditions for drift-space simulation.

\begin{tabular}{|c|c|}
\hline Beam Voltage & $170 \mathrm{kV}$ \\
\hline Beam Current & $18 \mathrm{~A}$ \\
\hline Frequency & $700 \mathrm{MHz}$ \\
\hline Time Step & $0.5 \times 10^{-12} \mathrm{~s}$ \\
\hline$\Omega / \omega$ Ratio & 1.4 \\
\hline $\mathbf{x}_{\mathbf{0}}$ & $2.02656 \times 10^{-4} \mathrm{~m}$ \\
\hline $\mathbf{y}_{\mathbf{0}}$ & $-9.14656 \times 10^{-4} \mathrm{~m}$ \\
\hline $\mathbf{v}_{\mathbf{x} 0}$ & $5715279.62 \mathrm{~m} / \mathrm{s}$ \\
\hline $\mathbf{v y}_{\mathbf{0}}$ & $815644.35 \mathrm{~m} / \mathrm{s}$ \\
\hline $\mathbf{v}_{\mathbf{z} 0}$ & $1.9808 \times 10^{8} \mathrm{~m} / \mathrm{s}$ \\
\hline
\end{tabular}

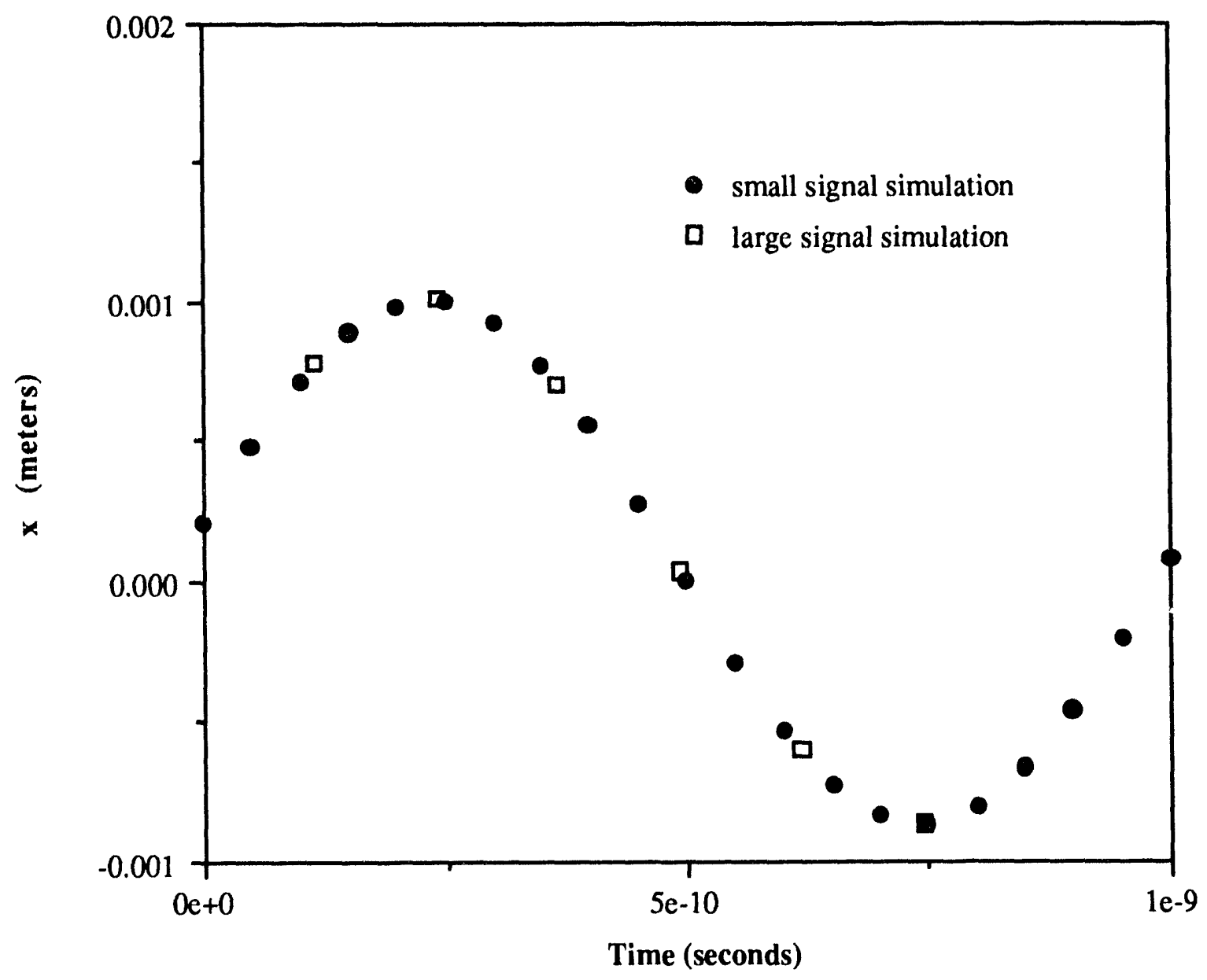

Figure 5.5. Drift-space comparison for displacement in $x$. 


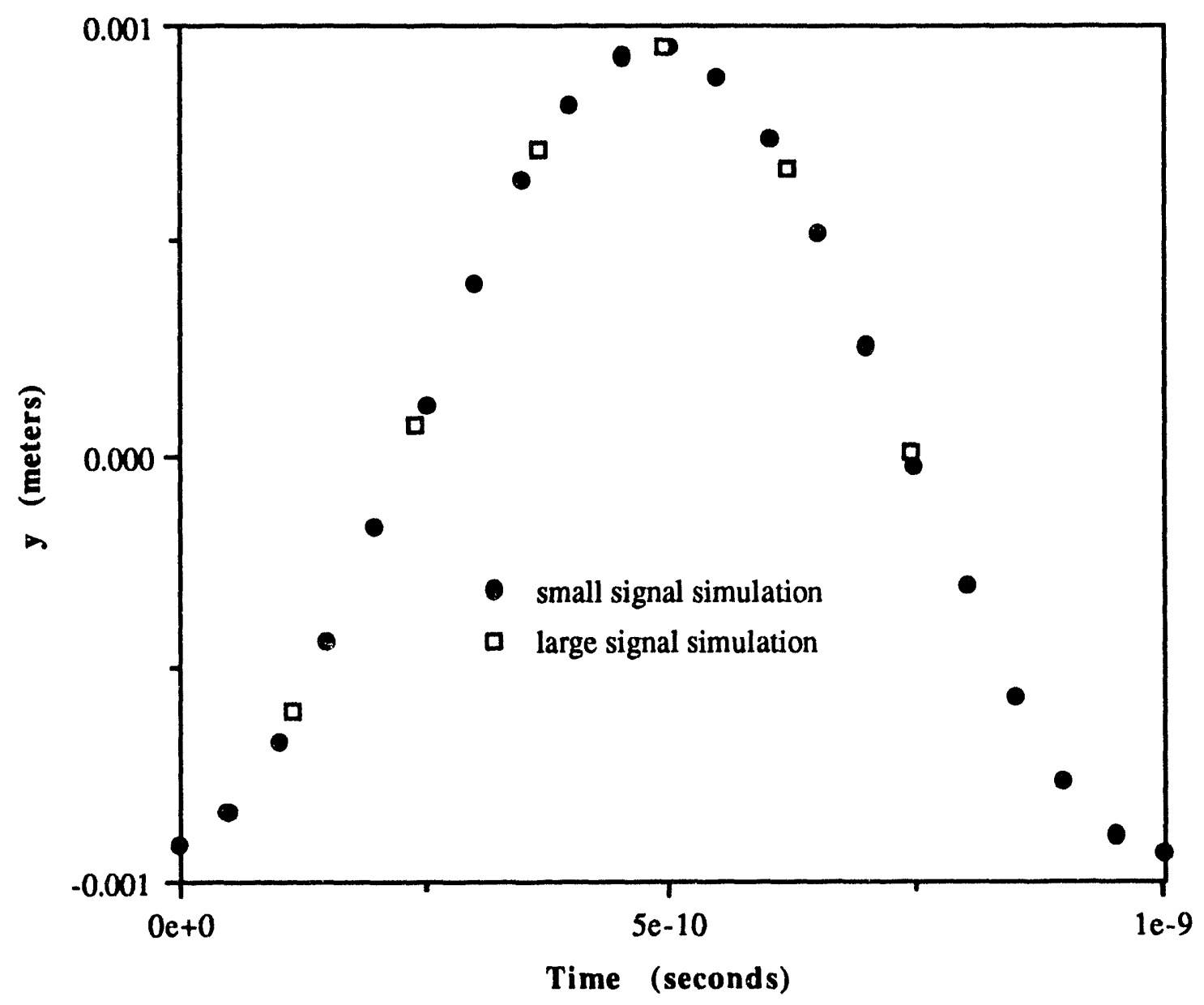

Figure 5.6. Drift-space comparison for displacement in $y$. 


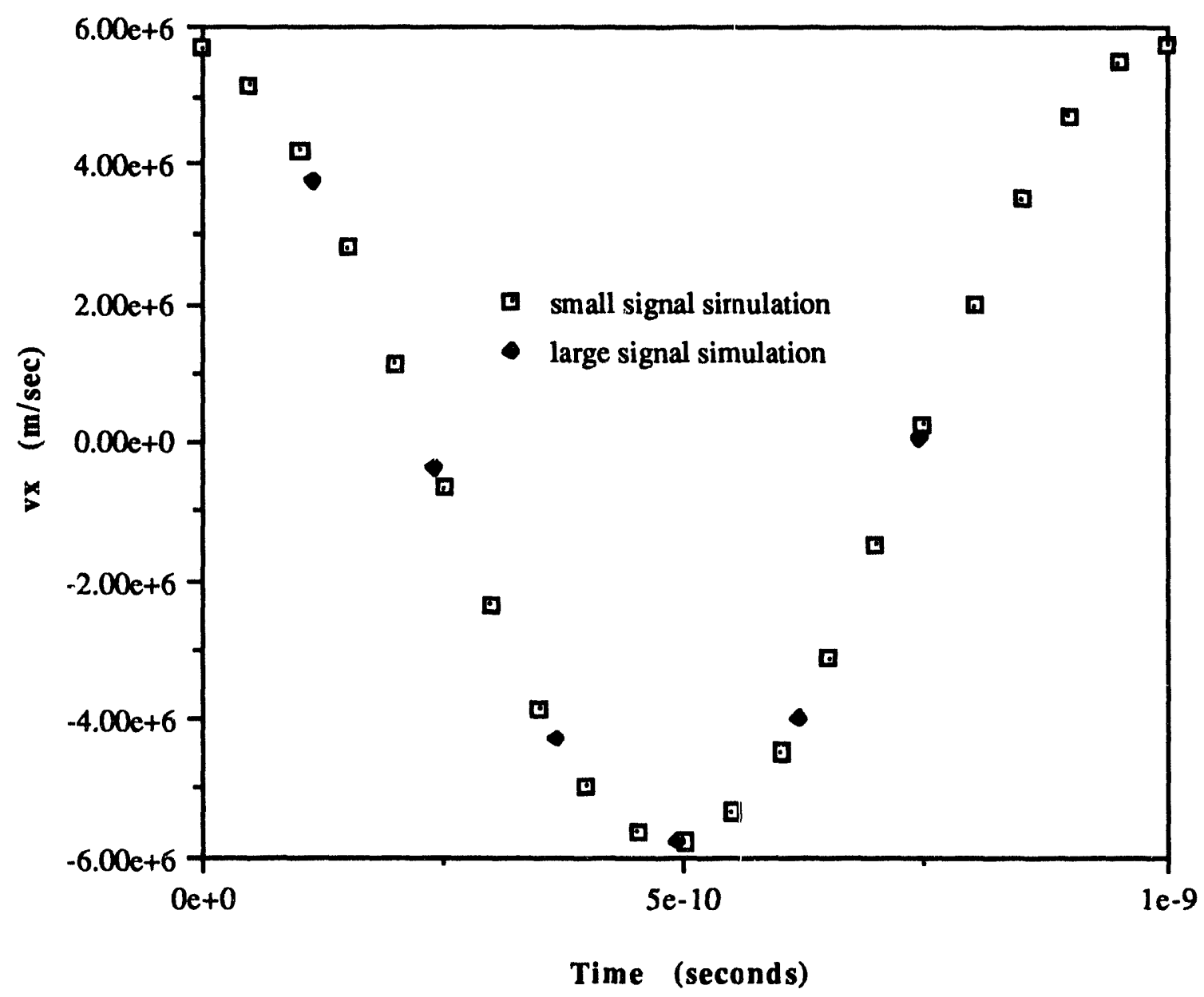

Figure 5.7. Drift-space comparison for velocity in $x$. 


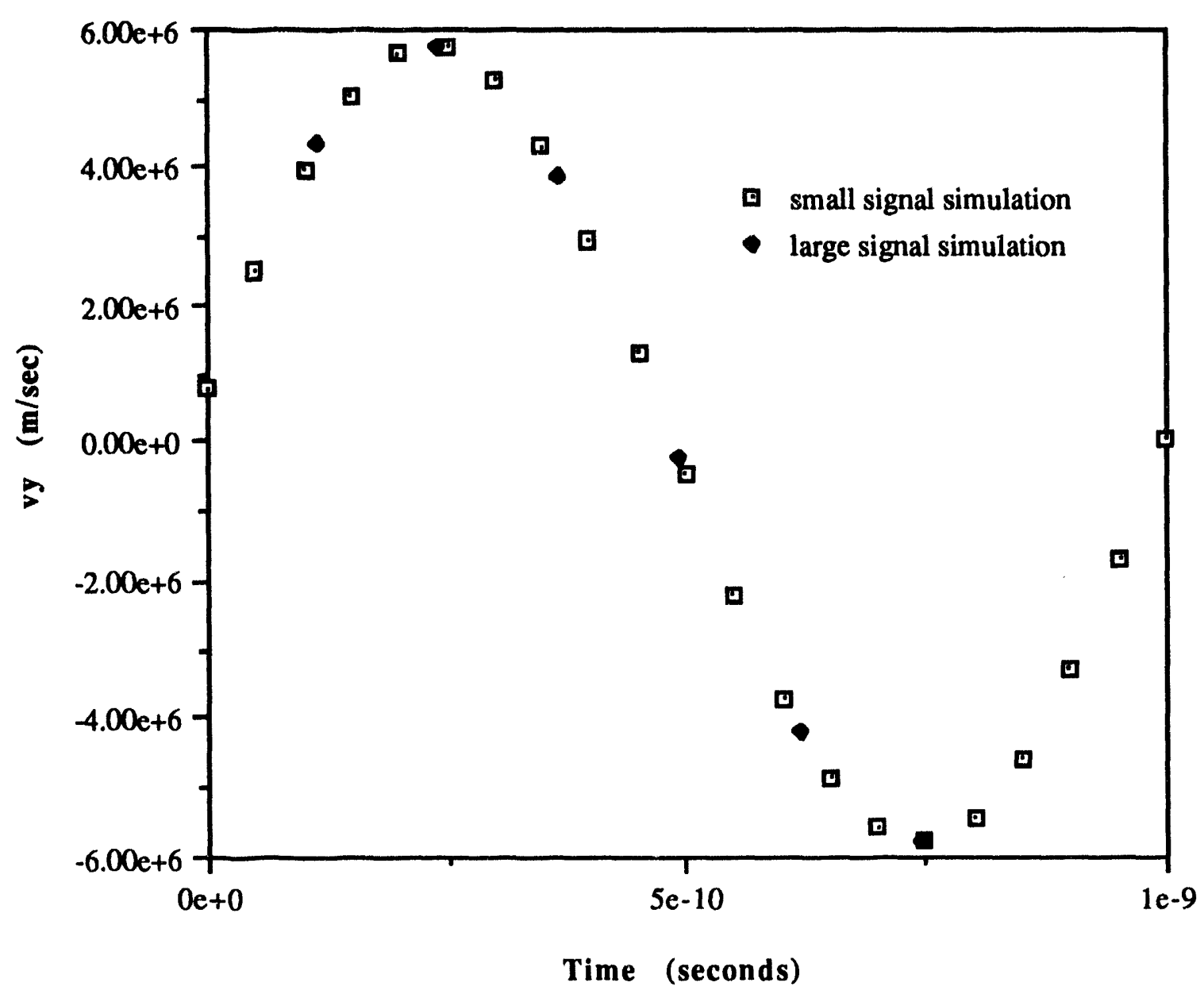

Figure 5.8. Drift-space comparison for velocity in $y$.

Figures 5.9 through 5.12 demonstrate the agreement between the small-signal theory and the large-signal simulation for the passive cavity. Figures 5.9 and 5.10 illustrate transverse displacement, while Figs. 5.11 and 5.12 illustrate transverse velocity. Figures 5.9 through 5.12 were generated under the conditions listed in Table 5.3. The passive-cavity length is approximately $0.2 \mathrm{~m}$. 
Table 5.3 Conditions for passive-cavity simulation.

\begin{tabular}{|c|c|}
\hline Beam Voltage & $170 \mathrm{kV}$ \\
\hline Beam Current & $18 \mathrm{~A}$ \\
\hline Frequency & $700 \mathrm{MHz}$ \\
\hline Time Step & $0.5 \times 10^{-12} \mathrm{sec}$ \\
\hline$\Omega / \omega$ Ratio & 1.4 \\
\hline $\mathbf{x}_{\mathbf{0}}$ & $-5.9231 \times 10^{-4} \mathrm{~m}$ \\
\hline $\mathbf{y}_{0}$ & $-3.5077 \times 10^{-4} \mathrm{~m}$ \\
\hline $\mathbf{v}_{\mathbf{x} 0}$ & $2253045.16 \mathrm{~m} / \mathrm{sec}$ \\
\hline $\mathbf{v}_{\mathbf{y}}$ & $-5315399.94 \mathrm{~m} / \mathrm{sec}$ \\
\hline $\mathbf{v}_{\mathbf{z O}}$ & $1.9808 \times 10^{8} \mathrm{~m} / \mathrm{sec}$ \\
\hline
\end{tabular}




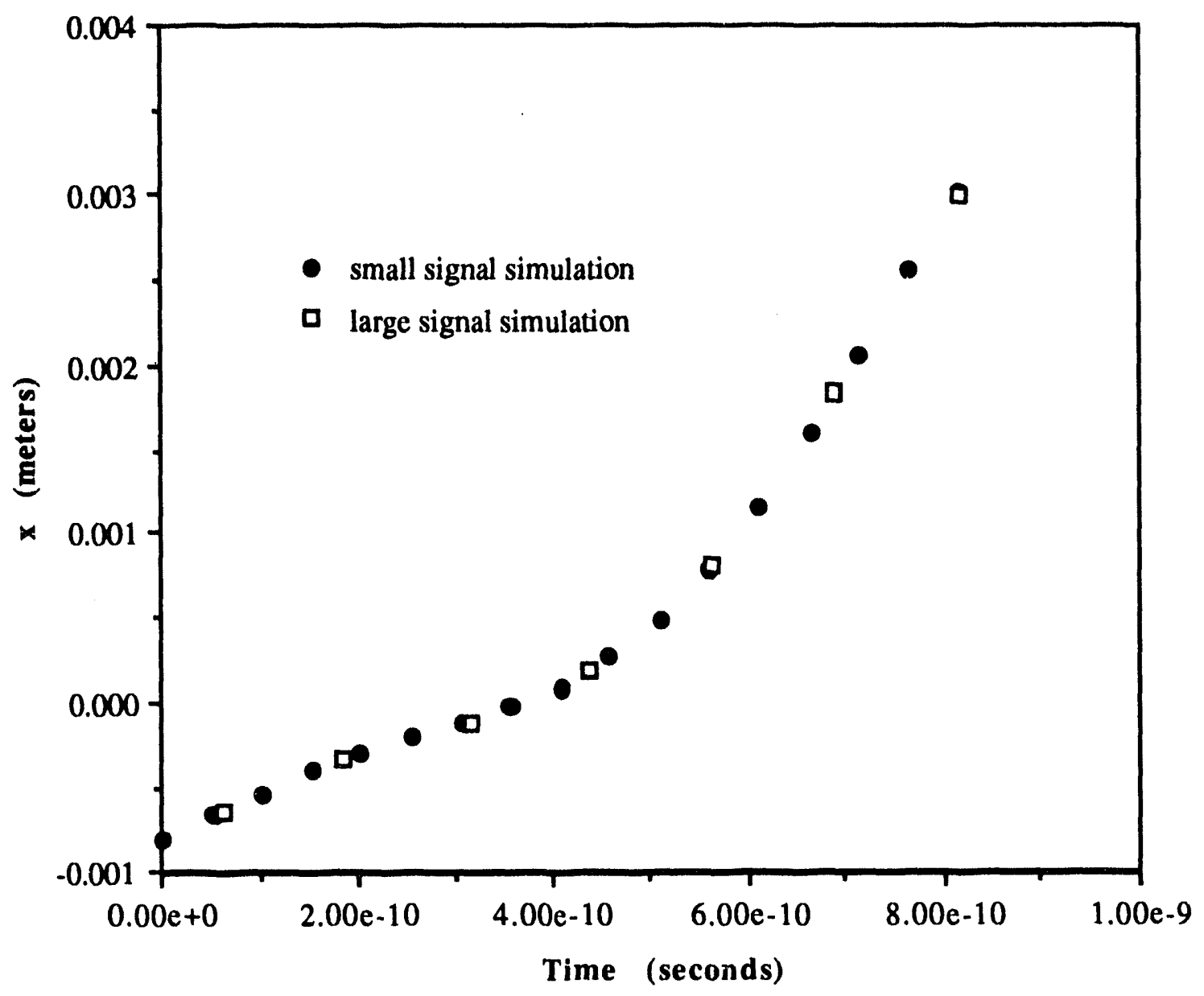

Figure 5.9. Passive-cavity comparison for displacement in $x$. 


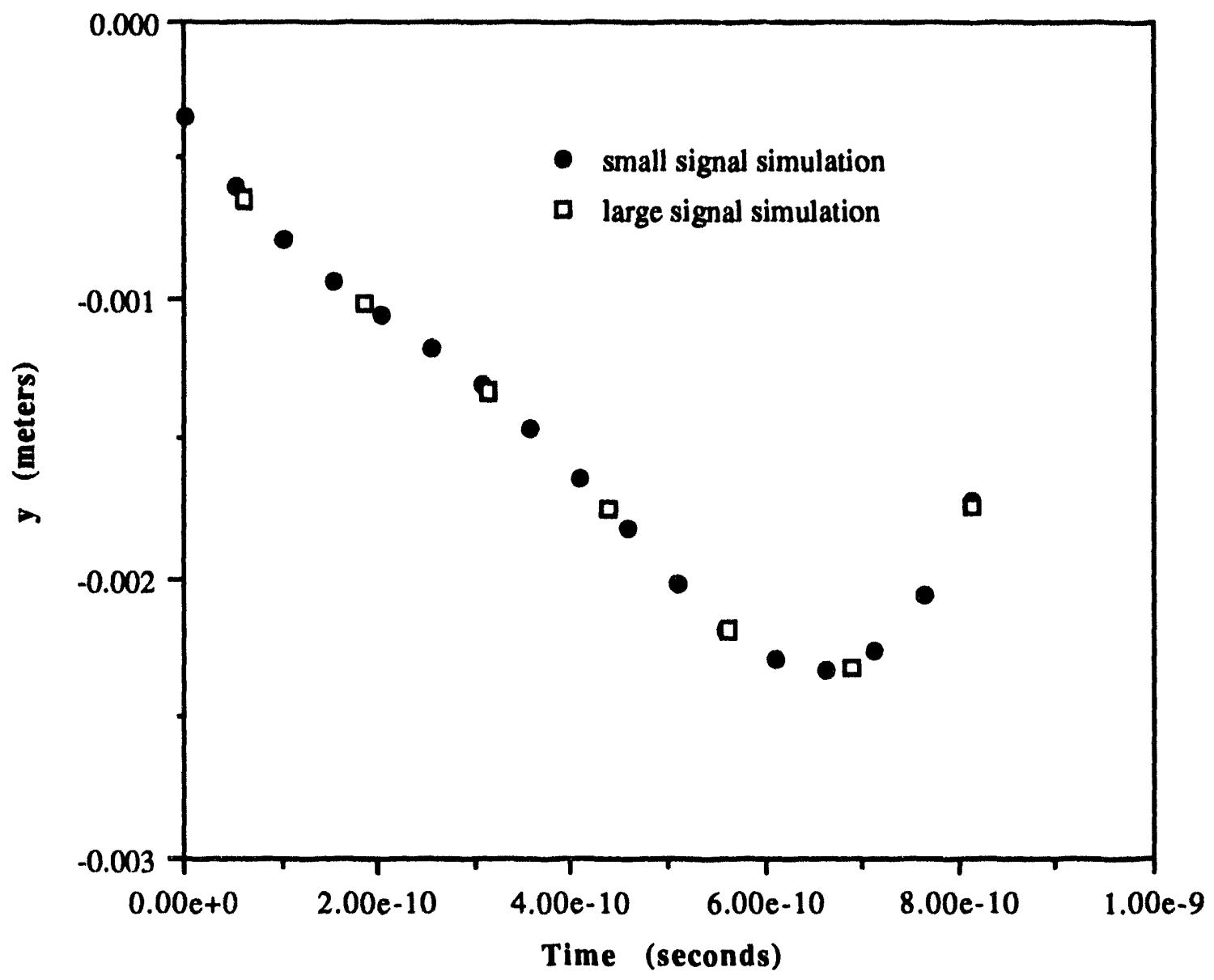

Figure 5.10. Passive-cavity comparison for displacement in $y$. 


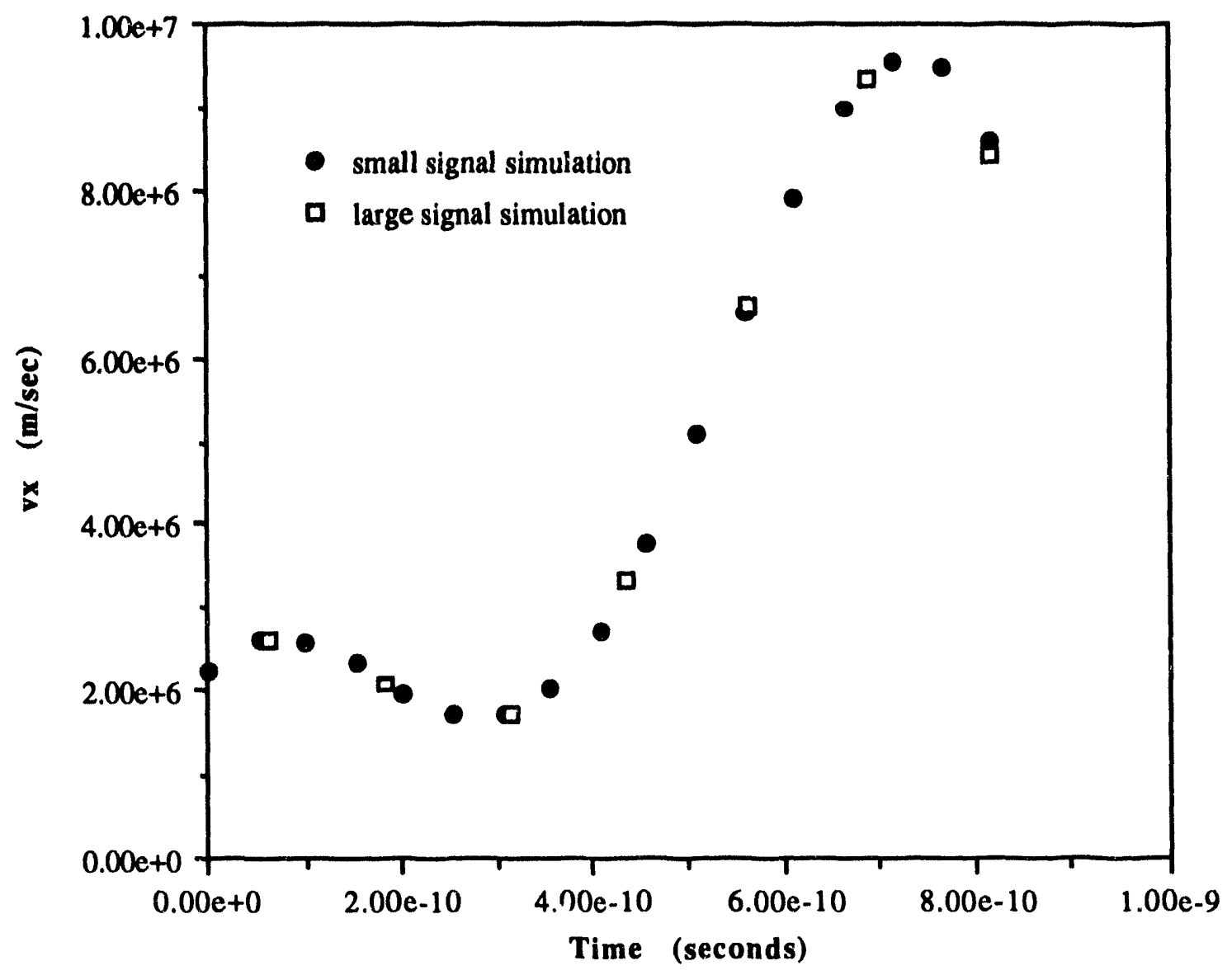

Figure 5.11. Passive-cavity comparison for velocity in $x$. 


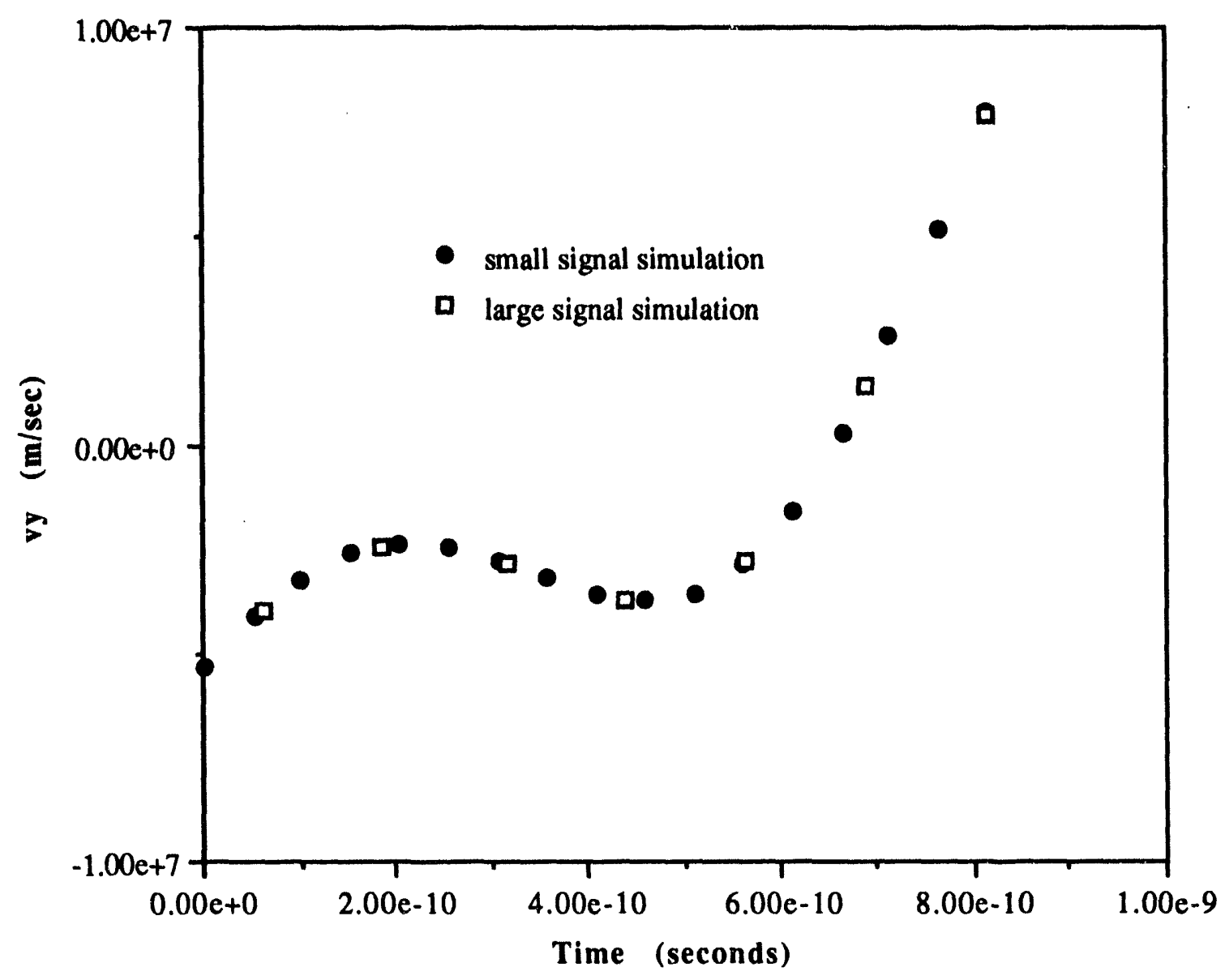

Figure 5.12. Passive-cavity comparison for velocity in $y$.

Even a cursory glance at these figures confirms that there is excellent agreement between the small-signal and large-signal simulation. (This is not unexpected, because the large-signal simulations were conducted using cavity field strengths that would result in trajectories in the small-signal regime.) These figures also serve as a validation of the rigid-beam model. The data for the small-signal simulation was generated on a spreadsheet, while the large-signal simulation data was generated using the large-signal code implementing the rigid-beam model. Due to the restrictive nature of the boundary conditions for the small-signal theory, minor large-signal code modifications were required to zero the time when the beam entered the drift space or passive cavity. However, no changes to algorithms in the large-signal code were required. 


\subsection{Optimum drive-cavity length}

The optimum drive-cavity length was calculated in Section 3.2.1.1 using smallsignal theory. It is expected that the small-signal theory will be valid in the drive cavity because the electron beam stays very near the axis. This section will verify that expectation as well as investigate the effect of drive field levels that are no longer in the small-signal regime. While these drive fields may be interesting in themselves, the magnitudes required to evoke large-signal behavior would limit the magnicon to very low power gain $(<10 \mathrm{~dB})$. Therefore, this does not represent a design that anyone would ever seek to physically realize. Except where noted, all simulations in this section use the baseline set of parameters described in Section 2.3.

Section 3.2.1.1 indicated that the cavity transit time that maximizes the conversion of longitudinal to radial velocity is

$$
t=\frac{\pi}{\Omega-\omega} .
$$

The cases investigated in this section are for $\Omega / \omega=1.0,1.4$, and 1.8 . For a $700-\mathrm{MHz}$ if frequency, the optimum cavity transit angles calculated from small-signal theory are:

$$
\begin{aligned}
& \text { for } \frac{\Omega}{\omega}=1.0 \quad t=\infty, \\
& \text { for } \frac{\Omega}{\omega}=1.4 \quad t=1.79 \times 10^{-9} \text { seconds, }
\end{aligned}
$$

and

$$
\text { for } \frac{\Omega}{\omega}=1.8 \quad t=8.93 \times 10^{-10} \text { seconds . }
$$

In the figures presented below, the drive-cavity output angle is plottec as a function of drive-cavity transit time. The corresponding drive pu wer required to achieve the transit time and output angle is also illustrated in the figures. Figures 5.13 and 5.14 are for $\Omega / \omega=1.0$. Figure 5.13 shows the drive-cavity output angle for three values of cavity field: $0.1 \mathrm{MV} / \mathrm{m}, 0.7 \mathrm{MV} / \mathrm{m}$, and $3 \mathrm{MV} / \mathrm{m}$. Figure 5.14 shows the corresponding drive power for the $0.1-\mathrm{MV} / \mathrm{m}$ drive-cavity field case. This field level was selected because it is consistent with the design of a moderate-gain magnicon. The other field levels require a drive level that is a significant portion of the total beam energy. Therefore, they do not allow for the realization of a high-gain device. In the other figures 
shown below, I do include some plots of the drive power for drive-cavity field levels in excess of $0.1 \mathrm{MV} / \mathrm{m}$. This results from the fact that the drive power required for higher field excitations decreases with increasing values of $\Omega / \omega$. In all plots an effort is made to describe only those drive power curves that would provide a reasonable level of gain for the magnicon, as specified by the baseline parameters.

The power required to support the drive-cavity fields is calculated from two components. The first component comes from Eq. (3.76). The power calculated using this equation is doubled to account for the two $\mathrm{TM}_{110}$ modes that comprise the rotating fields. The other component of drive power is calculated from the increase in the particle gamma as the electrons in the beam cross the drive cavity. This component is calculated according to

$$
\frac{\gamma_{\text {exit }}-\gamma_{\text {entrance }}}{\gamma_{\text {entrance }}}=\frac{\text { Pbeam }_{\text {exit }}-\text { Pbeam }_{\text {entrance }}}{\text { Pbeam }_{\text {entrance }}} .
$$

This second component is equal to the change in beam power and is added to the first component to determine the drive power required to support the drive cavity field. 


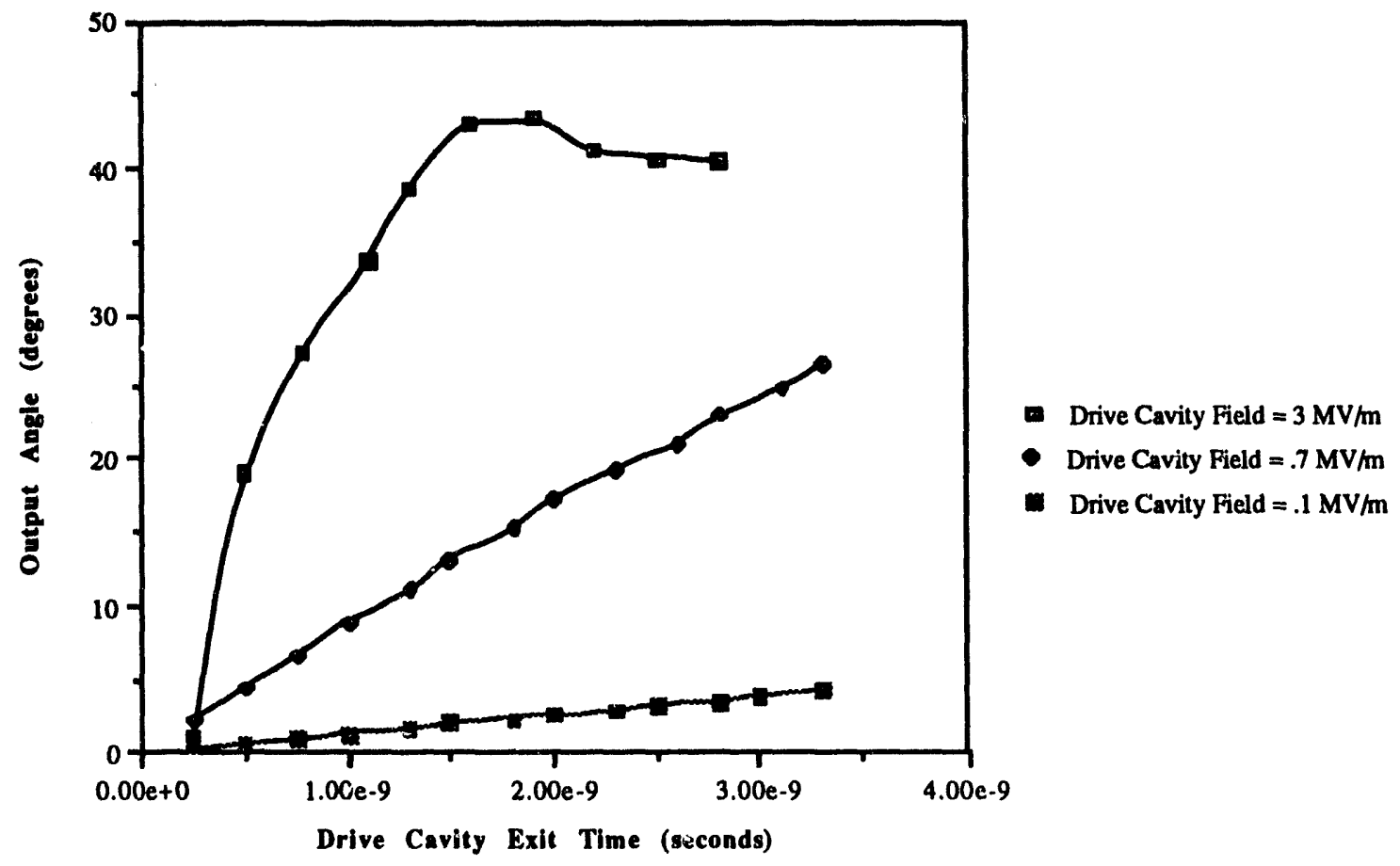

Figure 5.13. Output angle as a function of drive cavity exit time for $\Omega / \omega=1.0$. 


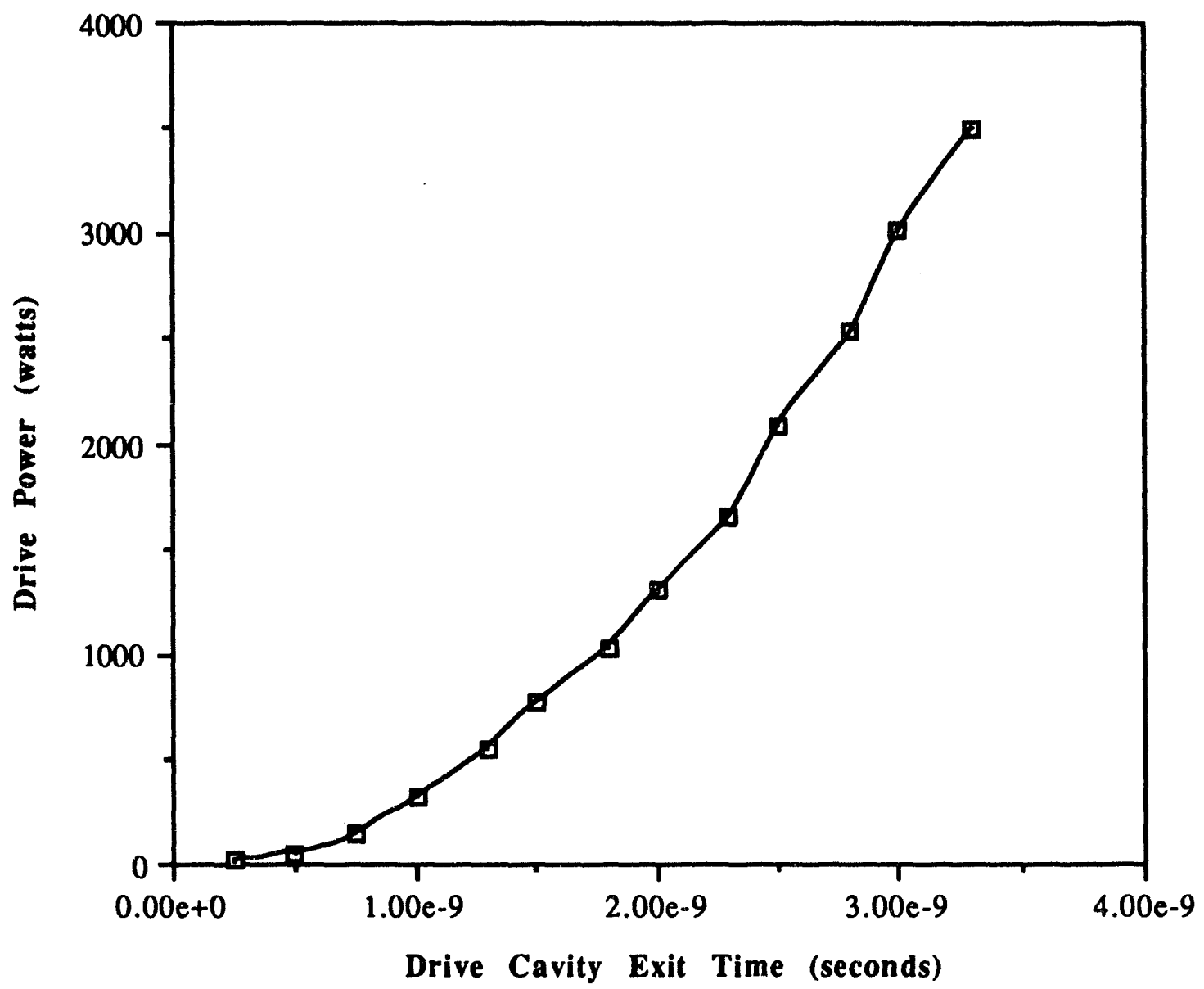

Figure 5.14. Drive power for the $0.1-\mathrm{MV} / \mathrm{m}$ curve of Fig. 5.15 versus cavity exit time plotted against the same abscissa as Fig. 5.15.

Figure 5.13 illustrates what is expected from the small-signal theory: that the optimum cavity length is infinite in length. This is seen in the two curves on Fig. 5.13 representing the lower field values. This is an expected result. Because the rf and cyclotron frequency are in phase, the electron beam experiences a constant deflecting force. This is only true in the small-signal regime, however. In the large-signal regime, the rf and cyclotron frequency will no longer be in phase, because the beam gains energy from the drive-cavity fields and the beam gamma changes. As is also seen in the curve corresponding to $3 \mathrm{MV} / \mathrm{m}$ in Fig. 5.13, the large-signal effects do result in an optimum length for high drive-cavity fields. 
Figure 5.15 illustrates the output angle as a function of the cavity transit time for the same values of drive-cavity field as Fig. 5.13, except that $\Omega / \omega=1.4$. Note that for the lower values of the cavity field, the optimum cavity transit time is consistent with the time predicted by the small-signal iheory. Comparing Figs. 5.13 and 5.15 shows that more longitudinal velocity is converted into radial velocity for lower values of $\Omega / \omega$. The drive-power requirements for the two lowest-field curves of Fig. 5.15 are shown in Figs. 5.16a and 5.16b. By comparing Figs. 5.14 and 5.16a, it can be seen that the lower value of $\Omega / \omega$ requires a substantially increased drive-power level to obtain the same output angle. For example, for a drive-cavity field of $0.3 \mathrm{MV} / \mathrm{m}$ to obtain an output angle of $0.7^{\circ}, 70 \mathrm{~W}$ of drive power is required at $\Omega / \omega=1.0,56 \mathrm{~W}$ of drive power is required at $\Omega / \omega=1.4$, and $45 \mathrm{~W}$ of drive power is required at $\Omega / \omega=1.8$. (This is also consistent with the sm.21l-signal analysis beam-loading calculations for the drive cavity.)

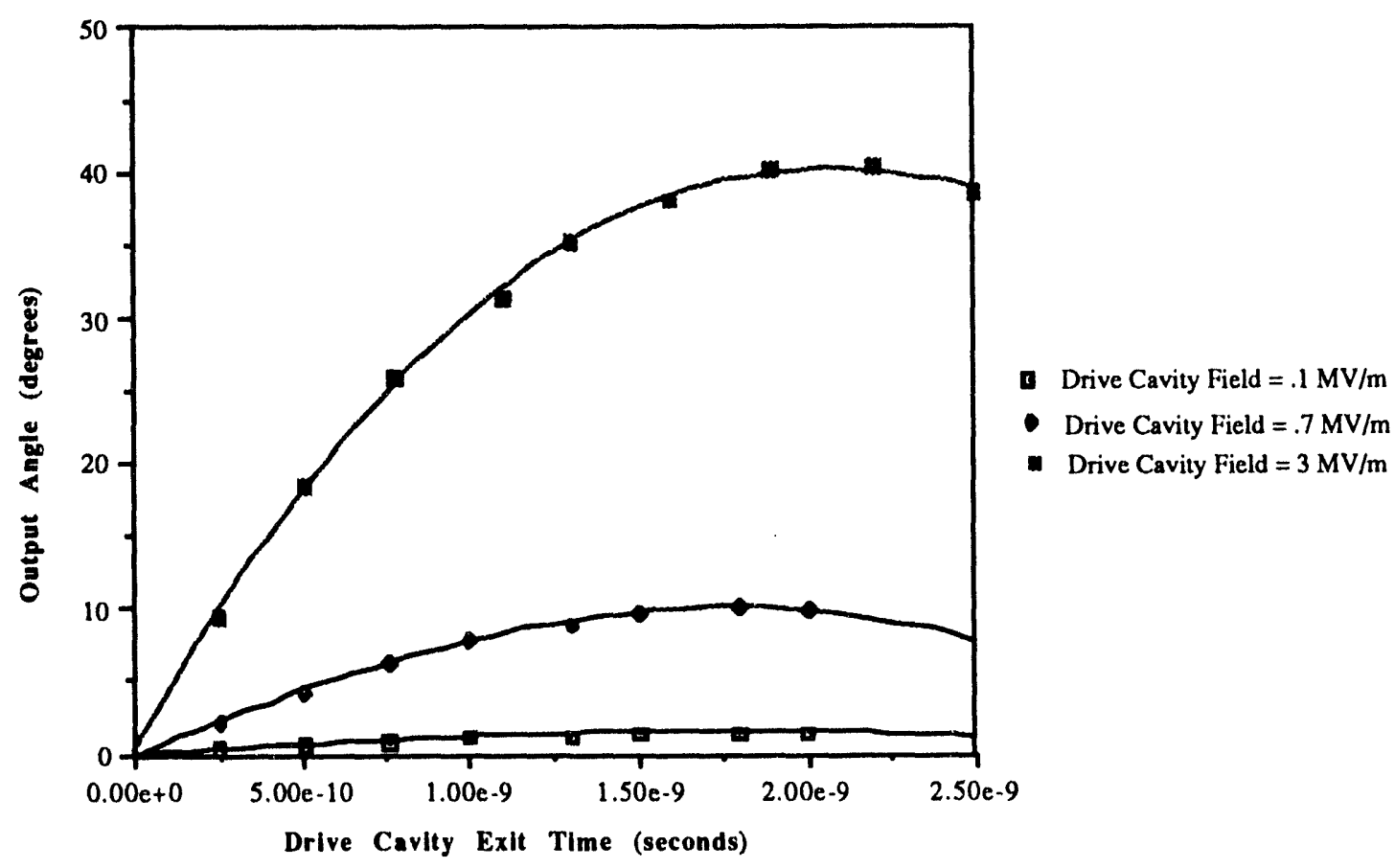

Figure 5.15. Output angle as a function of drive cavity exit time for $\Omega / \omega=1.4$. 


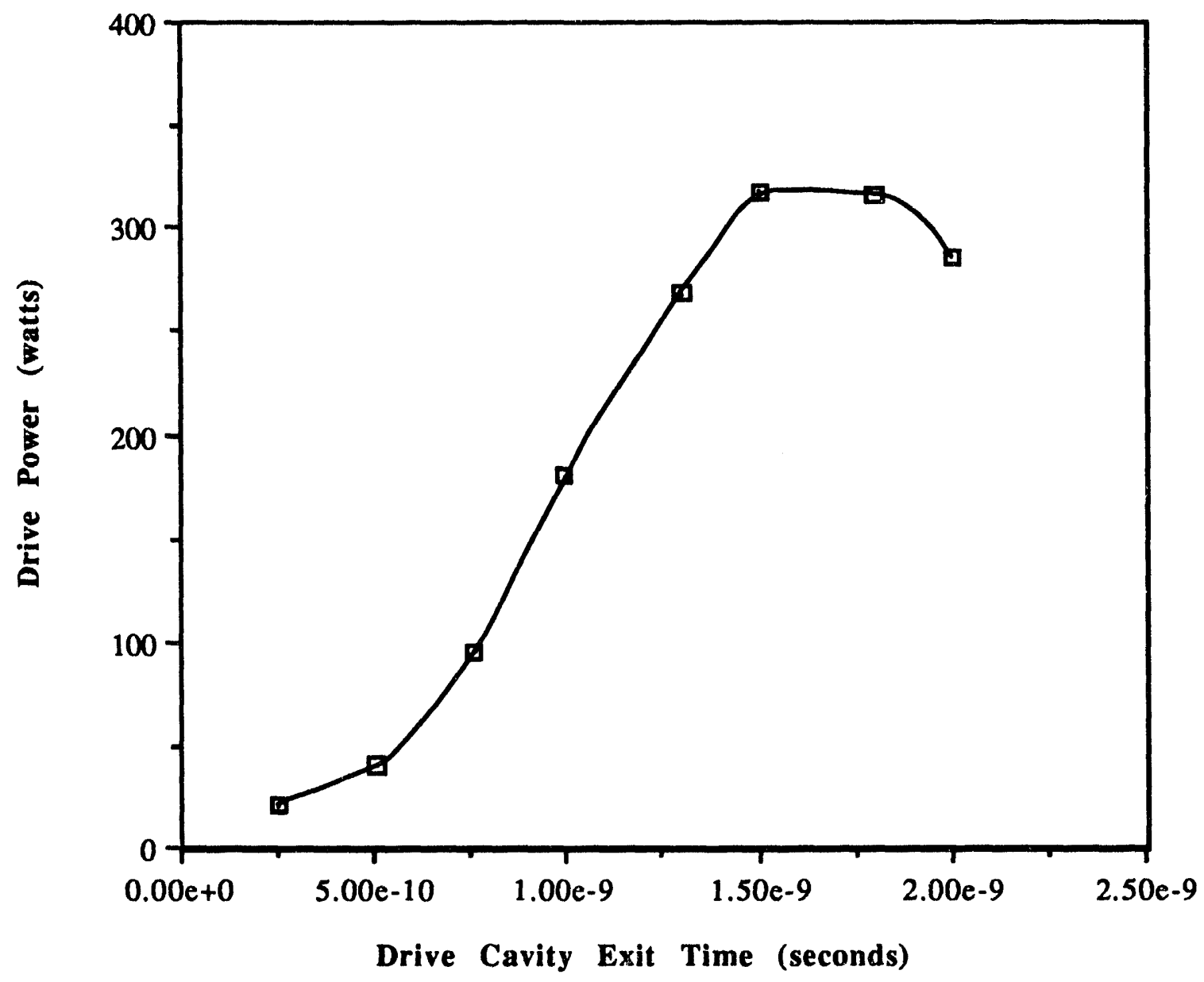

Figure 5.16a. Drive power for the $0.1-\mathrm{MV} / \mathrm{m}$ curve of Fig. 5.15 versus cavity exit time plotted against the same abscissa as Fig. 5.15. 


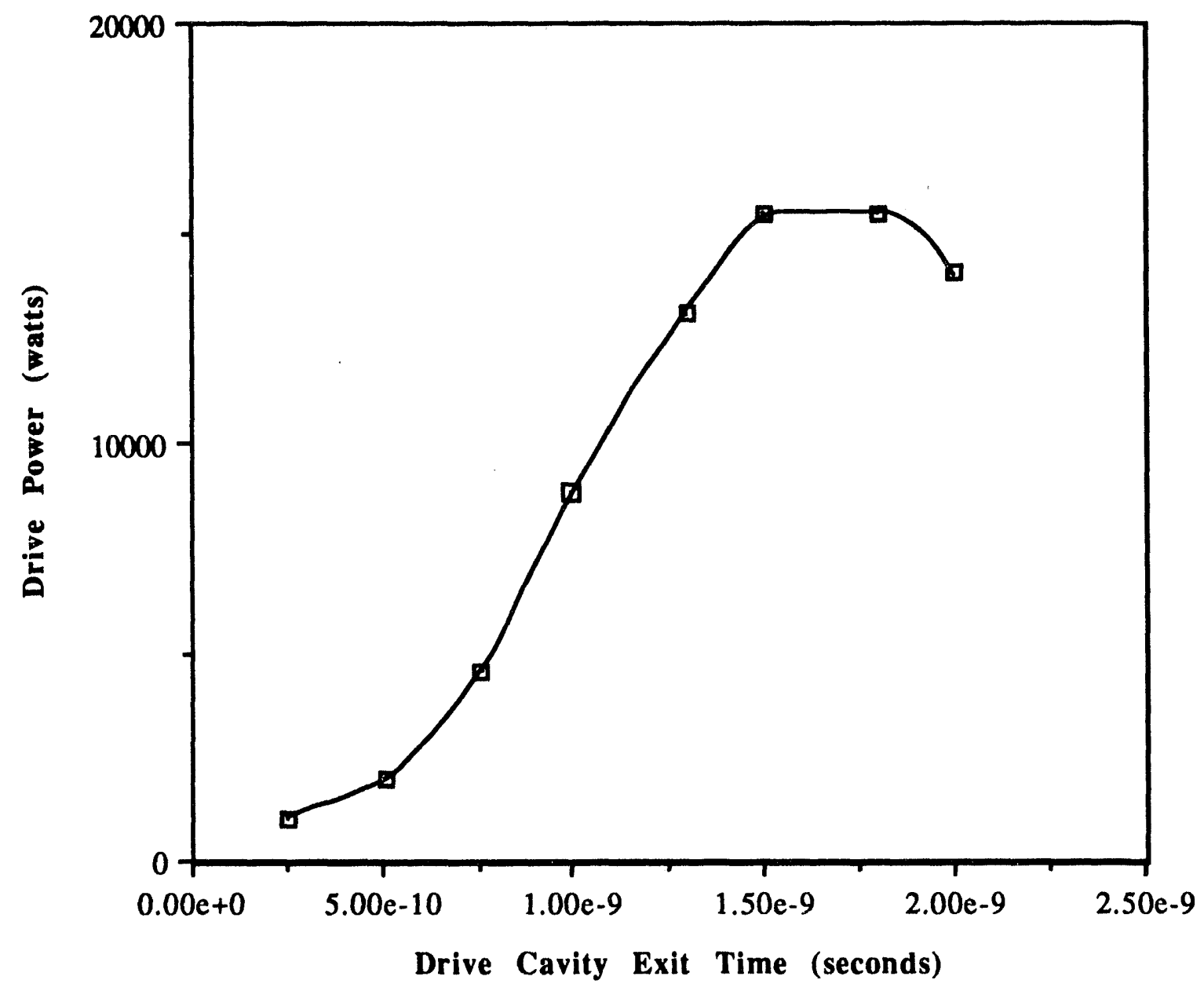

Figure 5.16b. Drive power for the $0.7-\mathrm{MV} / \mathrm{m}$ curve of Fig. 5.15 versus cavity exit time plotted against the same abscissa as Fig. 5.15.

Figure 5.17 illustrates the output angle as a function of cavity transit time for four values of cavity field, the three used in Figs. 5.13 and 5.15 and $0.7 \mathrm{MV} / \mathrm{m}$. The same trends identified above continue to apply, and the small-signal optimum transit time is in agreement with the results presented in Fig. 5.17. Figure 5.18 illustrates the drive power requirements to support the three lower values of the drive cavity field of Fig. 5.17. 


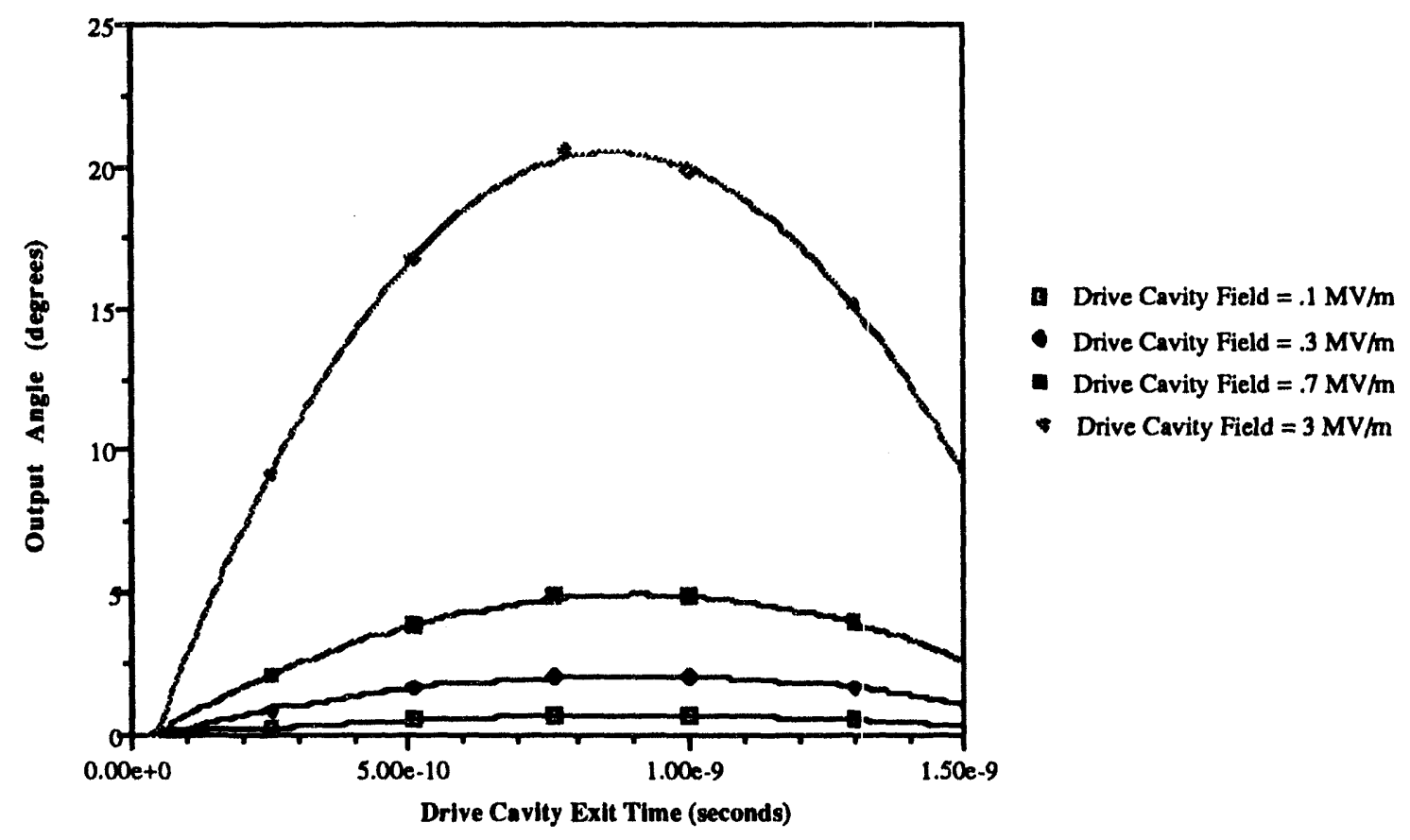

Figure 5.17. Output angle as a function of drive cavity exit time for $\Omega / \omega=1.8$.

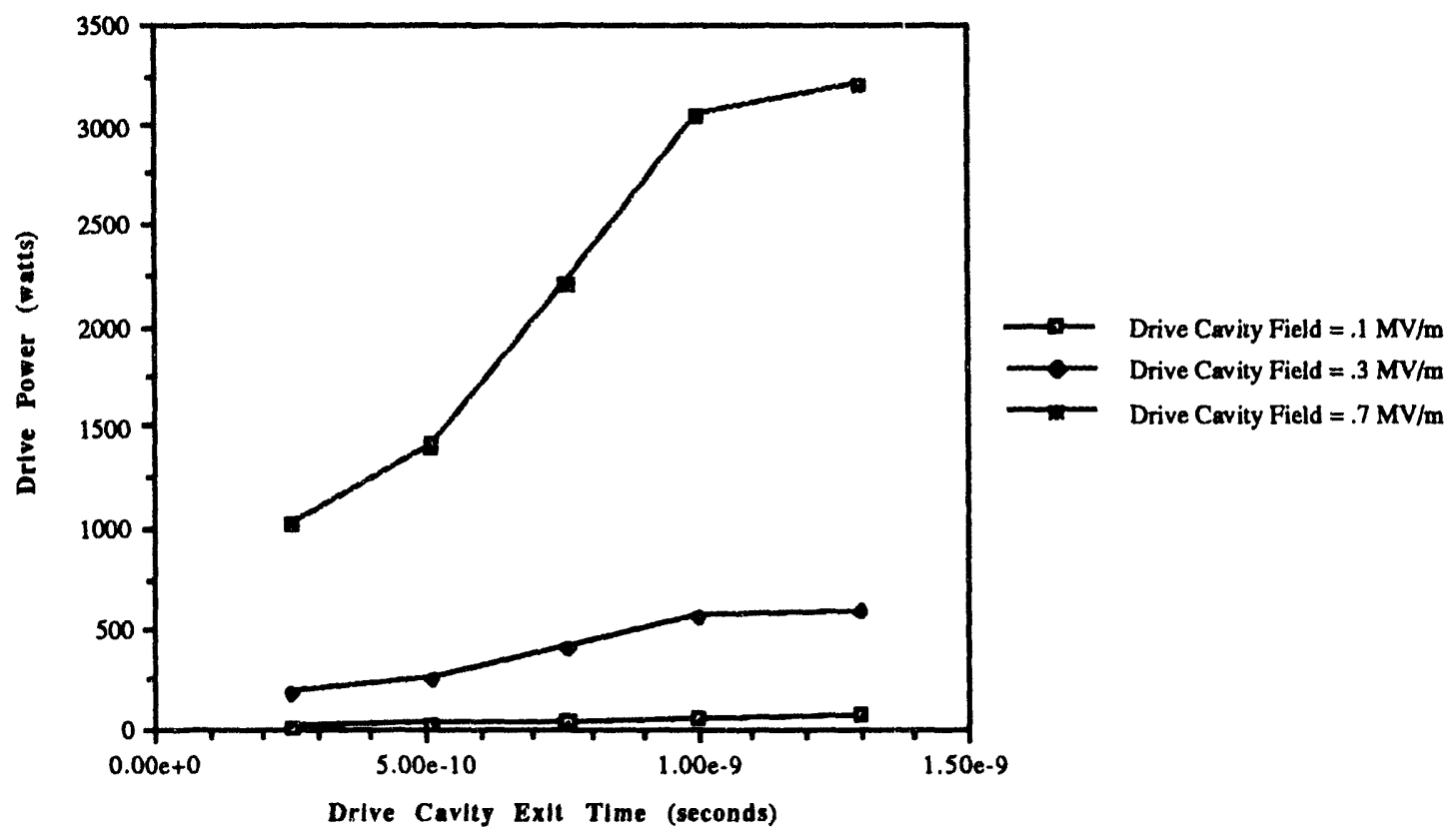

Figure 5.18. Drive power for the curves of Fig. 5.19 versus cavity exit time plotted against the same abscissa as Fig. 5.19. 


\subsection{Investigation of parameter variation on simple passive cavity performance}

Results of the rigid-beam modeling are now used to provide insight into the effect of passive-cavity length on performance and the effect of off-axis beam injection. The results presented are specific to the beam energy of the baseline model $(170 \mathrm{kV})$, although the results are presented in general units.

\subsubsection{Effect of variation of cavity length and focusing-field strength}

The velocity conversion for a range of cavity lengths is examined for three focusing field strengths $\left(\Omega / \omega=1.0,1.4\right.$, and 1.8) and four input angles $\left(\alpha_{\text {in }}=2.9^{\circ}, 8.7^{\circ}\right.$, $14.6^{\circ}$, and $20.7^{\circ}$ ). These values were selected to provide a sampling of the available parameter space and to allow for interpolation between known values. Several types of data are presented. One set of data depicts cavity gain versus transit angle as it is referenced to both the $\mathrm{rf}$ and cyclotron frequencies for each value of focusing field. In these cases, cavity gain is defined as

$$
\text { Cavity Gain }=10 \log _{10}\left(\frac{\alpha_{\text {out }}}{\alpha_{\text {in }}}\right) \text {. }
$$

The angles in this equation are measured from the magnicon longitudinal axis. Therefore, the greater the angle, the larger the transverse velocity. The gain variation and self-consistent cavity-field variation resulting from varying the focusing-field strength are then examined for a fixed input angle. The relationship between power lost in the passive-cavity walls and cavity gain is presented. The scaling of results to different beam energies is discussed and so is gain variation with electrical length (rather than transit angle).

Figures 5.19 and 5.20 illustrate the cavity gain as a function of entrance angle. Four input angles were selected between $2^{\circ}$ and $21^{\circ}$. This spread of input angles is typical of what angles would be seen by simple, cylindrical magnicon passive cavities in a high-efficiency design. The corresponding specific numerical values for the simulation calls for a bean with a gamma of 1.3322 , a beam current of $18 \mathrm{~A}$, and transverse input velocities of $0.1 \times 10^{8}, 0.3 \times 10^{8}, 0.5 \times 10^{8}$, and $0.7 \times 10^{8} \mathrm{~m} / \mathrm{s}$. Several interesting observations can be made by examining the figures. For instance, it can be seen that for the $2.9^{\circ}$ and $8.7^{\circ}$ input angle, the gain curves overlay each other almost exactly. However, for the $14.6^{\circ}$ and $20.7^{\circ}$ input angles, the curves begin to broaden and the peak gain decreases. This indicates that there is a saturation effect. The input angle increases 
as a result of an increase in input transverse velocity. As the transverse velocity is increased, it is physically impossible with a passive structure to maintain the same ratio of velocity conversion from the longitudinal to transverse direction. It can also be seen that as the cavity gain exhibits the saturation effect, the peak of the response shifts to higher transit angles.

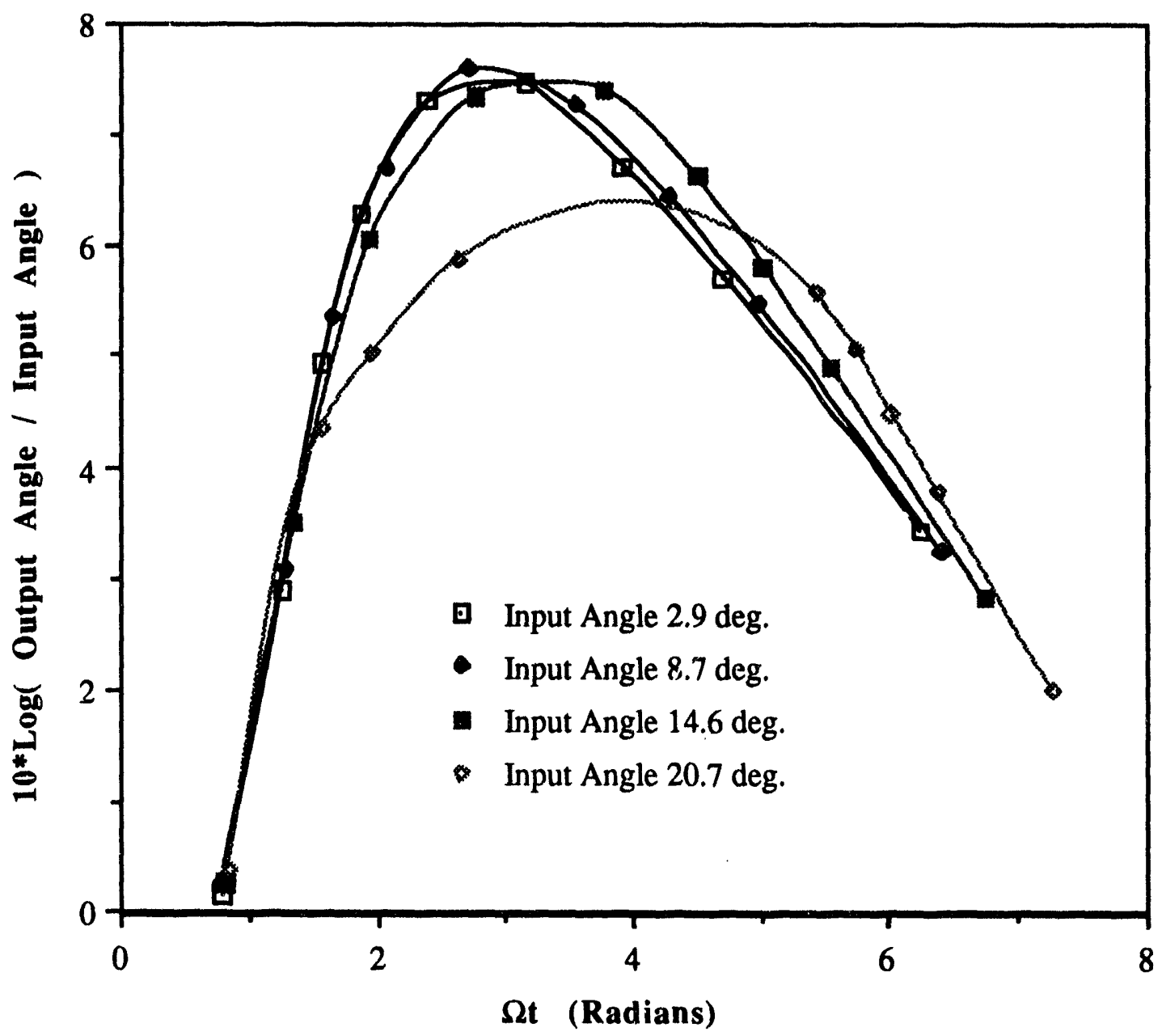

Figure 5.19. Angle gain as a function of transit angle referenced to cyclotron frequency $(\Omega / \omega=1.4)$. 


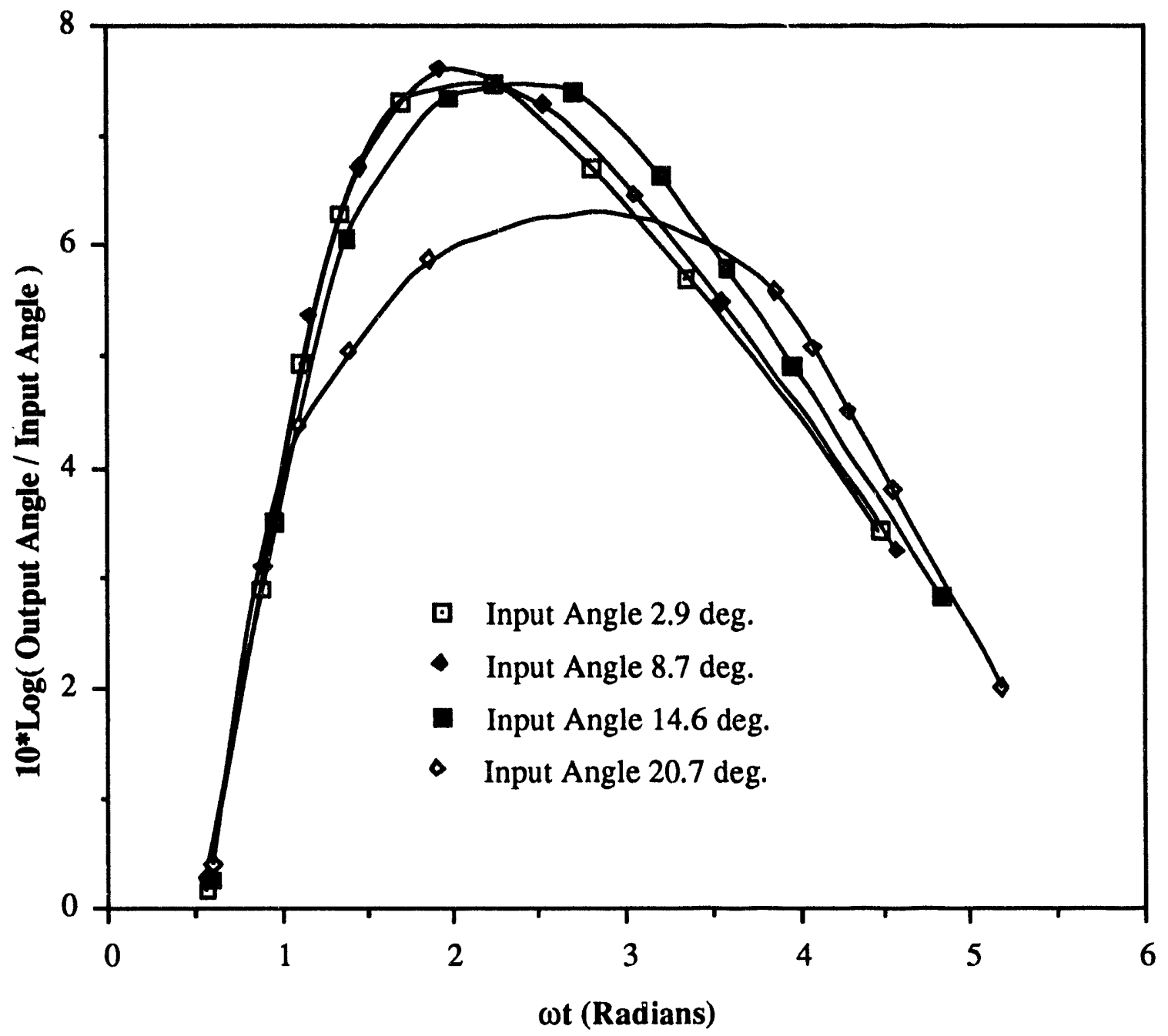

Figure 5.20. Angle gain as a function of transit angle referenced to rf frequency $(\Omega / \omega=1.4)$.

Figure 5.21 illustrates the relationship between power dissipated in the cavity walls and cavity gain for the $2.9^{\circ}$ angle, with the ordinate referenced to the cyclotron frequency. This figure is representative of the cavity's behavior at all angles and is intended to illustrate a specific point rather than serve as a design tool to determine specific geometries. The left vertical axis of the figure is cavity gain and the right vertical axis is power dissipated as a fraction of initial beam energy. Two curves are seen in the figure. The top curve represents cavity gain and the bottom rurve dissipated power. The figure shows that when designing for a specific cavity gain, two transit angles can be selected. The longer transit angle (longer cavity length) decreases the power dissipated in the cavity and, therefore, would be the better design choice. 


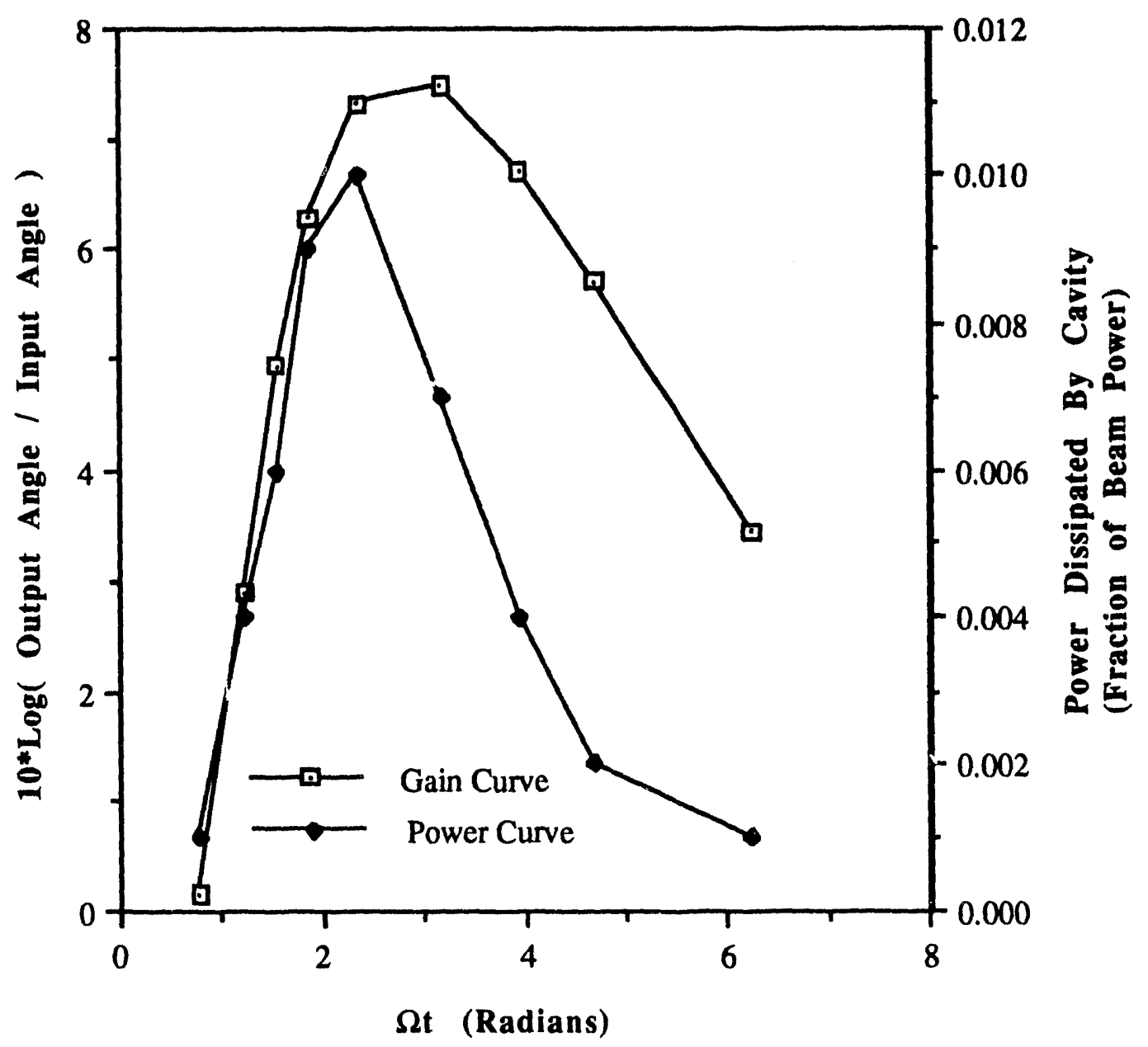

Figure 5.21. Relationship between cavity gain and dissipated power for $2.9^{\circ}$ input angle $(\Omega / \omega=1.4)$.

Figure 5.22 illustrates the trend of self-consistent cavity voltages as a function of input angle and transit angle for $\Omega / \omega=1.4$. Only three input angles are examined. The $20.7^{\circ}$ input angle is not included on the graph, because the loss associated with this angle does not inake it usable for a high-efficiency design. From the graph it can be observed that the cavity voltage can approximately scale with transverse input velocity. This is especially true for the two lowest input angles, but it is only approximately true for the largest input angle, where the saturation effects begin to be observed. 


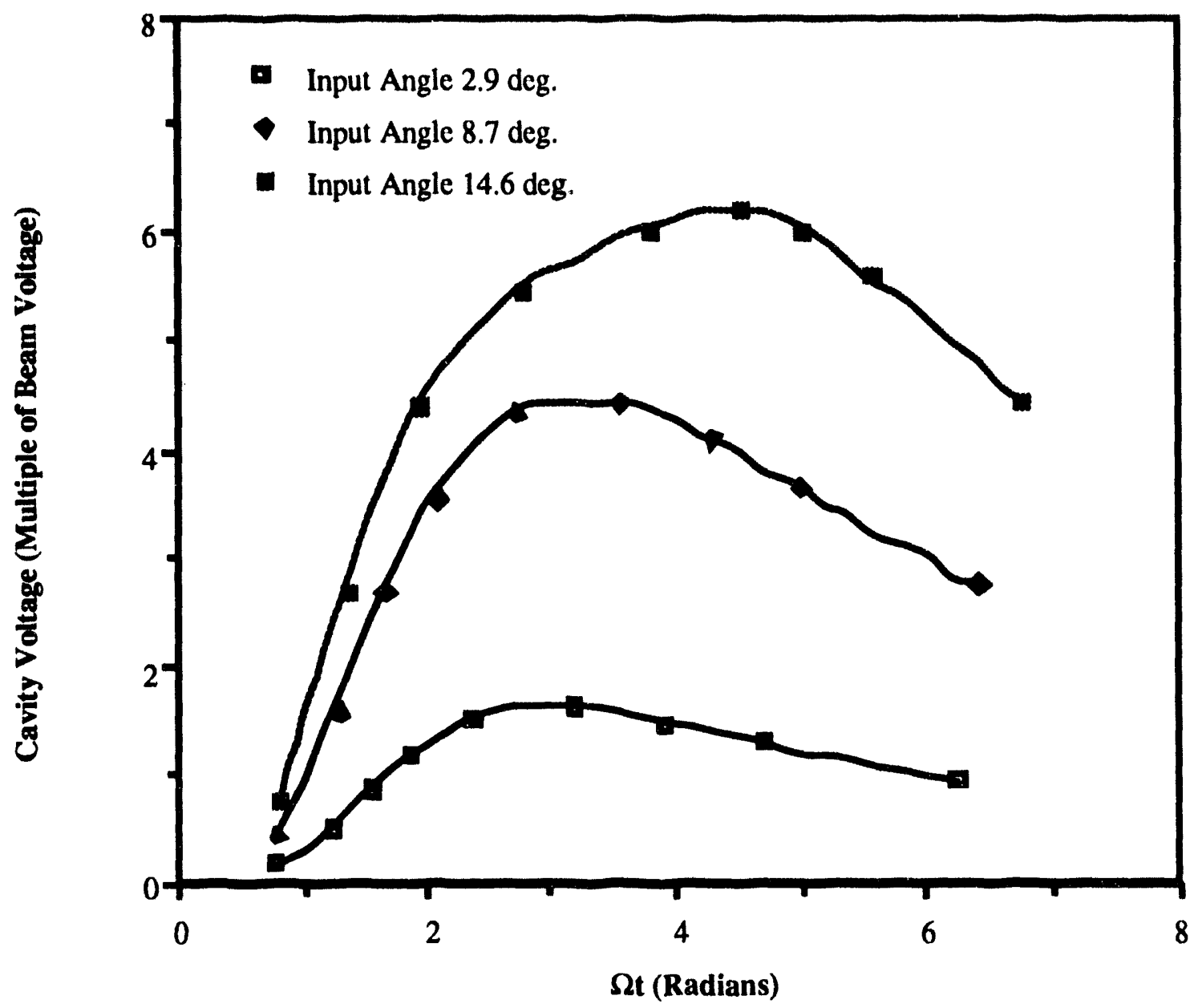

Figure 5.22. Self-consistent passive-cavity voltage excited for cavities with different transit angles for input angles in the linear range $(\Omega / \omega$ = 1.4).

Figure 5.23 is similar to Figs. 5.19 and 5.20, except that only one figure is presented because the cyclotron frequency is equal to the if frequency. Figure 5.23 displays the same four entrance angles displayed in Figs. 5.19 and 5.20. Note that for $\Omega / \omega=1.0$ only the largest input angle begins to exhibit the saturation effect. This results from the fact that the peak gain of the passive cavity for $\Omega / \omega=1.0$ is less than that for $\Omega / \omega=1.4$, so saturation is not evident until the highest value of input angle. A comparison of Fig. 5.23 with Figs. 5.19 and 5.20 also shows that the transit angle associated with the peak gain is smaller for $\Omega / \omega=1.0$ than it is for $\Omega / \omega=1.4$. 


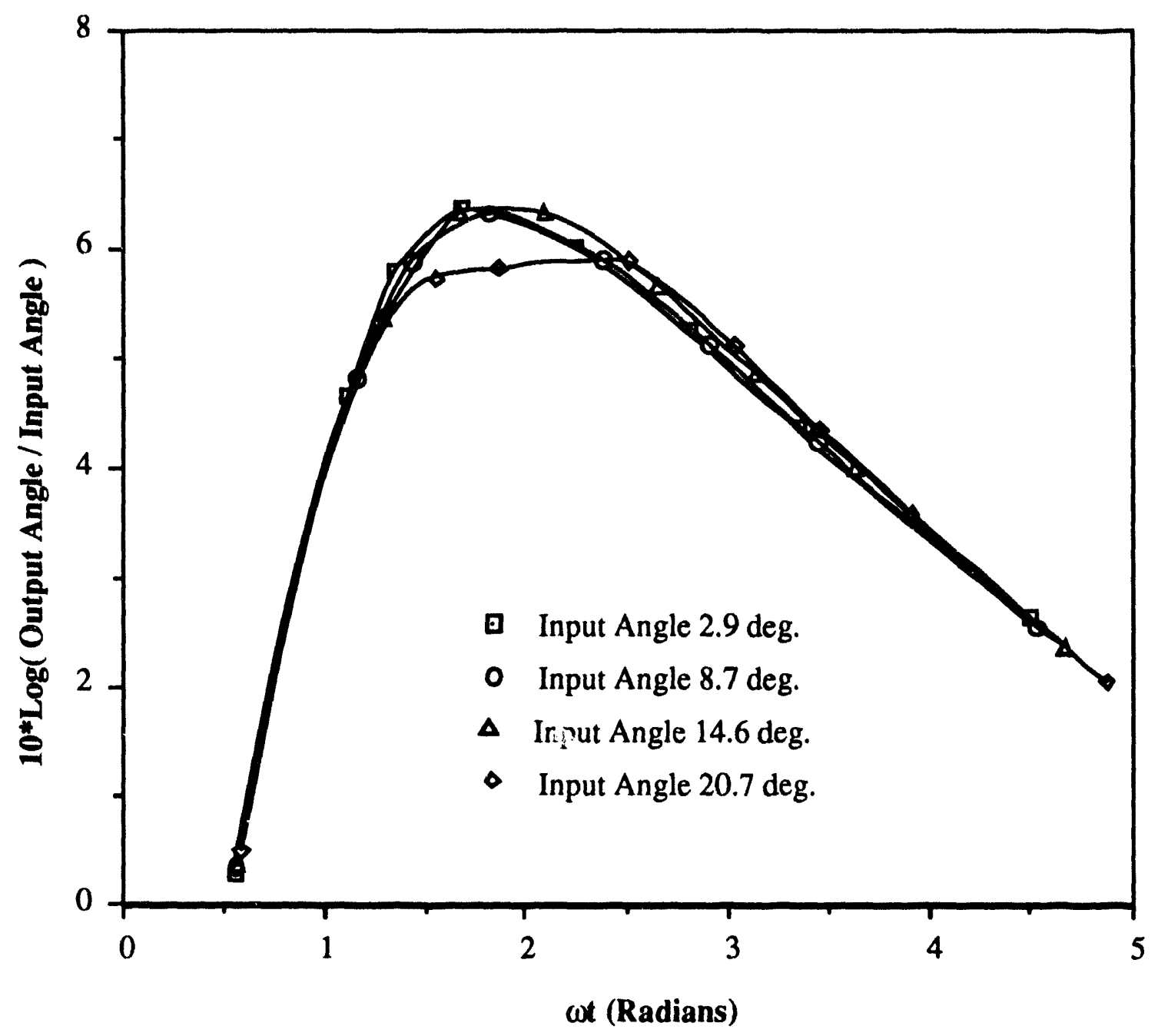

Figure 5.23. Angle gain as a function of transit angle referenced to rf frequency $(\Omega / \omega=1.0)$.

Figures 5.24 and 5.25 are similar to Figs. 5.19, 5.20, and 5.23, except they represent the case where $\Omega / \omega=1.8$. In Figs. 5.24 and 5.25 the satura/ion effect is even more pronounced when compared with the other figures. This effect results from an increased peak gain over the cases depicted in Figs. 5.19, 5.20, and 5.23. The spread of entrance angles in Fig. 5.23 is reduced to a maximum input angle of $14.6^{\circ}$. For the $20.7^{\circ}$ input angle, the dissipation at the ransit angles of maximum gain was in excess of $30 \%$ of the beam power and, therefore, not consistent with a high-efficiency device. 


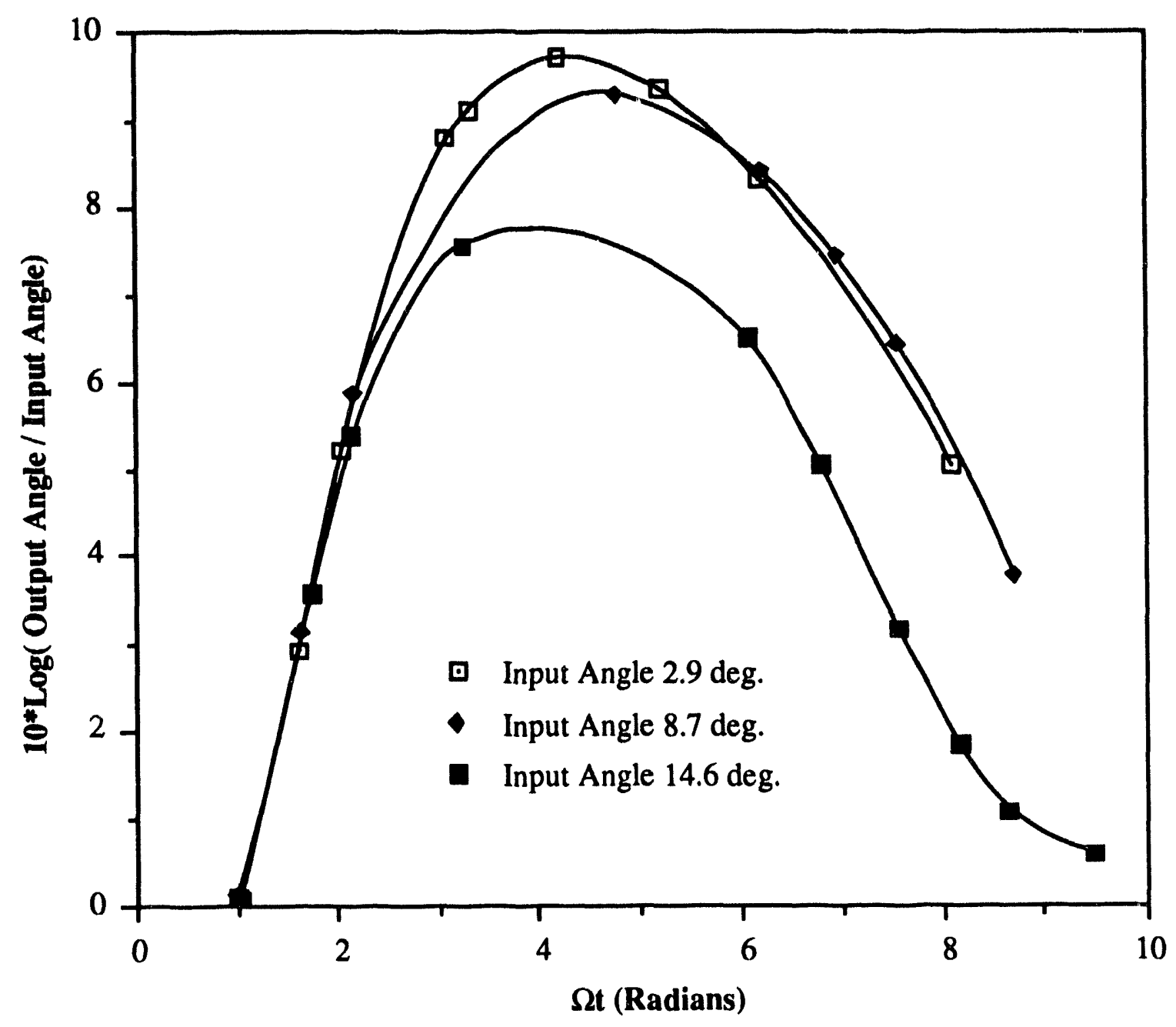

Figure 5.24. Angle gain as a function of transit angle referenced to cyclotron frequency $(\Omega / \omega=1.8)$. 


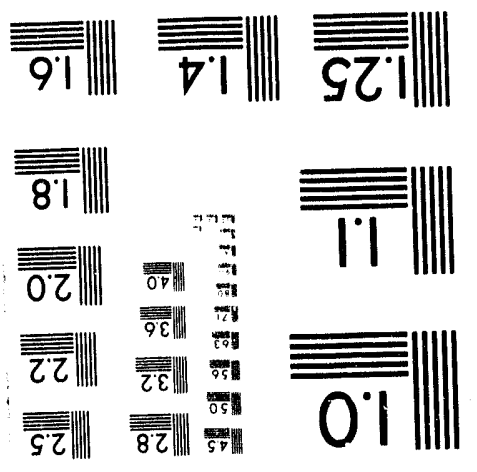



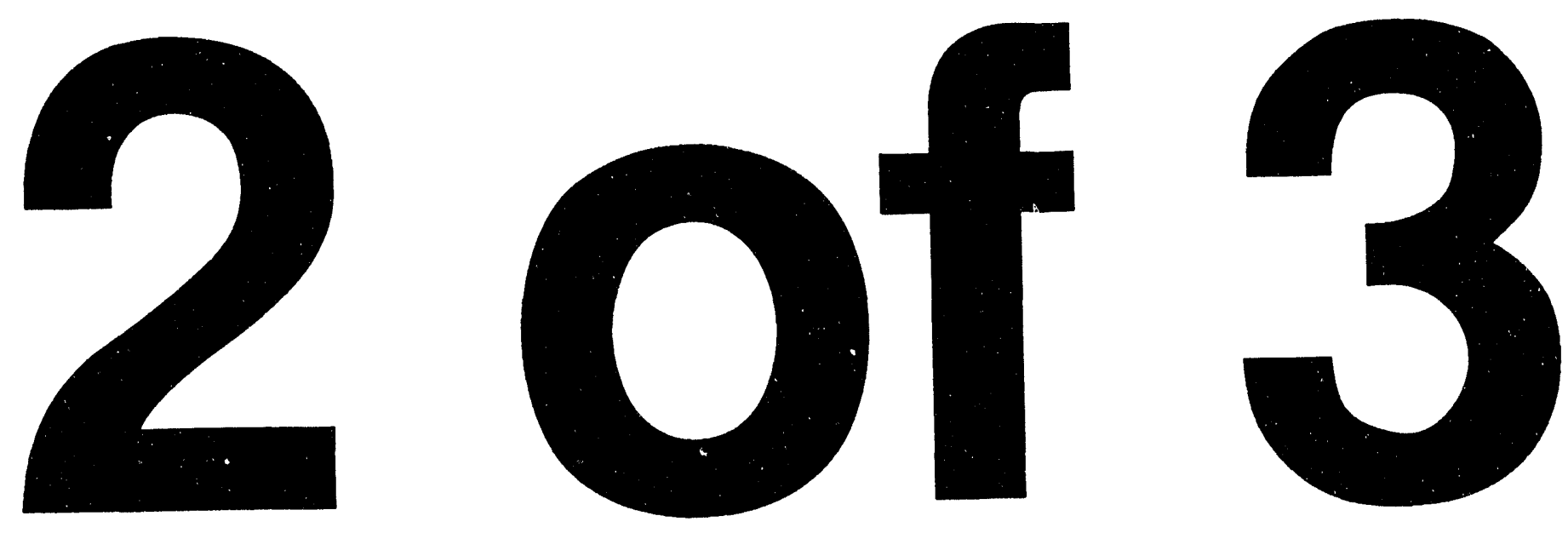


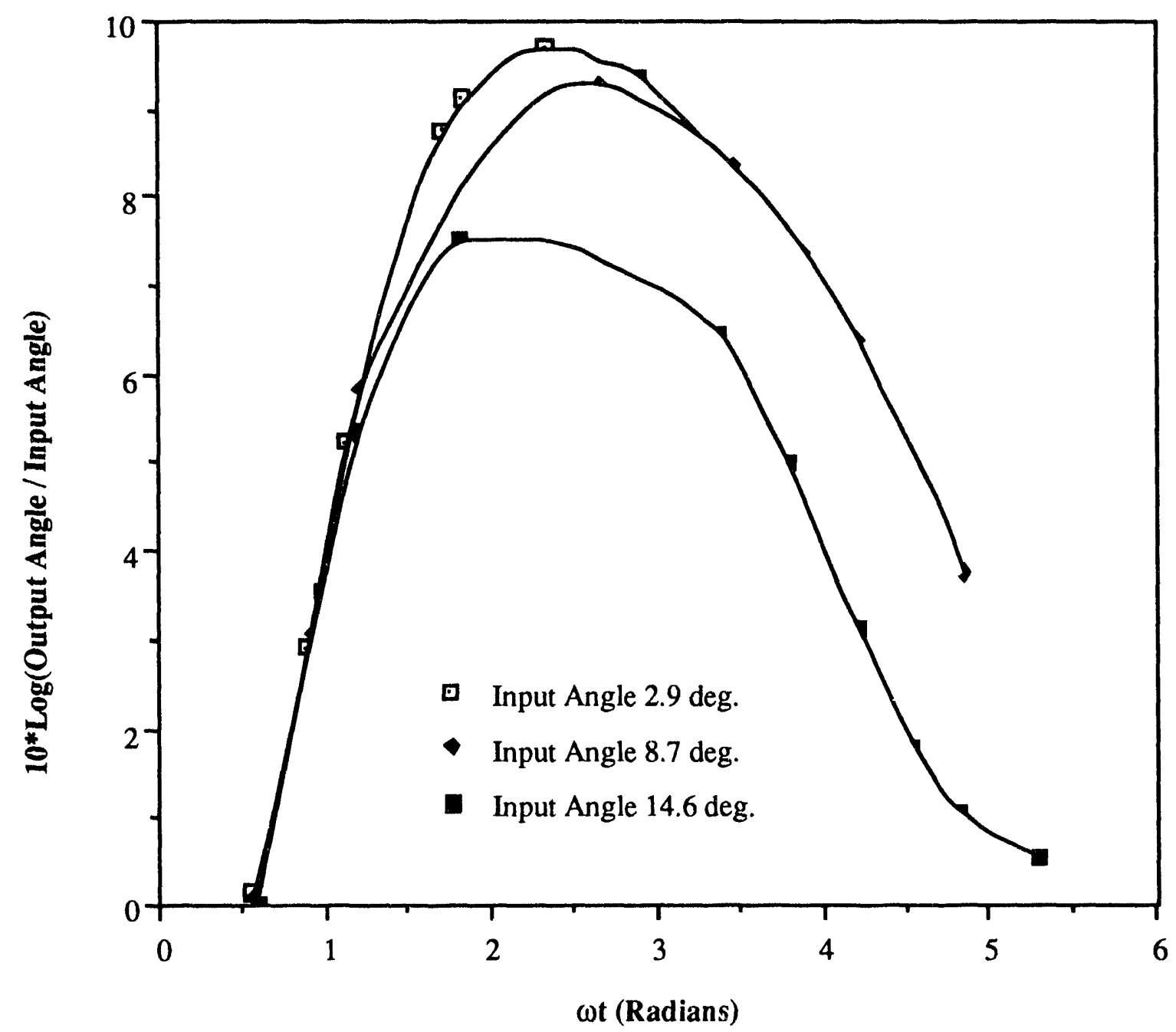

Figure 5.25. Angle gain as a function of transit angle referenced to $\mathrm{rf}$ frequency $(\Omega / \omega=1.8)$.

Figures 5.26 and 5.27 compare the plots presented on Figures 5.19, 5.20, 5.23, 5.25 , and 5.26. They illustrate the cavity gain as a function of the transit angle referenced to the cyclotron and rf frequency for the smallest input angle. From the figures it can be seen that the gain and optimum transit angle increases with increasing values of $\Omega / \omega$. They also show that the change in cavity gain is significant as the focusing-field strength changes. 


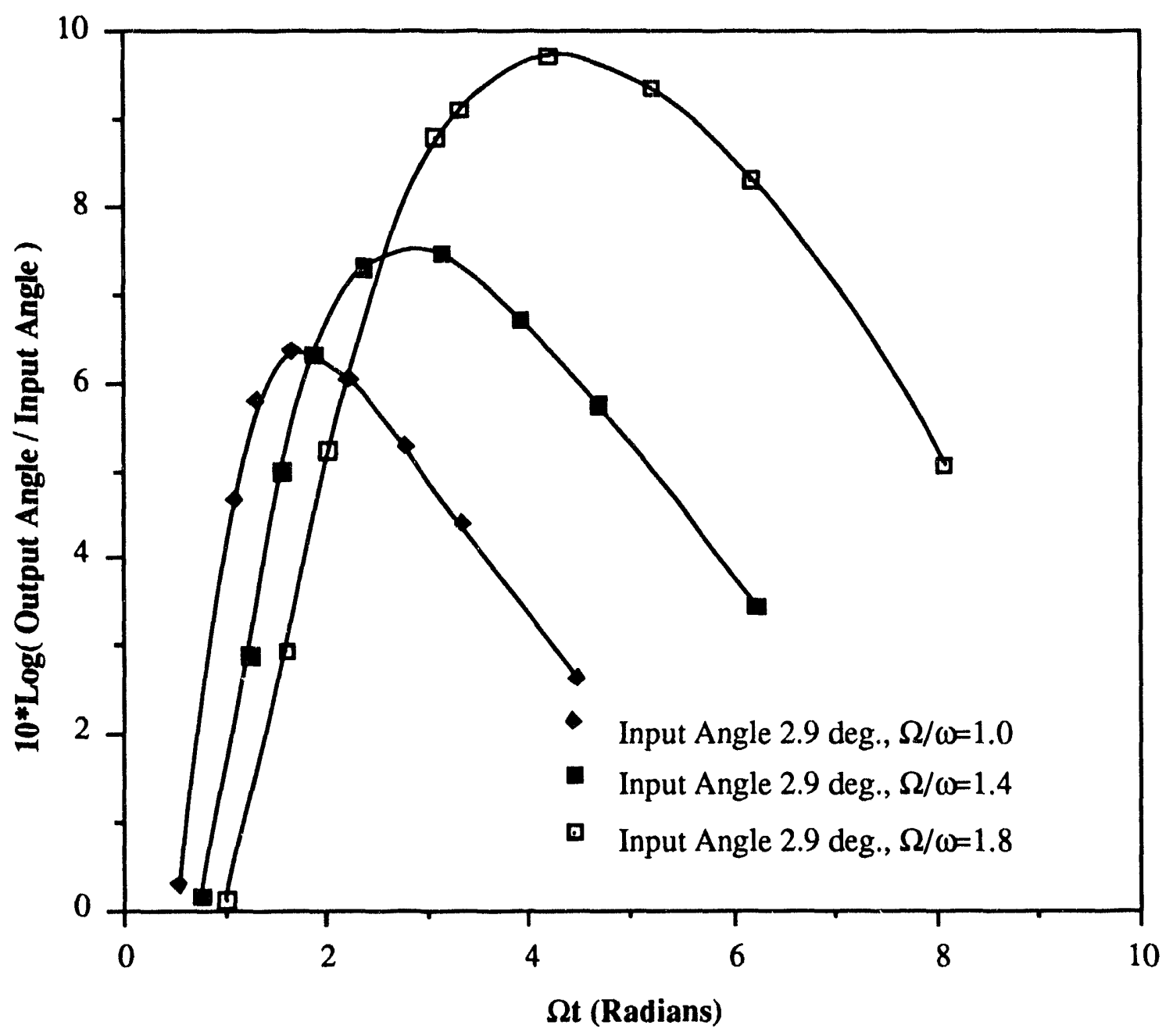

Figure 5.26. Angle gain versus transit angle referenced to cyclotron frequency for a fixed input angle of $2.9^{\circ}$. 


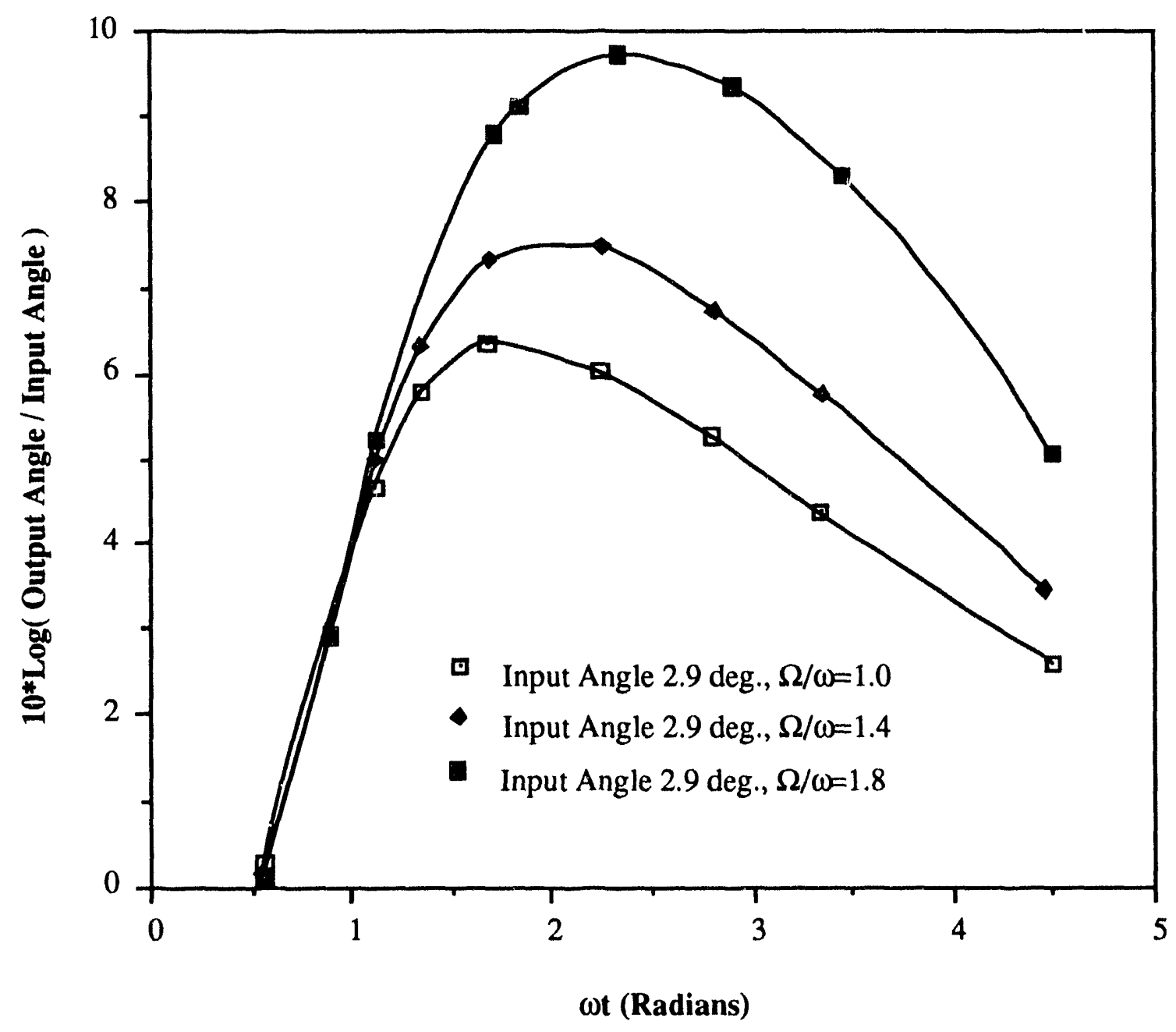

Figure 5.27. Angle gain versus transit angle referenced to $\mathrm{rf}$ frequency for a fixed input angle $2.9^{\circ}$.

Figure 5.28 illustrates the power lost as a function of transit angle for the three curves of Figs. 5.26 and 5.27. It illustrates that the point of maximum power loss is not the point of maximum energy conversion, as might be expected. It also shows that the way to achieve peak gain of the $\Omega / \omega=1.0$ curve in Fig. 5.26 is to lengthen the cavity beyond optimal length with the focusing-field strength set such that $\Omega / \omega=1.4$ or 1.8 . This would provide a cavity that achieves the same gain with a lower dissipated power. 


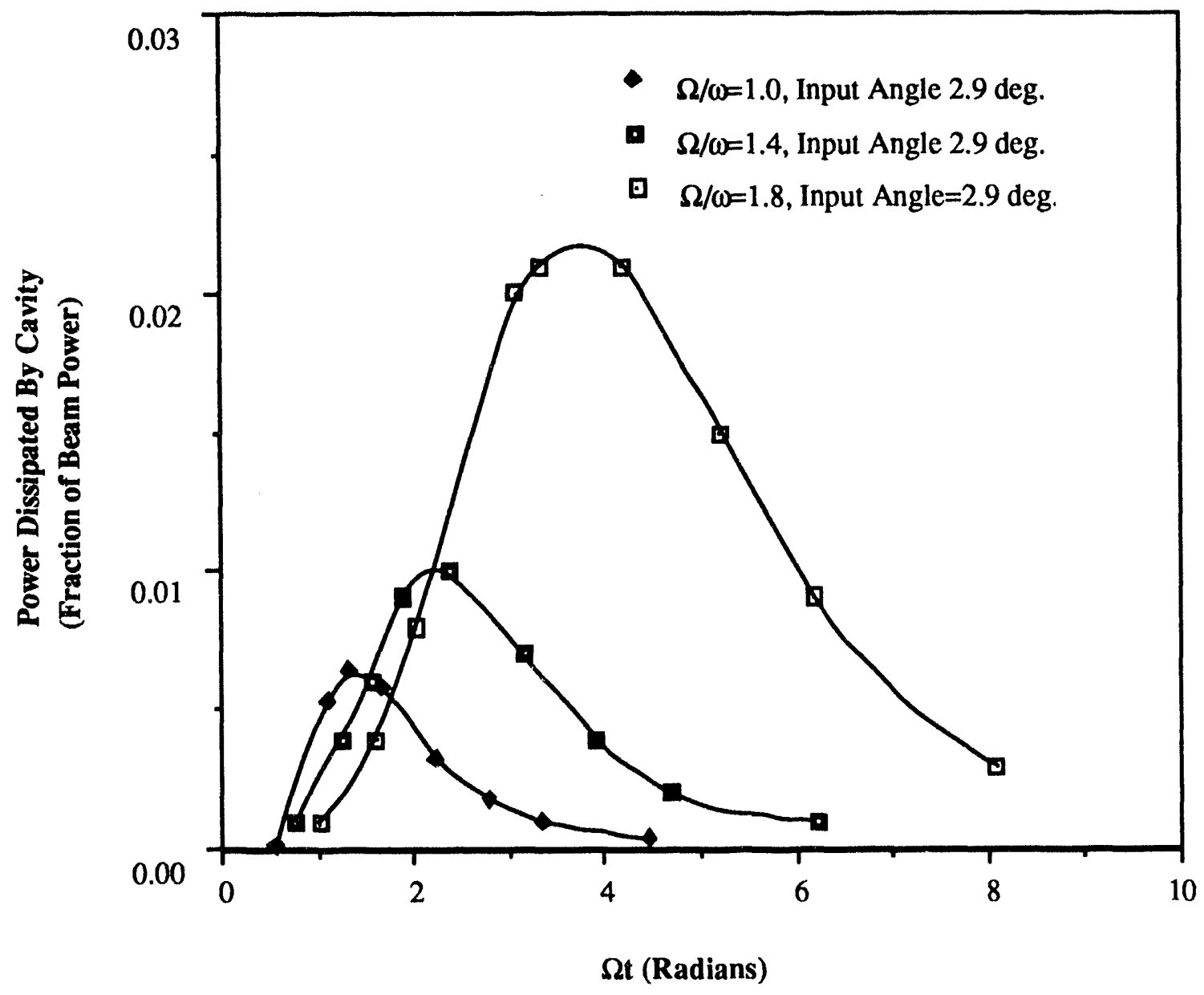

Figure 5.28. Power loss versus cavity transit angle corresponding to Figs. 5.26 and 5.27.

Figure 5.29 is used to quantify the beam-energy dependence of the results. It is the same type of plot as Fig. 5.19, except that the two curves in Fig. 5.29 represent two beam energies with gammas of 1.3322 and 1.155. Both plots have the same input angle, $14.7^{\circ}$. From the plots it can be seen that both responses peak at approximately the same transit angle. However, the gain differs. This observation does not apply to all magnicon passive cavities. Other parameters would affect the shape of the gain response. For instance, in the case of Fig. 5.29, the same beam current was maintained for the two curves. If the gain had been different, the shape of the higher-gain curve may be affected by saturation effects, while the lower-gain curve would not.

Figure 5.29 data is provided again in Fig. 5.30, except that the ordinate in Fig. 5.30 is a fraction of the rf wavelength rather than transit angle. This parameter was chosen to provide insight into the optimal cavity lengths. Figure 5.31 displays a similar 
plot. However, in this figure the curves nearly overlay each other. In Fig. 5.31, the higher gain curve has been corrected by adjusting it for the difference in gamma according to

$$
\text { Corrected Gain }=\text { Cain }-10 \log \left(\frac{\gamma_{1}}{\gamma_{2}}\right) \text {. }
$$

Figure 5.32, which is similar to Fig. 5.29 where the beam current is constant between the two cases, provides further insight into scaling the results between beam energies. In Fig. 5.32, the beam current is changed between the two cases to maintain a constant perveance. Figures 5.29, 5.31, and 5.32 indicate that linear scaling between beam energies is not possible. The correction would have to be a function of transit angle. Therefore, the results presented in this section are for a specific beam energy and current, but they could be scaled in frequency for the same beam energy, beam current, and input angles.

Demonstration of frequency scaling is provided in Figure 5.33, which illustrates the gain curves for frequencies of $700 \mathrm{MHz}$ and $1400 \mathrm{MHz}$, a $14.6^{\circ}$ input angle, $\Omega / \omega=$ 1.4 , and the same beam energy and beam current. Note the excellent agreement between the curves. The same agreement can be seen in Figs. 5.34 and 5.35, which compare the self-consistent cavity voltage and dissipated power between the two frequency curves of Fig. 5.33. It is important to note that the excellent agreement between frequencies resulted frol. the assumption of a frequency-independent surface resistivity, $R_{S}$. Reference [1] suggests that for a good conductor, $R_{S}$ is approximately

$$
R_{s}=\sqrt{\frac{\omega \mu}{2 \sigma}}
$$

where $\sigma$ is the conductivity. If $R_{S}$ were varied, the self-consistent cavity voltage would vary, the trajectory would vary, and frequency scaling would no longer be possible due to the nonlinear nature of the trajectories with variation in cavity field. 


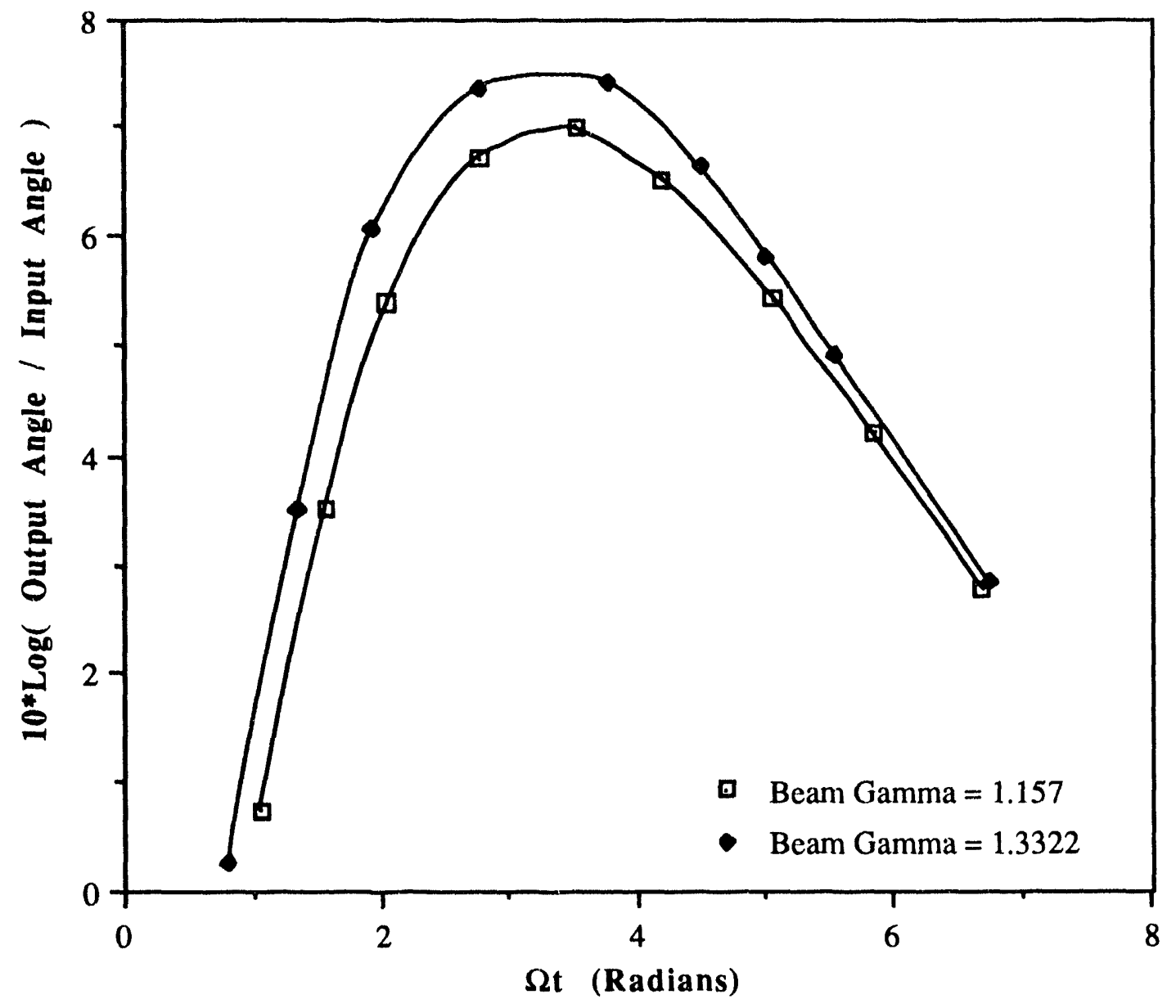

Figure 5.29. Comparison of angle gain versus transit angle for two beam energies with the same input angle $(\Omega / \omega=1.4)$. 


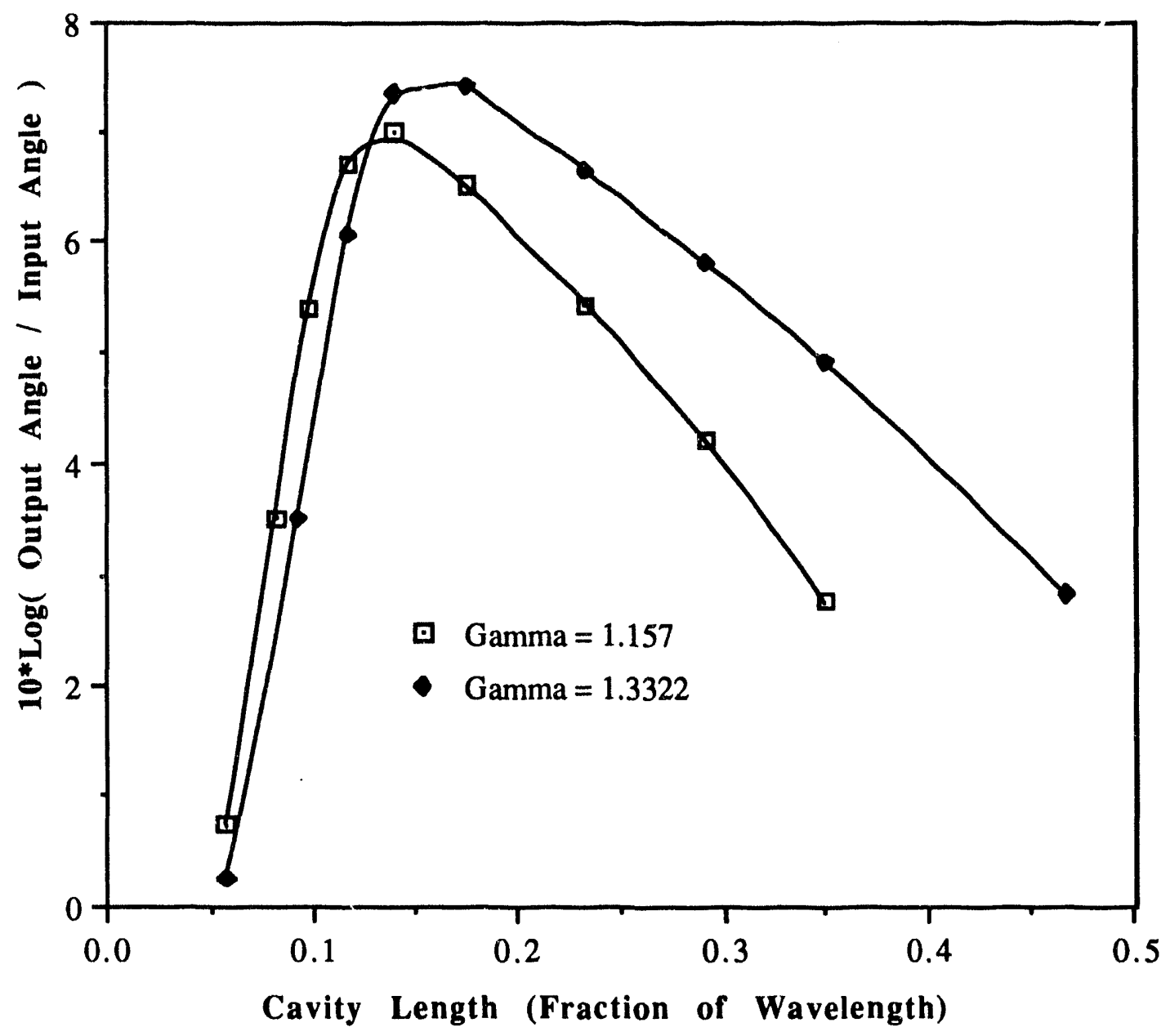

Figure 5.30. Angle gain for two beam energies with the same input angle versus cavity electrical length $(\Omega / \omega=1.4)$. 


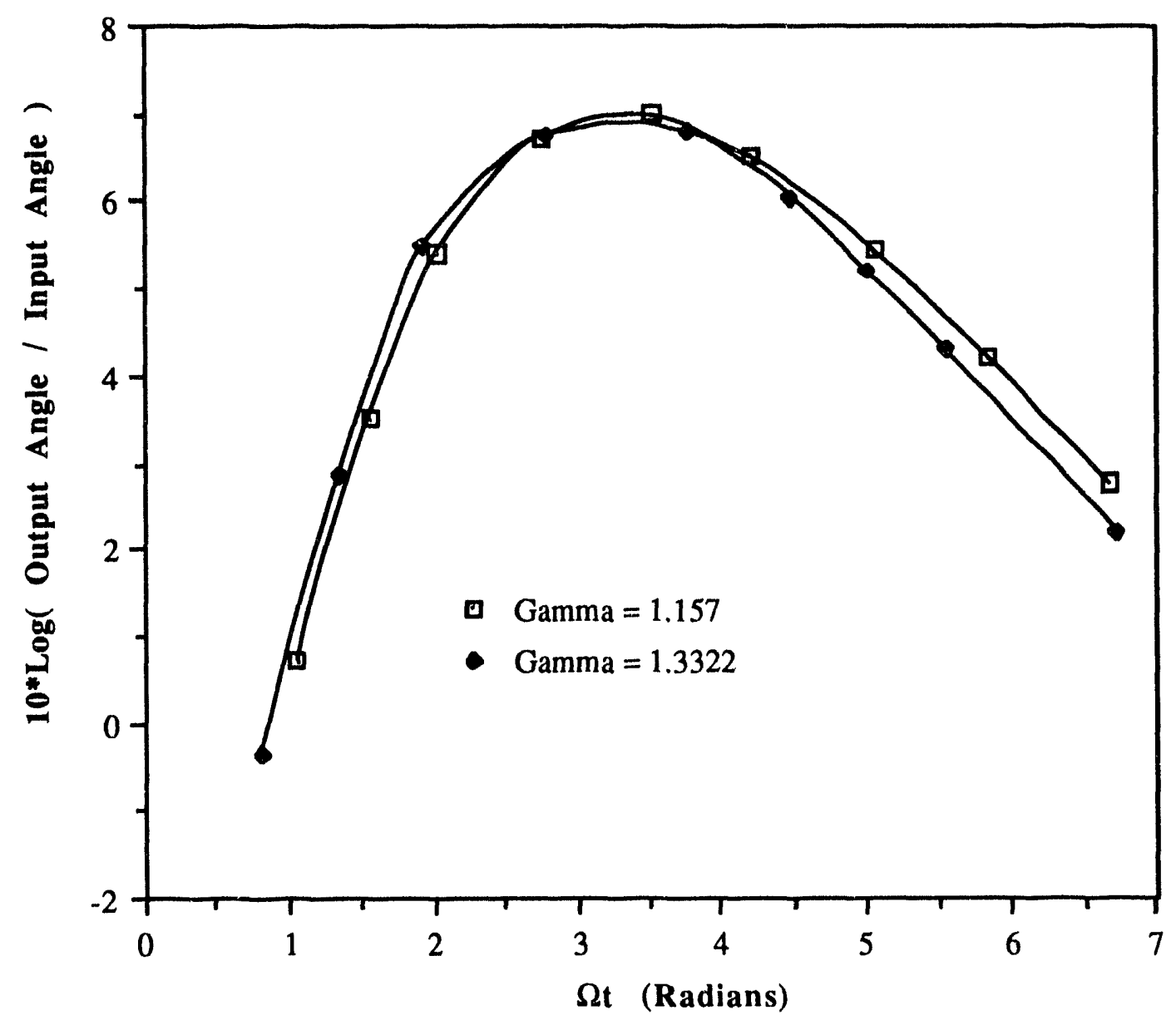

Figure 5.31. Angle gain versus transit angle referenced to the cyclotron frequency for two beam energies with the same input angle $\left(14.6^{\circ}\right)$ corrected for the difference in gamma $(\Omega / \omega=1.4)$. 


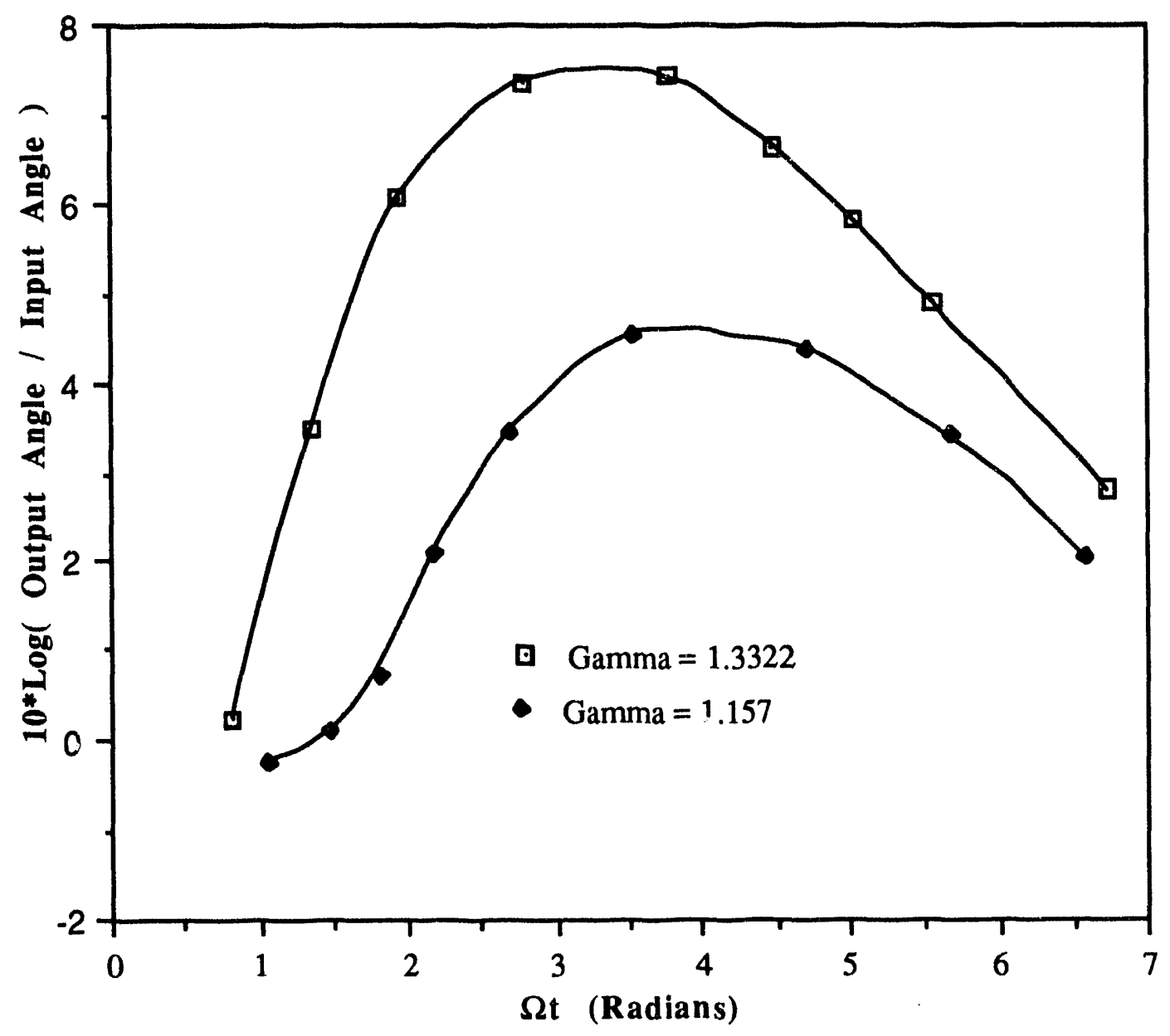

Figure 5.32. Gain comparison for two beam energies with the same input angle $\left(14.6^{\circ}\right)$ and beam perveance $(\Omega / \omega=1.4)$. 


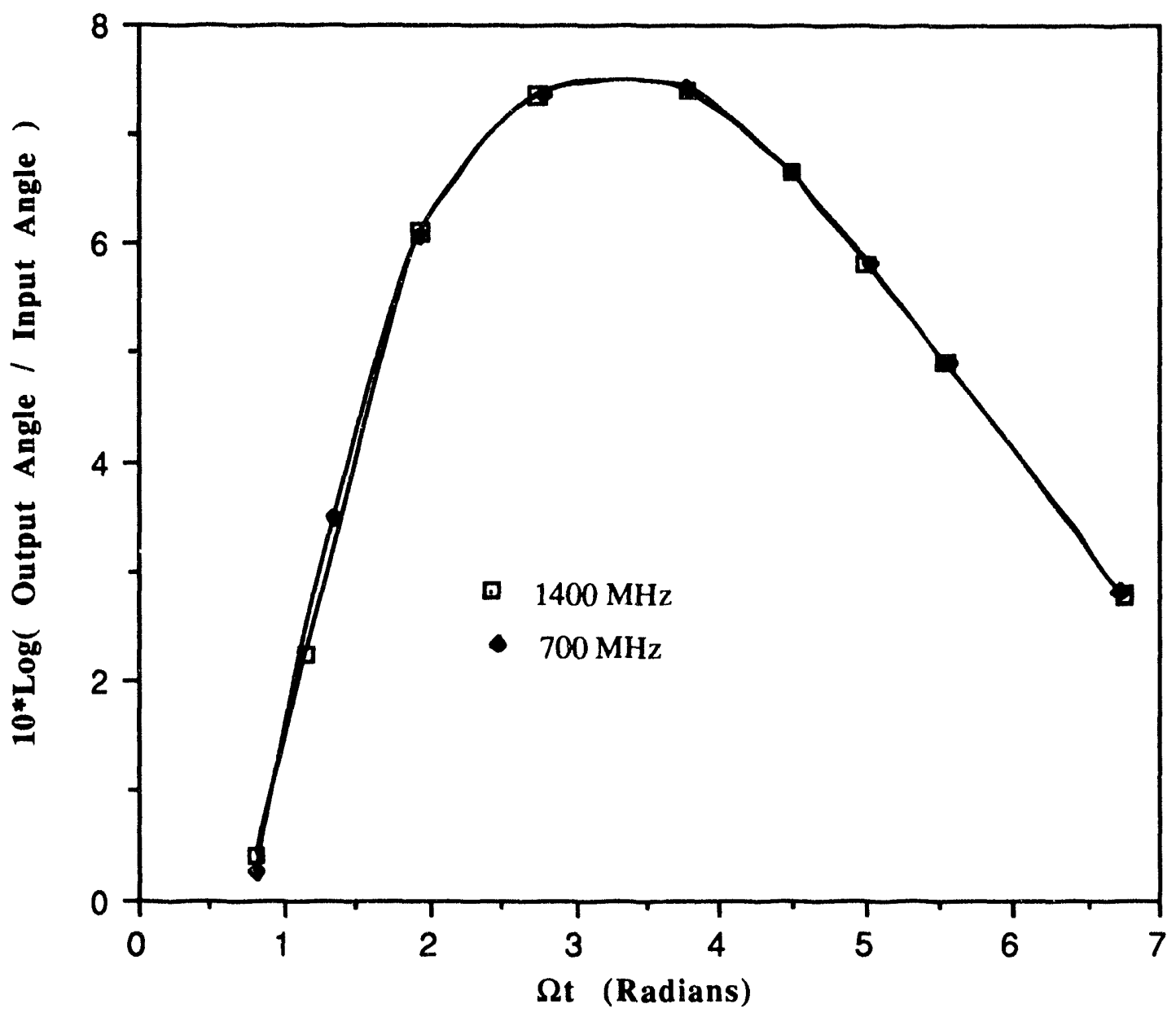

Figure 5.33. Gain curves as a function of cyclotron transit angle for $700 \mathrm{MHz}$ and $1400 \mathrm{MHz}$ for a $14.6^{\circ}$ input angle and constant beam energy and current $(\Omega / \omega=1.4)$. 


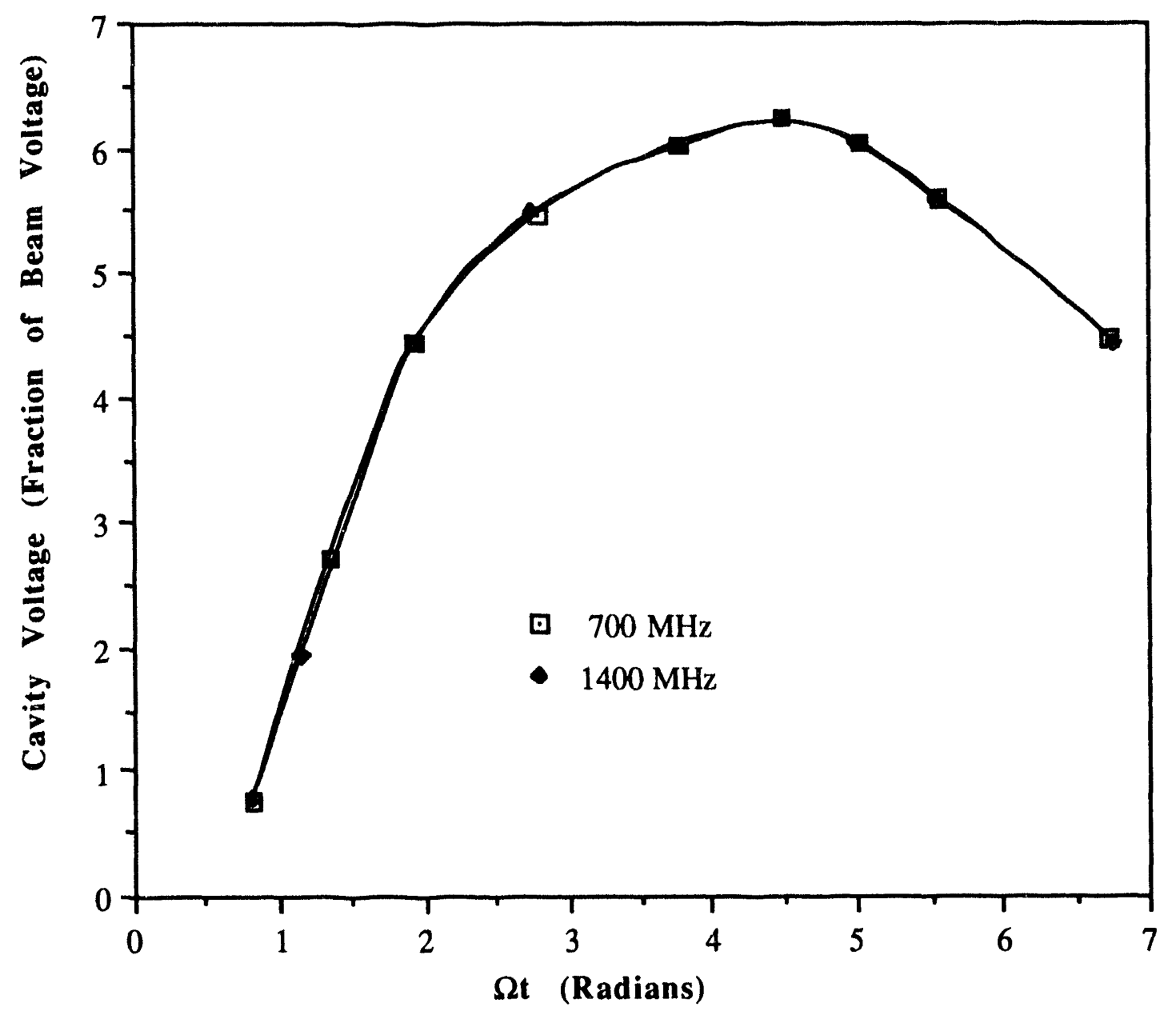

Figure 5.34. Cavity voltage as a fraction of the beam voltage versus cyclotron transit angle for conditions of Figure $5.33(\Omega / \omega=1.4)$. 


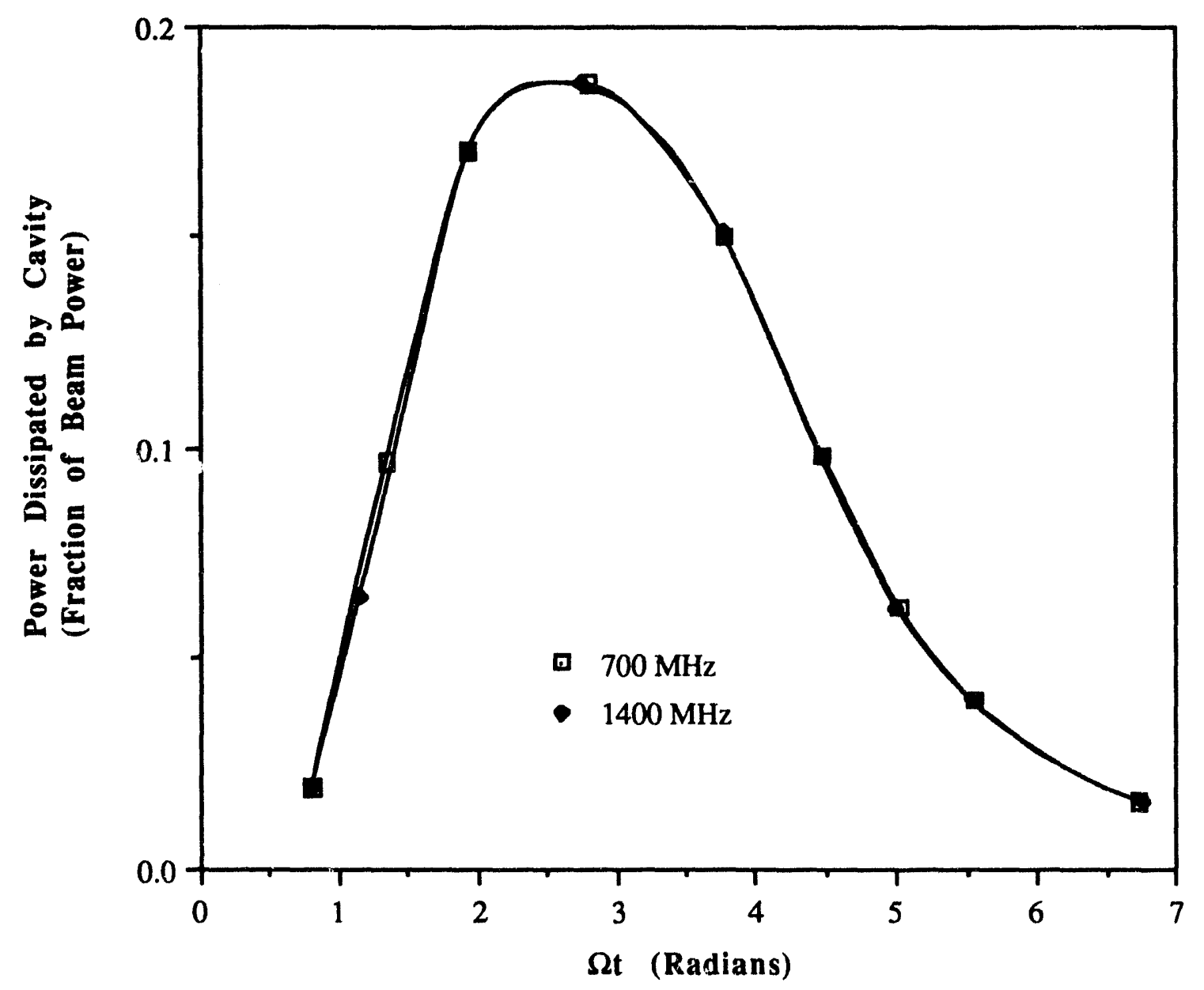

Figure 5.35. Power dissipated in cavity walls as a fraction of beam power versus cyclotron transit angle for conditions of Figure $5.33(\Omega / \omega=1.4)$.

\subsubsection{Beam entrance position}

In this section the effect of an off-axis entrance radius of the beam into a cylindrical, beam-driven cavity is evaluated. The evaluation considers a range of cavity lengths and entrance angles for three $\Omega / \omega$ ratios: $1.0,1.4$, and 1.8 . The results of the data presented in this section are used to determine the appropriate length of the drift spaces separating the cylindrical cavities in the deflection system in order to maximize conversion of longitudinal-to-transverse velocity in the deflection system (or to maximize 
deflection-system gain). All performance plots in this section were calculated using the nominal operating conditions specified in Chapter 2, Section 3, except as otherwise noted. (Note: A uniform length was not used for all curves. Some curves do not consider the full range of distances off the axis. From a physical point of view, only those points 0.05 wave'engths or less are useful for design. Some curves terminate at this point while others terminate when the gain gets too low to be usable.)

The first family of plots is presented in Figures 5.36 through 5.44. These plots illustrate the cavity angle gain for different values of $\Omega / \omega$ as a function of the radial displacement of the beam at the cavity entrance. Figures 5.36, 5.37, and 5.38 are for $\Omega / \omega=1.0$. Figure 5.36 is for an entrance angle of $2.9^{\circ}$. Figure 5.37 is for an entrance angle of $8.7^{\circ}$. Figure 5.38 is for an entrance angle of $14.6^{\circ}$. Each plot contains four curves corresponding to the four cavity lengths as specified in the graph legends.

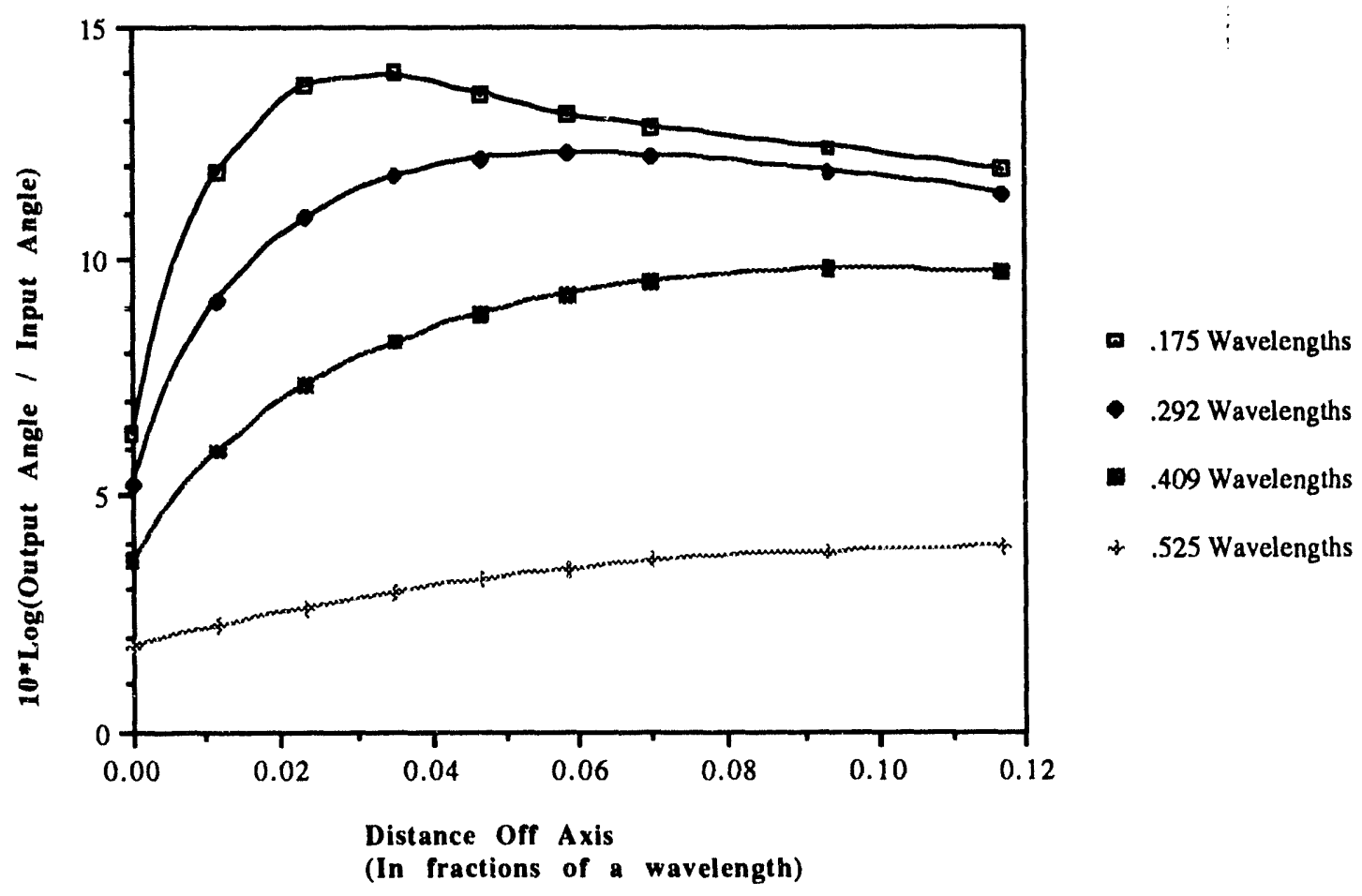

Figure 5.36. Cavity angle gain as a function of radial entrance position of beam for $\Omega / \omega=1.0$ and cavity entrance angle of $2.9^{\circ}$. 


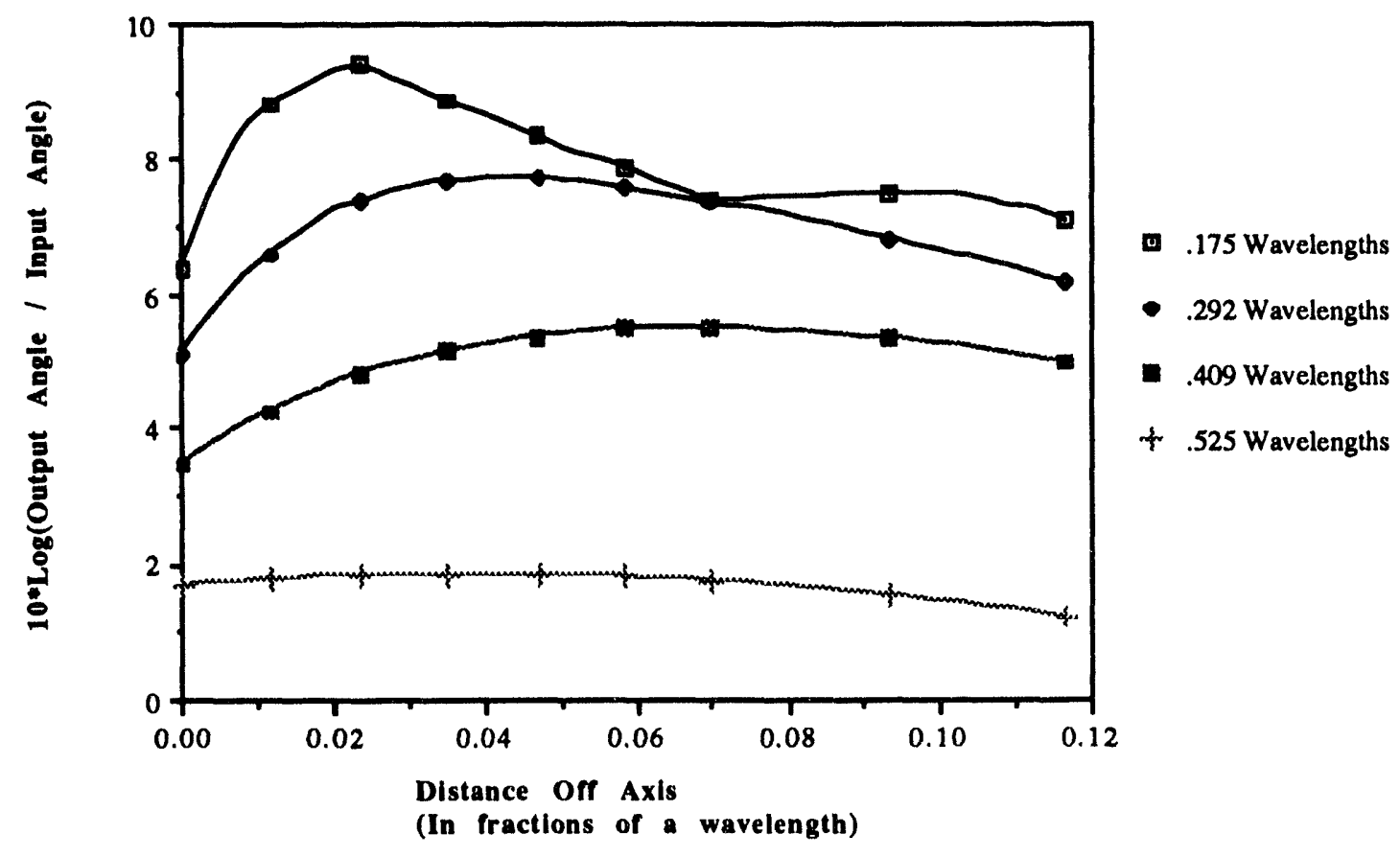

Figure 5.37. Cavity angle gain as a function of radial entrance position of beam for $\Omega / \omega=1.0$ and cavity entrance angle of $8.7^{\circ}$. 


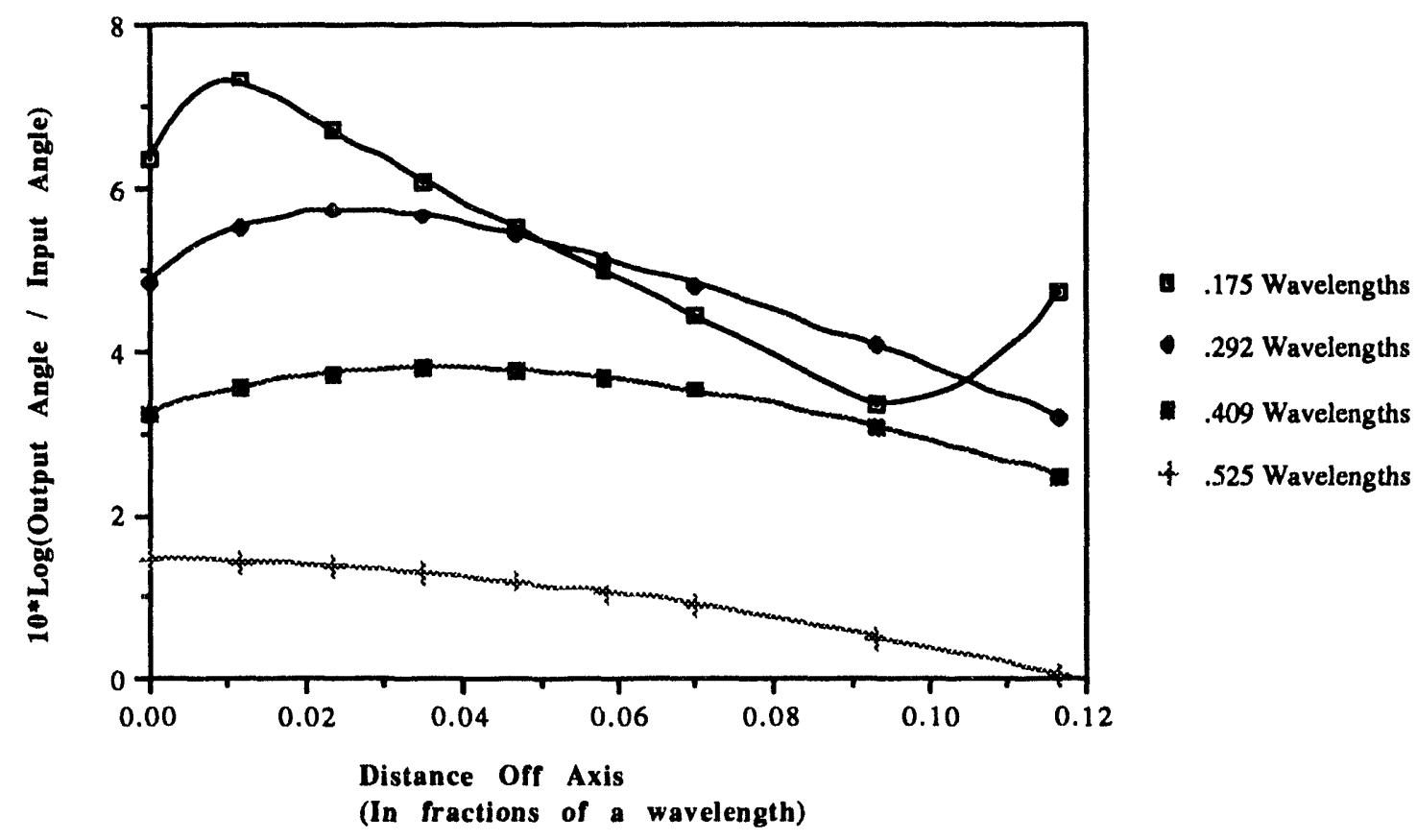

Figure 5.38. Cavity angle gain as a function of radial entrance position of beam for $\Omega / \omega=1.0$ and cavity entrance angle of $14.6^{\circ}$.

Figure 5.39, 5.40, and 5.41 are the same as Figs. 5.36, 5.37, and 5.38, except that $\Omega / \omega=1.4$. 


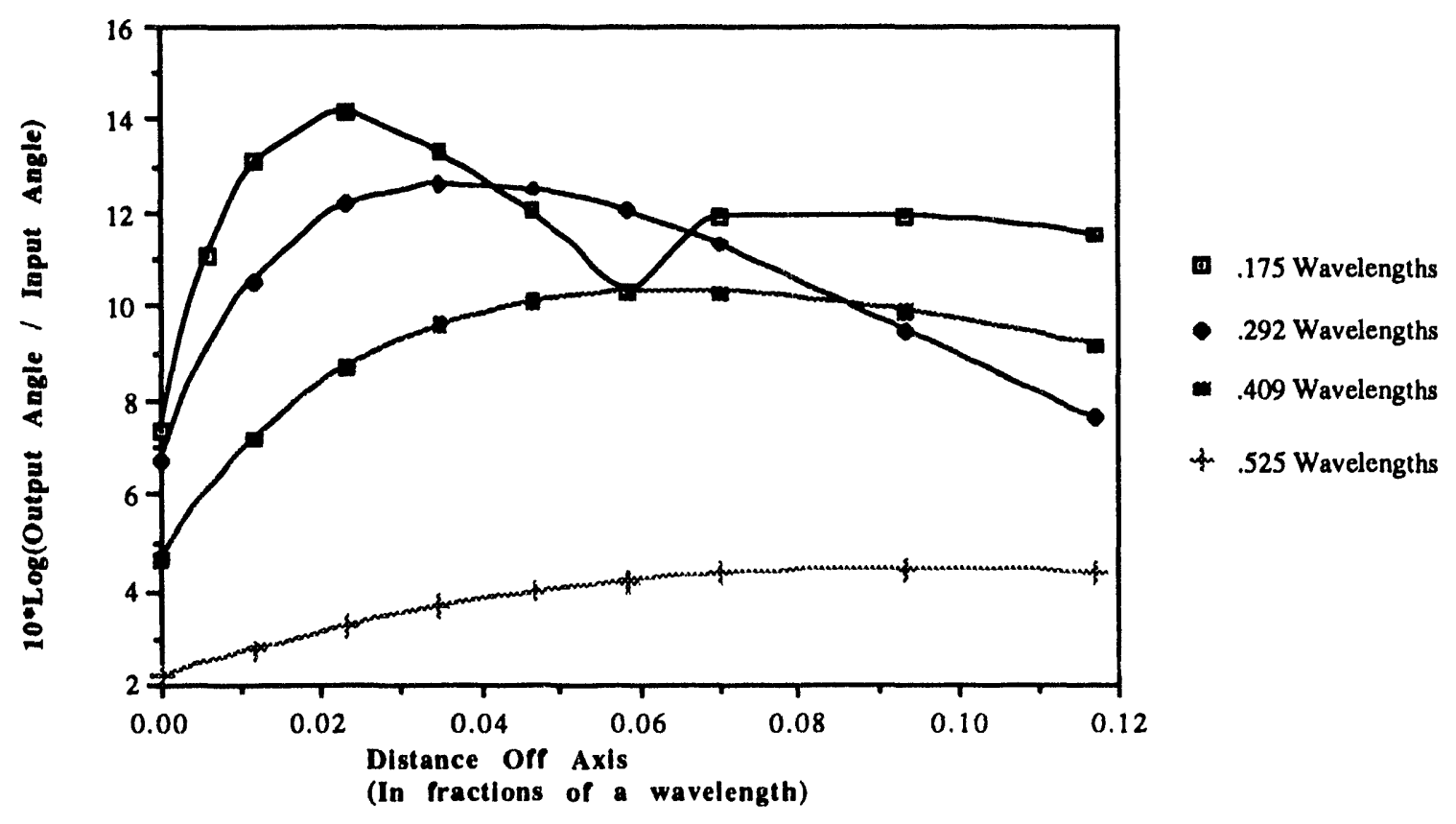

Figure 5.39. Cavity angle gain as a function of radial entrance position of beam for $\Omega / \omega=1.4$ and cavity entrance angle of $2.9^{\circ}$. 


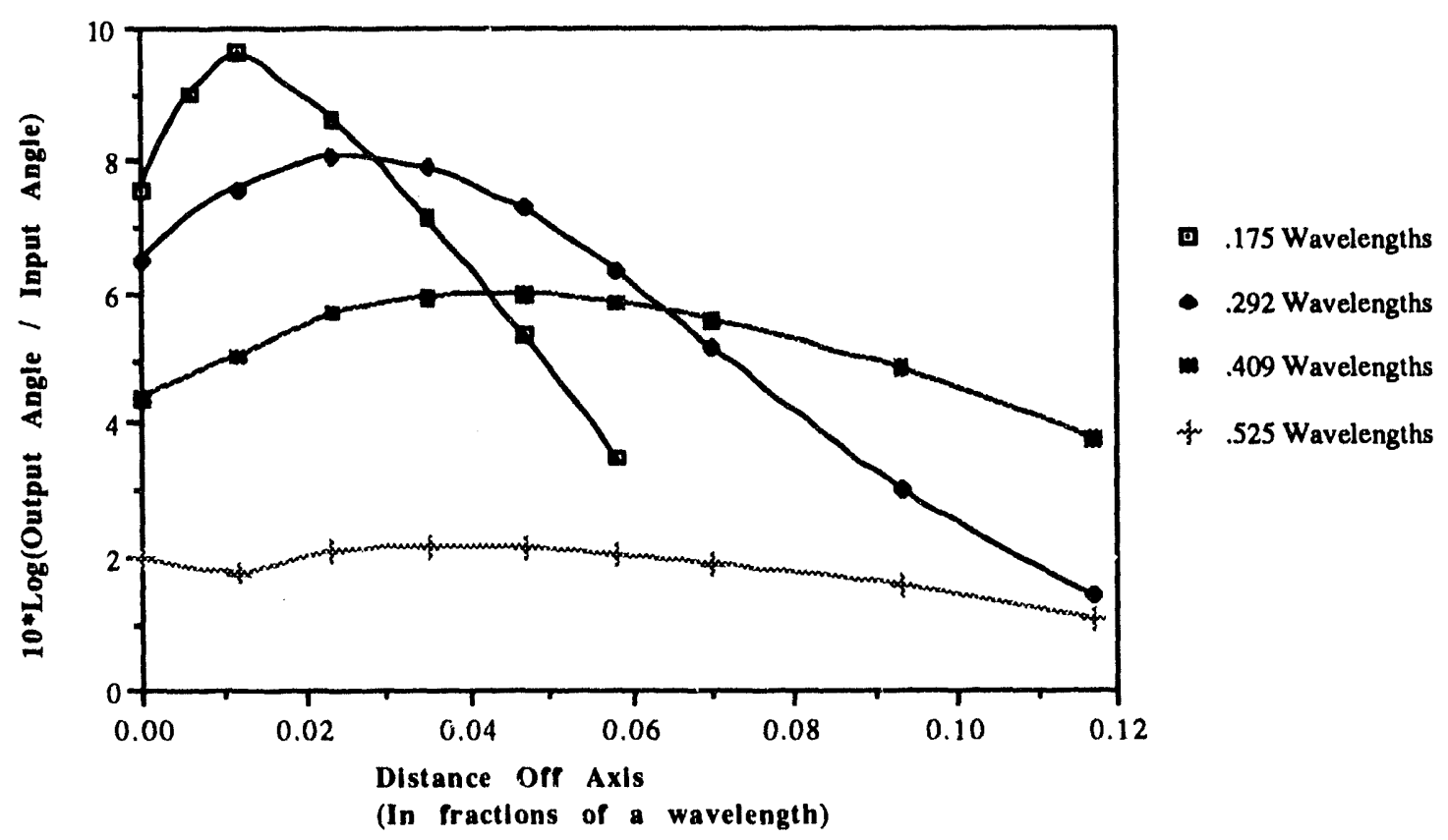

Figure 5.40. Cavity angle gain as a function of radial entrance position of beam for $\Omega / \omega=1.4$ and cavity entrance angle of $8.7^{\circ}$. 


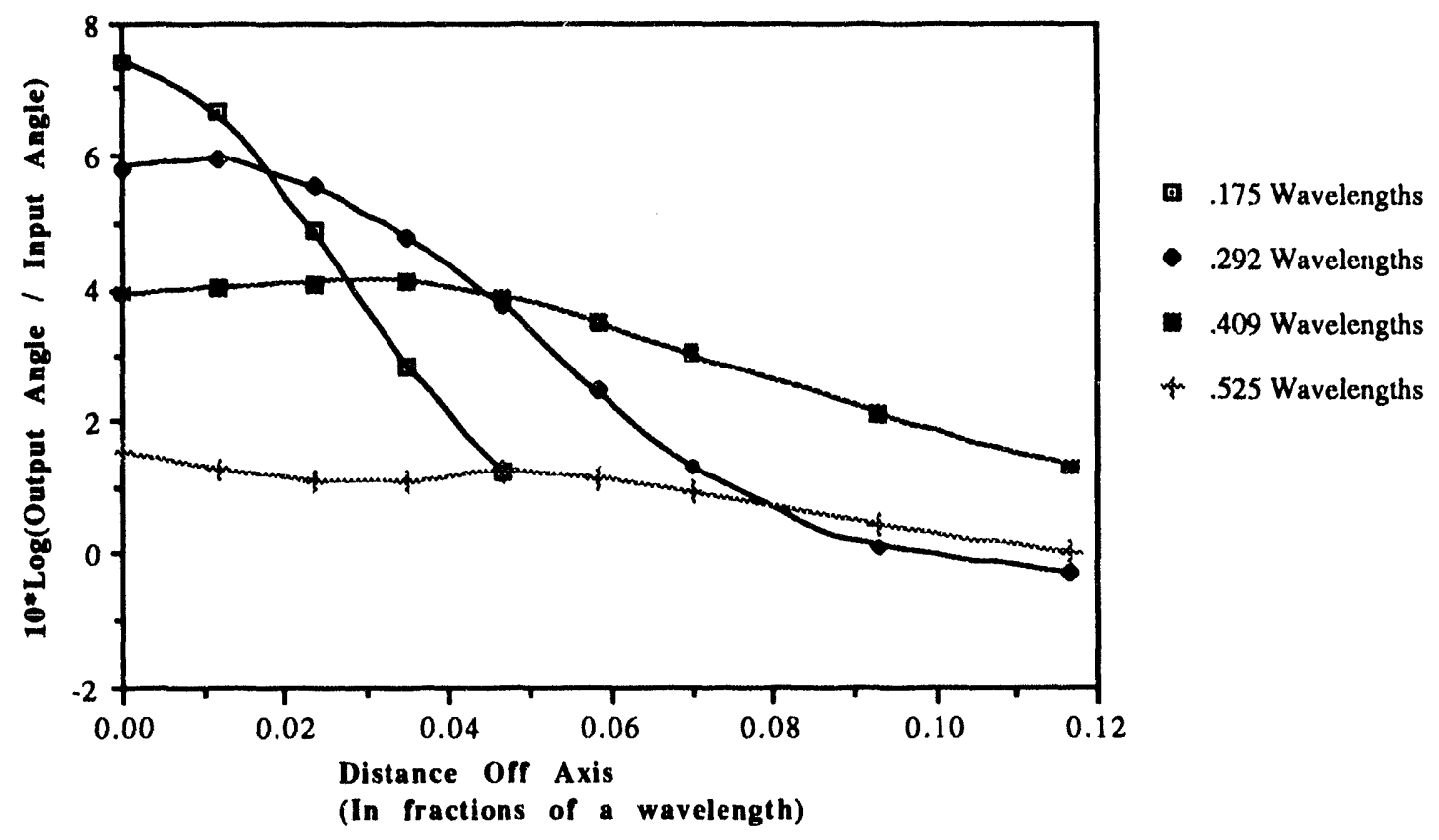

Figure 5.41. Cavity angle gain as a function of radial entrance position of beam for $\Omega / \omega=1.4$ and cavity entrance angle of $14.6^{\circ}$.

Figures 5.42, 5.43, and 5.44 also follow the same format, except that $\Omega / \omega=1.8$. 


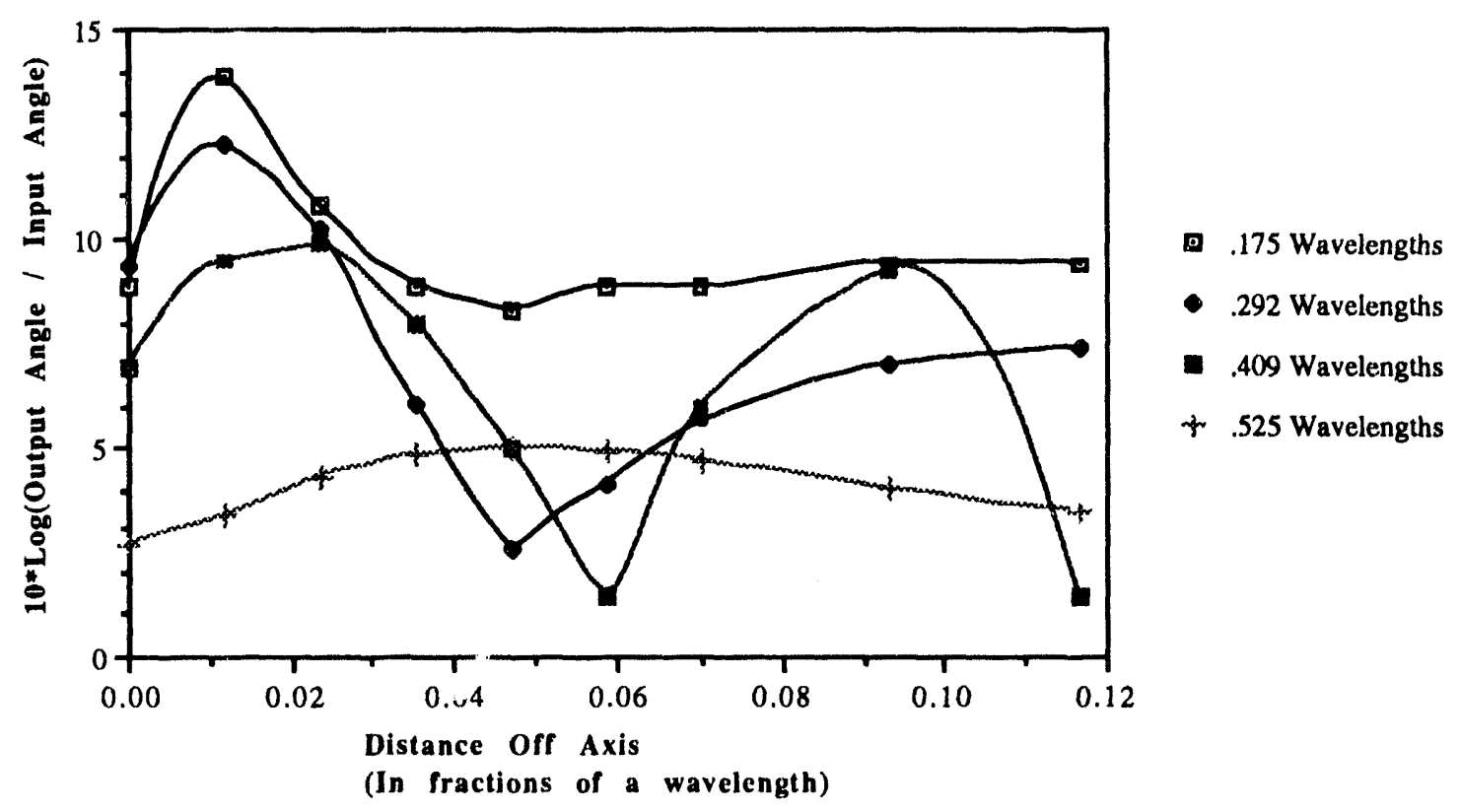

Figure 5.42. Cavity angle gain as a function of radial entrance position of beam for $\Omega / \omega=1.8$ and cavity entrance angle of $2.9^{\circ}$. 


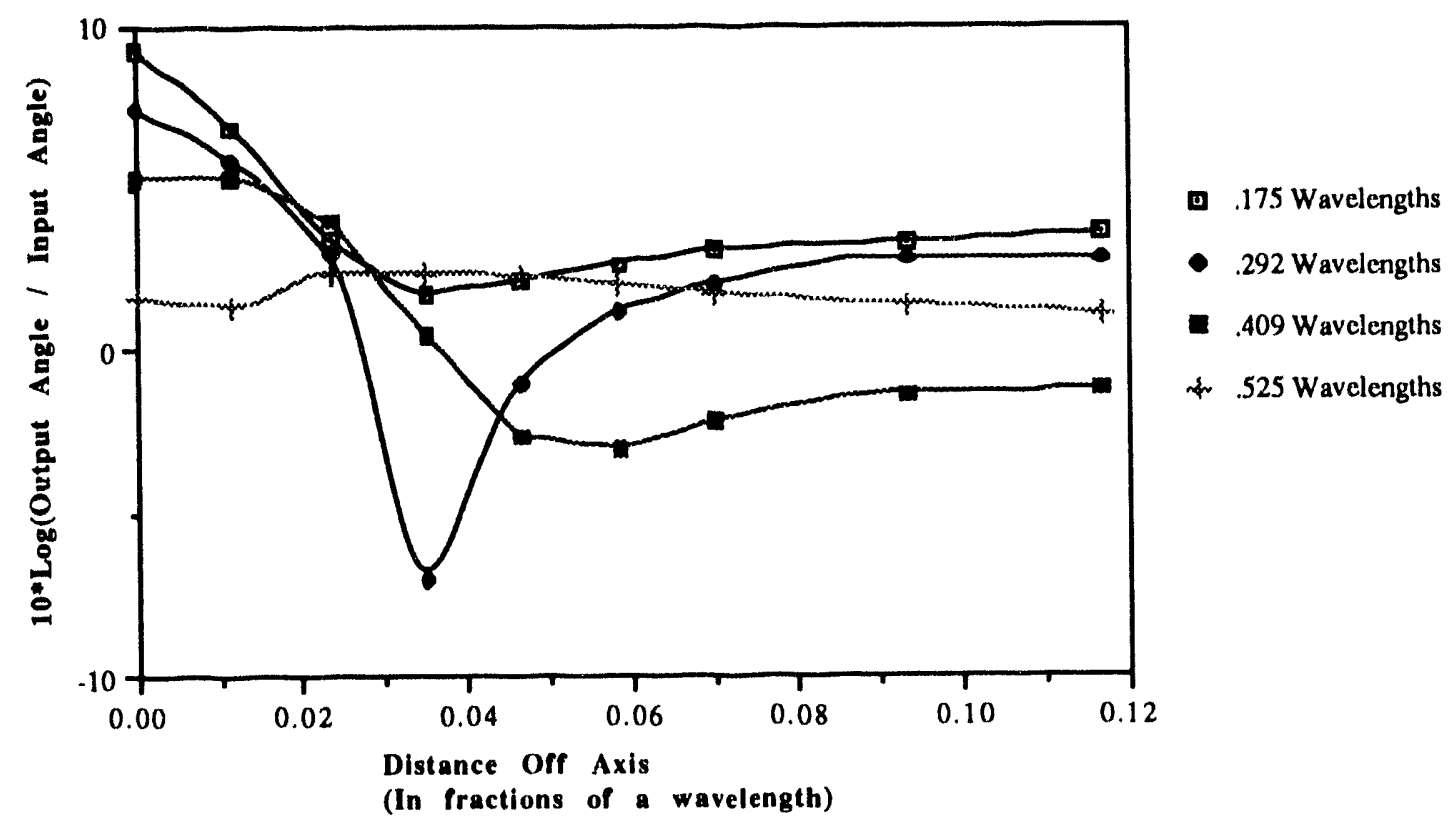

Figure 5.43. Cavity angle gain as a function of radial entrance position of beam for $\Omega / \omega=1.8$ and cavity entrance angle of $8.7^{\circ}$. 


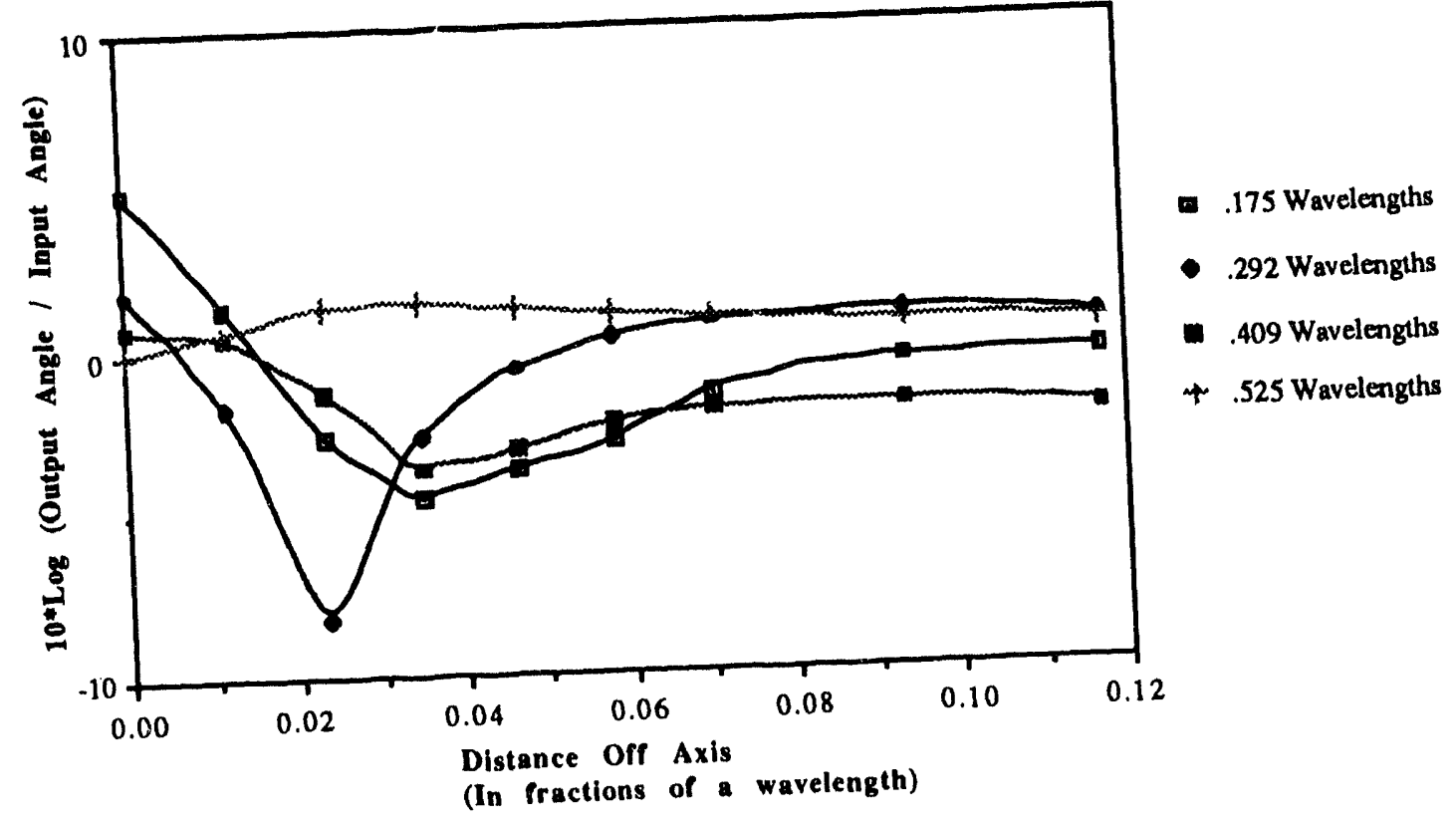

Figure 5.44. Cavity angle gain as a function of radial entrance position of beam for $\Omega / \omega=1.8$ and cavity entrance angle of $14.6^{\circ}$.

From Figs. 5.36 through 5.44, the following conclusions can be drawn:

- For smaller values of input angle, injection off-axis increases cavity angle gain over on-axis injection.

- The entrance radius that maximizes cavity-angle gain decreases with increasing input angle and with increasing $\Omega / \omega$ ratio. In fact, as the input angle increases and $\Omega / \omega$ ratio increases, the optimum injection radius is on the cavity axis.

The remaining 24 plots present the same data from a different perspective. Figures 5.45 through 5.56 are grouped in four sets of three and illustrate beam cavity angle gain versus beam entrance displacement off the longitudinal axis. The first set of three figures (Figs. 5.45, 5.46, and 5.47) is for a cavity length of $0.175 \lambda$. The second set of three figures (Figs. 5.48, 5.49, and 5.50) is for a cavity length of $0.292 \lambda$. The third set of three figures (Figs. 5.51, 5.52, and 5.53) is for a cavity length of $0.409 \lambda$. And the fourth set of three figures (Figs. 5.54, 5.55, and 5.56) is for a cavity length of $0.525 \lambda$. These four sets each contain three figures corresponding to $\Omega / \omega=1.0,1.4$, and 1.8. On 
each of these three figures are three traces corresponding to the three beam entrance angles as defined in the graph legends.

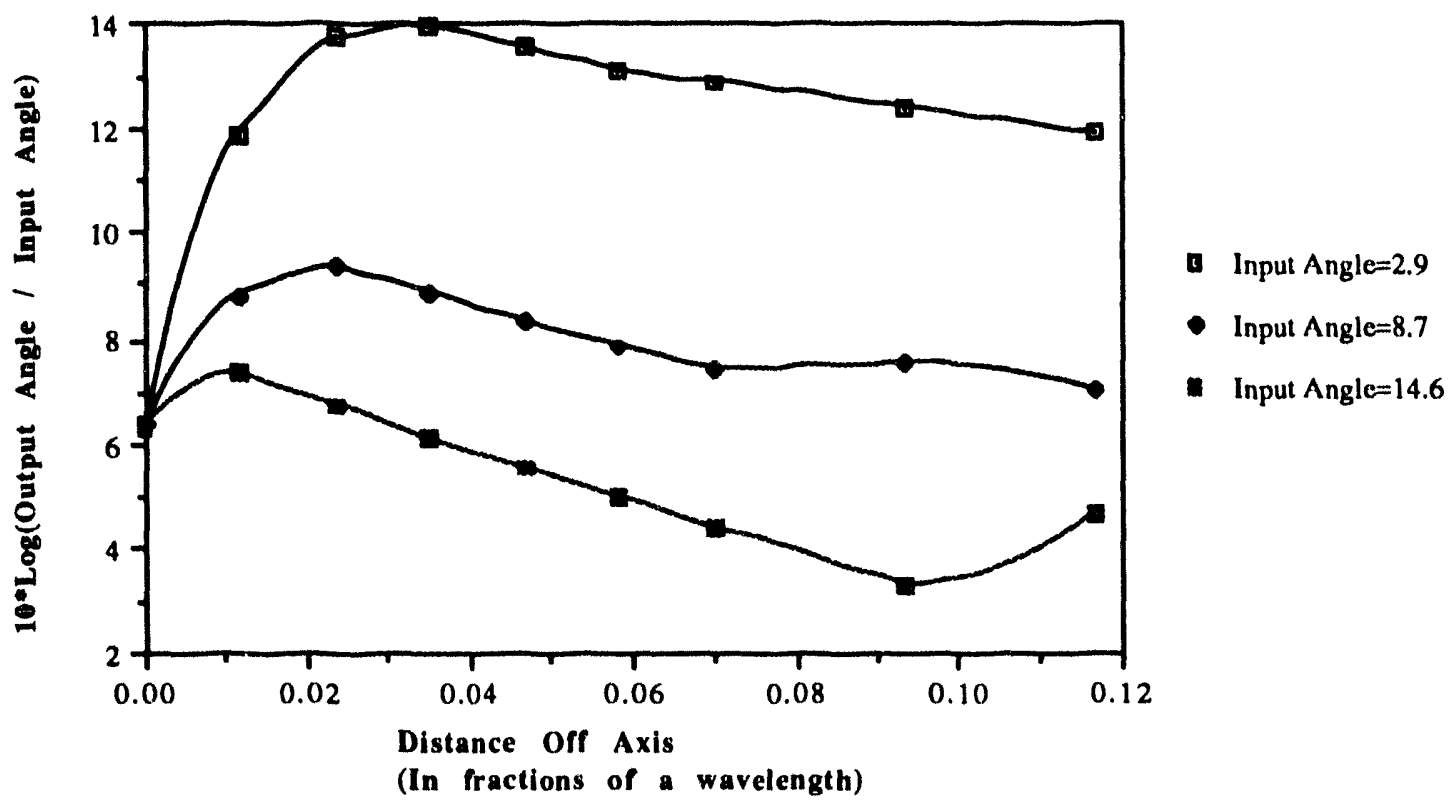

Figure 5.45. Cavity angle gain versus beam entrance displacement off the longitudinal axis for a cavity length of $0.175 \lambda$ and $\Omega / \omega=1.0$. 


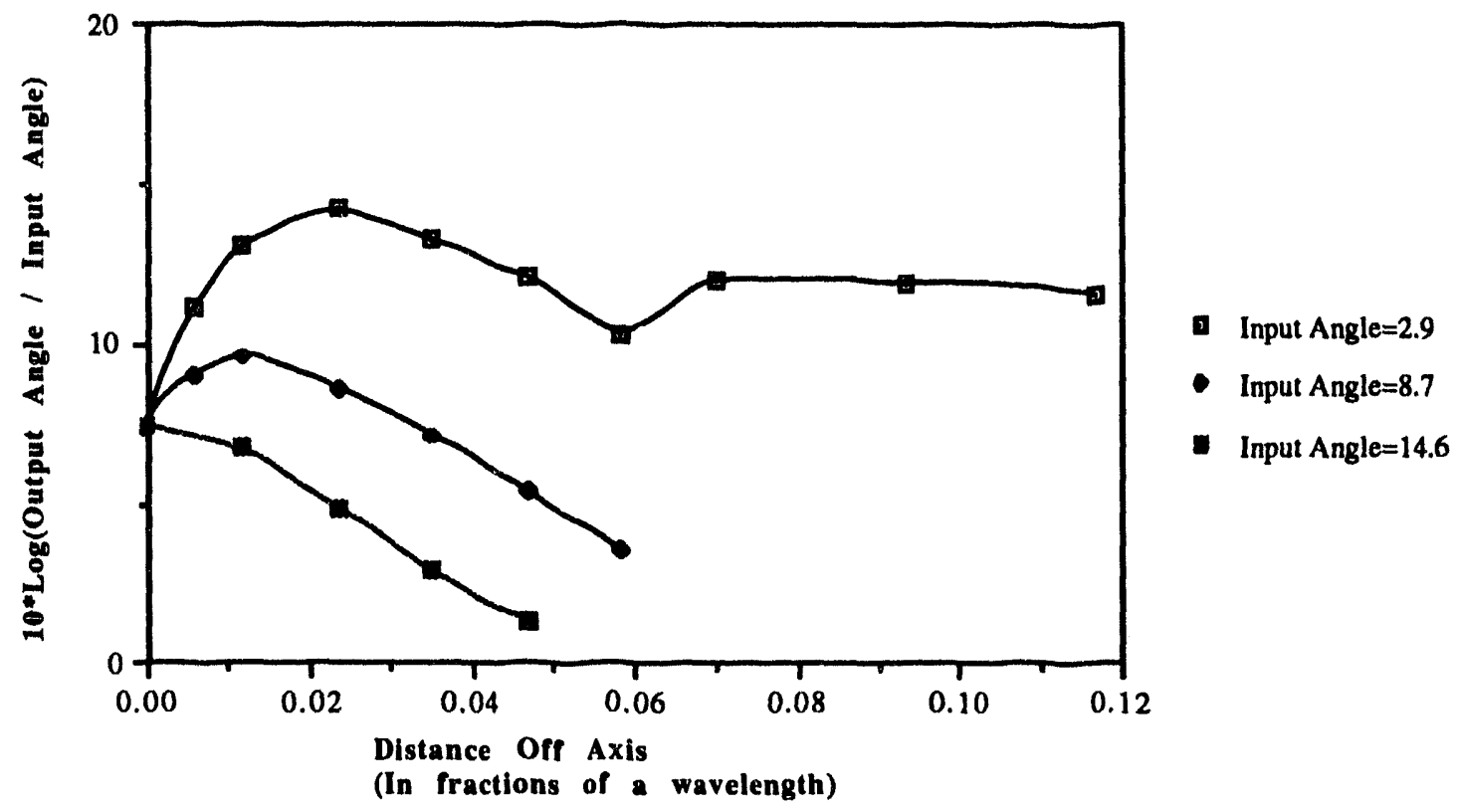

Figure 5.46. Cavity angle gain versus beam entrance displacement off the longitudinal axis for a cavity length of $0.175 \lambda$ and $\Omega / \omega=1.4$.

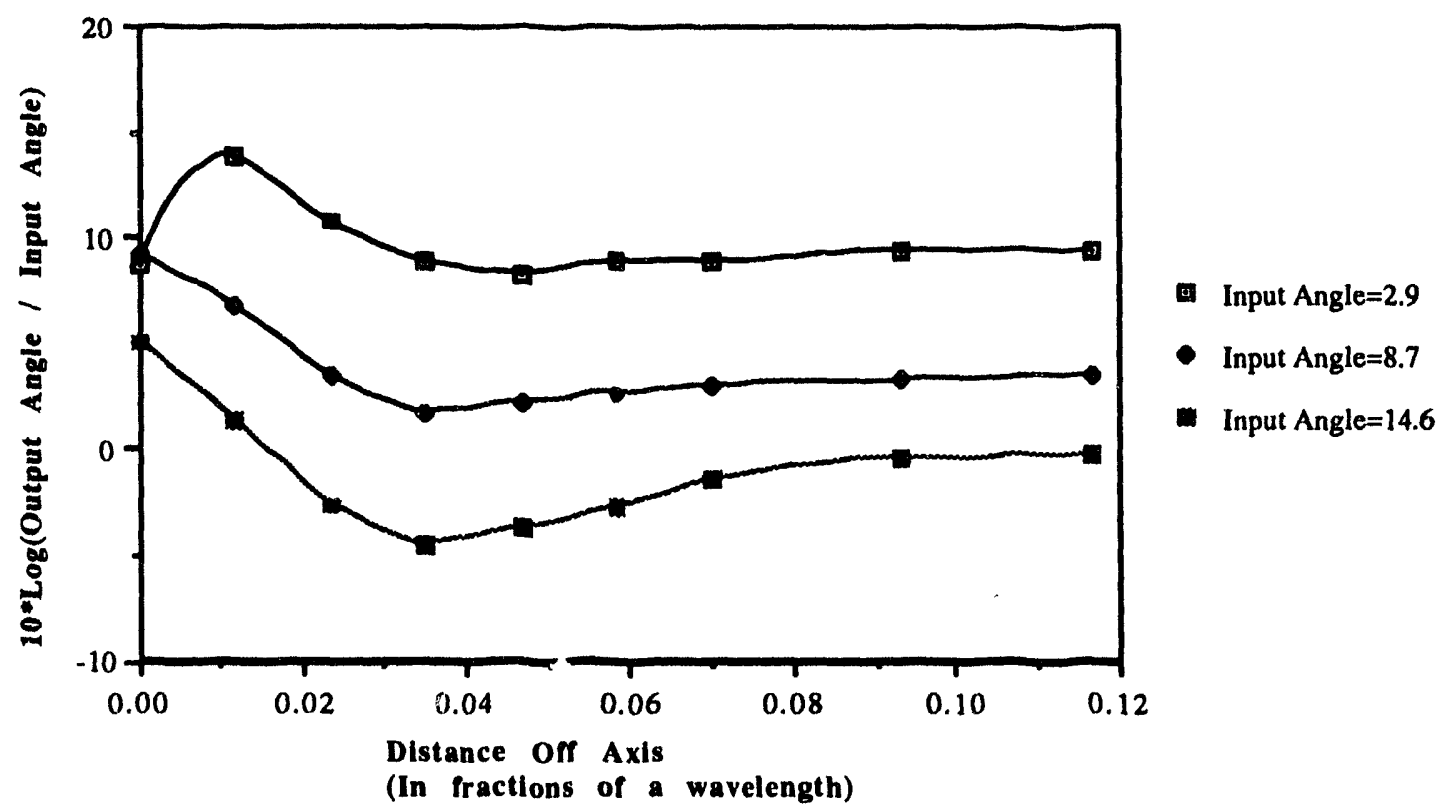

Figure 5.47. Cavity angle gain versus beam entrance displacement off the longitudinal axis for a cavity length of $0.175 \lambda$ and $\Omega / \omega=1.8$. 


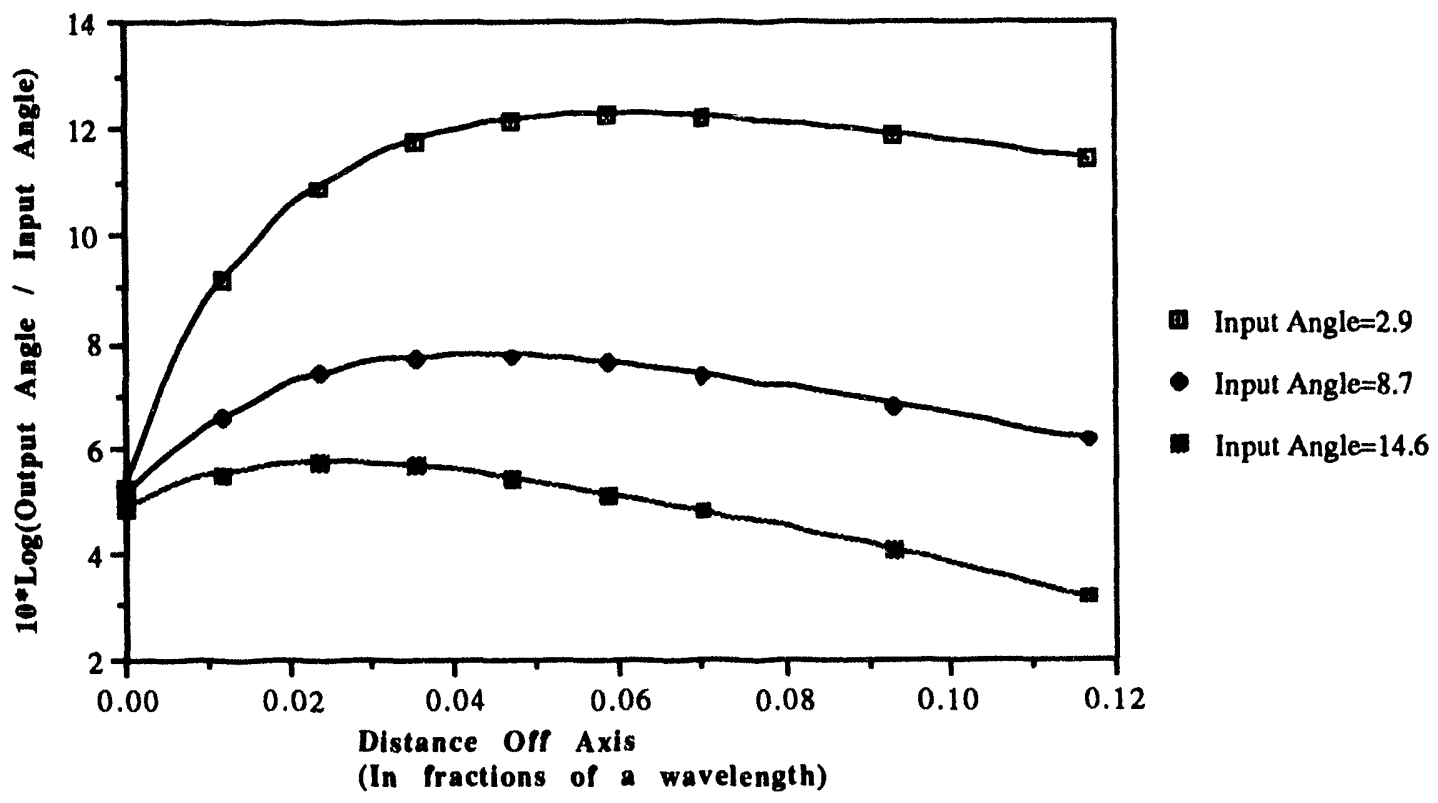

Figure 5.48. Cavity angle gain versus beam entrance displacement off the longitudinal axis for a cavity length of $0.292 \lambda$ and $\Omega / \omega=1.0$.

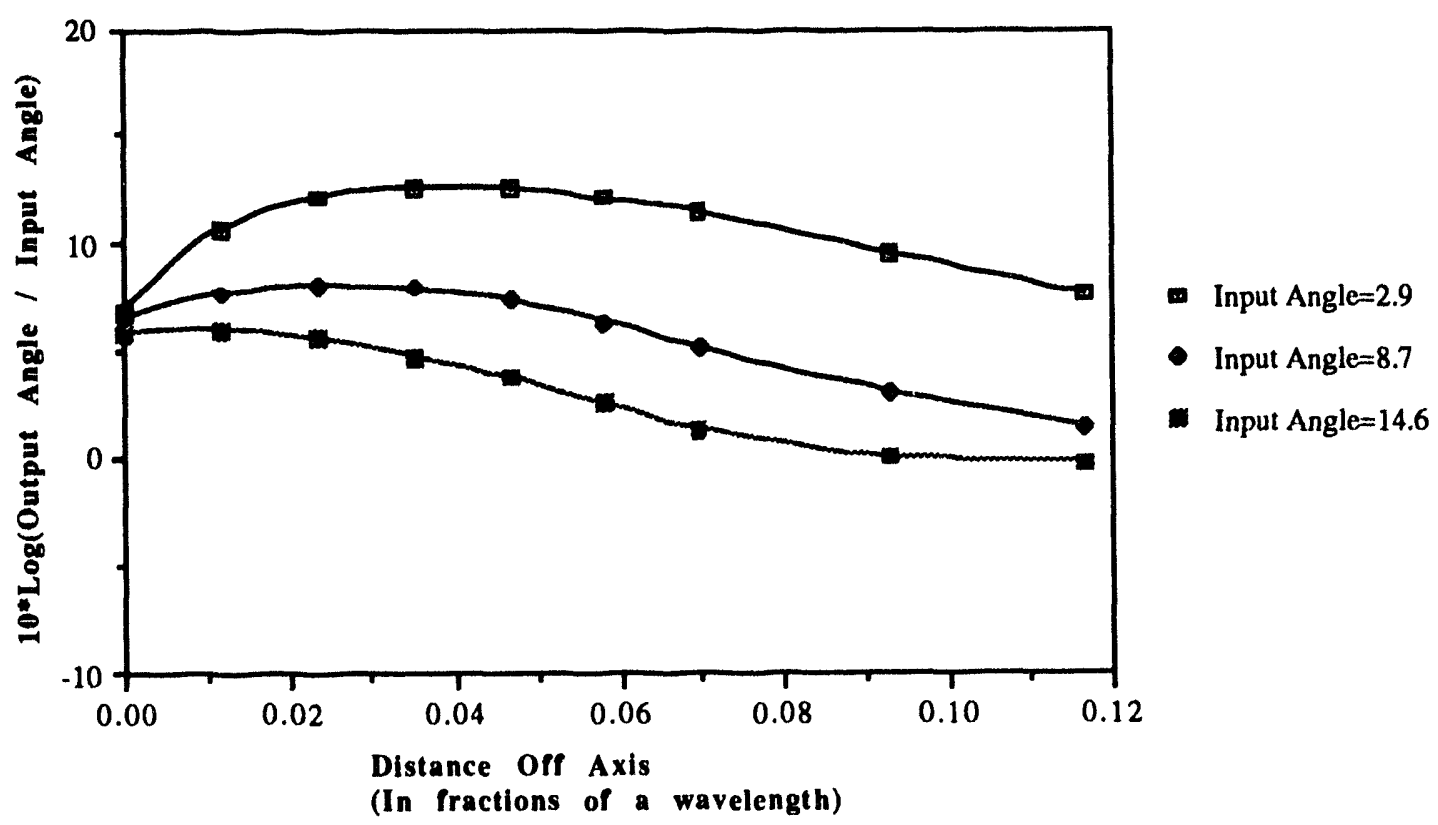

Figure 5.49. Cavity angle gain versus beam entrance displacement off the longitudinal axis for a cavity length of $0.292 \lambda$ and $\Omega / \omega=1.4$. 


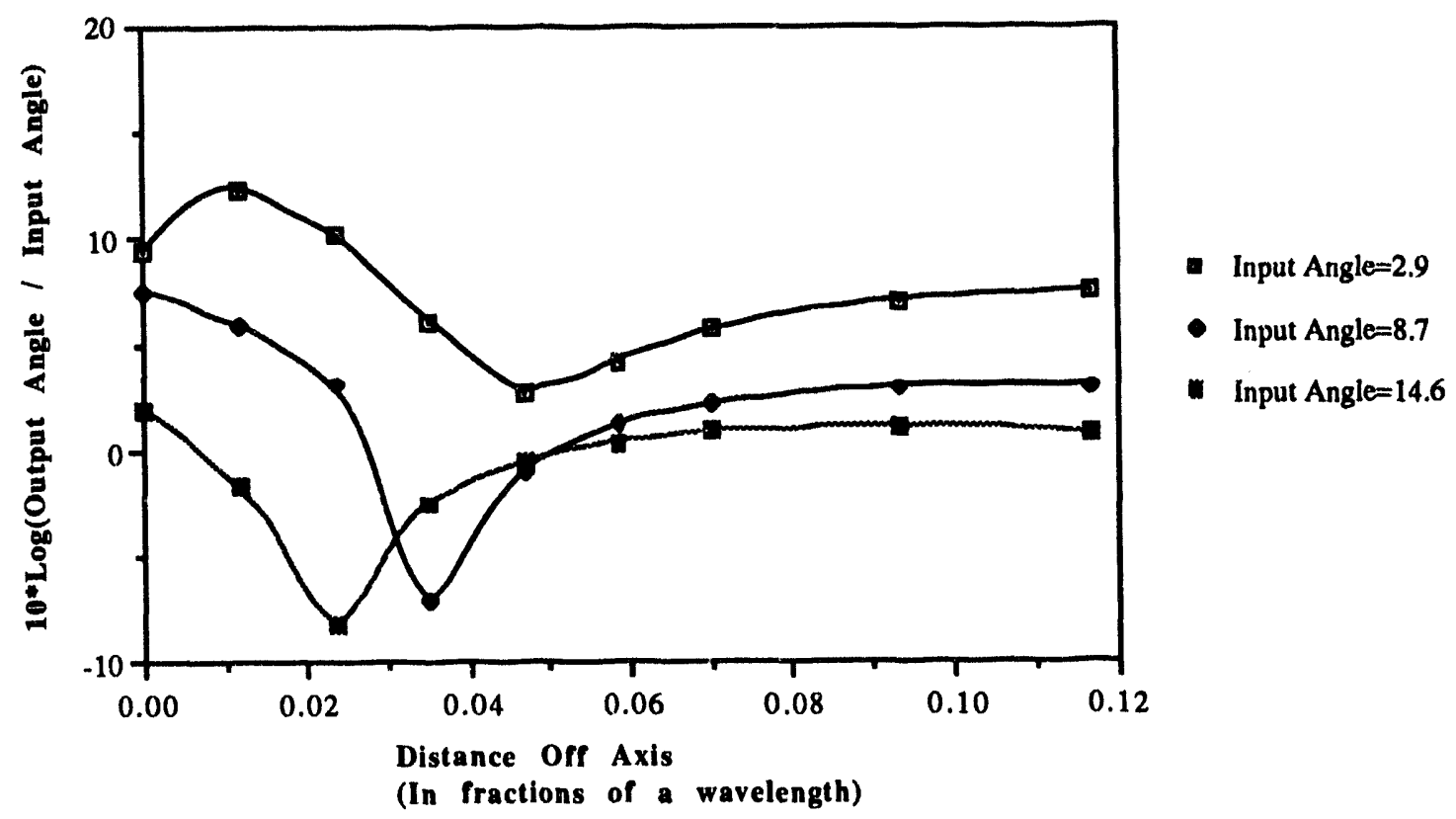

Figure 5.50. Cavity angle gain versus beam entrance displacement off the longitudinal axis for a cavity length of $0.292 \lambda$ and $\Omega / \omega=1.8$.

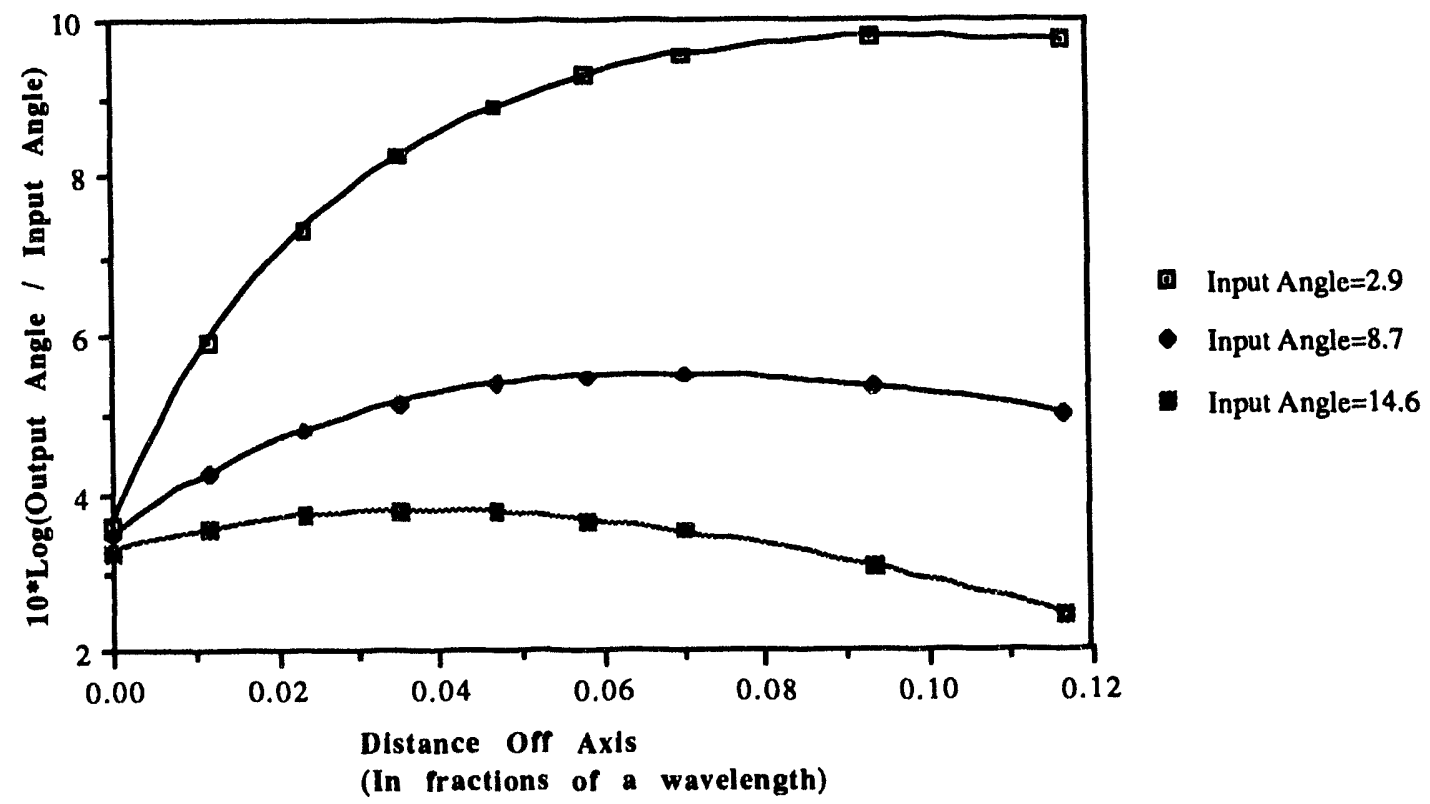

Figure 5.51. Cavity angle gain versus beam entrance displacement off the longitudinal axis for a cavity length of $0.409 \lambda$ and $\Omega / \omega=1.0$. 


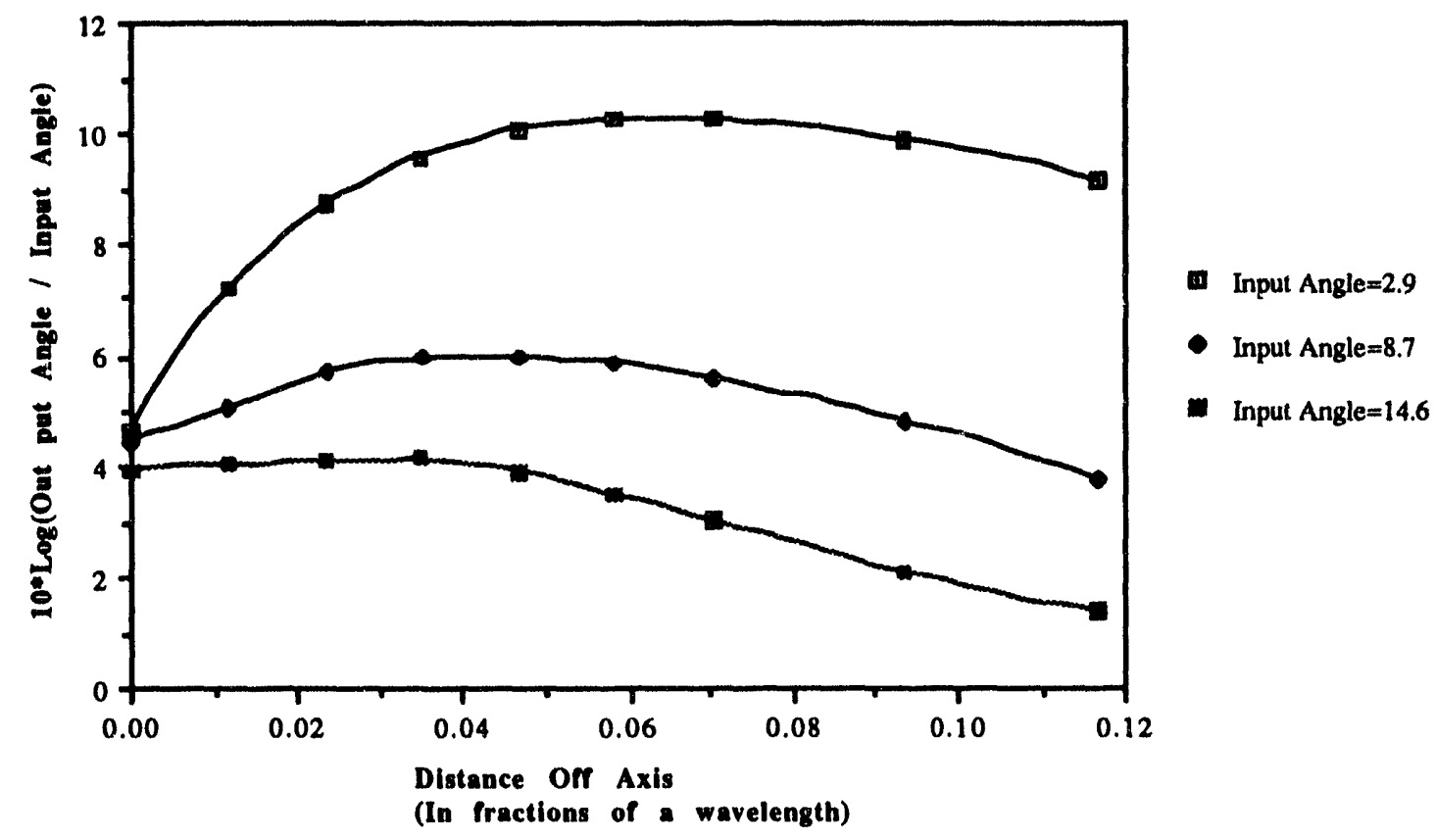

Figure 5.52. Cavity angle gain versus beam entrance displacement off the longitudinal axis for a cavity length of $0.409 \lambda$ and $\Omega / \omega=1.4$. 


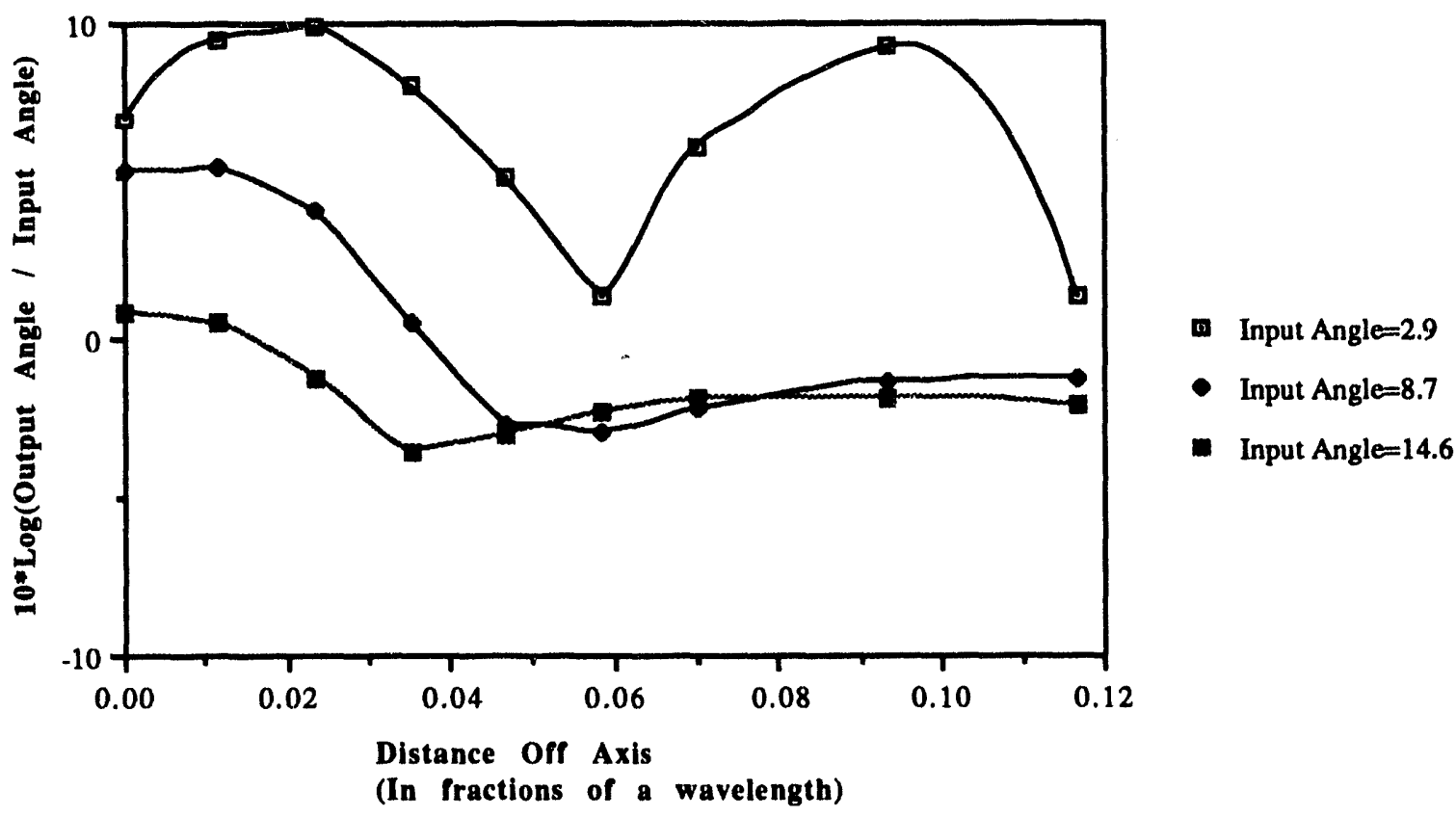

Figure 5.53. Cavity angle gain versus beam entrance displacement off the longitudinal axis for a cavity length of $0.409 \lambda$ and $\Omega / \omega=1.8$. 


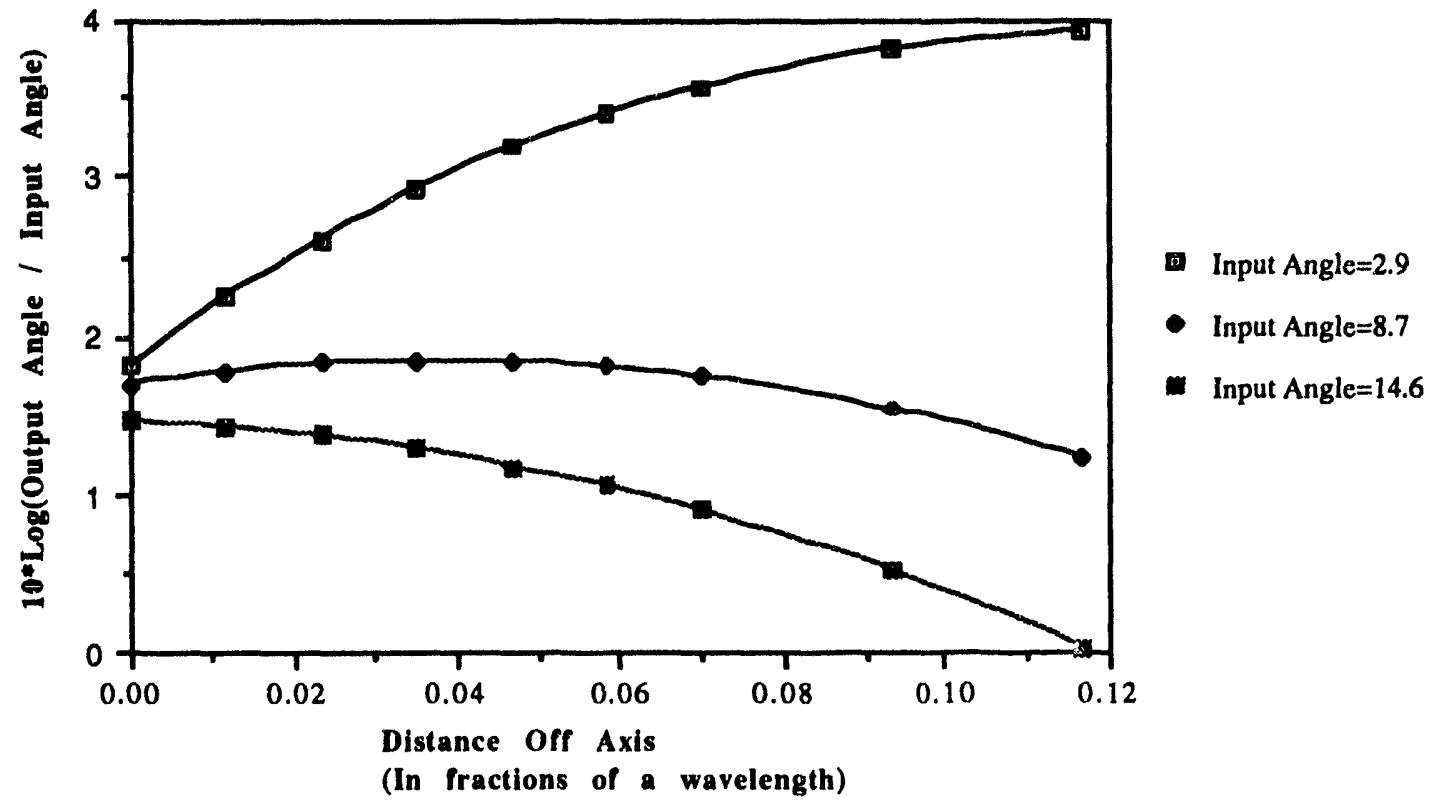

Figure 5.54. Cavity angle gain versus beam entrance displacement off the longitudinal axis for a cavity length of $0.525 \lambda$ and $\Omega / \omega=1.0$. 


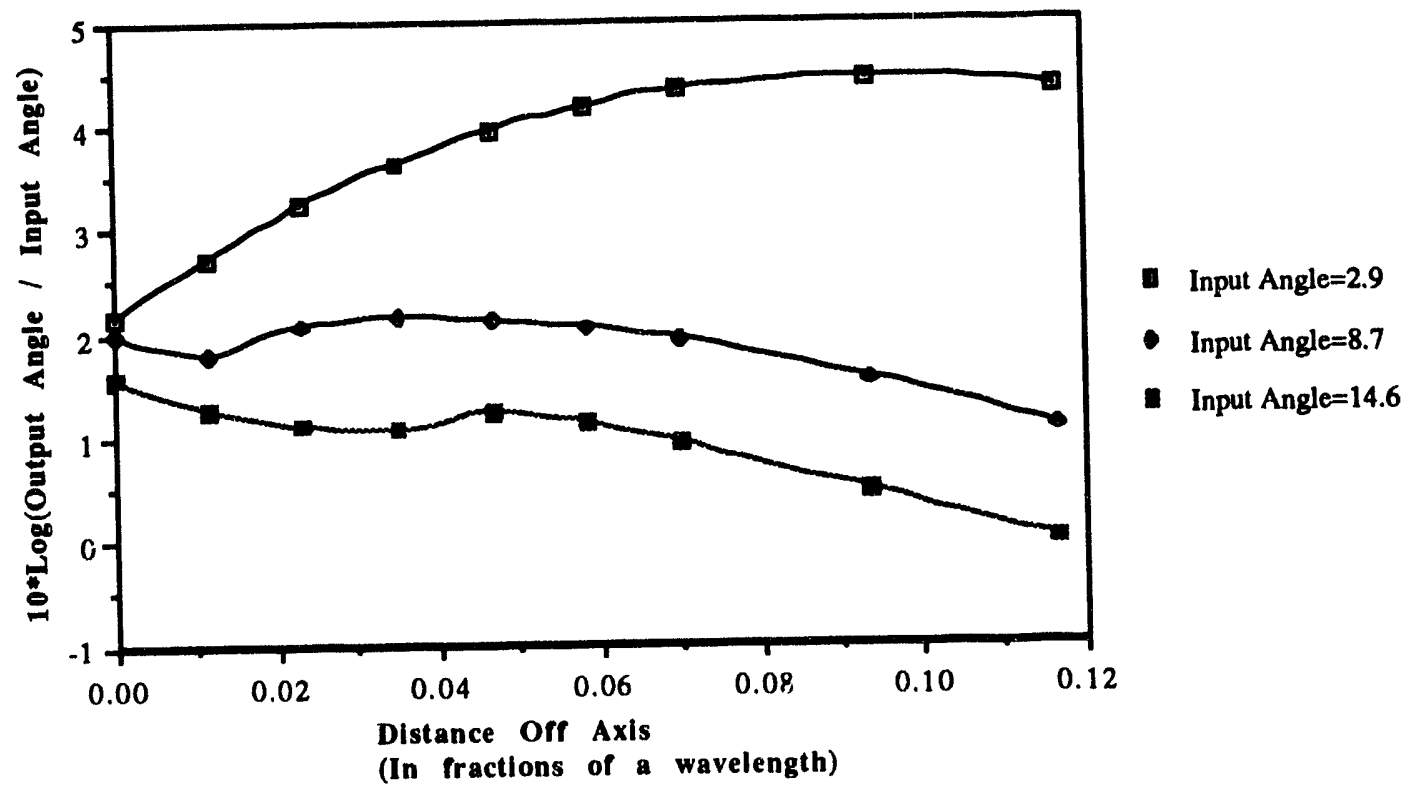

Figure 5.55. Cavity angle gain versus beam entrance displacement off the longitudinal axis for a cavity length of $0.525 \lambda$ and $\Omega / \omega=1.4$. 


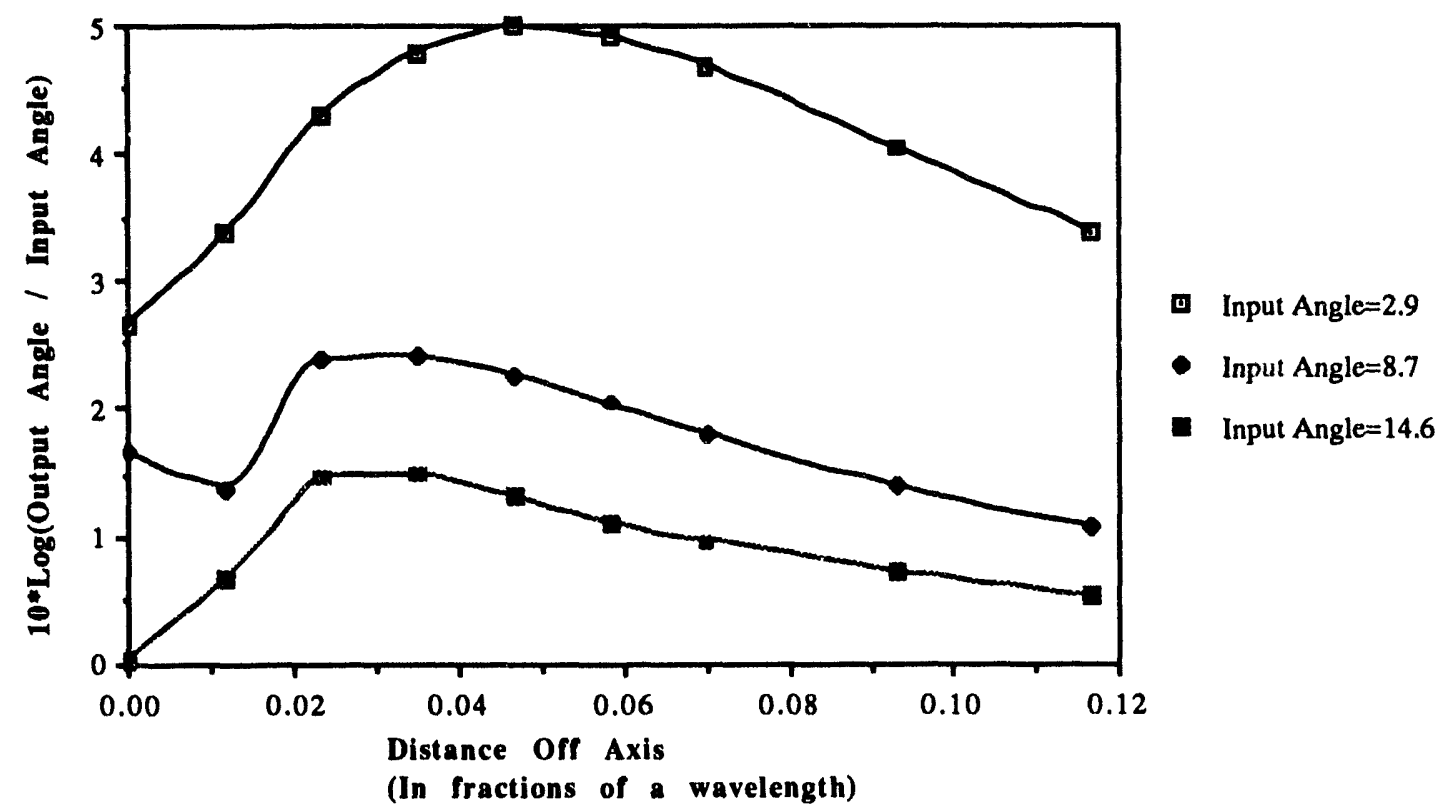

Figure 5.56. Cavity angle gain versus beam entrance displacement off the longitudinal axis for a cavity length of $0.525 \lambda$ and $\Omega / \omega=1.8$.

Figures 5.45 through 5.56 illustrate again that as the input angle increases, the increases in angle gain that can be realized through off-axis injection into the cavity are reduced or disappear. These figures also illustrate that even though the on-axis angle gain is similar for different input angles where the other parameters remain constant (or exactly the same for small angles), the gain corresponding to an off-axis injection is quite different and is a strong function of the input angle.

Figures 5.57 through 5.68 are very similar to Figs. 5.45 through 5.56. They show cavity angle gain versus beam entrance displacement off the longitudinal axis. The same four sets of graphs are distinguished by cavity length, and each set is made up of three graphs. However, in Figs. 5.57 through 5.68 each graph represents a different input angle, and the three traces on each of these three graphs represent different values of $\Omega / \omega$ $(1.0,1.4$, and 1.8). 


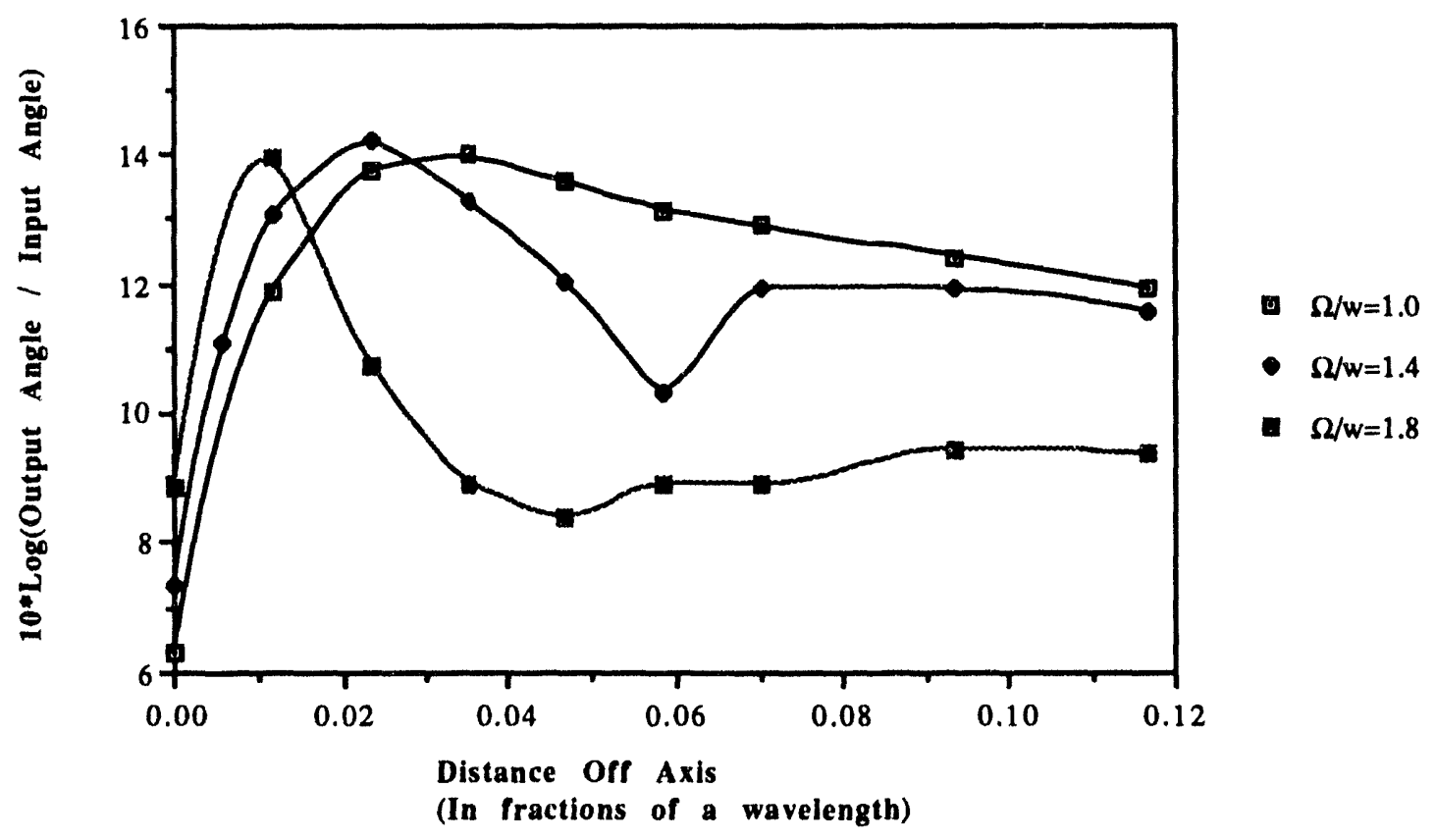

Figure 5.57. Cavity angle gain versus beam entrance displacement off the longitudinal axis for a cavity length of $0.175 \lambda$ and input angle of $2.9^{\circ}$. 


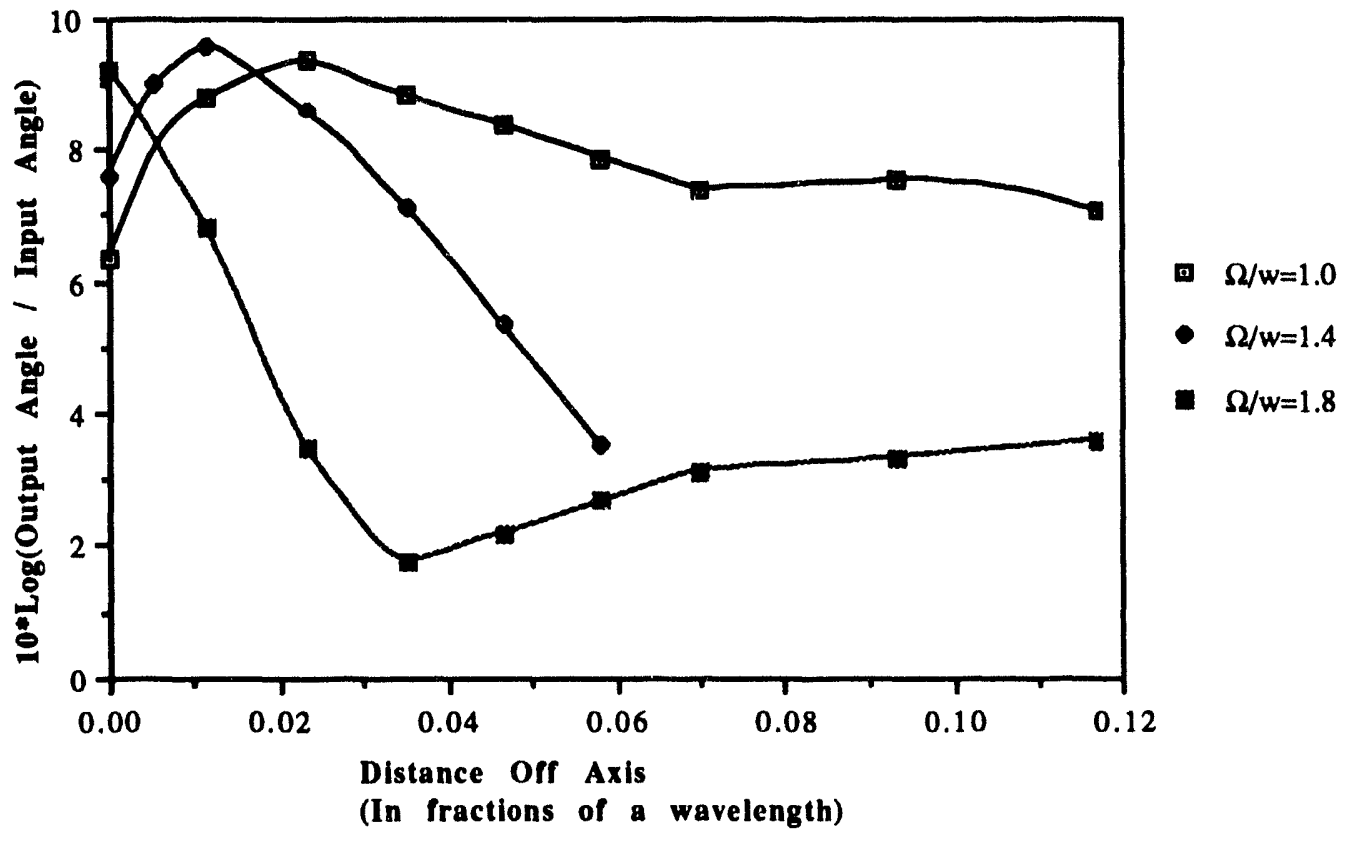

Figure 5.58. Cavity angle gain versus beam entrance displacement off the longitudinal axis for a cavity length of $0.175 \lambda$ and input angle of $8.7^{\circ}$. 


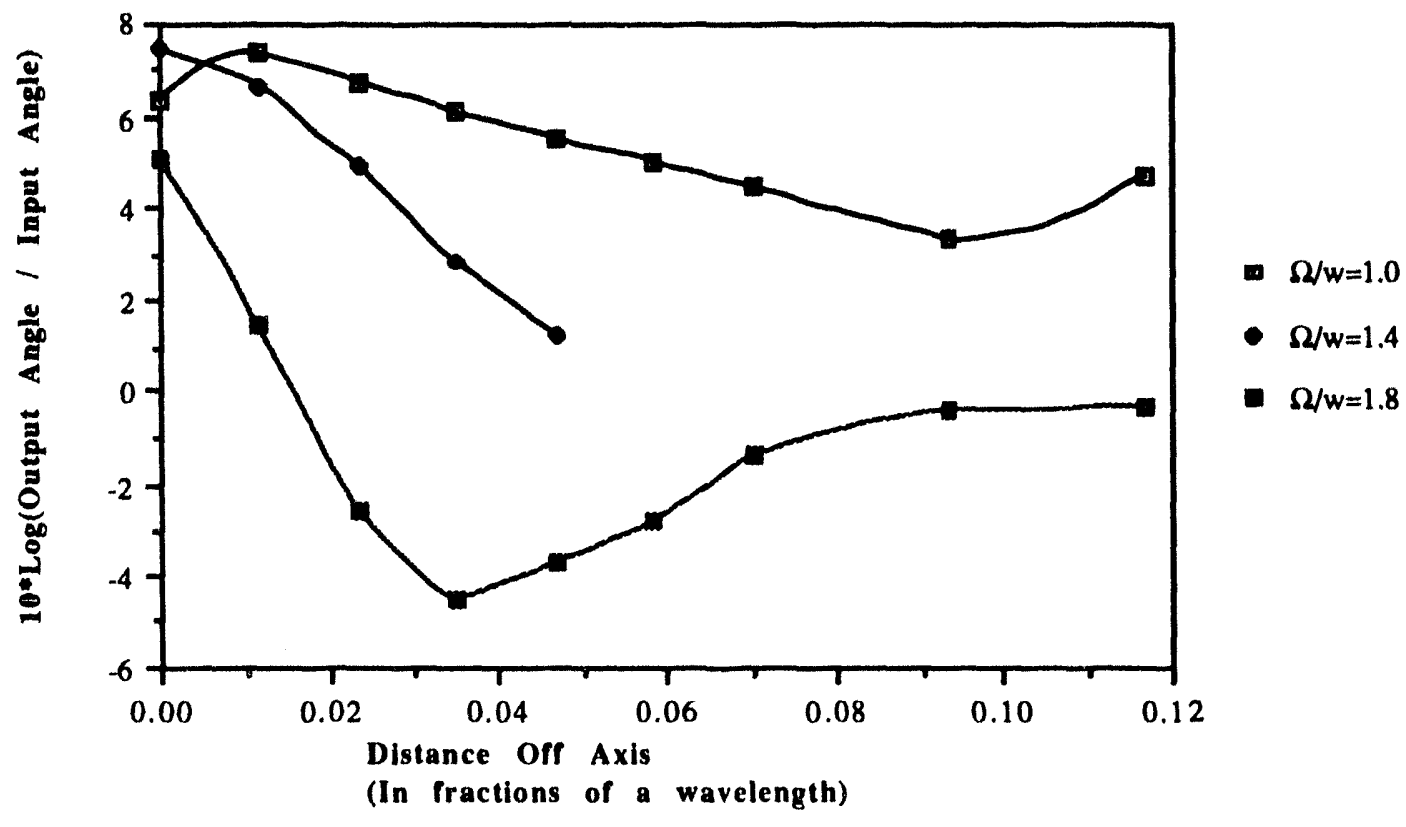

Figure 5.59. Cavity angle gain versus beam entrance displacement off the longitudinal axis for a cavity length of $0.175 \lambda$ and input angle of $14.6^{\circ}$. 


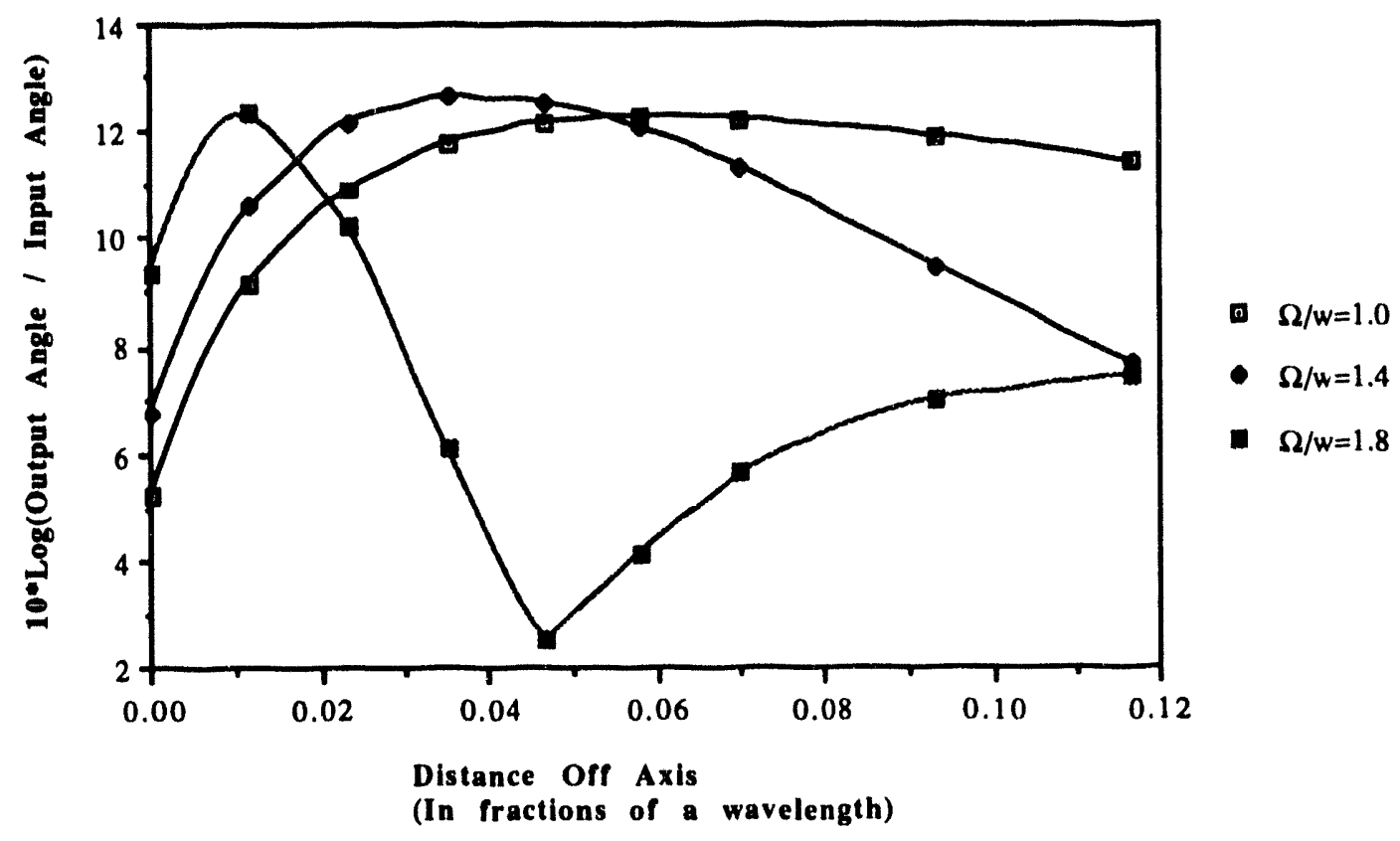

Figure 5.60. Cavity angle gain versus beam entrance displacement off the longitudinal axis for a cavity length of $0.292 \lambda$ and input angle of $2.9^{\circ}$. 


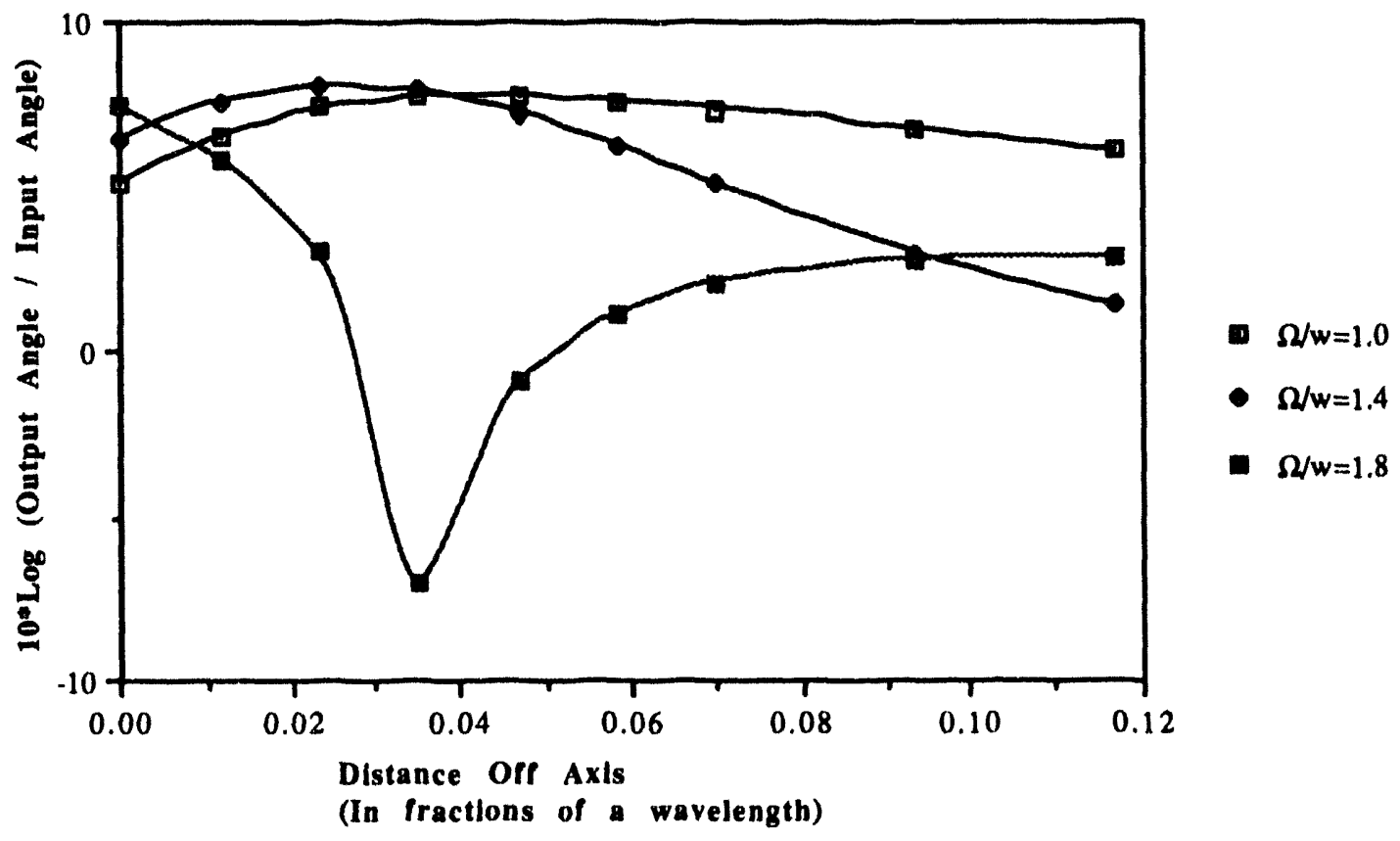

Figure 5.61. Cavity angle gain versus beam entrance displacement off the longitudinal axis for a cavity length of $0.292 \lambda$ and input angle of $8.7^{\circ}$. 


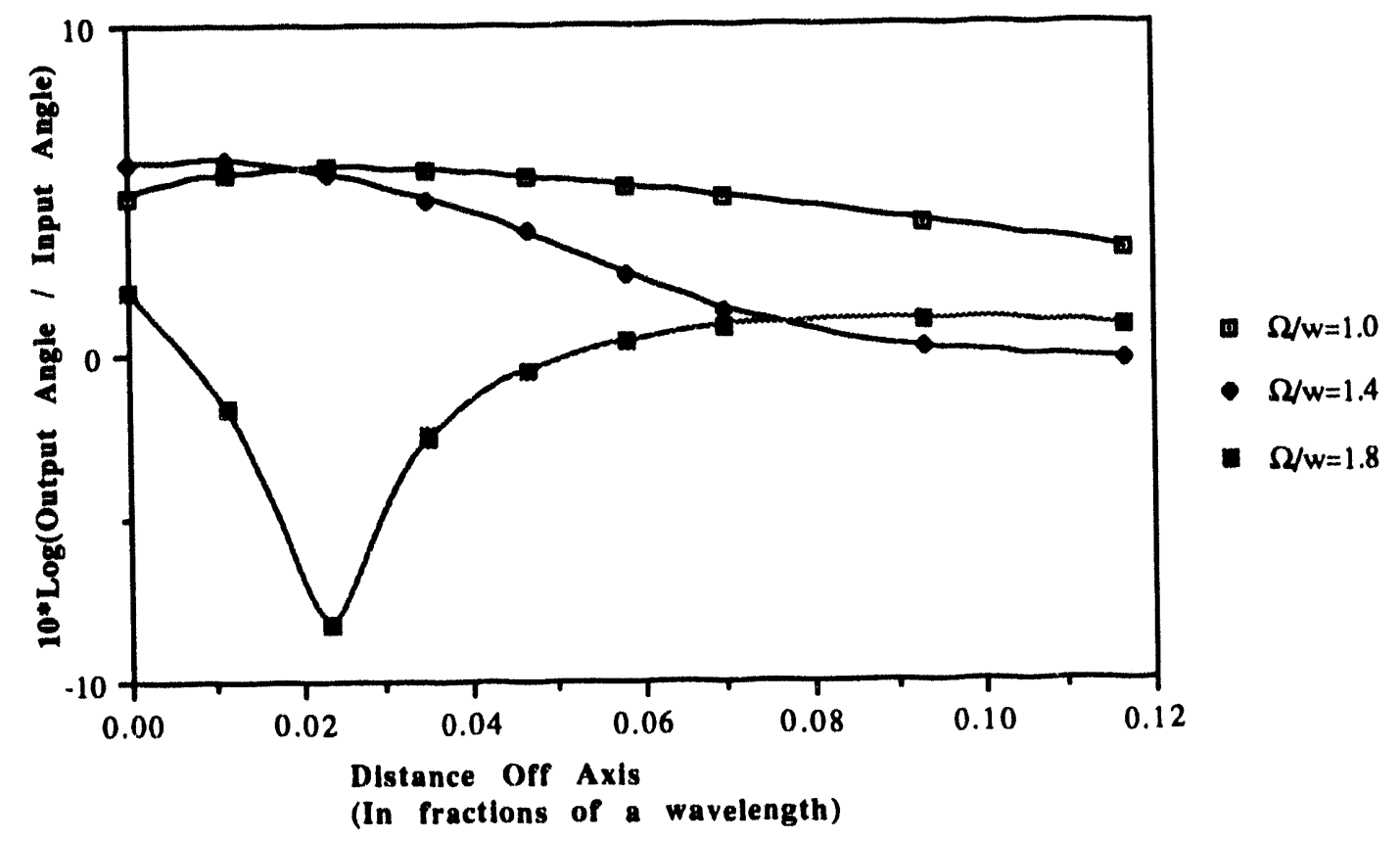

Figure 5.62. Cavity angle gain versus beam entrance displacement off the longitudinal axis for a cavity length of $0.292 \lambda$ and input angle of $14.6^{\circ}$. 


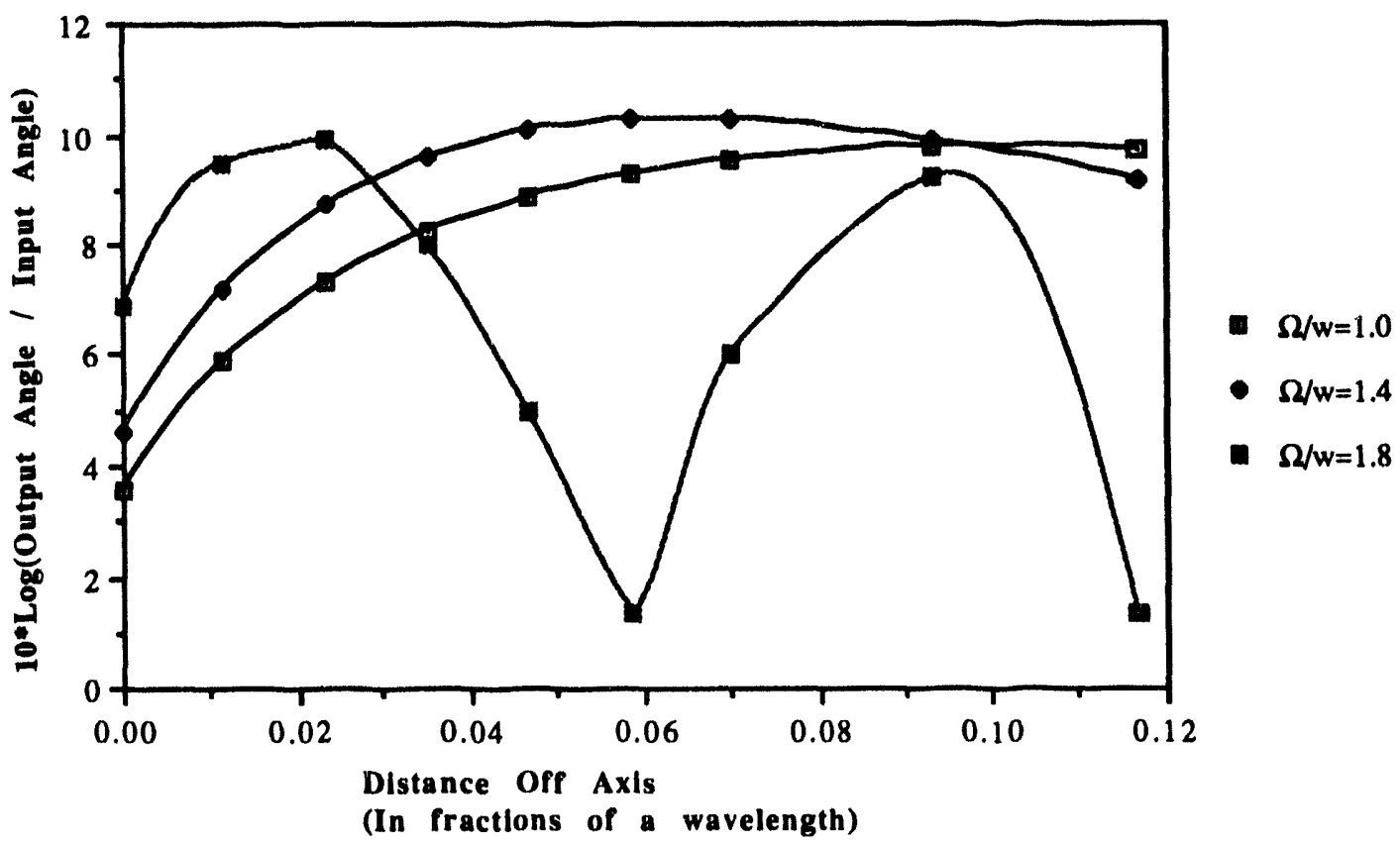

Figure 5.63. Cavity angle gain versus beam entrance displacement off the longitudinal axis for a cavity length of $0.409 \lambda$ and input angle of $2.9^{\circ}$. 


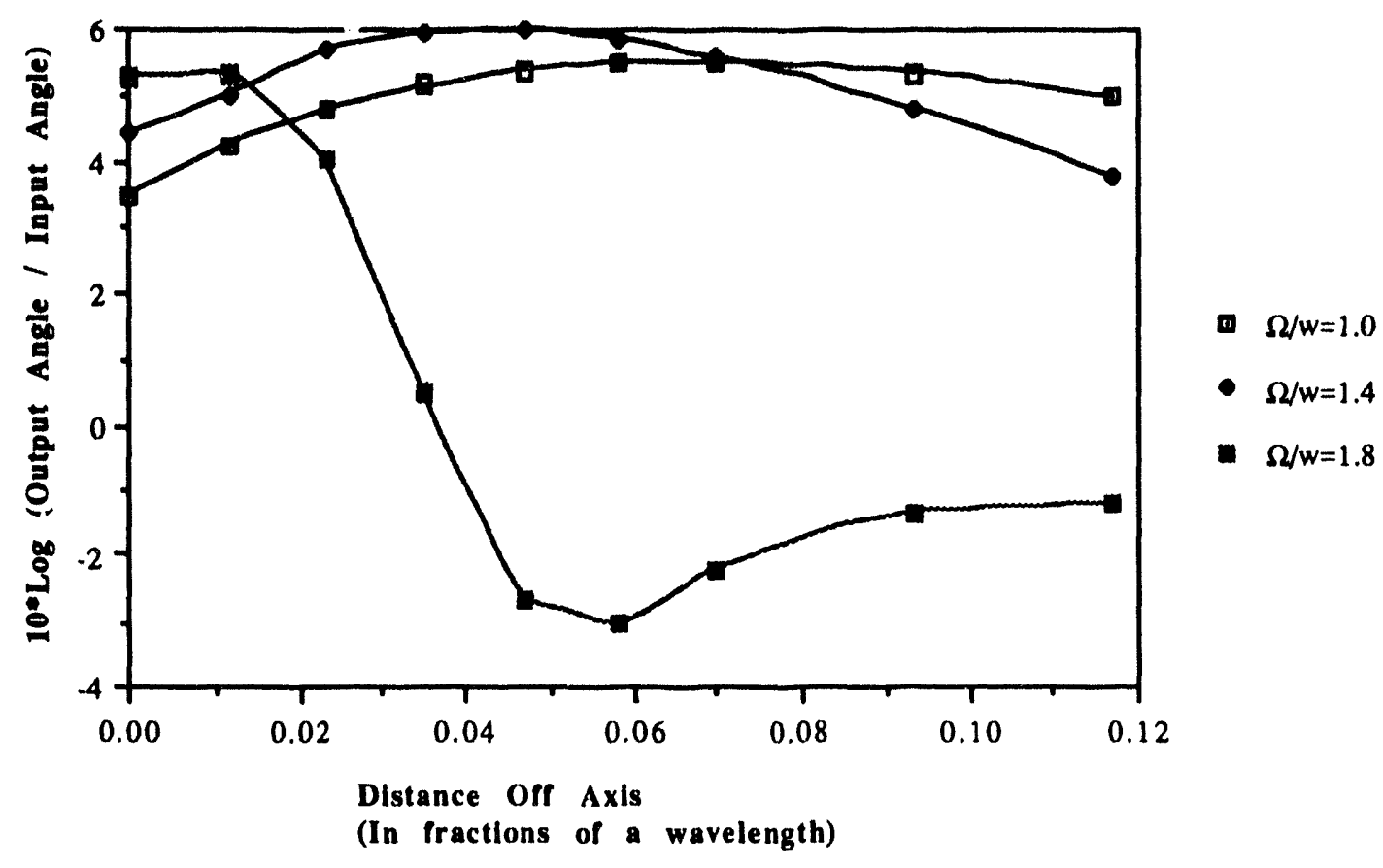

Figure 5.64. Cavity angle gain versus beam entrance displacement off the longitudinal axis for a cavity length of $0.409 \lambda$ and input angle of $8.7^{\circ}$. 


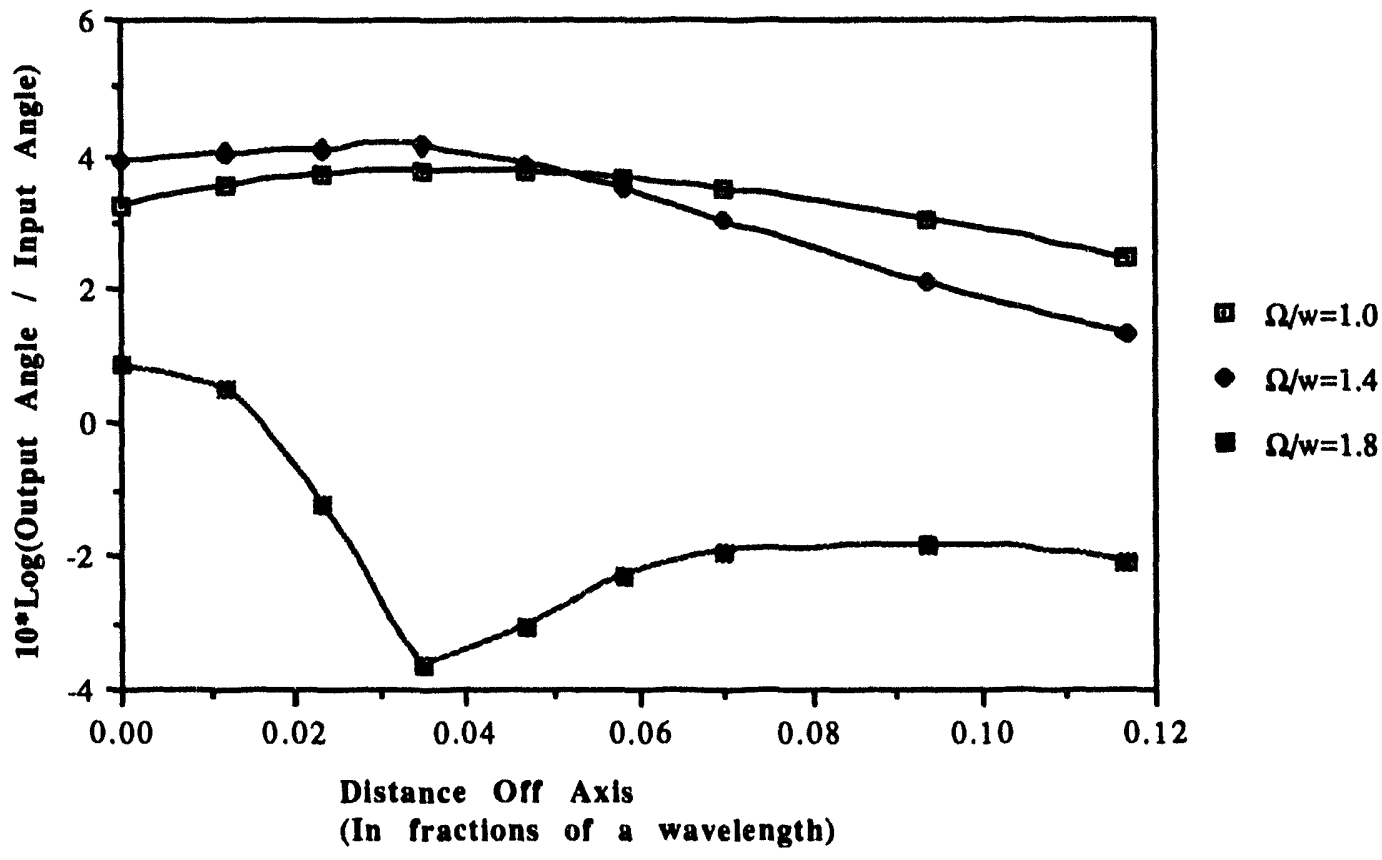

Figure 5.65. Cavity angle gain versus beam entrance displacement off the longitudinal axis for a cavity length of $0.409 \lambda$ and input angle of $14.6^{\circ}$. 


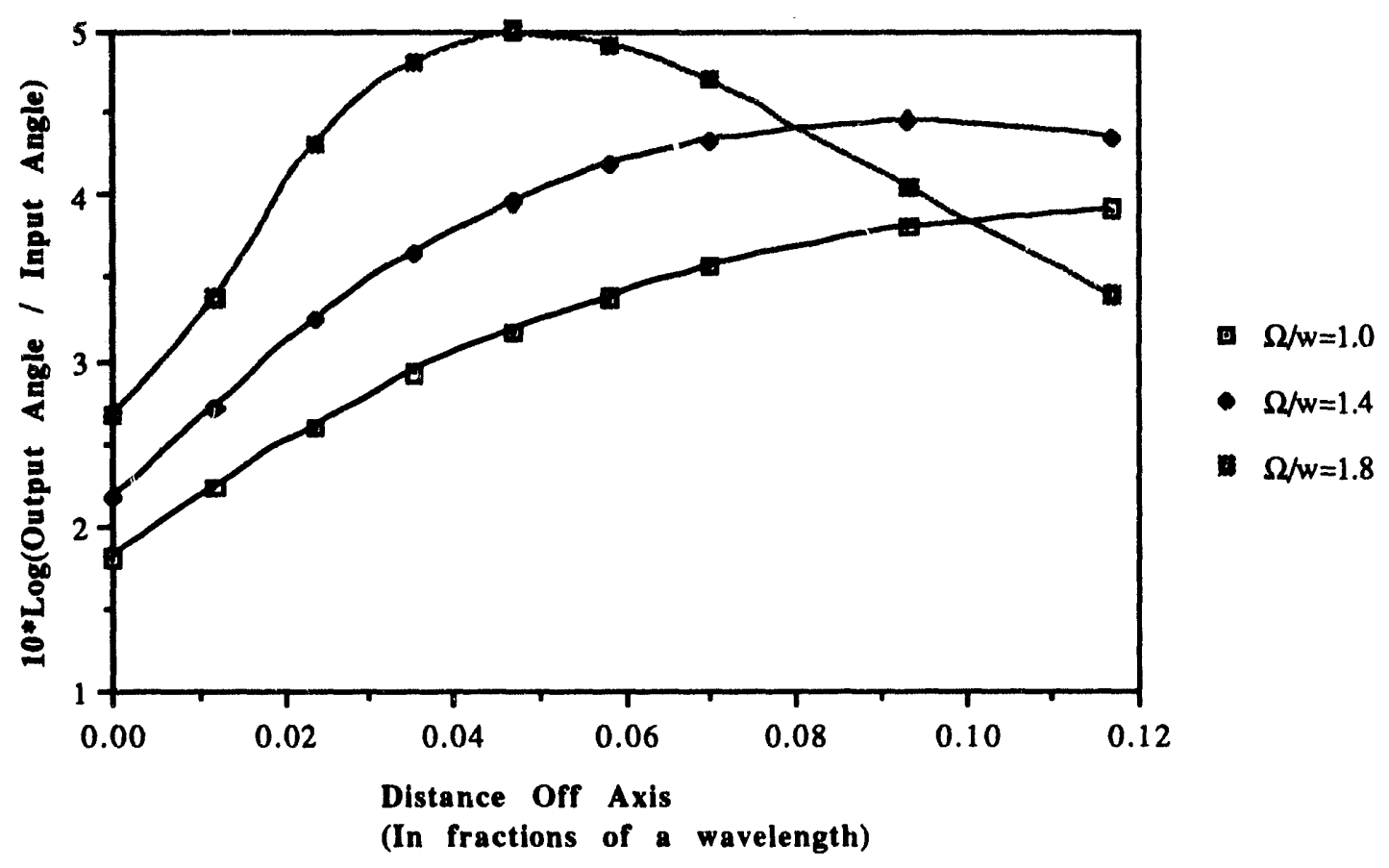

Figure 5.66. Cavity angle gain versus beam entrance displacement off the longitudinal axis for a cavity length of $0.525 \lambda$ and input angle of $2.9^{\circ}$. 


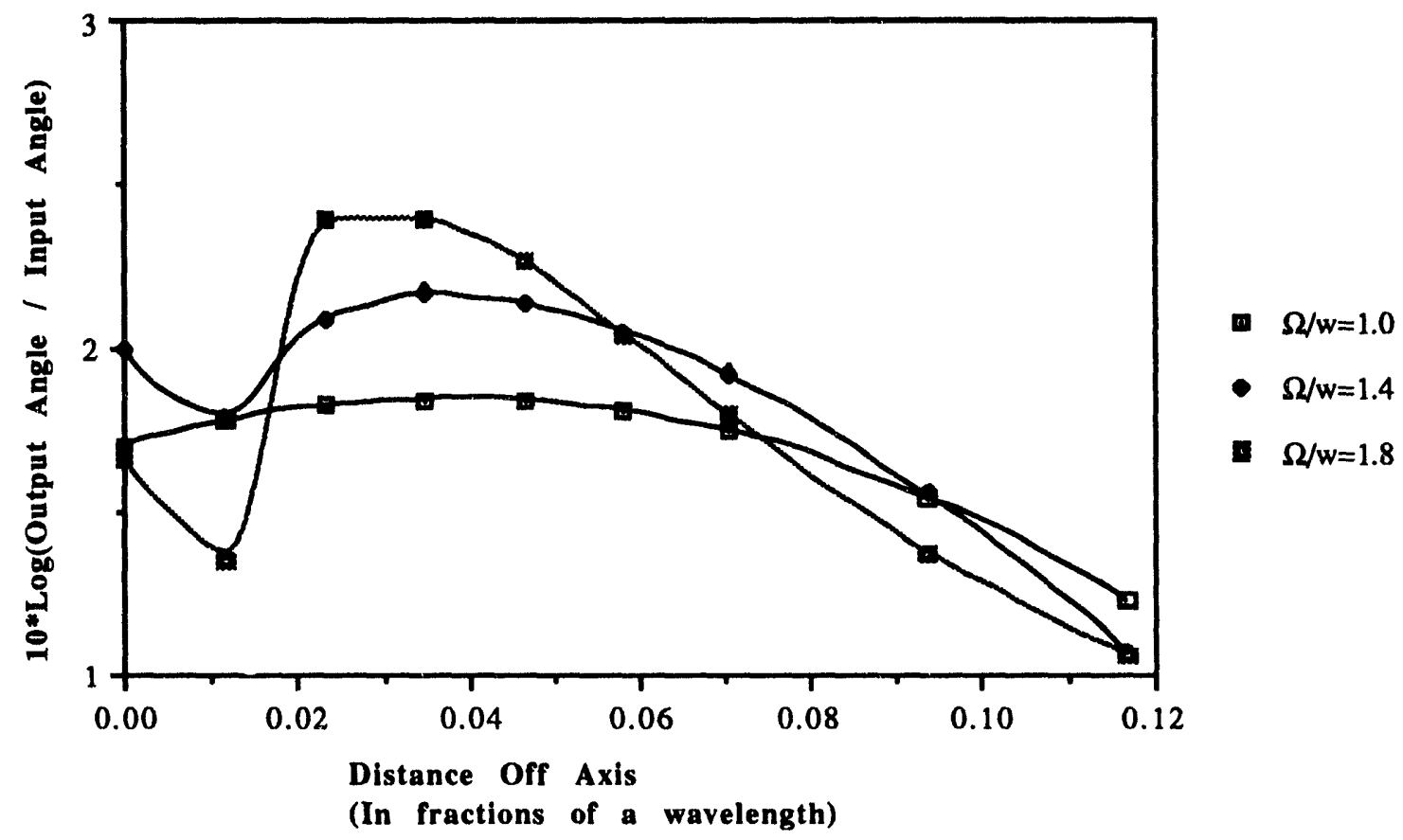

Figure 5.67. Cavity angle gain versus beam entrance displacement off the longitudinal axis for a cavity length of $0.525 \lambda$ and input angle of $8.7^{\circ}$. 


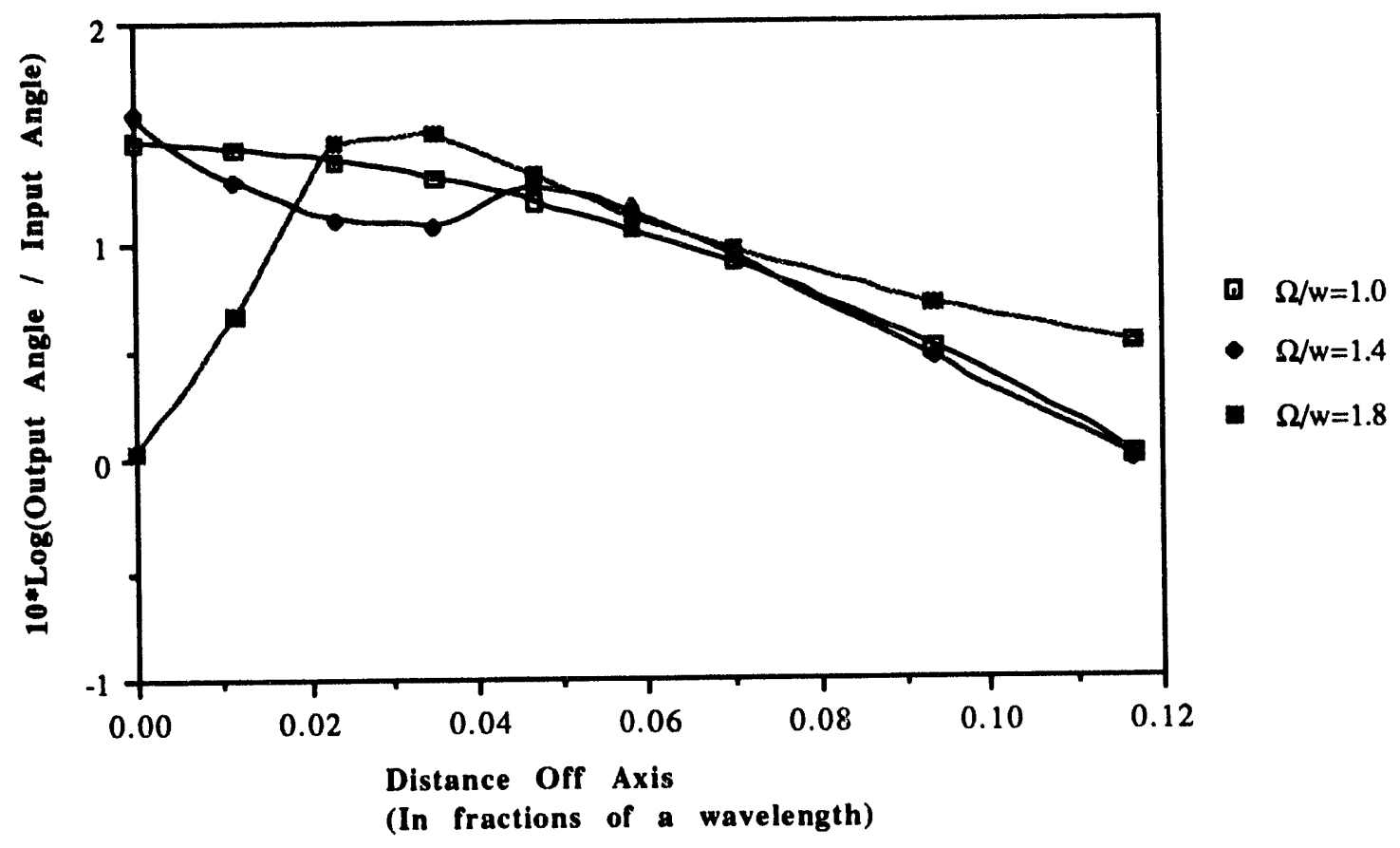

Figure 5.68. Cavity angle gain versus beam entrance displacement off the longitudinal axis for a cavity length of $0.525 \lambda$ and input angle of $14.6^{\circ}$.

This last set of graphs suggests the same conclusions of the previous set, with one addition. Through the inspection of Figs. 5.57, 5.58, 5.59, 5.60, 5.61, and 5.62, it can be seen that the peak gain achieved for small values of input angle is independent of the $\Omega / \omega$ ratio. From these figures it can be seen that if the input radius of the beam into the cavity is adjusted, then the same peak gain can be achieved. This is an unexpected result and leads to an additional question: what is the most effective combination of parameters to achieve the desired peak gain?

To determine this, the power dissipated in the cavity walls is examined for Figs. 5.57 and 5.60 in Figs. 5.69 and 5.70, respectively. Figures 5.69, 5.70, 5.71, and 5.72 show cavity angle gain versus power dissipated in the walls for $\Omega / \omega=1.0,1.4$, and 1.8. Because these figures are scatter piots taken from a limited data base, they are not perfect. But they do illustrate an important point: by injecting the beam into the passive cavity off-axis it is possible to realize the same cavity gain with lower wall losses as is realized for higher values of $\Omega / \omega$. 


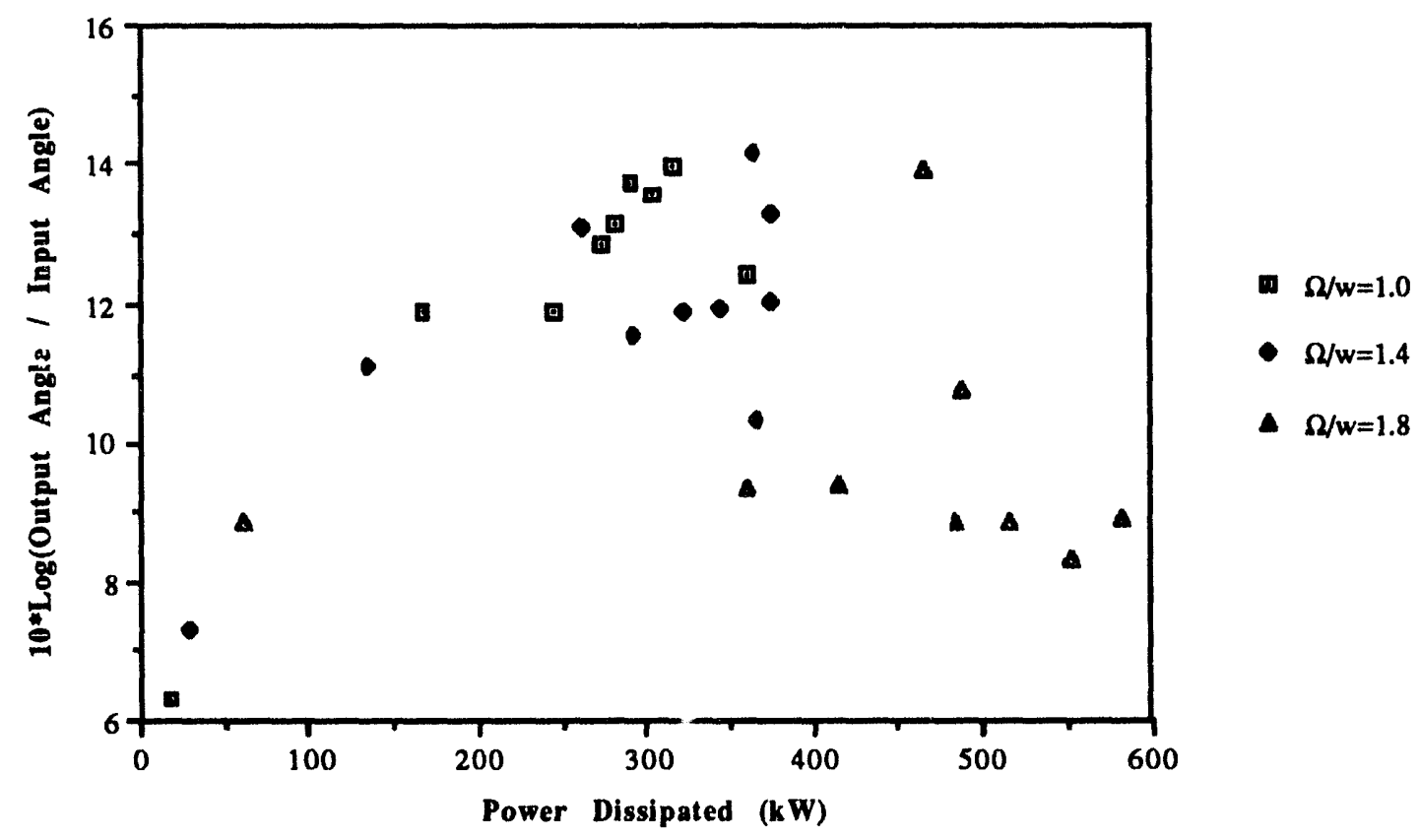

Figure 5.69. Scatter plot of angle gain versus power lost for $\Omega / \omega=1.0,1.4$, and 1.8 with a cavity length of $0.175 \lambda$ and a $2.9^{\circ}$ input angle. 


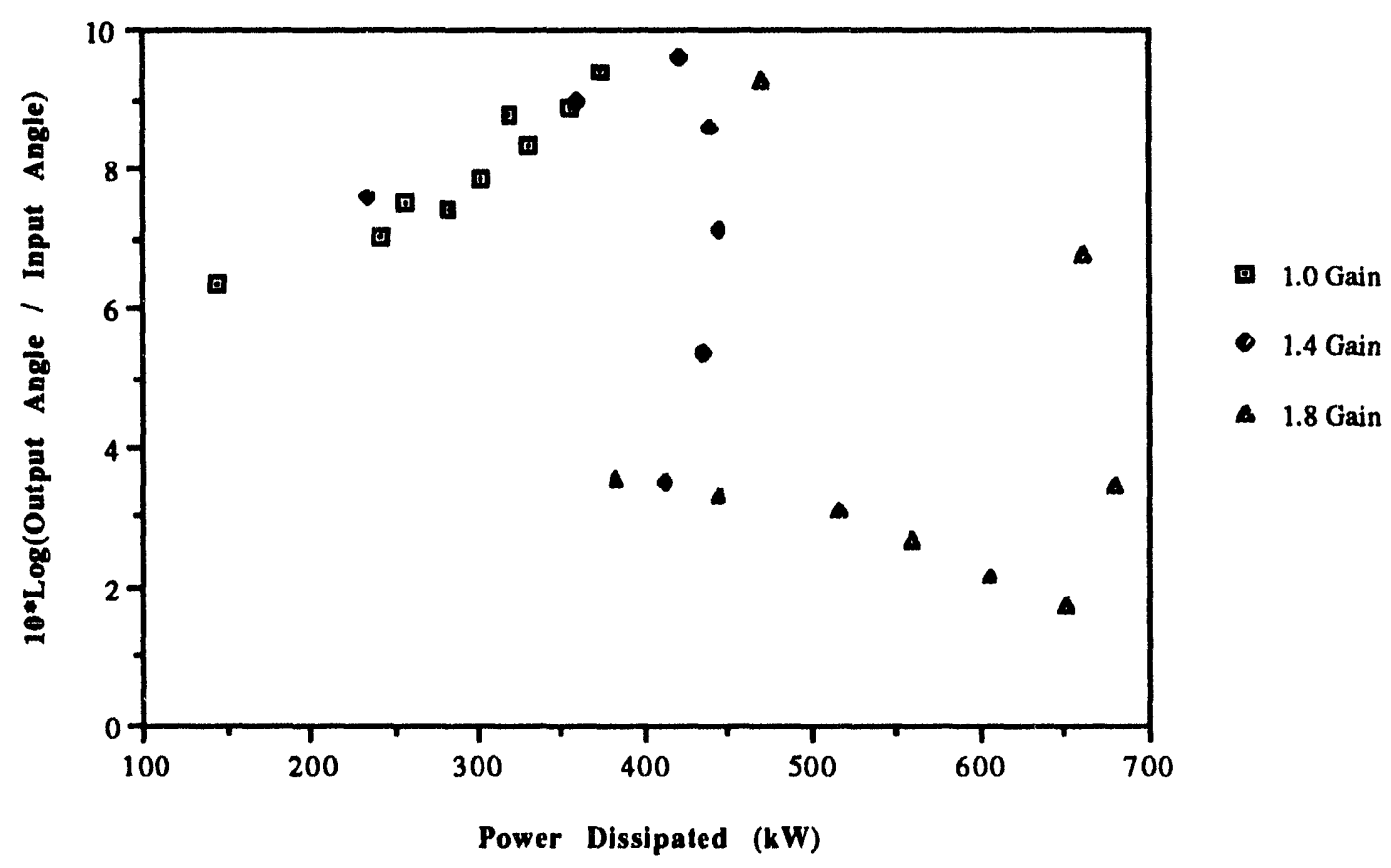

Figure 5.70. Scatter plot of angle gain versus power lost for $\Omega / \omega=1.0,1.4$, and 1.8 with a cavity length of $0.175 \lambda$ and a $8.7^{\circ}$ input angle. 


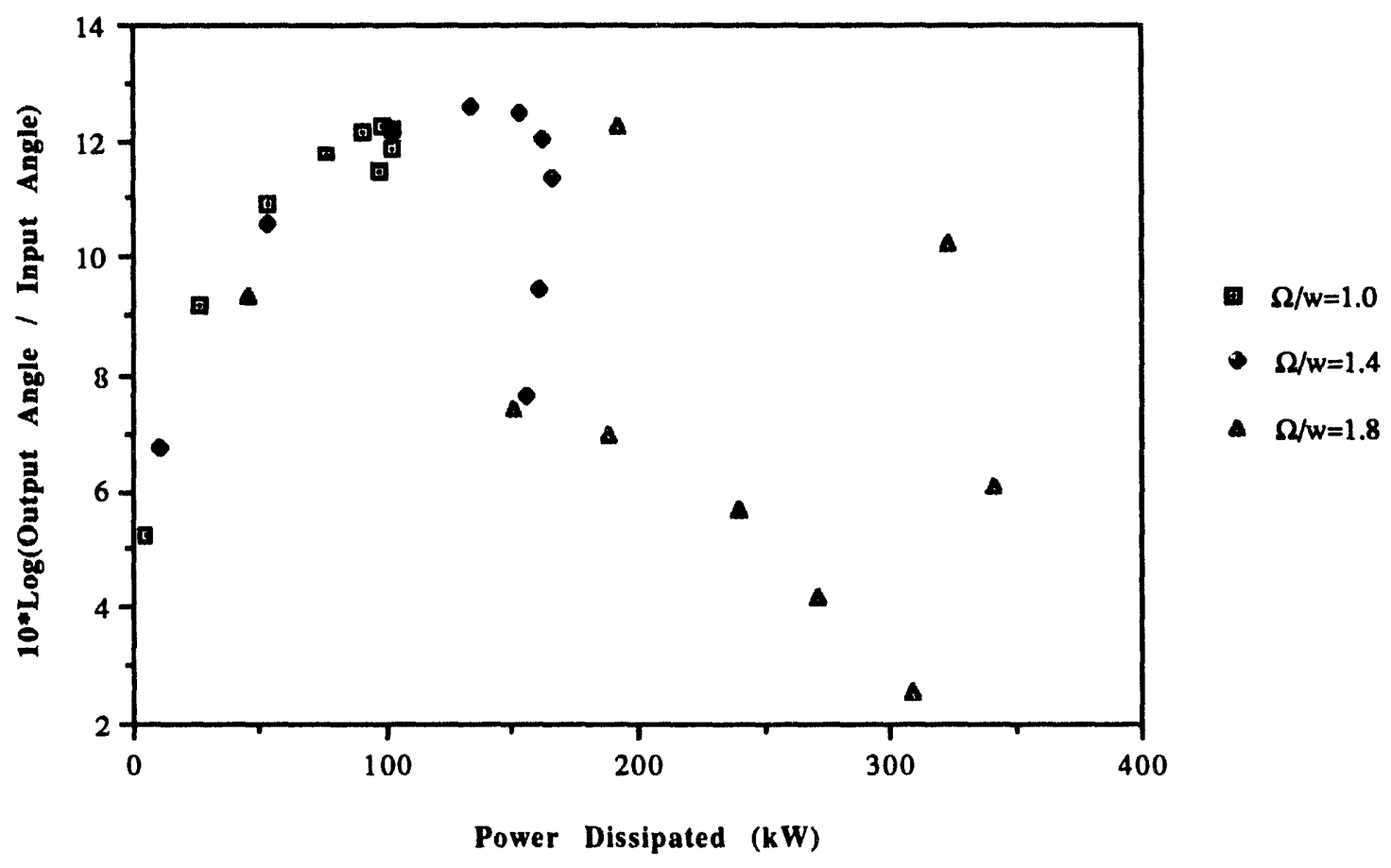

Figure 5.71. Scatter plot of angle gain versus power lost for $\Omega / \omega=1.0,1.4$, and 1.8 with a cavity length of $0.292 \lambda$ and a $2.9^{\circ}$ input angle. 


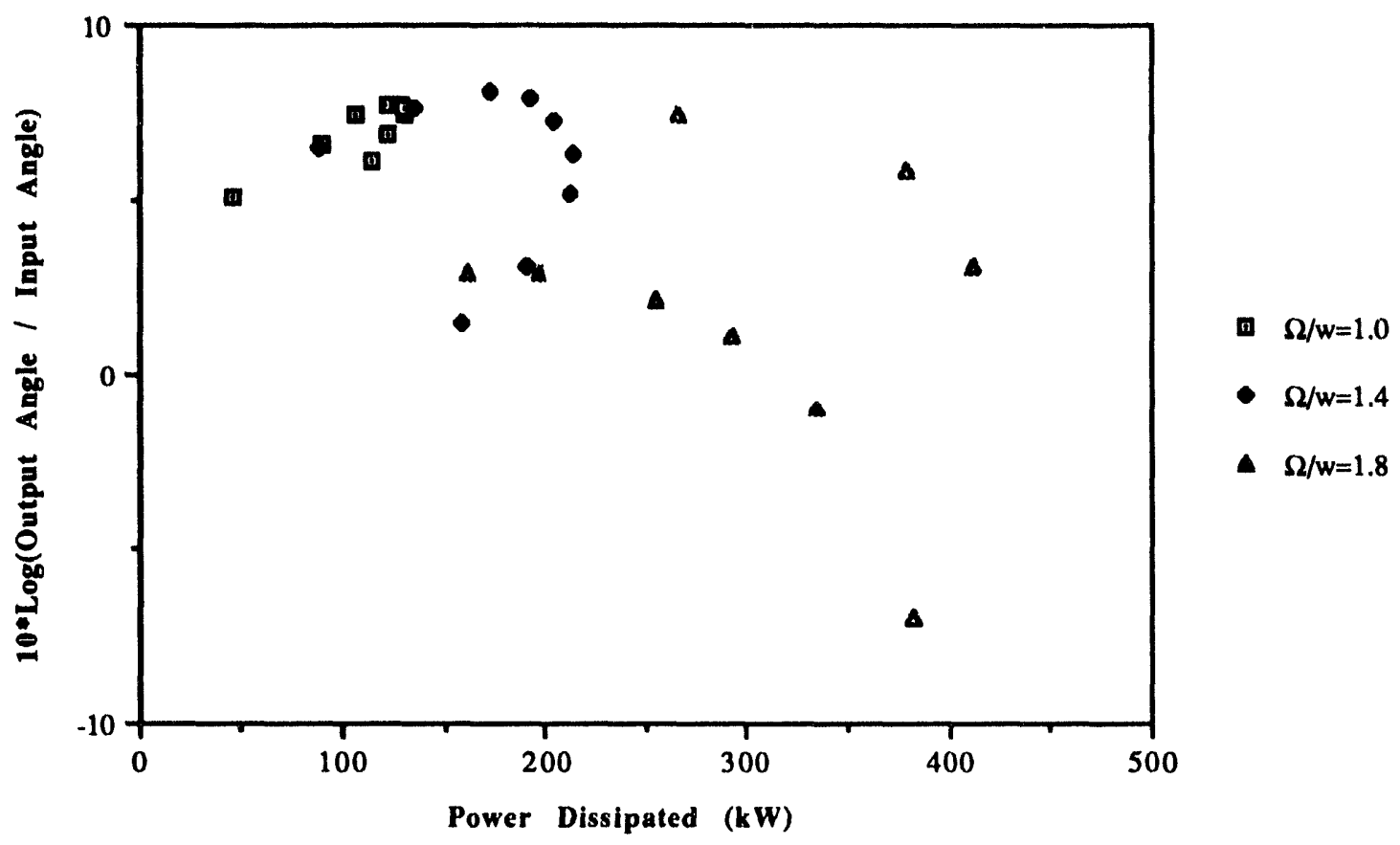

Figure 5.72. Scatter plot of angle gain versus power lost for $\Omega / \omega=1.0,1.4$, and 1.8 with a cavity length of $0.292 \lambda$ and a $8.7^{\circ}$ input angle.

In addition to the conclusions drawn above, Figs. 5.36 through 5.68 taken collectively provide a data base for the design of the portion of the deflection system consisting of simple passive cavities. (It would be possible to interpolate between these figures to determine parameters for those cases that fall in between the figures.) The scalability of these results would be identical to those results presented in Section 5.3.1.

\subsubsection{Beam-loaded $Q$ and cavity detuning}

The induced-current method described in Section 4.1.2 earlier will now be used to calculate the beam-loaded $\mathrm{Q}$ and cavity detuning. The data provided in this section was implemented only for a simple cylindrical passive cavity using ideal fields and the rigidbeam assumption. The algorithm for calculating the self-consistent solution was implemented as described in Section 4.1.2. Based on an initial estimate of cavity field and phase, a single particle is advanced through the cavity. At each time step, the position of the particle is stored in an array. It is then possible to extract the full beam 
position at any instant in time from the data stored in the array and calculate the instantaneous power provided by the beam at any given time.

It is necessary to define two time variables, $t_{\text {sample }}$ and $t_{\text {exit. }}$. The first represents the time corresponding to one estimate of the induced current that makes up the discrete Fourier transform. This variable is used to calculate the fundamental component of the induced current and has a value between $\omega t=0$ and $2 \pi$. The second variable is the exit time of the single particle in the cylindrical cavity sample corrected for sample time. These times are given by

$$
t_{\text {sample }}=i * d t_{\text {sample }}
$$

and

$$
t_{\text {sxit }}=t_{\text {out }}+t_{\text {sample }}
$$

where $i$ represents a single sample number of the samples that makes up the discrete Fourier transform, $t_{\text {out }}$ is the time the single particle exited the cylindrical cavity, and $d t_{\text {sample }}$ is the time step of the sample by which an $\omega t=2 \pi$ interval is sampled for the discrete Fourier transform.

For a given sample time, it is only necessary to correct the angular polar coordinate of the generated positional array by pushing a single particle through the cavity. The array contains particle radius, angle (in conventional cylindrical coordinates), time, and longitudinal position. The fact that only the polar angle needs to be corrected is another result of the rotating nature of the fields and beam. (This phenomenon was previously described in Section 4.1.1.) The angular position is corrected according to the following five equations:

$$
\begin{aligned}
& \text { phsadj } j_{1}=\omega^{*} d t^{*}(j-1)+\omega^{*} t_{\text {sample }}, \\
& \text { phsadj } j_{2}=\omega^{*} d t *(j)+\omega^{*} t_{\text {sample }}, \\
& \text { phi }=\text { phsadj }+ \text { phi }(\text { ncoord }-j), \\
& \text { phi } i_{2}=\text { phsadj }_{2}+\text { phi }(\text { ncoord }-j-1),
\end{aligned}
$$




$$
p h i=\frac{1}{2}\left(p h i_{1}+p h i_{2}\right) .
$$

These five equations correct the polar angle of two adjacent array entries based on the time difference between the particle designated by the array pointer, $j$, the exit time, and the sample time. Two adjacent array entries are corrected so that the average value can be used to calculate the instantaneous power provided by the beam. The average radius is also used. The field value is calculated according to Eq. (3.28) and the power extracted from the beam is given by

$$
\text { Beam Power }=\sum_{j=1}^{\text {ncoord }} E_{1}\left(\rho_{\text {avg }}(j), \phi_{\text {avg }}\left(j, t_{\text {exit }}\right), t_{\text {exi }}\right) * I_{o} *[z(j)-z(j-1)] \text {. }
$$

In this equation, a time-centered approach to calculating the beam power is used. Applying this equation, it can be seen that each sample point of induced current is calculated according to

$$
i_{\text {indeced }}\left(t_{\text {exit }}\right)=\frac{\text { Beam Power }}{V_{\text {gap }} \cos \left(\omega t_{\text {exit }}+\varphi\right)},
$$

where $V_{g a p}$ is the product of the field magnitude and cavity length, and $\varphi$ is the rf phase. The induced-current time samples are used to calculate the fundamental component of induced current using a discrete Fourier transform. The Fourier transform coefficients are calculated according to Ref. [2] as

$$
\begin{aligned}
& A_{n}=\frac{2}{n \text { beam }} \sum_{n=1}^{\text {nbeam }} i_{\text {induced }}(n) \cos \left(\omega t_{\text {exi }}\right), \\
& B_{n}=\frac{2}{\text { nbeam }} \sum_{n=1}^{n b e a m} i_{\text {induced }}(n) \sin \left(\omega t_{\text {exit }}\right),
\end{aligned}
$$

and

$$
C_{n}=A_{n}-j B_{n},
$$

where $C_{n}$ is the complex Fourier coefficient, nbeam is the number of samples that are taken within an $\omega t=2 \pi$ interval of the induced-current function, and $t_{\text {exit }}$ is the exit time corresponding to beam $n$. The coefficient for the fundamental mode is the fundamental component of induced current. Using $Z_{c a v}$ from Eq. (4.15), the new value for the cavity voltage is calculated from 


$$
V_{\text {now }}=\frac{I_{\text {ind furdamencal }}}{Z_{\text {cav }}}
$$

This new value is used in conjunction with the original guess or current value to generate the next value. The next value, $V_{\text {next }}$ is generated according to

$$
V_{\text {next }}=\chi V_{\text {new }}+(1-\chi) V_{\text {old }} \text {, }
$$

where $\chi$ is a number between +1 and -1 . The selection of $\chi$ controls the convergence of the algorithm. In the generation of the figures provided below, I observed that the algorithm converged quite rapidly off-resonance when large positive values of $\chi$ were used; however, as the resonant frequency was approached it was necessary to greatly reduce the value of $\chi$, or use negative values, in order to get the algorithm to converge. This condition worsened as the beam-loaded $Q$ increased. In fact, for very high $Q$ 's near resonance, it was necessary to start with a very accurate initial value for the algorithm to converge.

Figures 5.73, 5.74, 5.75, 5.76, and 5.77 illustrate the self-consistent cavity field amplitude and phase as a function of frequency for a simple cylindrical cavity $0.41 \lambda$ in length for an input angle of $2.89^{\circ}$, an input radial velocity of $0.1 \times 10^{8} \mathrm{~m} / \mathrm{s}$, and longitudinal velocity of $1.978 \times 10^{8} \mathrm{~m} / \mathrm{s}$. All plots have a common ordinate, and it can be seen that as $\Omega / \omega$ increases, the bandwidth of the response decreases while the peak amplitude increases. 


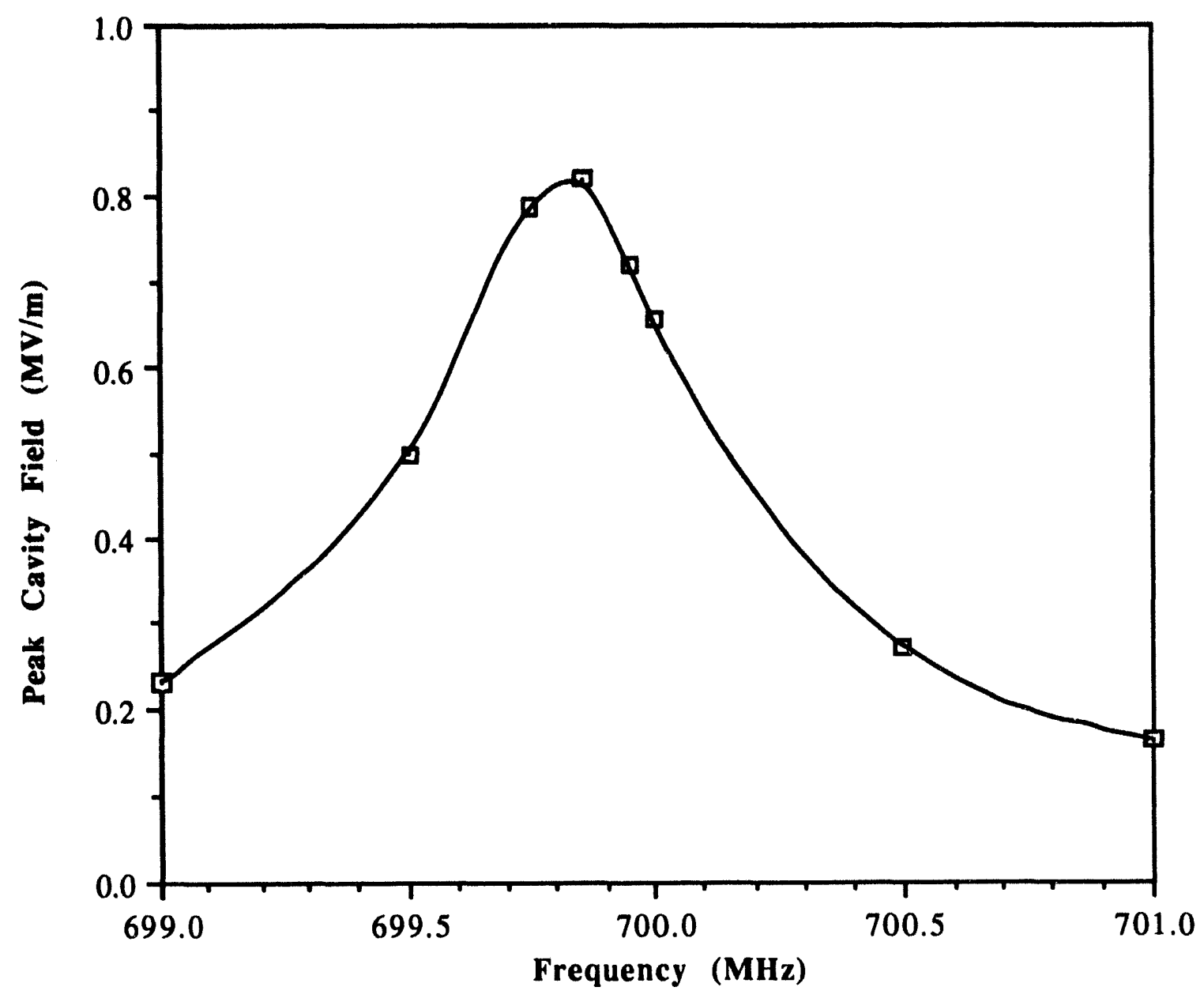

Figure 5.73a. Cavity field amplitude response for $\Omega / \omega=1.0$. 


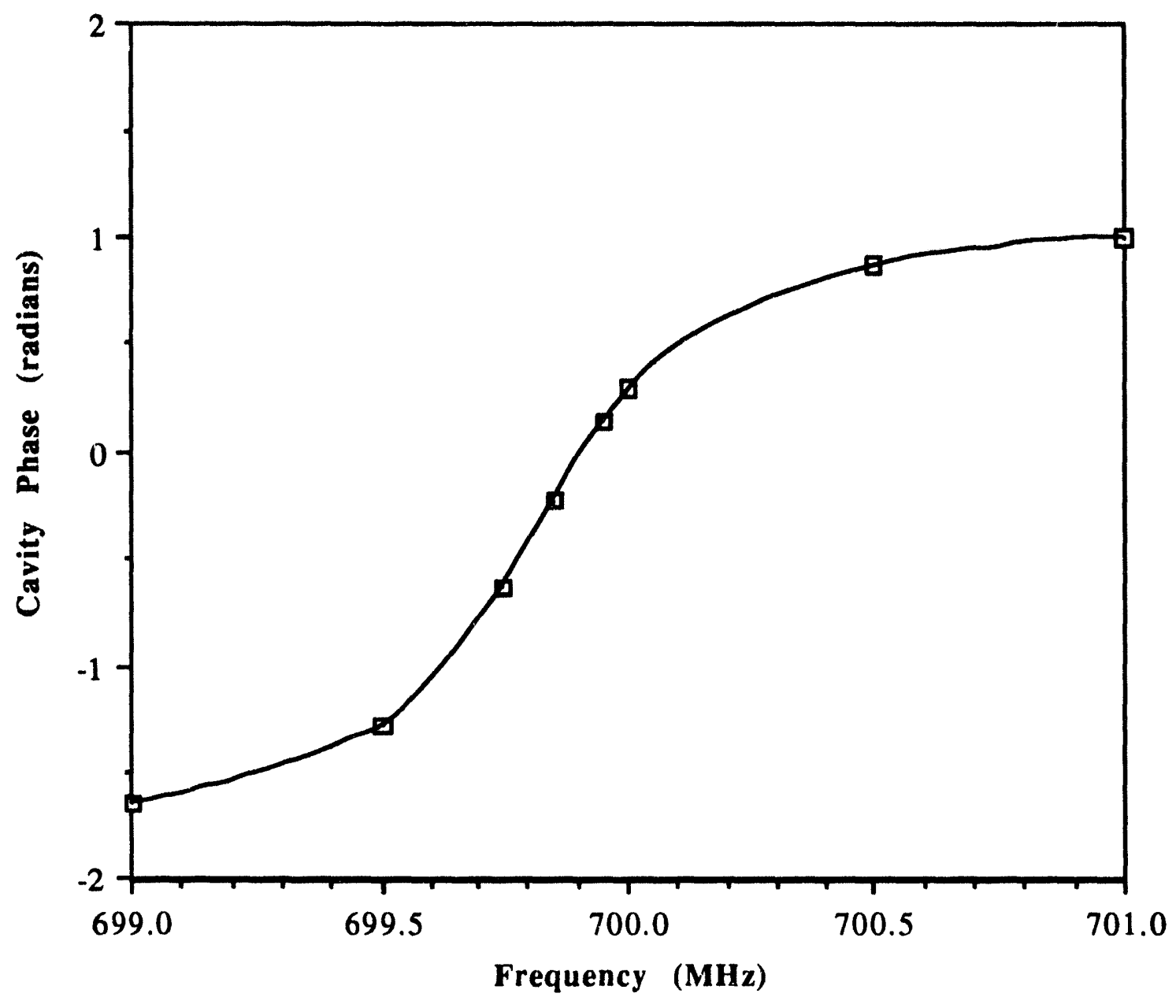

Figure 5.73b. Cavity field phase response for $\Omega / \omega=1.0$. 


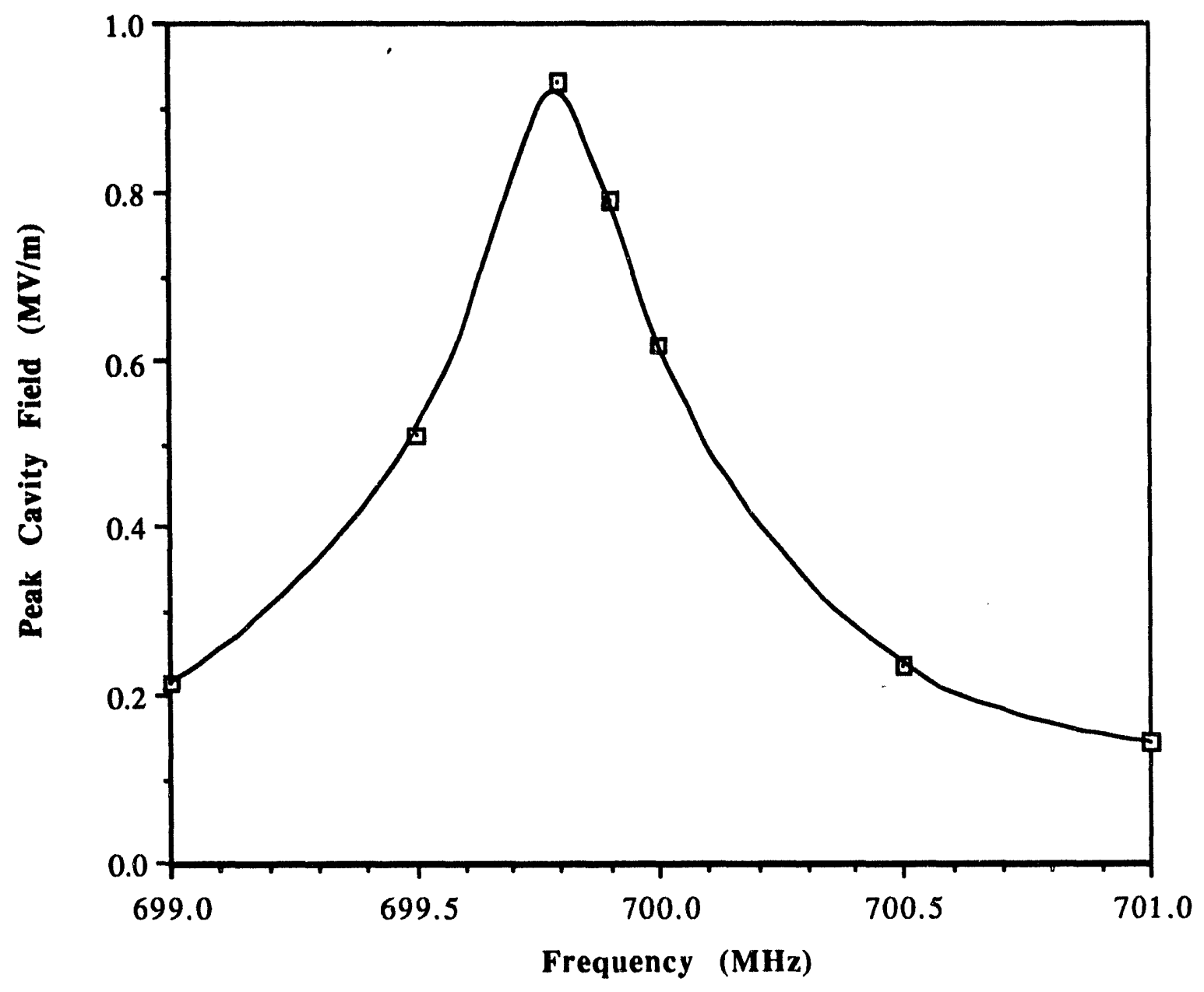

Figure 5.74a. Cavity field amplitude response for $\Omega / \omega=1.2$. 


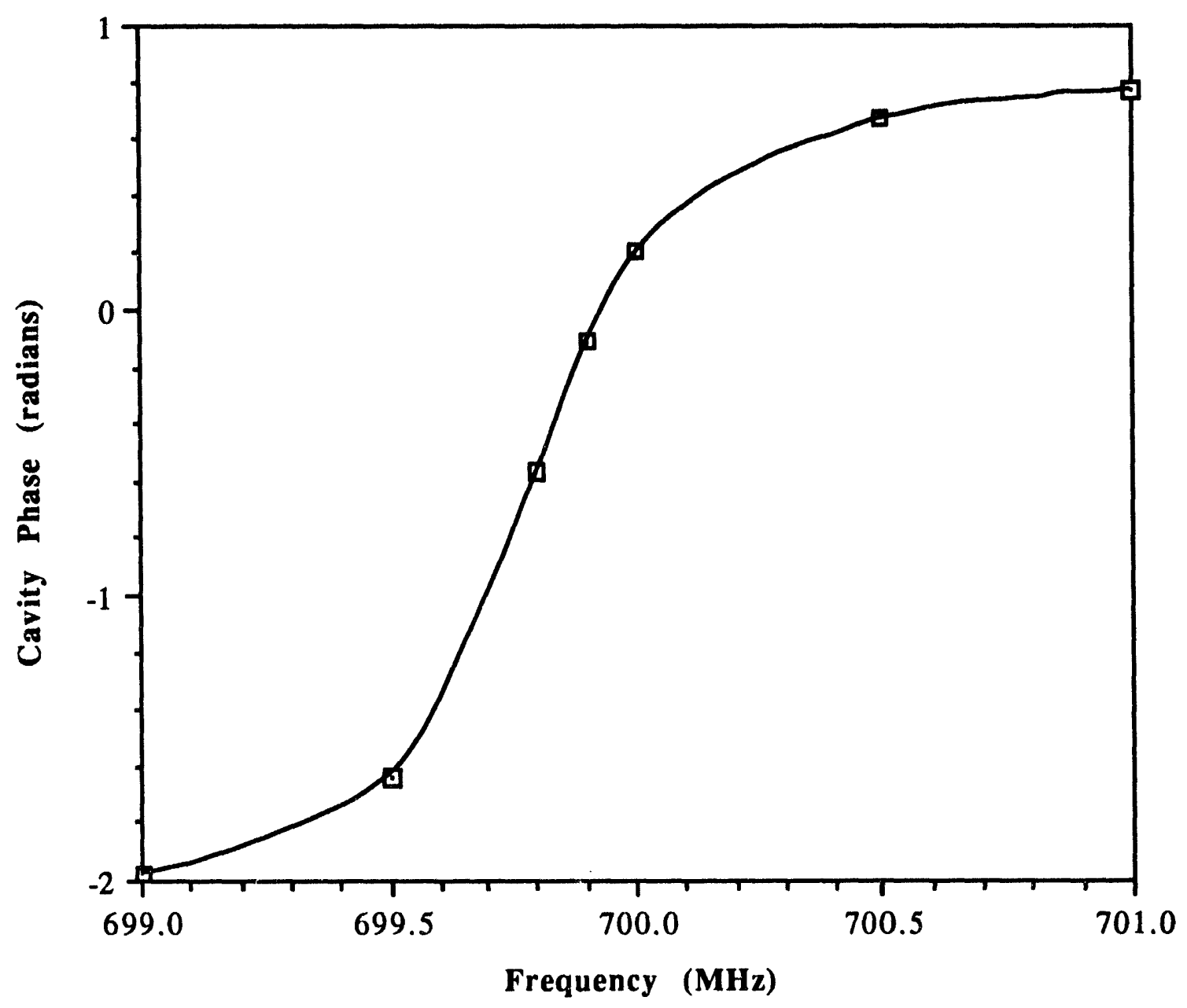

Figure 5.74b. Cavity field phase response for $\Omega / \omega=1.2$. 


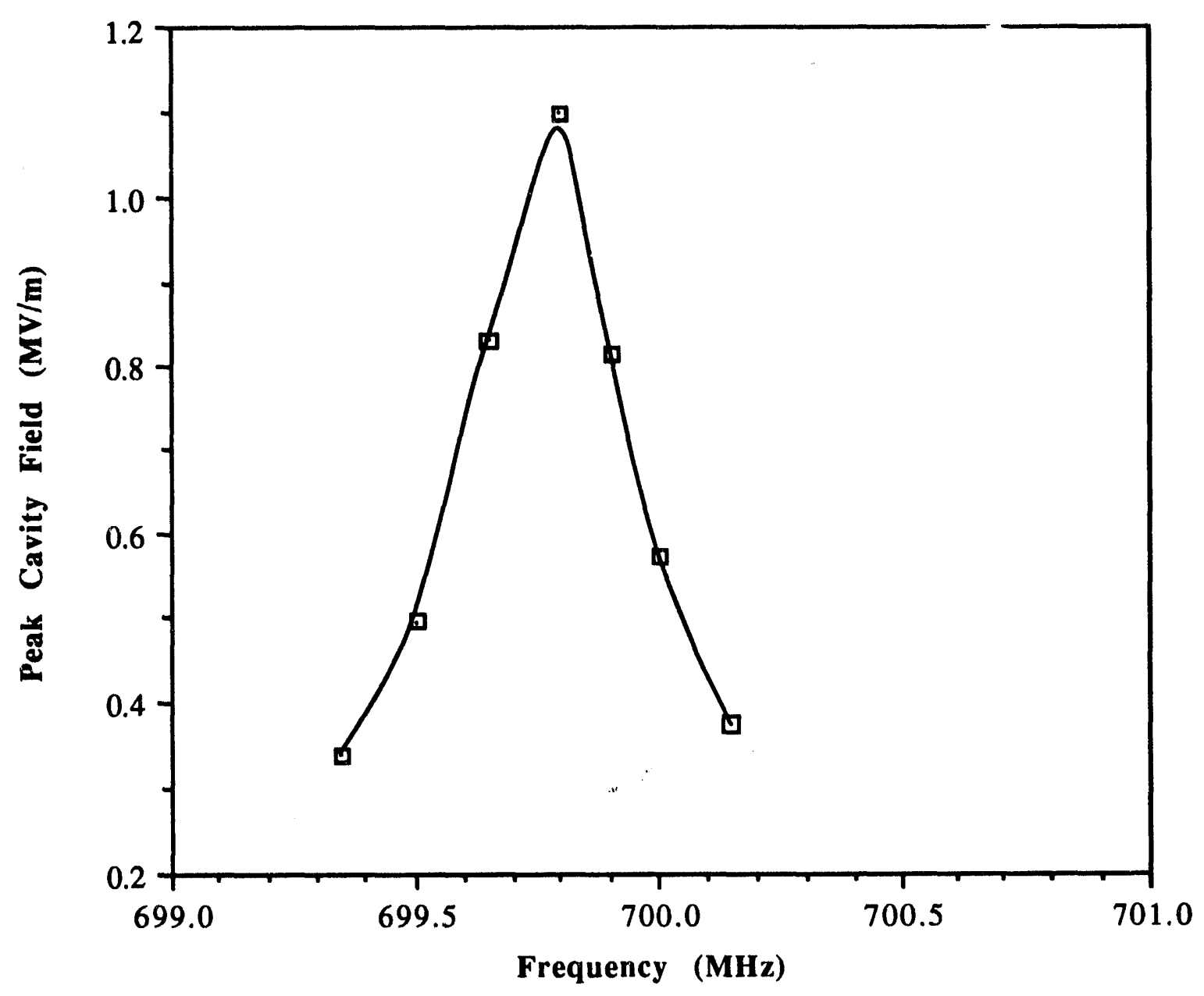

Figure 5.75a. Cavity field amplitude response for $\Omega / \omega=1.4$. 
146

Applications of Rigid-Beam Model for Magnicon Structures

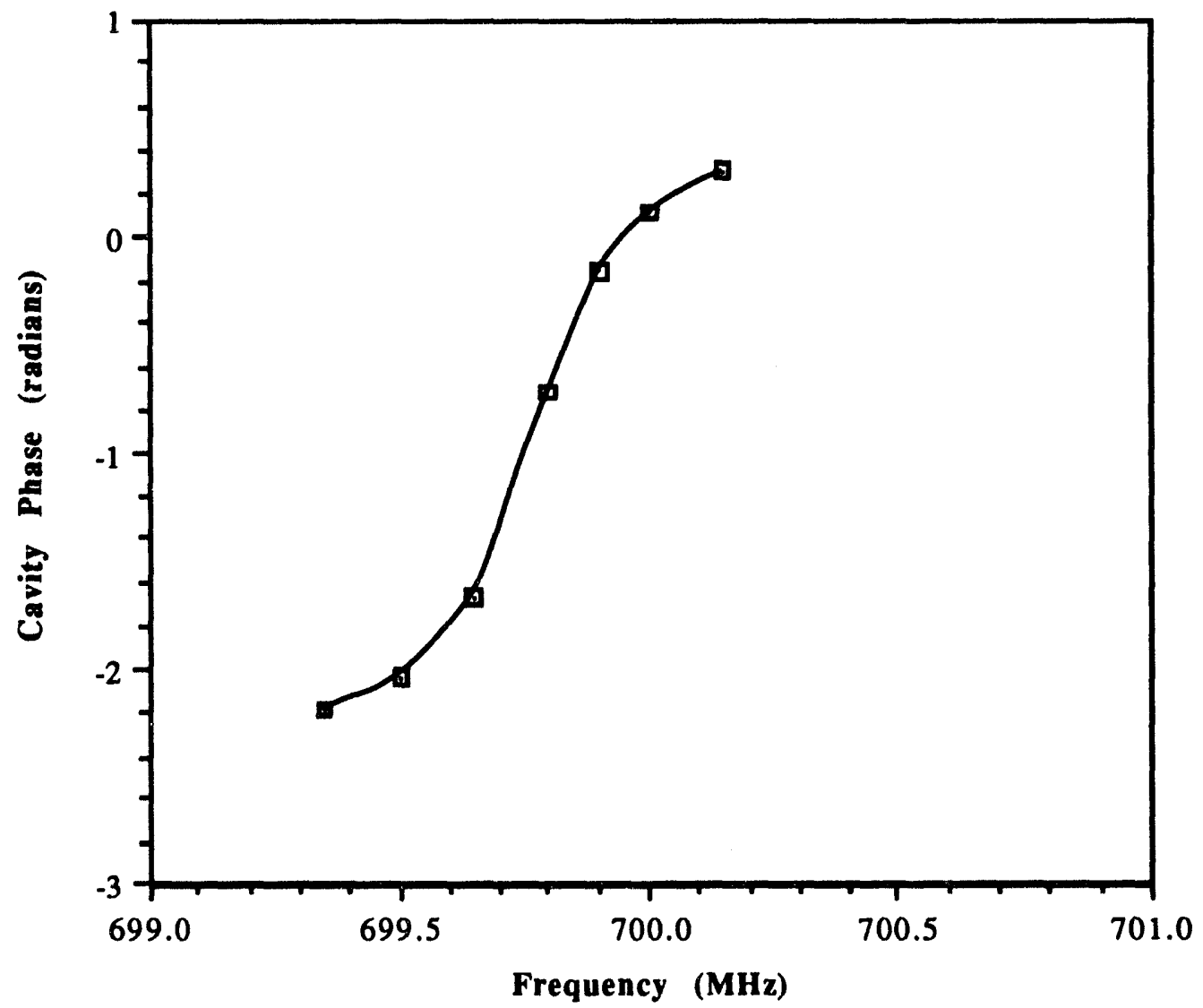

Figure 5.75b. Cavity phase amplitude response for $\Omega / \omega=1.4$. 


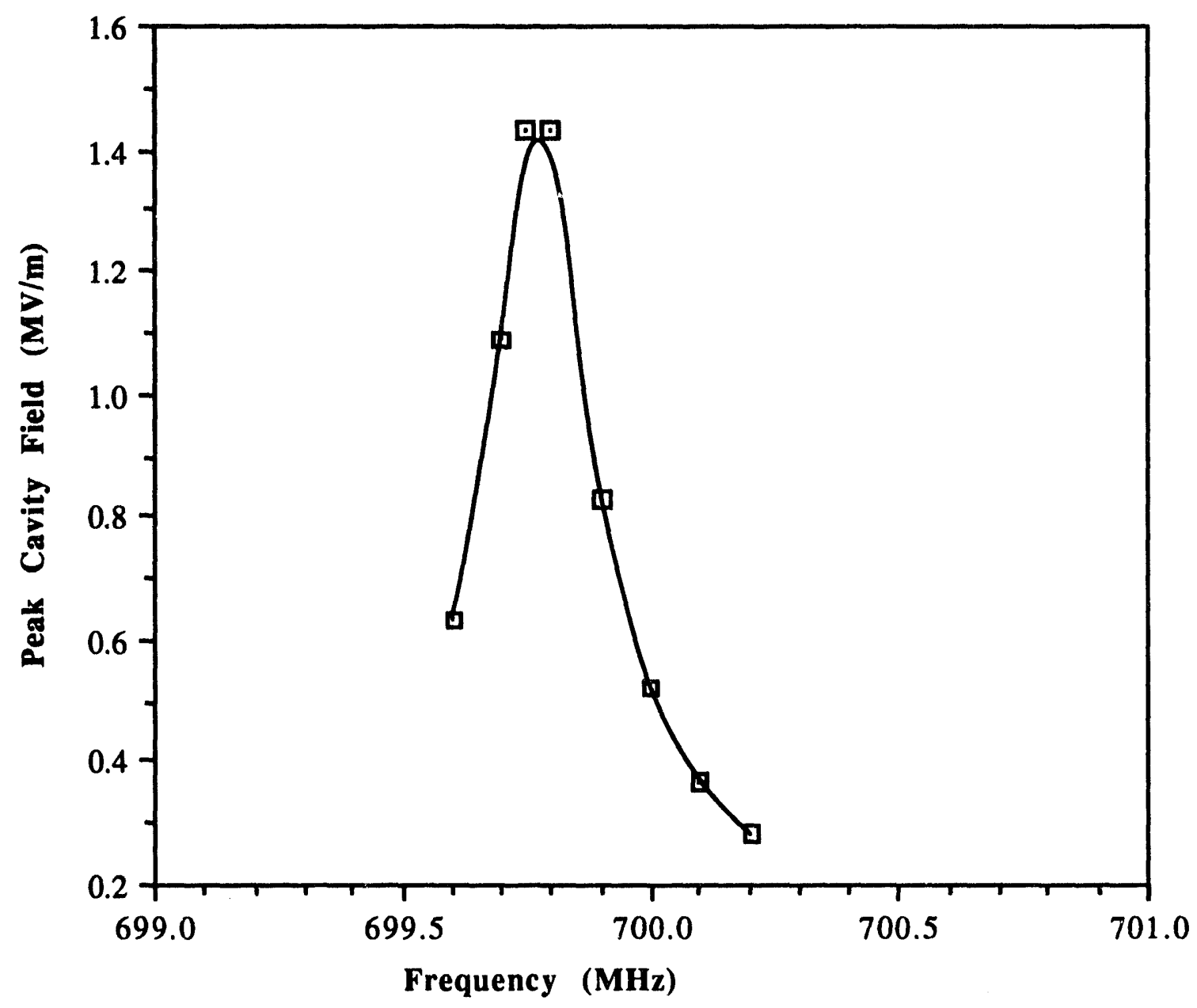

Figure 5.76a. Cavity field amplitude response for $\Omega / \omega=1.6$. 


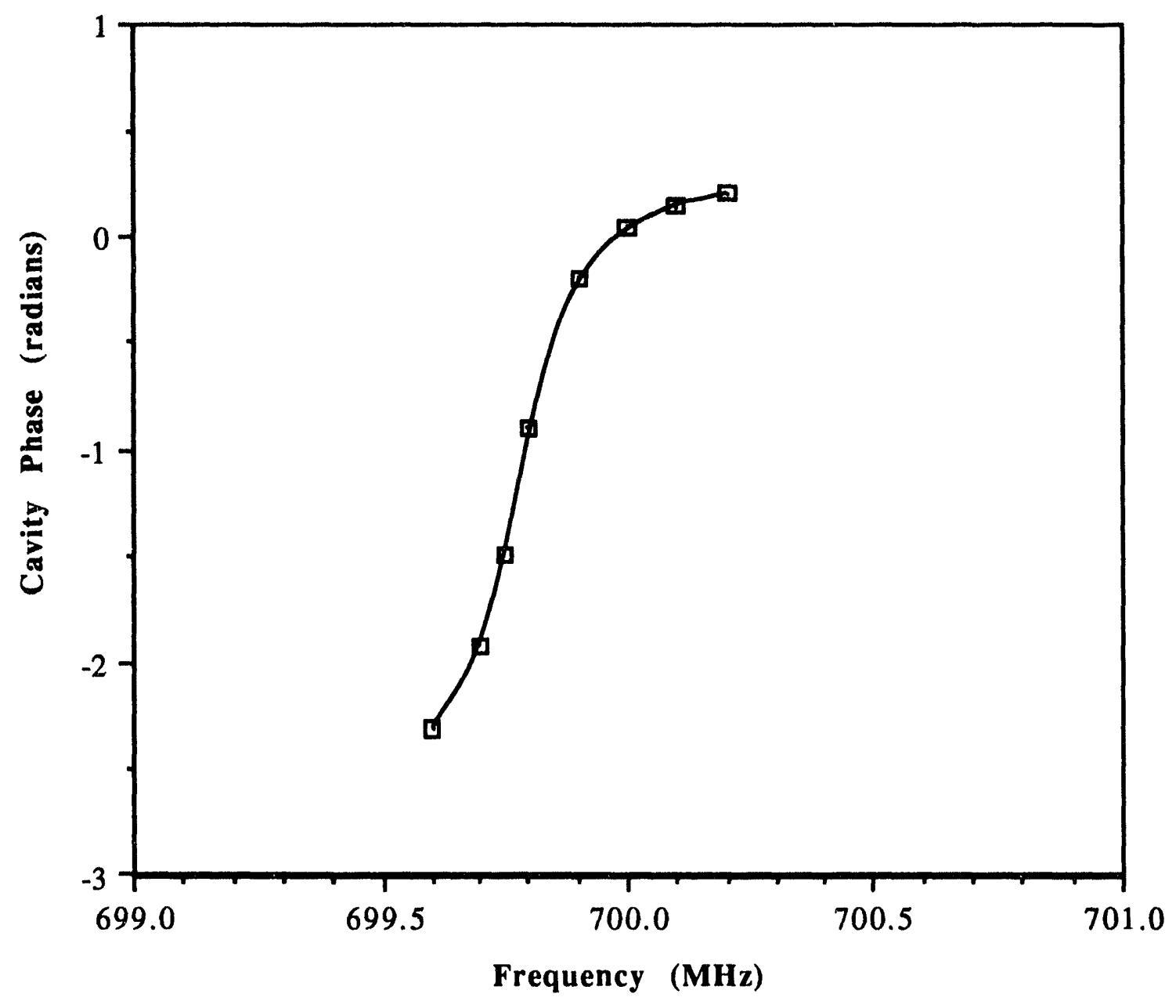

Figure 5.76b. Cavity field phase response for $\Omega / \omega=1.6$. 


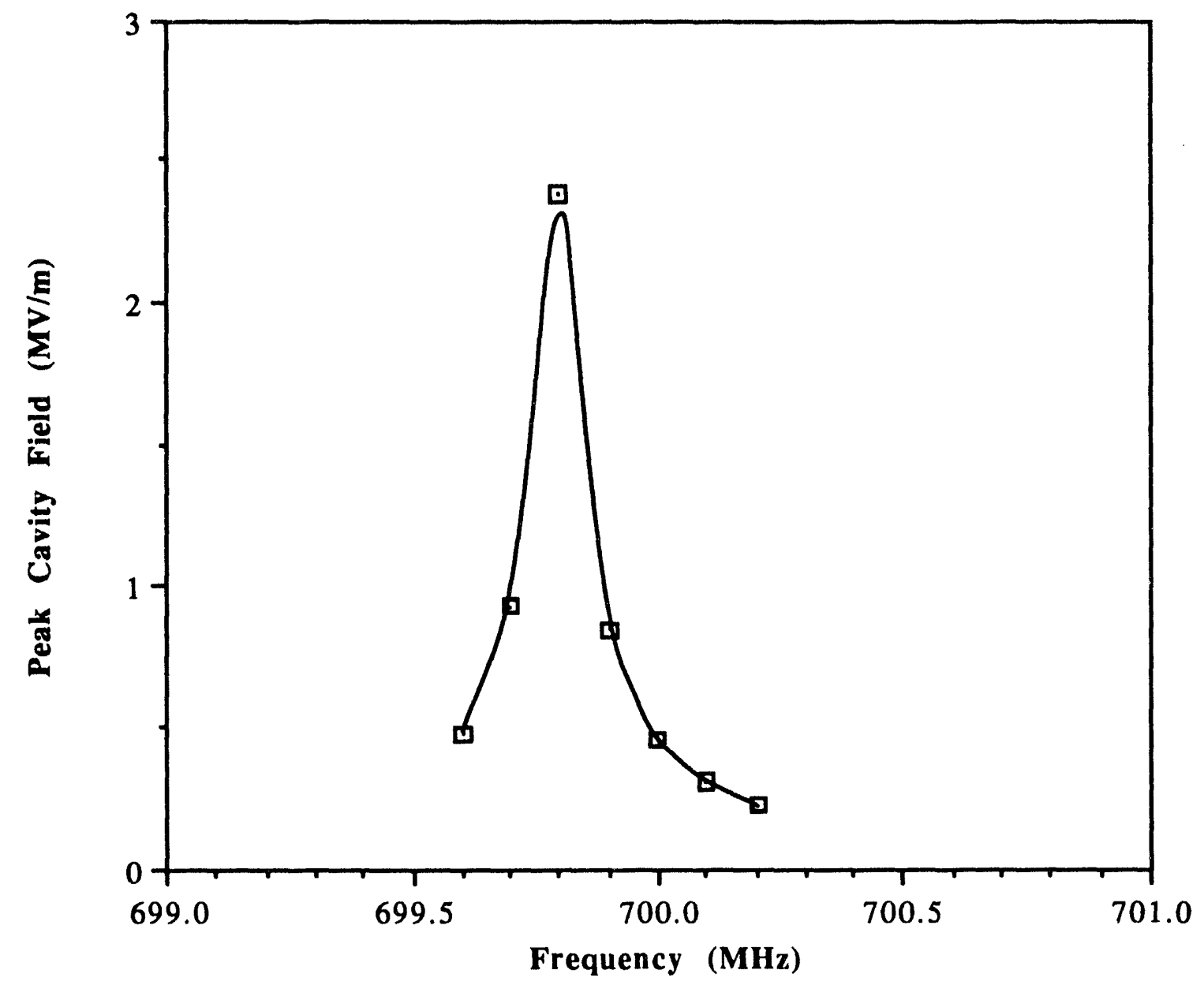

Figure 5.77a. Cavity field amplitude response for $\Omega / \omega=1.8$ 


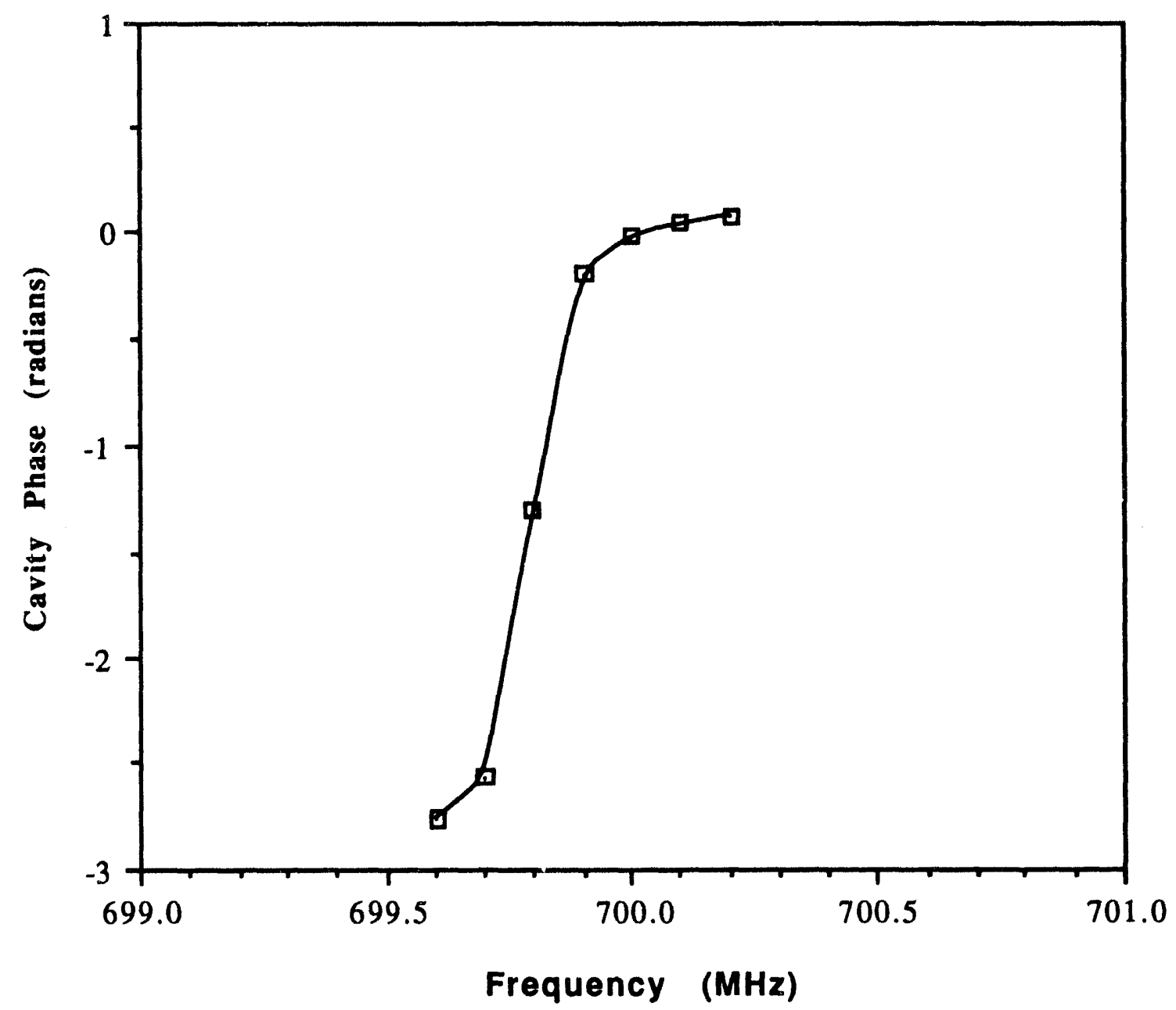

Figure 5.77b. Cavity field phase response for $\Omega / \omega=1.8$.

The beam-loaded $Q_{\text {hot }}$ can be calculated from the half-width of the amplitude response in the figures according to

$$
Q_{\text {hot }}=\frac{\omega_{0}}{\Gamma}
$$

where $\Gamma$ is the half-width (in terms of power) of the response. Gamma can be determined from the voltage response. It is the width where the voltage is equal to 0.7071 times the peak voltage. Table 5.4 contains the power half-width of the responses, the cyclotron-to- 
RF-frequency ratio, and the loaded (or hot) $Q$ calculated from Figs. 5.73, 5.74, 5.75, 5.76, and 5.77 .

Table 5.4. Beam-loaded $Q$ as a function of $\Omega / \omega$.

\begin{tabular}{|c|c|c|}
\hline$\Omega / \omega$ & Power Half-Width (MHz) & Beam-Loaded Q \\
\hline 1.0 & 0.45 & 1556 \\
\hline 1.2 & 0.36 & 1944 \\
\hline 1.4 & 0.30 & 2333 \\
\hline 1.6 & 0.20 & 3500 \\
\hline 1.8 & 0.10 & 7000 \\
\hline
\end{tabular}

Table 5.5 compares the self-consistent solution calculated using the inducedcurrent modeling and the self-consistent solution calculated using the energy-balance approach.

Table 5.5. Comparison of self-consistent solutions.

\begin{tabular}{|c|c|c|c|c|}
\hline & \multicolumn{2}{|c|}{ Induced-Current } & \multicolumn{2}{c|}{ Energy-Balance } \\
\hline$\Omega / \omega$ & $\begin{array}{c}\text { Cavity } \\
\text { Amplitude } \\
(\mathbf{M V} / \mathbf{m})\end{array}$ & $\begin{array}{c}\text { Cavity Phase } \\
\text { (radians) }\end{array}$ & $\begin{array}{c}\text { Cavity } \\
\text { Amplitude } \\
\text { (MV/m) }\end{array}$ & $\begin{array}{c}\text { Cavity Phase } \\
\text { (radians) }\end{array}$ \\
\hline 1.0 & 0.86 & -0.4 & 0.82 & -0.36 \\
\hline 1.2 & 0.95 & -0.68 & 0.92 & -0.64 \\
\hline 1.4 & 1.1 & -0.9 & 1.12 & -0.88 \\
\hline 1.6 & 1.5 & -1.15 & 1.48 & -1.17 \\
\hline 1.8 & 2.4 & -1.4 & 2.38 & -1.41 \\
\hline
\end{tabular}

The agreement between the two solutions is excellent, considering the data in the table for the induced-current solution was read from the graphs.

Figure 5.78 illustrates the cavity amplitude response versus frequency for three cavity lengths: $0.18 \lambda, 0.29 \lambda$, and $0.41 \lambda$. These graphs were used to determine how beam-loaded $Q$ varies with length for $\Omega / \omega=1.0$. The entrance condition of the beam for the generation of this figure is the same as was used for the generation of Figs. 5.73 through 5.77 . 


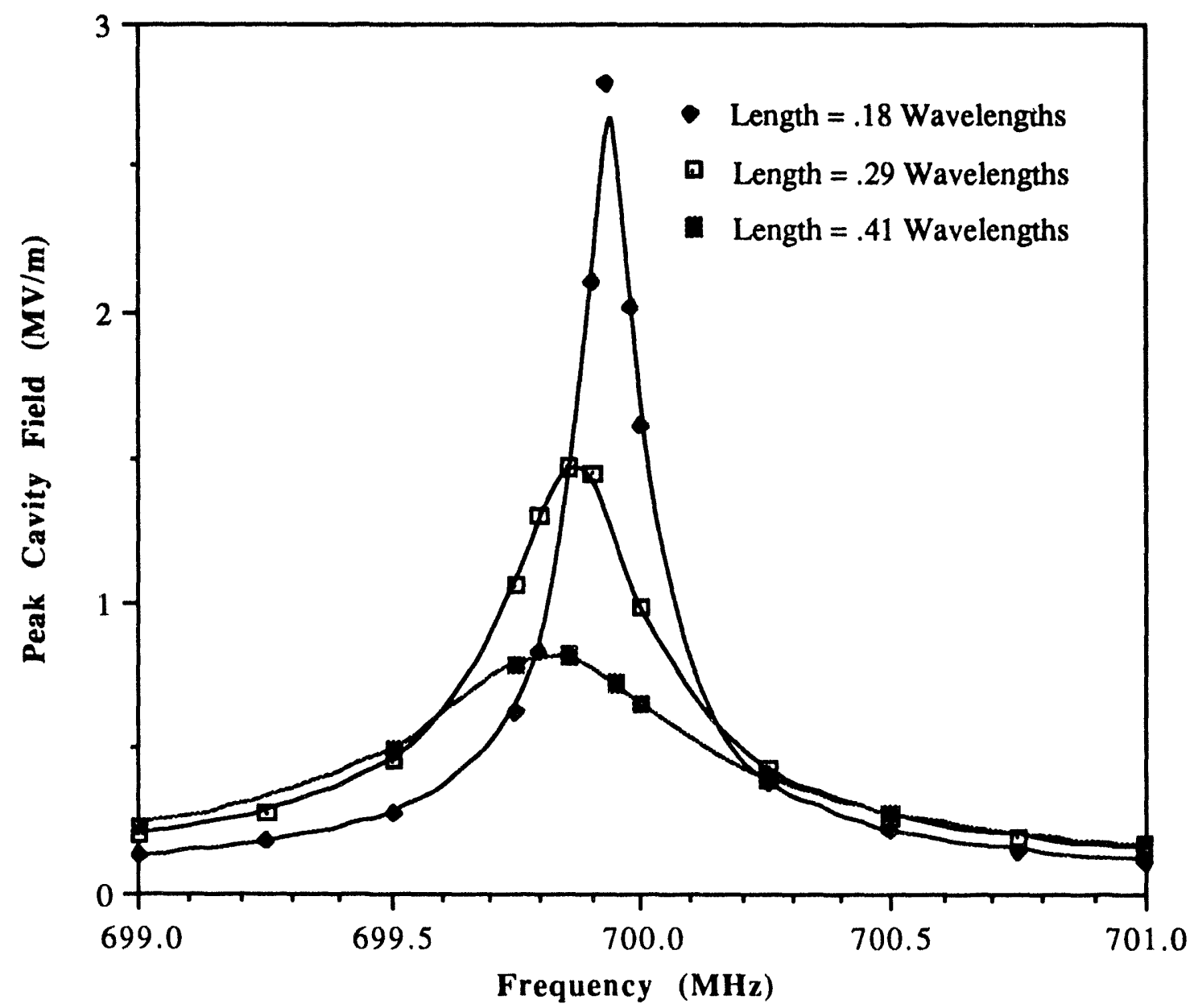

Figure 5.78. Cavity amplitude response for three lengths and $\Omega / \omega=1.0$.

The beam-loaded $Q$ for the three cases is provided in Table 5.6 and plotted in Fig. 5.79 .

Table 5.6. Beam-loaded $Q$ for different cases.

\begin{tabular}{|c|c|c|}
\hline Cavity Length & Power Half-Width (MHz) & Beam-Loaded Q \\
\hline $0.18 \lambda$ & 0.10 & 7000 \\
\hline $0.29 \lambda$ & 0.25 & 2800 \\
\hline $0.41 \lambda$ & 0.45 & 1556 \\
\hline
\end{tabular}




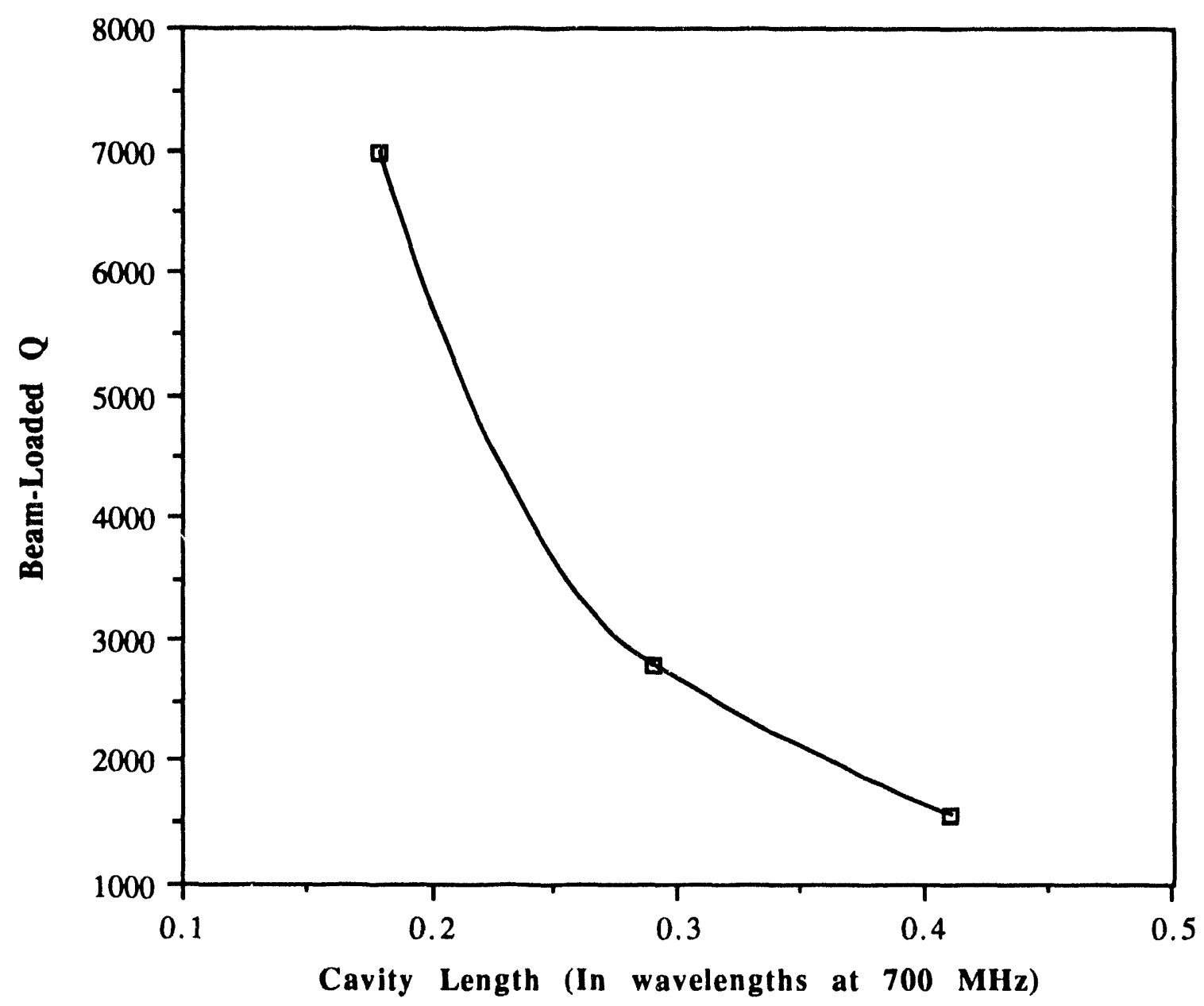

Figure 5.79. Beam-loaded $Q$ versus cavity length at $700 \mathrm{MHz}$ for $\Omega / \omega=1.0$.

From Figs. 5.78 and 5.79 and Table 5.6 it can be seen that beam-loaded Q increases with decreasing cavity length. This phenomenon would essentially limit the maximum achievable gain out of a cavity if a minimum instantaneous bandwidth is desired.

The figures and tables above indicate that a magnicon with a reasonable bandwidth would use a lower ratio of cyclotron frequency to rf frequency and a cavity of larger than optimal length. Using the data from Table 5.4 , an $\Omega / \omega$ of 1.6 would provide a 
maximum instantaneous bandwidth of $200 \mathrm{kHz}$ for the $700 \mathrm{MHz}$ magnicon (or $0.03 \%$ bandwidth). I would feel uncomfortable designing a device with a loaded $Q$ in excess of this value based on a fear that thermal effects would require some type of dynamic tuning be applied to the passive cavities in order to keep them in tune. Also, if the magnicon were used in a linear accelerator application, a bandwidth much smaller than $200 \mathrm{kHz}$ would compromise the ability of a low-level rf control system to control the accelerating cavity amplitude and phase with a rapid response time. Based on these results, I am going to try to use low values of $\Omega / \omega$ in the magnicon design with longer-than-optimum lengths.

\subsection{Investigation of parameter variation on coupled-cavity performance}

This section describes the performance of the coupled deflection cavity and the effect of varying the parameters of the coupled cavity: length, magnetic focusing-field strength, and entrance position.

First, coupled-cavity angle gain and power dissipation will be presented for a beam entering on-axis with an entrance angle of $8.7^{\circ}$. This plot is intended to illustrate the general performance characteristics of the coupled-cavity structure. Specific geometries are then evaluated over a range of $\Omega / \omega$ ratios, and the loss of the coupledcavity structure is recorded for a range of angle gains. In the next section these losses will be compared to the losses of simple cylindrical cavities that provide approximately the same ang!e gain. Specific geometries will then be identified that have desirable gain and power dissipation characteristics. For the case where $\Omega / \omega=1.4,1.2$, and 1.0 with the input angle of $14.6^{\circ}$, the effect of moving the entrance position of the beam off the longitudinal axis will be evaluated in order to predict the general trend. Several specific cases showing desirable on-axis angle gains will be examined. The simulation parameters for this section are those given in Section 2.3, except where otherwise noted.

Figures 5.80 and 5.81 are three-dimensional plots illustrating the coupled-cavity angle gain and corresponding power dissipated in the walls. The plots were generated from the data points shown in the contour plots of Figs. 5.82 and 5.83. In the figures $\Omega$ and $Z$ represent the length of the first and second cavity in the coupled-cavity structure. 


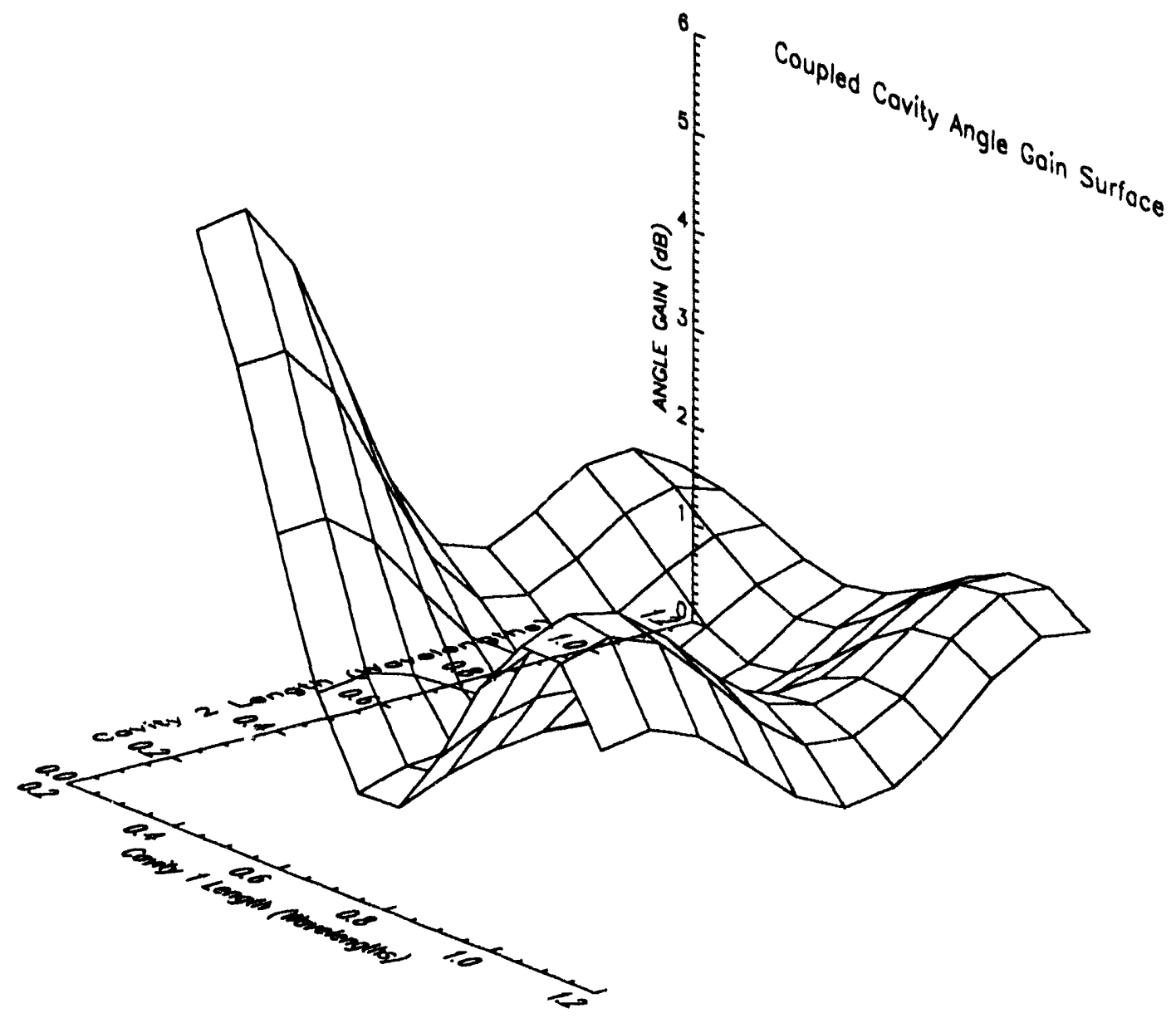

Figure 5.80. Three-dimensional plot of coupled-cavity angle gain as a function of the length of the two cavities, $l$ and $R$, which make up the coupledcavity structure. (Plot generated using commercial software package Precision Visuals-Wave. Special thanks to Stuart Bowling who helped with data manipulation, plotting, and graphing.) 


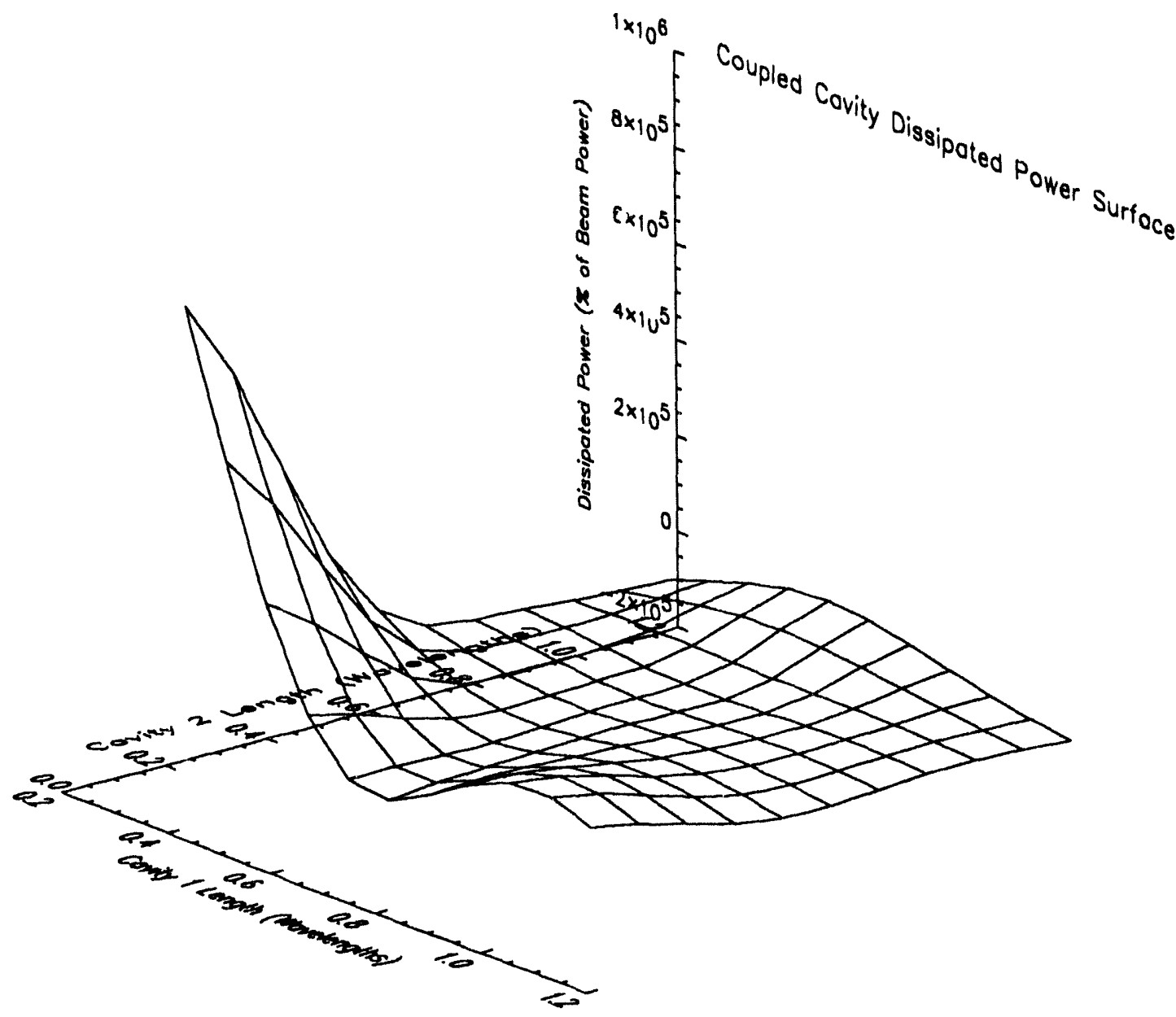

Figure 5.81. Three-dimensional plot of the power dissipated in the coupled-cavity walls as a function of the length of the two cavities, $l$ and $R$, which make up the coupled-cavity structure. This curve is to be used in conjunction with the gain given in Fig. 5.80. (Plot generated using commercial software package Precision Visuals-Wave. Special thanks to Stuart Bowling who helped with data manipulation, plotting, and graphing.) 
Figure 5.80 illustrates four regions of operation that maximize gain. In order to understand these regions, it is best to assume that the rf fields are stationary, such as might be the case for a simple, one-dimensional magnetic deflector. By making this assumption, we can see that maximum deflection could be achieved from a structure with a transit angle of $\pi$ in each of the cavities that comprise the coupled-cavity structure. Likewise, if either cavity had a transit angle of $3 \pi$ while the other cavity had a transit angle of $\pi$ (or both cavities had a transit angle of $3 \pi$ ), a local maximum in deflection would occur. Unfortunately, with the cyclotron motion caused by both the magnetic focusing field and the rotating if fields, a simple physical picture is not possible. However, I believe the regions of maximum deflection illustrated in Fig. 5.80 are analogous to the regions described for the simplified passive case.

Figures 5.82 and 5.83 are plots of cavity angle gain and dissipated power as a fraction of beam power. In these plots, regions of constant performance are plotted against the two cavity lengths that make up the coupled-cavity structure. They illustrate that the maxima corresponding to coupled cavities that are made up of two short sections have the greatest angle gain. They also show that no real savings in dissipated power is achieved for this particular case by operating cavities in the vicinity of one maxima versus another. For a numerical justification of this observation, look at Figs. 5.82 and 5.83. In the regions where there is an angle gain of 3.1 to $4.1 \mathrm{~dB}$, the dissipated power for the corresponding regions demonstrate areas of equal gain. 


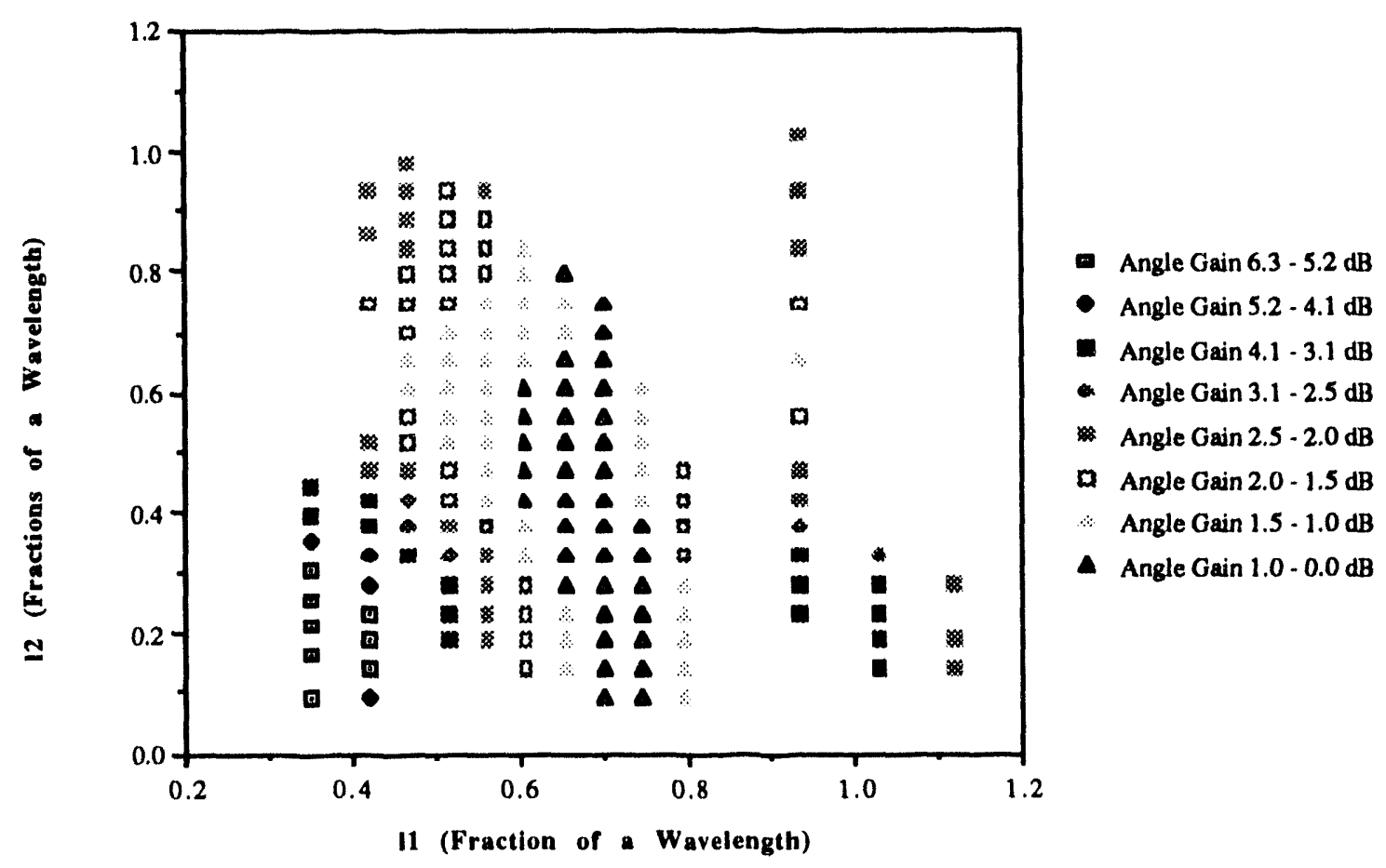

Figure 5.82 Plot of angle gain for different coupled-cavity length combinations. 


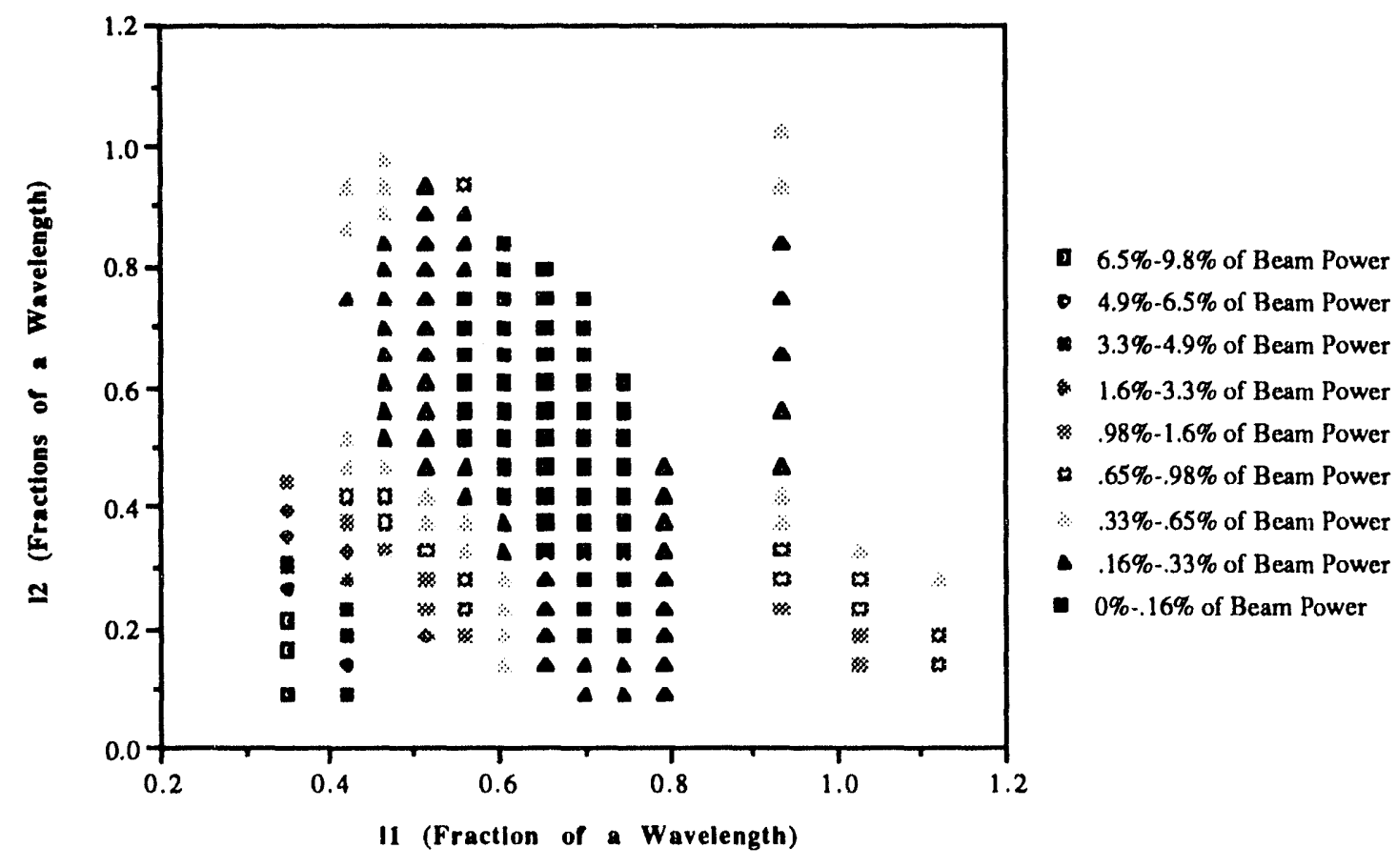

Figure 5.83. Plot of power dissipated in the walls as a fraction of beam power for different coupled-cavity length combinations.

Because searching the parameter space is further complicated by the addition of a second cavity in the coupled-cavity structure-as opposed to the single cylindrical cavity structure evaluated in Section 5.3-all of the potential parameter variations were not explored. Instead, localized simulations were conducted in regions that showed desirable gain and dissipated power characteristics. (Moreover, Ref. [3] has determined that stability issues exist in the coupled-cavity structure for higher values of $\Omega / \omega$, and induced-current modeling has demonstrated the effect of high $\Omega / \omega$ ratios on instantaneous bandwidth, so only coupled-cavity structures with values of $\Omega / \omega$ less than 1.4 were evaluated for performance and the impact of parameter variation.)

Table 5.7 summarizes the most desirable coupled-cavity structures at $\Omega / \omega$ values of 1.4, 1.2, and 1.0. The selection criterion was based on a large angle gain for a value of dissipated power that does not drastically impact overall magnicon efficiency. These values were calculated for an input angle of $14.62^{\circ}$ (a input radial velocity of $0.5 \times 10^{8} \mathrm{~m} / \mathrm{s}$ and a longitudinal input velocity of $1.9166 \times 10^{8} \mathrm{~m} / \mathrm{s}$ ). This input angle was selected 
because it could result in output angles in the $45^{\circ}$-to- $65^{\circ}$ range. This particular range in output angles was selected because the classical application of the coupled-cavity structure in gyrocons has been as the last cavity in the deflection system. As such, it is responsible for setting the input angle into the output structure. Section 5.6 will show that an output-cavity entrance angle in the range mentioned above is necessary for highefficiency extraction of the beam energy by the rf fields in the output cavity.

Table 5.7. High-performance coupled-cavity geometries and corresponding performance.

\begin{tabular}{|l|l|l|l|l|l|l|l|}
\hline $\begin{array}{l}\text { Cavity 1 } \\
\text { Length } \\
\text { (Fraction } \\
\text { of } \lambda \text { ) }\end{array}$ & $\begin{array}{l}\text { Cavity 2ength } \\
\text { (Fraction } \\
\text { of } \lambda \text { ) }\end{array}$ & $\begin{array}{l}\text { Cavity } \\
\text { Field } \\
(\mathbf{M V} / \mathrm{m})\end{array}$ & $\begin{array}{l}\text { Input } \\
\text { Angle } \\
\text { (Deg.) }\end{array}$ & $\begin{array}{l}\text { Output } \\
\text { Angle } \\
\text { (Deg.) }\end{array}$ & $\begin{array}{l}\text { Angle } \\
\text { Gain } \\
\text { (dB) }\end{array}$ & $\begin{array}{l}\text { Wall } \\
\text { Power } \\
\text { (Fraction } \\
\text { of beam } \\
\text { power) }\end{array}$ & $\begin{array}{l}\text { Ratio } \\
\text { Ration }\end{array}$ \\
\hline 0.397 & 0.303 & 4.79 & 14.62 & 53.5 & 5.6 & $4.6 \%$ & 1.4 \\
\hline 0.467 & 0.163 & 6.37 & 14.62 & 46.3 & 5.0 & $6.8 \%$ & 1.2 \\
\hline 0.887 & 0.327 & 2.95 & 14.62 & 38.5 & 4.2 & $1.9 \%$ & 1.0 \\
\hline
\end{tabular}

From this table it can be seen that the angle gain achievable diminishes with decreasing values of $\Omega / \omega$. (The data in this table will be used in a subsequent section on magnicon designs and will be compared at that time to simple cylindrical cavities of similar gain and $\Omega / \omega$ value.) These specific geometries are evaluated below for the impact of an off-axis beam-entrance position.

Figure 5.84 illustrates the impact of an off-axis injection on the angle gain of the data presented in Table 5.7. From the figure it can be observed that some values of $\Omega / \omega$ result in an increase in angle gain with off-axis beam entry while other values result in little change or a loss in angle gain. 


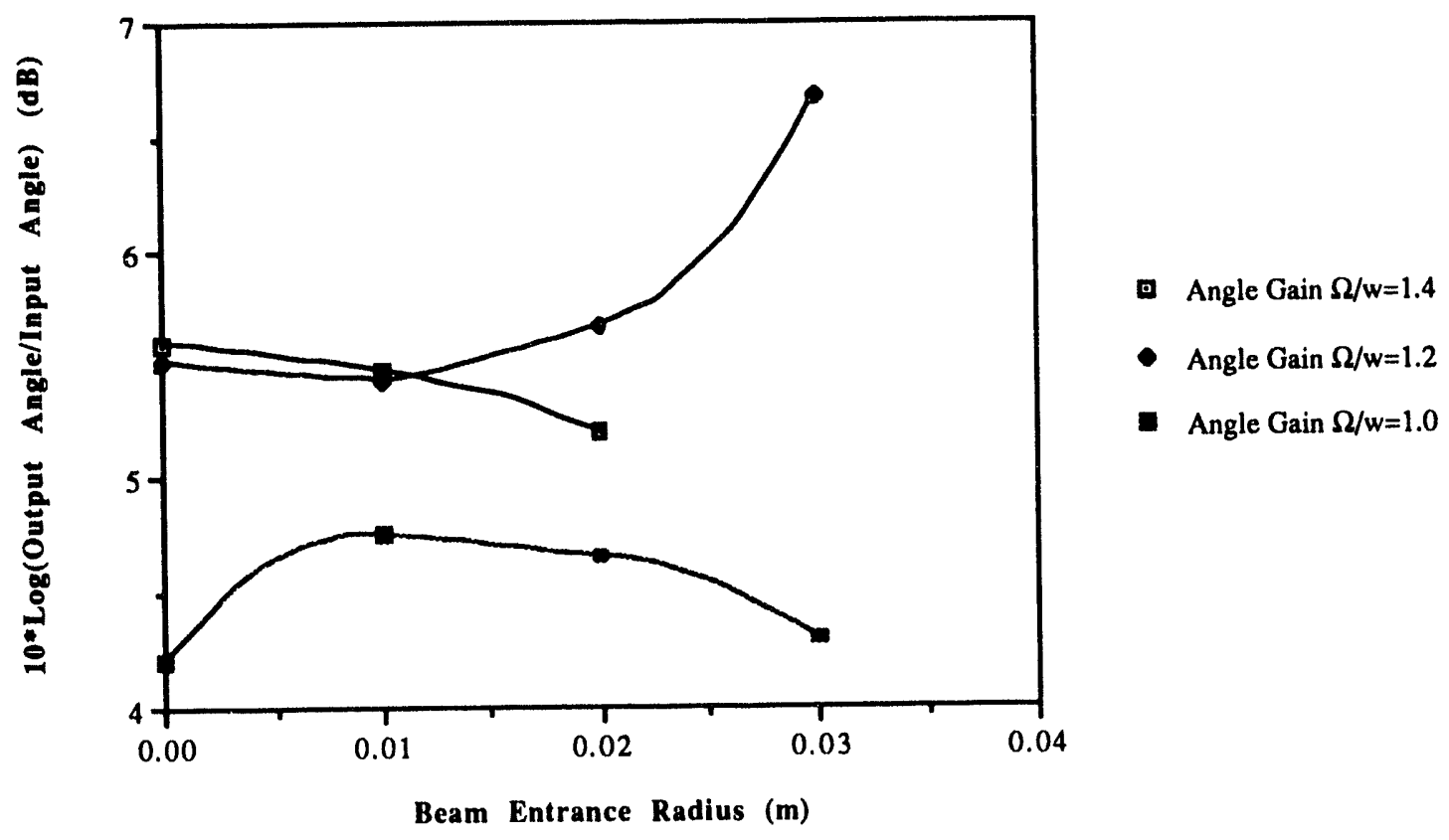

Figure 5.84. The impact of off-axis injection on angle gain.

\subsection{Comparison of coupled cavity and simple passive cavity}

In this section the performance of the coupled cavity and the simple cylindrical passive cavity is compared and contrasted. The coupled-cavity data is taken from Section 5.4, and the simple passive cavity data is taken from Section 5.3. (In some instances, the data from Section 5.3 was used to interpolate a value, and this value was then refined through further simulation.) The rigid-beam model forms the basis of the comparison.

The primary parameter being compared is the power dissipated in the cavity walls. The goal is to identify which structure provides the most gain for the smallest dissipated power penalty at three values of $\Omega / \omega(1.0,1.2$, and 1.4). For this comparison, only the case with the $14.6^{\circ}$ input angle with the beam entering on the longitudinal axis will be evaluated. This is based on the fact that only the power dissipated in the final cavity in the deflection system appreciably contributes to decreasing the overall magnicon efficiency. The power lost in the preceding cavities does not significantly impact overall magnicon performance. A $14.6^{\circ}$ input angle is approximately the one 
required to achieve a large entrance angle into the output cavity, a condition necessary for efficient conversion of the beam energy into the rf fields.

Table 5.8 displays eight columns. The first is the $\Omega / \omega$ ratio. The next three are for the simple cylindrical cavity and represent cavity length, cavity angle gain, and corresponding dissipated power, respectively. The last four columns are for the coupledcavity structure and represent the length of the two cavities in the coupled-cavity structure, the coupled-cavity angle gain, and the corresponding dissipated power, respectively. (The two columns for gain are included because even though every attempt is made to compare two cases of identical gain, it is not possible to find two cases with exactly the same gain.)

Table 5.8. Comparison between coupled-cavity structures and simple single cavity structures with comparable gains.

\begin{tabular}{|l|l|l|l|l|l|l|l|}
\hline$\Omega / \omega$ & $\begin{array}{l}\text { Simple } \\
\text { Cavity } \\
\text { Length } \\
(\text { Fract. } \\
\text { of } \lambda)\end{array}$ & $\begin{array}{l}\text { Simple } \\
\text { Cavity } \\
\text { Angle } \\
\text { Gain } \\
(\mathbf{d B})\end{array}$ & $\begin{array}{l}\text { Simple } \\
\text { Cavity } \\
\text { Dis. } \\
\text { Power } \\
(\% \text { of } \\
\text { beam } \\
\text { power) }\end{array}$ & $\begin{array}{l}\text { Coupled } \\
\text { Cavity } \\
\text { (Fract. } \\
\text { of } \lambda)\end{array}$ & $\begin{array}{l}\text { Coupled } \\
\text { Cavity } \\
\boldsymbol{2} \\
\text { (Fract. } \\
\text { of } \lambda)\end{array}$ & $\begin{array}{l}\text { Coupled } \\
\text {-Cavity } \\
\text { Angle } \\
\text { Gain } \\
\text { (dB) }\end{array}$ & $\begin{array}{l}\text { Coupled } \\
\text {-Cavity } \\
\text { Dis. } \\
\text { Power } \\
(\% \text { of } \\
\text { beam } \\
\text { power) }\end{array}$ \\
\hline 1.0 & 0.33 & 4.33 & 2.7 & 0.89 & 0.33 & 4.21 & 2 \\
\hline 1.2 & 0.28 & 5.53 & 5.0 & 0.42 & 0.16 & 5.50 & 10 \\
\hline 1.4 & 0.29 & 5.83 & 6.2 & 0.37 & 0.3 & 5.80 & 4.6 \\
\hline
\end{tabular}

It is difficult to make generalized statements based only on the data of Table 5.8. Nevertheless, it is evident that as $\Omega / \omega$ increases, a savings in dissipated power results for the coupled-cavity structure. This savings can result in higher-efficiency magnicons. At lower values for $\Omega / \omega$, the saving in dissipated power is reduced or disappears. However, at values of $\Omega / \omega$ approaching unity, another parameter becomes important: device length. For the coupled-cavity structure at low values of $\Omega / \omega$, larger cavity lengths are required to provide high gain with low dissipated power.

Referring now to Fig. 5.80 in Section 5.4, it can be seen that at lower values of $\Omega / \omega$, the optimum operating region is located at the gain peaks farthest from the origin. Specifically, investigating for $\Omega / \omega=1.0$ and $\Omega / \omega=1.2$ found that operating at the lowerright-hand region of maximum gain instead of the gain maximum near the origin resulted in a considerable saving in dissipated power, which allowed for the achievement of the desired gain. However, as is seen in the table above, this strategy can add considerable 
length to the device at lower frequencies. The length can become an issue both from the points of view of beam expansion and physical layout.

Based on this argument and the data above, I do not believe it is practical to employ a coupled-cavity structure for values of $\Omega / \omega$ less than 1.4. At values for $\Omega / \omega$ smaller than 1.4, I would use the single-cavity structure. (An ancillary consideration that is beyond the scope of this investigation is the impact of the rf fringe fields at the coupling aperture between the two cavities of the coupled-cavity structure. The hole is large compared to the entrance hole in the single-cavity structures, and the resulting radial component of electric field could interact with a finite-sized beam, spreading the beam and reducing efficiency.)

\subsection{Investigation of parameter variation on output-cavity performance}

The output-cavity performance is investigated by varying the parameters that govern output-cavity performance, specifically, the effects of cavity voltage, cavity length, cavity focusing field, the transverse-beam entrance velocity, and the displacement off-axis of the beam-entrance position. All these are evaluated to determine which combination maximizes the conversion efficiency of the output cavity. The approach taken to evaluate the broad parameter space defined above is to fix an entrance angle of $65^{\circ}$ and evaluate the conversion efficiency for different cavity lengths at a range of cavity voltages for a beam-entrance position on-axis and off-axis. Then, for the on-axis and offaxis beam injection, the entrance vector is varied $\left(45^{\circ}, 55^{\circ}\right.$, and $\left.65^{\circ}\right)$ to evaluate the dependence of output-cavity conversion efficiency on entrance angle. (Also, for the case of on-axis injection for a $65^{\circ}$ input angle, the output-cavity focusing field is varied around the field level that maximizes conversion efficiency.)

The angle values described above were selected as values that would result in high conversion efficiency consistent with a high-efficiency magnicon design. All other parameters of the high-efficiency simulation are consistent with the parameters described in Section 2.3. The range of values evaluated for the output-cavity field were all less than 2.5 MV/m, which is a smaller focusing field than that for a high-power klystron. According to Ref. [4], an order-of-magnitude evaluation of the peak output-cavity field in a high-power klystron can be determined using a peak voltage that is the product of beam voltage and $\sqrt{ } 2$, with a transit angle of $1 \mathrm{rad}$. When applied to a $1.25-\mathrm{MW}, 85-\mathrm{kV}$ klystron of $55 \%$ efficiency that is being employed at Los Alamos National Laboratory, these approximate equations determined its output-cavity field was $4.2 \mathrm{MV} / \mathrm{m}$. 
Therefore, because the interaction in the magnicon output cavity is extended-and remember that one of the advantages the magnicon has over the klystron is an extension of the output-cavity power capability - the magnicon's high efficiency must be achieved at a substantially lower output-cavity field than the klystron. Based on the comparison presented above, a magnicon peak output-cavity field that is approximately one-quarter that of a 55\%-efficient klystron will provide output-conversion efficiency in excess of 90\%. (Remember when comparing fields that the peak magnicon field is $E_{0}$ multiplied by the maximum value of $J_{1}$, or approximately 52 .)

The output-cavity evaluation neglects the effect of the sharp magnetic transition that can occur between the deflection system and the output cavity. Even though the transition is ideally a half-cusp, in practice the shape of the pole piece at the input to the output cavity and the size of the entrance hole in the output cavity can affect beam trajectories in the region of the output cavity. In essence, the actual region in which radial components of the magnetic-field transition exist are several pole-piece radii on each side of the cavity opening. This factor affects the input transverse velocity and consequently the input angle, making it difficult to realistically include the effects of the magnetic transition and still evaluate the full parameter space with a methodical approach. Therefore, this analysis assumes that the magnetic transition is very sharp and has occurred before the beam enters the output cavity. (Only longitudinal components of the magnetic focusing field are considered.)

In Fig. 5.85 the conversion efficiency in the output cavity is presented as a function of output-cavity field for several output-cavity lengths and a $65^{\circ}$ input angle. The magnetic focusing-field strength is $0.0292 \mathrm{~T}$, which corresponds to a cyclotron frequency of $612.7 \mathrm{MHz}$. As was indicated in earlier sections, the cyclotron frequency needs to be equal to the if frequency in the output cavity. Obviously, $612.7 \mathrm{MHz}$ is not equal to $700 \mathrm{MHz}$. The difference arises from the selection of gamma in the equation for cyclotron frequency. From Ref. [5], the cyclotron frequency can be determined from

$$
\Omega=\frac{|e| B_{o}}{m_{o}}
$$

As the beam energy is given up to the rf fields in the output cavity, the gamma of the beam changes from its initial gamma to approximately 1 at the end of the output cavity. Therefore, the cyclotron frequency of the particles in the output cavity is a 
function of length. The $612.7-\mathrm{MHz}$ cyclotron frequency given above is based on using the initial gamma (1.3322) for the calculation, and this will be the convention followed in presenting the results of this section. However, if the average gamma (1.166) is used, the cyclotron frequency is equal to $700 \mathrm{MHz}$-exactly the if frequency. As expected, this value of magnetic focusing field maximizes extraction efficiency in the output cavity and is used for most of the results presented below. However, two other values of magnetic field-one $15 \%$ higher than this nominal value and the other $15 \%$ lower-are also evaluated and presented.

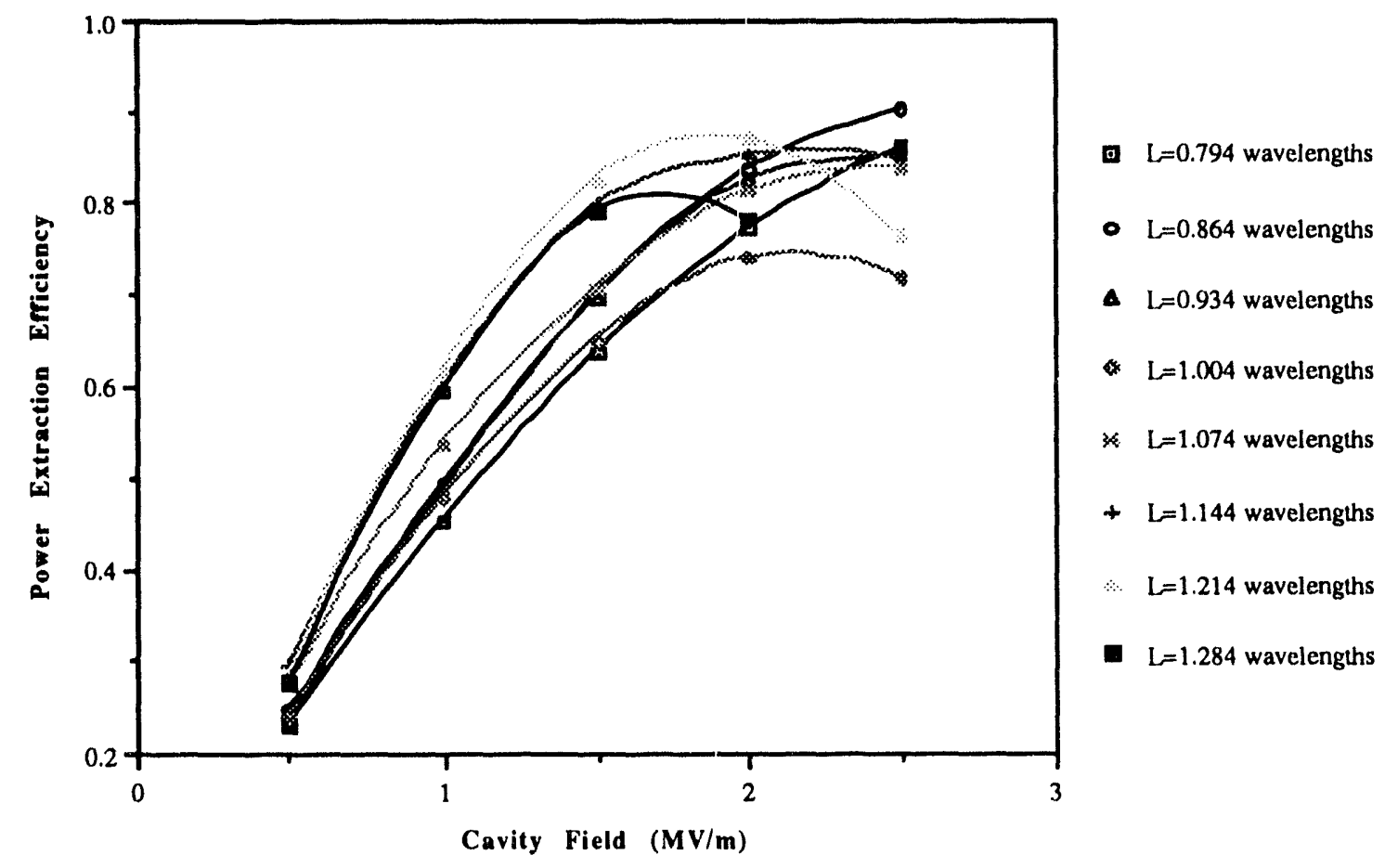

Figure 5.85. Conversion efficiency for input angle of $65^{\circ}$ for eight lengths as a function of output-cavity field. Initial cyclotron frequency is 612.7 $\mathrm{MHz}$.

Figure 5.85 illustrates that the conversion efficiency in the output cavity for an input angle of $65^{\circ}$ for a beam injected on-axis approaches $90 \%$. It also suggests that for field values in excess of the self-imposed linit of $2.5 \mathrm{MV} / \mathrm{m}$, higher efficiencies may be achievable. 
Figure 5.86 illustrates what happens to the conversion efficiency when the same magnetic field and input angle are used for a beam injected into the output cavity, but the beam is now $2.5 \mathrm{~cm}$ off-axis. (This is approximately $10 \%$ of the output-cavity radius.)

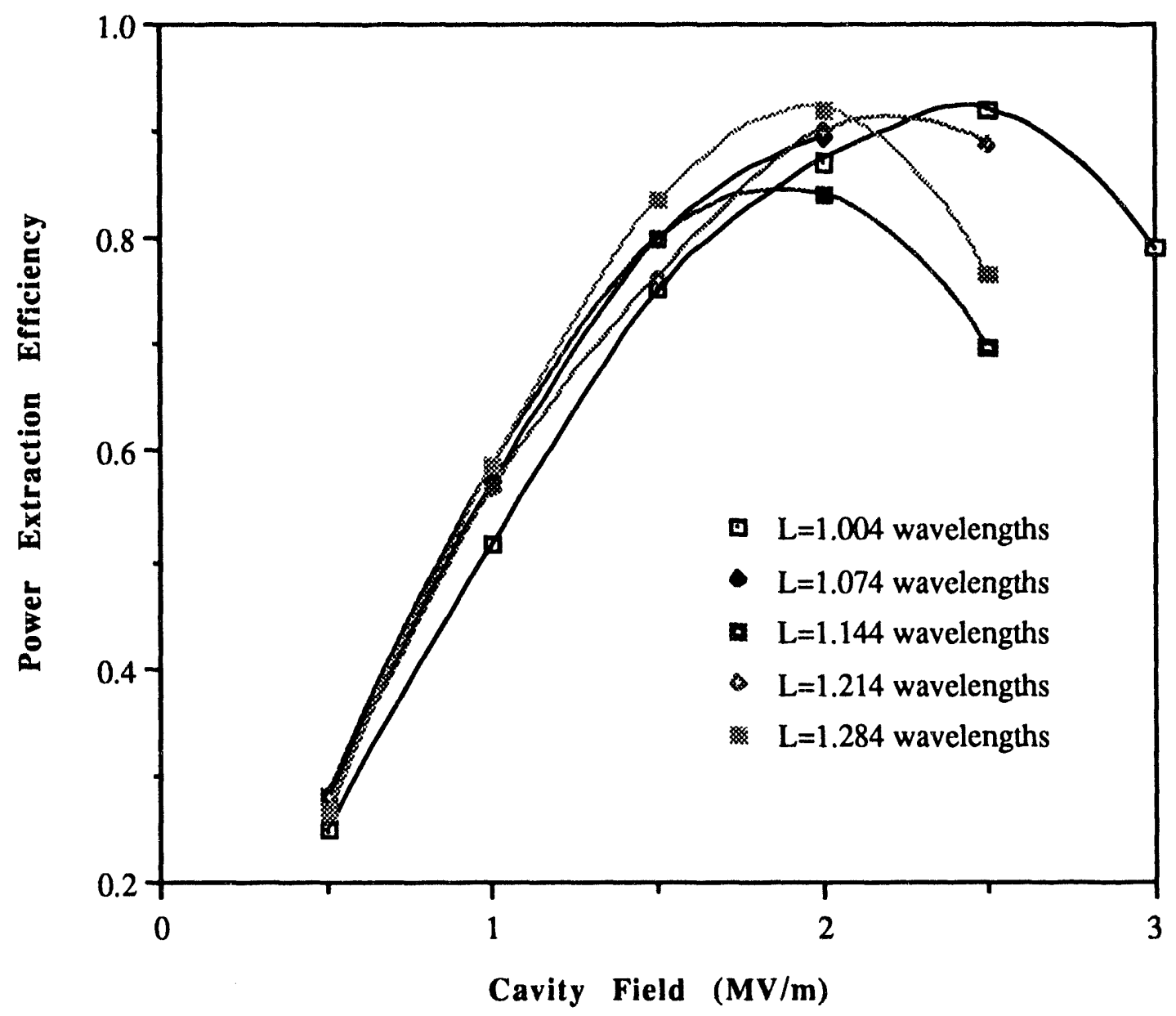

Figure 5.86. Conversion efficiency for input angle of $65^{\circ}$ for five lengths as a function of output-cavity field. Initial cyclotron frequency is 612.7 $\mathrm{MHz}$ and the beam-injection radius is $2.5 \mathrm{~cm}$.

This figure shows that the extraction efficiency is improved by injecting the beam into the output cavity off-axis, and it demonstrates extraction efficiencies in excess of $90 \%$. 
Figure 5.87 illustrates the same graph with a beam-injection radius of $5 \mathrm{~cm}$ (or approximately $20 \%$ of the cavity radius).

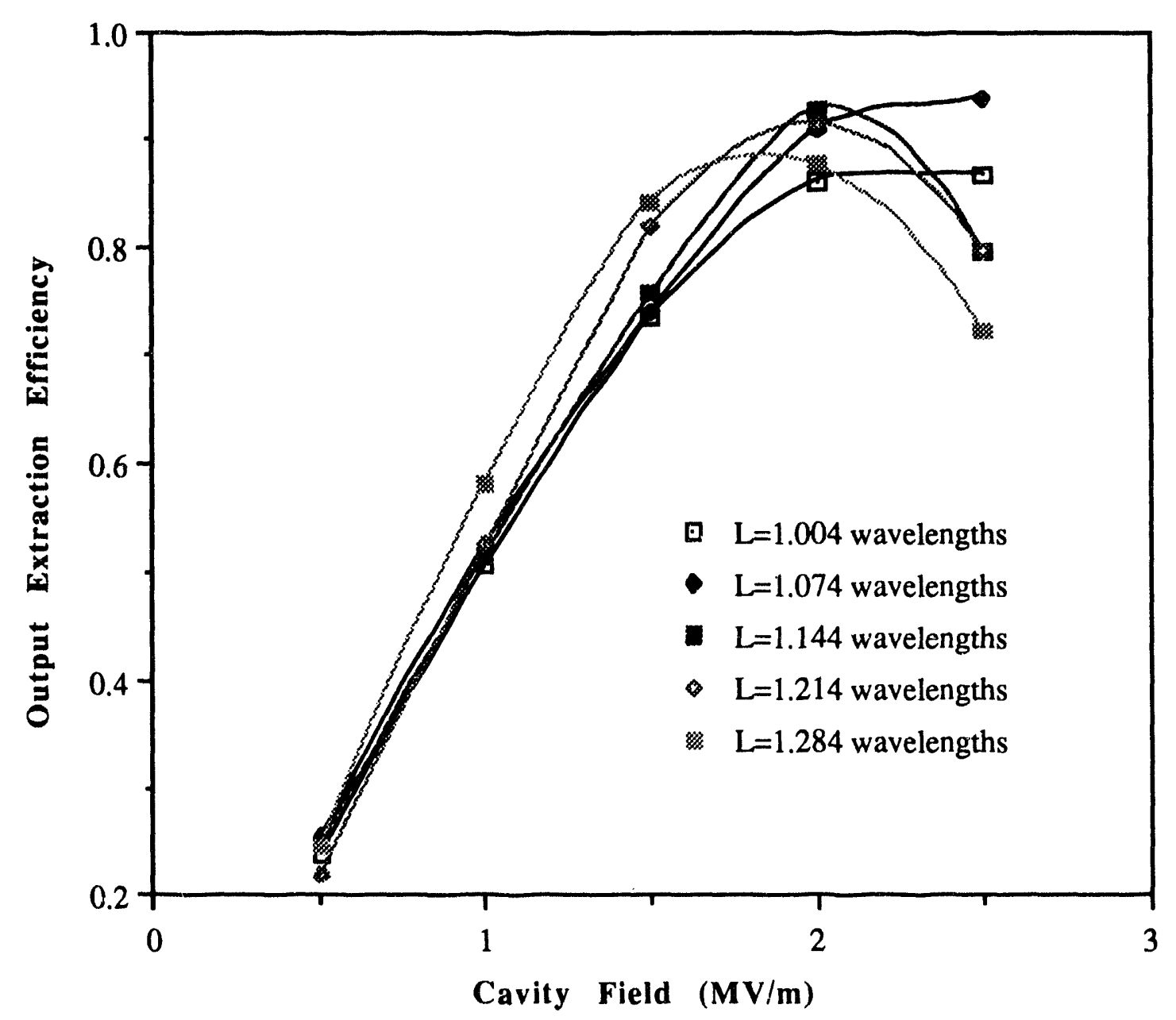

Figure 5.87. Conversion efficiency for input angle of $65^{\circ}$ for five lengths as a function of output-cavity field. Initial cyclotron frequency is 612.7 $\mathrm{MHz}$ and the beam-injection radius is $5.0 \mathrm{~cm}$.

This figure again shows that the extraction efficiency can be improved by injecting the beam into the output cavity off-axis. The extraction efficiency in Fig. 5.87 is again in excess of $90 \%$ and slightly higher than the extraction efficiency for the $2.5-\mathrm{cm}$ injection 
radius. Figures 5.88, 5.89, and 5.90 are similar to Figs. 5.85, 5.86, and 5.87, except that the entrance angle for these figures is $55^{\circ}$.

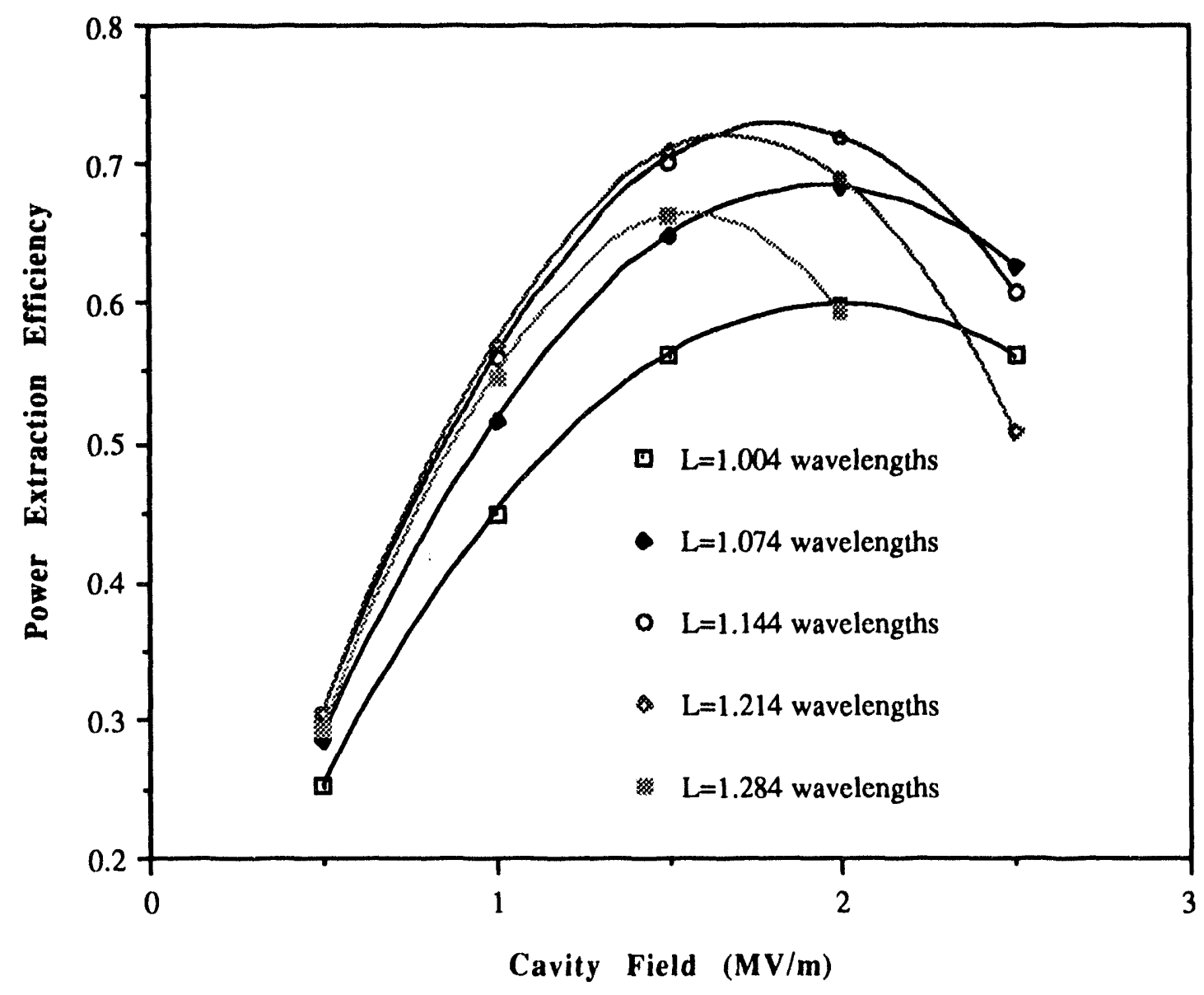

Figure 5.88. Conversion efficiency for input angle of $55^{\circ}$ for five lengths as a function of output-cavity field. Initial cyclotron frequency is 612.7 $\mathrm{MHz}$ and the beam is injected on the longitudinal axis. 


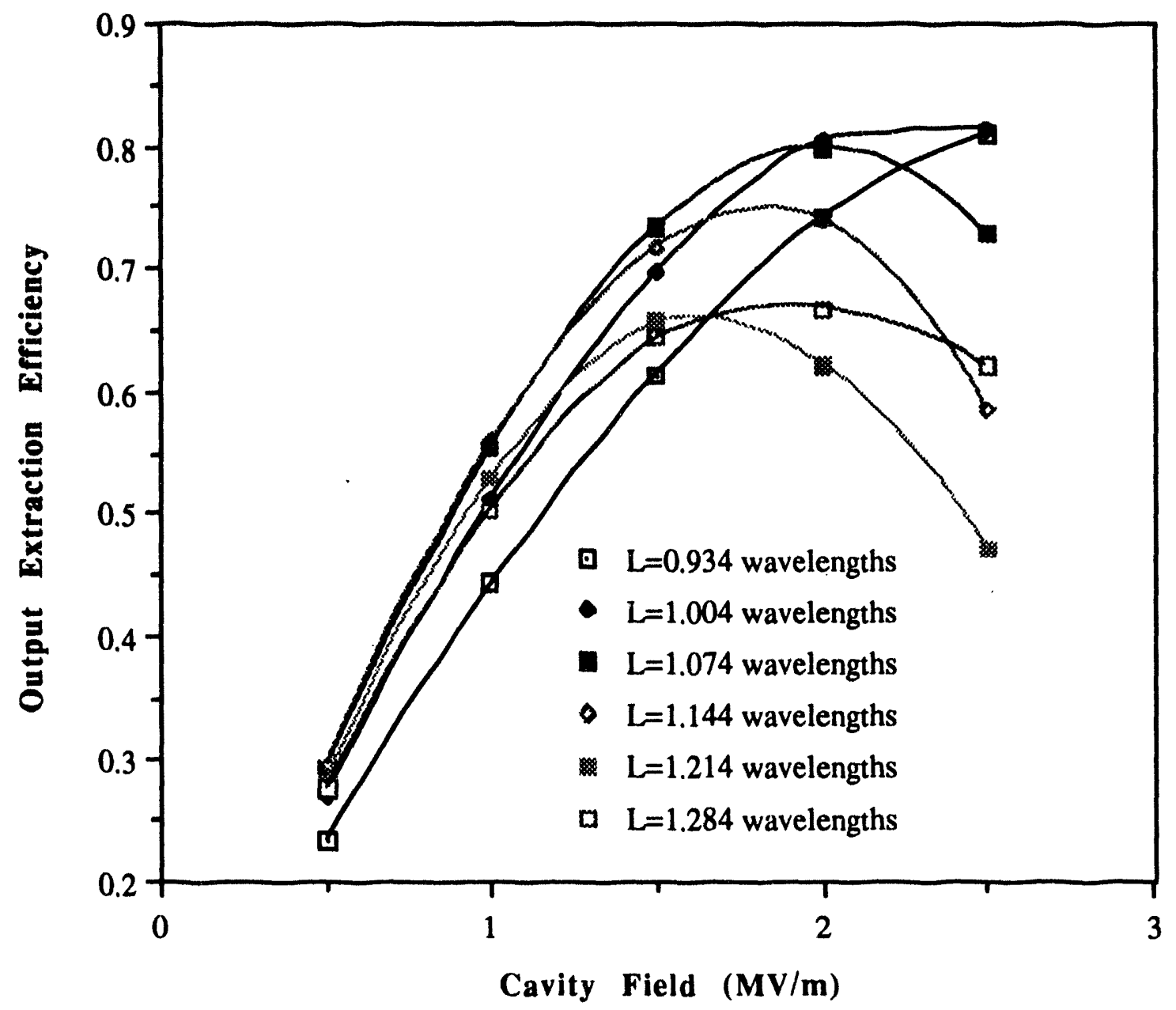

Figure 5.89. Conversion efficiency for input angle of $55^{\circ}$ for six lengths as a function of output-cavity field. Initial cyclotron frequency is 612.7 $\mathrm{MHz}$ and the beam-injection radius is $2.5 \mathrm{~cm}$. 


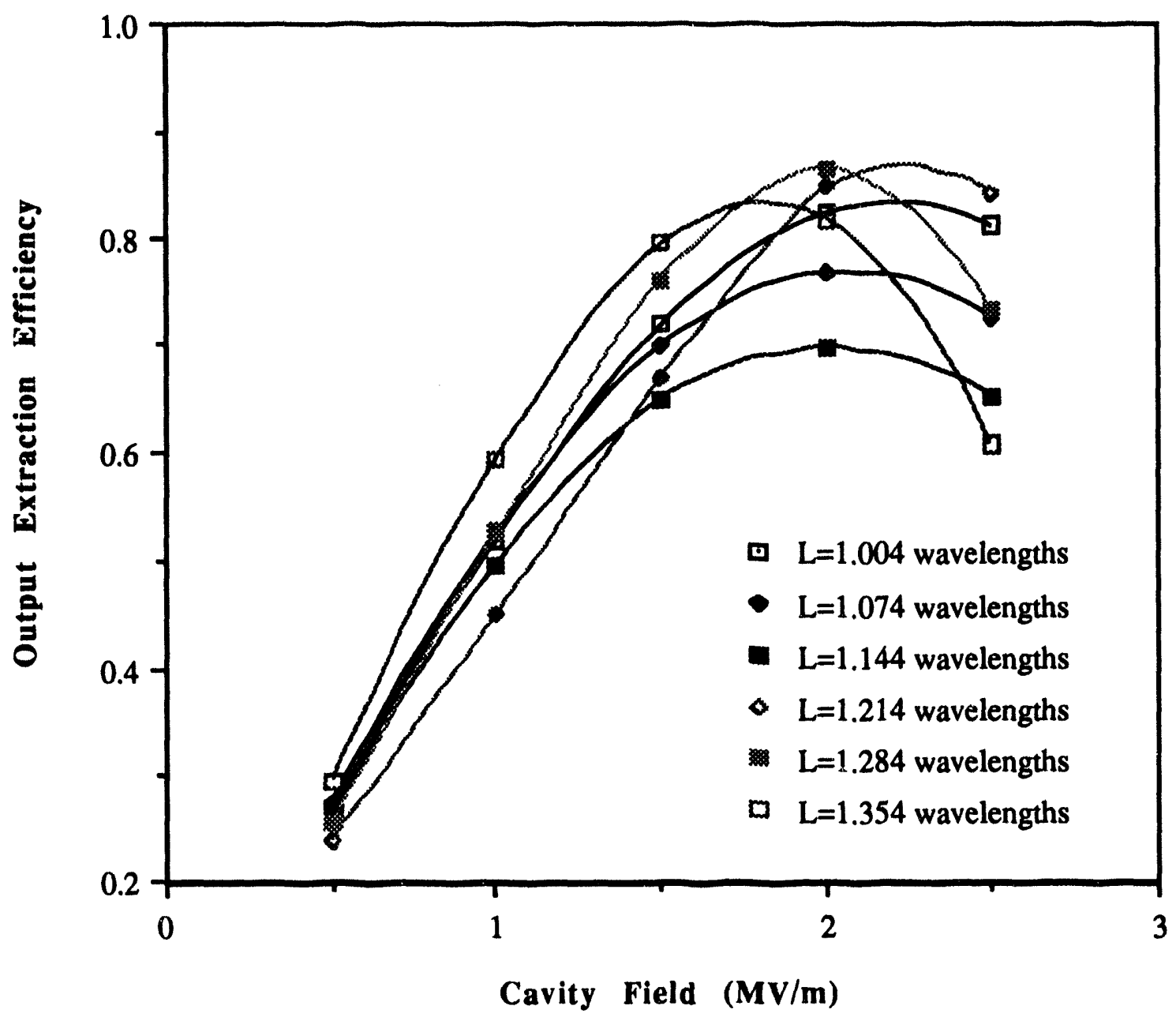

Figure 5.90. Conversion efficiency for input angle of $55^{\circ}$ for six lengths as a function of output-cavity field. Initial cyclotron frequency is 612.7 $\mathrm{MHz}$ and the beam-injection radius is $5.0 \mathrm{~cm}$.

These three figures illustrate the same general trend shown in Figs. 5.85, 5.86, and 5.87; the output-cavity extraction efficiency increases as the beam-entrance position into the output cavity is displaced off-axis, with the maximum extraction efficiency approaching $72 \%$ for on-axis beam injection, $81 \%$ for a beam injection $2.5 \mathrm{~cm}$ off-axis, and $86 \%$ for beam injection $5 \mathrm{~cm}$ off-axis. The maximum output-cavity extraction efficiencies are lower than those for the $65^{\circ}$ entrance angle. Later plots will compare these maximum extraction efficiencies and try to fit analytical functions to these curves. 
Figures 5.91, 5.92, and 5.93 are similar to Figs. 5.85 through 5.90, except they are for an entrance angle of $45^{\circ}$. Again, the output-cavity extraction efficiency increases as the beam moves off-axis, and the maximum achievable extraction efficiency is lower at $45^{\circ}$ than for the $55^{\circ}$ or $65^{\circ}$ entrance angle. The maximum calculated output-cavity extraction efficiency for the $45^{\circ}$ input angle is $57 \%, 60 \%$, and $73 \%$ for beam-injection radii of $0.0 \mathrm{~cm}, 2.5 \mathrm{~cm}$, and $5.0 \mathrm{~cm}$, respectively.

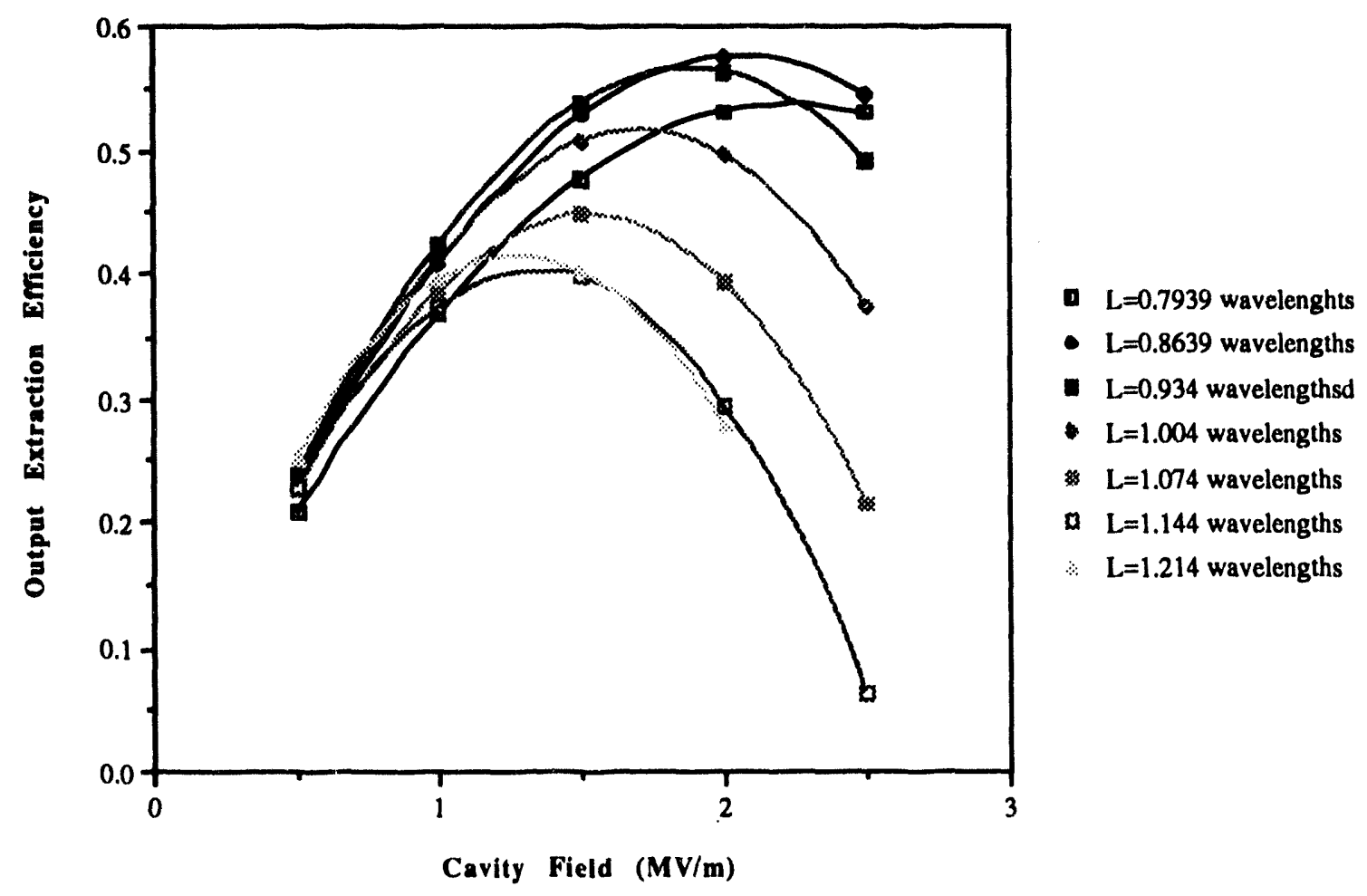

Figure 5.91. Conversion efficiency for input angle of $45^{\circ}$ for seven lengths as a function of output-cavity field. Initial cyclotron frequency is 612.7 $\mathrm{MHz}$ and the beam is injected on the longitudinal axis. 


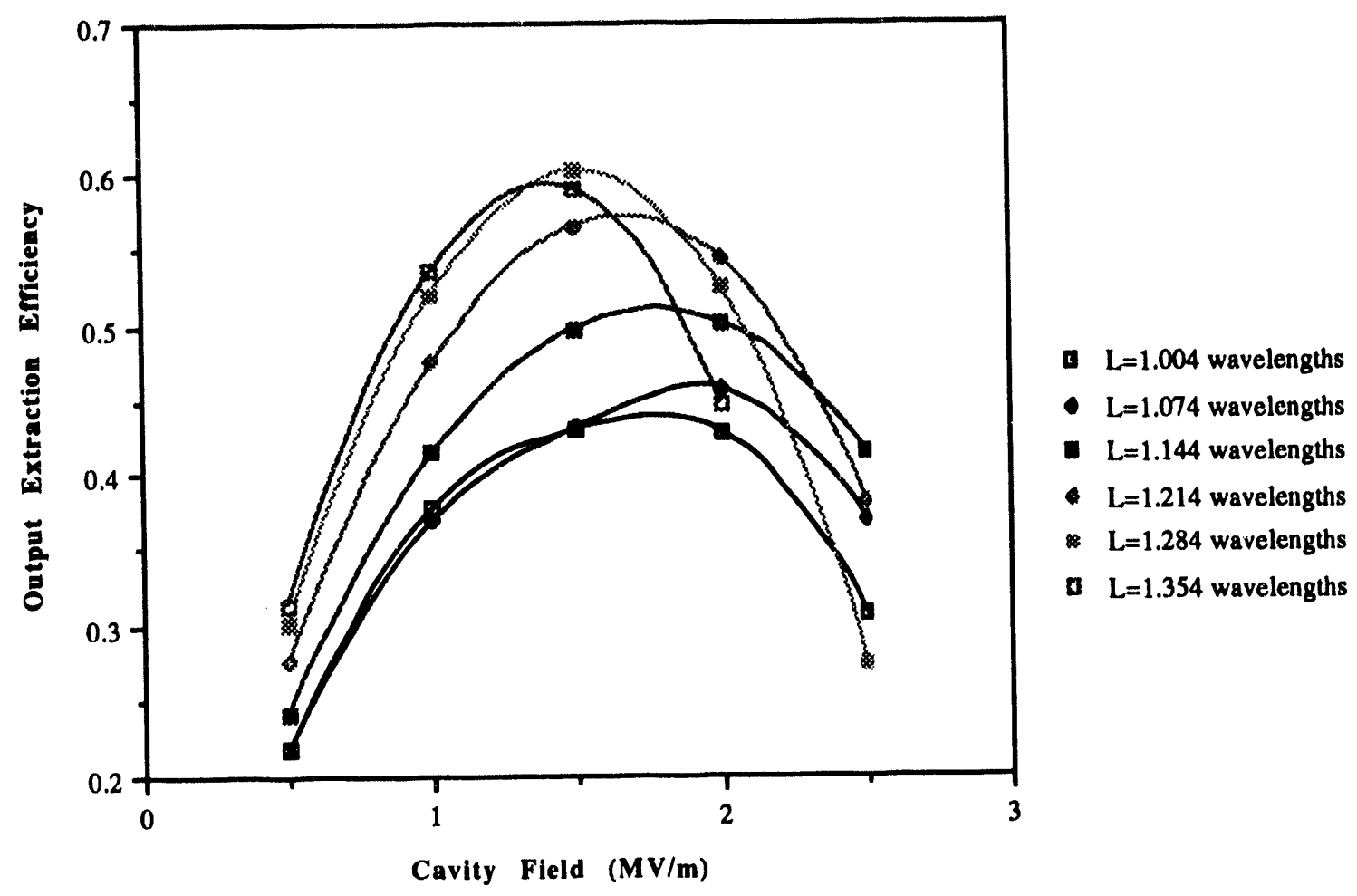

Figure 5.92. Conversion efficiency for input angle of $45^{\circ}$ for six lengths as a function of output-cavity field. Initial cyclotron frequency is 612.7 $\mathrm{MHz}$ and the beam-injection radius is $2.5 \mathrm{~cm}$. 


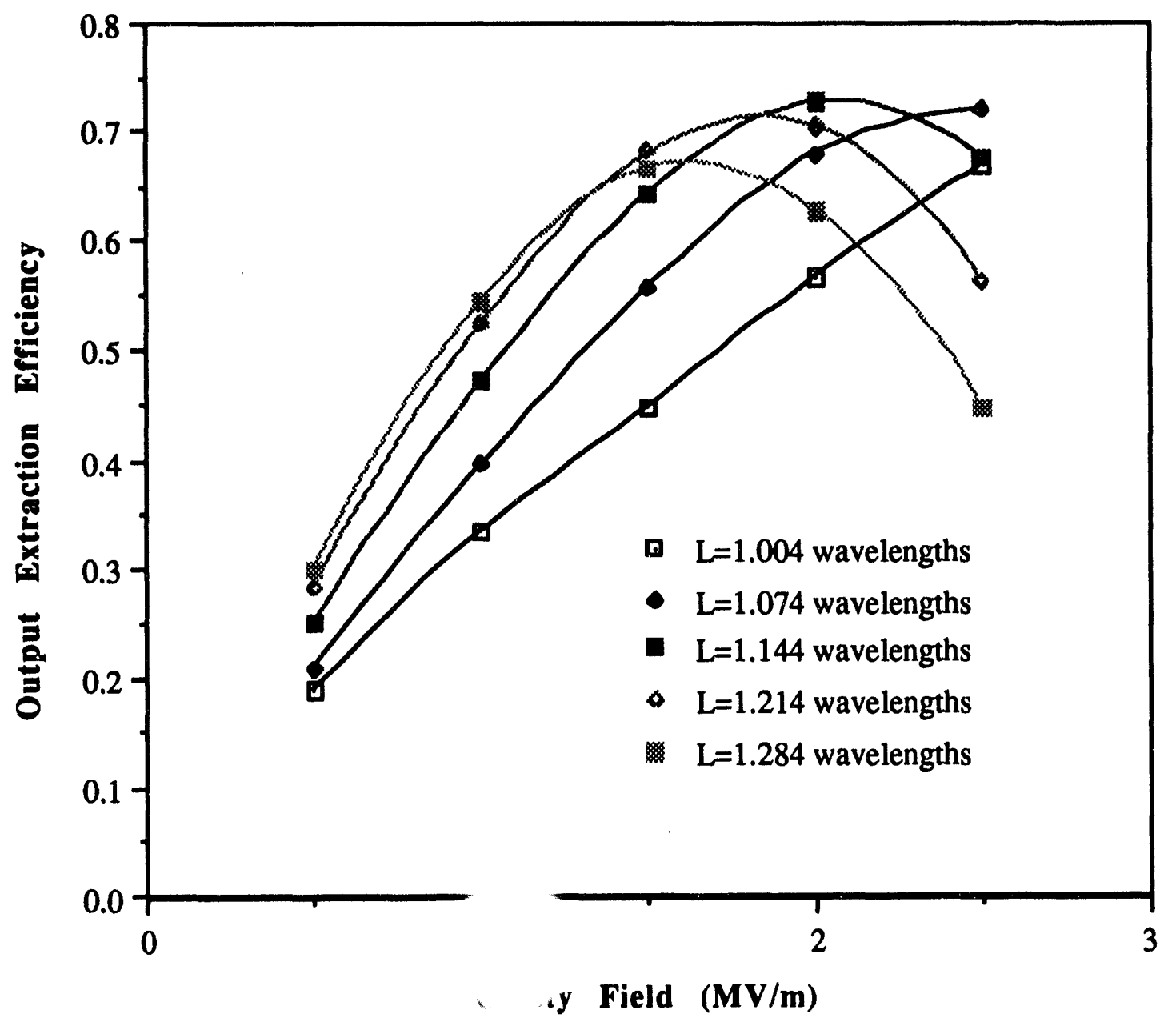

Figure 5.93. Conversion efficiency for input angle of $45^{\circ}$ for five lengths as a function of output-cavity field. Initial cyclotron frequency is 612.7 $\mathrm{MHz}$ and the beam-injection radius is $5.0 \mathrm{~cm}$. 


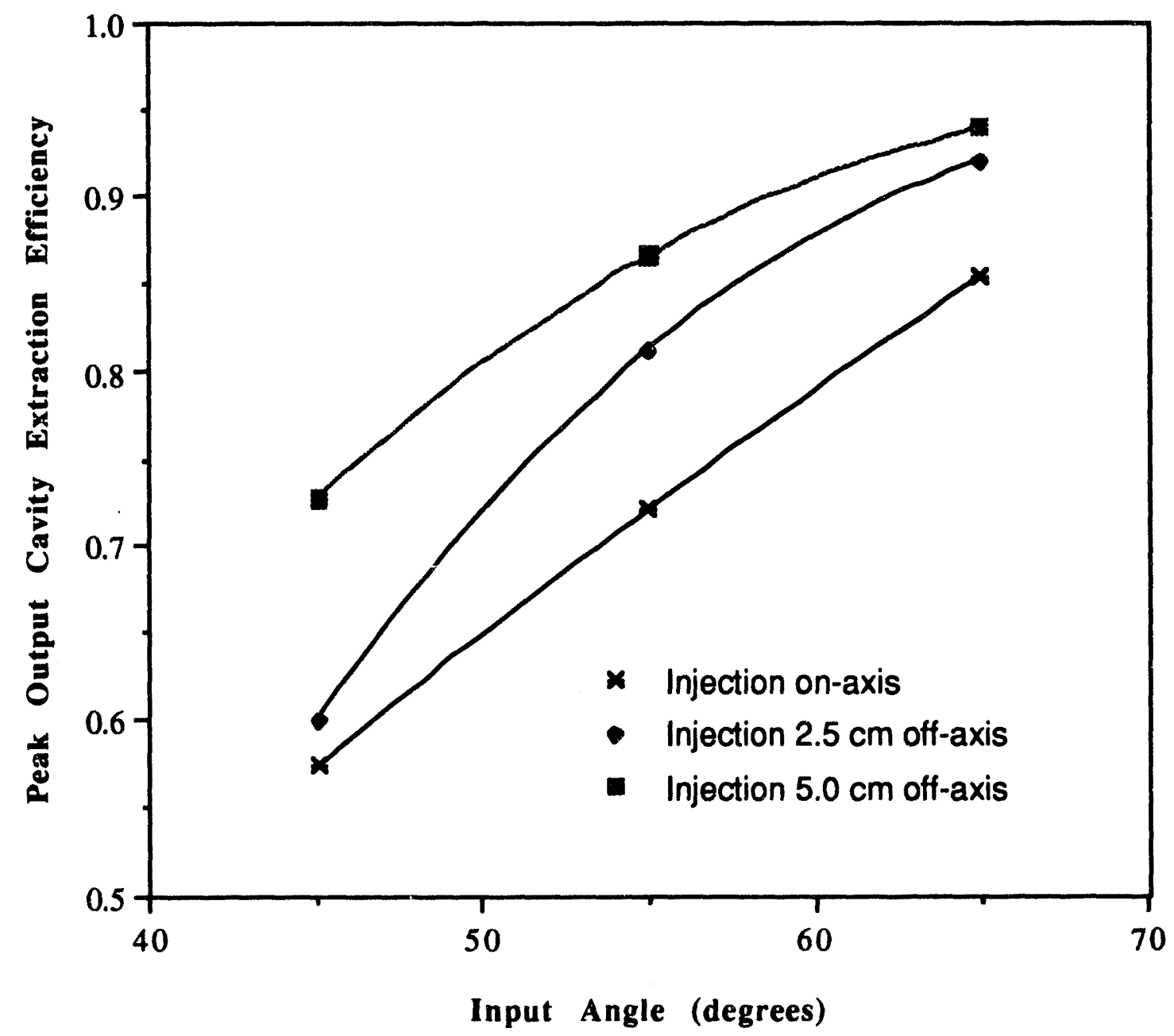

Figure 5.94. Peak output-cavity extraction efficiency for three output-cavity beam-entrance positions as a function of input angle.

Figure 5.94 illustrates three curves that are each composed of three points. The curves correspond to the three beam-entrance positions and $c$ ach point on the three curves corresponds to a beam-entrance angle. To better understand the shape of these curves, a - . top and side view of the particle trajectories are illustrated in Figs. 5.95; 5.96, and 5.97 for an input angle of $65^{\circ}$. Figures $5.95 \mathrm{a}$ and $5.95 \mathrm{~b}$ are for beam injection on-axis. Figures 5.96a and 5.96b are for beam injection $2.5 \mathrm{~cm}$ off-axis, and Figs. $5.97 \mathrm{a}$ and $5.97 \mathrm{~b}$ are for beam injection $5.0 \mathrm{~cm}$ off-axis. 
Figures 5.85 through 5.94 are all based on a magnetic focusing-field strength that results in an average cyclotron frequency equal to the rf frequency. (This also is the magnetic-field strength that maximizes output-cavity extraction efficiency for lower output-cavity-field values.) Figures 5.95, 5.96, and 5.97 illustrate particle trajectories and change in gammas for a $55^{\circ}$ input angle with the beam injected on-axis, $2.5 \mathrm{~cm}$ off-axis, and $5 \mathrm{~cm}$ off-axis, respectively. For these double figures, each figure "a" is the radius of a single particle of the beam as it passes through the output cavity, figure " $b$ " is a top view of the particle trajectory as it passes through the output cavity, and figure "c" is the gamma of a single particle of the beam as it passes through the output cavity. These figures are intended to help illustrate the differences in the output-cavity extraction efficiency for different injection radii, as well as provide insight into the nature of the interaction. They illustrate that off-axis injection positions result in a larger maximum radius for a beam particle, thereby subjecting the particle to a higher decelerating field. This results in more energy exchange. This is why there is an increase in extraction efficiency with off-axis injection. It is also interesting to note that the transverse energy decreases as the particle travels through the output cavity. This can be observed by the proximity of the data points that are separated in time by a uniform time step at the tail end of the top view of the particle trajectory (figure " $b$ "). The velocity relationships are examined in more detail in a later figure. 


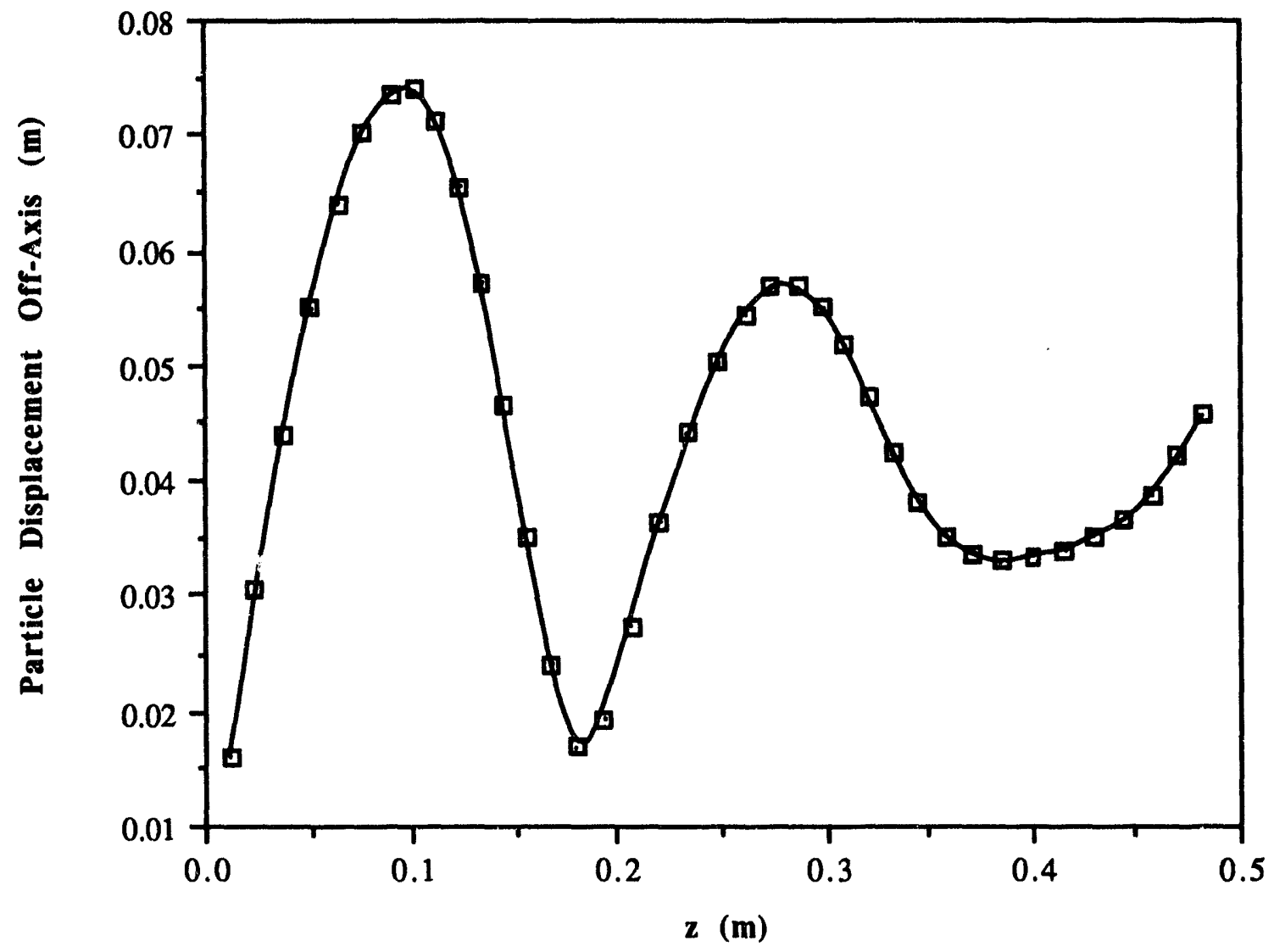

Figure 5.95a. Particle displacement off-axis as a function of longitudinal cavity position for $55^{\circ}$ entrance with the beam entering on-axis. The cavity length is 1.144 wavelengths and the peak electric field is $2.0 \mathrm{MV} / \mathrm{m}$. 


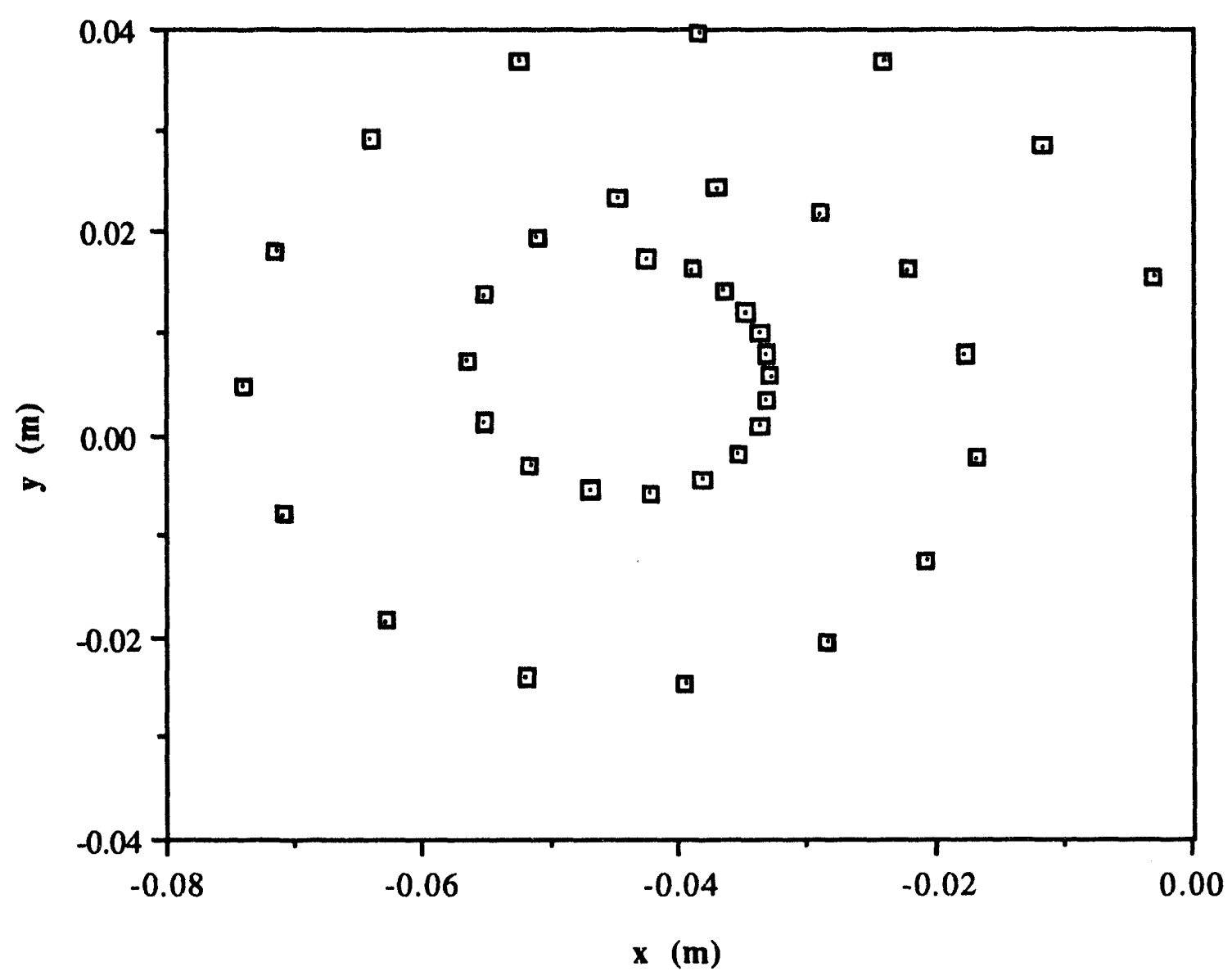

Figure 5.95b. Top view of particle trajectory in the output cavity for $55^{\circ}$ entrance with the beam entering on-axis. The cavity length is 1.144 wavelengths and the peak electric field is $2.0 \mathrm{MV} / \mathrm{m}$. 


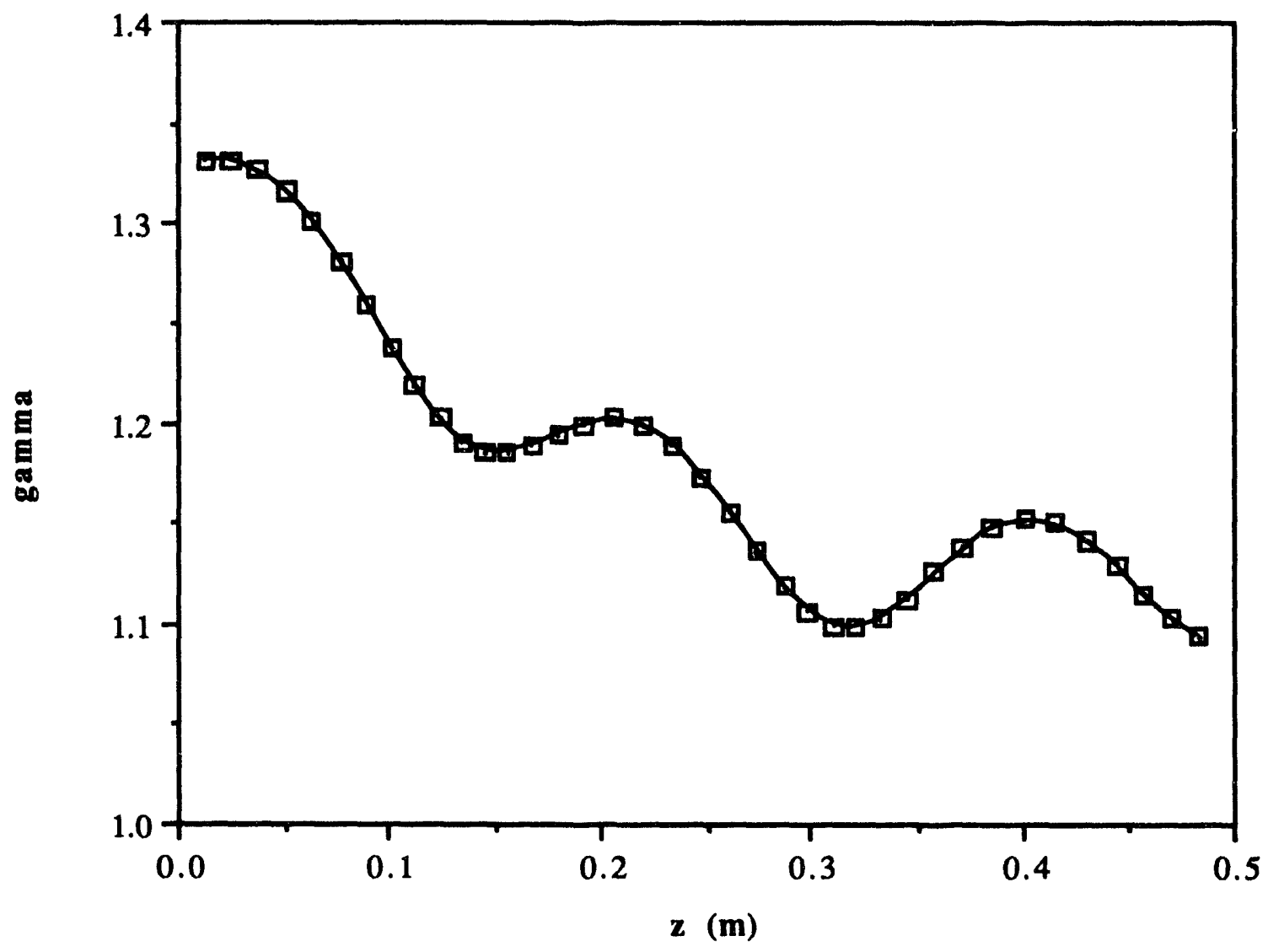

Figure 5.95c. Particle gamma as a function of longitudinal cavity position for $55^{\circ}$ entrance with the beam entering on-axis. The cavity length is 1.144 wavelengths and the peak electric field is $2.0 \mathrm{MV} / \mathrm{m}$. 


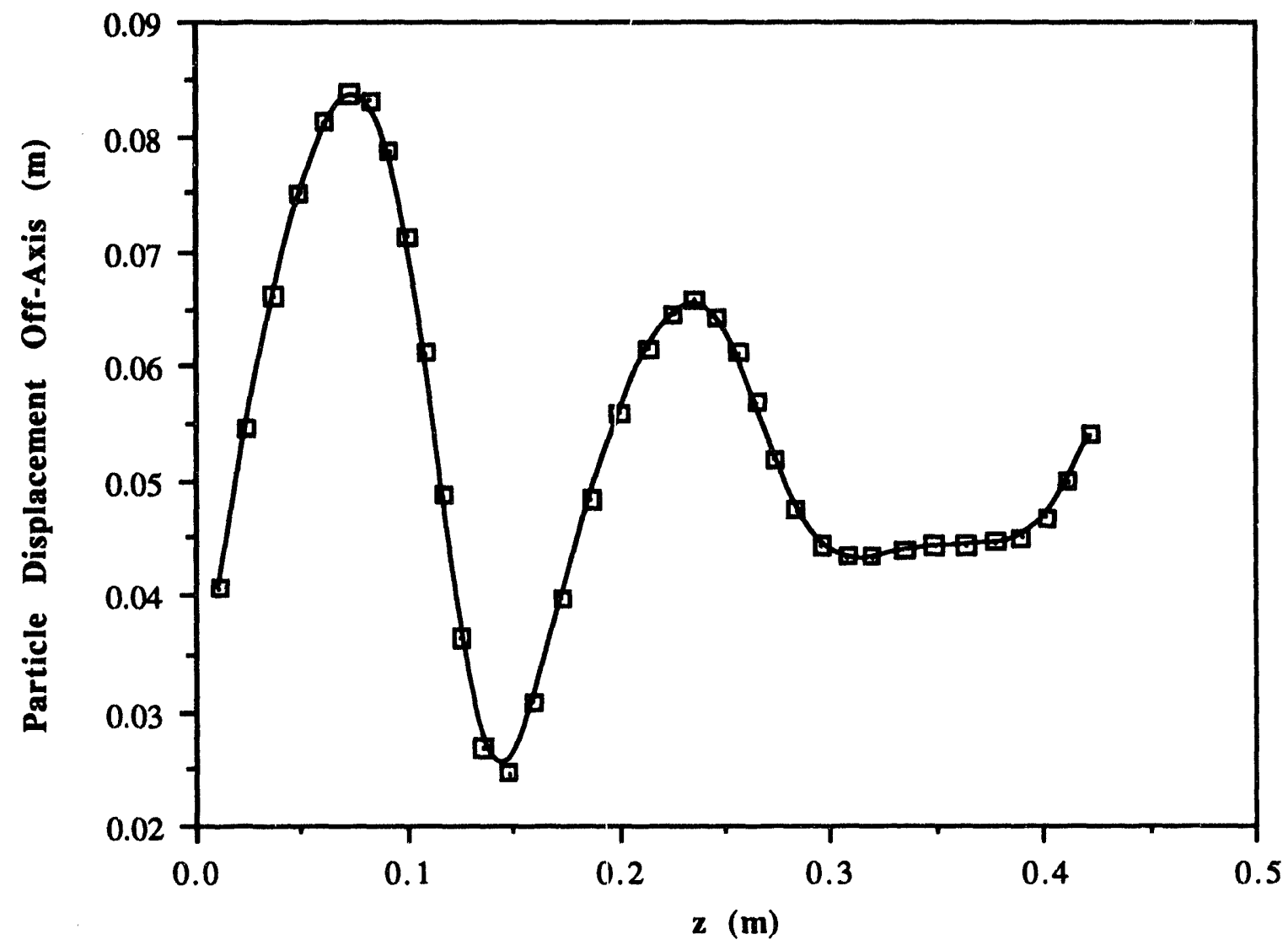

Figure 5.96a. Particle displacement off-axis as a function of longitudinal cavity position for $55^{\circ}$ entrance with the beam entering $2.5 \mathrm{~cm}$ off-axis. The cavity length is 1.0104 wavelengths and the peak electric field is $2.5 \mathrm{MV} / \mathrm{m}$. 


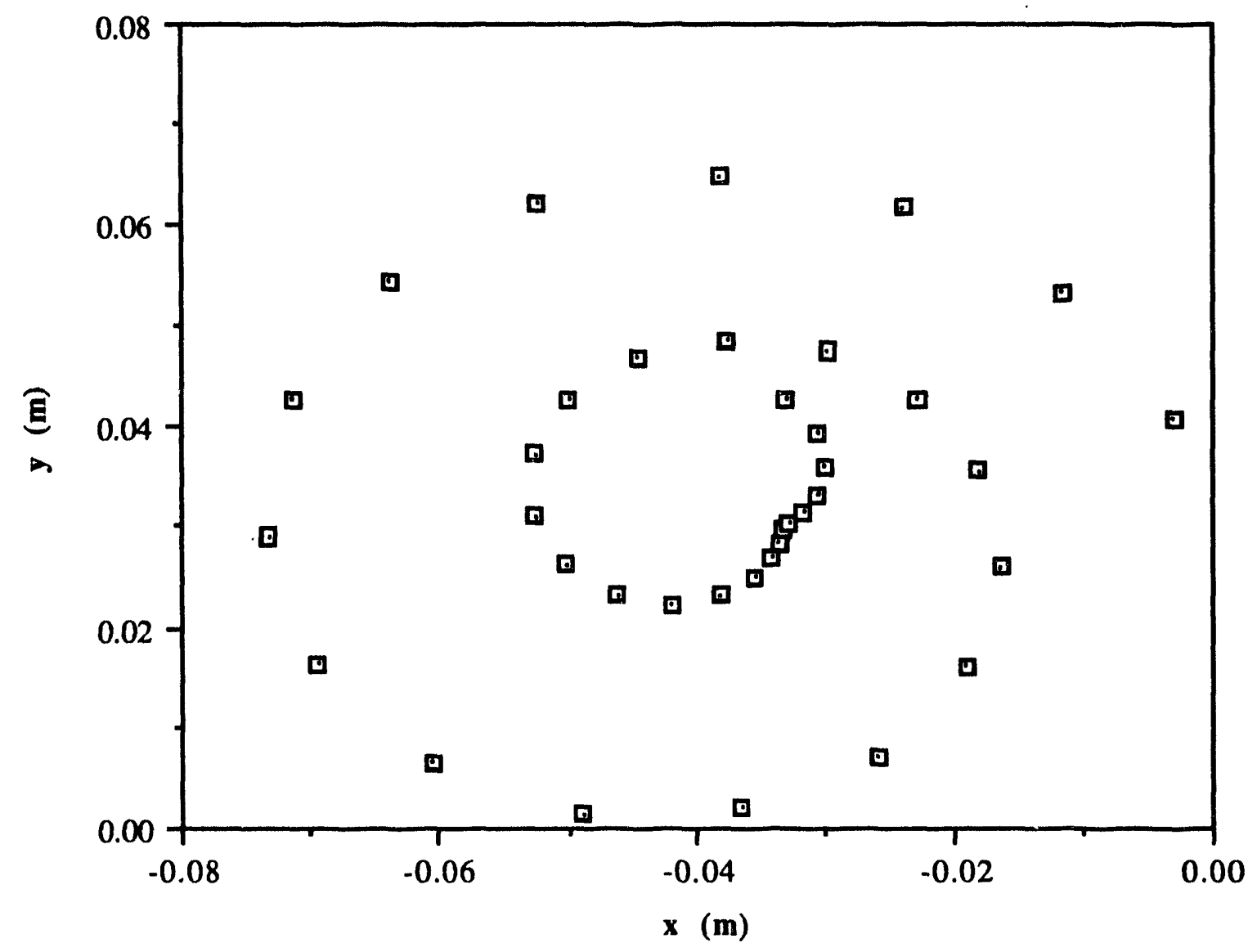

Figure 5.96b. Top view of particle trajectory in the output cavity for $55^{\circ}$ entrance with the beam entering $2.5 \mathrm{~cm}$ off-axis. The cavity length is 1.004 wavelengths and the peak electric field is $2.5 \mathrm{MV} / \mathrm{m}$. 


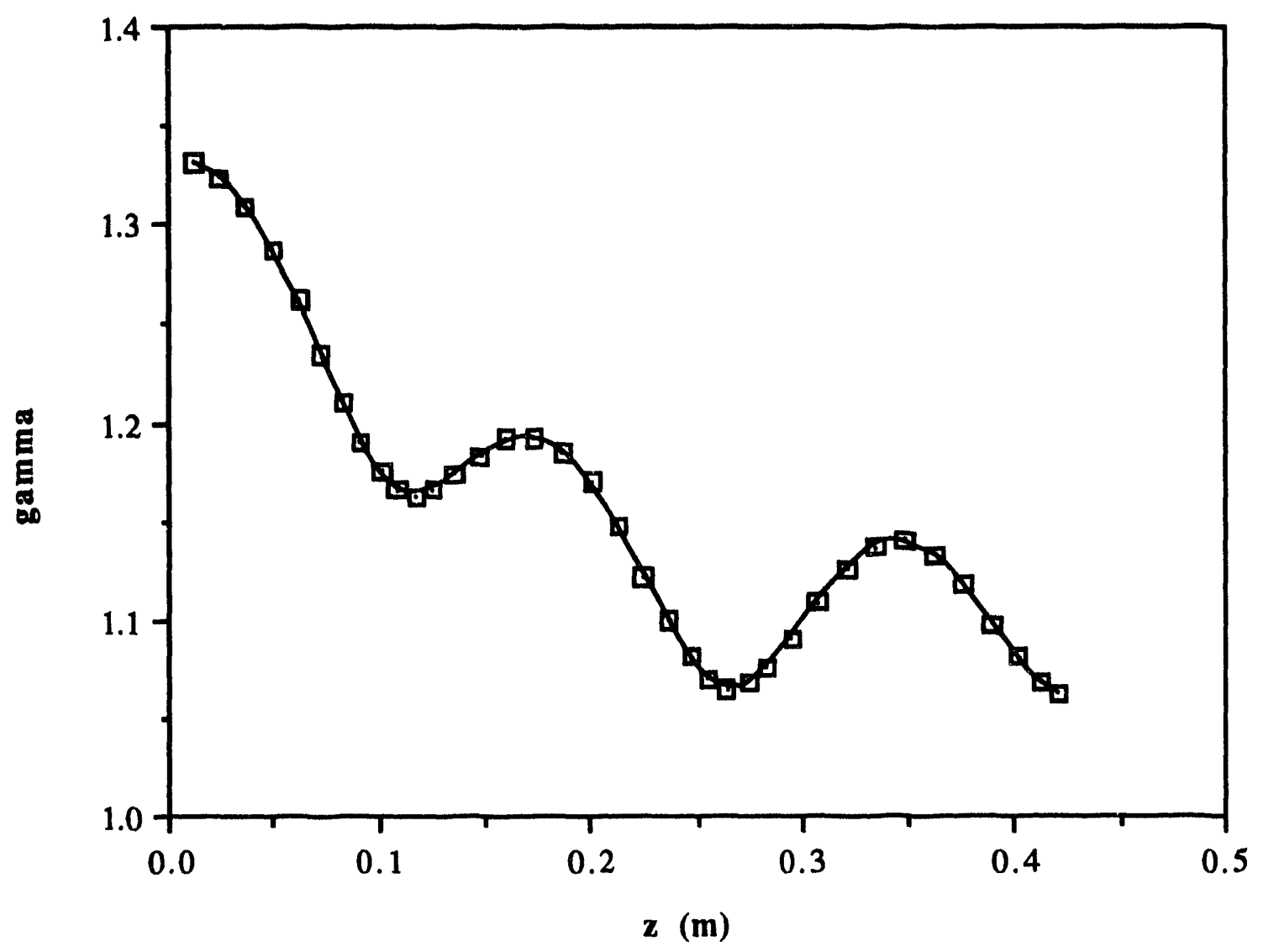

Figure 5.96c. Particle gamma as a function of longitudinal cavity position for $55^{\circ}$ entrance with the beam entering $2.5 \mathrm{~cm}$ off-axis. The cavity length is 1.004 wavelengths and the peak electric field is $2.5 \mathrm{MV} / \mathrm{m}$. 


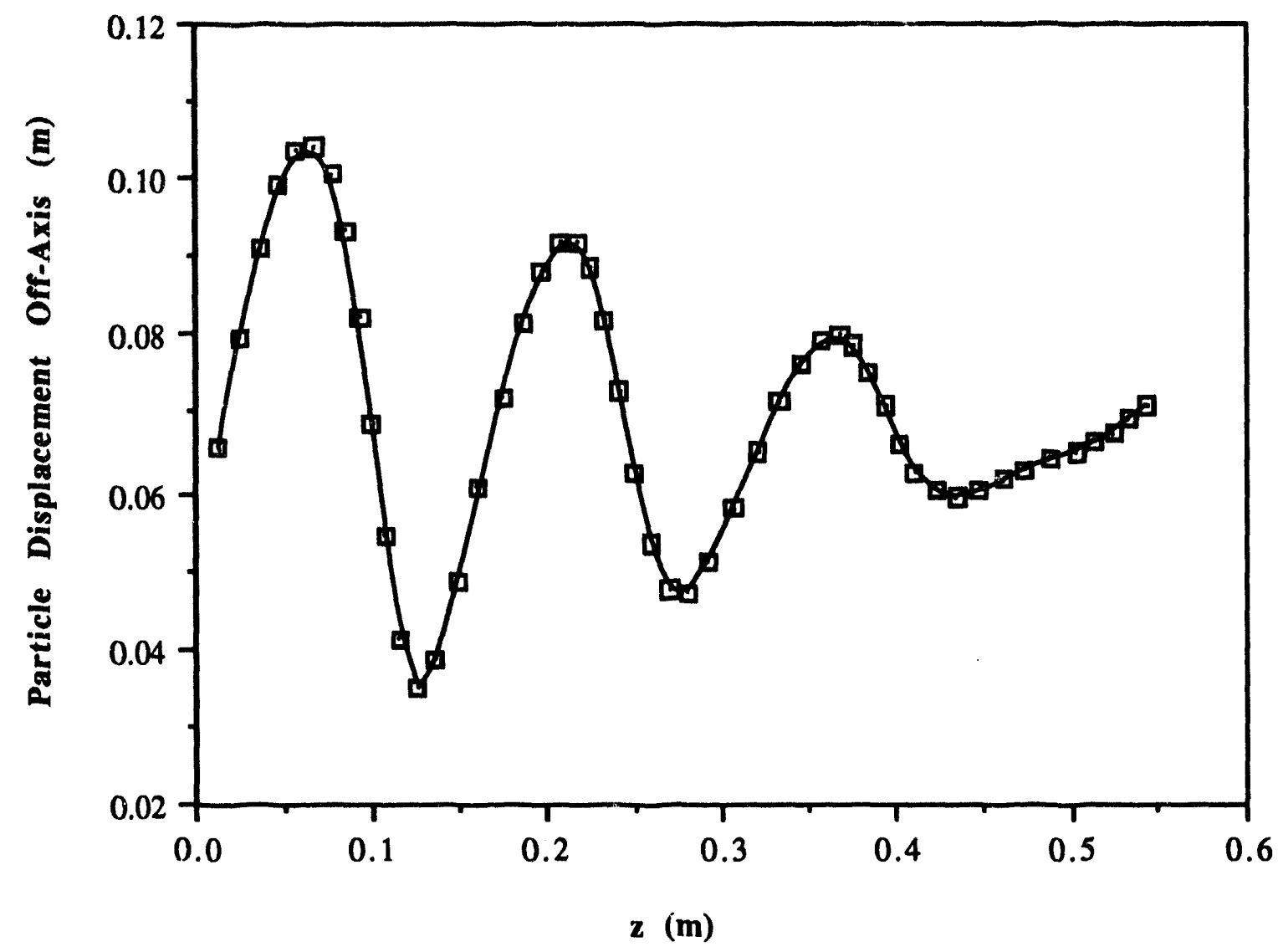

Figure 5.97a. Particle displacement off-axis as a function of longitudinal cavity position for $55^{\circ}$ entrance with the beam entering $5.0 \mathrm{~cm}$ off-axis. The cavity length is 1.284 wavelengths and the peak electric field is $2.0 \mathrm{MV} / \mathrm{m}$. 


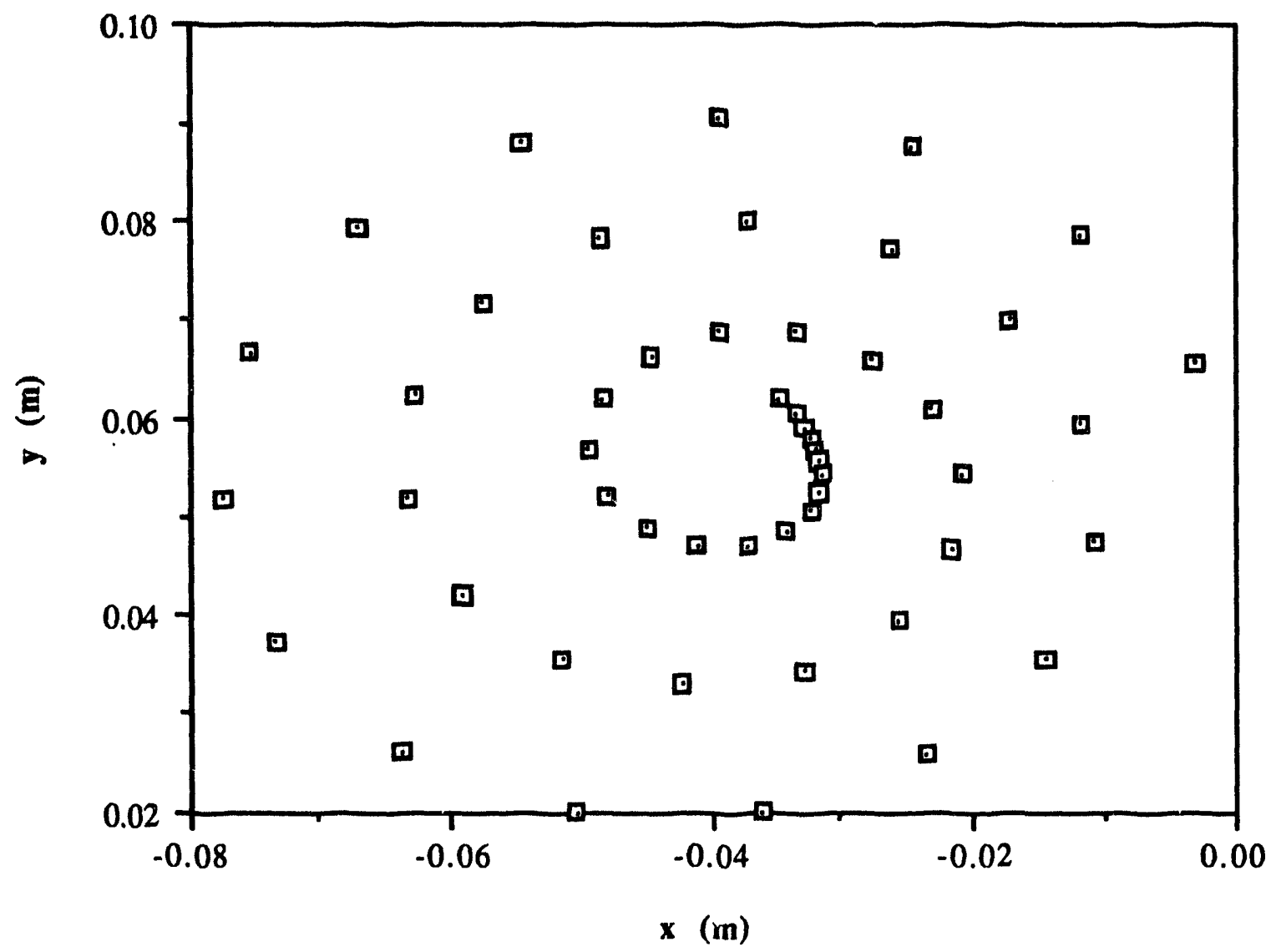

Figure 5.97b. Top view of particle trajectory in the output cavity for $55^{\circ}$ entrance with the beam entering $5.0 \mathrm{~cm}$ off-axis. The cavity length is 1.284 wavelengths and the peak electric field is $2.0 \mathrm{MV} / \mathrm{m}$. 


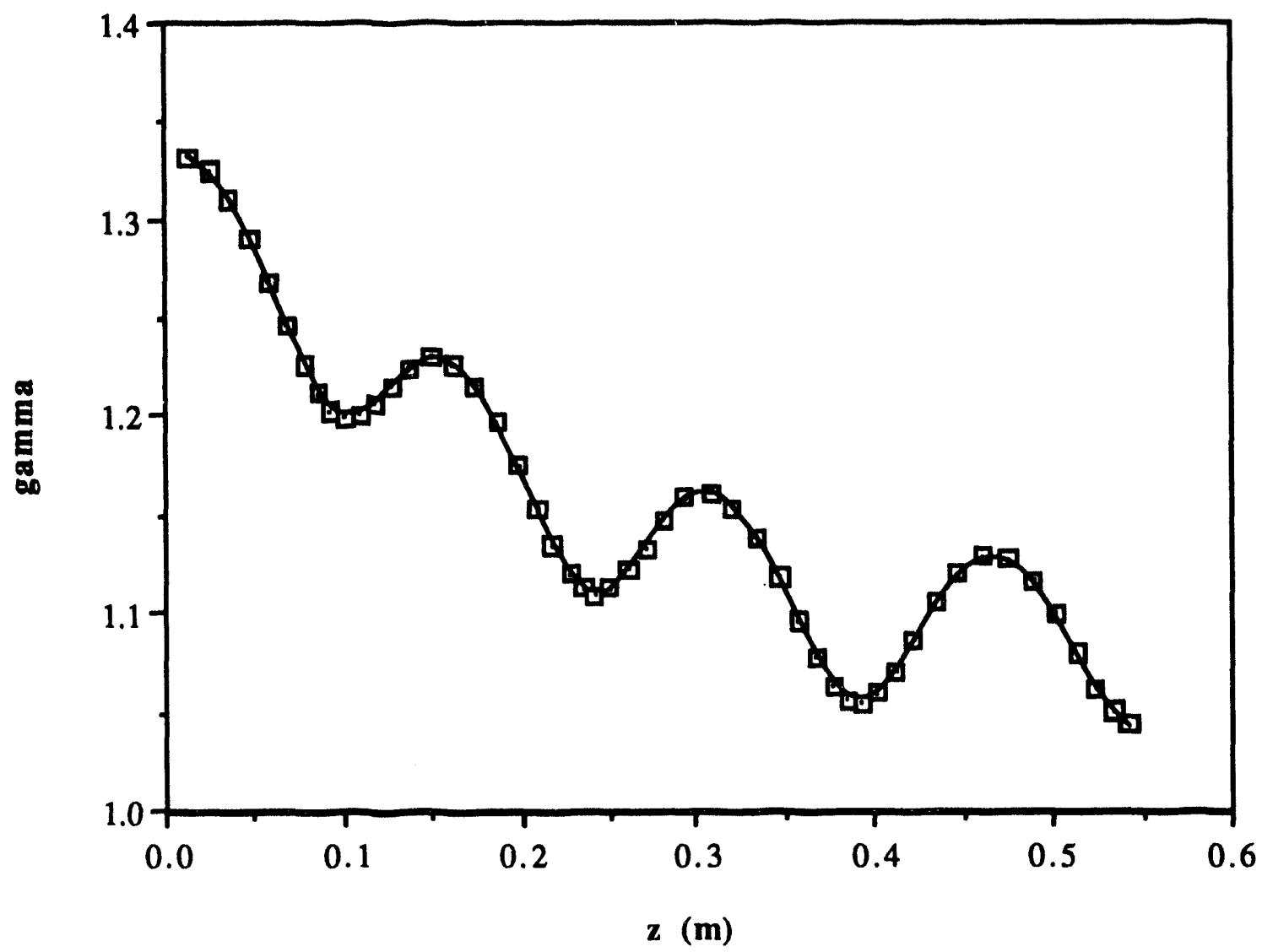

Figure 5.97c. Particle gamma as a function of longitudinal cavity position for $55^{\circ}$ entrance with the beam entering $5.0 \mathrm{~cm}$ off-axis. The cavity length is 1.284 wavelengths and the peak electric field is $2.0 \mathrm{MV} / \mathrm{m}$. 


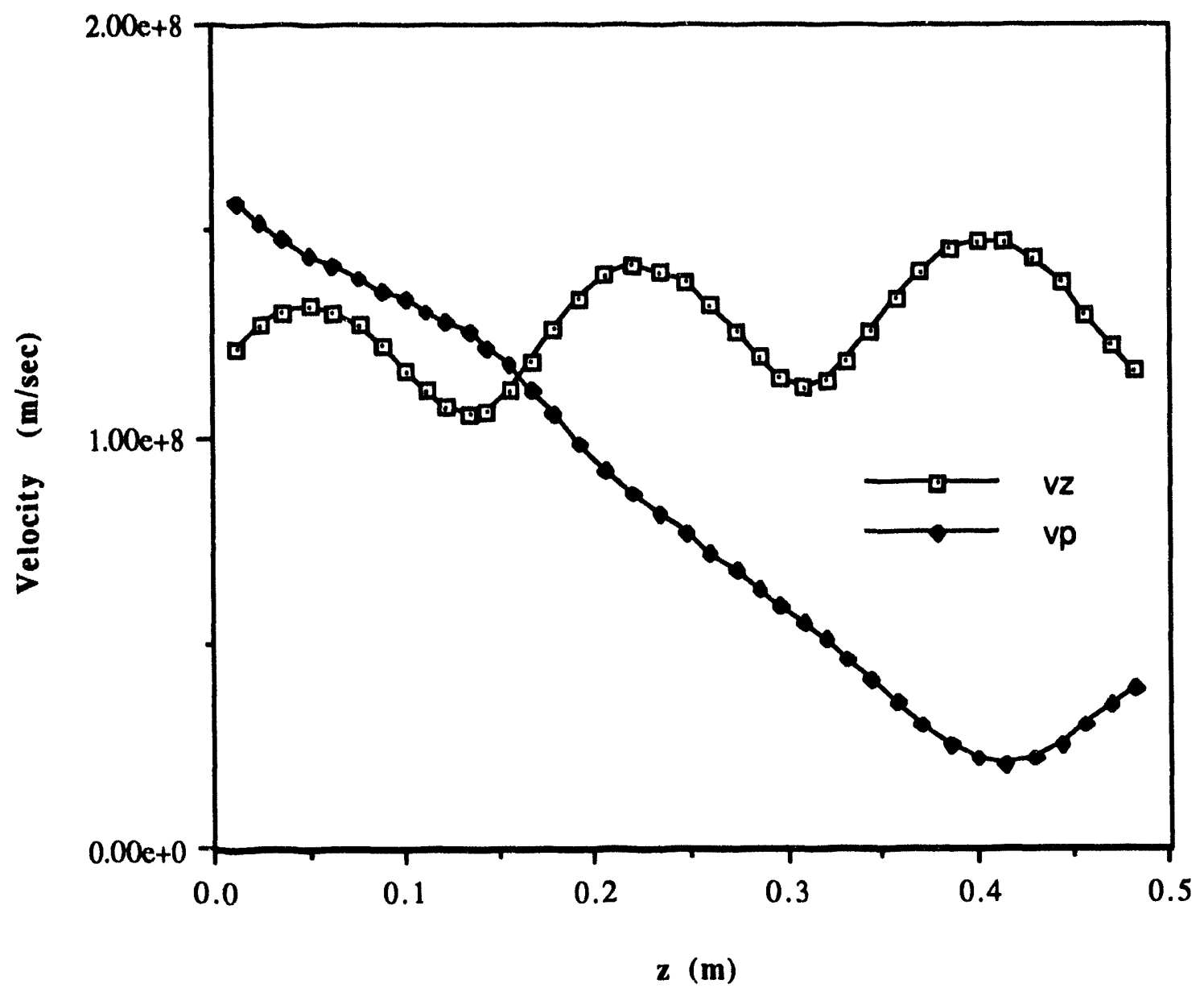

Figure 5.98. Particle radial velocity (vp) and longitudinal velocity ( $(1 / \mathrm{z})$ as a function of longitudinal cavity position for $55^{\circ}$ entrance with the beam entering on-axis. The cavity length is 1.144 wavelengths and the peak electric field is $2.0 \mathrm{MV} / \mathrm{m}$.

Figure 5.98 illustrates the transverse and longitudinal velocity of a single particle as it passes through the output cavity. As can be noted from this graph, the longitudinal velocity (energy) stays approximately constant while.the transverse velocity (energy) is converted into the if fields.

Figures 5.99 and 5.100 illustrate the effect of varying the magnetic field $\pm 15 \%$ from this nominal value. Figure 5.99 is for a magnetic field of $0.02482 \mathrm{~T}$, or an initial cyclotron frequency of $521 \mathrm{MHz}$ (or an average cyclotron frequency of $595 \mathrm{MHz}$ ). 
Figure 5.100 is for a magnetic field of $0.03358 \mathrm{~T}$, or an initial cyclotron frequency of 705 $\mathrm{MHz}$ (or an average cyclotron frequency of $804.9 \mathrm{MHz}$ ).

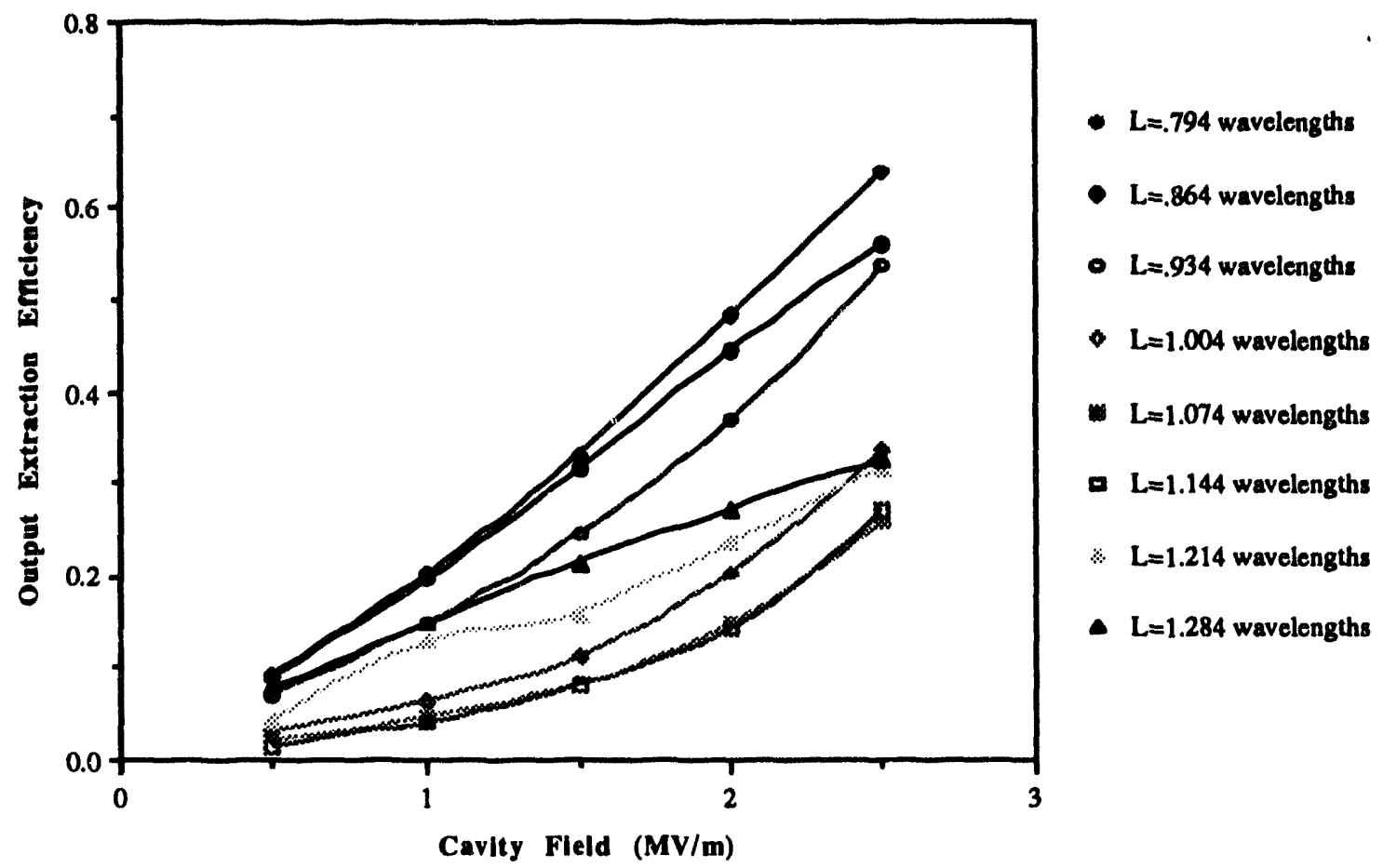

Figure 5.99. Output-cavity extraction efficiency as a function of peak rf cavity field for a number of lengths with a magnetic field $15 \%$ less than the nominal field. 


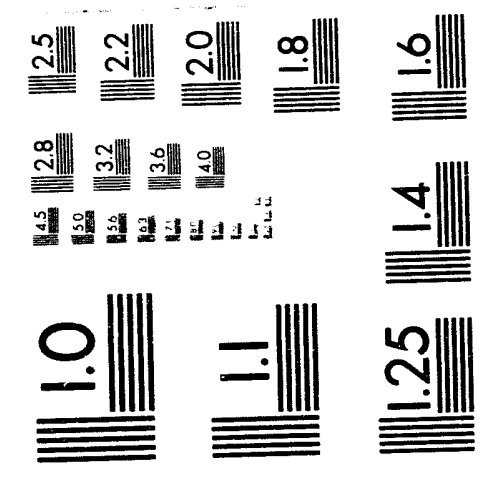



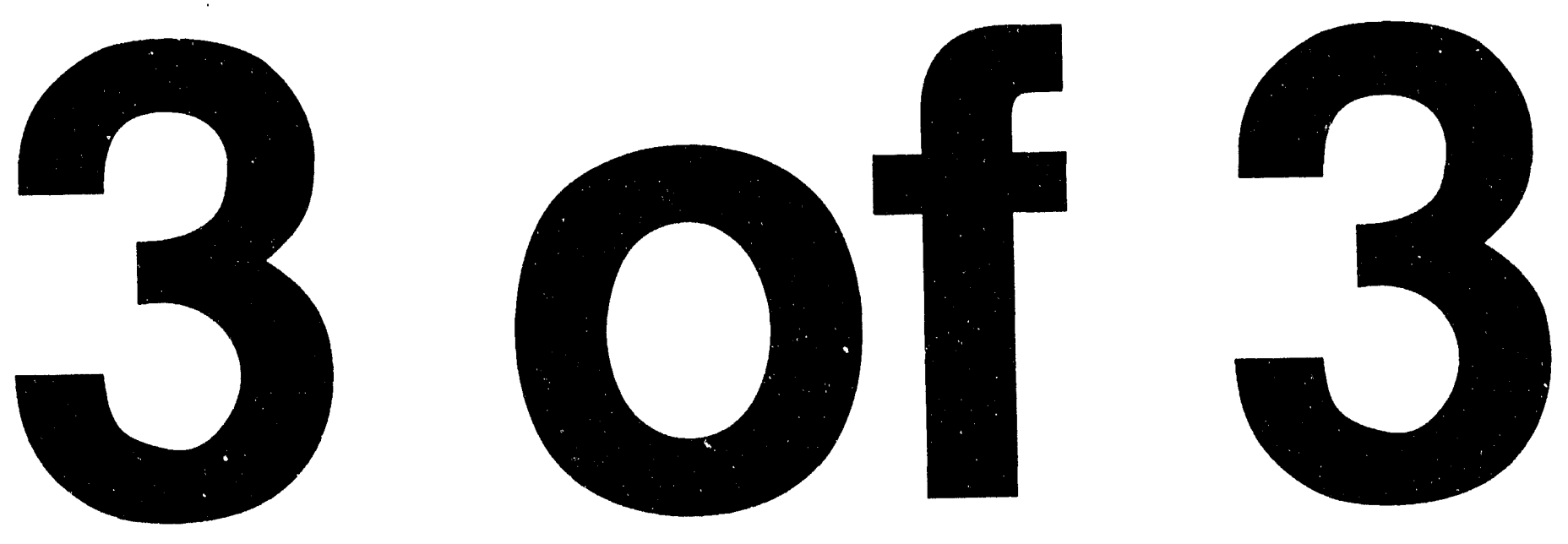


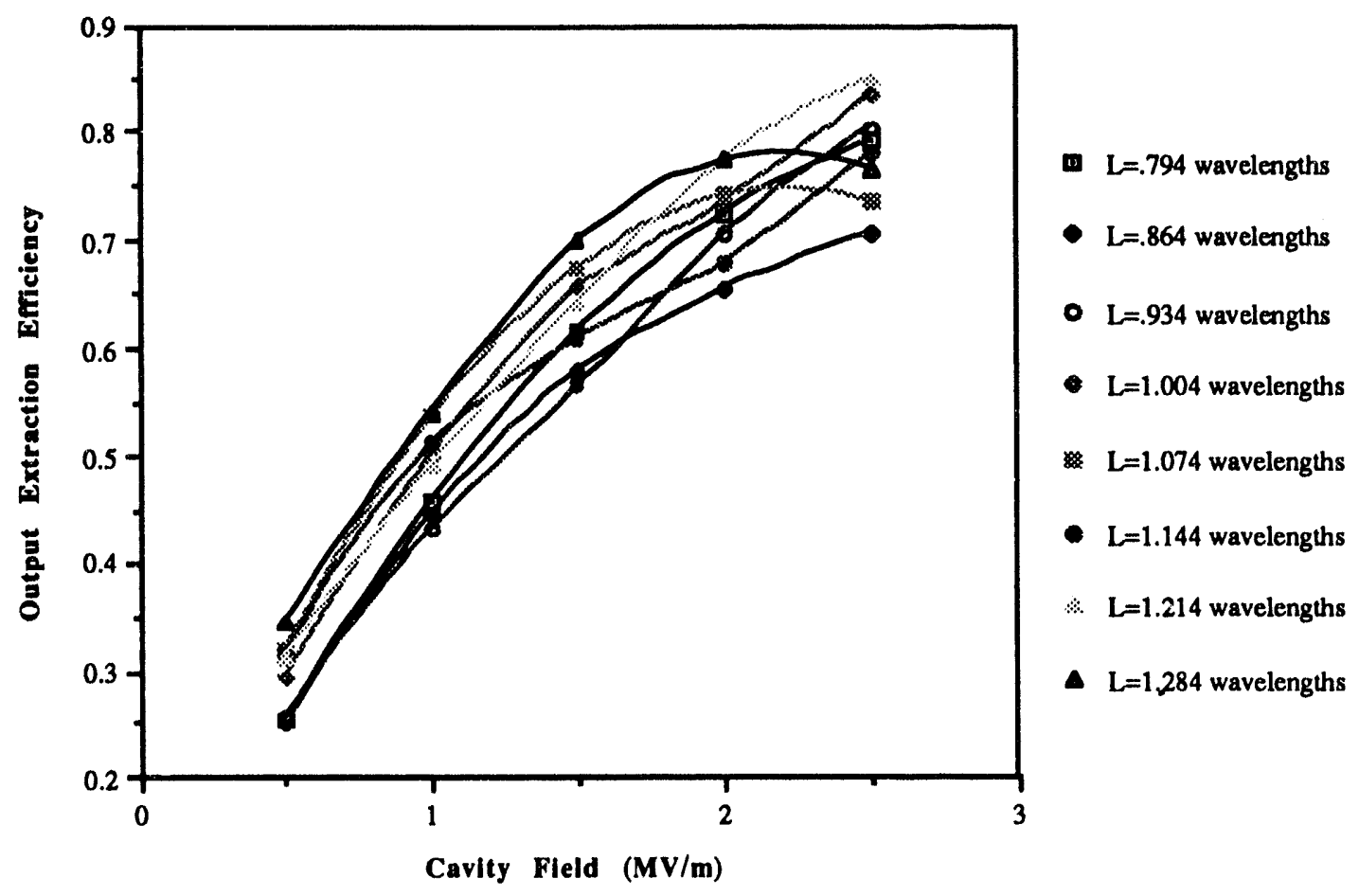

Figure 5.100. Output-cavity extraction efficiency as a function of peak rf cavity field for a number of lengths with a magnetic field $15 \%$ greater than the nominal field.

From Fig. 5.99 it can be seen that a $15 \%$ reduction in the magnetic field over the nominal case drastically reduces the output conversion efficiency for the peak electric field values of interest. From inspection of the figure, it can be seen that the maximum output-cavity conversion efficiency is reduced to $64 \%$ and appears to peak at higher values of peak if electric field. From Fig. 5.100 it can be seen that the $15 \%$ increase in magnetic field does not cause as large a reduction in output cavity conversion efficiency wih respect to the nominal case ( $85 \%$ versus $90 \%$, respectively). However, it does increase the optimum length of the output cavity (1.214 wavelengths for $0.03358 \mathrm{~T}$ versus 0.864 wavelengths for $0.0292 \mathrm{~T}$ ). 


\section{Application of Rigid-Beam Model for Magnicon Design}

\subsection{Design rules}

This section summarizes how the results of the component investigations presented in Chapter 5 are used to determine magnicon designs. I shall apply these results to two types of magnicons: the conventional magnicon and a new "uniform-field" magnicon, which has a revised design.

The first step in applying the results of Chapter 5 is to designate the beam entrance angle into the output cavity. Figure 5.94 in Section 5.6 illustrates the relationship between the output-cavity extraction efficiency and the entrance angle into the output cavity. It can be used to determine the entrance angle. Other plots in Section 5.6 can then be used to determine the output-cavity peak electric field and output-cavity focusing field. (Typically, this value is selected to equal the rf frequency, which equals the cyclotron frequency based on an average gamma.)

With the output-cavity entrance angle determined, it is next possible to design the deflection system. Two deflection systems are possible: one for the conventional magnicon and one for the uniform-field magnicon.

To design the deflection system, my approach is first to determine the lowest instantaneous bandwidth I can tolerate. For a uniform-field magnicon, where the $\Omega / \omega$ ratio is equal to the if frequency over the entire length of the device, the instantaneous bandwidth (or, correspondingly, the beam-loaded Q) can be determined by using Tables 5.4 and 5.6 in order to interpolate the smallest cavity length. Figure 5.23 can be used to calculate the gain of the cavity. Figure 5.21 illustrated that two lengths can provide the same gain if the instantaneous bandwidth constraint allows it. If two lengths are possible, Fig. 5.21 demonstrated that the longer length should be used in order to minimize dissipated power. If a conventional magnicon geometry with a half-cusp magnetic-field transition between the deflection system and output cavity is used, as large an $\Omega / \omega$ ratio as possible should be selected, within instantaneous-bandwidth constraints, to maximize gain. (This is based on the data presented in Section 5.3.3.) The data in Section 5.3.1 can then be used to determine lengths within the bounds set by instantaneous bandwidth in order to maximize gain and minimize power loss. 
The data of Section 5.3.2 can be used with either the traditional magnicon design or the uniform-field magnicon, but it must be used carefully. All the off-axis data presented in Section 5.3.2 used a beam-entrance-velocity vector that was entirely in the radial direction. Usually this is not the case, and the entrance-velocity vector also has a $\phi$-directed component. This will change the results of Section 5.3.2. The best technique I have found for optimizing drift-space length is numerical and involves adjusting the length (and consequently the entrance position into the next cavity) in order to maximize the gain of the next passive cavity; it has substantial benefits for larger values of $\Omega / \omega$.

Section 5.4 and 5.5 provided insight for the design of a coupled deflection cavity to serve as the final cavity in the deflection system. These sections allowed for the determination of geometry parameters required to provide a specific angle gain and the prediction of dissipated power. The coupled-cavity performance was also compared to the performance of a simple cylindrical passive cavity.

As a final note, it should be stated that the conventional magnicon design generated in this fashion must be modified by large-signal modeling. This is due to the fact that Section 5.6 does not consider the effects of the half-cusp magnetic transition, which provides further conversion of longitudinal velocity into the transverse direction. Therefore, it is not necessary for the deflection system to provide all of the longitudinalenergy conversion.

\subsection{Comparison to Russian high-perveance magnicon design}

In February of 1992 the inventor of the magnicon, Oleg Nezhevenko, spent one month at the Stanford Linear Accelerator Center (SLAC) collaborating with representatives of Los Alamos National Laboratory and SLAC on a proposal for lowfrequency, high-efficiency magnicon to be used on the proposed SLAC "beauty factory" and two proposed Los Alamos projects: Accelerator Production of Tritium and Accelerator Transmutation of Waste. In this section, the large-signal, rigid-beam model is used to evaluate the specific architecture proposed by Nezhevenko for these projects. This magnicon architecture serves as a convenient starting point from which to examine a number of magnicon geometries.

Nezhevenko's drawing, shown in Fig. 6.1, was intended to be an aid to the reader of the proposals to focus attention on the geometry of a high-perveance device and to estimate the device's overall length. Before proceeding, however, it is important to realize that the magnicon architecture shown in the figure is based on Nezhevenko's 
experience with a low-perveance magnicon operating at $915 \mathrm{MHz}$ and small-signal approximate expressions. However, in spite of these constraints, the calculated efficiency using the rigid-beam model of his proposed design is very similar to his predictions. The only major discrepancy is the overall gain of the magnicon, which can be explained by the difference in assumptions in modeling the half-cusp magnetic field between the deflection and output cavities.

Figure 6.1 shows Nezhevenko's high-perveance magnicon architecture. The proposed magnicon consists of one drive cavity, two single cylindrical passive cavities, a coupled cavity, a magnetic-field-free drift space separating the deflection system and output cavity, and an output cavity. The parameters of the proposed magnicon are listed in Table 6.1. 


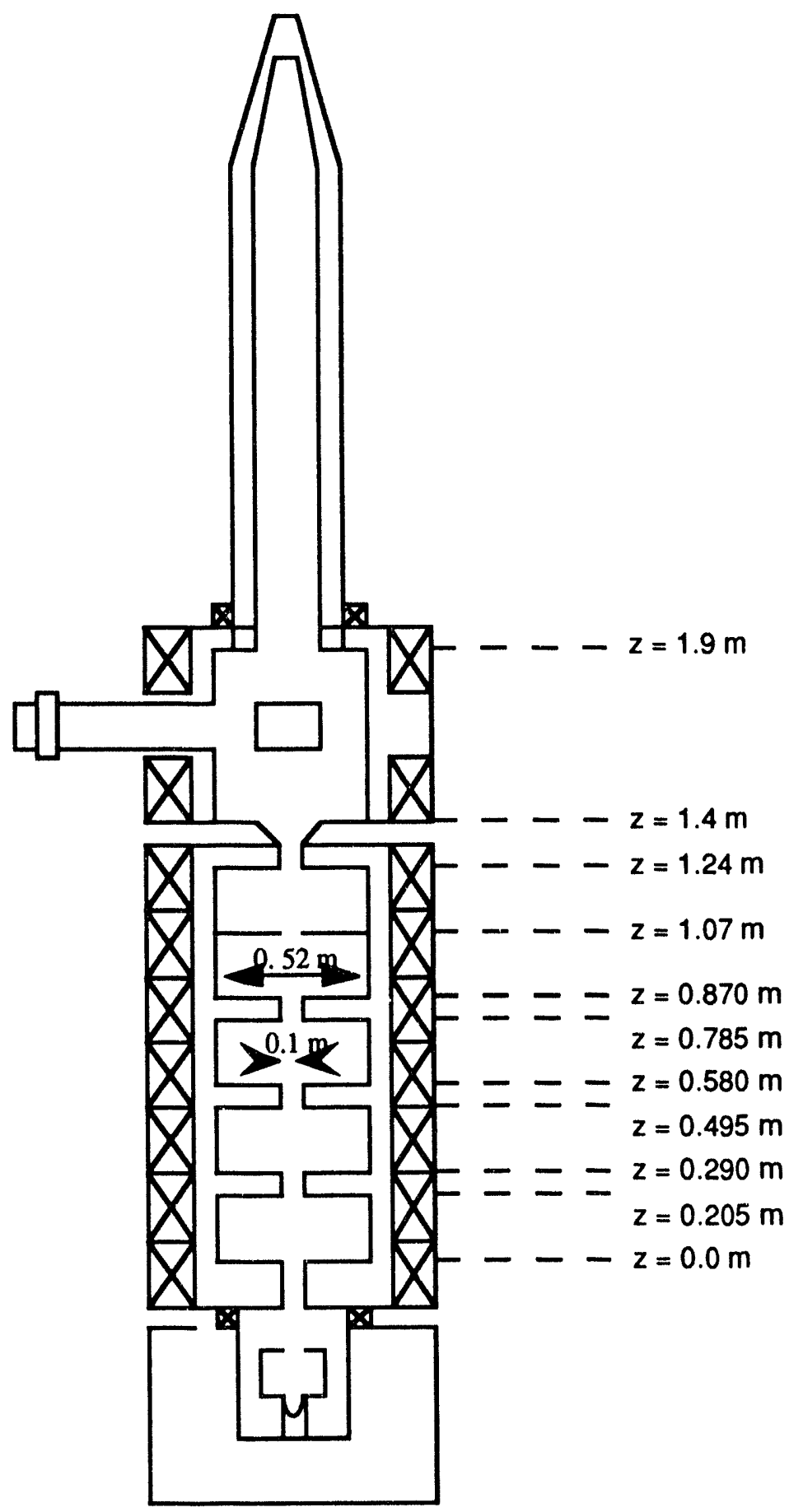

Figure 6.1. Nezhevenko's high-perveance magnicon architecture. 
Table 6.1. Proposed magnicon parameters.

\begin{tabular}{|l|c|}
\hline Beam Voltage (kV) & 170 \\
\hline Beam Current (A) & 18 \\
\hline Efficiency $(\%)$ & 82.5 \\
\hline Gain (dB) & 44.0 \\
\hline Output Cavity Field $(\mathrm{MV} / \mathrm{m})$ & 2.31 \\
\hline Deflection System $\Omega / \omega$ Ratio & 1.4 \\
\hline
\end{tabular}

I modeled a magnicon with these parameters and the geometry defined in Fig. 6.1 using the rigid-beam model. The approximation described in Section 3.3 was used to model the magnetic-field transition region between the deflection system and output cavity. This approximation is quite different fro' $n$ the pole-piece shape illustrated by Nezhevenko in Fig. 6.1 and accounts for some of the difference in gain between the predicted and calculated results. The results of the rigid-beam model are given in Table 6.2 .

Table 6.2. Calculated magnicon parameters.

\begin{tabular}{|l|c|}
\hline Beam Voltage (kV) & 170 \\
\hline Beam Current (Amps) & 18 \\
\hline Calculated Efficiency $(\%)$ & 81.1 \\
\hline Calculated Gain $(\mathbf{d B})$ & 25.0 \\
\hline Output Cavity Field $(\mathrm{MV} / \mathrm{m})$ & 1.6 \\
\hline Deflection System $\Omega / \omega$ Ratio & 1.4 \\
\hline
\end{tabular}

Figures 6.2 through 6.8 illustrate the particle trajectories and velocities of a single particle of the beam. Figures 6.2 and 6.3 illustrate the $x$ and $y$ position, respectively, as the particle moves along the longitudinal $z$ axis of the magnicon. Figure 6.4 is a scatter plot illustrating the top view of the particle trajectory over the transit time of the entire magnicon. Figures 6.5 and 6.6 illustrate the transverse velocity, $v_{x}$ and $v_{y}$, respectively, of a single particle as the particle moves along the longitudinal $z$ axis of the magnicon. Figure 6.7 shows the longitudinal velocity as the particle moves along the longitudinal axis of the magnicon. Figure 6.8 shows the variation of gamma as a function of the longitudinal position of the particle. 


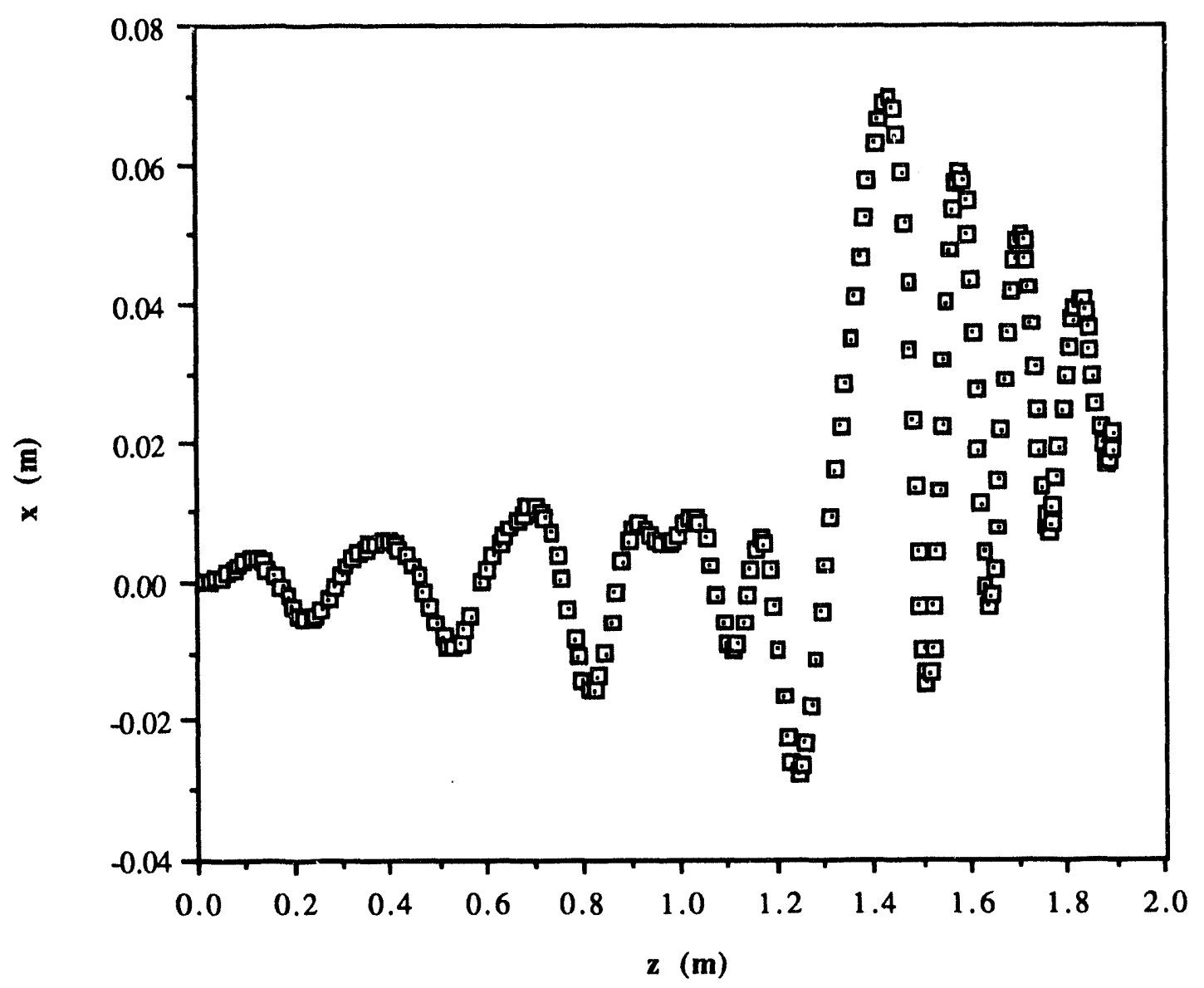

Figure 6.2. Transverse $x$-axis displacement versus longitudinal position. 


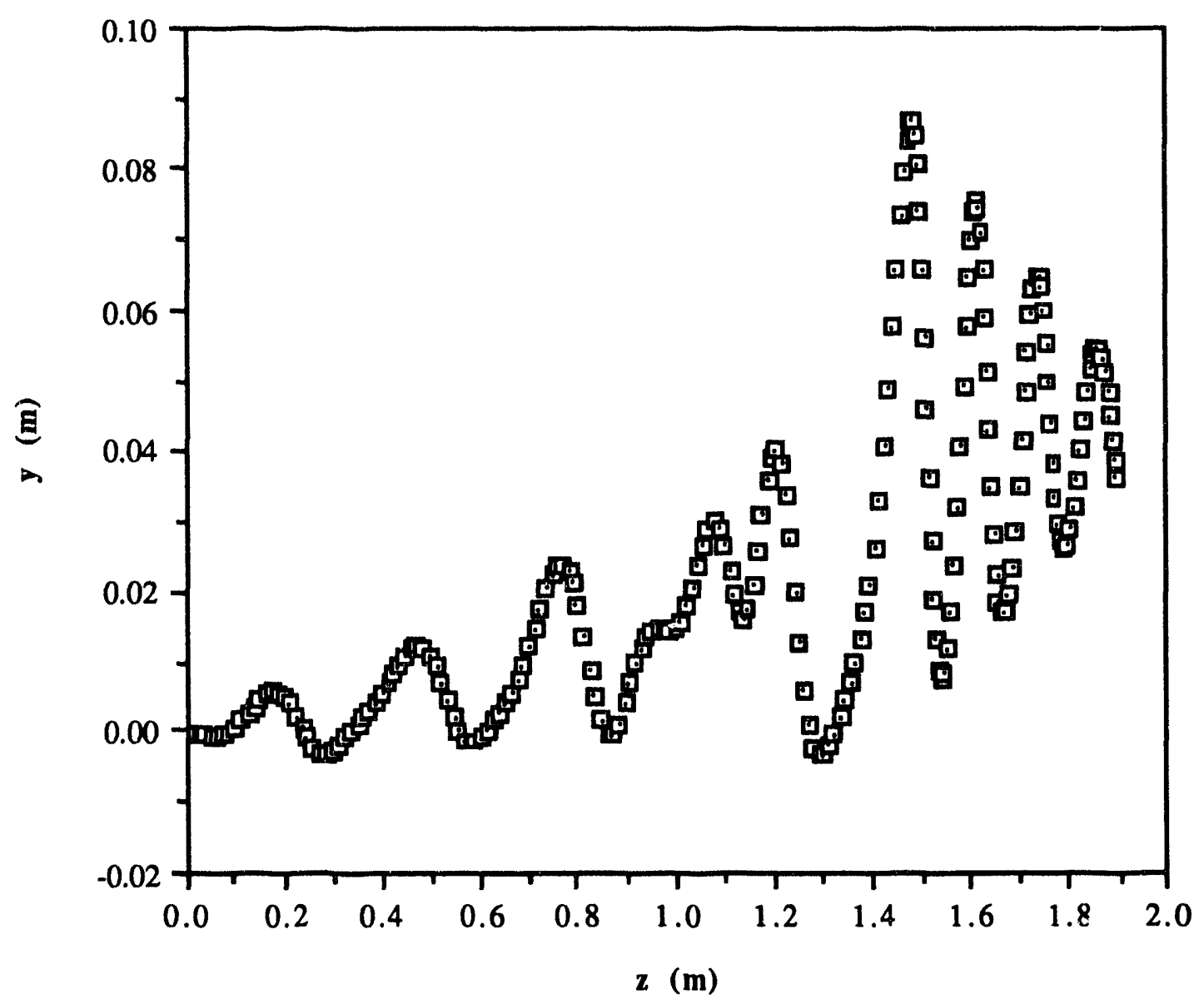

Figure 6.3. Transverse $y$-axis displacement versus longitudinal position. 


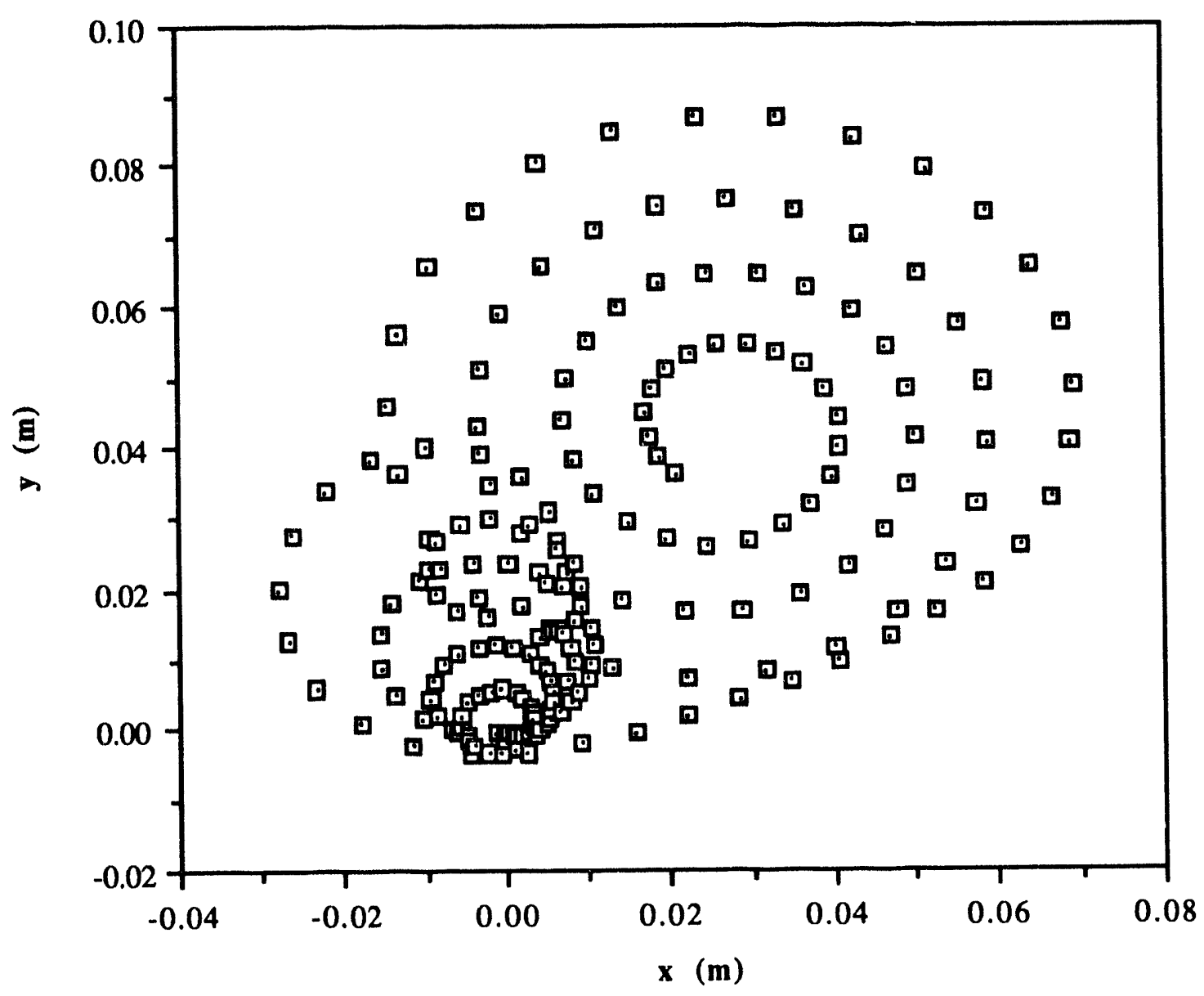

Figure 6.4. Scatter plot of transverse particle position. 


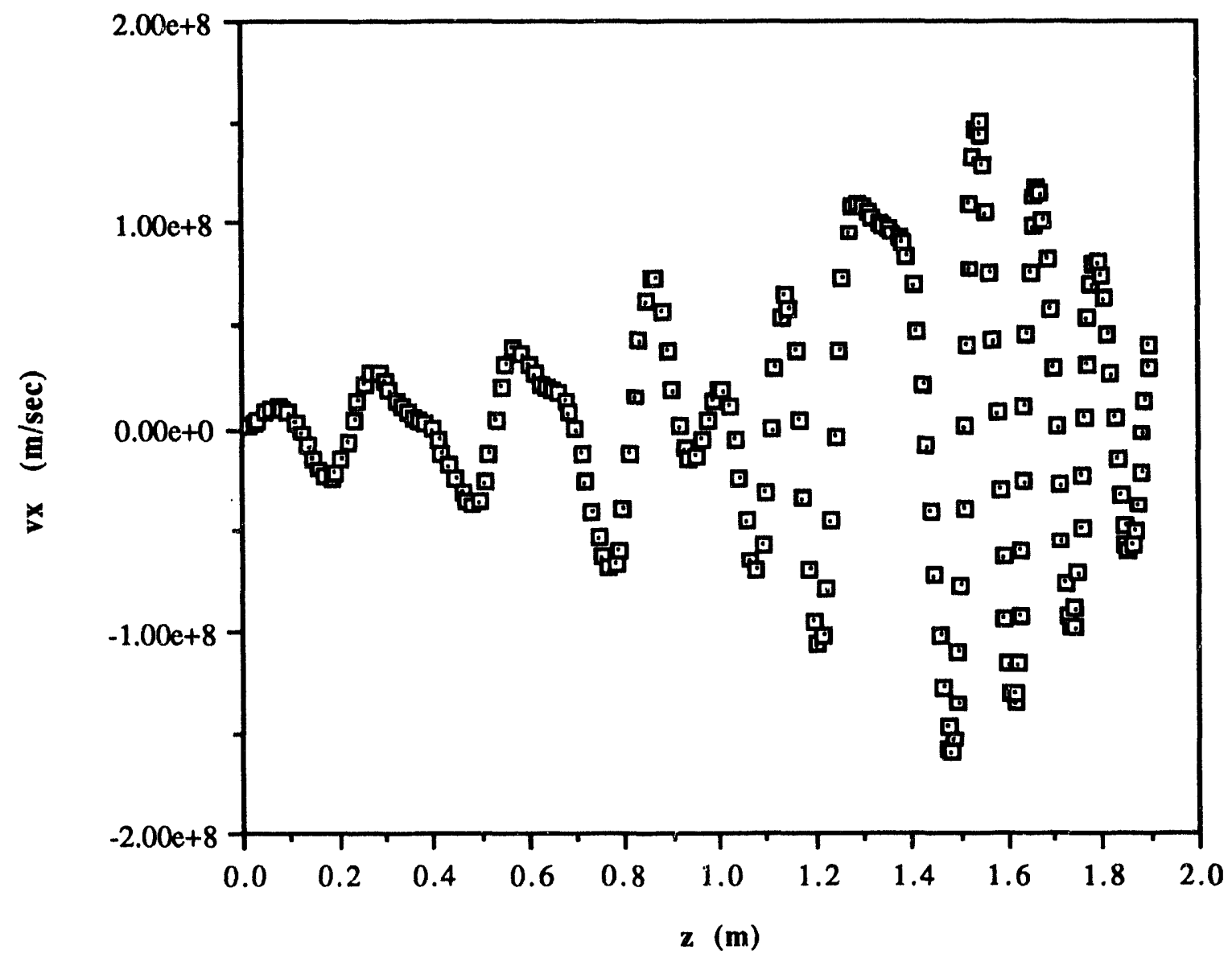

Figure 6.5. Transverse $x$-directed velocity versus longitudinal position. 

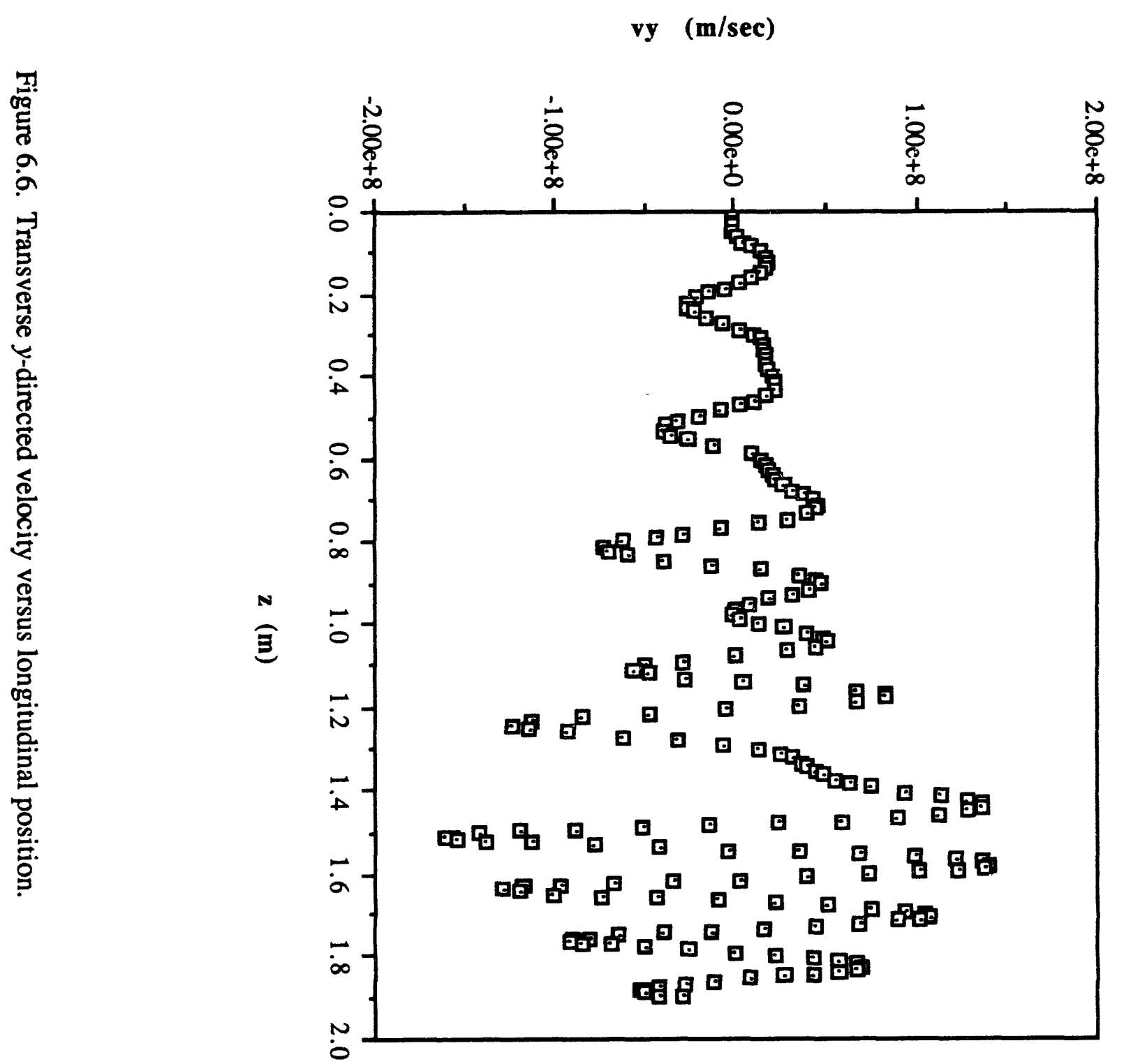


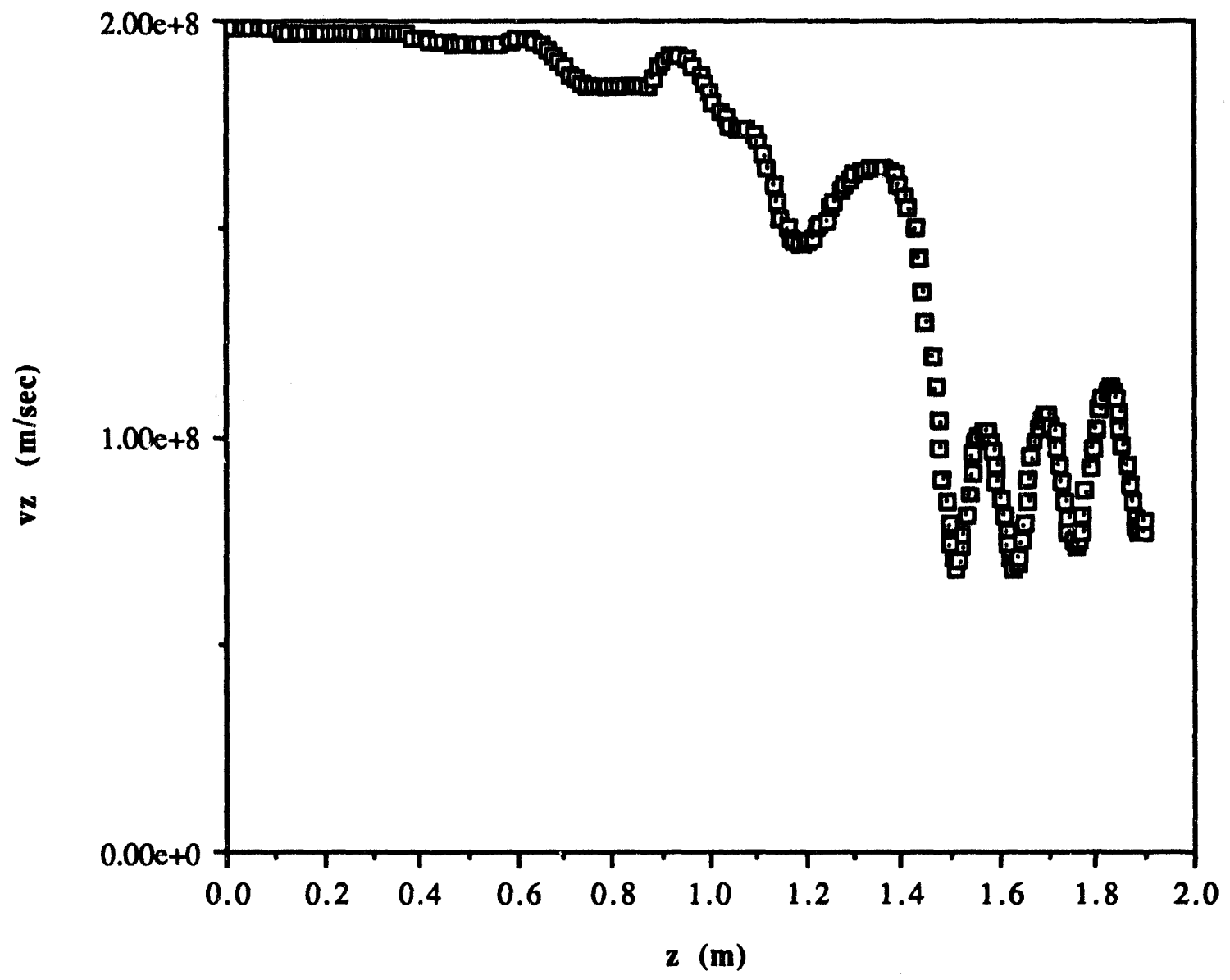

Figure 6.7. Longitudinal $z$-directed velocity versus longitudinal position. 


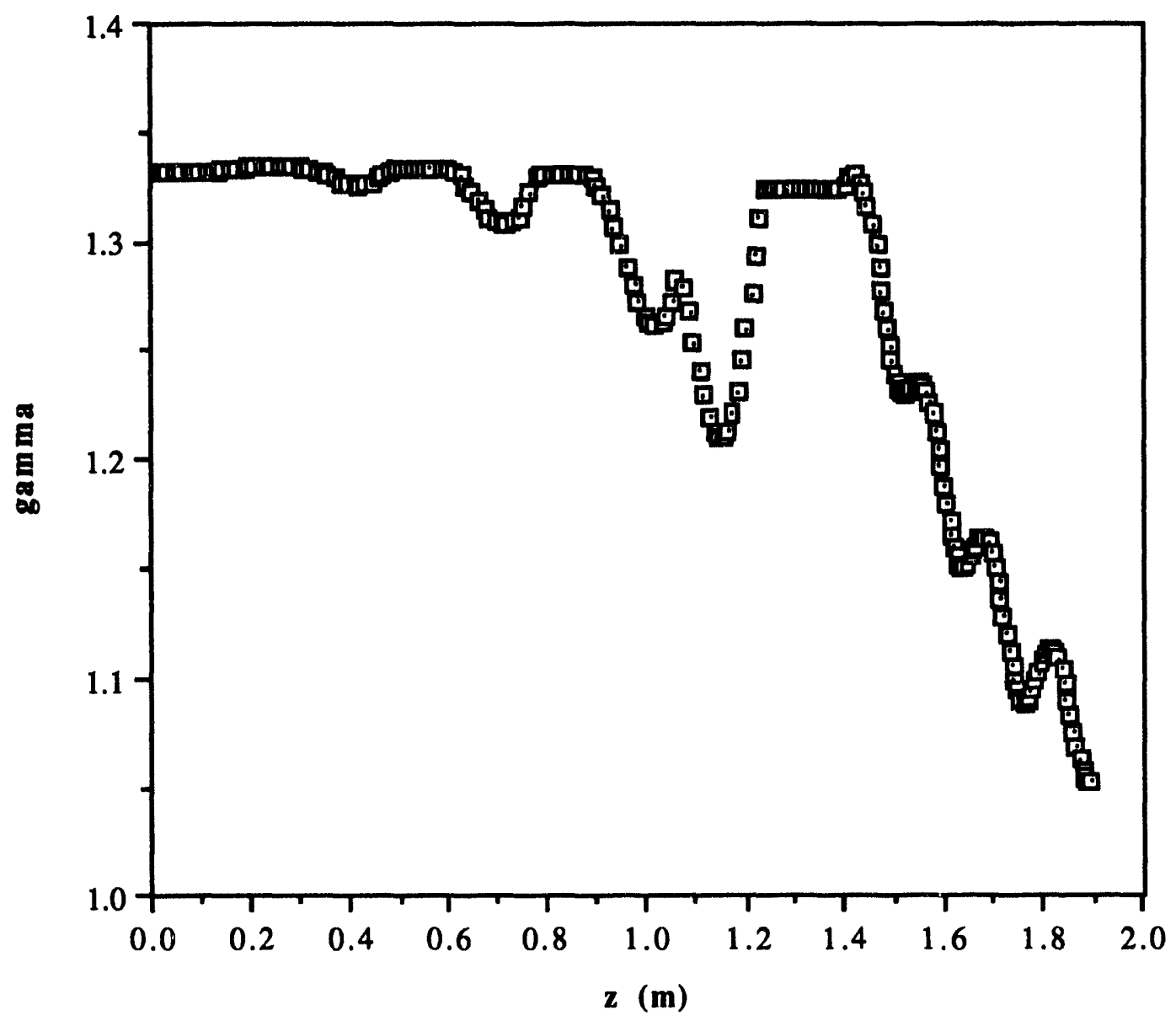

Figure 6.8. Particle gamma versus longitudinal position.

Figures 6.2 and 6.3 illustrate the transverse position of a particle as it moves along the longitudinal axis of the magnicon. When used in conjunction with Fig. 6.1, this information makes it possible to extract particle behavior in each of the magnicon structures (i.e., drive cavity, passive cavity, drift space, etc.). These figures show that the particle is displaced off-axis in the deflection system and then gains off-axis displacement as it moves through the half-cusp field at the input to the output cavity. The displacement off-axis then decreases as the particle gives up its transverse energy to the rf field. Figure 6.4 shows a scatter plot of the particle position over the transit time for the magnicon. Two distinct regions of circular motion are observed. The one starting from the origin 
shows the particle position in the deflection system, while the upper-right-hand corner of the plot shows the particle position as the energy is extracted in the output cavity. Figures 6.5 and 6.6 illustrate the transverse velocity of the particle as it moves along the axis. Note that the particle gains transverse velocity in the input structure and then loses this transverse velocity to the rf fields in the output cavity. Figure 6.7 shows the zdirected velocity component as the particle moves through the magnicon. Note that the $z$ directed velocity decreases in the deflection system as it is transferred to transverse velocity and that the bulk of velocity transfer actually occurs in the half-cusp field between the deflection system and output cavity. Thus, the difference between the theoretical model used here and Nezhevenko's experimental experience could account for the difference in gain. Also, the velocity is approximately constant in the output cavity, with a slight upward trend.

This constant $z$-directed velocity makes the magnicon a prime candidate for depressed-collector technology, which would further increase the efficiency of the device. Figure 6.8 illustrates the particle gamma as a function of longitudinal device position. As the illustration shows, after a slight increase in gamma in the drive cavity, the particle gamma decreases as the beam excites the passive cavities. Regions of constant gamma represent the drift spaces separating the cavities. The extended nature of the output interaction can be observed by noting the sinusoidal variation of gamma in the output cavity, which results in a net decrease in energy as the particle gives up its energy to the rf fields.

\subsection{Comparison with experimental results}

The NRL magnicon development has progressed to an experimental phase. Their engineers have fabricated and tested the first two cavities of the deflection system: the drive cavity and the first passive cavity. A 3-mm-diameter beam at $500 \mathrm{kV}$ and approximately $170 \mathrm{~A}$ has been deflected by the drive cavity and has excited a field in the first passive cavity. The beam-excited field of the passive cavity was measured, and the gain calculated by comparison to the drive cavity field. The NRL experimental data was provided in Ref. [1].

The architecture of the NRL magnicon is shown in Fig. 6.9, and the parameters are listed in Table 6.3. In Fig. 6.9 two types of protrusions are made into the cavities. The sample pins have a very small coupling coefficient and are used to measure the coldcavity $\mathrm{Q}$. The coupling pins have a larger coupling coefficient and are used to excite the 
drive cavity, sample the beam-excited fields in the passive cavity, and lower the passivecavity $\mathrm{Q}$.
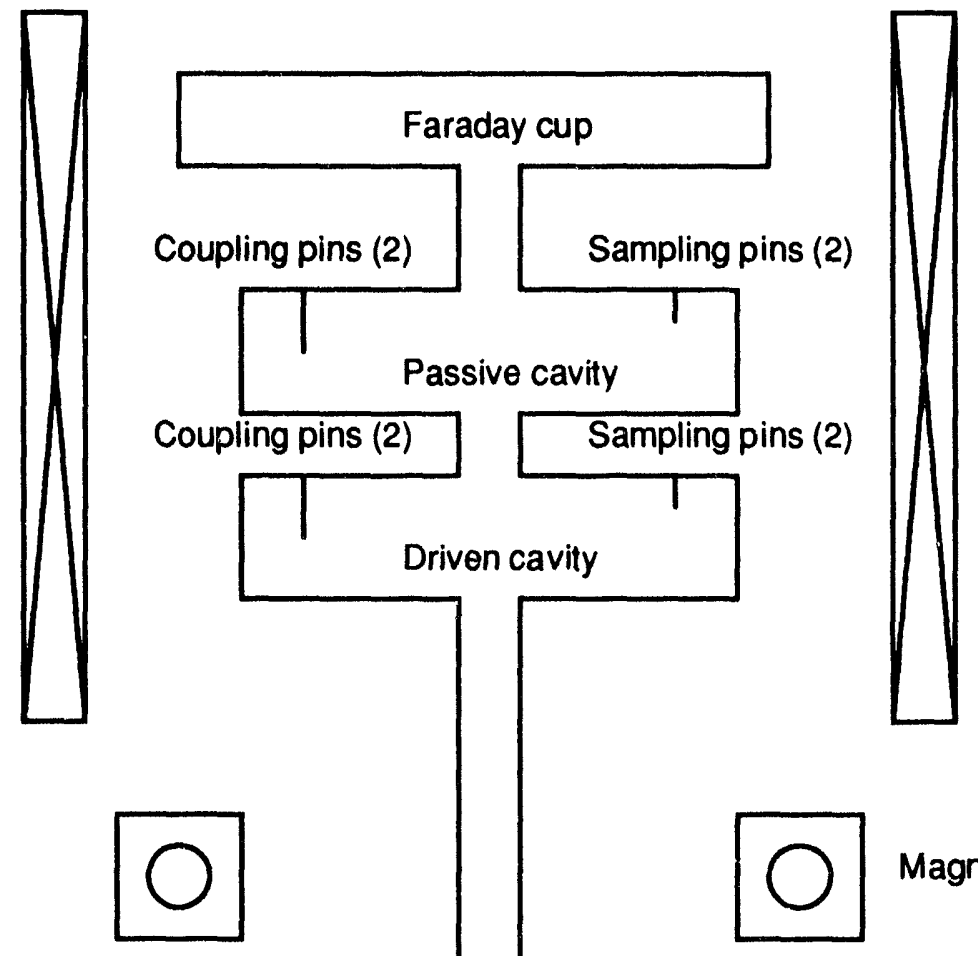

Magnet winding

Magnet winding

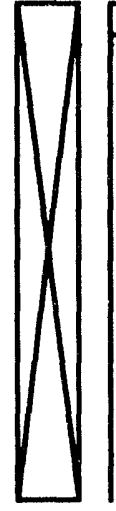

Graphite anode

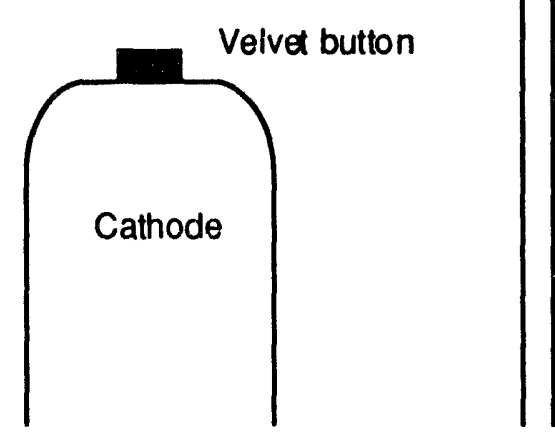

Magnet winding

Figure 6.9. NRL magnicon experimental magnicon. 
Table 6.3. NRL magnicon parameters.

\begin{tabular}{|l|c|}
\hline Parameter & Value \\
\hline Voltage & $500 \mathrm{kV}$ \\
\hline Beam Current & $\sim 170 \mathrm{~A}$ \\
\hline Cavity Radius & $3.2 \mathrm{~cm}$ \\
\hline Drive and Passive Cavity Length & $2.26 \mathrm{~cm}$ \\
\hline Cavity Quality Factor & 1000 \\
\hline Drift Space Length & $1.13 \mathrm{~cm}$ \\
\hline Input Power & $78 \mathrm{~W}$ \\
\hline Drive Frequency & $5.68 \mathrm{GHz}$ \\
\hline Magnetic Field & $0.81 \mathrm{~T}$ \\
\hline Drive Cavity Field & $0.08 \mathrm{MV} / \mathrm{m}$ \\
\hline Effective Resistivity & $0.2981 \Omega$ \\
\hline Output Cavity Field & $0.3838 \mathrm{MV} / \mathrm{m}$ \\
\hline Gain $=20$ log (E & $13.6 \mathrm{~dB}$ \\
\hline
\end{tabular}

Some of the parameters in Table 6.3-drive frequency, drive power, and effective resistivity-require some additional explanation. The drive frequency in the experimental NRL work varied from $5.67 \mathrm{GHz}$ to $5.69 \mathrm{GHz}$ based on the measured coldcavity resonance. The desired frequency was $5.712 \mathrm{GHz}$. The NRL limited the drive power for the experimental verification to $10-400 \mathrm{~W}$. I chose a comparison with a drivepower level of $78.3 \mathrm{~W}$. (Both my theoretical comparison and NRL's experimental results are in the small-signal regime.) Finally, the comparison with the NRL results required that I use an effective resistivity. The NRL presented for the drive cavity a cold-cavity $Q$, a coupled-cavity $Q$, and a total $Q$. The cold-cavity $Q$ was very high $(>90,000)$, while the coupled-cavity $\mathrm{Q}$ lowered the overall $\mathrm{Q}$ to around 1100 for the drive cavity and to around 1000 for the passive cavity. Couple-cavity $Q$ in this context refers to the effect on the $Q$ of the coupling pin in Fig. 6.9. Power dissipated in the coupling ports drastically lowered the overall $Q$.

To simulate this effect, I distributed this additional power dissipation over the entire surface of the cavity by increasing the cavity shunt resistance. I did this so I could 
model the effect of power being dissipated in the coupled ports when I calculated the selfconsistent passive-cavity fields using the energy-balance approach.

The rigid-beam model predicts a cavity gain of $13.6 \mathrm{~dB}$. This result agrees favorably with both the theoretical and experimental NRL results. The theoretical NRL results predict a cavity gain of $13.5 \mathrm{~dB}$. The NRL separately measured and calculated the two standing waves that make up the rotating field in the passive cavity and computed the gains for the two standing waves separately. One of the standing-wave polarizations provided a gain of approximately $12 \mathrm{~dB}$ while the other standing-wave polarization provided a gain of approximately $15 \mathrm{~dB}$. The NRL attributes the imbalance in the two standing-wave modes in part to calibration error and possibly an asymmetry in the cavity excitation (elliptical polarization), which is potentially caused by a small misalignment of the electron beam or an asymmetry in the mode of the drive cavity.

\subsection{New design for a standard magnicon}

In the following sections alternate magnicon geometries will be analyzed using the analytical half-cusp-field model and fields calculated by the code POISSON (Ref. [2]). The geometries for these models will be supported by the simulation data of previous sections and will be contrasted with the inventor's model, which was described above.

The magnicon architecture shown below in Fig. 6.10 builds on the geometry defined in Section 6.2 to increase the efficiency and increase the gain. The fundamental structure is the same except for the following changes:

1. The drive-cavity length has been increased to the optimum size as calculated in Section 5.2.

2. The passive-cavity and coupled-cavity lengths were shortened to $0.175 \mathrm{~m}$, or 0.4086 wavelengths.

3. An additional passive cavity was added.

4. The drift-space distance was optimized between each passive cavity to maximize the gain of the passive cavity following the drift space. 
5. The outfut-cavity length and field were optimized to extract the most rf energy from the beam in the output cavity.

Each of these changes is explored below in more detail.

The drive cavity length was increased from $0.205 \mathrm{~m}$ in Nezhevenko's design to $0.35 \mathrm{~m}$, which is the optimum length predicted in Section 5.2. The passive-cavity length and coupled-cavity lengths were shortened, thus increasing the gain of these cavities and increasing the beam-loaded $Q$. However, Section 5.3.3 predicts for a passive-cavity length of $0.175 \mathrm{~m}$ an instantaneous bandwidth of $0.3 \mathrm{MHz}$, which is suitable for accelerator application. The addition of the passive cavity helped increase the overall gain of this magnicon geometry. The large-signal numerical simulation was used to adjust the drift-space distances in order to maximize the gain of the following cavity by changing the beam's entrance position into the cavity. A localized parameter search was conducted to determine the values of cavity field and length that maximized efficiency. As a result of these changes, the efficiency was increased from $81 \%$ to $88 \%$.

The operating parameters of the magnicon geometry of Fig. 6.10 are included in Table 6.4. 


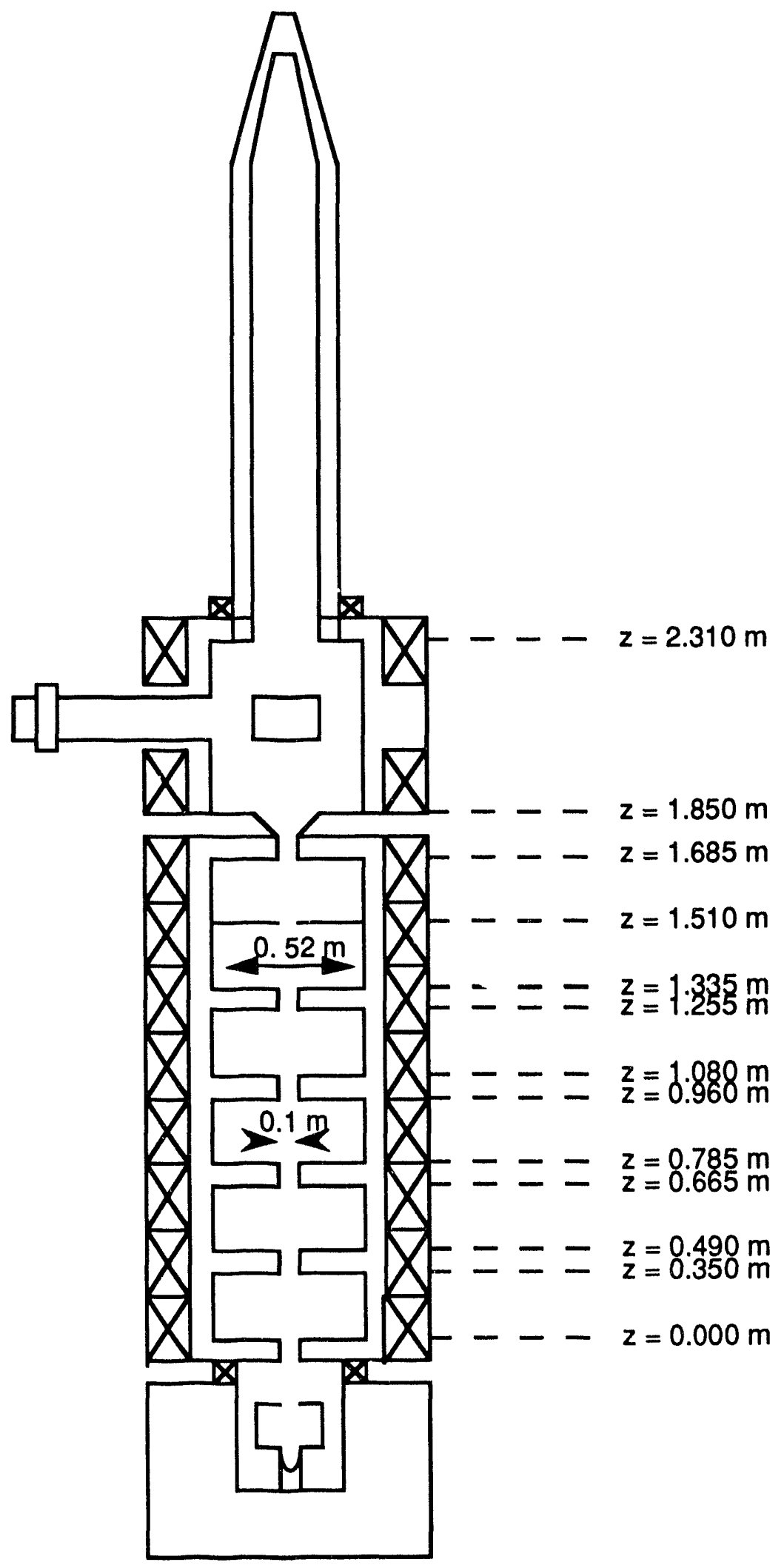

Figure 6.10. Proposed alternate magnicon geometry. (Note: picture is not to scale.) 
Table 6.4. Alternate geometry operating conditions.

\begin{tabular}{|l|c|}
\hline Operating Parameter & Value \\
\hline Beam Voltage & $170 \mathrm{kV}$ \\
\hline Beam Current & $18 \mathrm{~A}$ \\
\hline Gain & $41 \mathrm{~dB}$ \\
\hline Efficiency & $88 \%$ \\
\hline Output Cavity Field & $2.05 \mathrm{MV} / \mathrm{m}$ \\
\hline Deflection System $\Omega / \omega$ Ratio & 1.4 \\
\hline Output Cavity Magnetic Field & $0.0292 \mathrm{~T}$ \\
\hline Initial Particle Gamma & 1.33219 \\
\hline Output Power & $2.688 \mathrm{MW}$ \\
\hline
\end{tabular}

Figures 6.11 through 6.17 illustrate the particle trajectories and velocity conversion as a single particle of the beam moves through the magnicon. Figures 6.11 and 6.12 show the particles positioned off the longitudinal axis as a function of the longitudinal position. Figure 6.13 shows a transverse plot of the particle position during transit of the particle. Figures $6.14,6.15$, and 6.16 show the longitudinal and transverse velocity components as a function of longitudinal position. Figure 6.17 shows the particle gamma as a function of longitudinal position. 


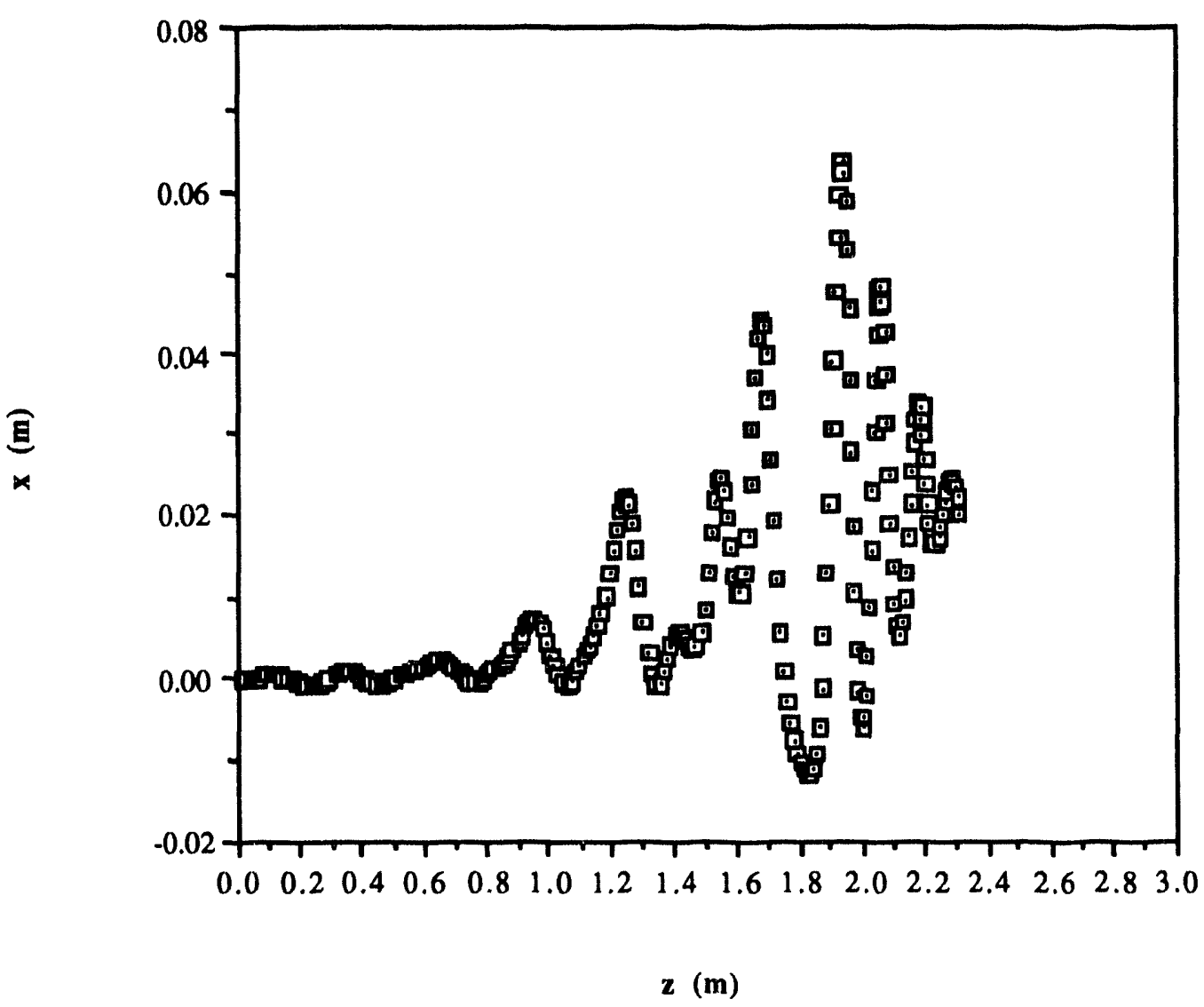

Figure 6.11. Particle $x$-axis displacement as a function of longitutinal position. 


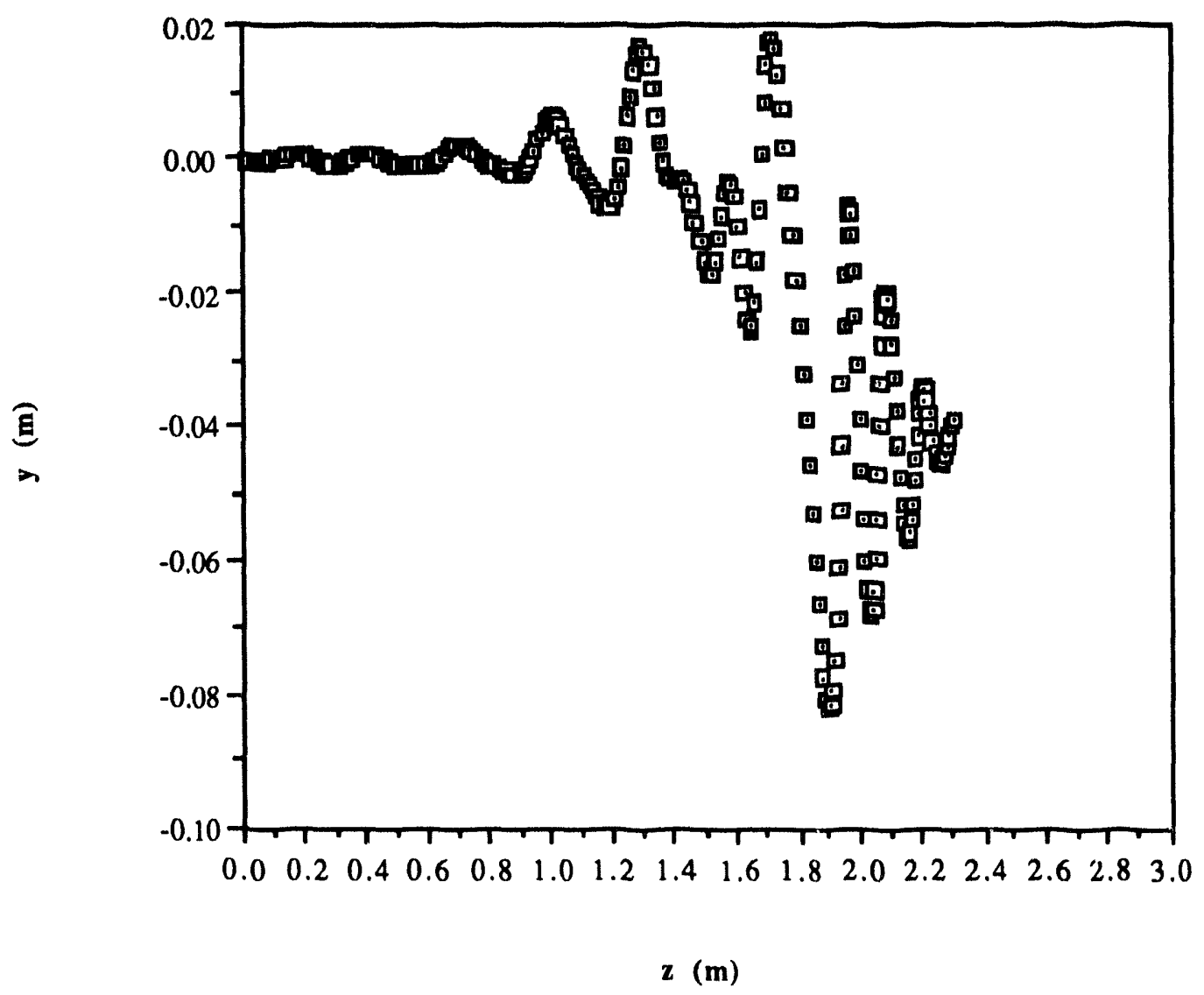

Figure 6.12. Particle $y$-axis displacement as a function of longitudinal position. 


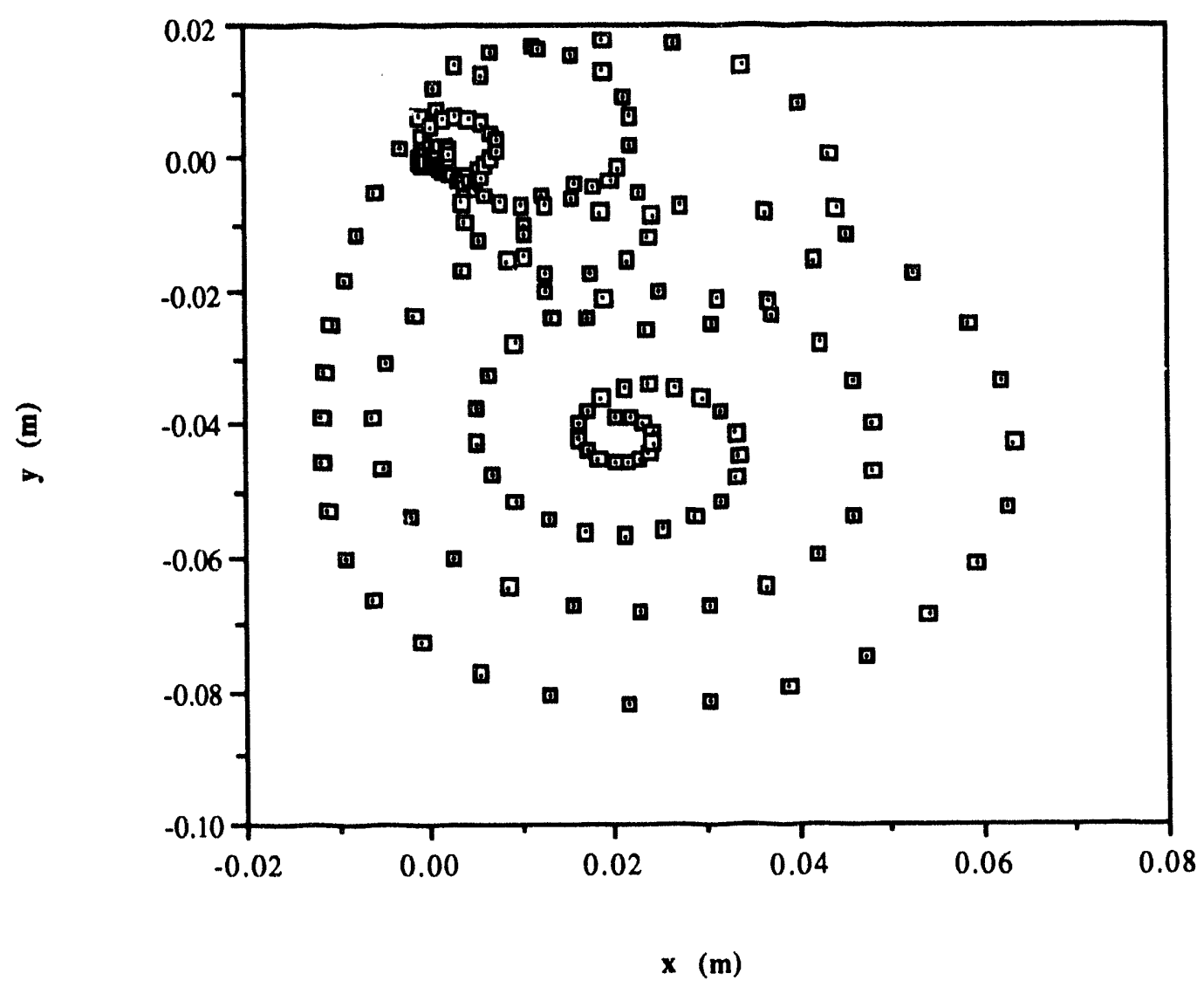

Figure 6.13. Scatter plot of particle transverse displacement during transit of magnicon. 


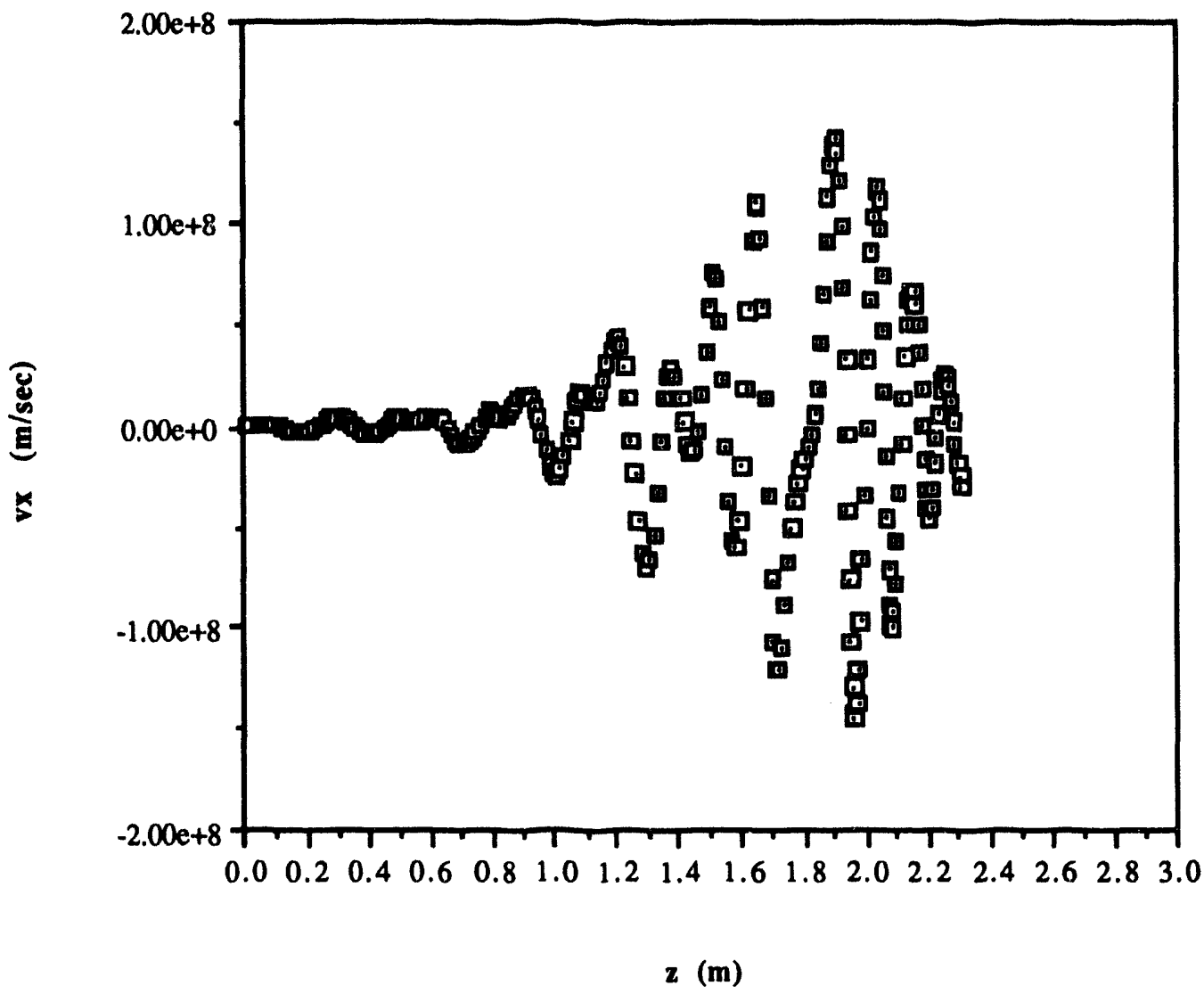

Figure 6.14. Particle $x$-directed velocity as a function of longitudinal position. 


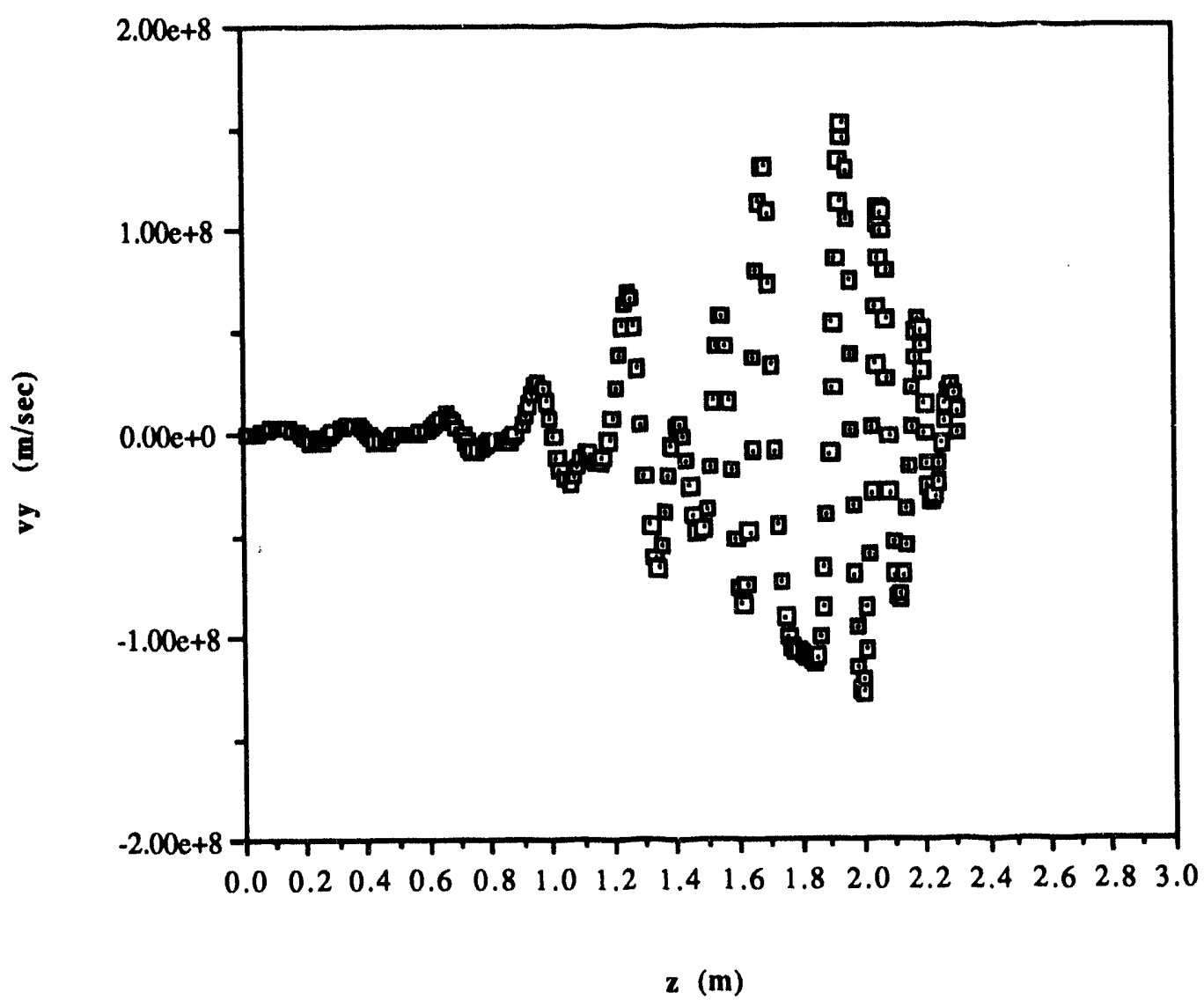

Figure 6.15. Particle $y$-directed velocity as a function of longitudinal position. 


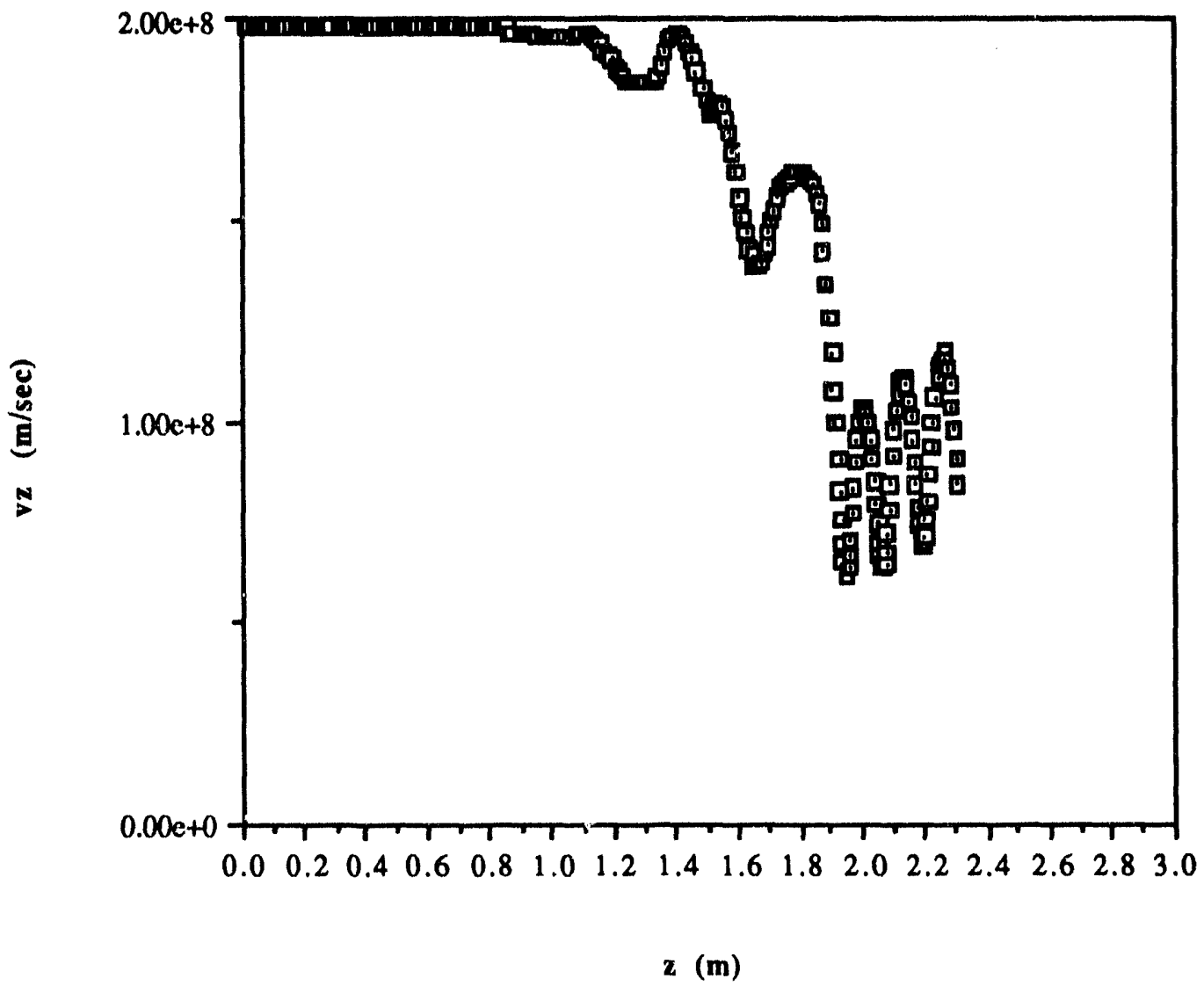

Figure 6.16. Particle $z$-directed velocity as a function of longitudinal position. 


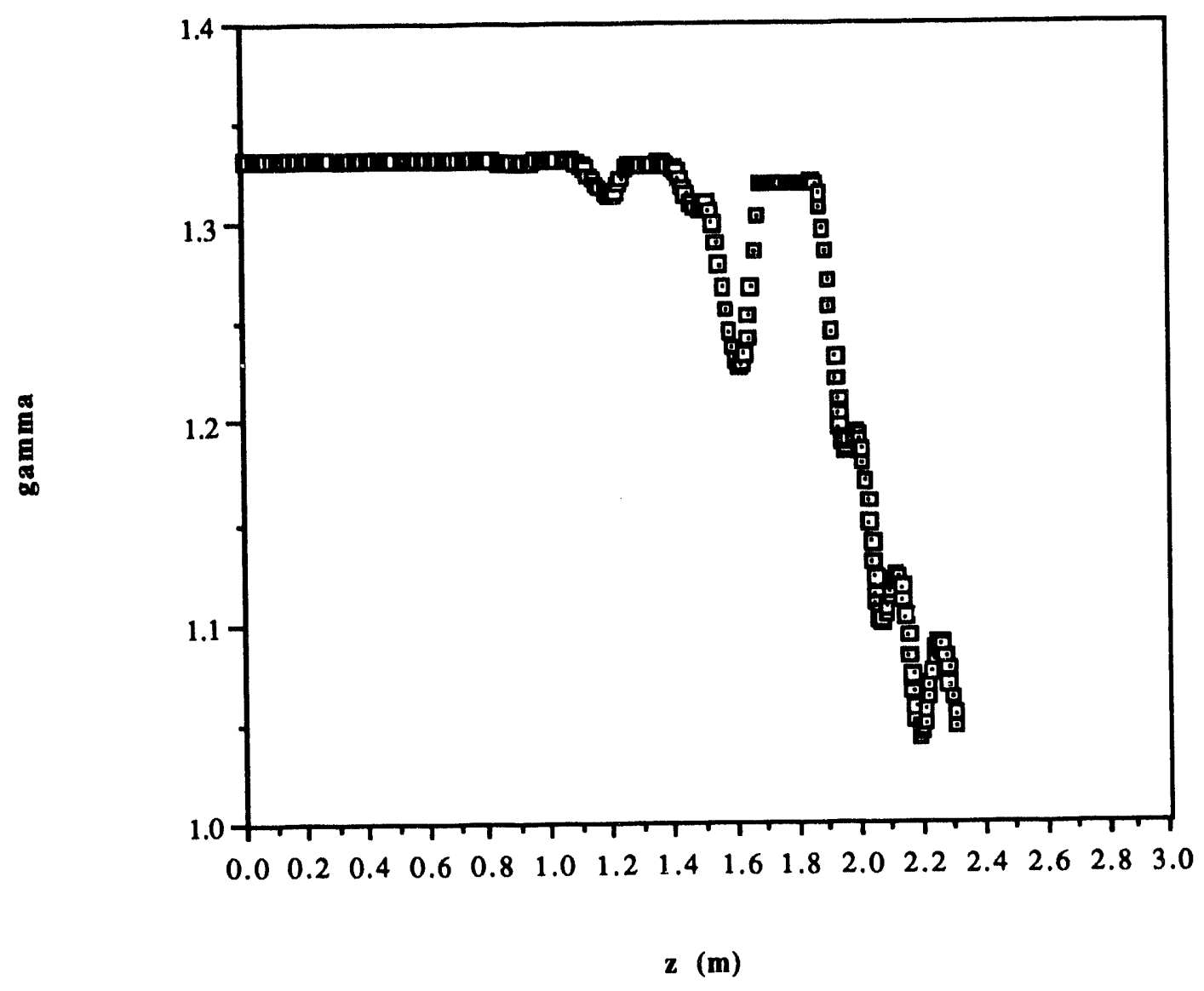

Figure 6.17. Particle gamma as a function of longitudinal position.

The comments presented in Section 6.2. on the trajectories of the proposed Russian geometry also apply to this geometry. Even though this geometry does demonstrate very high efficiency in the rigid-beam approximation, I do not believe that either this geometry or the proposed Russian geometry is optimum. This belief is based on the fact that during the interaction with the sharp magnetic-field transitions, a finitesized beam will suffer significantly from energy spread, and the energy spread translates into efficiency loss. Also, the drift space separating the deflection and output cavities would result in beam expansion due to space-charge forces. Again, this would have a negative impact on efficiency. These assertions will be demonstrated in subsequent sections using a finite-beam-size modeling technique. The alternate geometry presented 
above will also be evaluated by applying transition-region fields generated from the numerical code POISSON, which is given in the next section.

\subsubsection{Geometry with POISSON half-cusp fields.}

The magnicon of Fig. 6.10 will be analyzed using a variation of the method described in the previous section. Because the magnetic fields in the transition regions have a significant impact on magnicon performance, the approximate, analytical method of modeling the half-cusp region of Section 6.4 will be replaced by a numerical technique. This technique uses the code POISSON, which is cited in Ref. [2], to calculate the magnetic fields in the transition region between the deflection system and output cavity. A pictorial representation of the POISSON geometry and fields is illustrated in Fig. 6.18. 


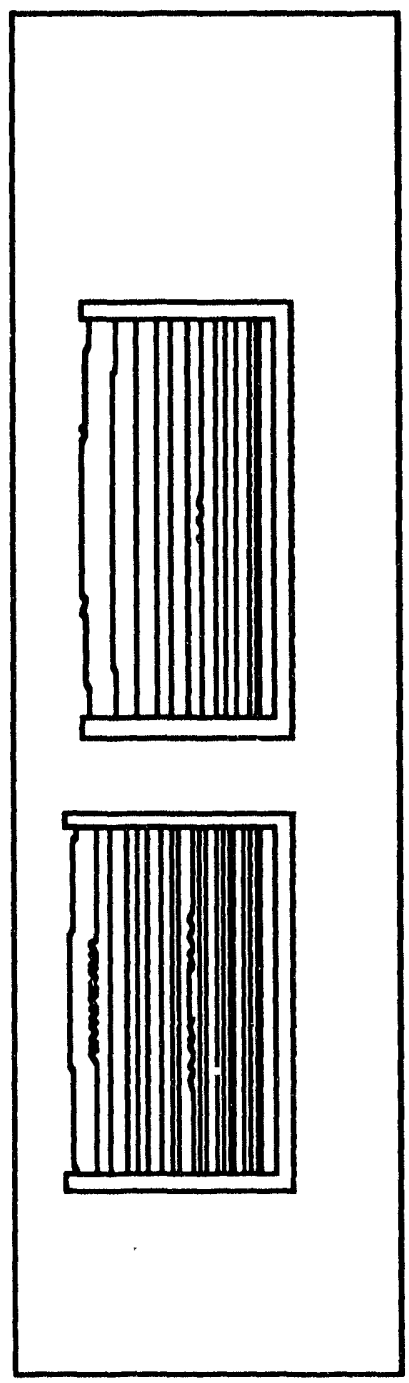

Figure 6.18. Plot of geometry and POISSON calculated fields.

The POISSON problem made two assumptions: that the pole piece was made of infinite-permeability iron and that the actual lengths of the two pole-piece assemblies were shorter than that required by the cavity length. (Note that the reduced length does not compromise the results, because the POISSON fields are applied only in the region of the magnetic-field transitions.) POISSON is used to generate a table of radial and longitudinal magnetic-field values in the region between the coupled cavity and a point that is two entrance-hole radii into the output cavity. Bilinear interpolation described in Ref. [3] is used to interpolate between the magnetic-field values in the radial-coordinatebased POISSON table because the problem is azimuthaily symmetrical. 
Table 6.5 contains the operating parameters for the magnicon. Note that the results of Table 6.5 are very close to the results of Table 6.4 in the previous section. This fact tends to validate both approaches. Figures 6.19 through 6.25 are trajectory plots that are identical to the trajectory plots from the previous section.

Table 6.5. Operating parameters of magnicon.

\begin{tabular}{|l|c|}
\hline Operating Parameter & Value \\
\hline Beam Voltage & $170 \mathrm{kV}$ \\
\hline Beam Current & $18 \mathrm{~A}$ \\
\hline Gain & $41 \mathrm{~dB}$ \\
\hline Efficiency & $86.2 \%$ \\
\hline Output-Cavity Field & $2.05 \mathrm{MV} / \mathrm{m}$ \\
\hline Initial Particle Gamma & 1.33219 \\
\hline Deflection-System $\Omega / \omega$ Ratio & 1.4 \\
\hline Output-Cavity Magnetic Field & $0.0292 \mathrm{~T}$ \\
\hline Output Power & $2.64 \mathrm{MW}$ \\
\hline
\end{tabular}




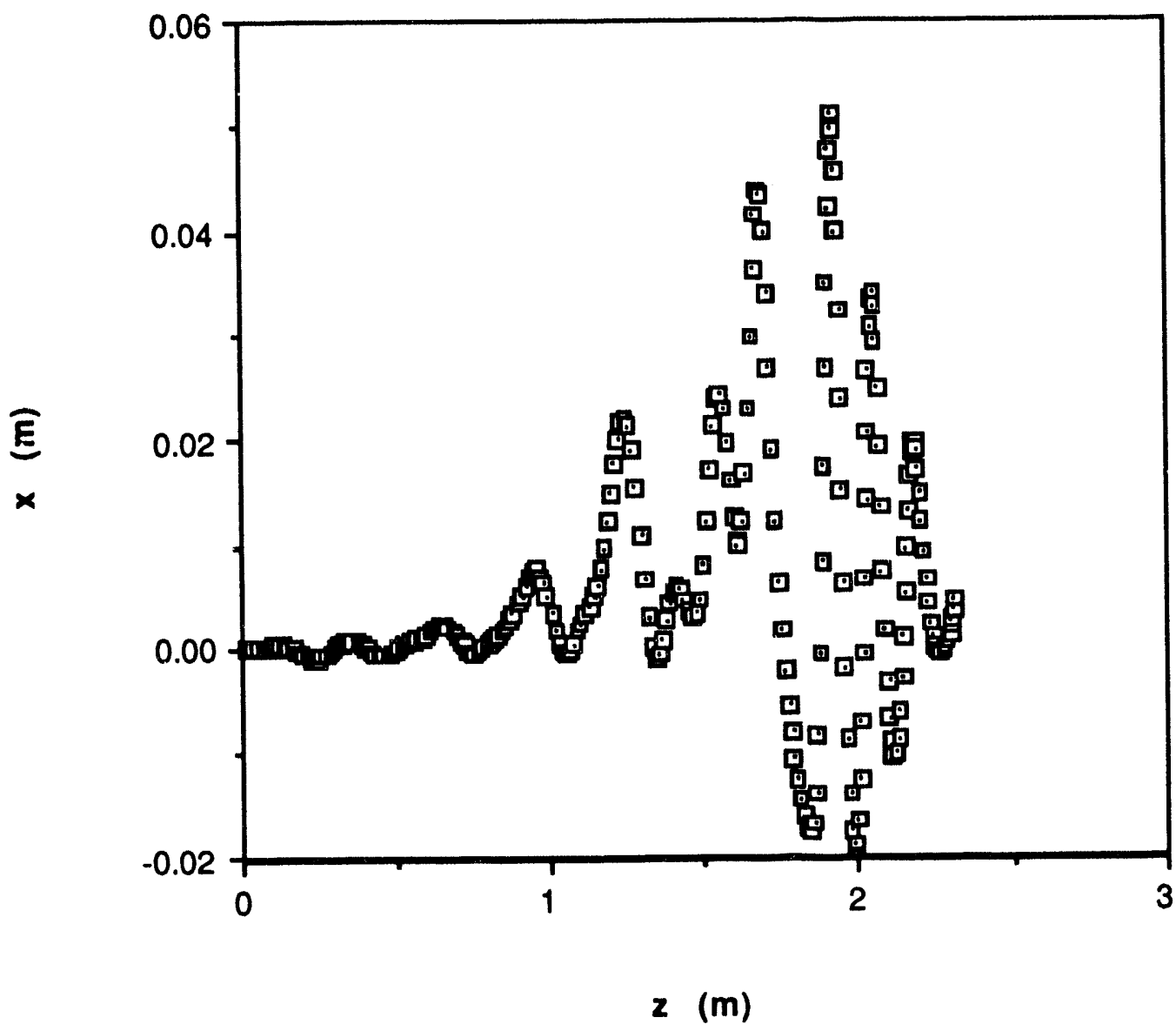

Figure 6.19. Particle $x$-axis displacement as a function of longitudinal position. 


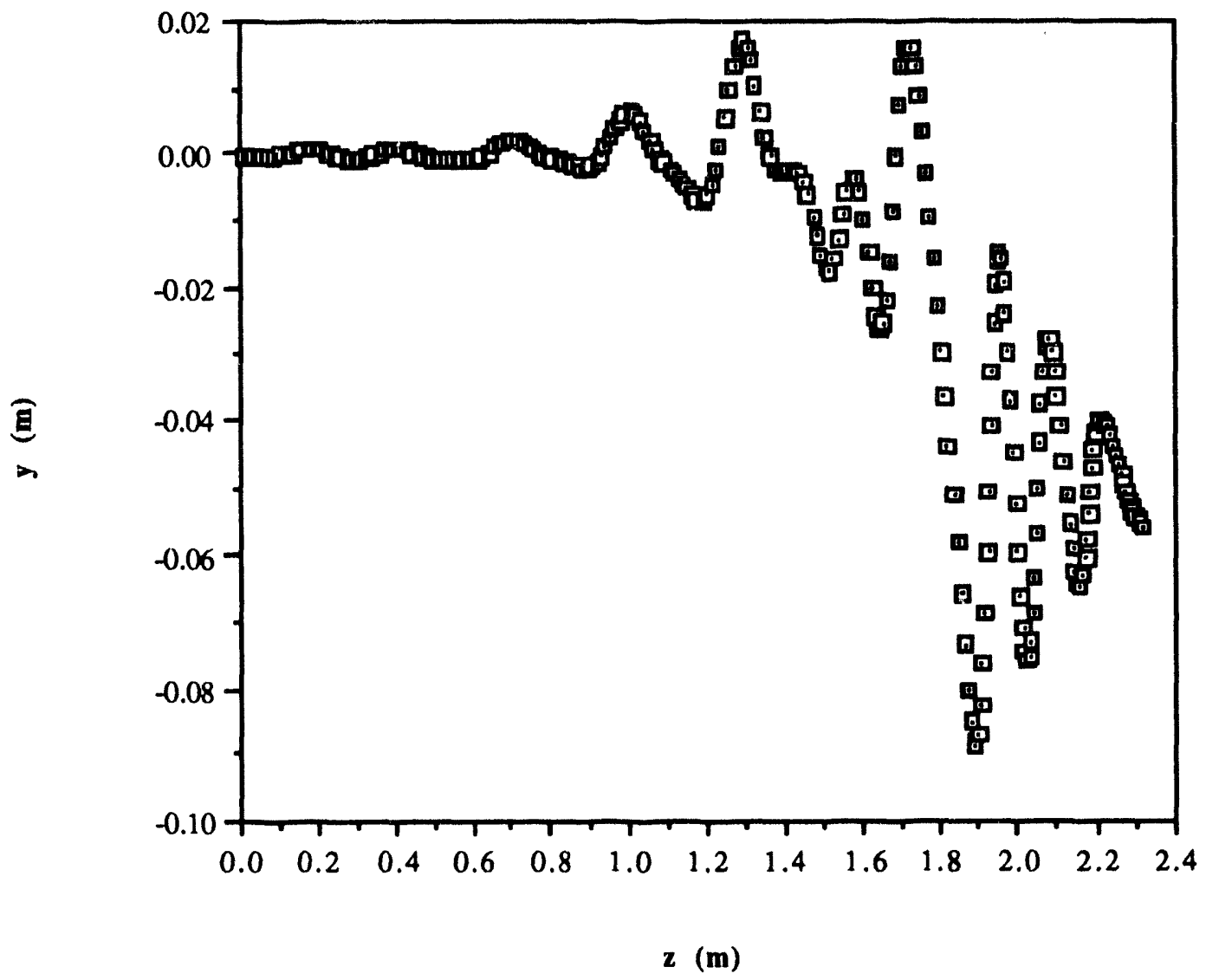

Figure 6.20. Particle $y$-axis displacement as a function of longitudinal position. 


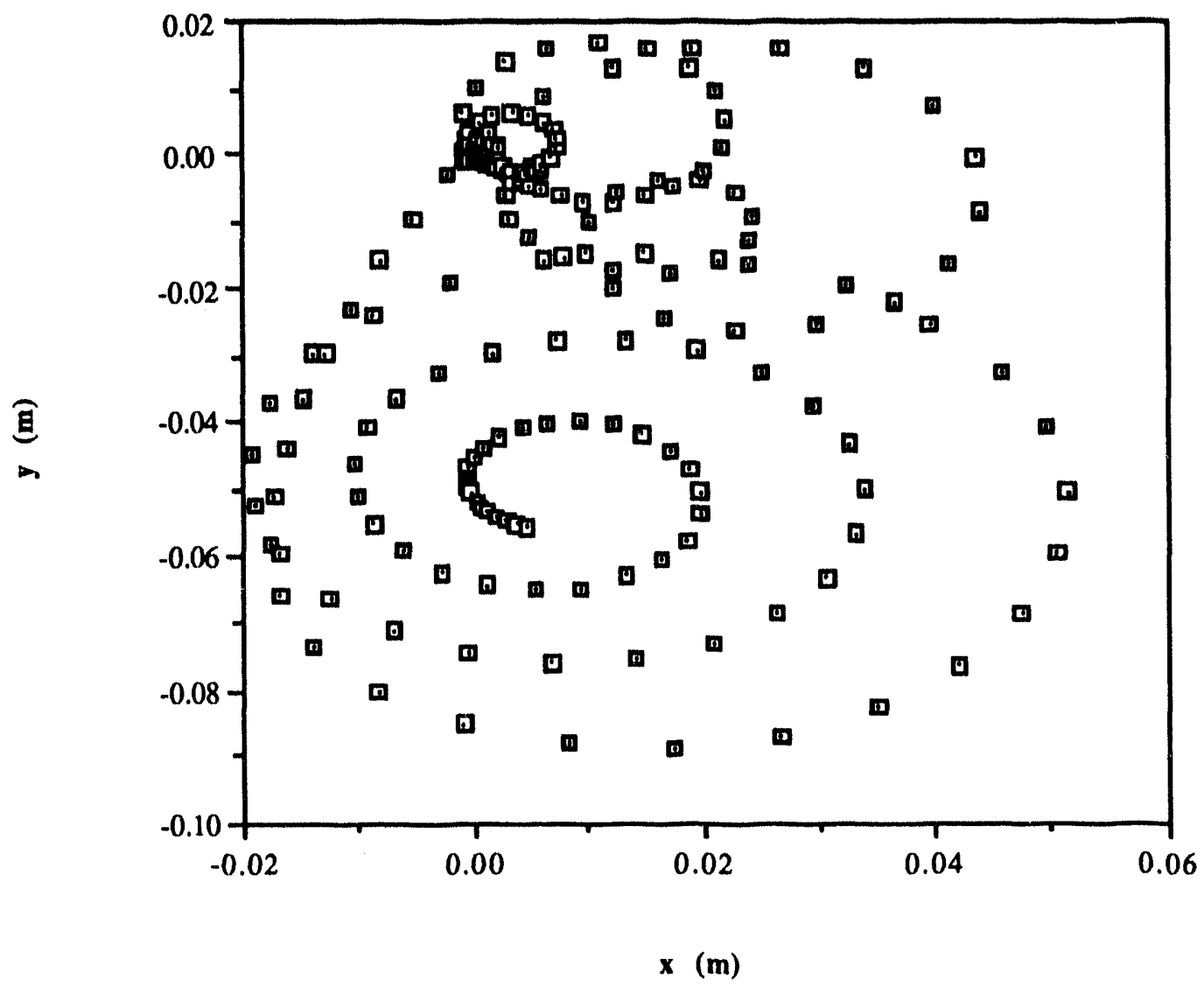

Figure 6.21. Scatter plot of particle transverse displacement during transit of the magnicon. 


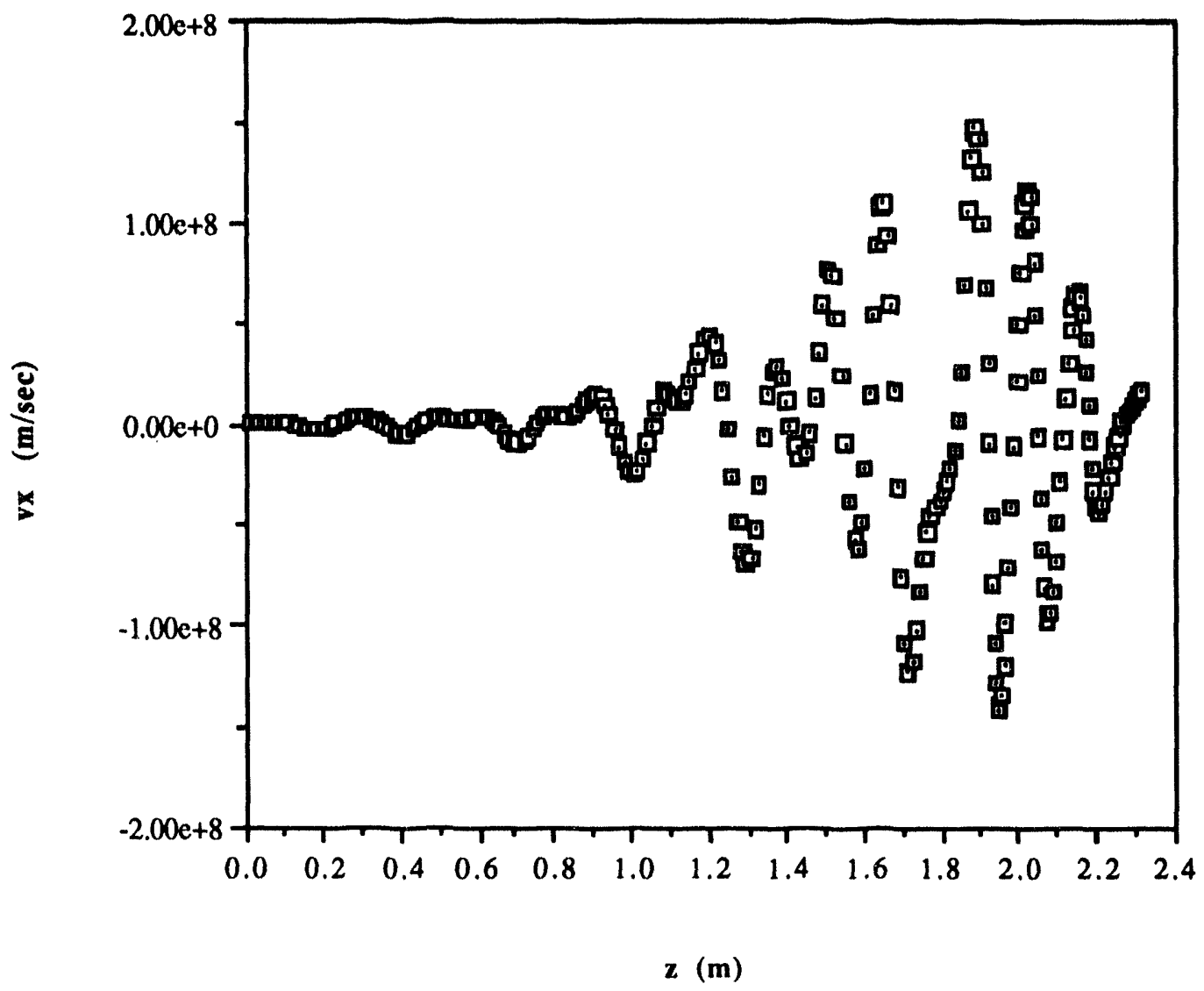

Figure 6.22. Particle $x$-directed velocity as a function of position. 


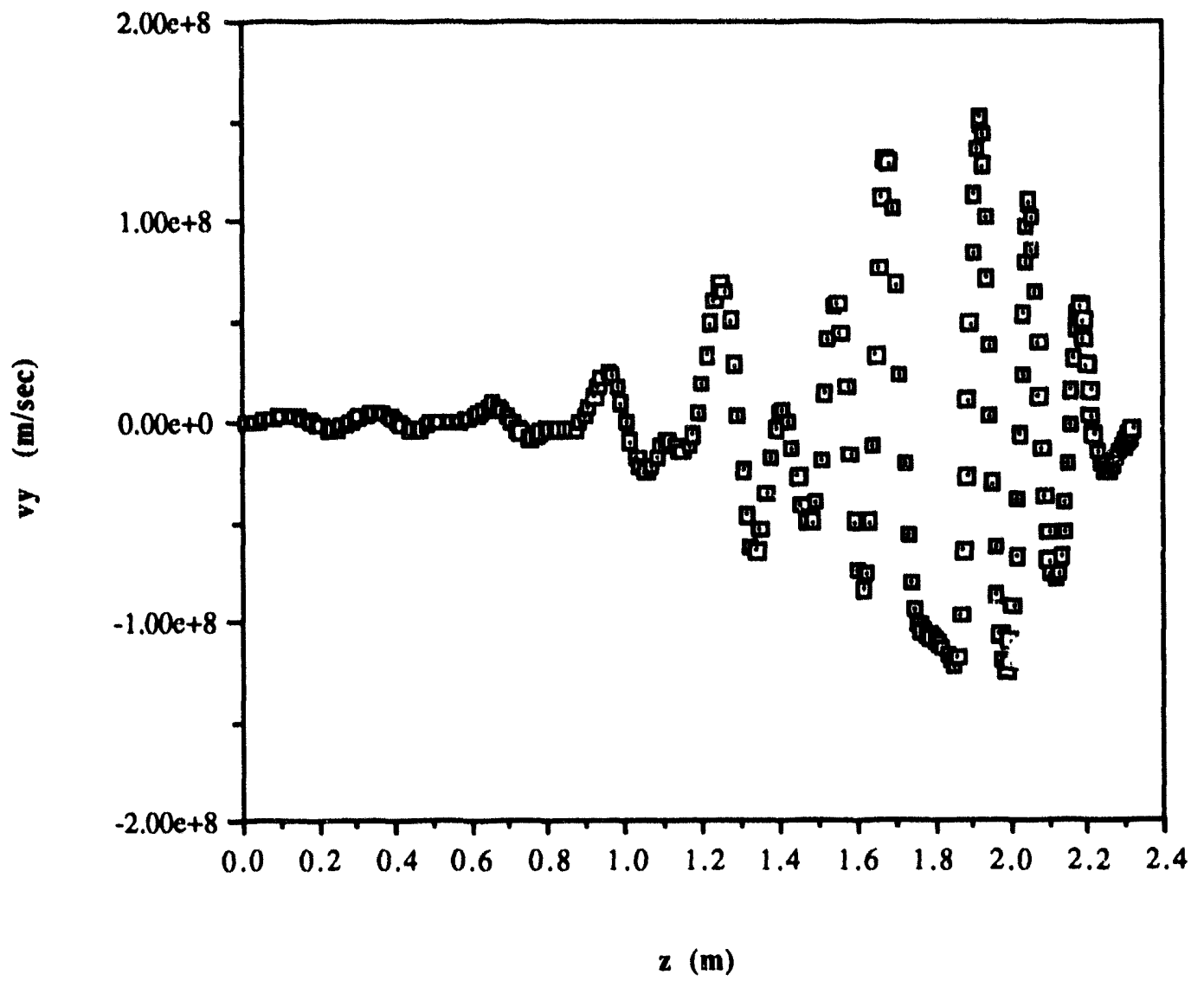

Figure 6.23. Particle $y$-directed velocity as a function of longitudinal position. 


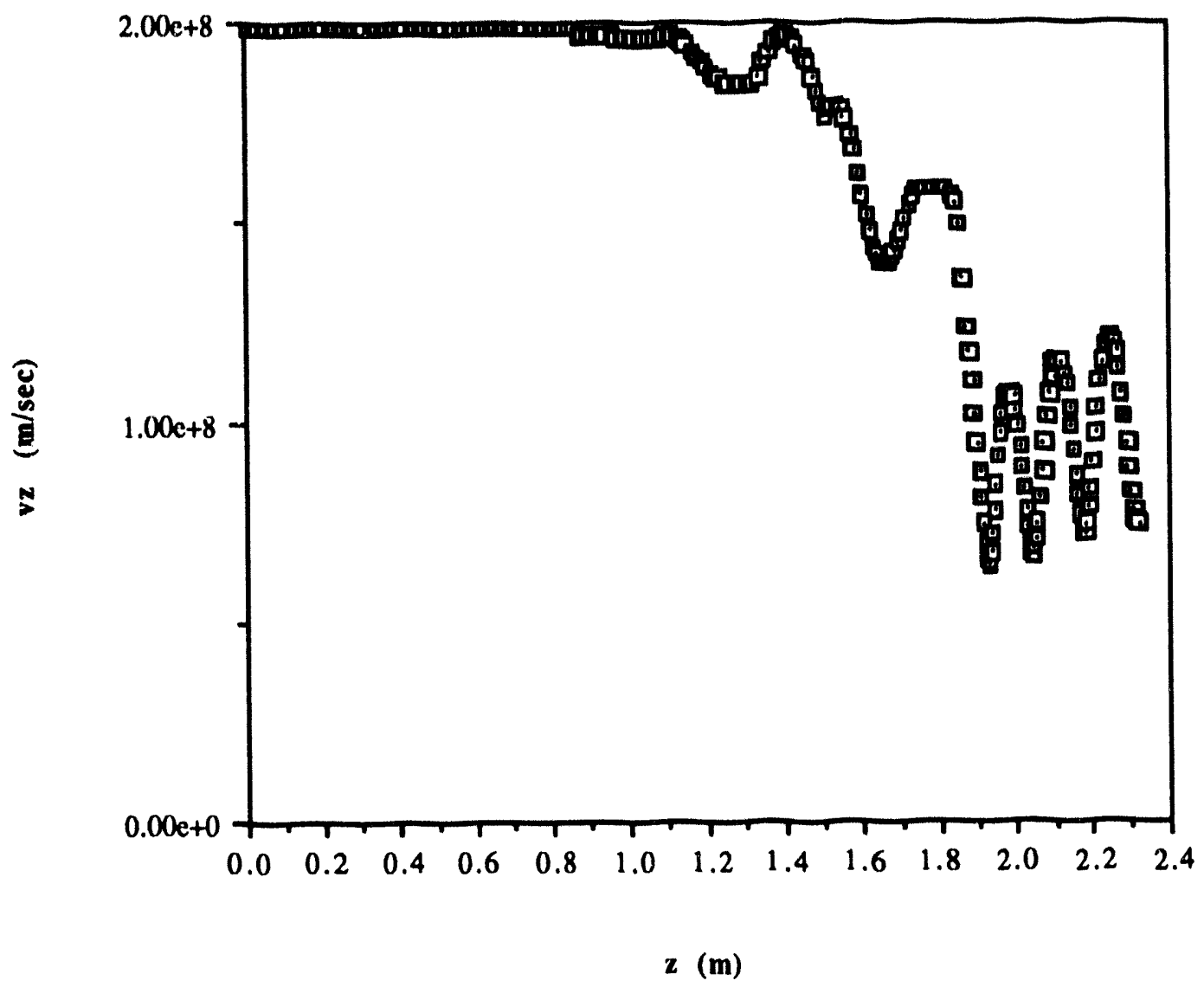

Figure 6.24. Particle $z$-directed velocity as a function of longitudinal position. 


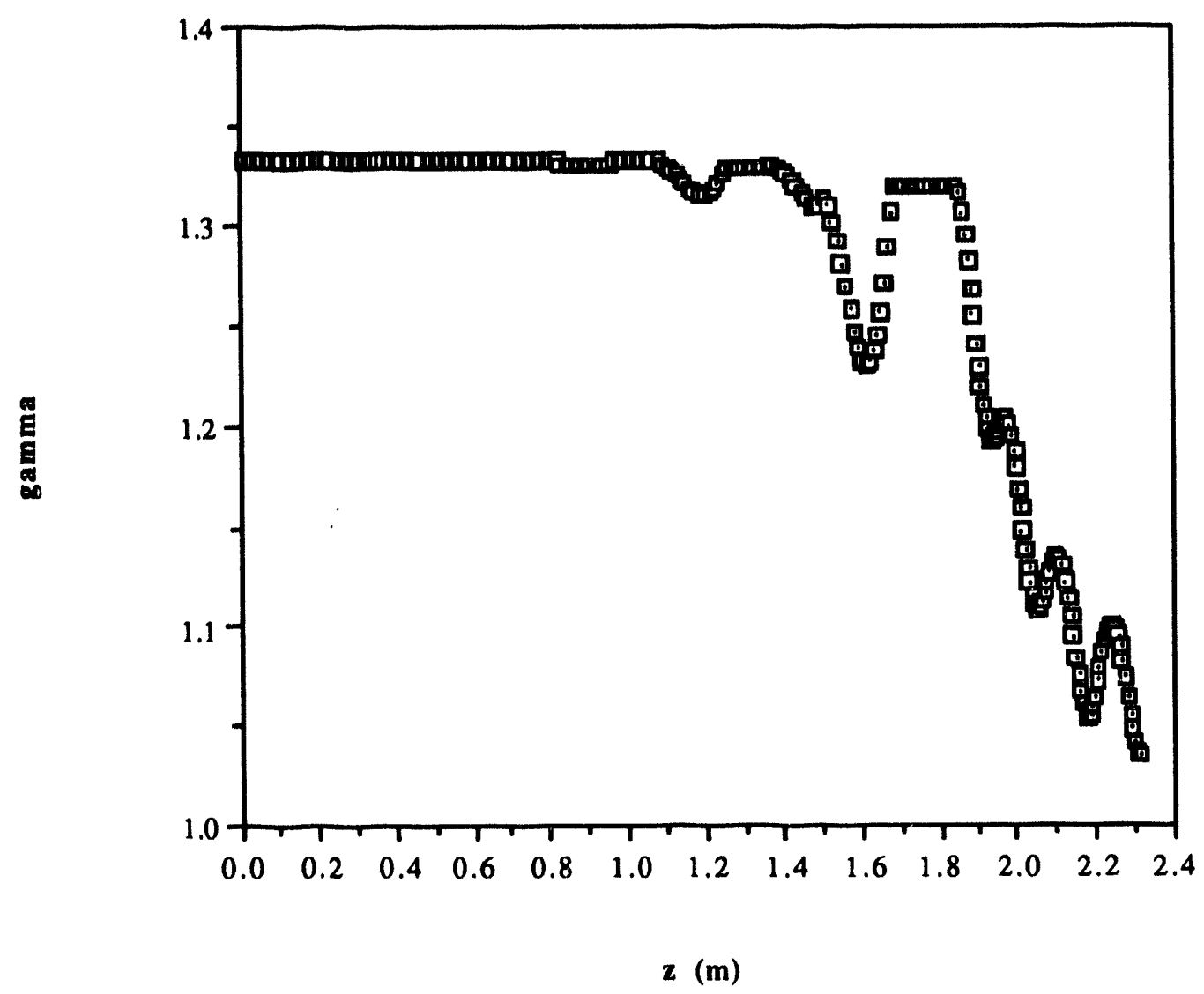

Figure 6.25. Particle gamma as a function of longitudinal position.

\subsection{The uniform-field magnicon: an improved geometry}

The geometry proposed below is a modification of the typical magnicon geometry. It is being proposed to improve the tolerance of the magnicon to highperveance beams. Unlike Russian magnicons, the geometry analyzed in this section has no magnetic transition between the deflection system and the output cavity, and the $\Omega / \omega$ ratio is much smaller than that employed in Russian magnicon and gyrocon work. This change from the Russian geometry will be shown in later sections to be less sensitive to finite beam size and more suitable for large beams and a high-perveance magnicon design. The implications of this change are listed below. 
1. All transverse deflection must be accomplished by if interactions with deflection cavities. The half-cusp transition no longer exists and will not provide any additional deflection.

2. The beam is always immersed in a confining magnetic field, so beam expansion due to beam-generated forces in the field-free drift space separating the deflection system and output cavity is no longer an issue.

3. The $\Omega / \omega$ ratio in the deflection system is based on the initial gamma is 0.875 , resulting in shorter cavities to maintain a gain similar to a magnicon with an $\Omega / \omega$ ratio of 1.4 .

4. Because there is no magnetic transition at the entrance to the output cavity, the concern of the magnetic transition spreading the beam energy during interaction with the beam is no longer a concern.

The uniform-field magnicon architecture is presented in Fig. 6.26. Note the length of the passive cavities has been decreased to offset the decrease in gain caused by the reduction in $\Omega / \omega$ ratio. The passive-cavity length was decreased until the results of Section 5.3.3 indicated the instantaneous bandwidth was being decreased below a reasonable level. The compromise between gain and bandwidth resulted in passive cavities with an instantaneous bandwidth of $200 \mathrm{kHz}$. A variable length between passive cavities is also used in an attempt both to minimize input radius (in order to keep the entrance holes in the cavities small) and to maximize passive-cavity gain. 


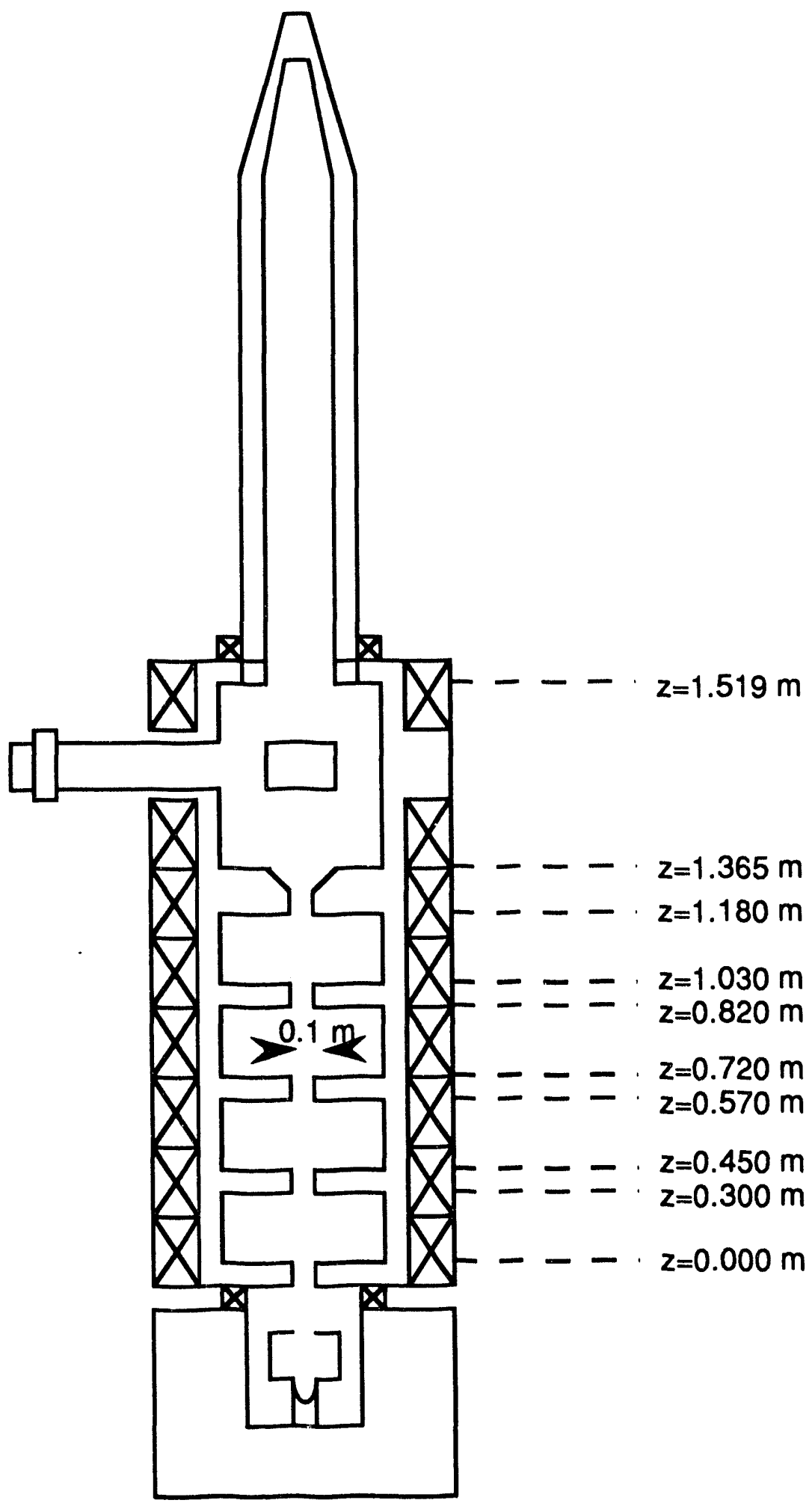

Figure 6.26. Uniform-field magnicon geometry. This geometry has no half-cusp magnetic field and different dimensions and $\omega / \Omega$ ratio from Figure 6.10. (Note: drawing is not to scale.) 
Table 6.6 shows the operating parameters of the uniform-field magnicon geomatry. From the table it is observed that the gain of this magnicon geometry is lower than the other geometries explored in previous sections. However, the output power and efficiency are comparable. The gain loss could be compensated by the addition of extra passive cavities. The other significant change in parameters is the output-cavity field. The 4.57 MV/m field given here is higher than for previous geometries. The peak cavity field can be determined by multiplying this value by the peak of the Bessel function in the electric-field expression (0.52), resulting in a peak cavity field of $2.37 \mathrm{MV} / \mathrm{m}$, which is similar to those in klystrons.

Table 6.6 Operating parameters for uniform-field magnicon architecture.

\begin{tabular}{|l|c|}
\hline Operating Parameter & Value \\
\hline Beam Voltage & $170 \mathrm{kV}$ \\
\hline Beam Current & $18 \mathrm{~A}$ \\
\hline Gain & $30.2 \mathrm{~dB}$ \\
\hline Efficiency & $87.9 \%$ \\
\hline Output Cavity Field & $5.0 \mathrm{MV} / \mathrm{m}$ \\
\hline Initial Particle Gamma & 1.33219 \\
\hline Deflection System $\Omega / \omega$ Ratio & 0.875 \\
\hline Output Cavity Magnetic Field & $0.0292 \mathrm{~T}$ \\
\hline Output Power & $2.69 \mathrm{MW}$ \\
\hline
\end{tabular}

Rigid-beam-model trajectory plots are shown below. Figures 6.27 and 6.28 illustrate the $x$ and $y$ displacement of the particle off the longitudinal axis as it passes through the magnicon. Figure 6.29a and 6.29b are scatter plots of the particle's $x$ and $y$ position during one transit interval. Figure $6.29 \mathrm{a}$ is a scatter plot of the entire transit interval while Fig. 6.29b is a scatter plot only of the deflection system, where different symbols designate different regions in the deflection system according to the provided legend. This convention was adopted for these graphs, which are not the same type presented in previous sections, because these graphs show more interesting trajectories and are more difficult to decipher. Specifically, Figs. 6.29a and 6.29b show drastic changes in trajectory direction, which is different than the behavior observed in the previous two sections. However, when evaluating the following transverse-velocity plots, the observer can see that the slope of the plots varies drastically in short time intervals, so sudden changes in the particle trajectories should be expected. Figures 6.30, 6.31 , and 6.32 show the particle's $x-, y-$, and $z$-directed velocity components as a function 
of longitudinal position. Figure 6.33 shows the particle gamma as a function of longitudinal position.

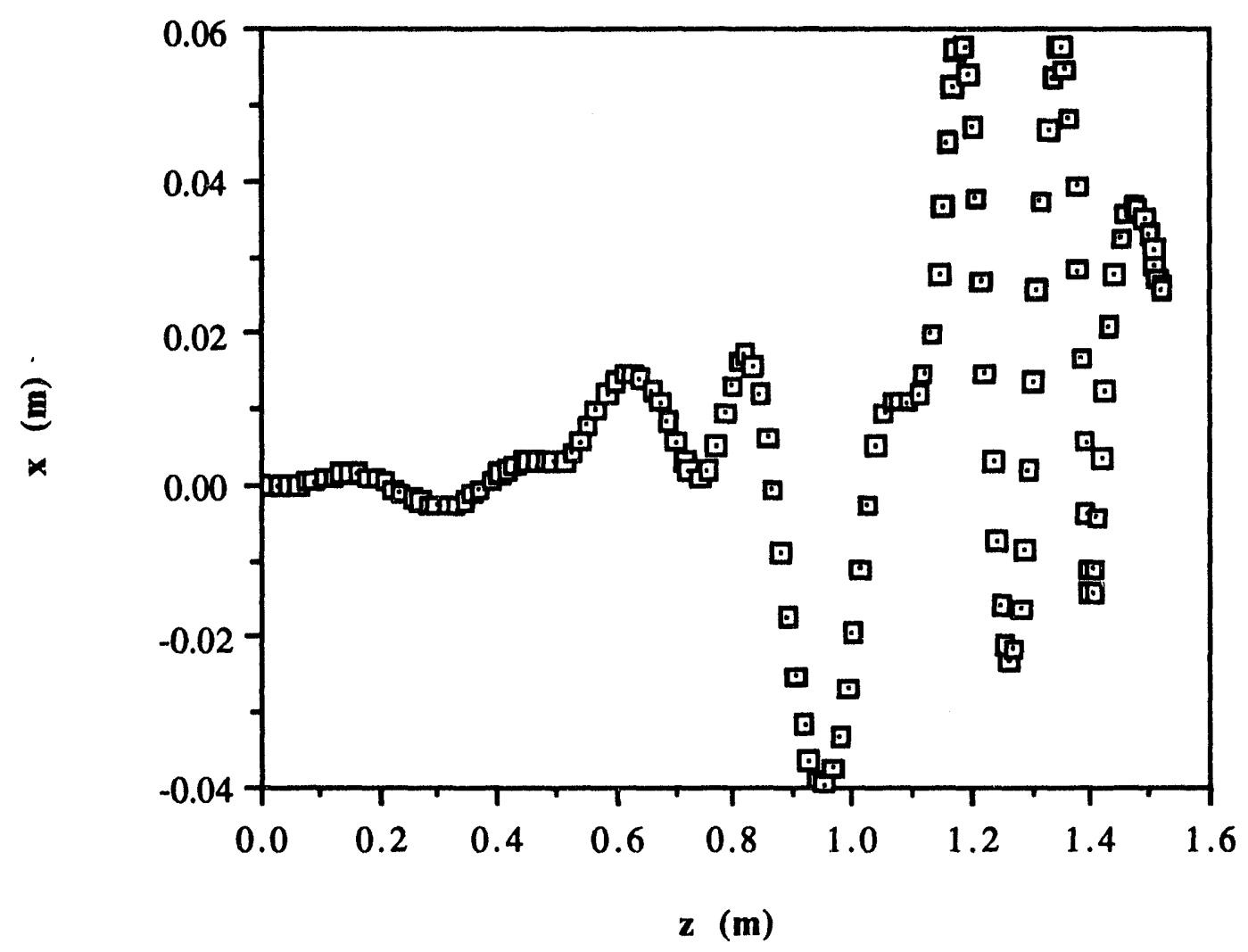

Figure 6.27. Particle $x$ displacement off axis as a function of longitudinal position. 


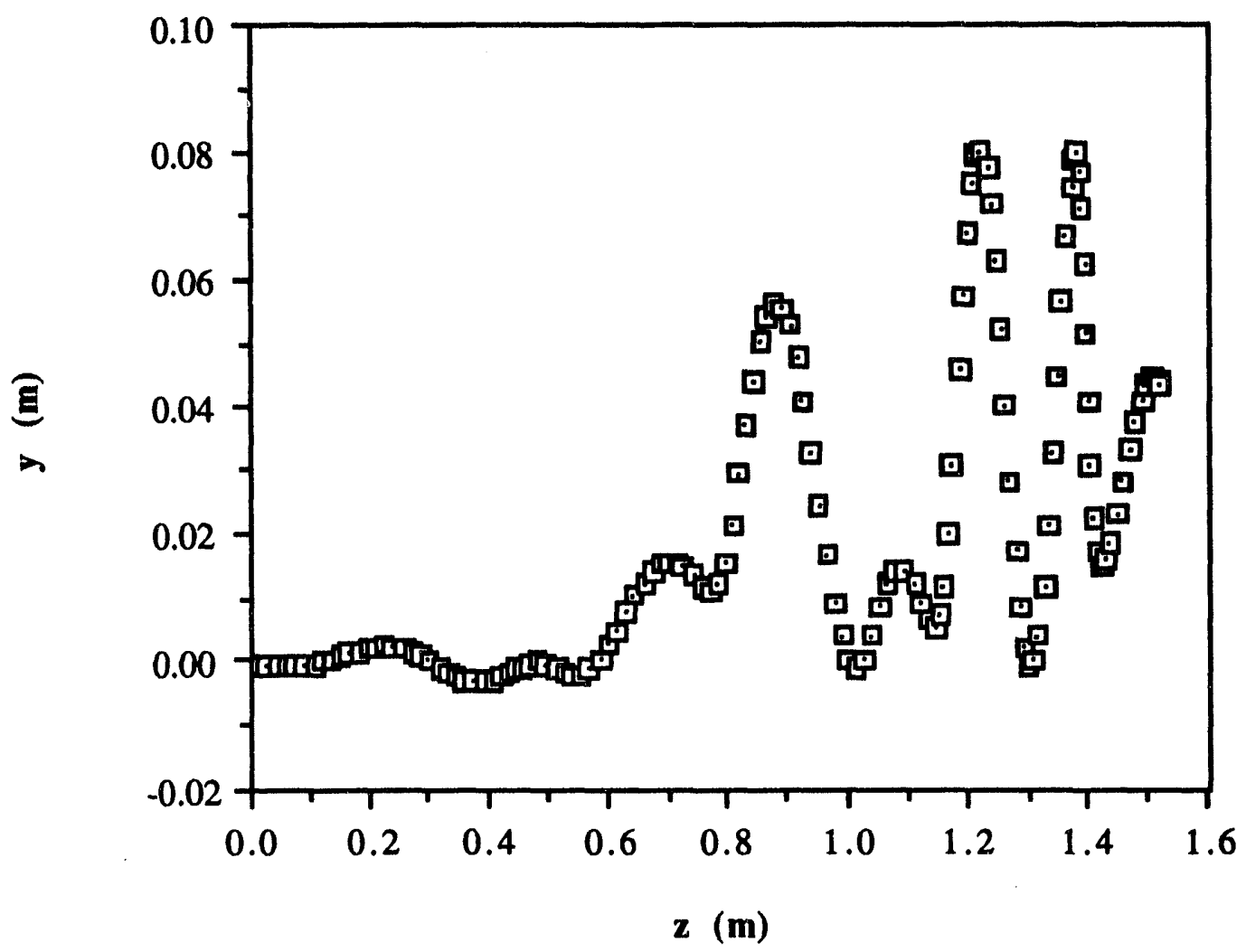

Figure 6.28. Particle $y$ displaccment as a function of longitudinal position. 


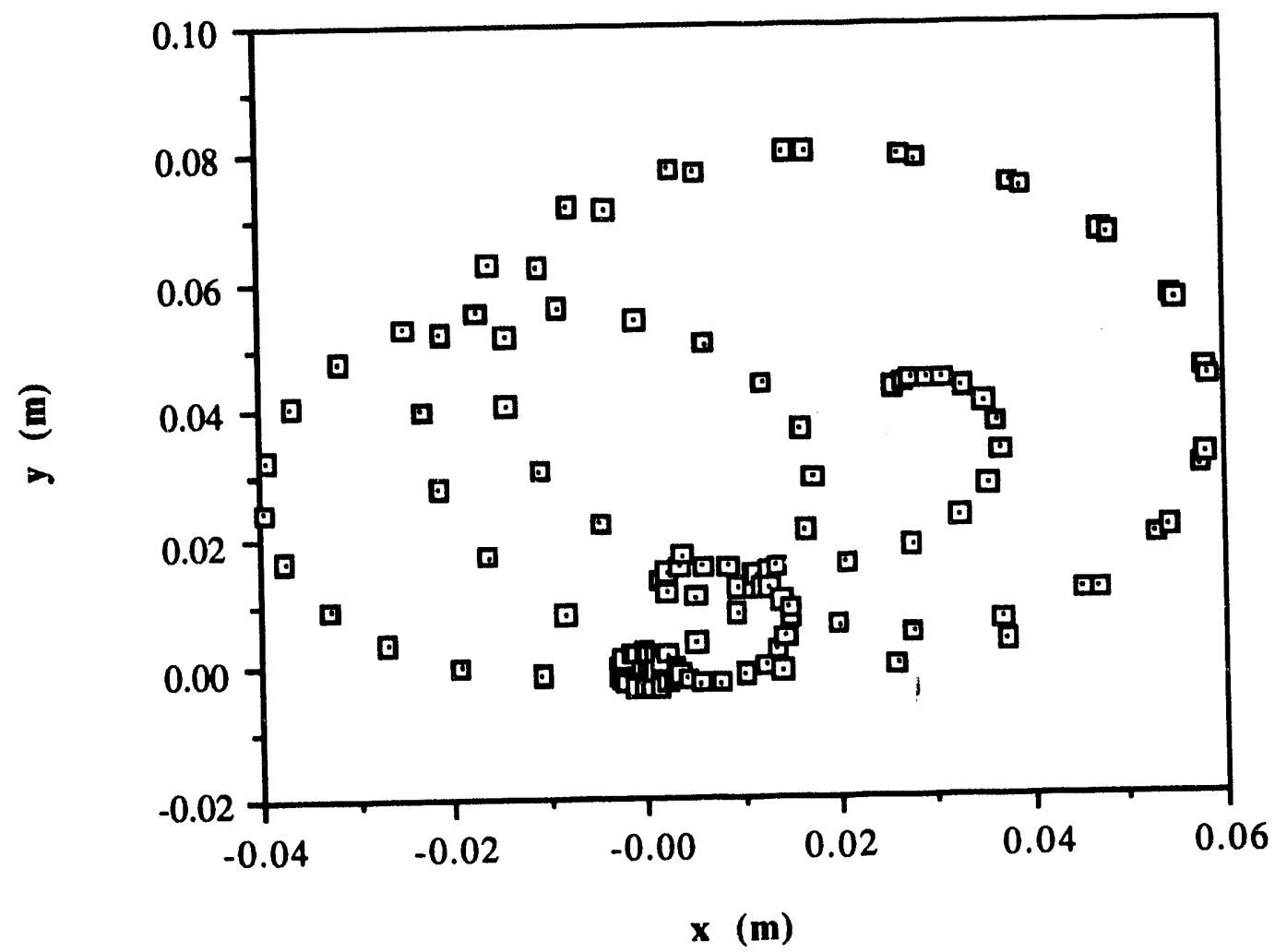

Figure 6.29a. Scatter plot of the entire transit interval 


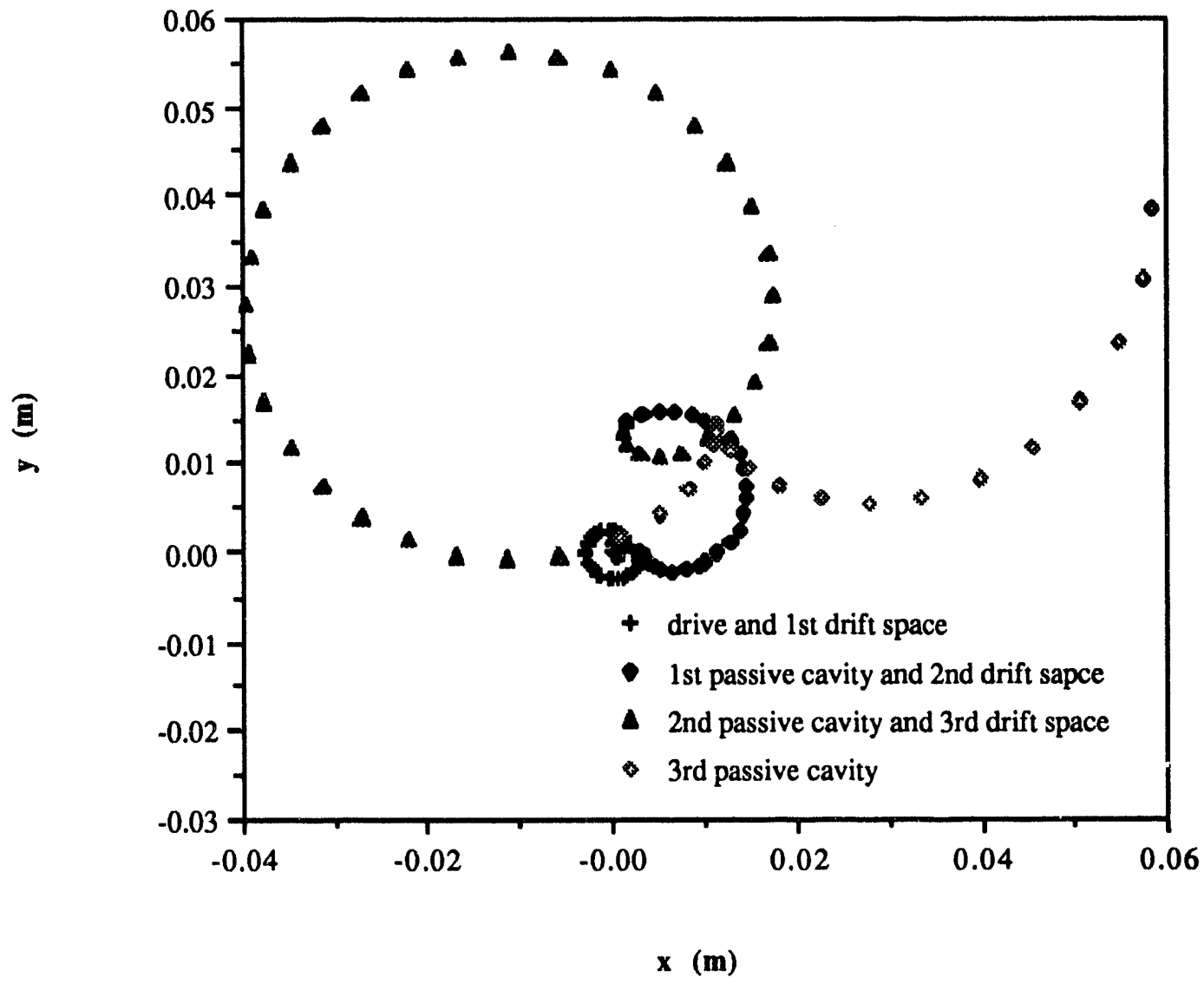

Figure 6.29b. Scatter plot of only the deflection system 


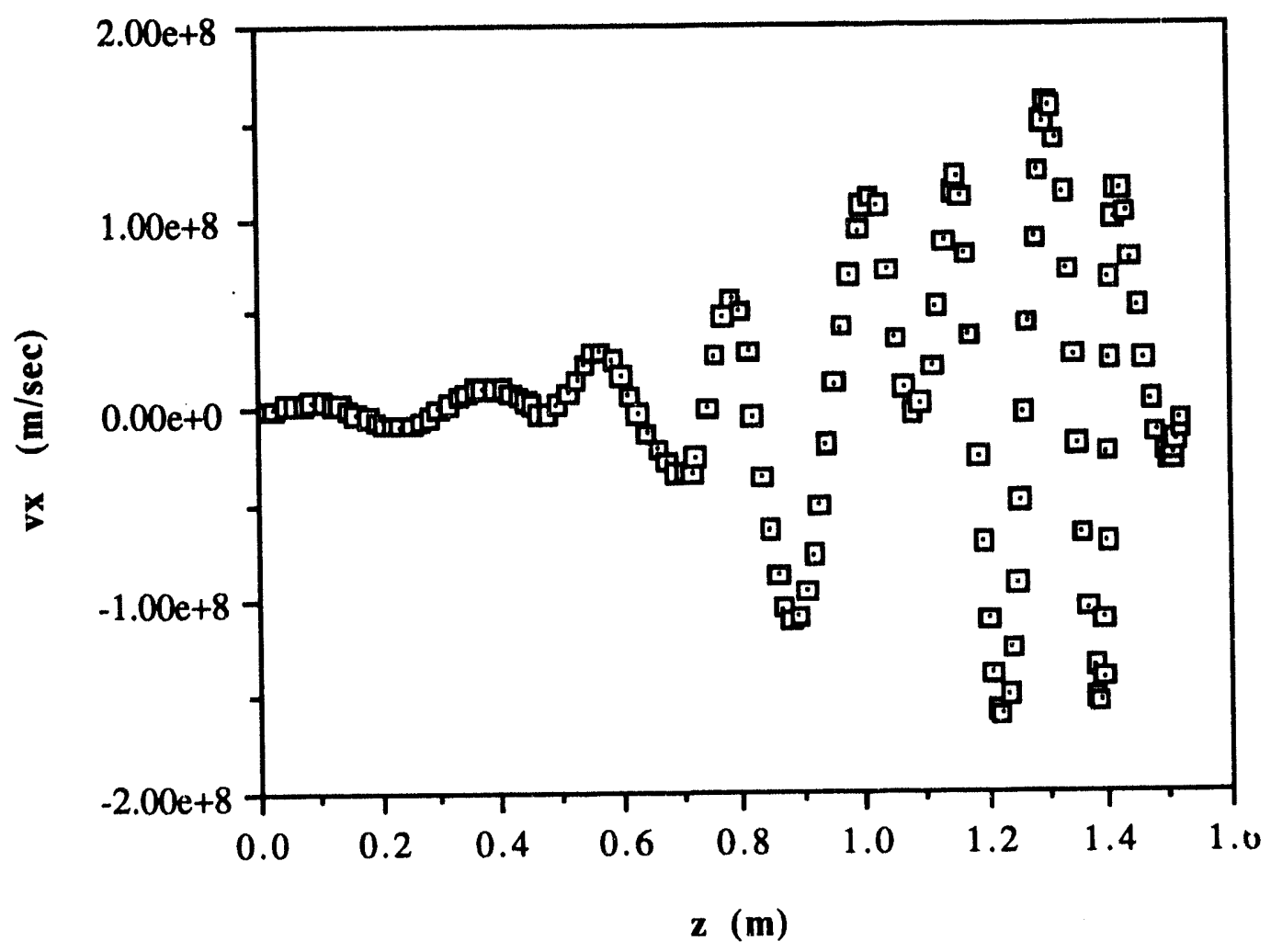

Figure 6.30. Particle $x$-directed velocity as a function of longitudinal position. 


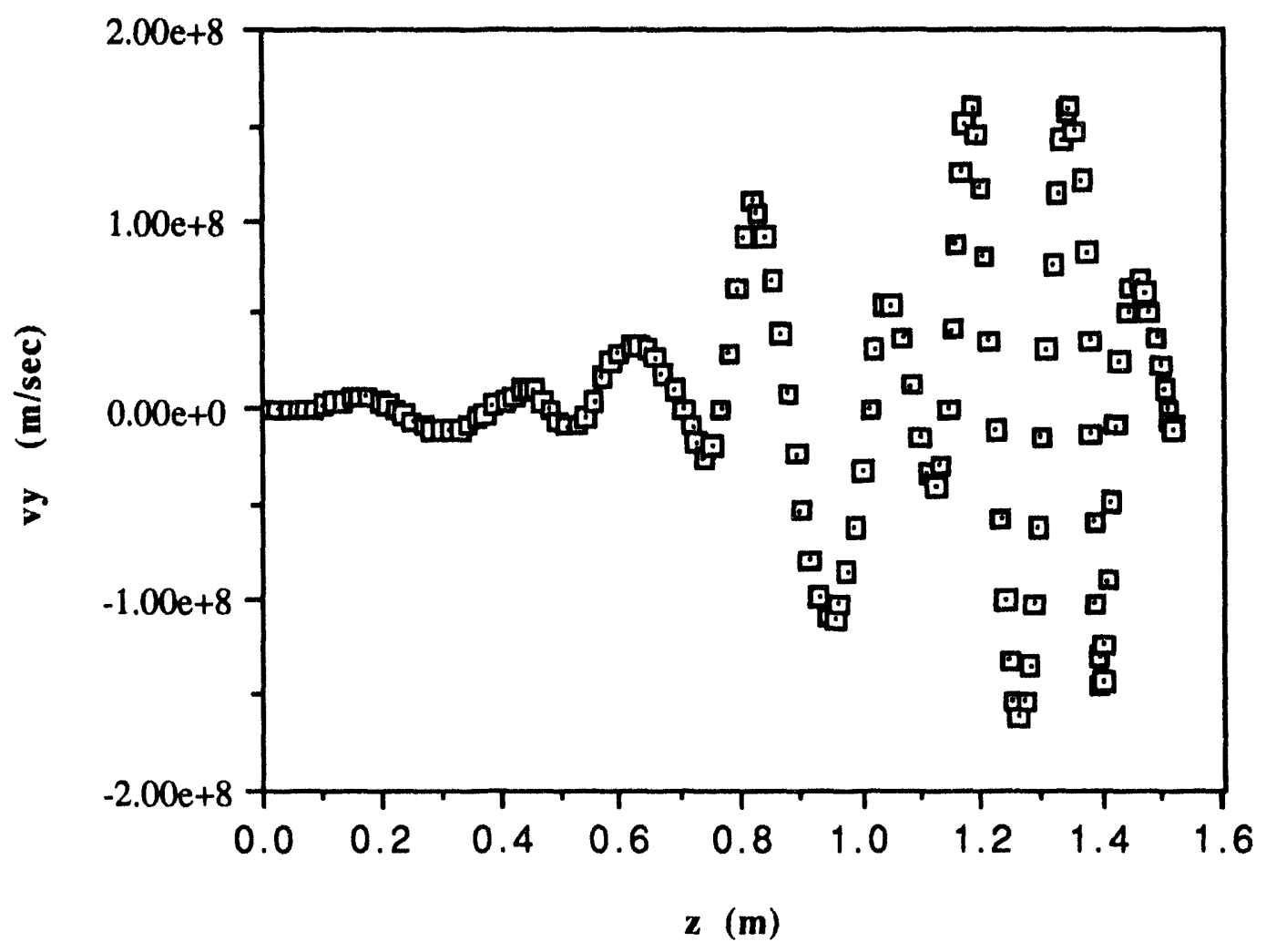

Figure 6.31. Particle $y$-directed velocity as a function of longitudinal position. 


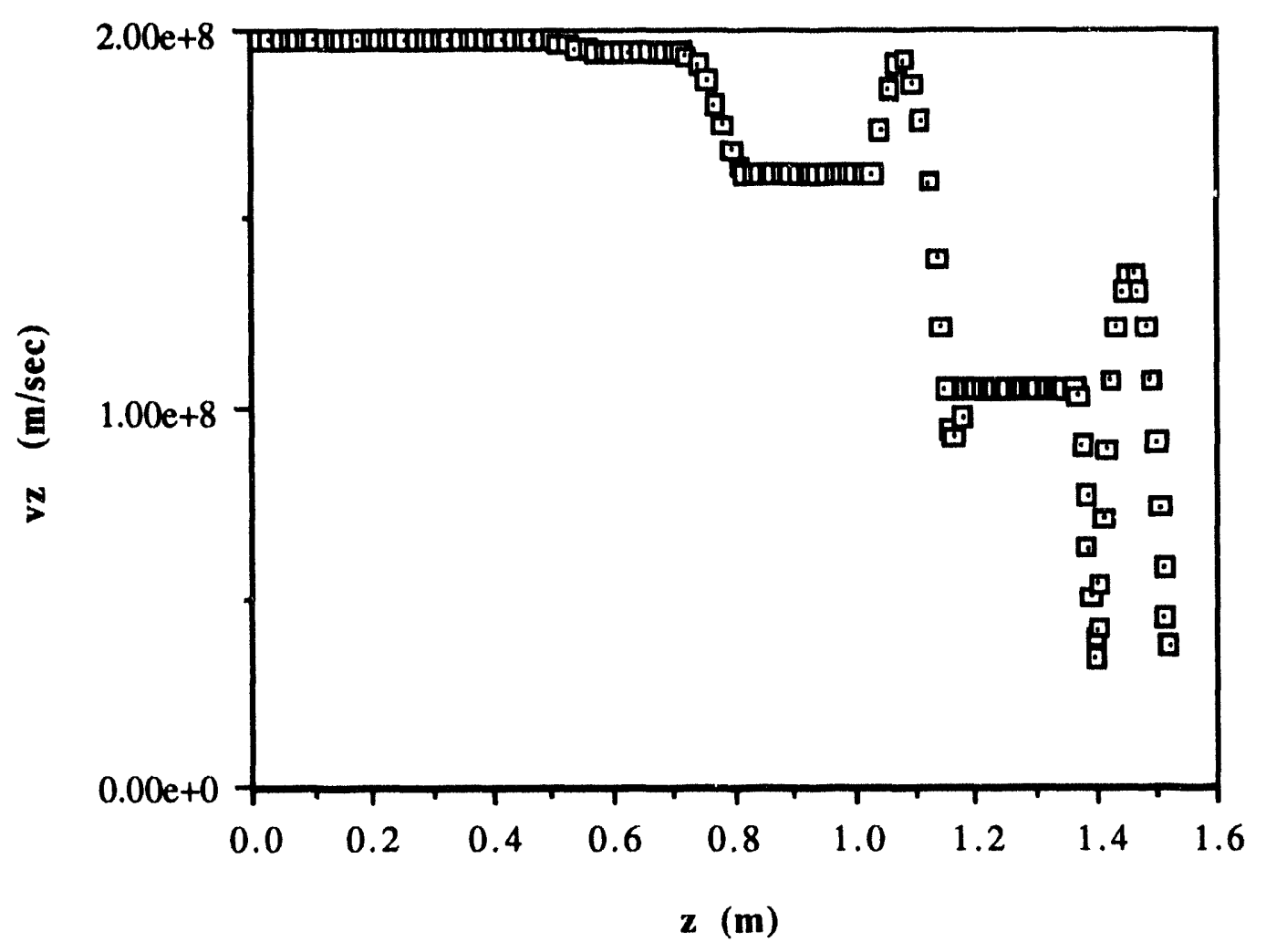

Figure 6.32 Particle longitudinal velocity as a function of longitudinal position. 


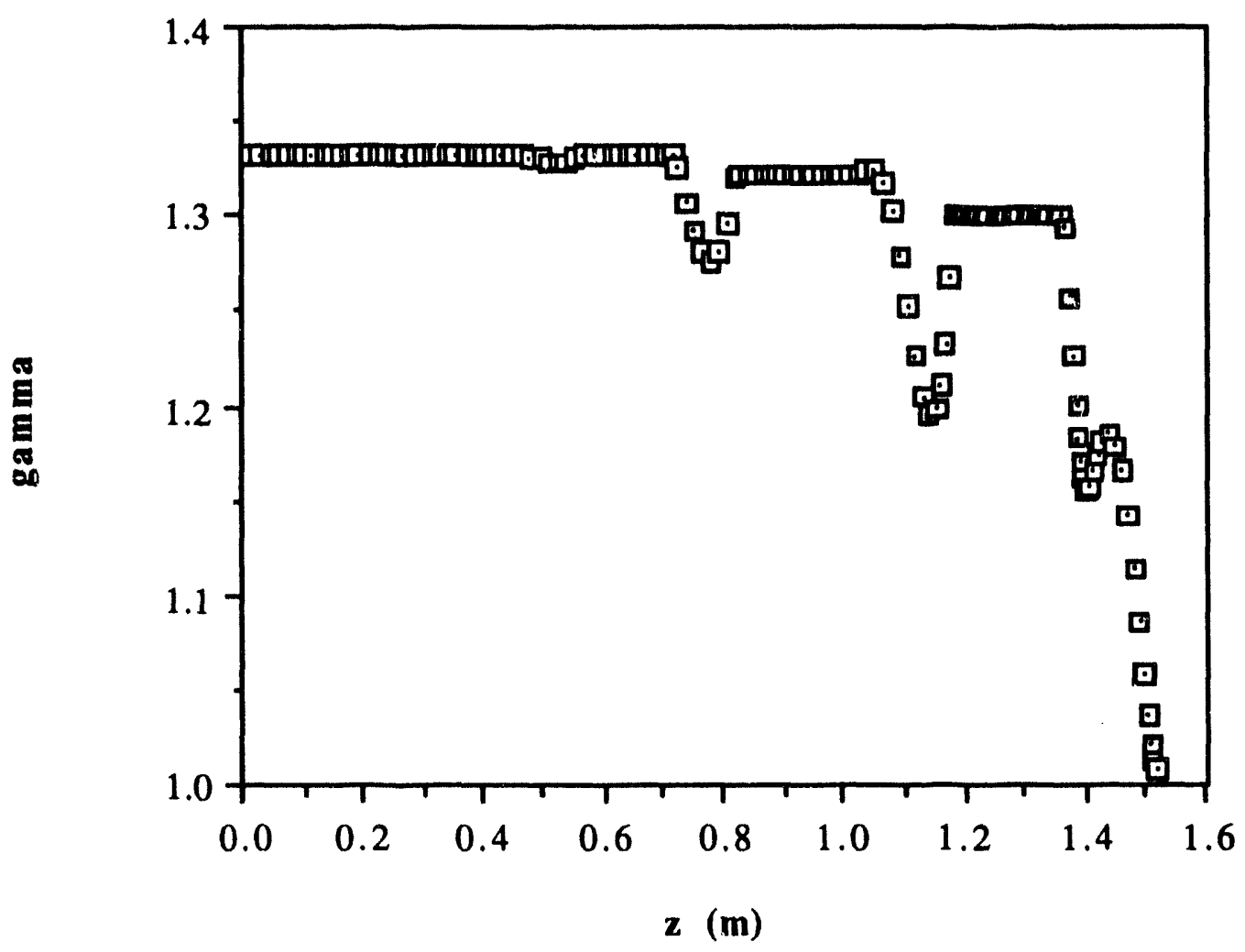

Figure 6.33. Particle gamma as a function of longitudinal position.

The geometry and results presented above are not as good as the previous rigidbeam results from either an efficiency or gain standpoint. However, it will be shown in the finite-beam-sized modeling that this architecture is superior for beams with a finite diameter when compared to the geometries with a magnetic-field transition between the deflection system and output cavity. 


\section{Finite-Beam Effects on Magnicon Efficiency}

The implementation, structure, and routines in the rigid-beam model form the basis for the finite-beam-size model. The important distinction between the finite-beamsize model and the rigid-beam model is that the finite-beam-size model is not selfconsistent. Instead, it uses the self-consistent solution from the rigid-beam models to advance the particles that make up the finite-diameter beam. This assumption is quite accurate when the beam size is small enough so that the field varies in a linear fashion over the region of the beam. This assumption becomes compromised, however, as the beam size increases. In the magnicon's case, this assumption is most bothersome in the output cavity, where the interaction of the finite beam with the sharp magnetic transition at the entrance to the output cavity has tended to spread the beam size and energy. In order to encompass the worst-case error caused by this assumption, the self-consistent phase for the output cavity computed by the rigid-beam model will be compared to the self-consistent phase computed for a finite-beam size. (The comparison will show that the resulting error is small.) In the cavities that make up the deflection system, the particles of the finite-beam cross section maintain a close proximity. Therefore, the field shapes that affect them are approximately linear, so the average energy exchanged from the beam to the cavity should be consistent with the value of the centroid position calculated in the rigid-beam model.

The starting point for the implementation of the finite-beam-size model was the rigid-beam model. Those portions of the code for the rigid-beam model that determined the self-consistent solution were removed. A routine that input the starting coordinates of the particles composing the finite-beam cross section was added, and a loop was inserted around the rigid-beam routines. Each particle of the finite-beam cross section was then advanced individually through the magnicon geometry. The first particle completed its journey before the second particle was advanced. Trajectories were written to data files, and the energy extracted from each particle in the output cavity was saved in arrays for summation after all particles had been passed through the magnicon model.

Essentially, the finite-beam-size model treats the beam as a collection of rigid beams. The beam is divided into $n$ equal-area parts, and the finite-beam-size model assumes a rigid beam with a current of $I_{o} / n$, where $I_{o}$ is the full beam current, which is initially located at the center of each of these $n$ regions. These are the initial coordinates of each particle that composes the finite-sized beam. Each of these small rigid beams is 
then advanced through the magnicon. The method used to quantize the beam is described in more detail in a subsequent section. (This approach essentially assumes a uniform distribution of charge across the beam-uniform current density - and by quantizing the beam into equal-area regions, it becomes valid to represent the quantized beam by $n$ equal-current regions. This approach could also be extended to any charge profile across the beam.)

As has been stated in previous sections, the finite-beam model uses threedimensional fields that neglect the beam entrance and exit holes in the cavities, and it is relativistic. Space-charge forces are not considered in the model but are discussed in Section 7.4.

\subsection{Modeling finite-beam effects}

For the finite-beam simulation, the beam is quantized into an $n$ number of equalarea sections. Each section is then represented by a filamentary beam. For uniform current density, each filamentary beam has a current value equal to the total current divided by the number of filamentary beams. The method of beam quantization is described below for $n=49$. This quantization level was chosen as a trade-off between accuracy and the time required to run the simulation. A visual inspection of the position of the 49 filamentary beams shows a complete sampling of the beam cross-section. (In Section 7.3, where the finite-beam modeling is used to present results, the value of $n$ is varied to demonstrate the impact of $n$ on the accuracy of the simulation.)

The initial coordinates of the filamentary beams that compose the beam cross section are calculated by dividing the beam into rings. An example of the calculation of the initial conditions is presented for $n=49$. This example will illustrate the method used, but it does not provide generalized equations suitable for any value of $n$. The complexity comes from the determiration of the number of rings. In general, my approach was to place a sample point every $30^{\circ}$ (or 12 sample points per ring) with the sample points on four successive rings offset by $15^{\circ}$. An additional sample point was placed on the center point. Thus, I have considered values of $n$ equal to 13, 25, 37, 49, 61 , etc. (An argument could also be made for a selecting sainple points at every $20^{\circ}$ or $10^{\circ}$. I selected the number of sample points per ring by looking at samplings that were visually pleasing.)

For $n=49$, one filamentary beam is placed at the center and the other 48 are arranged around four rings, with 12 filamentary beams per ring. Each filamentary beam 
represents an equal area of the circular beam cross-section. $R$ represents the beam radius, and the radii $r_{1}, r_{2}, r_{3}$, and $r_{4}$ represent the rings of the beam cross section used to define the initial positions of the filamentary beams. Figure 7.1 illustrates $r_{1}, r_{2}, r_{3}$, and $r_{4}$ for $n=49$ and $R=1 \mathrm{~cm}$. The area corresponding to the point at the center is determined from

$$
\frac{\pi R^{2}}{49}=\pi r_{1}^{2}
$$

or

$$
r_{1}=\frac{R}{7} .
$$

The outermost ring radius, $r_{4}$, is determined from

$$
\pi R^{2}-\pi r_{4}^{2}=\frac{12}{49} \pi R^{2}
$$

or

$$
r_{4}=\frac{\sqrt{37}}{7} R
$$

The next ring radius, $r_{3}$, is defined by

$$
\pi r_{4}^{2}-\pi r_{3}^{2}=\frac{12}{49} \pi R^{2}
$$

or

$$
r_{3}=\sqrt{r_{4}^{2}-\frac{12}{49} R^{2}}
$$

Using the same approach, $r_{2}$ is given by

$$
r_{2}=\sqrt{r_{3}^{2}-\frac{12}{49} R^{2}}
$$




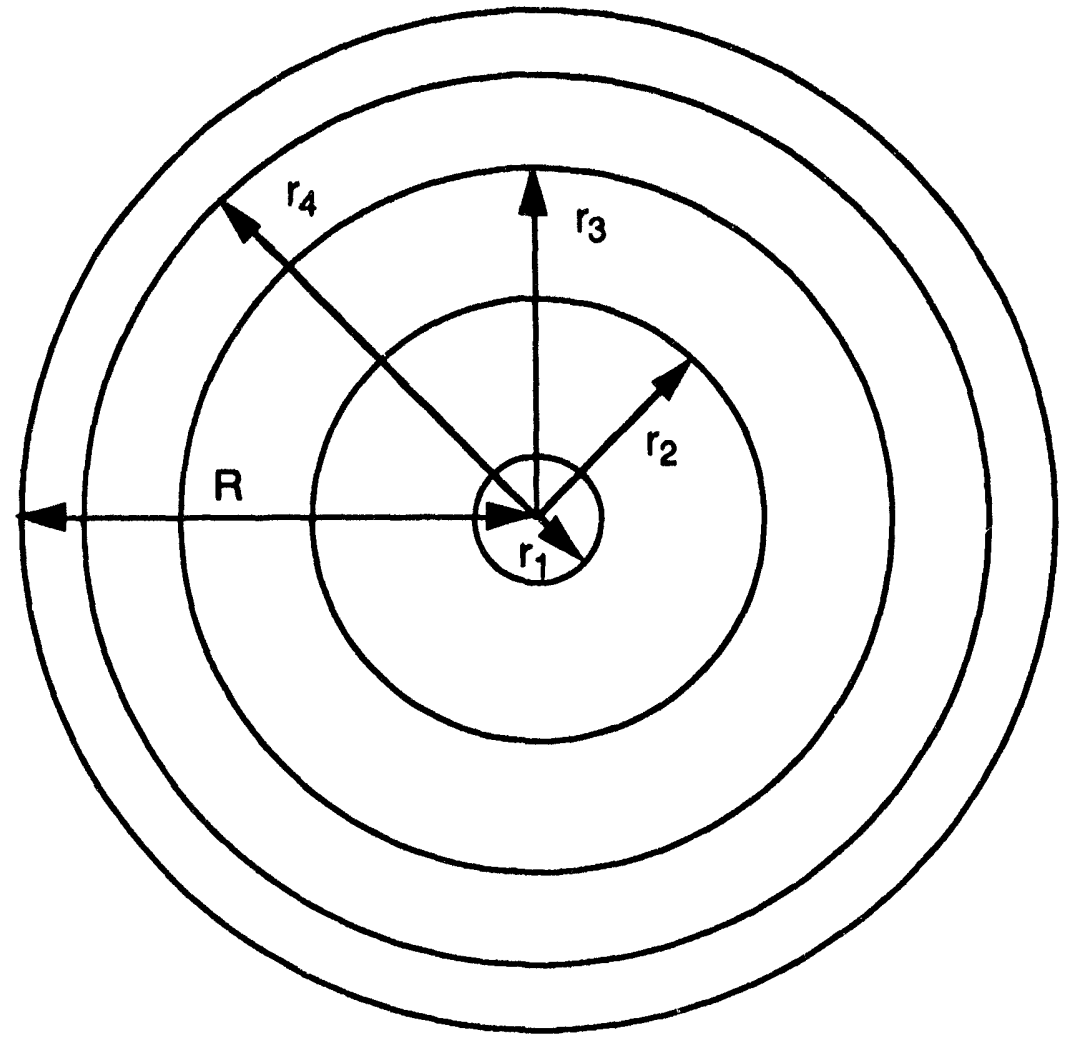

Figure 7.1. Illustration of ring regions in beam cross section.

The initial position of the filamentary beams are plotted on the scatter plot in Fig. 7.2 for $n=49$. 


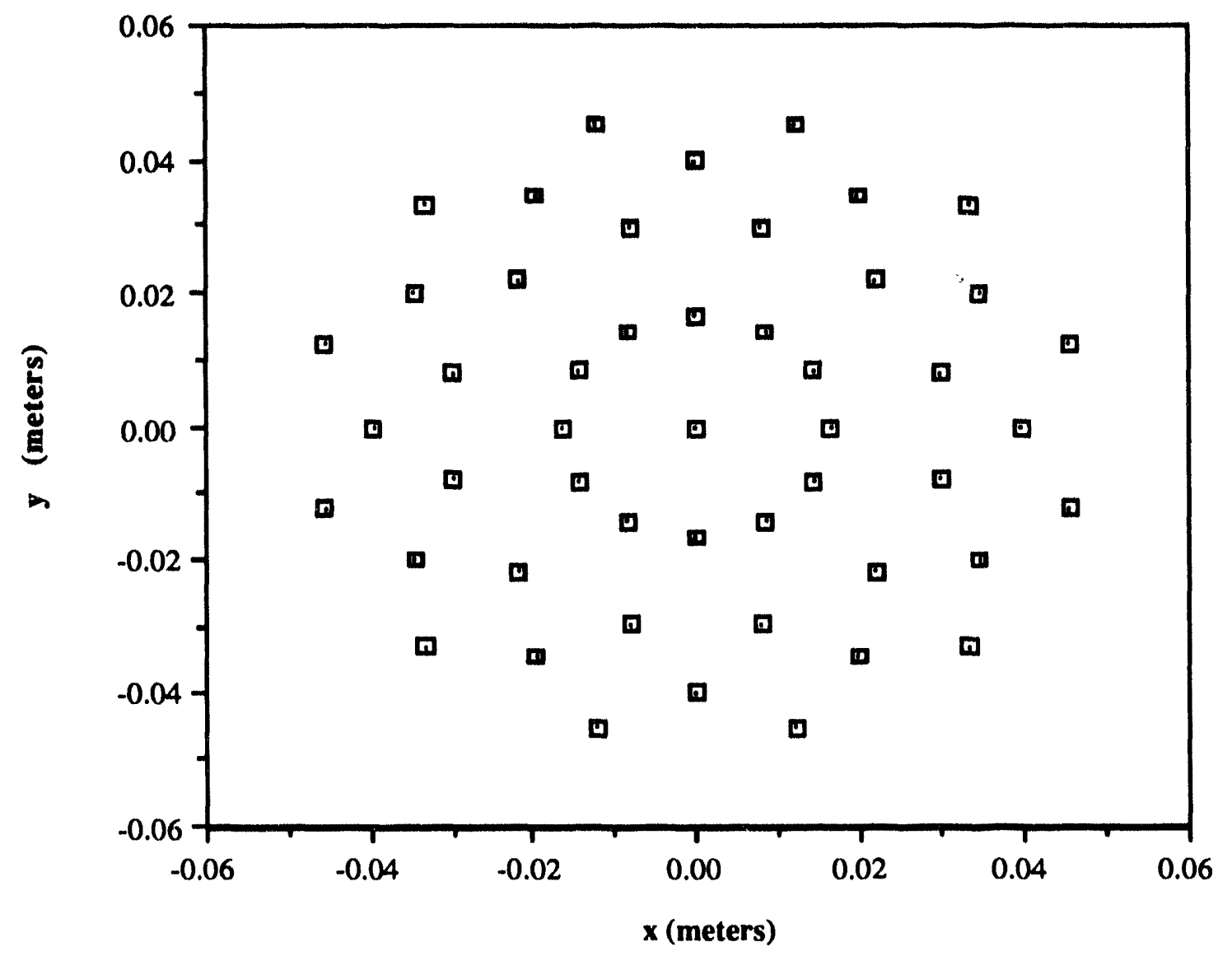

Figure 7.2. Initial filamentary beam positions that make up beam cross section.

As implemented in the finite-beam-size model, the electron representing each filamentary beam is initially given the same longitudinal velocity set by the beam voltage. It has no transverse velocity components (a zero-emittance beam) and represents a filamentary current $1 / n$th of the original current. By assuming that the electrons representing the filamentary beam initially all have the same longitudinal velocity potential, any depression resulting from the beam-generated fields is ignored. An approximate estimate of the potential depression can be calculated by considering a cylindrical beam on-axis in an infinitely long cylindrical pipe whose radius is either the beam pipe or cylindrical cavity. Using Gauss' law, the fields due to a line source of charge $Q_{l}$ are calculated from

$$
2 \pi r l \varepsilon_{o} E_{r}=l Q_{l} .
$$

When $r$ is less than the beam radius $\left(r<r_{b}\right)$, 


$$
Q_{1}=-e n_{0} \pi r^{2}
$$

and when $r$ is greater than the beam radius $\left(r>r_{b}\right)$,

$$
Q_{l}=-e n_{0} \pi r_{b}^{2}
$$

Using these values, we can define the electric fields inside and outside the beam as

$$
E_{r}=-\frac{e n_{o} r}{2 \varepsilon_{o}} \quad r \leq r_{b}
$$

and

$$
E_{r}=-\frac{e n_{o} r_{b}^{2}}{2 r \varepsilon_{o}} \quad r \geq r_{b}
$$

The voltage difference between an electron at the center of the beam and one at the edge of the beam is given by

$$
V=-\int_{0}^{r_{b}} E \cdot d l=\int_{0}^{r_{b}} \frac{e n_{o} r}{2 \varepsilon_{0}}=\frac{e n_{o} r_{b}^{2}}{4 \varepsilon_{o}} .
$$

The voltage difference between an electron at the edge of the beam and the cavity walls is

$$
V=-\int_{r_{b}}^{r_{w}} E \cdot d l=\int_{r_{b}}^{r_{w}} \frac{e n_{o} r_{b}^{2}}{2 r \varepsilon_{o}}=\frac{e n_{o} r_{b}^{2}}{2 \varepsilon_{o}} \ln \left(\frac{r_{w}}{r_{b}}\right) .
$$

If these expressions are evaluated numerically for the baseline parameters $(I=$ $18 \mathrm{~A}$ ) and we use a typical case where $r_{w}=0.05 \mathrm{~m}$ in the beam pipe (or $r_{w}=0.26 \mathrm{~m}$ in a cavity) and $r b=0.005 \mathrm{~m}$ as the potential across the beam, $V_{\text {beam }}$ is calculated to be

$$
V_{\text {beam }}=817.1 \mathrm{~V} \text {, }
$$

and the voltage between the edge of the beam and the cavity wall, $V_{\text {beam-wall, }}$ is

$$
V_{\text {beam-wall }}=6457 \mathrm{~V} \text {, }
$$

while the potential between the beam and beam pipe is

$$
V_{\text {beam-beam pipe }}=3763 \mathrm{~V} \text {. }
$$


This result can be interpreted to mean two things. First, the potential energy of an electron at the center of the beam in an infinitely long deflection cavity is the sum of Eqs. (7.15) and (7.16) and is $7274 \mathrm{~V}$, or approximately $4 \%$ of the beam voltage. The change in voltage across the beam indicates that the electrons' longitudinal velocity is a function of their radius within the beam, but the magnitude of the voltage change across the beam $(0.44 \%)$ makes the velocity difference negligible. The approach for self-consistent solutions described previously ignores the potential energy that the beam must supply. This approach is validated by the magnitude of these numbers. In addition, the potential across the deflection cavity would actually be less than what was calculated above because the actual cavity length is short and the beam-generated electric fields would have a longitudinal component near the cavity axis as the beam entered and exited the deflection cavity.

\subsection{Efficiency degradation in standard magnicon}

The results of the finite-beam-size modeling of a standard magnicon are presented below. The parameters of the model are identical to the rigid-beam model of Section 6.4 with the geometry illustrated in Fig. 6.10 and operating conditions given in Table 6.4. The self-consistent solutions from the rigid-beam model are used by the finite-beam model. Table 7.1 summarizes the results of the finite-beam-size modeling. The first column shows beam diameter and the second efficiency.

Table 7.1. Relationship between beam size and efficiency.

\begin{tabular}{|c|c|}
\hline Beam Diameter (m) & Efficiency (\%) \\
\hline$\sim 0.000$ & 86.2 \\
\hline 0.001 & 84.1 \\
\hline 0.002 & 79.6 \\
\hline 0.003 & 75.0 \\
\hline 0.004 & 72.8 \\
\hline 0.005 & 68.7 \\
\hline 0.006 & 63.2 \\
\hline 0.007 & 54.5 \\
\hline 0.008 & 46.3 \\
\hline 0.009 & 39.8 \\
\hline
\end{tabular}


From the table, it can be seen that a beam diameter of $2.0-3.0 \mathrm{~mm}$ will provide a significant improvement over the state-of-the-art $67.0 \%$-efficient klystrons.

Figures 7.3 through 7.8 illustrate the trajectory and velocities of the electron cloud as it moves through the magnicon. These plots are for a beam diameter of $9.0 \mathrm{~mm}$. Figures 7.3 and 7.4 illustrate the trajectory of the electron cloud as it moves through the magnicon. Figures 7.5, 7.6, and 7.7 illustrate the $x-, y$-, and $z$-directed velocity components as a function of longitudinal position. Figure 7.6 illustrates the gamma of the electron cloud as a function of longitudinal position. These plots (particularly Figs. 7.7 and 7.8) illustrate that the decrease in efficiency with large beam diameter is due primarily to two factors: energy spread as the beam passes through the half-cusp magnetic field and a magnetic mirroring effect at this same position. The simulation deals with the beams that are mirrored by adding their contribution to the output cavity energy for as long as they remain within the rf structure. As soon as they are turned about and exit the input hole of the output cavity (or hit the wall), the simulation stops including their energy contributions to the overall total and moves onto the next beam. 


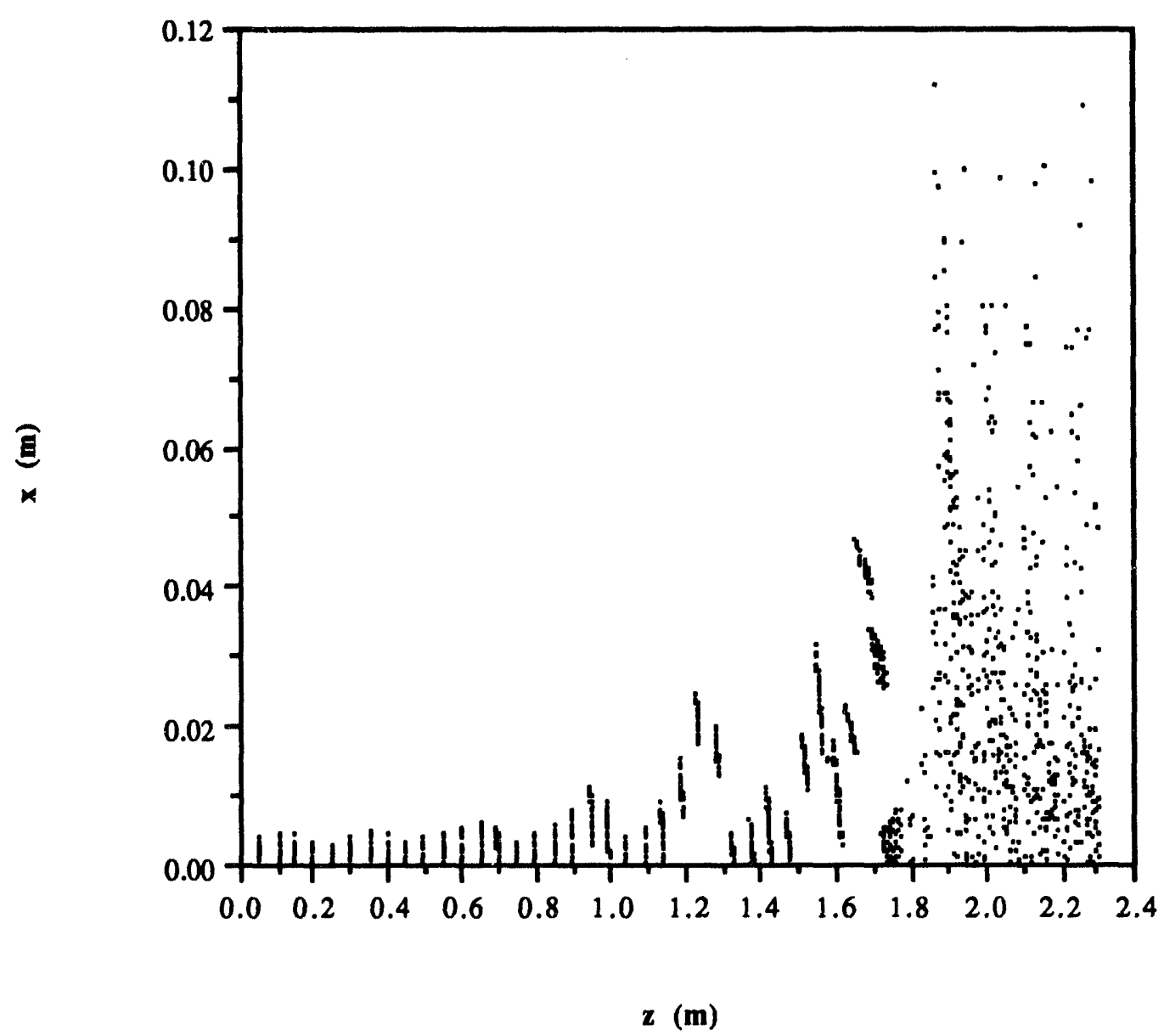

Figure 7.3. $X$-axis location of electron cloud as a function of longitudinal position. 



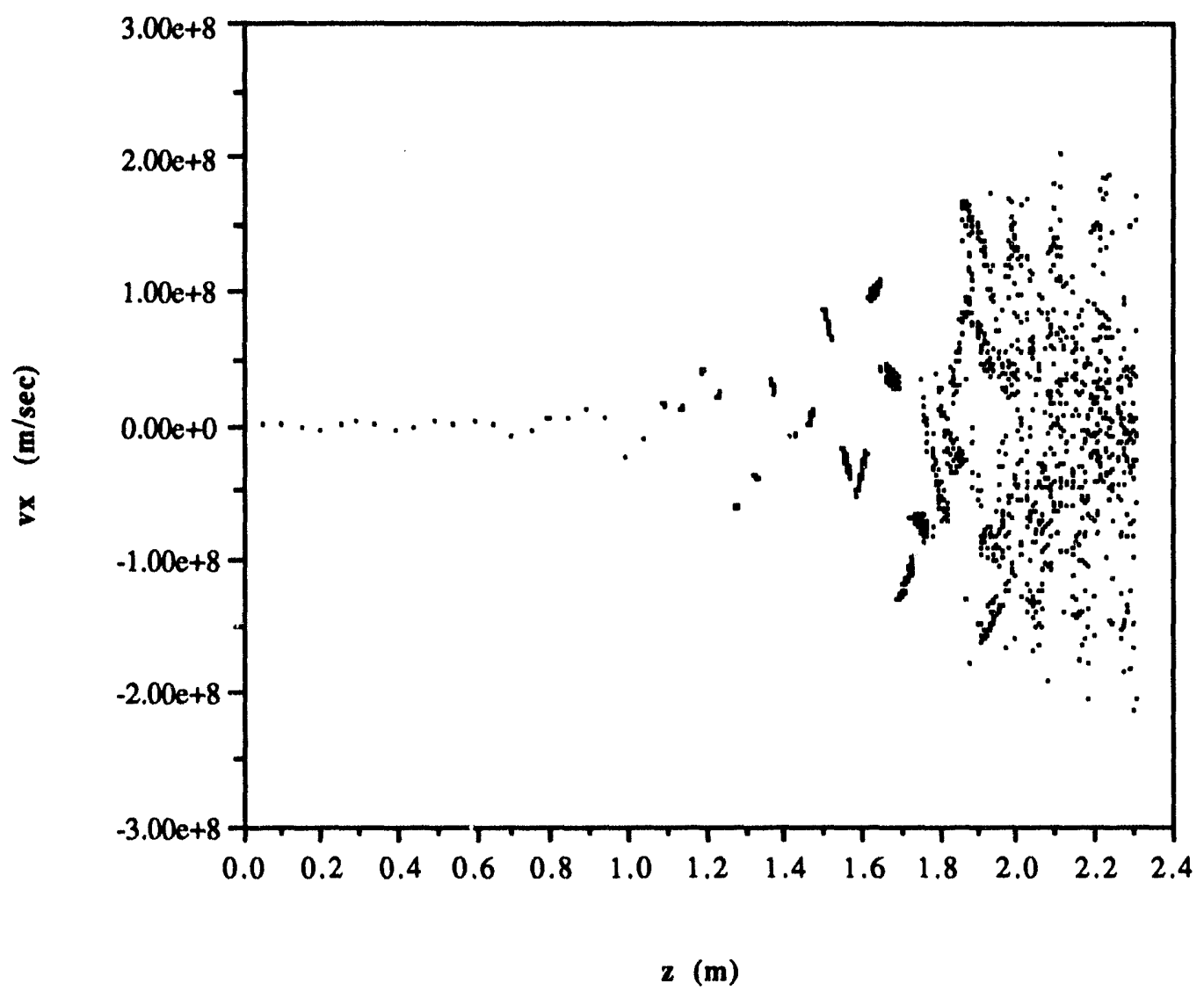

Figure 7.5. $X$-directed velocity as a function of longitudinal position. 


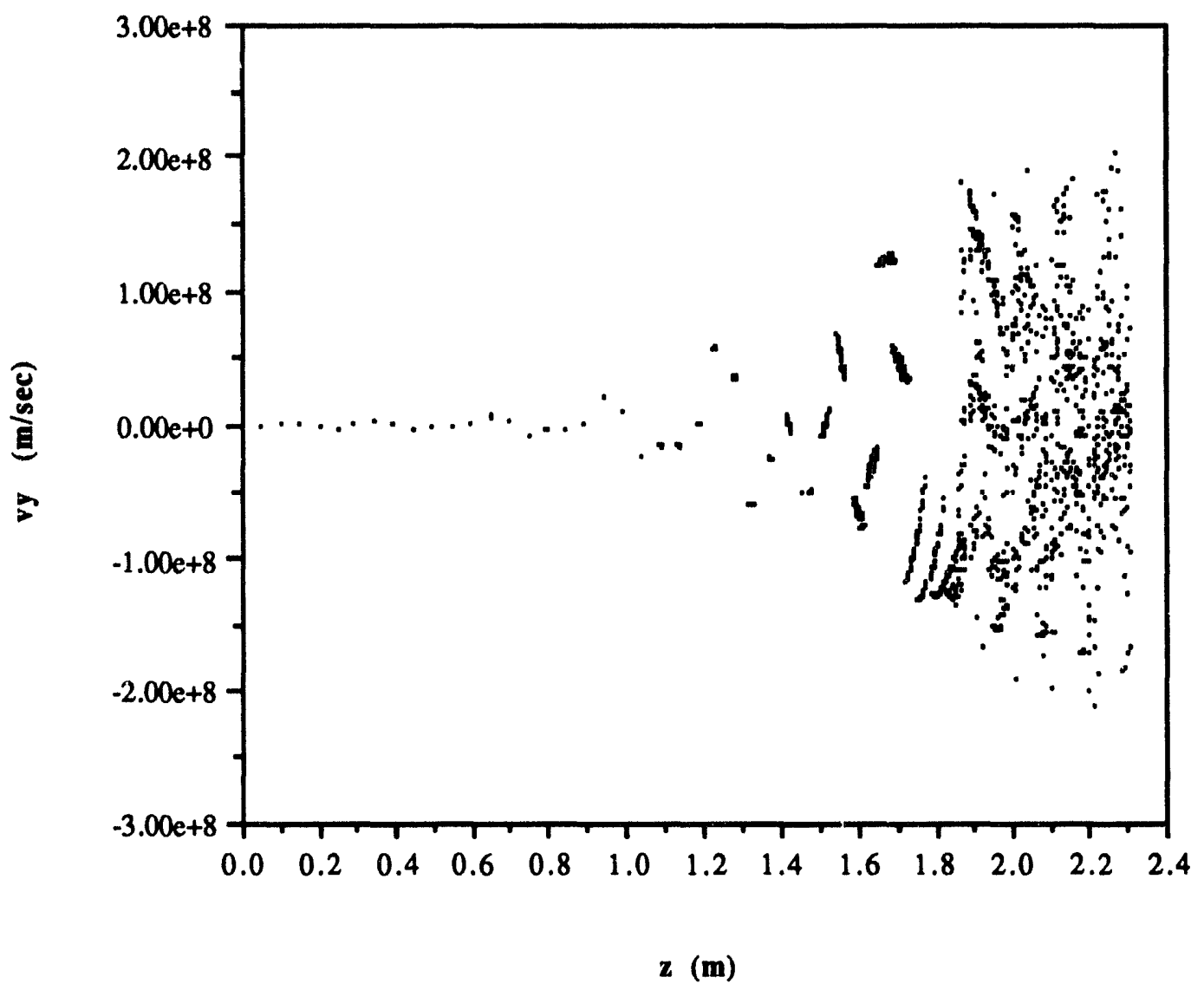

Figure 7.6. $Y$-directed velocity as a function of longitudinal position. 


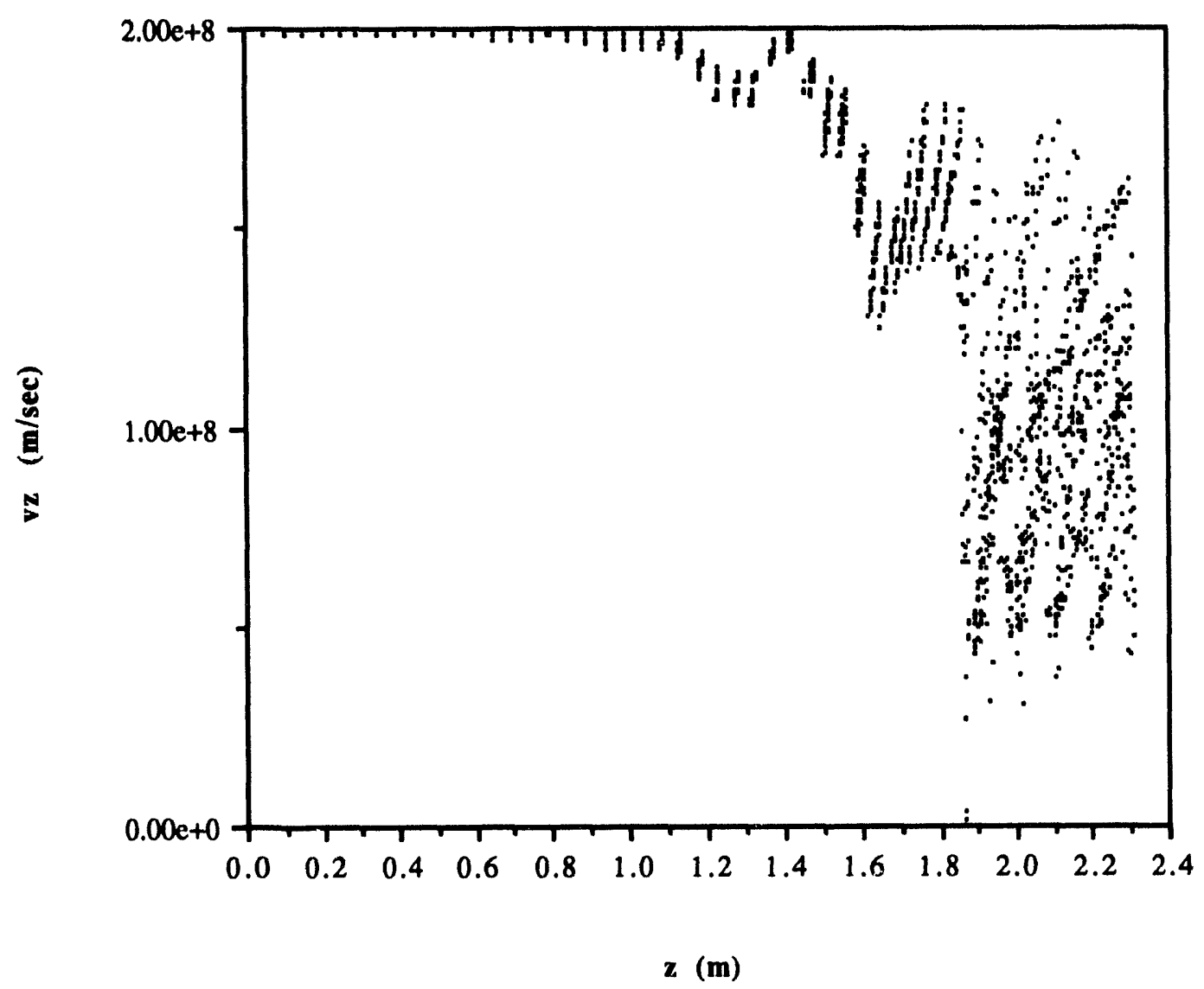

Figure 7.7. Z-directed veiocity spread of the electron cloud as a function of longitudinal position. 


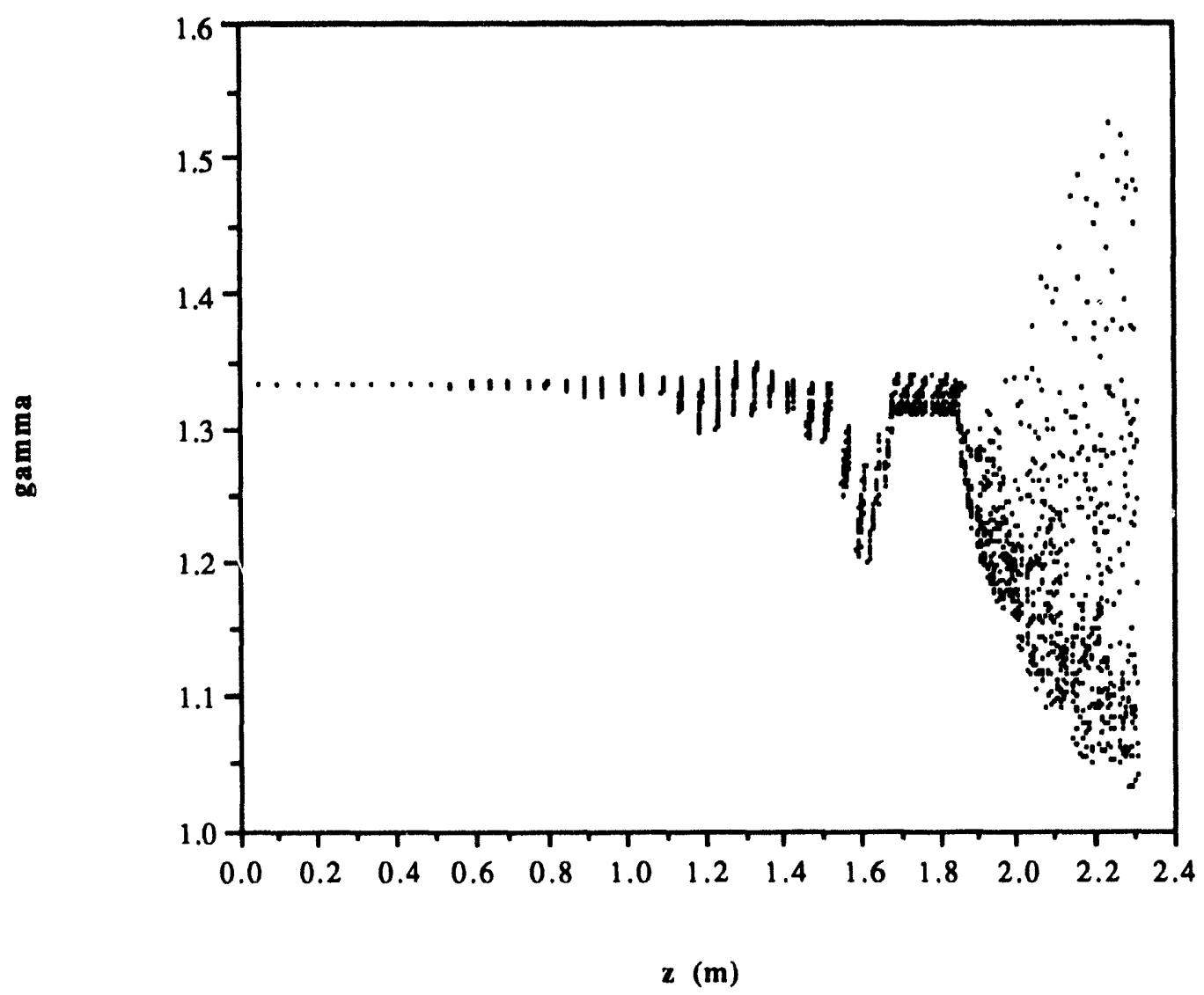

Figure 7.8. Gamma of electron cloud as a function of longitudinal position.

Figures 7.3 through 7.8 show the energy spread and positional spread of the electron beam as it moves through the magnicon. The energy spread caused by the halfcusp magnetic-field transition is apparent in all velocity plots and the plot of gamma. The beam is observed to be tightly bunched before entering the half-cusp, and it is almost impossible to distinguish the bunches after the half-cusp. Figure 7.8 also illustrates that some of the sample beams that comprise the finite-diameter beam actually gain energy in the output cavity. This is explained by the "mirroring" effect. The beam mirrors itself over a finite length in $z$ and, in the process, traverses a region of the rf fields near the output-cavity entrance where the transverse velocity is increased before the beam leaves the output cavity by the entrance hole or impacts on the cavity walls. 


\subsection{Efficiency degradation in uniform-field magnicon}

The results of the finite-beam-size modeling of the uniform-field magnicon are presented below. The parameters of the model are identical to those of the rigid-beam model of the previous chapter (Section 6.4), and the self-consistent solutions from the rigid-beam model are used by the finite-beam model. Table 7.2 summarizes the results of the finite-beam-size modeling. The first column shows beam diameter and the second efficiency.

Table 7.2. Relationship between beam size and efficiency.

\begin{tabular}{|c|c|}
\hline Beam Diameter (m) & Efriciency \\
\hline$\sim 0.000$ & 87.9 \\
\hline 0.001 & 87.6 \\
\hline 0.002 & 86.6 \\
\hline 0.003 & 85.6 \\
\hline 0.004 & 85.0 \\
\hline 0.005 & 84.6 \\
\hline 0.006 & 84.5 \\
\hline 0.007 & 83.7 \\
\hline 0.008 & 81.7 \\
\hline 0.009 & 80.1 \\
\hline 0.010 & 78.3 \\
\hline
\end{tabular}

From the table, it is observed that a beam diameter of $1.0 \mathrm{~cm}$ will provide a significant improvement over the state-of-the-art 67.0-to-69.0\%-efficient klystrons.

Figures 7.9 through 7.14 illustrate the trajectory and velocities of the electron cloud as it moves through the magnicon. These plots are for a beam diameter of $1.0 \mathrm{~cm}$. Figures 7.9 and 7.10 illustrate the trajectory of the electron cloud as it moves through the magnicon. Figures $7.11,7.12$, and 7.13 illustrate the $x-, y-$, and $z$-directed velocity components as a function of longitudinal position. Figure 7.14 illustrates the gamma of the electron cloud as a function of longitudinal position 


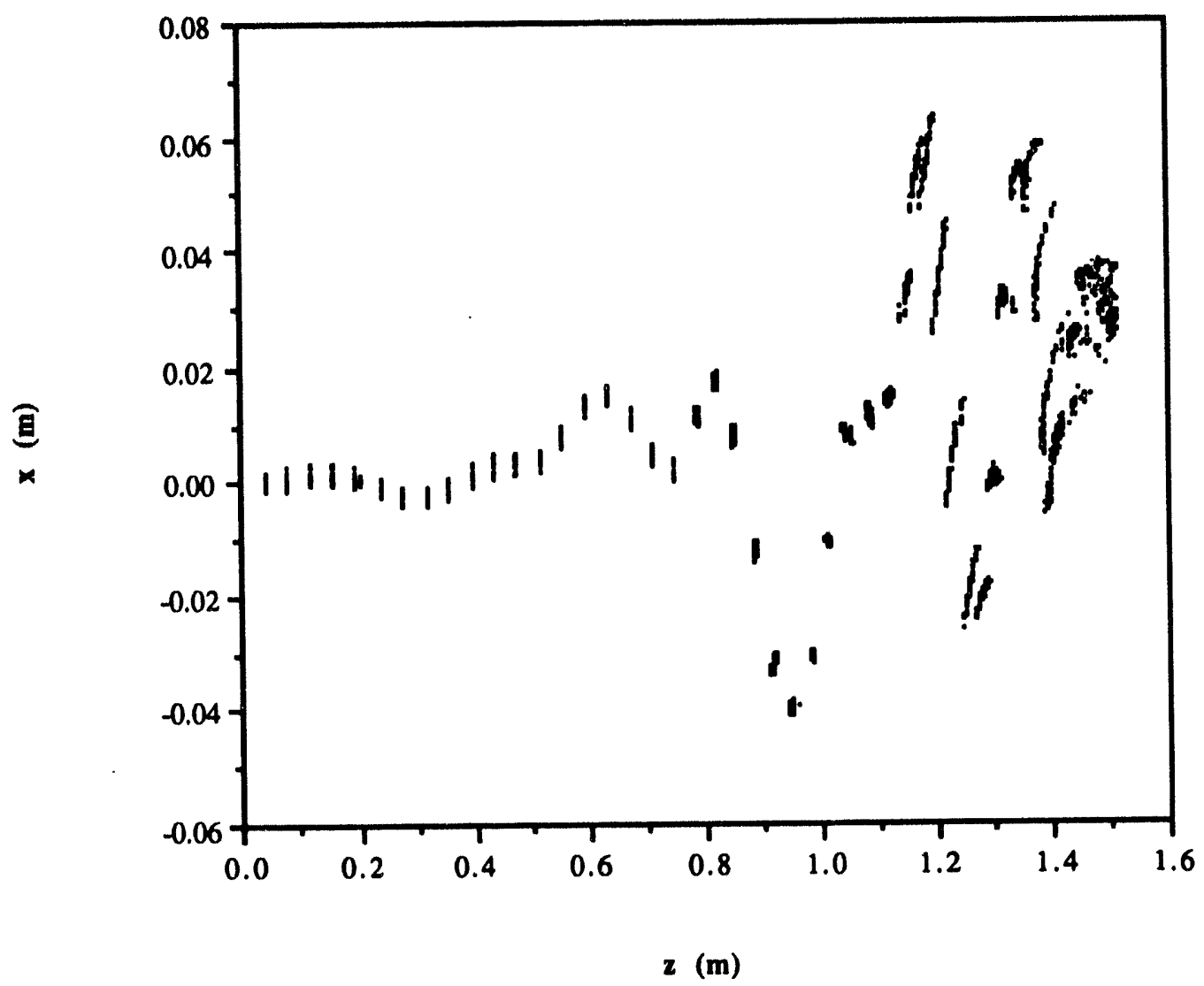

Figure 7.9. $X$-axis location of electron cloud as a function of longitudinal position. 


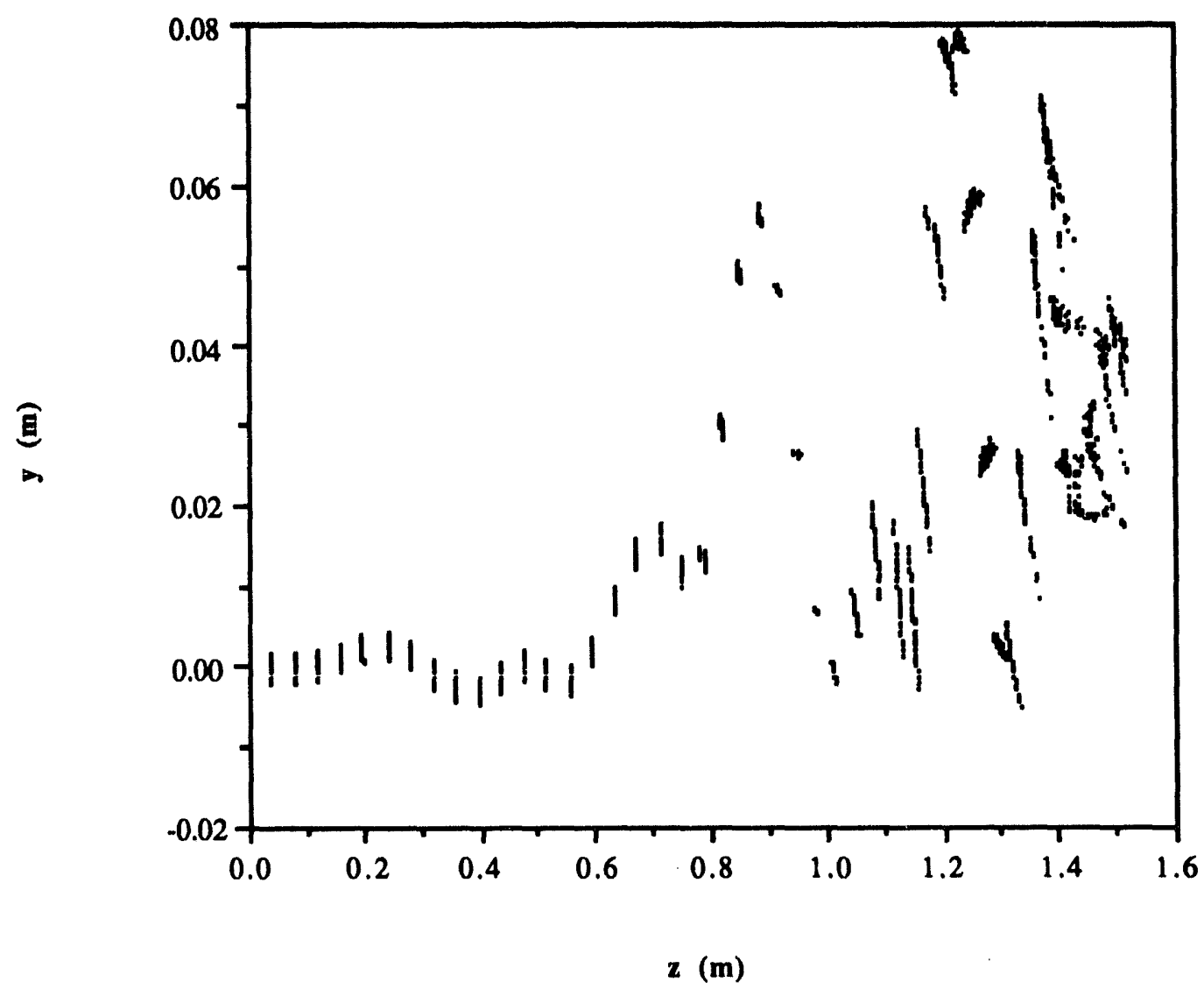

Figure 7.10. $Y$-axis location of electron cloud as a function of longitudinal position. 


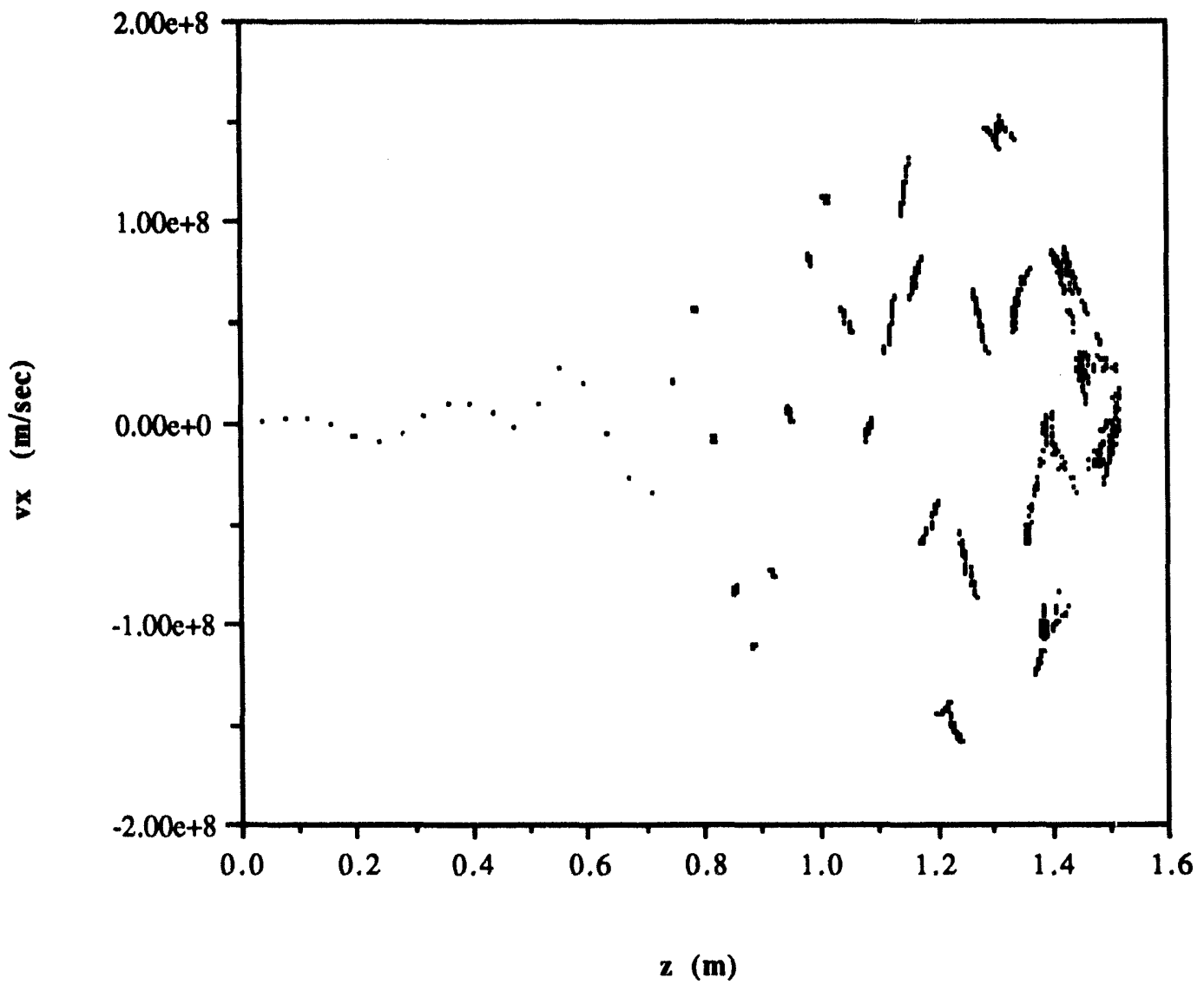

Figure 7.11. $X$-directed velocity as a function of longitudinal position. 


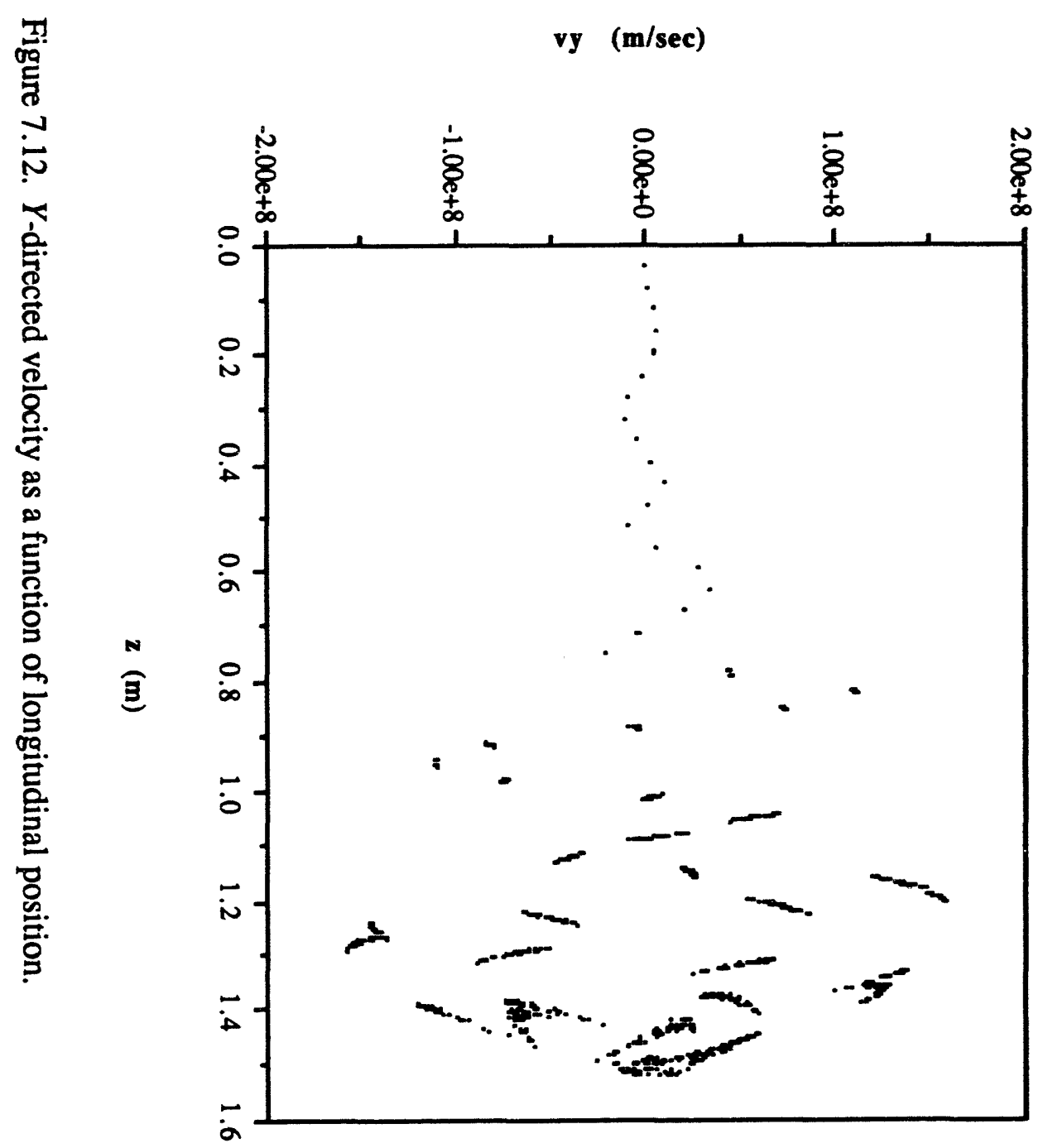

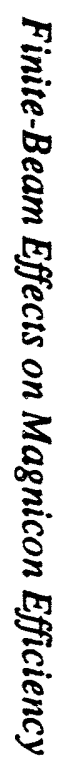




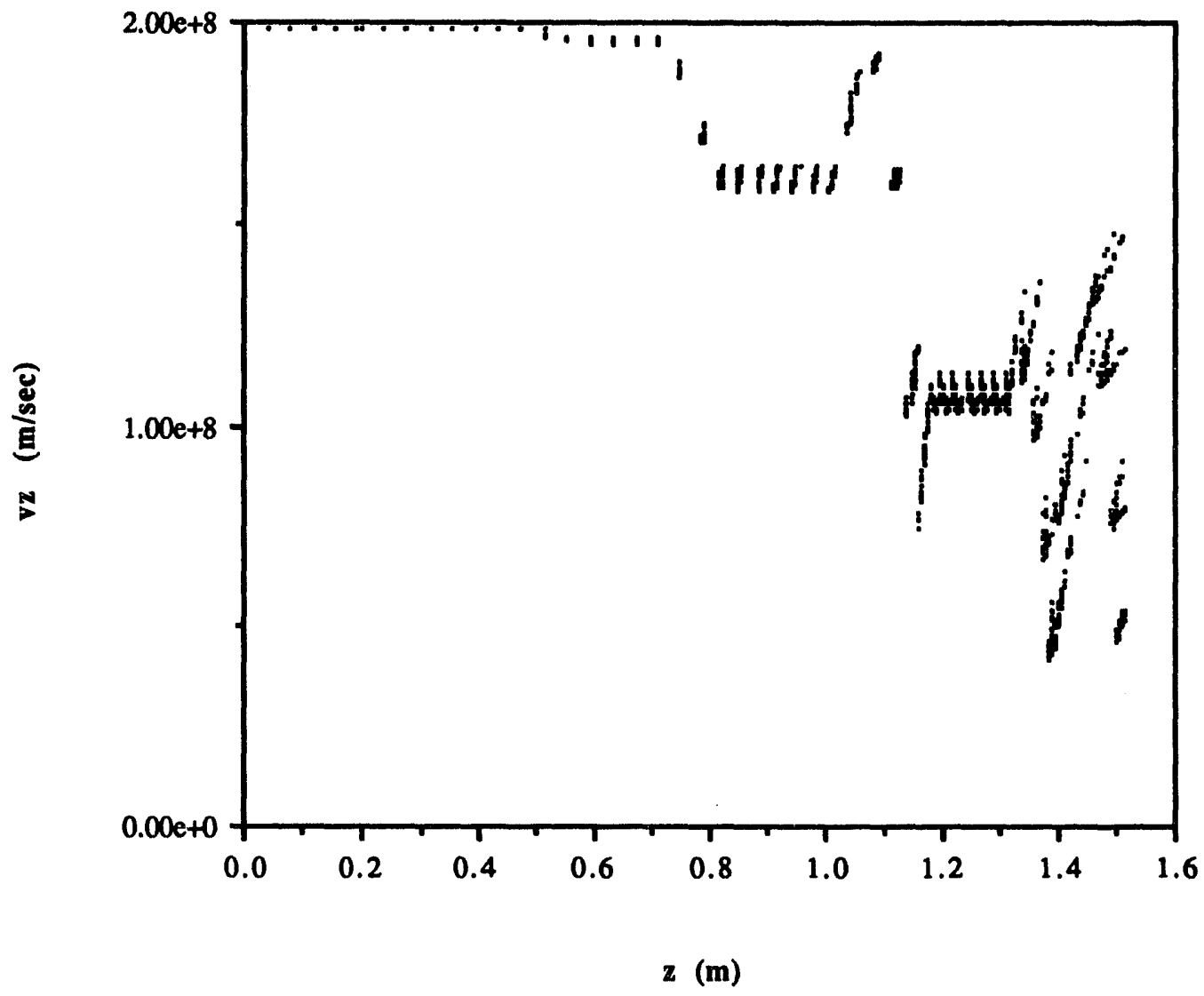

Figure 7.13. Z-directed velocity spread of the electron cloud as a function of longitudinal position. 


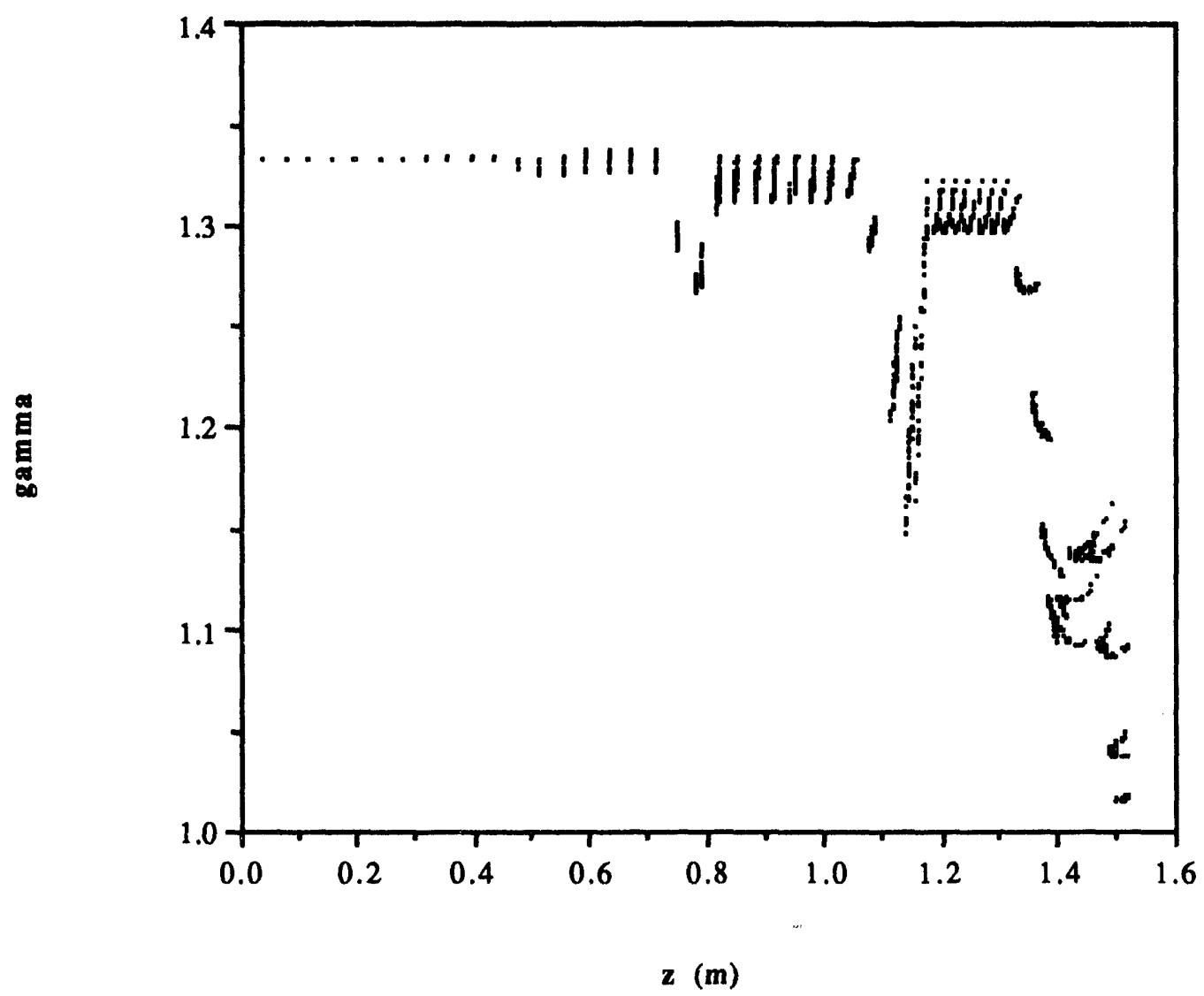

Figure 7.14. Gamma of electron cloud as a function of longitudinal position.

Figures 7.9 through 7.14 show the energy spread and positional spread of the electron beam as it moves through the magnicon. One very important characteristic is illustrated by Figs. 7.13 and 7.14. They show that even though there is some degree of spread in spent-electron energy, the electrons are still tightly grouped, which means that a depressed-collector design would be workable and would further increase magnicon efficiency. This phenomenon of tightly bunched electrons is most unusual for a highefficiency deflection- or velocity-modulated electron device, which generally creates a broad energy spread in the spent beam.

Section 7.1 indicated a numerical example would be provided to substantiate the choice for the beam quantization. To provide this support, the finite-beam model was used to determine the efficiency for a $1-\mathrm{cm}$ beam diameter. (The magnicon-structure 
model is that of the uniform-field magnicon described above.) The beam was quantized as described in Section 7.1 using four rings to subdivide the area of the beam. An additional small rigid beam was assigned as the center coordinate. Table 7.3 summarizes the results.

Table 7.3 displays three columns. The first represents the number of rigid beams used to model the finite-diameter beam. The second is the number of rigid beams assigned to each of the four rings. The third column shows the resulting efficiency.

Table 7.3. Effect of the number of rigid beams used to quantize the finite diameter beam for a $1-\mathrm{cm}$ beam diameter.

\begin{tabular}{|c|c|c|}
\hline $\begin{array}{c}\text { Total Number of } \\
\text { Rigid Beams }\end{array}$ & $\begin{array}{c}\text { Number of Rigid } \\
\text { Beams per Ring }\end{array}$ & Efficiency \\
\hline 49 & 12 & 78.3 \\
\hline 45 & 11 & 78.0 \\
\hline 41 & 10 & 77.8 \\
\hline 37 & 9 & 78.3 \\
\hline 33 & 8 & 78.0 \\
\hline 29 & 7 & 77.8 \\
\hline 25 & 6 & 77.8 \\
\hline 21 & 5 & 77.5 \\
\hline 17 & 4 & 78.1 \\
\hline 13 & 3 & 77.6 \\
\hline 9 & 2 & 75.9 \\
\hline
\end{tabular}

Table 7.3 shows that a quantization in excess of 33 rigid beams provides results where the efficiency varies only slightly with increased quantization. Therefore, a quantization level of 49 rigid beams certainly provides an adequate quantization interval.

\subsection{Results of modeling finite-beam effects}

The results of Sections 7.2 and 7.3 demonstrate the capability of the uniform-field magnicon to operate at a higher efficiency for larger-diameter beams than the conventional magnicon. However, for these results to be meaningful they must be evaluated in terms of the matched beam current that the solenoidal field for each magnicon architecture can support. From Ref. [4], the conditions for matched beam currents for electron beams with zero admittance are described as 


$$
I=\left[\frac{\pi \varepsilon e c}{2 m_{0}}\right](\beta \gamma)\left(B_{o} R\right)^{2}
$$

For the uniform-field magnicon geometry, the matched beam diameter for $18 \mathrm{~A}$ is 1.14 $\mathrm{cm}$; and for the standard magnicon geometry described Section 7.2, the matched beam diameter is $0.7 \mathrm{~cm}$. For any comparison to be physically significant, the efficiency of the two magnicon geometries should be compared at these beam diameters. At these diameters the uniform-field magnicon efficiency is $75.6 \%$ while the conventional magnicon efficiency is only $54.5 \%$.

Part of the rationale for neglecting space-charge forces in the modeling was the assumption that the beam current would be matched. In other words, the focusing force on the beam envelope would be balanced by the space-charge force. However, in the uniform-field magnicon this balance will only exist in the deflection system before the rf forces spread the beam, increasing its radius in the output cavity. Additional beamgenerated forces can be experienced by the beam whenever it is off-axis. Even so, these forces and their performance implications can be ignored for the following reason.

The force on a displaced filamentary beam due to the image current in the beam pipe can be calculated from Ref. [5] as

$$
F_{w} \cong\left[\frac{e I}{2 \pi \varepsilon r_{w}^{2} \beta \gamma^{2} c}\right] \delta r,
$$

where $\delta r$ is the distance off-axis and $r_{w}$ is the radius of the beam pipe (for a largest force calculation). A representative worst-case number for either magnicon architecture, assuming $\delta r$ as $0.5 \mathrm{~cm}$, is $2.95 \times 10^{-14} \mathrm{~N}$ in the radial direction. In contrast, assuming a radial velocity of $1.0 \times 10^{8} \mathrm{~m} / \mathrm{s}$-and a velocity much larger than this would actually be required to achieve the displacement assumed in the above calculation-the transverse force on the particle due to the solenoid focusing field would be $4.7 \times 10^{-13} \mathrm{~N}$, or a factor of 15.8 over the force caused by the wall. (This calculation was based on the focusing field of the uniform-field magnicon, which provides the weaker focusing.)

A problem unique to conventional magnicon geometry is that the beam must cross a magnetic-field-free drift space between the deflection system and output cavity. In this region the beam is free to expand as a result of space-charge forces, unhindered by a solenoid field. This means that if the drift space were $10 \mathrm{~cm}$ long, and the beam entering 
it had an initial beam diameter equal to the matched beam diameter described above $(0.7$ $\mathrm{cm}$ ), the radius of its beam envelope would increase from the $0.35 \mathrm{~cm}$ matched radius to $0.73 \mathrm{~cm}$ radius at the input to the output cavity. This calculation is based on the equations of Ref. [6]. This beam expansion represents approximately a 100\% growth in beam radius and would make an already-bad situation worse. In addition, the velocity spread caused by the interaction with this larger beam would greatly decrease efficiency below the low number presented above for the conventional magnicon geometry (Ref. [7]).

Finally, in the output cavity, the condition of matched beam current no longer exists, because the if fields have spread the beam to a large extent, as can be seen from the plots in Sections 7.2 and 7.3. These plots show that the beam diameter approaches 2 $\mathrm{cm}$. For a beam of this diameter, the space-charge radial force is approximately $1.47 \mathrm{x}$ $10^{14} \mathrm{~N}$, as given in Ref. [4]. In contrast, the radial force due to the solenoid field is a factor of 50 larger. In addition, if we treat the beam in the output cavity as a beam generated from an immersed cathode, then the ratio of the minimum to maximum envelope radius would be 1.2 , as calculated from Ref. [8]. This variation would have only a slight impact on efficiency because the average radius would be approximately 1.1 times the minimum radius, and the change in radial velocity due to this variation would be small compared with the radial velocity changes of the beam due to interactions with the rf fields. Summarizing, space-charge forces in the output cavity can be neglected because the beam is admittance dominated. 


\section{Conclusions}

The primary contribution of this work to deflection-modulated amplifier knowledge is the creation of a new magnicon architecture having increased dc-to-rf conversion efficiency for large diameter beams - in other words, a higher-perveance magnicon. This new magnicon architecture is called the uniform-field magnicon because it uses a constant-value magnetic focusing field along the entire length of the magnicon. A design for this magnicon is proposed and ready for experimental verification.

Through modeling, I was able to demonstrate a geometry capable of achieving an efficiency of $78 \%$ for a $1-\mathrm{cm}$ diameter beam at $700 \mathrm{MHz}$. In contrast, the conventional magnicon architecture I modeled, which uses a half-cusp magnetic transition between the deflection system and output cavity, achieved an efficiency of less than $50 \%$ under similar conditions. The primary reason for the increased efficiency of the uniform-field magnicon was the removal of this half-cusp magnetic-field transition.

As an intermediate step to generating the uniform-field magnicon geometry and optimizing the conventional magnicon geometry, I conducted exhaustive numerical modeling of the individual magnicon components (drive cavity, simple passive cavity, coupled passive cavity, magnetic-transition region, and output cavity). The results of this modeling are presented in this dissertation in a format intended to be used as a tool to generate magnicon designs. Sufficient detail is provided to permit, through the interpolation of presented data, design studies for magnicons over a range of operating conditions and performance specifications. The research also provides design rules for magnicon deflection-system design.

Two modeling techniques were used to generate the beam-driven cavity fields required for modeling magnicon performance. One technique, an energy-balance approach, was used for the bulk of the modeling. The energy-balance approach equates the energy given up by the beam with the energy required by the walls to maintain the cavity fields. The convergence of this approach to the self-consistent field solution was quite rapid and therefore became the method of choice. The weakness of the approach was that the self-consistent field solution was strictly a resonant solution. Evaluation of the off-resonant magnicon performance is not possible with the energy-balance approach to self-consistent solution. 
Because the desired magnicon bandwidth is an important parameter in determining both focusing-field strength and cavity length, it was necessary to make a calculation of the beam-loaded Q's of the magnicon's passive cavities. (Because the drive and output cavity are both externally loaded, the beam-loaded Q's of the passive cavities establish magnicon bandwidth.) In order to calculate the beam-loaded $Q$, a modeling technique known as the induced-current model was used. It calculated the beam-loaded $Q$ of the rotating modes in the cylindrical cavities. The induced-current method represents a new modeling technique for beam-driven rotating-mode cavities and for calculating the beam-loaded $Q$ in these cavities. (The technique has been used in the past to calculate beam loading in standing-wave cavities-like klystron cavities--but was adapted here and modified for application to rotating-mode cavities.) The modification involved the decomposition of the rotating mode into its standing-wave components and then calculation of individual solutions for each component mode.

In order to validate the model, the following steps were taken:

1. It was compared to the small-signal modeling results.

2. It was compared to the experimental and theoretical results of the NRL magnicon, which operates at approximately the same generalized perveance as the magnicon investigated in this work.

3. It was compared to magnicon inventor Oleg Nezhevenko's proposed highperveance designs, which use the same beam voltage and current as the magnicon investigated here.

4. It was compared to the self-consistent solutions for cavity fields generated through induced-current modeling and the energy-balance technique.

5. The numerically calculated cyclotron frequency was compared to the analytical cyclotron frequency.

6. The analytical half-cusp model was compared to the POISSON-calculated half-cusp model.

In all six cases these comparisons resulted in excellent agreement and, therefore, verify the accuracy of the numerical model. 
The modeling and results presented in the paper focused on a 700-MHz magnicon with a beam voltage of $170 \mathrm{kV}$ and a beam current of $18 \mathrm{~A}\left(2.6 \times 10^{-7}\right.$ pervs). These selected parameters were based on the interest of Los Alamos National Laboratory, the funding agency for this work. The Laboratory is interested in pursuing the magnicon as a high-efficiency source of $\mathrm{CW}$, if power for future particle-accelerator applications. 


\section{Appendix A}

\section{A.1 Particle-pushing algorithm}

The numerical calculation of particle orbits uses a difference method, which is known as a time-centered method and is described in Ref. [A1]. The time-centered approach uses the position and velocity of the particles at time, $t$, to calculate the forces acting on the particles. These forces are used to advance the position of the particle half of a time step, $\Delta t / 2$. At the particle positions corresponding to $t+\Delta t / 2$ the forces are again calculated, and these time-centered forces are used to advance the particle positions and velocities at time, $i$, a full time step to $t+\Delta t$. The particle's position and velocity is advanced according to the following equations:

For the half-step

$$
\begin{aligned}
& x\left(t+\frac{\Delta t}{2}\right)=x(t)+v_{x}(t) \frac{\Delta t}{2}, \\
& y\left(t+\frac{\Delta t}{2}\right)=y(t)+v_{y}(t) \frac{\Delta t}{2}, \\
& z\left(t+\frac{\Delta t}{2}\right)=z(t)+v_{z}(t) \frac{\Delta t}{2}, \\
& v_{x}\left(t+\frac{\Delta t}{2}\right)=v_{x}(t)+\frac{d v_{x}(t)}{d t} \frac{\Delta t}{2}, \\
& v_{y}\left(t+\frac{\Delta t}{2}\right)=v_{y}(t)+\frac{d v_{y}(t)}{d t} \frac{\Delta t}{2},
\end{aligned}
$$

and

$$
v_{2}\left(t+\frac{\Delta t}{2}\right)=v_{z}(t)+\frac{d \nu_{2}(t)}{d t} \frac{\Delta t}{2}
$$

For the full-step

$$
\begin{aligned}
& x(t+\Delta t)=x(t)+v_{x}\left(t+\frac{\Delta t}{2}\right) \Delta t, \\
& y(t+\Delta t)=y(t)+v_{y}\left(t+\frac{\Delta t}{2}\right) \Delta t,
\end{aligned}
$$




$$
\begin{aligned}
& z(t+\Delta t)=z(t)+v_{z}\left(t+\frac{\Delta t}{2}\right) \Delta t, \\
& v_{x}(t+\Delta t)=v_{x}(t)+\frac{d v_{x}\left(t+\frac{\Delta t}{2}\right)}{d t} \Delta t, \\
& v_{y}(t+\Delta t)=v_{y}(t)+\frac{d v_{y}\left(t+\frac{\Delta t}{2}\right)}{d t} \Delta t,
\end{aligned}
$$

and

$$
v_{z}(t+\Delta t)=v_{z}(t)+\frac{d v_{z}\left(t+\frac{\Delta t}{2}\right)}{d t} \Delta t
$$

The advantage of the time-centered method is that it eliminates first-order errors. The positions and velocities calculated in Eqs. (A.1) through (A.12) can then be used to leapfrog position and velocity to $t+3 \Delta t / 2$ for the next advance.

\section{A.2 Bessel function algorithms}

Algorithms for the calculation of Bessel functions of the first kind, $J_{O}(x)$ and $J_{1}(x)$, are given in Ref. [A2] as follows:

for $-3 \leq x \leq 3$,

$$
\begin{gathered}
J_{0}(x)=1-2.2499997(x / 3)^{2}+1.2656208(x / 3)^{4}-.3163866(x / 3)^{6}+.0444479(x / 3)^{8} \\
-.0039444(x / 3)^{10}+.0002100(x / 3)^{12}+\varepsilon
\end{gathered}
$$

where

$$
|\varepsilon|<5 \times 10^{-8}
$$

and for $3 \leq x \leq \infty$,

$$
J_{0}(x)=x^{-\frac{1}{2}} f_{0}(x) \cos \theta_{0}(x)
$$

where 


$$
\begin{gathered}
f_{0}(x)=.79788456-.00000077(3 / x)-.00552740(3 / x)^{2}-.00009512(3 / x)^{3}+.00137237(3 / x)^{4} \\
-.00072805(3 / x)^{5}+.00014476(3 / x)^{6}+\varepsilon
\end{gathered}
$$

with

$$
|\varepsilon|<1.6 \times 10^{-8}
$$

and where

$$
\begin{gathered}
\theta_{0}(x)=x-.78539816-.04166397(3 / x)-.00003954(3 / x)^{2}+.00262573(3 / x)^{3} \\
-.00054125(3 / x)^{4}-.00029333(3 / x)^{5}+.00013558(3 / x)^{6}+\varepsilon
\end{gathered}
$$

with

$$
|\varepsilon|<7 \times 10^{-8} \text {. }
$$

$J_{l}(x)$ is determined, for $-3 \leq x \leq 3$, from

$$
\begin{gathered}
x^{-1} J_{1}(x)=\frac{1}{2}-.56249985(x / 3)^{2}+.21093573(x / 3)^{4}-.03954289(x / 3)^{6} \\
+.00443319(x / 3)^{8}-.00031761(x / 3)^{10}+.00001109(x / 3)^{12}+\varepsilon,
\end{gathered}
$$

where

$$
|\varepsilon|<1.3 \times 10^{-8}
$$

and for $3 \leq x \leq \infty$, from

$$
J_{1}(x)=x^{-\frac{1}{2}} f_{1}(x) \cos \theta_{1}(x),
$$


where

$$
\begin{gathered}
\theta_{1}(x)=x-2.35619449+.12499612(3 / x)+.00005650(3 / x)^{2}-.00637879(3 / x)^{3} \\
+.00074348(3 / x)^{4}+.00079824(3 / x)^{5}-.00029166(3 / x)^{6}+\varepsilon
\end{gathered}
$$

with

$$
|\varepsilon|<9 \times 10^{-8}
$$

and where

$$
\begin{gathered}
f_{1}(x)=.79788456+.00000156(3 / x)+.01659667(3 / x)^{2}+.00017105(3 / x)^{3} \\
-.00249511(3 / x)^{4}+.00113653(3 / x)^{5}-.00020033(3 / x)^{6}+\varepsilon,
\end{gathered}
$$

with

$$
|\varepsilon|<4 \times 10^{-8}
$$

\section{A.3 Lorentz force algorithms}

The Lorentz force algorithm implements a relativistic form of the force equations.

The equations, derived in Chapter 3, are given in Eqs. (3.85) through (3.88) as

$$
\begin{aligned}
& \frac{d v_{x}}{d t}=-\frac{e}{m m}\left(v_{y} B_{z}-v_{z} B_{y}-\frac{v_{x} v_{z} E_{z}}{c^{2}}\right), \\
& \frac{d v_{y}}{d t}=-\frac{e}{m m}\left(v_{z} B_{x}-v_{x} B_{z}-\frac{v_{y} v_{z} E_{z}}{c^{2}}\right), \\
& \frac{d v_{z}}{d t}=-\frac{e}{m m}\left(E_{z}+v_{x} B_{y}-v_{y} B_{x}-\frac{v_{z}^{2} E_{z}}{c^{2}}\right),
\end{aligned}
$$

and

$$
\frac{d x}{d t}=-\frac{e}{m^{2} c} v_{z} E_{3} .
$$




\section{A.4 Coupled-cavity equivalent-circuit model}

Figure A.1 shows an equivalent-circuit series-resonant model for the two cylindrical cavities that make up the coupled cavities and a equivalent-circuit model for the coupled cavities.

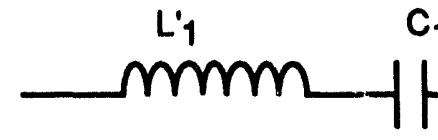

Cavity 1

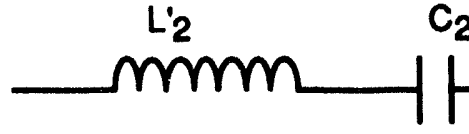

Cavity 2

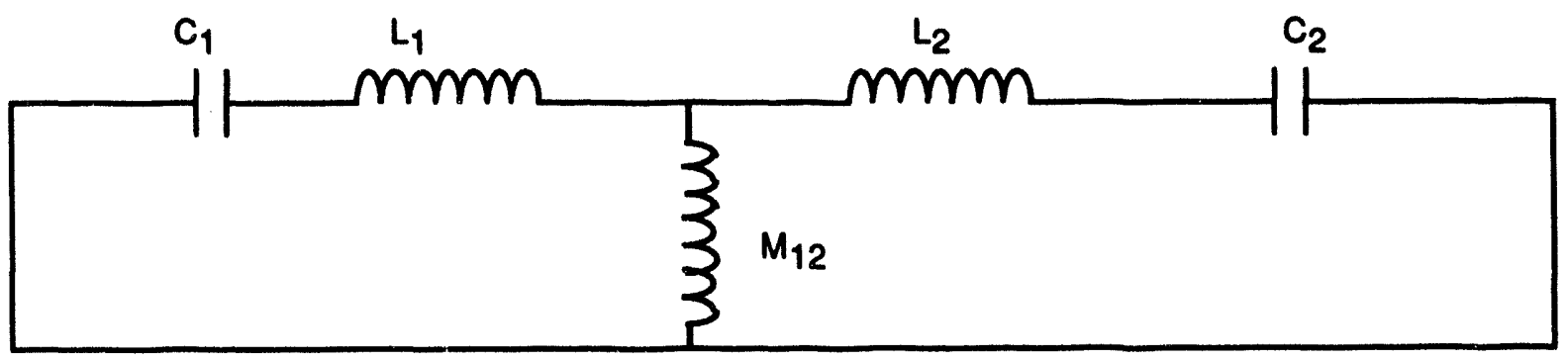

Cavities coupled together

Figure A.1 Equivalent-circuit models.

The development presented below makes use of the definitions

$$
\begin{aligned}
& L_{1}=L_{1}^{\prime}-M_{12}, \\
& L_{2}=L_{2}-M_{12}, \\
& \omega_{1}^{\prime}=\frac{1}{\sqrt{L_{1} C_{1}}},
\end{aligned}
$$

and

$$
\omega_{2}^{\prime}=\frac{1}{\sqrt{L_{2}^{\prime} C_{2}}} .
$$


The circuit equations for the coupled cavity are

$$
\left(j \omega L_{1}+\frac{1}{j \omega C_{1}}\right) i_{1}-j \omega M_{12} i_{2}=0
$$

and

$$
-j \omega M_{12} i_{1}+\left(j \omega L_{2}^{\prime}+\frac{1}{j \omega C_{2}}\right) i_{2}=0 .
$$

These equations can be rewritten as

$$
\left(1-\frac{\omega_{1}^{\prime 2}}{\omega^{2}}\right) i_{1}-\frac{M_{12}}{L_{1}} i_{2}=0
$$

and

$$
-\frac{M_{12}}{L_{2}} \dot{i}_{1}+\left(1-\frac{\omega_{2}^{\prime 2}}{\omega^{2}}\right) i_{2}=0
$$

These equations are further simplified to

$$
\left(1-\frac{\omega_{1}^{\prime 2}}{\omega^{2}}\right) x_{1}-k_{12} \chi_{2}=0
$$

and

$$
-k_{12} x_{1}+\left(1-\frac{\omega_{2}^{\prime 2}}{\omega^{2}}\right) x_{2}=0
$$

where

$$
\begin{aligned}
& \chi_{1}=i_{1} \sqrt{L_{1}^{\prime}}, \\
& \chi_{2}=i_{2} \sqrt{L_{2}^{\prime}},
\end{aligned}
$$

and

$$
k_{12}=\frac{M_{12}}{\sqrt{L_{1}^{\prime} L_{2}^{\prime}}} .
$$

Simultaneous solution of these equations results in 


$$
\left(1-\frac{\omega_{1}^{\prime 2}}{\omega^{2}}\right)\left(1-\frac{\omega_{2}^{\prime 2}}{\omega^{2}}\right)-k_{12}^{2}=0
$$

Assuming the uncoupled circuits have the same resonant frequency, $\omega$ ', then

$$
\omega_{1}^{\prime}=\omega_{2}^{\prime}=\omega^{\prime}
$$

and

$$
1-\frac{\omega^{\prime 2}}{\omega^{2}}= \pm k_{12}
$$

Using this equation, the resonant frequency for the zero and $\pi$ mode are given by

$$
\omega_{0}=\frac{\omega^{\prime}}{\sqrt{1-k_{12}}}
$$

and

$$
\omega_{n}=\frac{\omega^{\prime}}{\sqrt{1+k_{12}}}
$$

To verify that these equations are the zer - modes, they are substituted back into the original equations, resulting in

$$
i_{1} k_{12}=\frac{M_{12}}{L_{1}} i_{2} \text { (zero mode) }
$$

and

$$
-i_{1} k_{12}=\frac{M_{12}}{L_{1}^{\prime}} i_{2}(\pi \text { mode })
$$




\section{References}

\section{Chapter 1}

[1] N.M. Karliner et al., "The Magnicon-An Advanced Version of the Gyrocon," Nuclear Instruments and Methods in Physics Research A269, 459-473 (1988).

[2] Private conversation with George Lawrence, Accelerator Transmutation of Waste Program Manager, Los Alamos National Laboratory, February 1992.

[3] D. Rees, B. Carlsten, S. Humphries, Jr., and P. Tallerico, "Calculation of Beam Loading Using the Induced-Current Method in Passive Cavities," IEEE Trans. on Electron Devices 40, 543-1548 (1993).

\section{Chapter 2}

[1] H. Frischholz, "The LEP Main Ring High-Power RF System," in Proc. of the 1989 IEEE Particle Accelerator Conference, vol. 2, pp. 1131-1133.

[2] G.I. Budker et al., U.S. Patent No. 3,885,193 (1975).

[3] N.M. Karliner et al., "The Magnicon-An Advanced Version of the Gyrocon," Nuclear Instruments and Methods in Physics Research A269, 459-473 (1988).

[4] B. Hafizi et al., "Analysis of the Deflection System for a Magnetic-Field-Immersed Magnicon Amplifier," IEEE Trans. Plasma Sci., 20 (1992).

[5] Wallace M. Manheimer, "Theory and Conceptual Design of a High-Power, Highly Efficient Magnicon at 10 and $20 \mathrm{GHz}$," IEEE Trans. on Plasma Sci. 18 (1990).

[6] O.A. Nezhevenko, Ph.D. thesis, Institute for High-Energy Physics, Novosibirsk, Russia (1971).

[7] G.I. Budker et al. "The Gyrocon-An Efficient, Relativistic, High-Power VHF Generator," Particle Accelerators 10, $41-59$ (1979).

[8] V.E. Balakin et al., in Proc. 10th SVPAC, vol. 1, pp. 277-280 (1987).

[9] S.H. Gold et al., "The NRL 11.4 GHz Magnicon Amplifier Experiment," to be published in Proc. 1992 Bearrs Conf.

[10] B. Hafizi et al., "Analysis of the Deflection System for a Magnetic-Field-Immersed Magnicon Amplifier," IEEE Trans. Plasma Sci. 20 No.3, p. 232 (1992).

[11] O.A. Nezhevenko, "The Magnicon-A New RF Power Snurce for Accelerators," in Conference Rocord-1991 IEEE Particle Accelerator Conference, L. Lizama and J. Chew, Eds. (IEEE, New York), pp. 2933-2942 (1991).

\section{Chapter 3}

[1] C.A. Balanis, Advanced Engineering Electromagnetics (John Wiley and Sons, New York, 1989), pp. 471-499. 
[2] Victor L. Granatstein and Igor Alexeff, High-Power Microwave Sources (Artech House, Boston, 1987), p. 117.

[3] J.D. Jackson, Classical Electrodynamics, 2nd ed. (John Wiley and Sons, New York, 1975), pp. 121-127.

[4] Stanley Humphries, Jr., Charged Particle Beams (John Wiley and Sons, New York, 1990), pp. 110-113.

\section{Chapter 4}

[1] R.E. Collin, Foundations for Microwave Engineering (McGraw-Hill, New York, 1966), p. 376.

[2] Stanley Humphries, Jr., "Numerical Studies of the Scantron Microwave Amplifier," to be published in IEEE Trans. on Plasma Sci.

[3] J.W. Gewartowski and H.A. Watson, Principles of Electron Tubes (D. Van Nostrand Company, Inc., New York, 1965), pp. 183-188.

[4] M. Chodorow and C. Sussking, Fundamentals of Microwave Electronics (McGrawHill, New York, 1964), pp. 68-70.

[5]. B.E. Carlsten, A Self-Consistent Numerical Analysis of Klystrons Using LargeSignal Beam-Wave Interaction Simulations, Ph.D thesis, Stanford University (1985), pp. 251-261.

[6] R.E. Collin, Foundations for Microwave Engineering (McGraw-Hill, New York, 1966), pp. 351-359.

\section{Chapter 5}

[1] C.A. Balanis, Advanced Engineering Electromagnetics (John Wiley and Sons, New York, 1989), p. 376.

[2] Reference Data for Radio Engineers, 4th ed. (International Telephone and Telegraph Corporation, New York, 1956), pp. 1006-1007.

[3] Conversation with Dr. Stephen Gould, Naval Research Laboratory, Washington, D.C., May 1993.

[4] Private correspondence with Dr. Paul Tallerico, Los Alamos National Laboratory, July, 1993.

- [5] Stanley Humphries, Jr., Principles of Charged Particle Accelerators (John Wiley and Sons, New York, 1986), p. 55. 


\section{Chapter 6}

[1] S.H. Gold et al., "Study of Gain in C-Band Deflection Cavities for a FrequencyDoubling Magnicon Amplifier," Naval Research Laboratory, NRL/MR/4793-93-7197 (1993).

[2] Reference Manual for the POISSON/Superfish Group of Codes, Los Alamos National Laboratory, LA-UR-87-126 (1987).

[3] William Press, Brian Flannery, Saul Teukolsky, and William Vetterling, Numerical Recipes: the Art of Scientific Computing (Cambridge University Press, Cambridge, Massachusetts, 1986), p. 96.

[4] Stanley Humphries, Jr., Charged Particle Beams (John Wiley and Sons, New York, 1990), p. 412.

[5] Ibid., p. 475.

[6] Ibid., pp. 212-214.

[7] Ibid., p. 195.

[8] Ibid., pp. 441-443.

\section{Appendix A}

[A1] Stanley Humphries, Jr., Charged Particle Beams (John Wiley and Sons, New York, 1990), pp. 35-36.

[A2] Milton Abramowitz et al., Handbook of Mathematical Functions with Formulas, Graphs, and Mathematical Tables, National Bureau of Standards Applied Mathematics Series No. 55 (1965), pp. 369-370. 


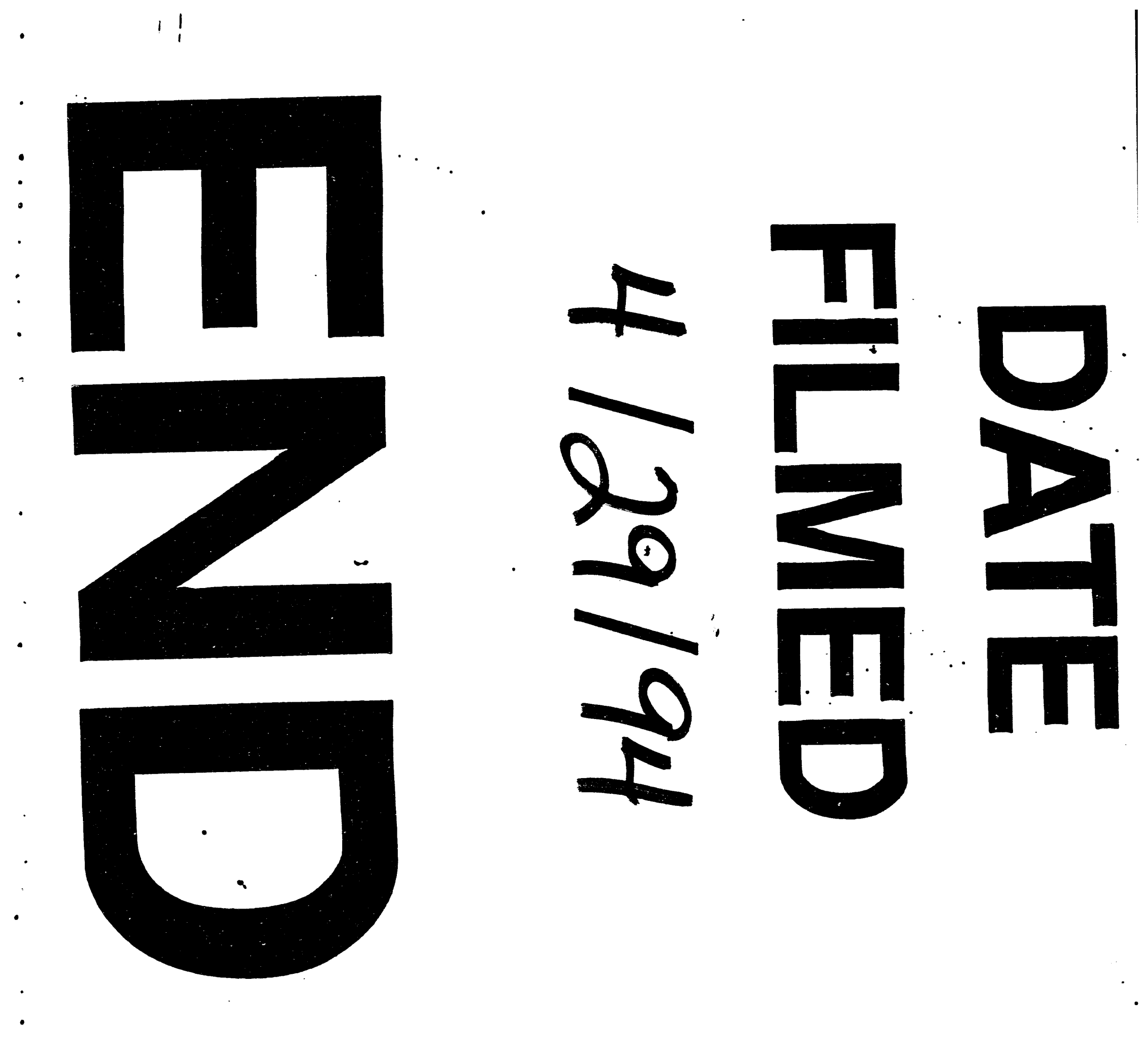


•

:

.

.

•

-

$\bullet$

$\underline{ }$

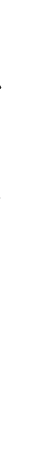

•
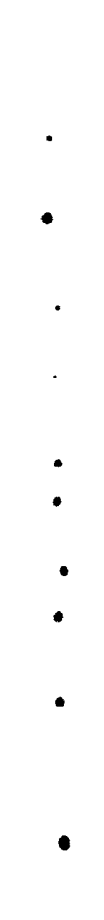

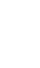

